\title{
IntechOpen
}

\section{Latest Research into \\ Quality Control}

Edited by Isin Akyar

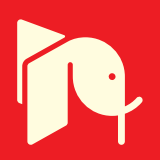





\section{LATEST RESEARCH INTO QUALITY CONTROL}

Edited by Isin Akyar 


\section{Latest Research into Quality Control}

http://dx.doi.org/10.5772/45955

Edited by Isin Akyar

\section{Contributors}

Sigrid Mennickent, Marta De Diego, Berta Schulz, Sunday Ameh, Alexandre Leal, Takuhiro Uto, Nguyen Huu Tung, Yukihiro Shoyama, Hiroyuki Tanaka, Isin Akyar, Donald R. Love, Stella Lai, Renate Marquis-Nicholson, Chuan-Ching Lan, Jonathan Skinner, Elba Lucia Cavalcanti Amorim, Valérium Castro, Joabe Melo, Tadeu José Da Silva Peixoto Sobrinho, Allan Jonathan Cernicchiaro, Stephen Inkoom, Osamu Morinaga, Huck, Saskia Van Ruth, Edoardo Capuano, Farzaneh Lotfipour, Somayeh Hallaj-Nezhadi, Bruna Chiari, Vera Isaac, Maria Gabriela José De Almeida, Marcos Antonio Corręa, Giuseppe Vermiglio, Giuseppe Acri, Barbara Testagrossa, Federica Causa, Maria Giulia Tripepi, Hoa Van Ba, Touseef Amna, Shihori Tanabe, Sun Ha Jee, Marcelo Gonzaga De Freitas Araujo, Tais Maria Bauab, Kung-Tien Liu, Jian-Hua Zhao, Lee-Chung Men, Chien-Hsin Chen, Qian Sen, Zhu Xuemin, Rodrigo Catharino, Felipe Ravagnani, Ana Faria, Daniel Saidemberg, Diogo Noin De Oliveira, Sabrina Sartor, Onur Karatuna

\section{(c) The Editor(s) and the Author(s) 2012}

The moral rights of the and the author(s) have been asserted.

All rights to the book as a whole are reserved by INTECH. The book as a whole (compilation) cannot be reproduced, distributed or used for commercial or non-commercial purposes without INTECH's written permission.

Enquiries concerning the use of the book should be directed to INTECH rights and permissions department (permissions@intechopen.com).

Violations are liable to prosecution under the governing Copyright Law.

\section{(cc) BY}

Individual chapters of this publication are distributed under the terms of the Creative Commons Attribution 3.0 Unported License which permits commercial use, distribution and reproduction of the individual chapters, provided the original author(s) and source publication are appropriately acknowledged. If so indicated, certain images may not be included under the Creative Commons license. In such cases users will need to obtain permission from the license holder to reproduce the material. More details and guidelines concerning content reuse and adaptation can be foundat http://www.intechopen.com/copyright-policy.html.

\section{Notice}

Statements and opinions expressed in the chapters are these of the individual contributors and not necessarily those of the editors or publisher. No responsibility is accepted for the accuracy of information contained in the published chapters. The publisher assumes no responsibility for any damage or injury to persons or property arising out of the use of any materials, instructions, methods or ideas contained in the book.

First published in Croatia, 2012 by INTECH d.o.o.

eBook (PDF) Published by IN TECH d.o.o.

Place and year of publication of eBook (PDF): Rijeka, 2019.

IntechOpen is the global imprint of IN TECH d.o.o.

Printed in Croatia

Legal deposit, Croatia: National and University Library in Zagreb

Additional hard and PDF copies can be obtained from orders@intechopen.com

Latest Research into Quality Control

Edited by Isin Akyar

p. cm.

ISBN 978-953-51-0868-9

eBook (PDF) ISBN 978-953-51-5141-8 


\section{We are IntechOpen, \\ the world's leading publisher of Open Access books}

Built by scientists, for scientists

\section{$4,000+$ \\ Open access books available \\ $116,000+$ \\ International authors and editors

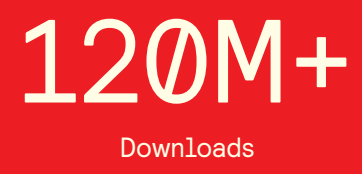

Our authors are among the

151

Countries delivered to

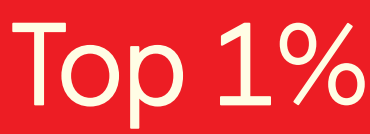

most cited scientists

Contributors from top 500 universities

$12.2 \%$

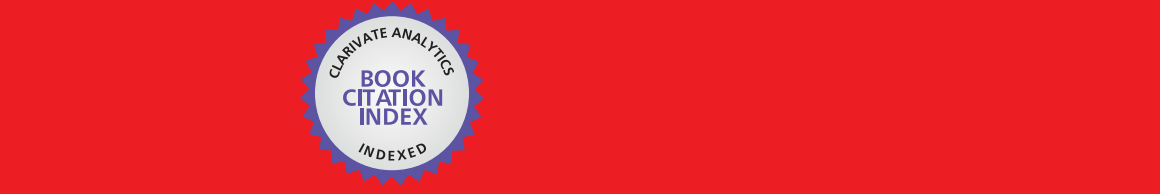

WEB OF SCIENCE ${ }^{\mathrm{M}}$

Selection of our books indexed in the Book Citation Index in Web of Science ${ }^{\mathrm{TM}}$ Core Collection (BKCI)

\section{Interested in publishing with us? \\ Contact book.department@intechopen.com}





\section{Meet the editor}

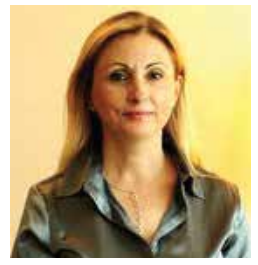

Isin Akyar graduated from School of Medicine, Cukurova University, Adana, Turkey in 1989. She finished her Medical Microbiology Specialty training in Gazi University, Ankara, Turkey in 1999. She joined the Acibadem Labmed Clinical Laboratories in Istanbul as a Specialist of Microbiology in 2004. She became Coordinator of Microbiology in 2007. Since 2004 she has had several Quality Control trainings. She works in the first accredited laboratory according to ISO 15189 for clinical laboratories in Turkey. In 2008 she joined the Department of Medical Microbiology at Acibadem University in Istanbul. In 2011 she was promoted to serve as an Assistant Professor. Her special interests are laboratory quality control, molecular microbiology, parasitology and proteomics studies. Currently she is working as both Microbiology Coordinator and Assistant Professor. She has been an Associate Editor for the Journal of Acibadem University Science of Health since 2009. 



\section{Contents}

Preface XII

Section 1 Quality Control of Herbal Medicine 1

Chapter 1 Quality Control of Rheum and Cassia Species by Immunological Methods Using Monoclonal Antibodies Against

Sennosides 3

Osamu Morinaga and Yukihiro Shoyama

Chapter 2 Applications of Anti-natural Compound Immunoaffinity Purification on Quality Control 29

Takuhiro Uto, Nguyen Huu Tung, Hiroyuki Tanaka and Yukihiro

Shoyama

Chapter 3 Standard Operating Procedures (SOP) for the Spectrophotometric Determination of Phenolic Compounds Contained in Plant Samples 47

Elba Lúcia Cavalcanti de Amorim, Valérium Thijan Nobre de Almeida de Castro, Joabe Gomes de Melo, Allan Jonathan

Chernichiarro Corrêa and Tadeu José da Silva Peixoto Sobrinho

Chapter 4 Microbial Quality of Medicinal Plant Materials 67

Marcelo Gonzaga de Freitas Araújo and Taís Maria Bauab

Chapter 5 Application of ISO 9001 Industrial Standard to Herbal Drug Regulation 83

Sunday Ameh, Florence Tarfa, Magaji Garba and Karniyus Gamaniel

Section 2 Quality Control in Food Science 109

Chapter 6 QA: Fraud Control for Foods and Other Biomaterials by Product Fingerprinting 111

Edoardo Capuano and Saskia M. van Ruth 
Chapter 7 Principle of Meat Aroma Flavors and Future Prospect 145

Hoa Van Ba, Inho Hwang, Dawoon Jeong and Amna Touseef

Chapter 8 Novel Analytical Tools for Quality Control in Food Science 177 Christian W. Huck

Section 3 Quality Control in Pharmaceutics 193

Chapter 9 Microbial Quality Concerns for Biopharmaceuticals 195 Farzaneh Lotfipour and Somayeh Hallaj-Nezhadi

Chapter 10 New Approachs in Drug Quality Control: Matrices and Chemometrics 215

Sigrid Mennickent, M. de Diego, B. Schulz, M. Vega and C. G. Godoy

Chapter 11 Quality Control of Formulated Medicines 227

Alexandre S. Leal, Maria Ângela de B. C. Menezes, Ilza Dalmázio, Fernanda P. Sepe, Tatiana C. B. Gomes, Amalia S. Santana, Luzia H. da Cunha and Radojko Jaćimović

Section 4 Quality Control in Radiology and Clinical Imaging 243

Chapter 12 Quality Assurance in Diagnostic Medical Exposures in Ghana A Medical Physicist's Perspective 245 Stephen Inkoom

Chapter 13 Quality by Design and Risk Assessment for Radiopharmaceutical Manufacturing and Clinical Imaging 255 Kung-Tien Liu, Jian-Hua Zhao, Lee-Chung Men and Chien-Hsin Chen

Chapter 14 Unified Procedures for Quality Controls in Analogue and Digital Mammography 293 Barbara Testagrossa, Giuseppe Acri, Federica Causa, Raffaele Novario, Maria Giulia Tripepi and Giuseppe Vermiglio

Section 5 Quality Control in Energy 317

Chapter 15 The Quality Management of The R\&D in High Energy Physics Detector 319

Xuemin Zhu and Sen Qian 


\section{Section 6 Quality Control in Cosmetics 335}

Chapter 16 Cosmetics' Quality Control 337

Bruna Galdorfini Chiari, Maria Gabriela José de Almeida, Marcos

Antonio Corrêa and Vera Lucia Borges Isaac

Section 7 Sops: What Are They Good For? 365

Chapter 17 Standard Operating Procedures (What Are They Good For ?) 367

Isin Akyar

Section 8 Quality Control in Clinical Laboratory Medicine 393

Chapter 18 Postmortem DNA: QC Considerations for Sequence and Dosage Analysis of Genes Implicated in Long QT Syndrome 395

Stella Lai, Renate Marquis-Nicholson, Chuan-Ching Lan, Jennifer M. Love, Elaine Doherty, Jonathan R. Skinner and Donald R. Love

Chapter 19 Quality Assurance in Antimicrobial Susceptibility Testing 413 Onur Karatuna

Chapter 20 The Investigation of Gene Regulation and Variation in Human Cancers and Other Diseases $\mathbf{4 3 5}$

Shihori Tanabe and Sun Ha Jee

Chapter 21 Quality Control Considerations for Fluorescence In Situ Hybridisation of Paraffin-Embedded Pathology Specimens in a Diagnostic Laboratory Environment 469

Lisa Duffy, Liangtao Zhang, Donald R. Love and Alice M. George

Chapter 22 Quality Control of Biomarkers: From the Samples to Data Interpretation 491

F. G. Ravagnani, D. M. Saidemberg, A. L. C. Faria, S. B. Sartor, D. N.

Oliveira and R. R. Catharino 



\section{Preface}

Quality control has an emerging importance in every field of life. Quality control is a process that is used to guarantee a certain level of quality in a product or service. It might include whatever actions a business deems necessary to provide for the control and verification of certain characteristics of a product or service. Most often, it involves thoroughly examining and testing the quality of products or the results of services. The basic goal of this process is to ensure that the products or services that are provided meet specific requirements and characteristics, such as being dependable, satisfactory, safe and fiscally sound. There are some standards which guarantee quality control. In those standards you've got to document everything and track it. You should write what you do, do what you write. Groups that engage in quality control typically have a team of workers who focus on testing a certain number of products or observing services being done. The goal of the quality control team is to identify products or services that do not meet a company's specified standards of quality. If a problem is identified, the job of a quality control team or professional might involve stopping production or service until the problem has been corrected. Depending on the particular service or product as well as the type of problem identified, production or services might not cease entirely. There should be well organized procedures and management for ensuring quality control.

With the improvement of technology everyday we meet new and complicated devices and methods in different fields. Quality control should be performed in all of those new techniques.

In this book "Latest Research Into Quality Control" our aim was to collect information about quality control in many different fields such as:

Quality Control in general: SOPs

Quality Control in Clinical Laboratory Medicine

Quality Control of Herbal Medicine

Quality Control in Food Science

Quality Control in Pharmaceutics

Quality Control in Radiology and Clinical Imaging

Quality Control in Energy

Quality Control in Cosmetics 
The aim of this book is to share useful and practical knowledge about quality control in several fields with the people who want to improve their knowledge.

Dr Isin Akyar

Acibadem University, School of Medicine, Department of Medical Microbiology, Istanbul, Turkey 
Section 1

Quality Control of Herbal Medicine 

Chapter 1

\title{
Quality Control of Rheum and Cassia Species by Immunological Methods Using Monoclonal Antibodies Against Sennosides
}

\author{
Osamu Morinaga and Yukihiro Shoyama \\ Additional information is available at the end of the chapter \\ http://dx.doi.org/10.5772/51272
}

\section{Introduction}

Recently, medical usage of Japanese traditional medicine has been expanded by reaching aging society and increasing various chronic diseases. Therefore, the demand of crude drugs prescribed for Japanese traditional medicine has been increased. However, over $90 \%$ of crude drugs are imported in our country, and those over $70 \%$ are supplied by the collection of wild species. It is well known that the natural resources bring the difficulty of quality control depending on collection season, cultivation place, a variety of species and so on. The other problem, shortage of crude drug comes up. For these general environment, micropropagation and clonal propagation systems using tissue and cell culture were investigated in this laboratry.

Sennoside A (SA) and B (SB) have the strong catharsis activity and contained in rhubarb and senna (Figure 1) [1]. The concentration of sennosides in rhubarb and senna is variously dependent on the genetic heterogeneity of species, differences in soil condition and climate influence. Sennosides are metabolized by intestinal bacteria to rheinanthrone which acts in the intestines as a direct purgatives [2,3] and functions as similar to a natural prodrug (Figure 2). Despite the rising availability of a number of synthetic cathartics, sennoside- containing prescriptions are still among the most widely used today, and their importance is increasing.

Rhubarb, the rhizome and root of Rheum spp. (Polygonaceae), is an important drug in traditional Japanese herbal medicine as well as in western medicine since ancient times. It was already recorded in Chinese Materia Medica 2000 years ago. It is used in many traditional Japanese herbal medicines prescribed with other herbal medicines for the syndrome of stasis of blood, as an anti-inflammatory, sedative agent and as a stomachic. Furthermore, it is widely 
used as cathartics in Japan. The main purgative principles of rhubarb have proved to be sennosides [1], identical with those isolated from senna leaves, and rheinosides, which were also isolated as purgatives of rhubarb, together with various kinds of phenolics, like tannins, stilbenes, naphthalenes and lindleyin. The quality of rhubarb is severely regulated by Japanese Pharmacopeia as rhubarb contains SA of over $0.25 \%$ dry weight in root [4].

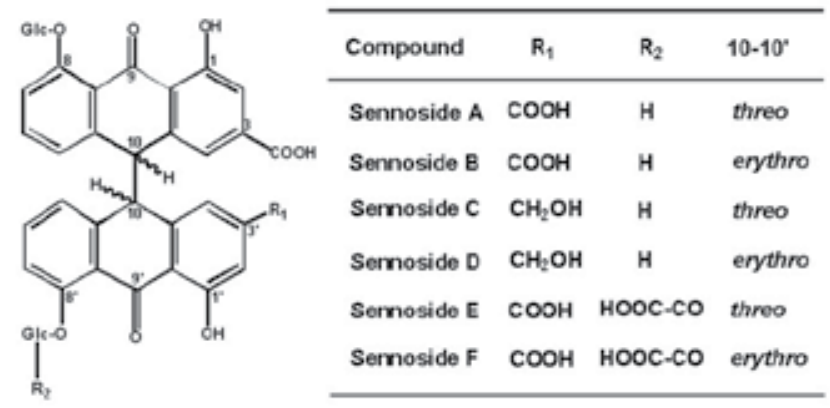

Figure 1. Structures of sennosides

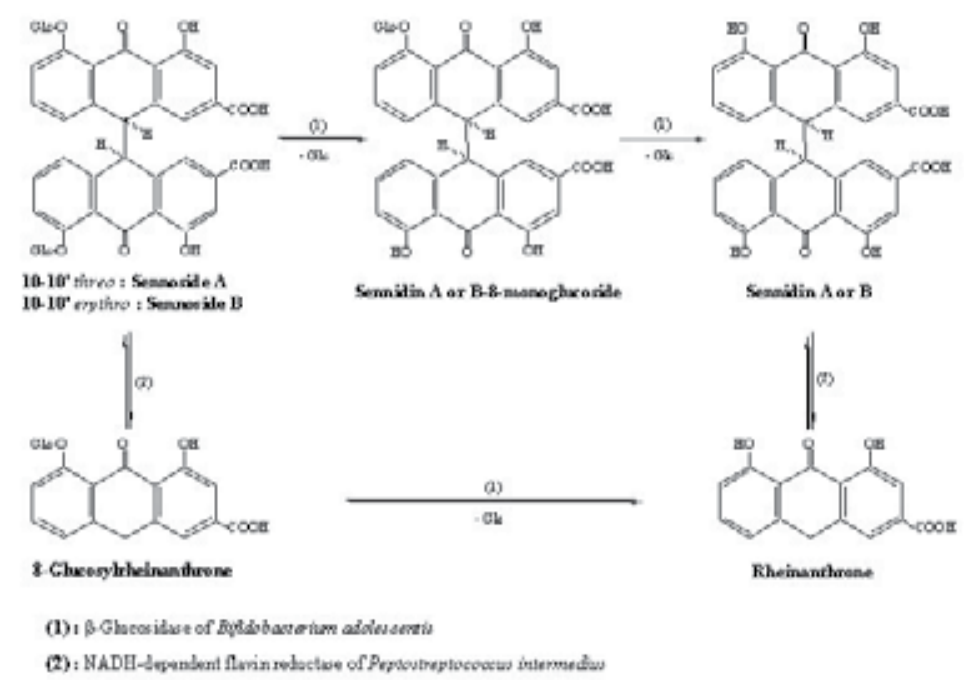

Figure 2. Metabolic pathways of sennosides by intestinal bacteria.

Senna, the leaf and pod of Cassia spp. (Leguminosae), is one of the most popular in herbal remedies and in health food industry. It has been widely used in cathartics for the relief of constipation prescribed with other health teas and dieter's teas in Japan, and often used as natural dietary supplements for enhancement of bloodflow and metabolism in USA, Europe and Australia. These pharmaceutical properties are due to sennosides, which are contained 
in Cassia acutifolia Delile and Cassia angustifolia Vahl. C. angustifolia listed in Japanese Pharmacopeia, and the quality is severely regulated as senna contains total sennosides (SA and SB) of over $1.0 \%$ dry weight in leaf [4].

In the breeding research on the plant, a lot of stages are required as follows : dedifferentiation, extension of mutation by the mutagen, redifferentiation, analysis of the redifferentiated plant, mass propagation of the higher yielding plant and transplanting to soil. Therefore, it is very important to study a large number of plant samples in the phytochemical field and a small sample size in vitro for the breeding of Rheum and Cassia species yielding high concentration of sennosides. Many analytical approaches have been investigated for the determination of sennosides in plant extracts. Among these methods, the use of high-performance liquid chromatography (HPLC) appears most frequently and widely today. However, when the assay of very low concentration of sennosides in the regenerated plantlets is needed, the HPLC method is not appropriate and efficient.

Recently, the immunological assay method is widely developed for the purpose of analysis for a small amount of constituent. In general immunological methodologies in particular enzyme-linked immunosorbent assay (ELISA) have promoted the development of higher sensitive assay system.

On the one hand, monoclonal antibodies (MAbs) have many potential uses in addition to immunological methods in plant sciences. MAbs are superior to polyclonal antibodies (PAbs) in the antigenic specificity and stability. Therefore, immunoassay using MAbs against pharmacologically active compound having small molecular weight has become an important tool for the studies on receptor binding analysis, enzyme assay and quantitative and/or qualitative analytical techniques in plants owing to its specific affinity, and possesses an extremely high possibility in the phytochemical analysis. Up to now, immunological approach for assaying quantities of sennosides in C. angustifolia using PAb against SB has been investigated by Atzorn et al [5]. However, since no success with MAbs against SA and SB has been reported, objectives of this work are shown as following.

1. Production of MAb against SA, its characterization and use for ELISA.

2. Production of MAbs against SB, their characterization and use for ELISA.

3. Establishments of a new eastern blotting, double staining and immunohistochemical staining using anti-SA and SB MAbs.

\section{Production of MAb against SA, its characterization and use for ELISA}

\subsection{Preface}

In the immunologically analytical methodology, there are two measuring methods using the antiserum (polyclonal antibody; $\mathrm{PAb}$ ) and MAb in general. PAb is a heterogeneous mixture of antibody molecules arising from a variety of constantly evolving B lymphocytes. Therefore, $\mathrm{PAb}$ can often show high affinity because different antibody populations react with the 
variety of epitopes that characterize the antigen. On the other hand, there are some problems of PAb that the extensive cross-reactivity occurs between the antibody and the multiple antigens which have the same antigenic determinant, and it is impossible to supply for identical antibody permanently. In the meantime, MAb is produced from a single B lymphocyte and can react with one antigenic determinant of the specific antigen. Besides MAb has identical specificity and affinity. There are some advantages that the complete purity of the immunized antigen is not required and the hybridoma cells can be preserved as freeze stock, and it is possible to get MAb depending on necessary respond.

There are several formats for ELISA like direct ELISA, competitive ELISA, sandwich ELISA and competitive ELISA according to the immune complexes formed during manipulation. Analysis of low molecular weight compound by immunoassay is still limited to competitive format.

Quality control of the Japanese herbal medicine is necessary because it is believed that approximately $70 \%$ of these crude drugs prescribed are collected from natural resource. Furthermore, since MAbs become necessary for the assay of concentrations of active constituents in our on-going plant biotechnological projects, we have already produced MAbs against natural compounds such as forskolin [6], solamargine [7], opium alkaloids [8], marihuana compounds [9], glycyrrhizin [10], crocin [11], ginsenoside Rb1 [12] and Rg1 [13], and developed individual competitive ELISAs. An immunological approach for assaying quantities of sennosides using a PAbs has been investigated by Atzorn et al.[5]. However, since no result of MAb related to sennosides has been reported yet, anti-SA MAb was produced as described [14].

\subsection{Experimental}

\subsubsection{Chemicals and immunochemicals}

SA was purchased from Wako Pure Chemical Ind., Ltd. (Osaka, Japan). 1-Ethyl-3-(3'-dimethylaminopropyl)-carbodiimide $\mathrm{HCl}$ (EDC) was purchased from Nacalai Tesque Inc. (Kyoto, Japan). BSA and HSA were provided by Pierce (Rockford, IL, USA). Peroxidase-labeled antimouse IgG was provided by Organon Teknika Cappel Products (West Chester, PA, USA). Enriched RPMI1640-Dulbecco's-Ham's F12 (eRDF) medium and RD-1 additives (containing $9 \mu \mathrm{g} / \mathrm{mL}$ insulin, $20 \mu \mathrm{g} / \mathrm{mL}$ transferrin, $20 \mu \mathrm{M}$ ethanolamine, $25 \mu \mathrm{M}$ sodium selenite) were purchased from Kyokuto Pharmaceutical Industrial Co., Ltd. (Tokyo, Japan). Hypoxanthineaminopterin-thymidine (HAT) additives were obtained from Sigma Chemical Company (St. Louis, MO, USA). Fetal calf serum (FCS) was purchased from Cambrex Corporation (Walkersville, MA, USA). All other chemicals were standard commercial products of analytical grade. Samples of various rhubarb roots were purchased from the Tochimototenkaido Corporation (Osaka, Japan). 


\subsubsection{Extraction of various rhubarb samples}

Dried samples (30 mg) of various rhubarb roots were powdered, and then extracted five times with $\mathrm{MeOH}$ containing $0.1 \%(\mathrm{w} / \mathrm{v}) \mathrm{NH}_{4} \mathrm{OH}(0.5 \mathrm{~mL})$ with sonication, filtered using a Cosmonice Filter W $(0.45 \mu \mathrm{m}$ Filter Unit, Nacalai Tesque Inc., Kyoto, Japan), and the combined extracts were diluted with $10 \mathrm{mM} \mathrm{NaHCO}_{3}$ to prepare a solution suitable for the ELISA.

\subsubsection{Synthesis of antigen conjugates}

To SA (6 mg) dissolved in $1 \mathrm{~mL}$ of tetrahydrofuran-20 mM phosphate buffer of $\mathrm{pH} 5.5$ (7:3), $0.3 \mathrm{~mL}$ of $20 \mathrm{mM}$ phosphate buffer ( $\mathrm{pH} 5.5$ ) containing $6 \mathrm{mg}$ of EDC was added. Then, 0.3 $\mathrm{mL}$ of $20 \mathrm{mM}$ phosphate buffer ( $\mathrm{pH}$ 5.5) containing $6 \mathrm{mg}$ of BSA was added, with stirring at room temperature for $14 \mathrm{hr}$. The reaction mixture was dialyzed five times against $\mathrm{H}_{2} \mathrm{O}$, and then lyophilized to give $5.8 \mathrm{mg}$ of SA conjugate (SA-BSA). SA-HSA conjugate was also synthesized in the same manner.

\subsubsection{Determination of hapten density in $S A$-carrier protein conjugate by matrix-assisted laser desorption/ionization (MALDI)-time of flight (TOF) mass spectrometry}

The hapten number in the SA-carrier protein conjugate was determined by MALDI-TOF mass spectrometry as previously described [15]. A small amount (1-10 pmol) of antigen conjugate was mixed with a $10^{3}$-fold molar excess of sinapinic acid in an aqueous solution containing $0.15 \%$ trifluoroacetic acid (TFA). The mixture was subjected to a JEOL Mass Spectrometers (JMS) time-of-flight (TOF) mass monitor (model Voyager Elite, PerSeptive Biosystems Inc., Framingham, MA, USA) and irradiated with a $\mathrm{N}_{2}$ laser (337 nm, $150 \mathrm{~ns}$ pulse). The ions formed by each pulse were accelerated by a $20 \mathrm{kV}$ potential into a $2.0 \mathrm{~m}$ evacuated tube and detected using a compatible computer as previously reported [15].

\subsubsection{Competitive ELISA for $S A$}

SA-HSA (five molecules of SA per molecule of HSA) $(100 \mu \mathrm{L}, 1 \mu \mathrm{g} / \mathrm{mL})$ dissolved in $50 \mathrm{mM}$ carbonate buffer ( $\mathrm{pH}$ 9.6) was adsorbed to the wells of a 96-well immunoplate then treated with $300 \mu \mathrm{L}$ S-PBS for $1 \mathrm{hr}$ to reduce non-specific adsorption. Fifty $\mu \mathrm{L}$ of various concentrations of SA or samples dissolved in $10 \mathrm{mM} \mathrm{NaHCO}_{3}$ solution were incubated with $50 \mu \mathrm{L}$ of MAb solution $(0.218 \mu \mathrm{g} / \mathrm{mL})$ for $1 \mathrm{hr}$. The plate was washed three times with T-PBS, and then incubated with $100 \mu \mathrm{L}$ of a 1:1000 dilution of POD-labeled anti-mouse IgG for $1 \mathrm{hr}$. After washing the plate three times with T-PBS, $100 \mu \mathrm{L}$ of substrate solution [0.1 M citrate buffer $\left(\mathrm{pH}\right.$ 4) containing $0.003 \% \mathrm{H}_{2} \mathrm{O}_{2}$ and $0.3 \mathrm{mg} / \mathrm{mL}$ of ABTS] was added to each well and incubated for $15 \mathrm{~min}$. The absorbance was measured by a micro plate reader at $405 \mathrm{~nm}$ and $490 \mathrm{~nm}$.

The cross-reactivities (CR) of sennosides and related compounds were determined as following.

$\mathrm{CR}(\%)=\frac{\mu g / \mathrm{mL} \text { of SA yielding } A / A_{0}=50 \%}{\mu g / \mathrm{mL} \text { of compound under investigation yielding } A / A_{0}=50 \%} \times 100$ 
where $A$ is the absorbance in the presence of the test compound and $A_{0}$ is the absorbance in the absence of the test compound.

\subsection{Results and discussion}

\subsubsection{Direct determination of SA-carrier protein conjugate by MALDI-TOF mass spectrometry}

In general, the low molecular weight compounds (hapten) like plant secondary metabolite have no immunogenicity. Therefore, it should be conjugated with some high molecular compound like protein resulting in immunogenic. The specificity of immunoassay method is dependent on the site of linkage between hapten and carrier protein moiety, and enumeration of hapten in immunogen conjugate. SA-BSA and SA-HSA conjugates were synthesized as immunogen and the immobilization antigen for ELISA, respectively. Figure 3 shows the typical synthetic pathway of SA-BSA conjugate. The commonly used methods to link carboxyl group and amino group in a hapten or carrier involve activation by carbodiimides, isobutylchloroformate or carbonyldiimidazole. Carbodiimides react with carboxyl groups to form an unstable $\mathrm{O}$-acetylisourea intermediate, which reacts with amines to form amide bonds. EDC can be used commonly as a carbodiimide. In this case, carrier protein combined directly to antigen as indicated in Figure 3.

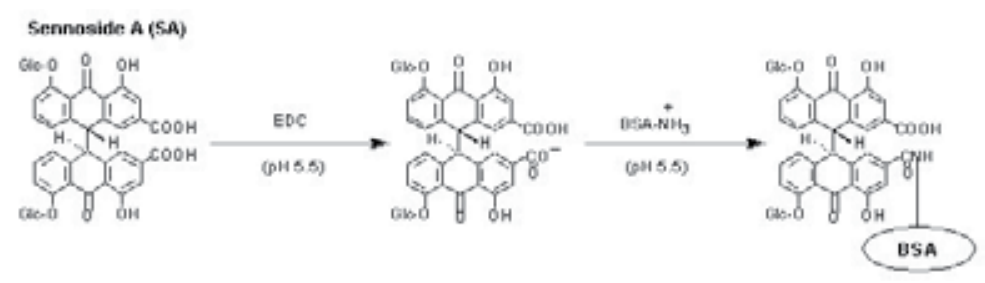

Figure 3. Typical synthetic pathway of SA-BSA. Carboxyl group of SA was activated by EDC and subsequently combined to amino residues of lysine and/or arginine on the protein to form amide bond.

Figure 4 shows the MALDI-TOF mass spectrum of the antigen, SA-BSA conjugate. A broad peak coinciding with the conjugate of SA and BSA appeared from $m / z 68,500$ to 73,500 centering at around $m / z 70,600$. Using experimental results and a molecular weight of 66,433 for BSA, the calculated values of SA component (MW 862) are 4,218 resulting in the range of two to eight molecules of SA (five on average) conjugated with BSA. In general eight to twenty five molecules of hapten conjugated with carrier protein in the conjugate were sufficient for immunization. Therefore, the hapten number was estimated to be sufficient for immunization because an antigen conjugate having a similar hapten number was sufficient for immunization in a previous study [10]. The number of SA contained in the SA-HSA conjugate was also determined to be around five molecules by its spectrum. 


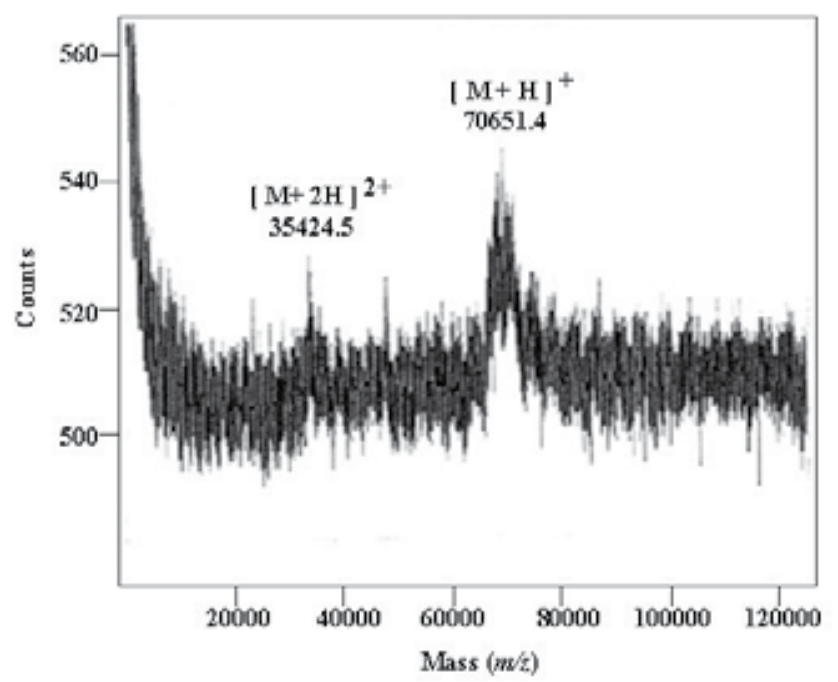

Figure 4. Direct determination of SA-BSA by MALDI-TOF MS. $[\mathrm{M}+\mathrm{H}]+,[\mathrm{M}+2 \mathrm{H}]^{2}+$ are single and double protonated molecules of SA-BSA, respectively.

\subsubsection{Production and characteristic of MAb against $S A$}

After the cell fusion and HAT selection, hybridoma producing MAb reactive to SA was obtained, and classfied into $\operatorname{IgG}_{1}$ which had $k$ light chains. Refined MAb was confirmed to be IgG compared to the MALDI-TOF MS measurement. The molecular weight of MAb was 151,396 calculated [16]. The reactivity of IgG type MAb 6G8 was tested for varying the antibody concentration and for performing a dilution curve in direct ELISA. The antibody concentration of $0.218 \mu \mathrm{g} / \mathrm{mL}$ showed the absorbance at 0.8 in direct ELISA, therefore it was selected for the competitive ELISA.

\subsubsection{Assay sensitivity and assay specificity}

The free MAb 6G8, following incubation with competing antigen, was bound to the polystyrene microtitre plates precoated with SA-HSA. Under these conditions, the full measuring range of the assay extended from 20 to $200 \mathrm{ng} / \mathrm{mL}$ as indicated in Figure 5.

SA is a unique anthraquinone having individual double of carboxylic acid-, hydroxyl-, carbonyl- and O-glucosyl-groups at C-3, C-1, C-9 and C-8 positions in a molecule, respectively. Moreover, SA possessed a threo- configuration between C-10 and C-10' positions as indicated in Figure 6. Therefore, a MAb should detect all these functions, and also the stereochemical recognition is needed for this complicated compound. Since the newly established ELISA against SA is expected to be applied for phytochemical investigations involving crude plant extracts, the assay specificity was checked by determining the cross-reactivities of MAb with various related compounds. The cross-reactivities of the MAb was examined by competitive ELISA. 


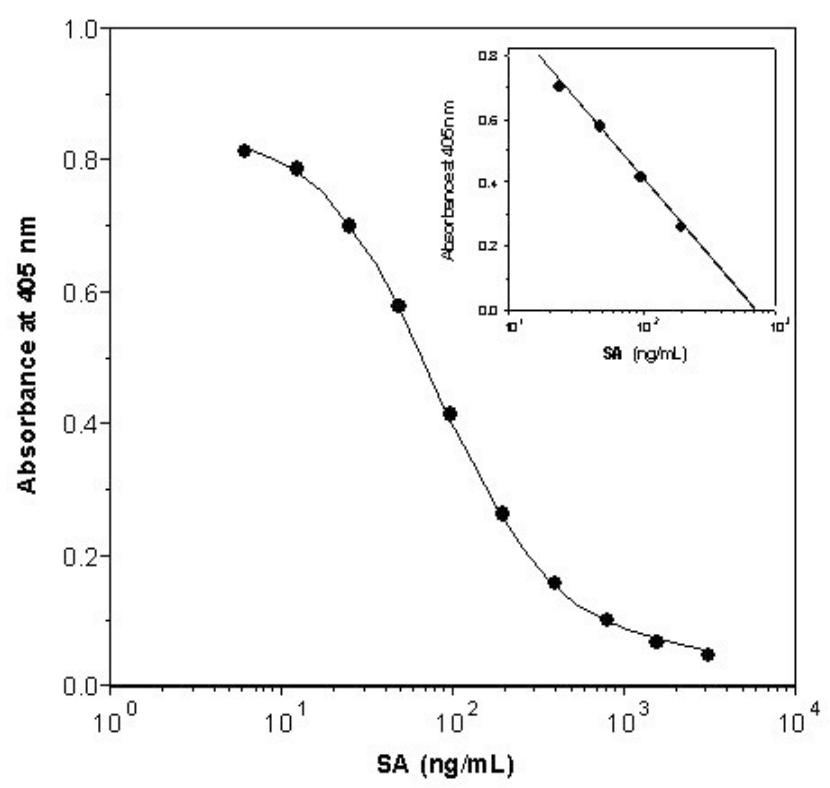

Figure 5. Calibration curve for SA.<smiles>CC1c2cc(C(=O)O)cc(O)c2C(=O)c2c(O)cccc2-c2c(O)cc(C(=O)O)cc21</smiles>

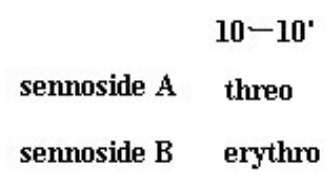<smiles>[R]c1cc(O)c2c(c1)C(=O)c1c(O)cc([R1])cc1C2=O</smiles>

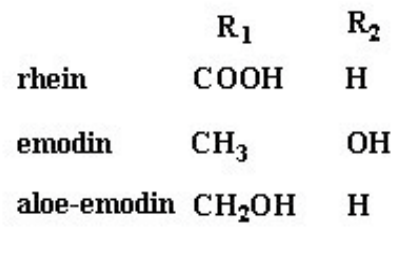

Figure 6. Chemical structures of SA, SB and its structurally related compounds.

Table 1 indicates the cross-reactivities of anti-SA MAb against related anthraquinone, anthrone and phenol carboxylic acid. MAb 6G8 cross-reacted with rhein and SB weakly; 0.28 and $0.35 \%$, respectively. However, the other related anthraquinone and anthrone did not 
have appreciable cross-reactivities. From these results it is suggested that a basal structure of rhein and sugar moiety caused immunization. In addition the most important property of MAb 6G8 is an ability of stereochemical recognition because the differences of structure between SA and SB are only the stereochemical configuration at the C-10 and C-10' positions. Therefore, it is suggested that threo-configurational structure of bisanthrone is indispensable as an immunodominant molecule for reactivity of MAb 6G8.

\begin{tabular}{ll}
\hline Compound & Cross-reactivities (\%) \\
\hline Anthraquinone and anthrone & 100 \\
\hline sennoside A & 0.28 \\
\hline sennoside B & 0.35 \\
\hline rhein & $<0.04$ \\
\hline emodin & $<0.04$ \\
\hline aloe-emodin & $<0.04$ \\
\hline barbaloin & $<0.04$ \\
\hline 1,4-dihydroxy-anthraquinone &
\end{tabular}

\section{Stilbene}

rhaponticin $<0.04$

\begin{tabular}{ll}
\hline Phenol carboxylic acid & $<0.04$ \\
\hline gallic acid & $<0.04$ \\
\hline vanillic acid & $<0.04$ \\
\hline caffeic acid & $<0.04$
\end{tabular}

Table 1. Cross-reactivities (\%) of MAb-6G8 against sennosides and other compounds.

2.3.4. Correlation of results of $S A$ determination in crude extracts of rhubarb roots between HPLC and ELISA using MAb 6 G8

The ELISA was utilized to measure the concentrations of SA in various rhubarb (Table 2). Oshio and Kawamura determined sennoside concentrations in various crude rhubarbs by HPLC [17]. More recently Seto et al. reported the comparative concentrations of sennosides determined by HPLC in various commercial rhubarbs [18]. They required a lager sample size compared to the newly established ELISA due to some pretreatments because the crude materials contained several kinds of phenolics such as tannins, stilbens, naphthalen derivatives and lindleyin as previously indicated.

Table 2 shows the SA concentrations in various rhubarbs. Shinshu Daio bred by crossing $R$. palmatum and $R$. coreanum in order to increase the concentration of SA in Japan, contained the highest SA; $13.69 \pm 0.69 \mu \mathrm{g} / \mathrm{mg}$ dry wt. Ga-wo which was estimated to be high grade, con- 
tained $6.62 \pm 0.42 \mu \mathrm{g} / \mathrm{mg}$ dry wt. The other three species showed almost the same concentrations of SA, around $3.3 \mu \mathrm{g} / \mathrm{mg}$ dry wt. These results are in good agreement with the previous reports [18]. The correlation between results from ELISA and HPLC is reasonable except for Kinmon Daio. The concentration analyzed by HPLC was very low compared to the others. The reason is still obscure although individual peaks separated by HPLC were analyzed by ELISA.

\begin{tabular}{|c|c|c|}
\hline \multirow[t]{2}{*}{ Sample } & \multicolumn{2}{|c|}{ Concentration ( $\mu \mathrm{g} / \mathrm{mg}$ dry wt. powder) } \\
\hline & ELISA & HPLC \\
\hline Shinshu Daio & $13.69 \pm 0.69$ & $12.28 \pm 0.41$ \\
\hline Ga-wo & $6.62 \pm 0.42$ & $6.93 \pm 0.02$ \\
\hline Kinmon Daio & $3.34 \pm 0.02$ & $0.85 \pm 0.04$ \\
\hline Itto-Ga-wo (powder) & $3.27 \pm 0.20$ & $3.69 \pm 0.32$ \\
\hline Itto-Ga-wo (refuse) & $3.43 \pm 0.16$ & $3.69 \pm 0.28$ \\
\hline
\end{tabular}

Table 2. SA concentrations in various rhubarb samples. Data are the means of triplicate assays.

\section{Production of MAbs against SB, their characterization and use for ELISA}

\subsection{Preface}

SB is a very important natural bioactive component of rhubarb and senna as well as SA. Total sennoside (SA and SB) concentrations are important, when rhubarb and senna are used as a raw material of medical supply and traditional Japanese herbal medicine for the purgative effect.

A number of methods for the quantification of SB have been published, most of which have been performed by HPLC [17]. Immunological approaches for assaying quantities of sennosides and SA using PAb and MAb have been investigated by Atzorn et al. [5] and by us [14], respectively. However, no success with MAb against SB has been reported. In here, production of anti-SB MAb and the competitive ELISA using anti-SA and SB MAbs for the direct determination of SA and SB in various samples are described [19].

\subsection{Experimental}

\subsubsection{Plant materials}

Samples of various rhubarb roots were purchased from the Tochimototenkaido Corporation (Osaka, Japan). Samples of leaves of Cassia plants were collected in Thailand. Traditional Japanese prescriptions were procured from Tsumura \& Co. (Tokyo, Japan). Dietary supplements (health teas and dieter's teas) were purchased from drug and department stores. 


\subsubsection{Sample preparation}

Dried samples $(30 \mathrm{mg}$ ) of various rhubarb roots, Cassia plant leaves, traditional Japanese prescriptions and dietary supplements were powdered, and then extracted five times with $\mathrm{MeOH}$ containing $0.1 \%(\mathrm{w} / \mathrm{v}) \mathrm{NH}_{4} \mathrm{OH}(0.5 \mathrm{~mL})$ with sonication, filtered using a Cosmonice Filter W (0.45 $\mu \mathrm{m}$ Filter Unit, Nacalai Tesque Inc., Kyoto, Japan), and the combined extracts were diluted with $10 \mathrm{mM} \mathrm{NaHCO}_{3}$ to prepare a solution suitable for the ELISA.

\subsubsection{Synthesisi of antigen conjugates}

To SB (6 mg) dissolved in $1 \mathrm{~mL}$ of tetrahydrofuran-20 mM phosphate buffer of $\mathrm{pH} 5.5$ (7:3), $0.3 \mathrm{~mL}$ of $20 \mathrm{mM}$ phosphate buffer ( $\mathrm{pH} 5.5$ ) containing $6 \mathrm{mg}$ of EDC was added. Then, 0.3 $\mathrm{mL}$ of $20 \mathrm{mM}$ phosphate buffer ( $\mathrm{pH}$ 5.5) containing $6 \mathrm{mg}$ of BSA was added, with stirring at room temperature for $14 \mathrm{hr}$. The reaction mixture was dialyzed five times against $\mathrm{H}_{2} \mathrm{O}$, and then lyophilized to give $5.5 \mathrm{mg}$ of SB-BSA conjugate. SB-HSA conjugate was also synthesized in the same manner.

\subsubsection{Determination of hapten density in SB-carrier protein conjugate by MALDI-TOF mass spectrometry}

The hapten number in the SB-carrier protein conjugate was determined by MALDI-TOF mass spectrometry as previously described [15].

\subsubsection{Competitive ELISA for $S B$}

SB-HSA (four molecules of SB per molecule of HSA) $(100 \mu \mathrm{L}, 1 \mu \mathrm{g} / \mathrm{mL})$ dissolved in $50 \mathrm{mM}$ carbonate buffer ( $\mathrm{pH}$ 9.6) was adsorbed to the wells of a 96-well immunoplate then treated with $300 \mu \mathrm{L}$ S-PBS for $1 \mathrm{hr}$ to reduce non-specific adsorption. Fifty $\mu \mathrm{L}$ of various concentrations of $\mathrm{SB}$ or samples dissolved in $10 \mathrm{mM} \mathrm{NaHCO}_{3}$ solution were incubated with $50 \mu \mathrm{L}$ of MAb solution $(0.121 \mu \mathrm{g} / \mathrm{mL})$ for $1 \mathrm{hr}$. The plate was washed three times with T-PBS, and then incubated with $100 \mu \mathrm{L}$ of a 1:1000 dilution of POD-labeled anti-mouse IgG for $1 \mathrm{hr}$. After washing the plate three times with T-PBS, $100 \mu \mathrm{L}$ of substrate solution [0.1 M citrate buffer ( $\mathrm{pH}$ 4) containing $0.003 \% \mathrm{H}_{2} \mathrm{O}_{2}$ and $0.3 \mathrm{mg} / \mathrm{mL}$ of ABTS] was added to each well and incubated for $15 \mathrm{~min}$. The absorbance was measured by a micro plate reader at $405 \mathrm{~nm}$ and $490 \mathrm{~nm}$.

\subsection{Results and discussion}

\subsubsection{Direct determination of SB-carrier protein conjugate by MALDI-TOF mass spectrometry}

It is well known that hapten number in an antigen conjugate is important for immunization against low molecular weight compounds. Figure 7 shows the MALDI-TOF mass spectrum of the antigen, SB-BSA conjugate. A broad peak coinciding with the conjugate of SB and BSA appeared from $m / z 67,300$ to 70,700 centering at around $m / z 68,900$. Using experimental results and a molecular weight of 66,433 for BSA, the calculated values of SB component 
(MW 862) are 2,500 resulting in the range of one to five molecules of SB (three on average) conjugated with BSA. This conjugate, although having a relatively low hapten number, proved sufficiently immunogenic in agreement with our previous results [10]. The number of SB contained in the SB-HSA conjugate was also determined to be around four molecules by its spectrum.

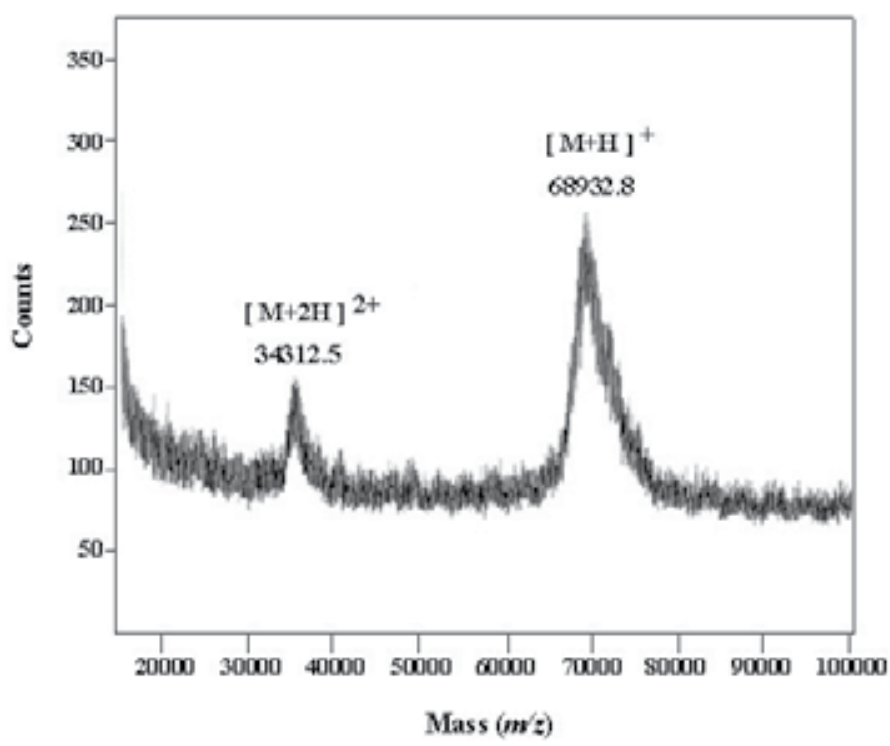

Figure 7. Direct determination of SB-BSA by MALDI-TOF MS.

\subsubsection{Production and characteristics of Mabs against $S B$}

The immunized BALB/c mice yielded splenocytes which were fused with P3-X63-Ag8-653 myeloma cells by the routinely established procedure in this laboratory [6]. Hybridoma producing MAbs reactive to SB were obtained, and classified as IgG1 (5G6, 7H12) and IgG2b (5C7) which had $k$ light chains. The reactivity of IgG type MAb 7H12 was tested by varying the antibody concentration and by performing a dilution curve in direct ELISA. The antibody concentration $(0.121 \mu \mathrm{g} / \mathrm{mL})$ at which the absorbance was about 1.0 in direct ELISA was selected for competitive ELISA.

\subsubsection{Assay sensitivity and assay specificity}

The free MAb 7H12 following competition was bound to the polystyrene microtitre plates precoated with SB-HSA. Under these conditions, the full measuring range of the assay extends from $0.5 \mathrm{ng} / \mathrm{mL}$ to $15 \mathrm{ng} / \mathrm{mL}$ as indicated in Figure 8 and the ELISA using a MAb 7H12 is more sensitive than those using MAb 5C7 and 5G6. 


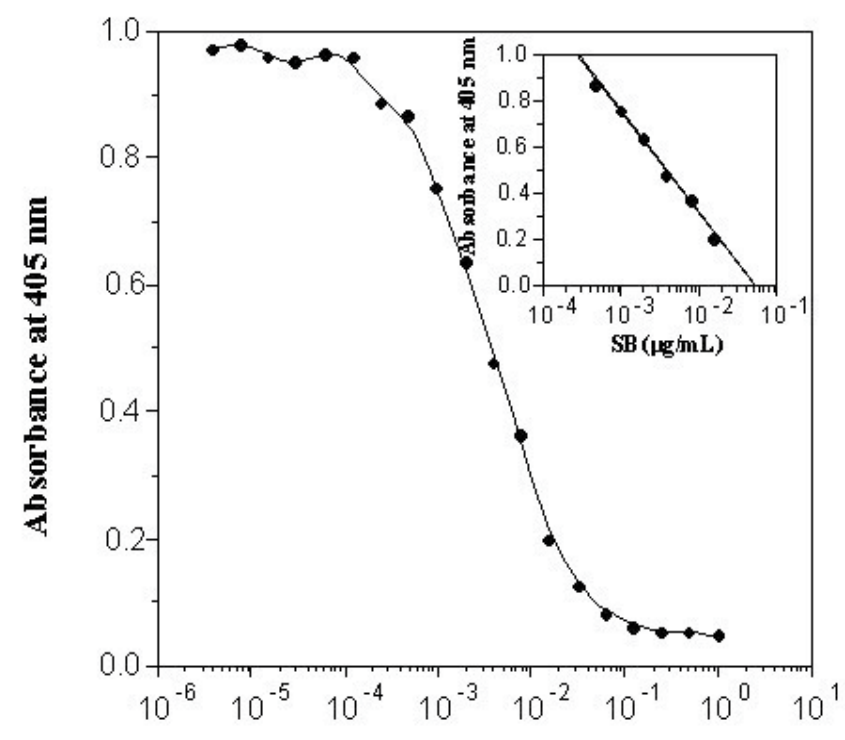

$\mathrm{SB}(\mu \mathrm{g} / \mathbf{m L})$

Figure 8. Calibration curve for SB.

$\mathrm{SB}$ is a unique anthraquinone having individual double-carboxylic acid-, hydroxyl-, carbonyl- and O-glucosyl-groups at C-3, C-1, C-9 and C-8 positions in the molecule, respectively. Moreover, SB possesses an erythro-configuration between C-10 and C-10' positions. Therefore, MAbs should distinguish all these functional groups, and also recognize the stereochemistry of this complicated compound. Since the newly established ELISA against SB is expected to be used for phytochemical investigations involving crude plant extracts, the assay specificity was checked by determining the cross-reactivities of the MAbs with various related compounds. The cross-reactivities of MAbs were examined by the competitive ELISA. Table 3 indicates the cross-reactivities of anti-SB MAbs against related anthraquinone, anthrone and phenol carboxylic acid. MAb 7H12 has weak cross-reactivities with SA $(2.45 \%)$ and rhein $(0.012 \%)$. However, the other related anthraquinone and anthrone did not have appreciable cross-reactivities. From these results it is suggested that the epitope consists of a basal structure of rhein and sugar moiety. In addition the most important property of MAb 7H12 is its ability to distinguish between SB and SA, which differ only in the stereochemical configuration at the C-10 and C-10' positions. Therefore, it is suggested that erythro-configurational structure of bisanthrone is indispensable as an immunodominant molecule for the reactivity of MAb 7H12. So the ELISA using a MAb 7H12 possesses apparently high sensitivity and specificity for SB. Because we have also prepared an anti-SA MAb having a weak cross-reactivity with SB $(0.28 \%)$ as already discussed, these two MAbs make it possible to investigate stereochemical recognition precisely. 


\begin{tabular}{llll}
\hline \multirow{2}{*}{ Compound } & \multicolumn{2}{l}{ Cross-reactivities (\%) } \\
\cline { 2 - 4 } Anthraquinone and anthrone & $\mathbf{7 H 1 2}$ & $\mathbf{5 G 6}$ & $\mathbf{5 C 7}$ \\
\hline sennoside B & 100 & 100 & 100 \\
\hline sennoside A & 2.45 & 2.30 & 8.53 \\
\hline rhein & 0.012 & 0.030 & 0.007 \\
\hline emodin & $<0.004$ & $<0.023$ & $<0.006$ \\
\hline aloe-emodin & $<0.040$ & $<0.023$ & $<0.006$ \\
\hline barbaloin & $<0.004$ & $<0.023$ & $<0.006$ \\
\hline 1,4-dihydroxy-anthraquinone & $<0.004$ & $<0.023$ & $<0.006$ \\
\hline Stilbene & $<0.004$ & $<0.023$ & $<0.006$ \\
\hline rhaponticin & $<0.004$ & $<0.023$ & $<0.006$ \\
\hline Phenol carboxylic acid & $<0.004$ & $<0.023$ & $<0.006$ \\
\hline gallic acid & $<0.004$ & $<0.023$ & $<0.006$ \\
\hline vanillic acid & $<0.004$ & $<0.023$ & $<0.006$ \\
\hline caffeic acid & $<0.03$ & $<$ \\
\hline homogentisic acid & & $<$ & \\
\hline
\end{tabular}

Table 3. Cross-reactivities of anti-SB MAbs against various compounds.

3.3.4. Correlation of results of SB determination in crude extracts of rhubarb roots between HPLC and ELISA using MAb $7 \mathrm{H} 12$

The concentrations of SB in various rhubarb samples were determined by ELISA (Table 4). Shinshu Daio, bred by crossing R. palmatum and R. coreanum in order to increase the level of SB concentration in Japan, contained the highest SB level of $6.01 \pm 0.18 \mu \mathrm{g} / \mathrm{mg}$ dry wt. Ga-wo, estimated to be high grade in the traditional Japanese medicine, contained SB level of $3.14 \pm 0.27 \mu \mathrm{g} / \mathrm{mg}$ dry wt. These results are in good agreement with previous reports [18]. The correlation between results from ELISA and HPLC is also good.

\begin{tabular}{lll}
\hline \multirow{2}{*}{ Sample } & \multicolumn{2}{l}{ Concentration $(\mu \mathrm{g} / \mathbf{m g}$ dry $w \mathbf{~ p o w d e r})$} \\
\cline { 2 - 3 } & ELISA & HPLC \\
\hline Shinshu Daio & $6.01 \pm 0.18$ & $6.15 \pm 0.59$ \\
\hline Ga-wo & $3.14 \pm 0.27$ & $3.80 \pm 0.16$ \\
\hline Kinmon Daio & $0.35 \pm 0.01$ & $0.38 \pm 0.02$ \\
\hline Itto-Ga-wo (powder) & $1.44 \pm 0.12$ & $1.52 \pm 0.18$ \\
\hline Itto-Ga-wo (refuse) & $1.42 \pm 0.07$ & $1.40 \pm 0.11$
\end{tabular}

Table 4. SB concentrations in various rhubarb samples. Data are the means of triplicate assays. 


\subsubsection{Determination of concentrations of $S A$ and $S B$ in various Cassia species}

The concentrations of SA and SB in leaves of various Cassia species were determined by ELISA using anti-SA and SB MAbs (Table 5). The results indicate that C. angustifolia contains $4.56 \pm 0.25 \mu \mathrm{g} / \mathrm{mg}$ dry wt. powder of $\mathrm{SA}$ and $5.10 \pm 0.15 \mu \mathrm{g} / \mathrm{mg}$ dry wt. powder of SB indicating higher amounts of SA and SB compared to the other species. C. alata contains $1.19 \pm 0.12 \mu \mathrm{g} / \mathrm{mg}$ dry wt. powder of SA and $1.16 \pm 0.15 \mu \mathrm{g} / \mathrm{mg}$ dry wt. powder of SB. C. fistula (A) (D) contain 0.10-2.04 $\mu \mathrm{g} / \mathrm{mg}$ dry wt. powder of SA and 0.13-2.05 $\mu \mathrm{g} / \mathrm{mg}$ dry wt. powder of SB, respectively.

\begin{tabular}{llll}
\hline Sample & \multicolumn{2}{l}{ Concentration $(\boldsymbol{\mu g} / \mathbf{m g}$ dry wt. powder) } & \\
\cline { 2 - 4 } & Sennoside A & Sennoside B & Total sennosides \\
\hline Cassia angustifolia & $4.56 \pm 0.25$ & $5.10 \pm 0.15$ & $9.66 \pm 0.40$ \\
\hline C. alata & $1.19 \pm 0.12$ & $1.16 \pm 0.15$ & $2.35 \pm 0.27$ \\
\hline C. bakeriana & $0.40 \pm 0.03$ & $0.44 \pm 0.02$ & $0.84 \pm 0.05$ \\
\hline C. fistula (A) & $1.14 \pm 0.08$ & $0.75 \pm 0.08$ & $1.89 \pm 0.16$ \\
\hline C. fistula (B) & $2.04 \pm 0.32$ & $1.52 \pm 0.12$ & $3.56 \pm 0.44$ \\
\hline C. fistula (C) & $1.90 \pm 0.16$ & $2.05 \pm 0.24$ & $3.95 \pm 0.40$ \\
\hline C. fistula (D) & $0.10 \pm 0.01$ & $0.13 \pm 0.00$ & $0.23 \pm 0.01$ \\
\hline C. mimosoides & $(1.30 \pm 0.24) \times 10^{-2}$ & $(1.88 \pm 0.29) \times 10^{-4}$ & $(1.32 \pm 0.24) \times 10^{-2}$ \\
\hline C. floribunda & $(2.78 \pm 0.11) \times 10^{-3}$ & $(1.04 \pm 0.03) \times 10^{-4}$ & $(2.88 \pm 0.11) \times 10^{-3}$ \\
\hline C. surattensis & $(1.15 \pm 0.18) \times 10^{-2}$ & $(2.44 \pm 0.17) \times 10^{-4}$ & $(1.17 \pm 0.18) \times 10^{-2}$ \\
\hline C. tora & $(2.13 \pm 0.21) \times 10^{-3}$ & $(3.64 \pm 0.21) \times 10^{-5}$ & $(2.17 \pm 0.23) \times 10^{-3}$ \\
\hline C. siamea & $(4.45 \pm 0.14) \times 10^{-3}$ & $(1.87 \pm 0.13) \times 10^{-3}$ & $(6.32 \pm 0.27) \times 10^{-3}$
\end{tabular}

Table 5. Total sennoside concentrations in leaves of various Cassia species. Data are the means of triplicate assays.

\section{Establishments of a new eastern blotting, double staining and immunohistochemical staining using anti-SA and SB MAbs}

\subsection{Preface}

Thin-layer chromatography (TLC) is most widely used for detection, separation and monitoring of small molecular compounds like sennosides. If the direct TLC immunostaining with $\mathrm{MAb}$ can be done, this procedure must be contributive to the development of structural analysis of small molecular compounds. However, this procedure cannot be used for the direct detection of small molecular compounds on a TLC plate because the silica gel is sloughed off from the plate and the compounds on the plate are easily washed out without fixing during treatment. If the compounds are transferred from the TLC plate to a plastic 
membrane with hydrophobic properties and immobilized on the membrane, these difficulties can be solved. Therefore, I examined the transfer of sennosides from a TLC plate to a plastic membrane. Towbin et al. first reported the transfer of glycosphingolipids using nitrocellulose membranes [20]. However, since its transfer efficiency was poor and reproducible results were not obtained, I tested various plastic membranes and transfer conditions resulting in a polyvinylidene difluoride (PVDF) membrane to be the best [21]. The membrane is very stable against heating and various organic solvents in addition to retaining sennosides with high efficiency. I named this new method as eastern blotting (EB), because theoretically same methodology compared to previous EB except the way of sennoside-BSA conjugation for fixing sennosides on the membrane [22]. I communicate here the EB procedure for sennosides and its application for analytical survey of sennosides [23].

\subsection{Experimental}

\subsubsection{Chemicals and immunochemicals}

Polyvinylidene difluoride (PVDF) membranes (Immobilon-N) were purchased from Millipore Corporation (Bedford, MA, USA). Glass microfiber filter sheets (GF/A) were purchased from Whatman International Ltd. (Maidstone, England). All other chemicals were standard commercial products of analytical grade.

\subsubsection{EB and Double staining}

Sennosides were applied to a TLC plate and developed with 1-propanol-ethyl acetate-wateracetic acid (40:40:30:1, by volume). The developed TLC plate was dried and then sprayed with a blotting solution mixture of isopropanol-methanol-water (1:4:16, by volume). It was placed on a stainless steel plate and then covered with a PVDF membrane sheet. After covering with a glass microfiber filter sheet, the whole assembly was pressed evenly for $70 \mathrm{~s}$ with a $120{ }^{\circ} \mathrm{C}$ hot plate as previously described with some modifications [24, 25]. The PVDF membrane was separated from the TLC plate and dried.

The blotted PVDF membrane was dipped in $20 \mathrm{mM}$ carbonate buffer solution ( $\mathrm{pH}$ 9.6) containing BSA $(1 \%)$ and EDC $(20 \mathrm{mg} / \mathrm{mL})$, and stirred at room temperature for $14 \mathrm{hr}$. After washing the PVDF membrane twice with T-PBS for $5 \mathrm{~min}$ and then treated with S-PBS for 3 $\mathrm{hr}$ to reduce non-specific adsorption. The PVDF membrane was washed with T-PBS twice for $5 \mathrm{~min}$, and then immersed in anti-SA MAb (6G8) and stirred at room temperature for 3 hr. After washing the PVDF membrane twice with T-PBS for $5 \mathrm{~min}$, a 1:1000 dilution of POD-labeled goat anti-mouse IgG in PBS cotaining $0.2 \%$ of gelatin (G-PBS) was added and stirred at room temperature for $1 \mathrm{hr}$. The PVDF membrane was washed twice with T-PBS and water, then exposed to $1 \mathrm{mg} / \mathrm{mL}$ 4-chloro-1-naphtol- $0.03 \% \mathrm{H}_{2} \mathrm{O}_{2}$ in PBS solution which was freshly prepared before use for $10 \mathrm{~min}$ at room temperature. The protocol of the EB technique is shown in Figure 9. 


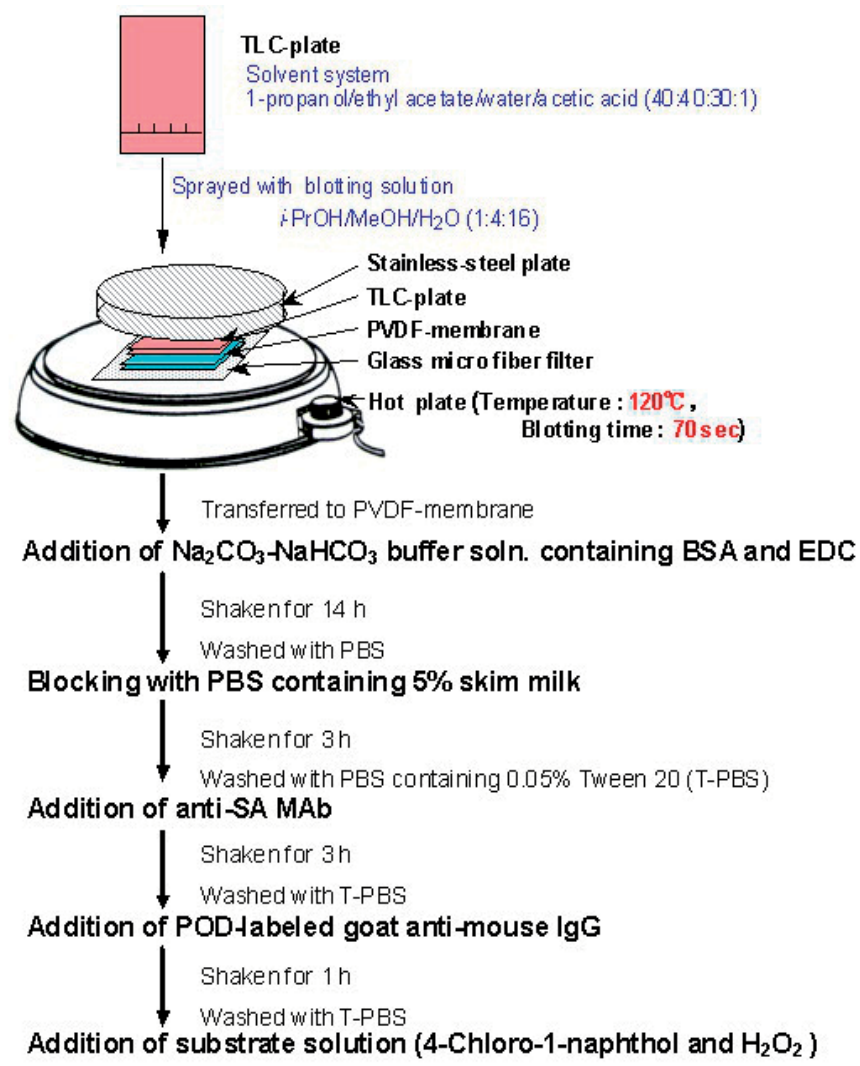

Figure 9. Eastern blotting protocol.

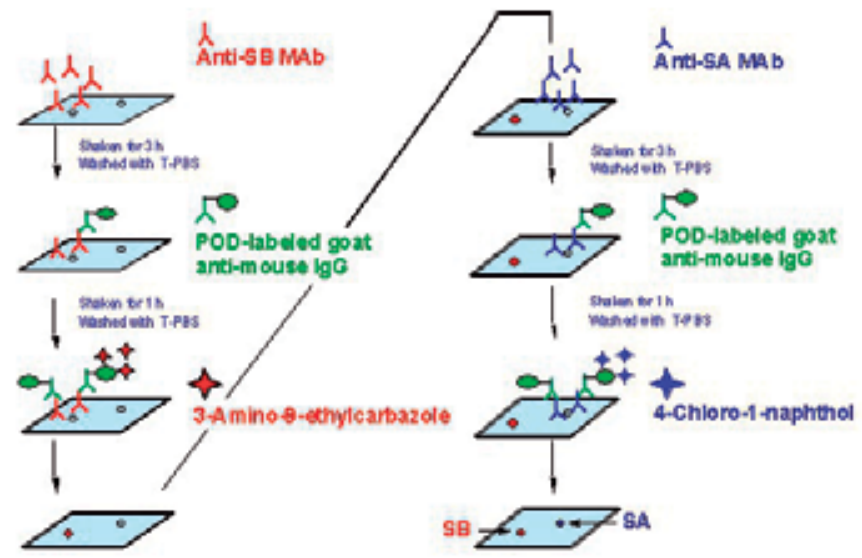

Figure 10. Double staining protocol. 
For successive staining by anti-SB MAb (7H12), the PVDF membrane stained by anti-SA MAb was treated in the same way as anti-SA MAb (6G8) except that it was exposed to $2 \mathrm{mg} / 10 \mathrm{~mL}$ 3-amino-9-ethylcarbazole- $0.03 \% \mathrm{H}_{2} \mathrm{O}_{2}$ in acetate buffer $(0.05 \mathrm{M}, \mathrm{pH} 5.0)$ containing $0.5 \mathrm{~mL}$ of $\mathrm{N}, \mathrm{N}$-dimethyl formamide. The protocol of double staining is shown in Figure 10.

\subsubsection{EB for immunohistochemical staining of $S A$}

A piece of PVDF membrane was placed on a glass microfiber filter sheet. A sliced fresh rhubarb root was placed on the PVDF membrane, and they were pressed together evenly for $1 \mathrm{hr}$. The blotted PVDF membrane was stained using the same procedure described for the EB method.

\subsection{Results and discussion}

\subsubsection{EB of $S A$ using anti-SA $M A b$}

Previously we established a new immunostaining method named as eastern blotting for several glycosides like solasodine glycosides [21], ginsenosides [26, 27] and glycyrrhizin [22, 28] by using individual MAbs. In this methodology we separated the sugar moiety in a molecule into two functions, the epitope part and fixation ability part on a membrane after blotted to a PVDF membrane from a TLC plate, since small molecular compounds can not be fixed on the membrane. Although I followed the previous methodology for SA, unfortunately staining was not succeeded. Therefore, a new blotting method onto a PVDF membrane from the developed TLC plate is required. SA was transferred to the PVDF membrane by the same way as previously described, and treated with EDC solution followed by the addition of BSA as indicated in Figure 9. This reaction enhanced the fixation of SA via SA-BSA conjugate on the PVDF membrane and the pathway was indicated diagrammatically in Figure 11. When the blotted PVDF membrane was incubated in the absence of EDC, it was essentially free of immunostaining (data not shown).

Figure 12 shows the EB of sennosides and other structurally related compounds using anti$\mathrm{SA} \mathrm{MAb}(\mathrm{A})$ and the $\mathrm{H}_{2} \mathrm{SO}_{4}$ staining (B). The EB indicated only limited staining of $\mathrm{SA}$ as shown in Figure 12A, lane 7. Moreover, the EB method was considerably more sensitive than that of $\mathrm{H}_{2} \mathrm{SO}_{4}$ staining. Since anti-SA MAb cross-reacts against $\mathrm{SB}$ and rhein as 0.28 and $0.35 \%$, respectively, they can be stained very weakly by anti-SA MAb, as described in the previous section. Previously Fukuda et al. succeeded the EB of ginsenoside Rb1 by using anti-ginsenoside $\mathrm{Rb} 1 \mathrm{MAb}$ resulting in staining together with ginsenoside $\mathrm{Rc}, \mathrm{Rd}, \mathrm{Re}$ and $\mathrm{Rg} 1$ $[26,27]$. The difference between the newly established EB and the previous methodology is combine system of sugar moiety to PVDF membrane. The sugar moiety in ginsenosides was oxidatively cleavaged to release aldehyde groups which were conjugated with a protein to fix on a PVDF membrane. Since it was evident that a part of sugar moiety in ginsenoside $\mathrm{Rb} 1$ was immunized, the cleavage of sugar moiety by $\mathrm{NaIO}_{4}$ expanded its cross-reactivity against other ginsenosides resulting in possibility of staining for ginsenoside $\mathrm{Rc}, \mathrm{Rd}, \mathrm{Re}$ and Rg1, though their cross reactivities are weak. On the other hand, the newly established EB in here does not hinder around sugar moiety in SA. Therefore, strength of staining for SA, SB and rhein was proportional to their cross-reactivities as described in ELISA. 


\section{Sennoside A}
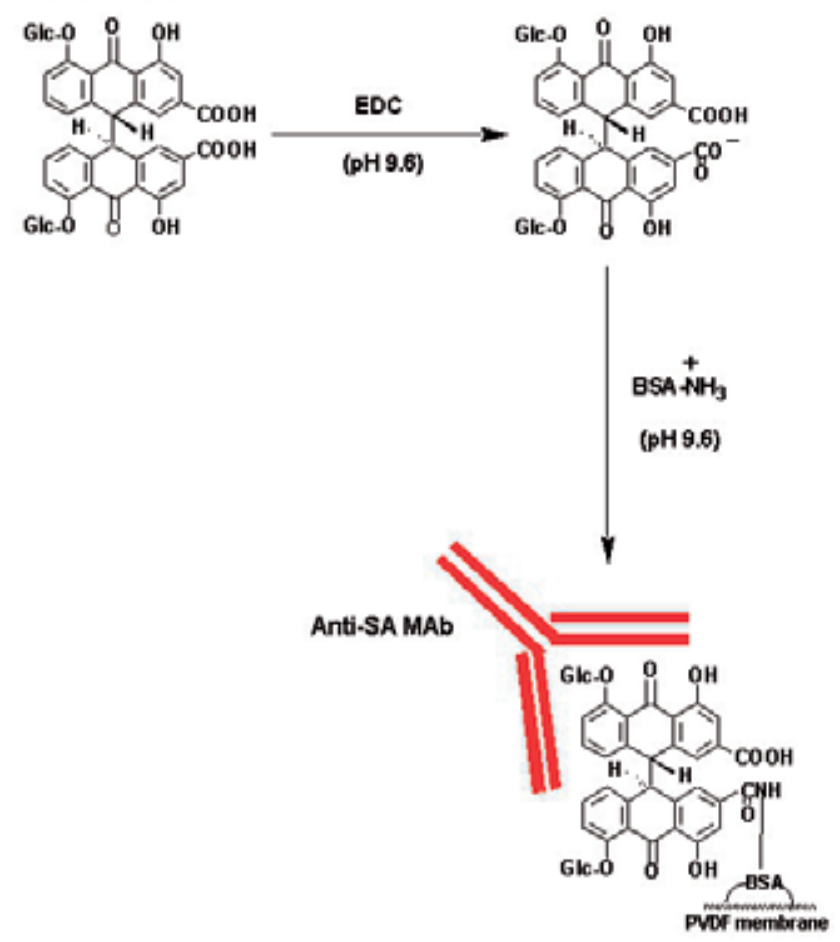

Figure 11. Schematic diagram illustrating the eastern blotting of SA onto the PVDF membrane and the detection using anti-SA MAb.

A

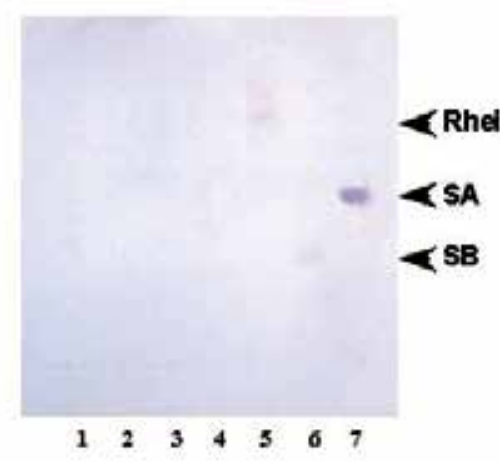

B

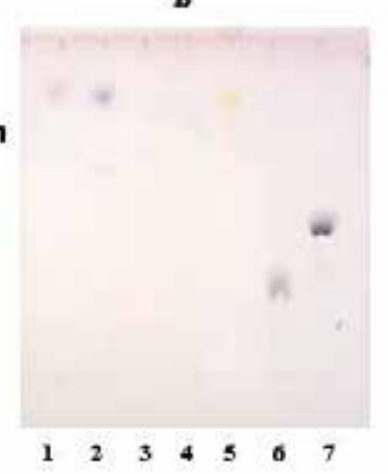

Figure 12. Eastern blotting of sennosides and related compounds stained by anti-SA MAb (A). B shows a TLC plate stained by $10 \% \mathrm{H}_{2} \mathrm{SO}_{4}$. Lanes $1,2,3,4,5,6$ and 7 indicate rhaponticin, barbaloin, aloe-emodin, emodin, rhein, SB and SA $(3 \mu \mathrm{g})$, respectively. 


\subsubsection{Double staining of sennosides using anti-SA and SB MAbs}

Previously, I used 4-chloro-1-naphthol for staining of SB. However, since it could not function well for SB, the combination of 4-chloro-1-naphthol and 3-amino-9-ethylcarbazole was selected to improve double staining of sennosides as indicated in Figure 10. SA and SB were stained clearly by the purple and red color, respectively (Figure 13). From this result both antibodies can distinguish stereochemical configurations, threo and erythro between C-10 and C-10' positions in a molecule on PVDF membrane stained as double coloring, respectively.

A

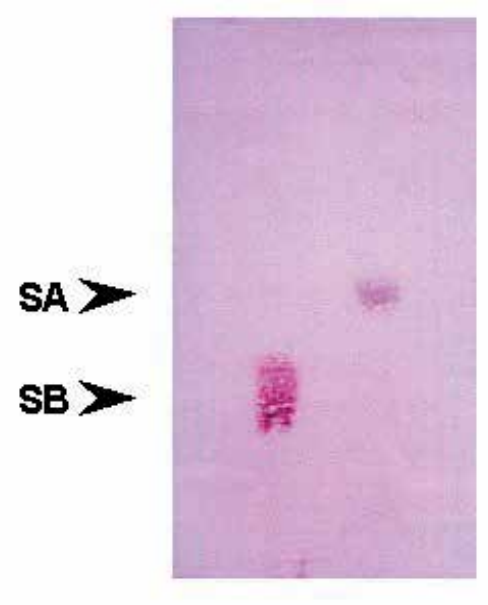

B

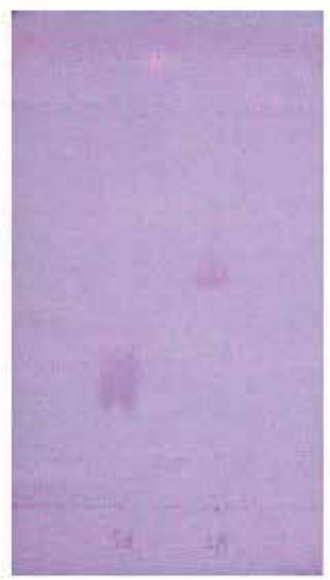

Figure 13. Double staining of sennosides using eastern blotting technique (A). B shows a result of $\mathrm{H}_{2} \mathrm{SO}_{4}$ staining. Red and purple colors were stained by anti-SB and SA MAb, respectively.

4.3.3. Detection of $S A$ and $S B$ in various Cassia species using double staining with a new EB technique

The crude extracts of various Cassia species were analyzed by the newly developed double staining system and TLC stained with $\mathrm{H}_{2} \mathrm{SO}_{4}$ as shown in Figure 14. Although $\mathrm{H}_{2} \mathrm{SO}_{4}$ staining (Figure 14B) detected many spots including probably sugars and different types of anthraquinone glycosides in various Cassia species, double staining (Figure 14A) detected clearly SA and SB, and very weakly other sennosides except appearance of chlorophylls around top. Band 1 indicated a purple color that means a threo-configuration between C-10 and C-10' positions detected by EB using anti-SA MAb as shown in Figure 14A. Moreover, its $\mathrm{Rf}$ value indicated that band 1 has one sugar moiety and $\mathrm{CH}_{2} \mathrm{OH}$ group instead of $\mathrm{COOH}$ group in a molecule. I surveyed the previous papers regarding sennosides in senna [1]. Judging from these evidences, I suggested that band 1 is sennoside C (SC) having threoconfiguration as indicated previously [1]. Band 2 was easily suggested to be erythro-configu- 
ration from its red color. The $R \mathrm{f}$ value clearly showed that band 2 includes one sugar moiety having a HOOC-CO group. From these results I supposed that band 2 is sennoside F (SF) that has erythro-configuration as indicated previously [1]. The double staining by EB indicates that $C$. angustifolia, C. alata, C. bakeriana and C. fistula contain a higher concentration of sennosides compared to the other species. This result has a good agreement with that of ELISA. The limit of detection by the double staining method was confirmed to be $48 \mu \mathrm{g} / \mathrm{mL}$ of both SA and SB.

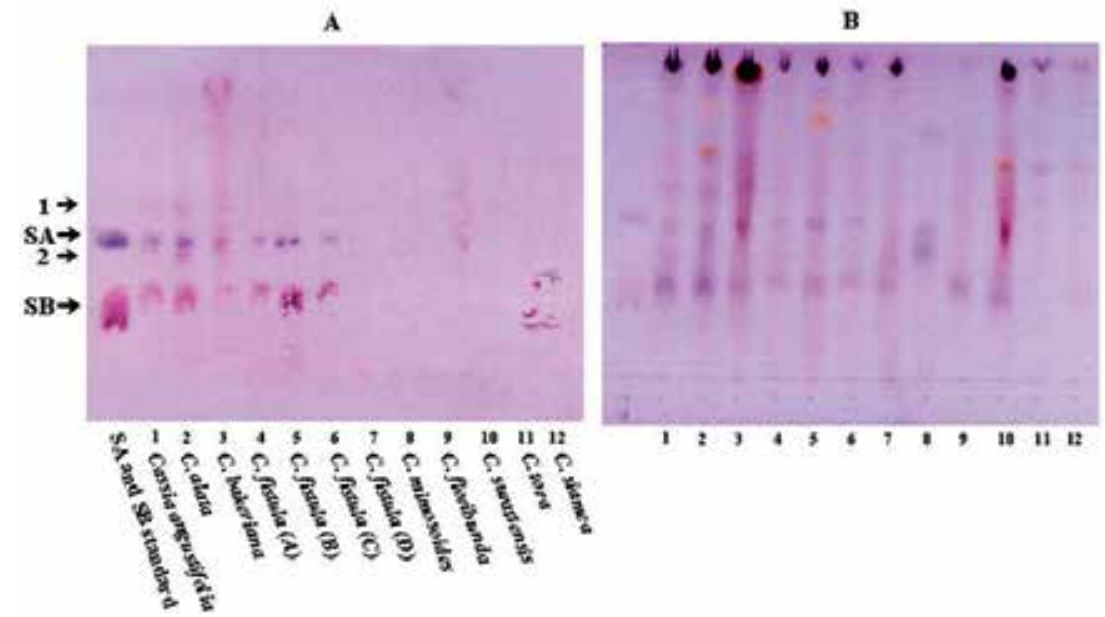

Figure 14. Double staining of $\mathrm{SA}$ and $\mathrm{SB}$ in various Cassia species (A). B shows a result of $\mathrm{H}_{2} \mathrm{SO}_{4}$ staining. Lefthand lane indicates SA $(4 \mu \mathrm{g})$ and SB $(3 \mu \mathrm{g})$. Lanes $1 \sim 12$ indicate various Cassia species $(3 \mu \mathrm{L})$.

\subsubsection{Validation of EB for immunohistochemical staining of $S A$}

As an other application of the EB method, the immunohistochemical staining of SA in rhubarb root, was investigated. A sliced fresh rhubarb root was placed on the PVDF membrane, and they were pressed together evenly for $1 \mathrm{hr}$. The blotted PVDF membrane was stained using the same procedure described for the EB method. Figure 15II illustrates the immunohistochemical staining of SA in fresh Hokkai Daio root. The phloem and cambium contained a higher concentration of SA compared to other tissues, pith and bud. To confirm this result, I analyzed these tissues individually by ELISA and HPLC. The concentrations of SA were determined by ELISA to determine $64.4 \pm 4.5,48.1 \pm 8.2,15.0 \pm 1.6$ and $1.8 \pm 0.3 \mathrm{ng} / \mathrm{mg}$ fresh wt. in phloem, cambium, pith and bud, respectively. This result was a good agreement with those of HPLC resulting in $58.4 \pm 2.6,49.0 \pm 3.9$ and $13.3 \pm 0.5 \mathrm{ng} / \mathrm{mg}$ fresh wt. in phloem, cambium and pith, respectively. 
I

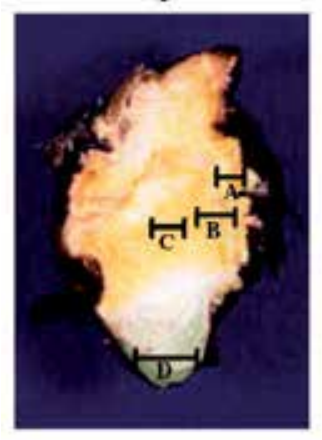

II

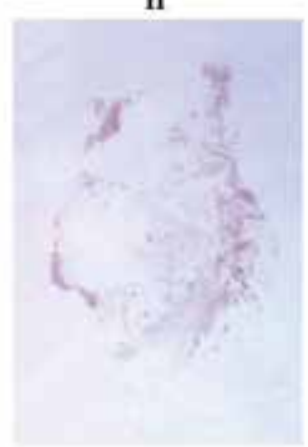

Figure 15. Immunohistochemical staining of SA using anti-SA MAb in rhubarb root. I, cross section of Hokkai Daio root; II, direct eastern blotting on PVDF membrane of a cross section of Hokkai Daio root. A, Phloem; B, Cambium; C, Pith; D, Bud, respectively.

\section{Conclusion}

The recent developments of molecular biosciences and their biotechnological applications have opened up many new avenues of pharmaceutical areas. MAbs have many potential uses in addition to immunological methods to plant sciences. Therefore, immunoassay system using MAbs against pharmacologically active natural products having low molecular weight have become an important tool for the studies on receptor binding analysis, enzyme assay, and quantitative and/or qualitative analytical techniques in plants owing to their specific affinity.

In order to analyze the stereochemical isomers, SA and SB in plants, medicaments, prescriptions, health foods and patients'sera, I have produced MAbs against them. These MAbs have the most important ability to distinguish between SA and SB, which differ only in the stereochemical configuration at the C-10 and C-10' positions, respectively. Moreover, they have no detectable cross-reaction with the other related anthraquinone and anthrone.

Analytical systems of SA and SB by competitive ELISA using anti-SA and SB MAbs were established. These ELISA systems are capable of measuring SA and SB in complex matrics without any pretreatments. Furthermore, these ELISA methods are approximately 2,000 times for SA and 10,000 times for SB more sensitive than that of HPLC method.

The newly developed EB methodology can be theoretically expanded for all compounds having carboxylic acid such as phenol carboxylic acids, glucuronides, furthermore compounds having only a carboxylic group in a molecule. A new double staining with EB method for sennosides using anti-SA and SB MAbs was established. SA and SB were stained purple and red color, respectively. This system visualized sennosides on a PVDF membrane. In fact, SA and SB in the crude extracts of various Cassia species were distinguished by their coloring and $\mathrm{Rf}$ values. Moreover, it could make it possible to survey the natural resour- 
ces of sennosides and quickly determine their structures. Furthermore, EB also can be used for the survey of distribution of SA and/or SB in the Rheum specimen by immunohistochemical staining.

\section{Acknowledgements}

We thank Dr. Hiroyuki Tanaka (Faculty of Pharmaceutical Sciences, Kyushu University) for useful suggestions in this work. This research was supported in part by Japan Science and Technology Agency, Grant-in-Aid from the Ministry of Education, Culture, Sports, Science and Technology of Japan, the research grant from Takeda Science Foundation.

\section{Author details}

Osamu Morinaga and Yukihiro Shoyama*

*Address all correspondence to: shoyama@niu.ac.jp

Department of Pharmacognosy, Faculty of Pharmaceutical Sciences, Nagasaki International University, Sasebo, Japan

\section{References}

[1] Oshio, H., Naruse, Y., \& Tsukui, M. (1978). Quantilative analysis of the purgative components of rhubarb and senna. Chemical Pharmaceutical Bulletin, 26, 2458-2464.

[2] Yang, L., Akao, T., Kobashi, K., \& Hattori, M. (1996). A sennoside-hydrolyzing $\beta$-glucosidase from Bifidobacterium sp.strain SEN is inducible. Biological Pharmaceutical Bulletin, 19, 701-704.

[3] Yang, L., Akao, T., Kobashi, K., \& Hattori, M. (1996). Purification and characterization of a novel sennoside-hydrolyzing $\beta$-glucosidase from Bifidobacterium sp. strain SEN, a human intestinal anaerobe. Biological Pharmaceutical Bulletin, 19, 705-709.

[4] Japanese Pharmacopoeia (2011). The Japanese Pharmacopoeia, 16. th ed.; Jiho

[5] Atzorn, R., Weiler, E. W., \& Zenk, M. H. (1981). Formation and distribution of sennosides in Cassia angustifolia, as determined by a sensitive and specific radioimmunoassay. Planta Medica, 41, 1-14.

[6] Sakata, R., Shoyama, Y., \& Murakami, H. (1994). Production of monoclonal antibodies and enzyme immunoassay for typical adenylate cyclase activator, forskolin. Cytotechnology, 16, 101-108. 
[7] Ishiyama, M., Shoyama, Y., Murakami, H., \& Shinohara, H. (1995). Production of monoclonal antibodies and development of an ELISA for solamargine. Cytotechnolo$g y, 18,153-158$.

[8] Shoyama, Y., Fukada, T., \& Murakami, H. (1995). Production of monoclonal antibodies and ELISA for thebaine and codeine. Cytotechnology, 19, 55-61.

[9] Tanaka, H., Goto, Y., \& Shoyama, Y. (1996). Monoclonal antibody based enzyme immunoassay for marihuana (cannabinoid) compounds. Journal of Immunoassay, 17, 321-342.

[10] Tanaka, H., \& Shoyama, Y. (1998). Formation of a monoclonal antibody against glycyrrhizin and development of an ELISA. Biological Pharmaceutical Bulletin, 21, 1391-1393.

[11] Xuan, L., Tanaka, H., Xu, Y., \& Shoyama, Y. (1999). Preparation of monoclonal antibody against crocin and its characterization. Cytotechnology, 29, 65-70.

[12] Tanaka, H., Fukuda, N., \& Shoyama, Y. (1999). Formation of monoclonal antibody against a major ginseng component, ginsenoside $\mathrm{Rb} 1$ and its characterization. Cytotechnology, 29, 115-120.

[13] Fukuda, N., Tanaka, H., \& Shoyama, Y. (2000). Formation of monoclonal antibody against a major ginseng component, ginsenoside Rg1 and its characterization. Monoclonal antibody for a ginseng saponin. Cytotechnology, 34, 197-204.

[14] Morinaga, O., Tanaka, H., \& Shoyama, Y. (2000). Production of monoclonal antibody against a major purgative component, sennoside $\mathrm{A}$, its characterization and ELISA. Analyst, 125, 1109-1113.

[15] Shoyama, Y., Sakata, R., Isobe, R., \& Murakami, H. (1993). Direct determination of forskolin-bovine serum albumin conjugate by matrix-assisted laser desorption ionization mass spectrometry. Organic Mass Spectrometry, 28, 987-988.

[16] Langone, J. J. (1980). A simple procedure to use whole serum as a source of either IgG- or IgM-specific antibody. Journal of Immunol Methods, 32, 51-58.

[17] Oshio, H., \& Kawamura, N. (1985). Determination of the laxative compounds in rhubarb by high performance liquid chromatography. Shoyakugaku Zasshi, 39, 131-38.

[18] Seto, T., Yasuda, I., Hamano, T., Takano, I., Kiyono, S., Nishijima, M., \& Akiyama, K. (1996). Determination method of sennoside A, sennoside B, rhein and rhein 8-glucoside in kampo or crude drug preparations and the comparison of these components in processed rhubarb. Natural Medicines, 50, 138-144.

[19] Morinaga, O., Nakajima, S., Tanaka, H., \& Shoyama, Y. (2001). Production of monoclonal antibodies against a major purgative component, sennoside $\mathrm{B}$, their characterization and use in ELISA. Analyst, 126, 1372-1376. 
[20] Towbin, H., Schoenenberger, C., Ball, R., Braun, D. G., \& Rosenfelder, G. (1984). Glycosphingolipid-blotting: an immunological detection procedure after separation by thin layer chromatography. Journal of Immunological Methods, 72, 471-479.

[21] Tanaka, H., Putalun, W., Tsuzaki, C., \& Shoyama, Y. (1997). A simple determination of steroidal alkaloid glycosides by thin-layer chromatography immunostaining using monoclonal antibody against solamargine. FEBS Letters, 404, 279-82.

[22] Shan, S. J., Tanaka, H., \& Shoyama, Y. (2001). Enzyme-linked immunosorbent assay for glycyrrhizin using anti-glycyrrhizin monoclonal antibody and an eastern blotting technique for glucuronides of glycyrrhetic acid. Analytical Chemistry, 73, 5784-5790.

[23] Morinaga, O., Uto, T., Sakamoto, S., Putalun, W., Lhieochaiphant, S., Tanaka, H., \& Shoyama, Y. (2009). Development of eastern blotting technique for sennoside A and sennoside B using anti-sennoside A and anti-sennoside B monoclonal antibodies. Phytochemical Analysis, 20, 154-158.

[24] Towbin, H., Staehelin, T., \& Gordon, J. (1979). Electrophoretic transfer of proteins from polyacrylamide gels to nitrocellulose sheets: procedure and some applications. Proceedings of the National Academy of Sciences of the United States of America, 76, 4350-4.

[25] Taki, K., Kasama, T., Handa, S., \& Ishikawa, D. (1994). A simple and quantitative purification of glycosphingolipids and phospholipids by thin-layer chromatography blotting. Analytical Biochemistry, 223, 232-8.

[26] Fukuda, N., Tanaka, H., \& Shoyama, Y. (1999). Western blotting for ginseng saponins, ginsenosides using anti-ginsenoside $\mathrm{Rb} 1$ monoclonal antibody. Biological Pharmaceutical Bulletin, 22, 219-20.

[27] Fukuda, N., Tanaka, H., \& Shoyama, Y. (2001). Double staining of ginsenosides by Western blotting using anti-ginsenoside $\mathrm{Rb} 1$ and $\mathrm{Rg} 1$ monoclonal antibodies. Biological Pharmaceutical Bulletin, 24, 1157-1160.

[28] Shan, S. J., Tanaka, H., \& Shoyama, Y. (1999). Western blotting method for the immunostaining detection of glucuronides of glycyrrhetic acid using anti-glycyrrhizin monoclonal antibody. Biological Pharmaceutical Bulletin, 22, 221-223. 

Chapter 2

\title{
Applications of Anti-natural Compound Immunoaffinity Purification on Quality Control
}

\author{
Takuhiro Uto, Nguyen Huu Tung, Hiroyuki Tanaka \\ and Yukihiro Shoyama
}

Additional information is available at the end of the chapter

http://dx.doi.org/10.5772/45955

\section{Introduction}

Worldwide demand of herbal medicines has increased in recent years owing to rising interest in the health benefits. Among with this, the quality control of plant extracts and plantderived medicines is growing in importance to ensure their efficacy and safety. Effective quality control of the traditional Chinese medicines (TCM) and plant crude extracts requires the rapid and sensitive methods for separation and quantification of bioactive compounds. Various methods have been employed for the separation and quantification of certain constituents in medicinal plants or herbal medicines. However, the current methods in use are not necessarily optimal approaches. For example, separation and quantification of glycyrrhizin (GC), the main active constituent in licorice (Glycyrrhiza spp.), have been used gas chromatography, high performance liquid chromatography (HPLC) and micellar trokinetic chromatography and so on [1,2]. Commercial purification of GC typically progressed through several steps, including crystallization, column chromatography, and liquid partitioning. These current methods are not sufficiently approaches because of insufficient sensitivity and reproducibility, large consumption of organic solvent for extraction and analysis, and long analysis time.

Immunoassay systems using monoclonal antibody (MAb) against drugs and small molecular weight bioactive compounds have become an important tool for studies on receptor binding assays, enzyme assays, and quantitative and qualitative analytical techniques both in vivo and in vitro studies. Although immunoaffinity purification against higher molecule analyte such as peptides and proteins are widely used in the research and commercial ways, there are too few cases of immunoaffinity purification targeting a small molecule compound such as natural compounds. Our laboratory has prepared many kinds of MAbs against 
naturally occurring bioactive compounds such as terpenoids [3-5], alkaloids [6,7], saponins [8-12], and phenolics [13-16], and developed several applications. One of the applications by using MAbs is immunoaffinity column conjugated with anti-natural compound-specific MAbs and work by specifically binding and removing the target compounds. We have been establishing several affinity columns against a kind of terpenoid, forskolin [17], solasodine glycosides [18], ginsenosides $\mathrm{Rb}_{1}$ [19], and GC [20]. Application of an immunoaffinity column to isolate and concentrate a natural compound may decrease the amount of solvent consumption and the number of purification steps, shorten analysis time, and simplify sample analysis compared to traditional cleanup techniques.

In this chapter, we focus on the immunoaffinity purification to separate and concentrate the target bioactive compounds from the crude extract. Our approaches effectively succeeded one-step purification of target compounds by MAb-conjugated immunoaffinity column, which leads to high-sensitivity detection and isolation of target compounds. In addition, the immunoaffinity column can prepare the knockout $(\mathrm{KO})$ extract which contains all components except an antigen molecule, and $\mathrm{KO}$ extract will be useful for the pharmacological investigation to reveal the real effects of bioactive compound in the crude extract.The information in this chapter may provide new insight into quality control of plant-derived medicines.

\section{Preparation of anti-ginsenoside $R b_{1}$ immunoaffinity column and its application}

Ginseng, the root of Panax ginseng, has been an important component in traditional medicines for more than 1000 years in Eastern Asia. It is now one of the most extensively used alternative medicines all over the world and appears in the pharmacopoeias of several countries. The biological and pharmacological activities of ginseng have been reported to have antiaging, anti-cancer, anti-inflammation, anti-diabetics, anti-stress, maintenance of homeostasis, and to affect on central nervous system and immune function [21]. The bioactive components responsible for ginseng actions are ginsenosides, which are triterpenes saponins that possess a dammarane skeleton with sugar moieties [22]. Up to now more than 60 kinds of ginsenosides have been isolated from Panax genus [23]. It is well-known that the concentrations of ginsenosides vary in the ginseng root or the root extracts depending on the method of extraction, subsequent treatment, or even the season of its collection [24,25]. Due to the importance of ginseng, a number of researches has been carried out to develop the methods for the identification, quantification and quality control of ginsenosides in raw plants materials, extracts and commercial products. Currently, analytical and preparative HPLC are commonly used to quantify and purify the individual ginsenosides from ginseng [26]. However, isolation of ginsenosides by HPLC requires the repeated purification steps, including cumbersome handling and lengthy analysis times, and may result in the decrease of the final yield.Thus, the developed approaches are required for quality control of ginseng in the field of TCM.

Ginsenoside $R b_{1}\left(G-R b_{1}\right)$ is one of the main ginsenosides responsible for many pharmaceutical actions of ginseng [27]. G- $\mathrm{Rb}_{1}$ has various biological activities, including facilitating acquisition and retrieval of memory [28], scavenging free radicals [29], inhibition of calcium over-influx into neurons [30], and preserving the structural integrity of the neurons [31]. 
In order to develop efficient quality control of ginseng, we have prepared anti-G- $R b_{1} M A b$, set up of enzyme-linked immunosorbent assay (ELISA), and a new immunostaining method named Eastern blotting [8,32]. Furthermore, we established an immunoaffinity column against $\mathrm{G}-\mathrm{Rb}_{1}$ and its application for one-step isolation from crude extract of ginseng root [19, 32]. Herein we describe the preparation of anti-G- $R b_{1}$ immunoaffinity column and it applications for identification and concentration of $\mathrm{G}-\mathrm{Rb}_{1}$.

\subsection{Preparation of $M A b$ and immunoaffinity column against $G-R b_{1}$}

\subsubsection{Analytical methodology for determination of hapten number in antigen, hapten-carrier protein} conjugate

The first step for the MAb production is the synthesis of a hapten-carrier protein conjugate. Bovine serum albumin (BSA) conjugated with $\mathrm{G}-\mathrm{Rb}_{1}$ was produced for the preparation of specific MAb in mouse [8]. There had been no direct and appropriate methods for the determination of haptens conjugated carrier proteins without differential UV analysis, radiochemical or chemical methods. Therefore, immunization by the injection of hapten-carrier protein conjugate was unreliable. Wengatez et al. determined the hapten density of immuno-conjugates by matrix-assisted UV laser desorption/ionization (MALDI) mass spectrometry [33]. We also reported the direct analytical method of hapten and carrier protein conjugates by a MALDI tof mass spectrometry using internal standard [3-16]. Figure 1 shows the MALDI tof mass spectra of $\mathrm{G}-\mathrm{Rb}_{1}$-BSA conjugate. A broad peak coinciding with the conjugate of $\mathrm{G}-\mathrm{Rb}_{1}$ and BSA appeared from $m / z 70,000$ to 90,000 centering at around $m / z 79,469$. Using experimental results and a molecular weight of 66,433 for $\mathrm{BSA}$, the calculated values of $\mathrm{G}-\mathrm{Rb}_{1}$ component (MW1,109) are from 3,327 to 23,289 resulting in the range of 3 to 21 (12 in average) molecules of $\mathrm{G}-\mathrm{Rb}_{1}$ conjugated with BSA [8]. This method is suitable for characterization of conjugates between small molecule natural compound and carrier protein conjugates.

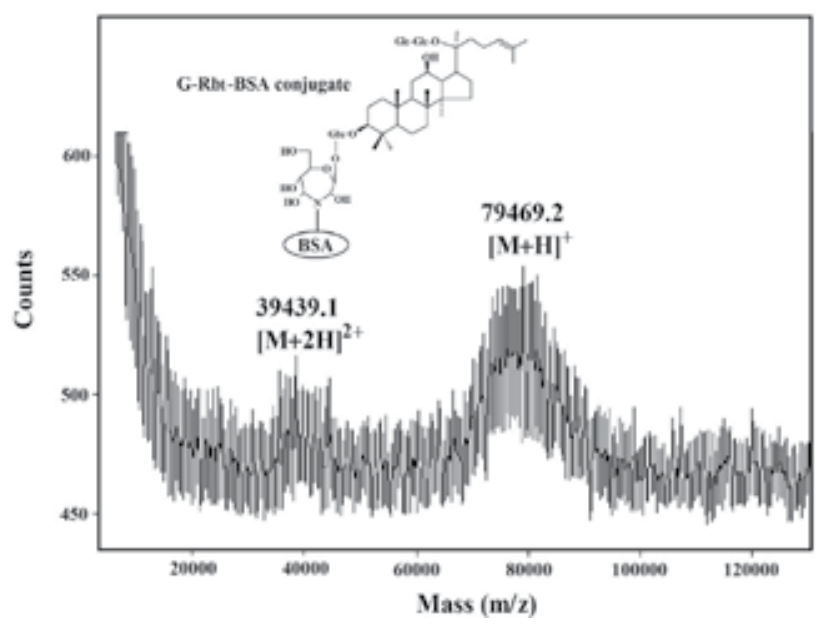

Figure 1. Direct detection of $\mathrm{G}-\mathrm{Rb} \mathrm{b}_{1}-\mathrm{BSA}$ conjugate by MALDI tof mass spectrometry. $[\mathrm{M}+\mathrm{H}]^{+},[\mathrm{M}+2 \mathrm{H}]^{2+}$ are single and double protonated molecules of $\mathrm{G}-\mathrm{Rb}_{1}$-BSA, respectively. 


\subsubsection{Preparation of anti-G-R $b_{1} M A b$ and ELISA as an assay system}

A hybridoma-producing $\mathrm{MAb}$ reactive to $\mathrm{G}-\mathrm{Rb}_{1}$ was obtained by general procedure and classified into IgG2b which had $\kappa$ light chains [8]. The reactivity of IgG type MAb, 9G7 was tested by varying antibody concentration and by performing a dilution curve. The antibody concentration was selected for competitive ELISA. The free MAb following competition is bound to polystyrene microtiter plates precoated with $\mathrm{G}-\mathrm{Rb}_{1}$-human serum albumin (HSA). Under these conditions, the full measurement range of the assay extends from 20 to 400 $\mathrm{ng} / \mathrm{mL}$. The cross-reactivity against G-Rc and G-Rd, which possess a diglucose moiety attached to the C-3 hydroxy group, were weak compared with G- $\mathrm{Rb}_{1}(0.024$ and $0.020 \%$, respectively). G-Re and G-Rg $g_{1}$ showed no cross-reactivity (less than $0.005 \%$ ). It is evident that the $\mathrm{MAb}$ reacted only with a small number of structurally related $\mathrm{G}-\mathrm{Rb}_{1}$ molecules, and very weakly and did not react with other steroidal compounds.

\subsubsection{Preparation of anti-G-R $b_{1}$ immunoaffinity column and appropriate buffer systems for separation of $G-R b_{1}$}

The purified IgG (10 mg) was treated by $\mathrm{NaIO}_{4}$ to give dialdehyde group in sugar moiety which was coupled to Affi-Gel Hz hydrazide gel resulting in a hydrozone-type immunoaffinity gel [32]. The immunoaffinity gel was packed into plastic mini-column (Figure 2). Due to examine the optimal conditions of adsorption and elution, $400 \mu \mathrm{g}$ of $\mathrm{G}-\mathrm{Rb}_{1}$ was dissolved in phosphate buffered saline (PBS) and loaded on anti-G-Rb $b_{1}$ affinity column. After washing with washing buffer ( $20 \mathrm{mM}$ PB containing $0.5 \mathrm{M} \mathrm{NaCl}$ ), various buffer solutions for elution were loaded on the column, and then the recovery efficiency was determined by ELISA. The $\mathrm{G}-\mathrm{Rb}_{1}$ concentration was somewhat increased by eluting with a $20 \mathrm{mM}$ phosphate buffer containing $0.5 \mathrm{M} \mathrm{KSCN}$ and $10 \% \mathrm{MeOH}$. When the $20 \mathrm{mM}$ phosphate buffer was changed to $100 \mathrm{mM}$ AcOH buffer ( $\mathrm{pH} 4$ ), the elution ability reached the optimal level. Although $20 \%$ $\mathrm{MeOH}$ could enhance the elution of $\mathrm{G}-\mathrm{Rb}_{1}$, higher $\mathrm{MeOH}$ concentration of over $20 \%$ was ineffective. Thus, $100 \mathrm{mM}$ AcOH buffer containing $0.5 \mathrm{M} \mathrm{KSCN}$ and $20 \% \mathrm{MeOH}$ could be used as an elution buffer in the immunoaffinity chromatography.

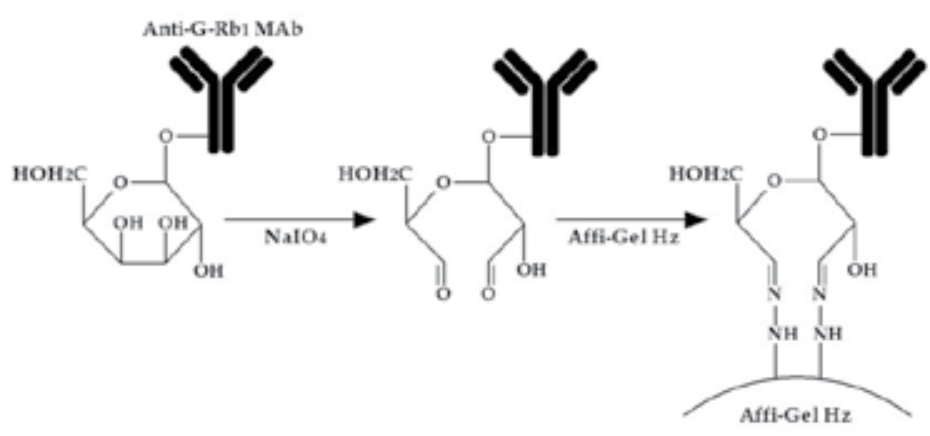

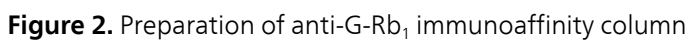




\subsection{Purification of $G-R b_{1}$ by immunoaffinity column}

2.2.1. One-step purification of $G-R b_{1}$ from crude extract of $P$. ginseng roots by anti-G-R $b_{1}$ immunoaffinity column

A crude extract $\left(3.8 \mathrm{mg}\right.$ ) of $P$. ginseng roots was loaded onto anti-G- $R \mathrm{~b}_{1}$ immunoaffinity column. The column was washed with the washing buffer (fractions 1-20), and then eluted with elution buffer (fractions 21-40). As shown in Figure 3, the fractions 1-8 contained the overloaded $\mathrm{G}-\mathrm{Rb}_{1}$, which determined by ELISA. The other ginsenosides such as $\mathrm{G}-\mathrm{Rg}_{1}, \mathrm{Rc}$, $\operatorname{Re}$ and $\mathrm{Rd}$ were also detected in these fractions by Eastern blotting procedure. After washing, a sharp peak was observed around fractions 21-24 of elution buffer, which contained G$\mathrm{Rb}_{1}$. However, these eluted fractions were still contaminated by a small amount of malonyl$\mathrm{G}-\mathrm{Rb}_{1}$ as detected by Eastern blotting. The malonyl-G- $\mathrm{Rb_{1 }}$ has almost the same crossreactivity with $\mathrm{G}-\mathrm{Rb}_{1}$ [32]. Therefore, the eluted fractions were treated with a mild alkaline solution $(0.1 \% \mathrm{KOH}$ in $\mathrm{MeOH})$ at room temperature to give pure $\mathrm{G}-\mathrm{Rb}_{1}$ [19]. Overcharged $\mathrm{G}-\mathrm{Rb}_{1}$ in washing solution (fractions $1-8$ ) was repeatedly loaded and finally isolated in pure form. The anti-G- $\mathrm{Rb}_{1} \mathrm{MAb}$ was stable during all procedures, and the immunoaffinity column showed almost no decrease in capacity ( $20 \mu \mathrm{g}$ of $\mathrm{G}-\mathrm{Rb}_{1} / \mathrm{ml}$ gel) after repeated use more than 10 times under same conditions, as reported for a one-step purification of forskolin from a crude extract of Coleus forslohlii root [17].

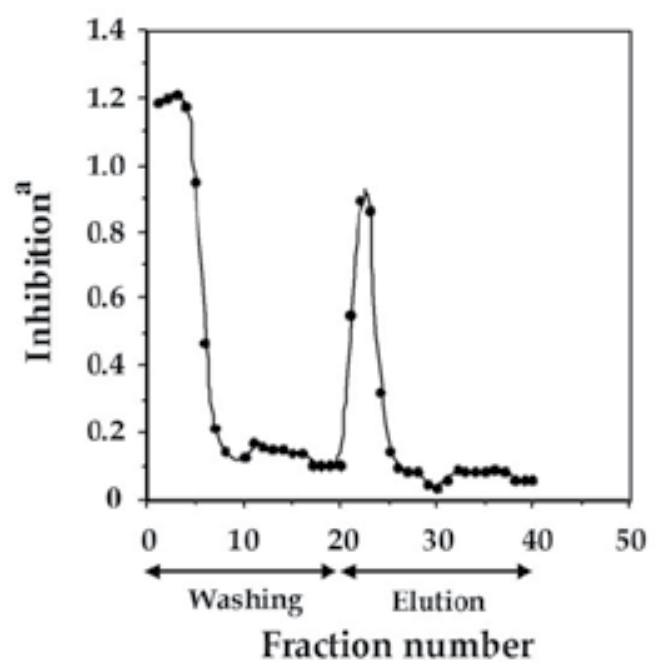

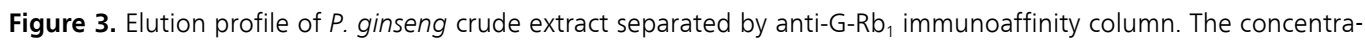
tion of $\mathrm{G}-\mathrm{R} \mathrm{b}_{1}$ in each fraction was monitoring by ELISA using anti-G-Rb, MAb. Individual fraction ( $2 \mathrm{~mL}$ ) were assayed by ELISA. Inhibition $=\left(A_{0}-A\right) / A_{0} ; A_{0}$ is the absorbance in the absence of the test compounds. $A$ is the absence in the presence the test compounds.

This methodology is effective for the rapid and simple purification of $G-\mathrm{Rb}_{1}$ and may open up a wide field of comparable studies with other families of saponins for which an acceptable method for one-step separation has not yet been developed. Furthermore, to separate the 
total ginseng saponins, a wide cross-reactive MAb against ginsenoside, like anti G-Re MAb which showed wide cross-reactivity, could be designed [34]. A combination of immunoaffinity column, Eastern blotting and ELISA could be used to survey low concentrations of ginsenoside $\mathrm{Rb}_{1}$ of plant origin and/or in experimental animals and human. In fact we have succeeded in the isolation of $\mathrm{G}-\mathrm{Rb}_{1}$ from a different plant, Kalopanax pictus Nakai, which was not known previously to contain ginsenosides, using this combination of methods [35].

\subsubsection{Isolation and determination of unknown compounds related to $G-R b_{1}$ by anti-G-R $b_{1}$ immunoaffinity column}

Several species of ginseng are known to exist and contain different amount and kinds of ginsenosides. P. japonicus is distributed in Japan and China and it is morphologically different from the other Panax species. Yahara et al. indicated that $\mathrm{G}-\mathrm{Rb}_{1}$ was not detected in P. japonicus, and isolated oleanane-type saponins called chikusetsusaponins and determined their structures [36]. Morita et al. reported the varieties of saponins in P. japonicus by chemical analysis. These results suggested that the concentration of $\mathrm{G}-\mathrm{Rb}_{1}$ might be trace level in $P$. japonicas [37]. We previously analyzed the $\mathrm{G}-\mathrm{Rb}_{1}$ concentration in several ginseng roots by ELISA using anti-G- $\mathrm{Rb}_{1} \mathrm{MAb}$ and HPLC after pre-treatment under mildly alkaline condition [32]. As shown in Table 1, G-R $\mathrm{b}_{1}$ concentrationsof $P$. ginseng, P. notoginseng and P. quinquefolius were correlated between ELISA and HPLC. However, the G-R $b_{1}$ of P. japonicus was higher concentrations compared with HPLC and previous reports [37]. This data suggest that anti-G- $\mathrm{Rb}_{1} \mathrm{MAb}$ using ELISA has the cross-reactivity with some unknown compounds contained in P. japonicus.

\begin{tabular}{llcc}
\hline & \multicolumn{2}{c}{$\begin{array}{c}\text { G-Rb } \text { concentration } \\
\text { Sample }\end{array}$} & \multicolumn{2}{c}{$(\mu \mathrm{g} / \mathrm{mg}$ dry weight powder $)$} \\
\cline { 3 - 4 } P. ginseng & White ginseng & $5.49 \pm 0.75$ & $4.96 \pm 0.05$ \\
& Red ginseng & $3.57 \pm 0.62$ & $3.93 \pm 0.34$ \\
& Fibrous ginseng & $64.44 \pm 3.64$ & $69.75 \pm 1.45$ \\
P. notoginseng & $47.08 \pm 3.34$ & $42.39 \pm 1.39$ \\
P. quinquefolium & $48.51 \pm 1.79$ & $47.96 \pm 1.04$ \\
P. japonicus & $1.37 \pm 0.34$ & $0.63 \pm 0.06$ \\
\hline
\end{tabular}

Table 1. G-Rb, concentration in various ginseng samples

To clarify the unknown compounds bound to anti-G- $\mathrm{Rb}_{1} \mathrm{MAb}$, the crude extract of $P$. japonicus was concentrated by immunoaffinity column using anti-G- $\mathrm{Rb}_{1} \mathrm{MAb}$. The crude root extract was loaded on the column and washed with the washing Buffer, followed by the elution buffer as already indicated. Figure 4 shows the $\mathrm{H}_{2} \mathrm{SO}_{4}$ staining (A) and the Eastern blotting (B) profiles of the washing fractions 1-4 and eluted fraction 5. Fraction 1 is first elut- 
ed fraction by the washing buffer, and showed many spots, indicating chikusetsusaponins, similar to the original extract of $P$. japonicus. After washing, the column was eluted by elution buffer (fraction 5), and then one spot was detected. As shown in Figure 4B, Eastern blotting indicated two different spots in washing fraction (Compound 1) and eluted fraction (Compound 2). These compounds bound with anti-G- $\mathrm{Rb}_{1} \mathrm{MAb}$ have a dammarane saponin having protopanaxadiol as a framework.

A

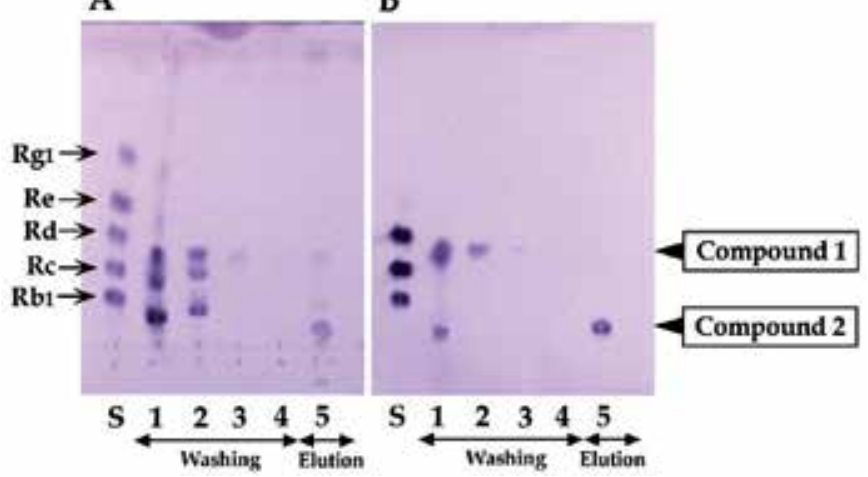

Figure 4. Purification and determination of ginsenosides of $P$. japonicus by the anti-G-Rb, immunoaffinity column. TLC (A) and Eastern blotting (B) profiles of the separated fractions from $P$. japonicus crude extract by the anti-G-Rb 1 immunoaffinity column. Lane $S$ indicates the standard of ginsenosides (G-Rd, G-Rc, G-Rb, G-Rg , and G-Re). Lane 1-4 and Lane 5 were the washing fractions and the eluted fraction, respectively.

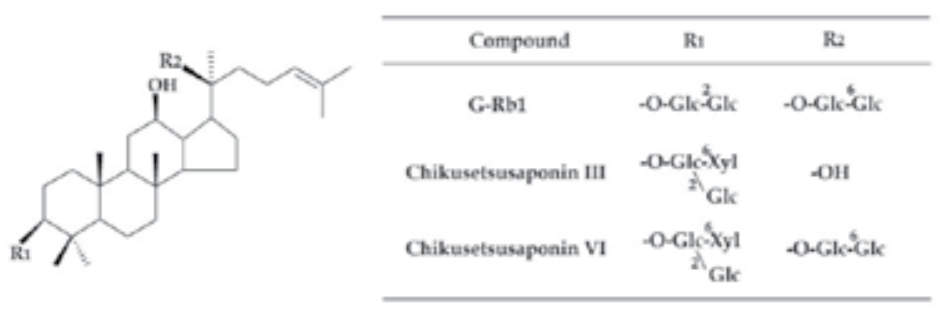

Figure 5. Chemical structures of $\mathrm{G}-\mathrm{Rb}_{1}$ and chikusetsusaponins purified from $P$. japonicus by the anti-G-Rb, immunoaffinity column.

Compound 1 has three sugar moieties in a molecule because that the $R_{\mathrm{F}}$ value closes to that of G-Rd, indicating that this compound is chikusetsusaponin III (Figure 5). Finally, we identified that this compound as chikusetsusaponin III in a direct comparison with authentic sample [32]. Another unknown spot, compound 2 appeared in fraction 5 of the eluted fraction. Thus, compound 2 has a similar molecular structure and high cross-reactivity with G$\mathrm{Rb}_{1}$, and seems to be related ginseng saponin having protopanaxadiol as an aglycone. Moreover, compound 2 might have the same sugar fragments and possess five sugar moiety compared with $\mathrm{G}-\mathrm{Rb}_{1}$, as indicated by their $R_{\mathrm{F}}$ value. From these evidences compound 2 
might be chikusetsusaponin III-20-O-gentiobiose, chikusetsusaponin VI (Figure 5), which has 5 sugars in a molecule in good agreement with the $R_{\mathrm{F}}$ value previously reported [38] and we confirmed that compound 2 is chikusetsusaponin VI by the direct comparison with authentic sample.

These data suggested that the anti-G- $R b_{1}$ immunoaffinity column could isolate some unknown structurally resemble compounds having cross-reactivity against anti-G- $R b_{1} \mathrm{MAb}$. Therefore, this purification system will be applied to survey new compounds related to target compound of MAb. In our previous studies, we demonstrated the immunoaffinity purification against all solasodine glycosides from crude extract by one-step purification. In this case, all solasodine glycoside have almost same cross-reactivity against anti-solamargine $\mathrm{MAb}$ [12].

\subsubsection{Preparation of $G-R b_{1}$ knockout extract by anti-G-R $b_{1}$ immunoaffinity column}

The capacity of this anti-G- $R b_{1}$ immunoaffinity column is $20 \mu \mathrm{g}$ of $\mathrm{G}-\mathrm{Rb} / \mathrm{ml}$ gel [32]. By loading the samples not to exceed the binding capacity against $\mathrm{G}-\mathrm{Rb}_{1}$, this immunoaffinity column becomes possible to remove all $\mathrm{G}-\mathrm{Rb}_{1}$ from crude ginseng extract. Figure 6 showed $\mathrm{H}_{2} \mathrm{SO}_{4}$ staining of TLC of the purification steps by the immunoaffinity column. Lane 1 and 2 were spotted the standard of ginsenosides (G-Rd, G-Rc, G- $\mathrm{Rb}_{1}, \mathrm{G}-\mathrm{Rg}_{1}$, and G-Re). Lane A, B, and $C$ were the crude extract, the washing fraction, and the eluted fraction, respectively. In the crude extract (lane A), all spots of ginsenosides were clearly detected. On the other hand, the washing fraction (lane B) contained all of the ginsenosides in the crude extract except G$R b_{1}$. Furthermore, the spot of $G-R b_{1}$ was detected in the eluted fractions (lane $C$ ). These data strongly indicated that $\mathrm{G}-\mathrm{R} \mathrm{b}_{1}$ molecule in the ginseng extract can be eliminated by an anti$\mathrm{G}-\mathrm{Rb}_{1}$ immunoaffinity column and the washing fractions was knockout only by the antigen molecule, G- $\mathrm{Rb}_{1}$. Thus, we named the washing fractions a knockout (KO) extract $[39,40]$. This KO extract may be useful for the determination of real pharmacologically active principle in the TCMs.
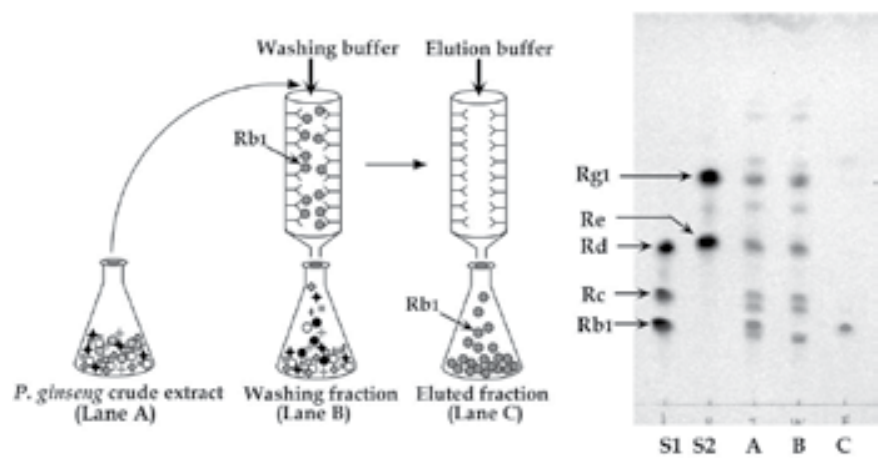

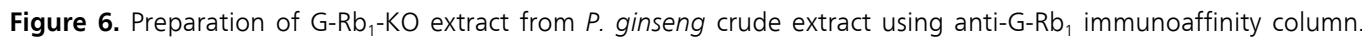
Lane S1 and S2 indicate the standard of ginsenosides (G-Rd, G-Rc, G-Rb,$G-R g_{1}$ and G-Re). Lane A, B, and C were the crude extract, the washing fraction, and the eluted fraction, respectively. 


\section{Glycyrrhizin-knockout extract and its application for in vitro assay}

Licorice (Glycyrrhiza spp.) is also important crude drug used in over $70 \%$ of the TCMs and Japanese Kampo medicines. It is prescribed with other herbal medicines as a demulcent in the treatment of sore throats, an expectorant for coughs and bronchial catarrh, an antitussive, a taste-modifying agent for relieving pain, an anti-inflammatory agent for anti-allergic reactions, rheumatism and arthritis, a prophylactic for liver disease and tuberculosis and adrenocorticoid insufficiency [41-43]. Accumulated evidence indicated that GC, a main saponin component of licorice, is one of the biologically active compounds. It has been reported that GC exhibits numerous pharmacological effects such as anti-inflammation, anti-ulcer, anti-tumor, anti-allergy, and hepatoprotective activities [44,45]. Clinically, GC has been used to treat patients with chronic hepatitis $[46,47]$. Although GC is supposed to be a major active principle in licorice crude extract, a number of studies by HPLC profiles suggested that licorice has many other bioactive components, including flavonoids, isoflavonoids and chalcones [43,48]. Biological studies showed that various flavonoid glycosides and their aglycones of licorice exhibit anti-inflammatory, anti-oxidative, anti-microbial, superoxide scavenging, and anti-carcinogenic activities [43,48]. In order to confirm the role of GC in TCM, we previously purified GC from TCM using an immunoaffinity column conjugated with anti-GC MAb [20]. In this section, we describe the preparation of GC-KO extract and its application for functional analysis of GC in licorice crude extract.

\subsection{Preparation of GC-KO extract by anti-GC immunoaffinity column and the characterization of GC-KO extract}

Our previous study demonstrated the preparation of anti-GC MAb [11]. The cross-reactivities of the anti-GC MAb against glycyrrhetic acid-3-O-glucuronide and glycyrrhetic acid were $0.585 \%$ and $1.865 \%$, respectively. The other related compounds (deoxycholic acid, ursolic acid, and oleanolic acid) were all less than $0.005 \%$. Moreover, we established competitive ELISA and Eastern blotting method using anti-GC MAb [11,49].

The immunoaffinity column against GC was prepared by coupling the purified $60 \mathrm{mg}$ of the anti-GC MAb to $25 \mathrm{ml}$ of an Affi-Gel Hz gel [11]. To eliminate GC from licorice extract, 12 mg of licorice crude extract (GC content: $1275.0 \mu \mathrm{g}$ ) in loading buffer $(5 \% \mathrm{MeOH})$ was applied on the anti-GC MAb immunoaffinity column, and then the loading buffer was continuously circulated through the column to enhance the binding efficiency. After overnight circulation at $4{ }^{\circ} \mathrm{C}$, the unbound fraction was separated. The column was washed with washing buffer $(5 \% \mathrm{MeOH})$ and then eluted with elution buffer $(20 \mathrm{mM}$ phosphate buffer containing $30 \% \mathrm{MeOH})$. After separation, each fraction was deionized and the solvent was lyophilized. Figure 7 showed the recovery ratio of GC checked by ELISA. In the unbound fraction, $3.50 \mu \mathrm{g}$ of GC ( $0.27 \%$ of the applied GC) was detected. On the other hand, 1269.26 $\mu \mathrm{g}$ of GC ( $99.55 \%$ of the applied GC) was obtained in the bound fraction. These data indicate that the anti-GC column could eliminate $99.55 \%$ of the loading GC. Thus, we named this unbound fraction "GC-knockout (GC-KO) extract" [50]. 


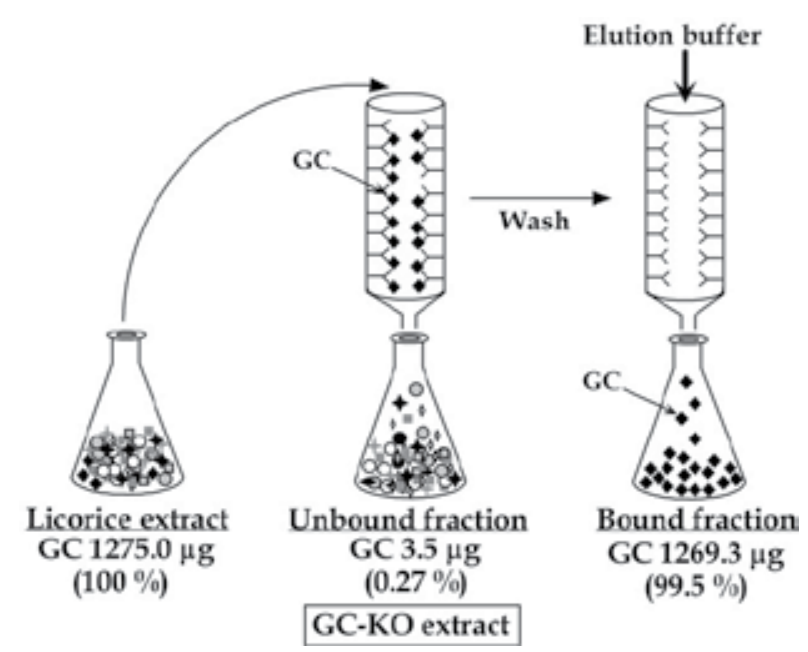

Figure 7. Preparation of GC-KO extract from licorice extract by anti-GC immunoaffinity column.

To further characterize GC-KO extract, the TLC analysis and Eastern blotting were performed [50]. As shown in Figure 8A, several spots including GC were detected in licorice extract (Lane B). However, the spot of GC was completely disappeared in GC-KO extract, although all other spots were clearly detected (lane C). Eastern blotting by antiGC MAb indicated that GC was detected in licorice extract (Figure 8B, lane B), but the spot of GC was disappeared in GC-KO extract (Figure 8B, lane C). Therefore, these data suggest that GC was specifically eliminated from licorice extract by anti-GC MAb immunoaffinity column.

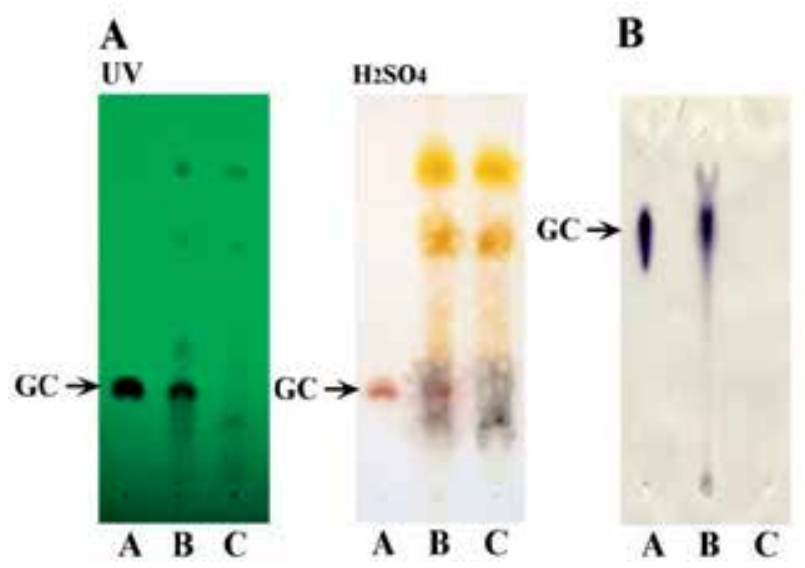

Figure 8. TLC profiles (A) and Eastern blotting by anti-GC MAb (B). Lane A, B, and C indicate GC, licorice extract, and GC-KO extract, respectively. 


\section{2. in vitro Assay by GC-KO extract prepared by anti-GC immunoaffinity column}

Nitric oxide (NO), synthesized by NO synthase (NOS) from L-arginine, is an important regulatory/modulatory mediator for several physiological processes [51]. However, during inflammatory process, a large amount of NO is produced by inducible NOS (iNOS) stimulated by bacterial lipopolysaccharide (LPS) and inflammatory cytokines participates in the pathogenesis of inflammatory diseases [52]. Overproduced NO synthesized by iNOS triggers a variety of inflammatory diseases including sepsis, psoriasis, arthritis, multiple sclerosis, and systemic lupus erythematosus [53]. Therefore, inhibiting NO production by blocking iNOS expression may be useful strategy to treat a variety of inflammatory diseases.

In LPS-treated mouse RAW264 macrophages, licorice extract inhibited NO production and iNOS expression. At $100 \mu \mathrm{g} / \mathrm{mL}$ of licorice extract, iNOS protein and mRNA were completely suppressed [50].

A

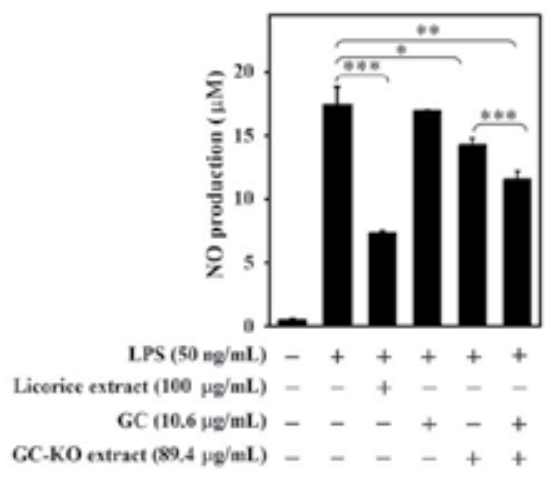

B

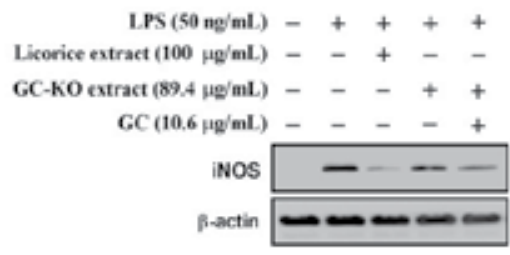

Figure 9. Effect of GC-KO extract and the combination of GC-KO extract and GC on NO production (A) and iNOS protein expression (B) in LPS-treated RAW264 cells.Each bar indicates the mean S.D. of four individual experiments. ${ }^{*} P<0.05,{ }^{* *} P<0.01,{ }^{* * *} P<0.001$ indicate significant differences from the LPS alone

We next examined the inhibitory effect of GC alone, GC-KO extract and the combined treatment with GC and GC-KO extract on NO production [50]. Since $100 \mu \mathrm{g}$ of licorice extract contains $10.6 \pm 0.618 \mu \mathrm{g}$ of GC, the cells were pre-treated with licorice extract $(100 \mu \mathrm{g} / \mathrm{ml})$, GC-KO extract $(89.4 \mu \mathrm{g} / \mathrm{ml})$, or the combination of GC-KO extract $(89.4 \mu \mathrm{g} / \mathrm{ml})$ and GC $(10.6$ $\mu \mathrm{g} / \mathrm{ml})$. Figure9A indicated that the treatment of licorice extract led to a marked suppression of NO production as compared to LPS treatment [inhibition ratio (IR) $57.7 \%$ ]. Interestingly, the inhibitory effect of GC-KO extract was lower (IR 17.8\% ) compared with licorice extract although GC alone could not block NO production as indicated above. On the other hand, the combined treatment with GC-KO extract and GC significantly improved the inhibitory ability (IR 33.5\% ). To determine whether the combinational effect of GC-KO extract and GC was related to iNOS expression, we performed Western blotting. As shown in Figure 9B, the treatment of GC-KO extract diminished the inhibitory ability of LE on iNOS expression, and addition of GC to GC-KO extract could improve it. These data suggest that GC alone cannot 
suppress iNOS expression, but combinational inhibition of iNOS expression may occur when GC coexists with the other constituents contained in licorice extract. The in vitro and in vivo analysis by using $\mathrm{KO}$ extract prepared by immunoaffinity column is a useful approach for determination of potential function of natural compound on in vitro and in vivo assays.

\section{Conclusion}

In this chapter, we introduce the unique strategy of one-step purification of target compounds from crude extract by anti-natural compound specific MAb-conjugated immunoaf-

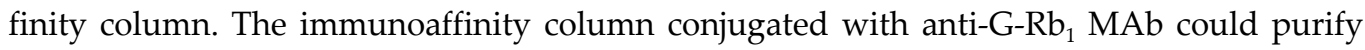
the $\mathrm{G}-\mathrm{Rb}_{1}$ from $P$. ginseng extract, and the washing fraction contained all compounds expect only $\mathrm{G}-\mathrm{Rb}_{1}$, which was named $\mathrm{G}-\mathrm{R} \mathrm{b}_{1}-\mathrm{KO}$ extract. By the use of the cross-reactivity of $\mathrm{MAb}$, the anti-G- $\mathrm{Rb}_{1}$ immunoaffinity column can identify new unknown compounds related to target compound of $\mathrm{MAb}$ and determine their structures. Furthermore, our data suggest that the combination of the immunoaffinity column and ELISA by using MAb provided a reliable and high sensitivity analysis for target compound in various TCMs and crude extract. We also demonstrated the in vitro assay by using GC-KO extract prepared by anti-GC immunoaffinity column from licorice extract. The $\mathrm{KO}$ extract may be able to support the pharmacological investigation for finding out a really active compound in a TCM and crude drug.

\section{Acknowledgements}

This work was funded by the Asahi Beer Science Promoting Foundation and Takeda Science Foundation. The research in this paper was also supported in part by Sasakawa Scientific Research Grant from Japan Science Society and "Science and Technology Research Partnership for Sustainable Development (SATREPS)" supported by the Japan Science and Technology Agency (JST) and the Japan International Cooperation Agency (JICA).

\section{Author details}

Takuhiro Uto ${ }^{1}$, Nguyen Huu Tung ${ }^{1}$, Hiroyuki Tanaka ${ }^{2}$ and Yukihiro Shoyama ${ }^{1 *}$

*Address all correspondence to: shoyama@niu.ac.jp

1 Faculty of Pharmaceutical Sciences, Nagasaki International University „, Japan

2 Faculty of Pharmaceutical Sciences, Kyushu University, Japan 


\section{References}

[1] Ong, E. S. (2002). Chemical assay of glycyrrhizin in medicinal plants by pressurized liquid extraction (PLE) with capillary zone electrophoresis (CZE). J Sep Sci, 25(13), 825-831.

[2] Tan, T. W., Huo, Q., \& Ling, Q. (2002). Purification of glycyrrhizin from glycyrrhiza uralensis fisch with ethanol/phosphate aqueous two phase system. Biochem Lett, 24(17), 1417-1420.

[3] Sakata, R., Shoyama, Y., \& Murakami, H. (1994). Production of monoclonal antibodies and enzyme immunoassay for typical adenylate cyclase activator, Forskolin. Cytotechnology, 16(2), 101-108.

[4] Xuan, L., Tanaka, H., Xu, Y., \& Shoyama, Y. (1999). Preparation of monoclonal antibody against crocin and its characterization. Cytotechnology, 29(1), 65-70.

[5] Lu, Z., Morinaga, O., Tanaka, H., \& Shoyama, Y. (2003). A quantitative ELISA using monoclonal antibody to survey paeoniflorin and albiflorin in crude drugs and traditional Chinese herbal medicines. Biol Pharm Bull, 26(6), 862-866.

[6] Shoyama, Y., Fukada, T., \& Murakami, H. (1996). Production of monoclonal antibodies and ELISA for thebaine and codeine. Cytotechnology, 19(1), 55-61.

[7] Kim, J. S., Tanaka, H., \& Shoyama, Y. (2004). Immunoquantitative analysis for berberine and its related compounds using monoclonal antibodies in herbal medicines. Analyst, 129(1), 87-91.

[8] Tanaka, H., Fukuda, N., \& Shoyama, Y. (1999). Formation of monoclonal antibody against a major ginseng component, ginsenoside $\mathrm{Rb}_{1}$ and its characterization. Cytotechnology; , 29(1), 115-120.

[9] Fukuda, N., Tanaka, H., \& Shoyama, Y. (2000). Formation of monoclonal antibody against a major ginseng component, ginsenoside $\mathrm{Rg}_{1}$ and its characterization Monoclonal antibody for a ginseng saponin. Cytotechnology, 34(3), 197-204.

[10] Zhu, S., Shimokawa, S., Tanaka, H., \& Shoyama, Y. (2004). Development of an assay system for saikosaponin a using anti-saikosaponin a monoclonal antibodies. Biol Pharm Bull, 27(1), 66-71.

[11] Shan, S. J., Tanaka, H., \& Shoyama, Y. (2001). Enzyme-linked immunosorbent assay for glycyrrhizin using anti-glycyrrhizin monoclonal antibody and an eastern blotting technique for glucuronides of glycyrrhetic acid. Anal Chem, 73(24), 5784-5790.

[12] Ishiyama, M., Shoyama, Y., Murakami, H., \& Shinohara, H. (1996). Production of monoclonal antibodies and development of an ELISA for solamargine. Cytotechnolo$g y, 18(3), 153-158$. 
[13] Morinaga, O., Tanaka, H., \& Shoyama, Y. (2000). Production of monoclonal antibody against a major purgative component, sennoside A, its characterization and ELISA. Analyst, 125(8), 1109-1113.

[14] Morinaga, O., Nakajima, S., Tanaka, H., \& Shoyama, Y. (2001). Production of monoclonal antibodies against a major purgative component, sennoside $\mathrm{B}$, their characterization and use in ELISA. Analyst, 126(8), 1372-1376.

[15] Tanaka, H., Goto, Y., \& Shoyama, Y. (1996). Monoclonal antibody based enzyme immunoassay for marihuana (cannabinoid) compounds. Immunoassay, 17(4), 321-342.

[16] Loungratana, P., Tanaka, H., \& Shoyama, Y. (2004). Production of monoclonal antibody against ginkgolic acids in Ginkgo biloba Linn. Am J Chin Med, 32(2), 33-48.

[17] Yanagihara, H., Sakata, R., Minami, H., Shoyama, Y., \& Murakami, H. (1996). Immunoaffinity column chromatography against forskolin using an anti-forskolin monoclonal antibody and its application. Anal Chim Acta, 335(1-2), 63 -70.

[18] Putalun, W., Tanaka, H., \& Yukihira, S. (1999). Rapid separation of solasodine glycosides by an immunoaffinity column using anti-solamargine monoclonal antibody. Cytotechnology, 31(1-2), 151-156.

[19] Fukuda, N., Tanaka, H., \& Shoyama, H. (2000). Isolation of the pharmacologically active saponin ginsenoside $\mathrm{Rb}_{1}$ from ginseng by immunoaffinity column chromatography. J Nat Prod, 63(2), 283-285.

[20] Xu, J., Tanaka, H., \& Shoyama, Y. (2007). One-step immunochromatographic separation and ELISA quantification of glycyrrhizin from traditional Chinese medicines. $J$ Chromatog B, 850(1-2), 53-58.

[21] Gillis, C. N. (1997). Panax ginseng pharmacology: a nitric oxide link? Biochem Pharmacol, 54(1), 1-8.

[22] Liu, C. X., \& Xiao, P. G. (1992). Recent advances on ginseng research in China. J. ethnopharmacol, 36(1), 27-38.

[23] Yu, H., Zhang, C., Lu, M., Sun, F., Fu, Y., \& Jin, F. (2007). Purification and characterization of new special ginsenosidase hydrolyzing multi-glycisides of protopanaxadiolginsenosides, ginsenosidase type I. Chem Pharm Bull (Tokyo), 55(2), 231-235.

[24] Kitagawa, I., Taniyama, T., Yoshikawa, M., Ikenishi, Y., \& Nakagawa, Y. (1989). Chemical studies on crude drug processing. IV. Chemical structures of malonyl-ginsenosides $\mathrm{Rb}_{1}, \mathrm{Rb}_{2}$, Re and $\mathrm{Rd}$ isolated from the root of Panax Ginseng C.A. Meyer. Chem Pharm Bull, 37(11), 2961-2970.

[25] Tanaka, O. (1989). Saponin-composition of Panax species. In: Shibata S, Ohtsuka Y, Saito H. (eds.) Recentadvances in ginseng studies. Tokyo, Hirokawa Publishing, 43-47.

[26] Fuzzati, N. (2004). Analysis methods of ginsenosides. J Chromatogr B Analyt Technol Biomed Life Sci, 812(1-2), 119 -33. 
[27] Washida, D., \& Kitanaka, S. (2003). Determination of polyacetylenes and ginsenosides in Panax species using high performance liquid chromatography. Chem Pharm Bull, 51(11), 1314-1317.

[28] Mook-Jung, I., Hong, H. S., Boo, J. H., Lee, K. H., Yun, S. H., Cheong, M. Y., Joo, I., Huh, K., \& Jung, M. W. (2001). Ginsenoside $\mathrm{Rb}_{1}$ and $\mathrm{Rg}_{1}$ improve spatial learning and increase hippocampal synaptophysin level in mice. J Neurosci Res, 63(6), 509-515.

[29] Lim, J. H., Wen, T. C., Matsuda, S., Tanaka, J., Maeda, N., Peng, H., Aburaya, J., Ishihara, K., \& Sakanaka, M. (1997). Protection of ischemic hippocampal neurons by ginsenoside $\mathrm{Rb}_{1}$, a main ingredient of ginseng root. Neurosci Res, 28(3), 191-200.

[30] Liu, M., \& Zhang, J. (1995). Effects of ginsenoside $\mathrm{Rb}_{1}$ and $\mathrm{Rg}_{1}$ on synaptosomal free calcium level, ATPase and calmodulin in rat hippocampus. Chin Med J (Engl), 108(7), 544-547.

[31] Jiang, K. Y., \& Qian, Z. N. (1995). Effects of Panax notoginseng saponins on posthypoxic cell damage of neurons in vitro. Zhongguo Yao Li Xue Bao, 16(5), 399-402.

[32] Fukuda, N., Tanaka, H., \& Shoyama, Y. (2000). Applications of ELISA, western blotting and immunoaffinity concentration for survey of ginsenosides in crude drugs of Panax species and traditional Chinese herbal medicines. Analyst, 125(8), 1425-1429.

[33] Wengatz, I., Schmid, R. D., Kreißig, S., Wittmann, C., Hock, B., Ingendoh, A., \& Hillenkamp, F. (1992). Determination of the hapten density of immuno-conjugates by matrix-assisted UV laser desorption/ionization mass spectrometry. Anal Lett, 25(11), 1983-1997.

[34] Morinaga, O., Tanaka, H., \& Shoyama, Y. (2006). Detection and quantification of ginsenoside Re in ginseng samples by a chromatographic immunostaining method using monoclonal antibody against ginsenoside Re. J Chromatography B, 830(1), 100-104.

[35] Tanaka, H., Fukuda, N., Yahara, S., Isoda, S., Yuan, C. S., \& Shoyama, Y. (2005). Isolation of ginsenoside $\mathrm{Rb}_{1}$ from Kalopanax pictus by eastern blotting using anti-ginsenoside $\mathrm{Rb}_{1}$ monoclonal antibody. Phytother Res, 19(3), 255-258.

[36] Yahara, S., Kasai, R., \& Tanaka, O. (1977). New dammarane type saponins of leaves of Panax japonicus C.A. Meyer. (1). Chikusetsusaponins $\mathrm{L}_{5}, \mathrm{~L}_{9 \mathrm{a}}$ and $\mathrm{L}_{10}$. Chem Pharm Bull, 25(8), 2041-2047.

[37] Morita, T., Tanaka, O., \& Kohda, H. (1985). Saponin composition of rhizomes of Panax japonicus collected in South Kyushu, Japan, and its significance in oriental traditional medicine. Chem Pharm Bull, 33(9), 3852-3858.

[38] Kohda, H., Tanaka, S., Yamaoka, Y., \& Ohhara, Y. (1991). Saponins from Amaranthus hypochondriacus. Chem Pharm Bull, 39(10), 2609-2612.

[39] Tanaka, H., Fukuda, N., \& Shoyama, Y. (2007). Eastern blotting and immunoaffinity concentration using monoclonal antibody for ginseng saponins in the field of traditional chinese medicines. J Agric Food Chem, 55(10), 3783-3787. 
[40] Wang, C. A., \& Shoyama, Y. (2006). Herbal medicine: identification, analysis, and evaluationstrategies. In: Yuan CS, Bieber EJ, Bauer BA (eds) Textbook of complementary and alternative medicine, second edition. United Kingdom Informa Healthcare, 51-70.

[41] Kim, S. C., Byun, S. H., Yang, C. H., Kim, C. Y., Kim, J. W., \& Kim, S. G. (2004). Cytoprotective effects of Glycyrrhizae radix extract and its active component liquiritigenin against cadmium-induced toxicity (effects on bad translocation and cytochrome c-mediated PARP cleavage). Toxicology, 197(3), 239-251.

[42] Fuchikami, J., Isohama, Y., Sakaguchi, M., Matsuda, M., Kucota, T., Akie, Y,k., Fujino, A., \& Miyata, T. (2004). Effect of glycyrrhizin on late asthmatic responses induced by antigen inhalation in guinea pigs. J Pharmacol Sci, 94(Suppl.1), 251.

[43] Asl, M. N., \& Hosseinzadeh, H. (2008). Review of pharmacological effects of Glycyrrhiza sp. and its bioactive compounds. Phytother Res, 22(6), 709-724.

[44] Jakkula, M., Boucher, T. A., Beyendorff, U., Conn, S. M., Johnson, J. E., Nolan, C. J., Peine, C. J., \& Albrecht, J. H. (2004). A randomized trial of Chinese herbal medicines for the treatment of symptomatic hepatitis C. Arch Intern Med, 164(12), 1341-1346.

[45] Yanagawa, Y., Ogura, M., \& Fujimoto, E. (2004). Effects and cost of glycyrrhizin in the treatment of upper respiratory tract infections in members of the Japanese maritime self-defense force: Preliminary report of a prospective, randomized, doubleblind, controlled, parallel-group, alternate-day treatment assignment clinical trial. Curr Ther Res Clin Exot, 65(1), 26-33.

[46] Schalm, S. W., Brouwer, J. T., Bekkering, F. C., \& van Rossum, T. G. (1999). New treatment strategies in non-responder patients with chronic hepatitis C. J Hepatol, 31(Suppl.1), $1184-1188$.

[47] Coon, J. T., \& Ernst, E. (2004). Complementary and alternative therapies in the treatment of chronic hepatitis C: a systematic review. J Hepatol, 40(3), 491-500.

[48] Chin, Y. W., Jung, H. A., Liu, Y., Su, B. N., Castoro, J. A., Keller, W. J., Pereira, M. A., \& Kinghorn, A. D. (2007). Anti-oxidant constituents of the roots and stolons of licorice (Glycyrrhiza glabra). J Agric Food Chem, 55(12), 4691-4697.

[49] Morinaga, O., Fujino, A., Tanaka, H., \& Shoyama, Y. (2005). An on-membrane quantitative analysis system for glycyrrhizin in licorice roots and traditional Chinese medicines. Anal Bioanal Chem, 383(4), 668-672.

[50] Uto, T., Morinaga, O., Tanaka, H., \& Shoyama, Y. (2012). Analysis of the synergistic effect of glycyrrhizin and other constituents in licorice extract onlipopolysaccharideinduced nitric oxide production using knock-out extract. Biochem Biophys Res Commun, 417(1), 473-478.

[51] Moncada, S., Palmer, R. M., \& Higgs, E. A. (1991). Nitric oxide: physiology, pathophysiology, and pharmacology. Pharmacol Rev, 43(2), 109-142. 
[52] Blantz, R. C., \& Munger, K. (2002). Role of nitric oxide in inflammatory conditions. Nephron, 90(4), 373-378.

[53] Kröncke, K. D., Fehsel, K., \& Kolb-Bachofen, V. (1998). Inducible nitric oxide synthase in human diseases. Clin Exp Immunol, 113(2), 147-156. 

Chapter 3

\title{
Standard Operating Procedures (SOP) for the Spectrophotometric Determination of Phenolic Compounds Contained in Plant Samples
}

\author{
Elba Lúcia Cavalcanti de Amorim, \\ Valérium Thijan Nobre de Almeida de Castro, \\ Joabe Gomes de Melo, \\ Allan Jonathan Chernichiarro Corrêa and \\ Tadeu José da Silva Peixoto Sobrinho
}

Additional information is available at the end of the chapter

http://dx.doi.org/10.5772/51686

\section{Introduction}

The quality control of raw materials and products from plants is one of the topics most discussed by universities and health surveillance agencies. One of the primary tools used to ensure the reliability of production processes is the use of Standard Operating Procedures (SOPs). SOPs sequentially describe the steps of a particular methodology so that it can be reproduced by different analysts, which minimises variations in their implementation and improves the standardisation of the final product.

Several techniques, such as high performance liquid chromatography, gas chromatography and mass spectrometry, can be used in SOPs to control the quality of plant phenolic compounds $[1,2]$. However, these compounds have a characteristic spectrum produced by the double bonds in the aromatic rings and substituent positions that facilitates their identification and the development of spectrometric analytical techniques is easily accomplished. In this sense, spectrophotometric methods are more practical, reproducible and inexpensive than other techniques and are therefore favoured for the development of analytical methodologies for such determinations.

In addition to producing compounds such as carbohydrates, lipids, proteins and nucleic acids directly involved in their essential growth functions, plants have an arsenal of enzymes capable 
of producing, processing and accumulating several other substances not necessarily related to the maintenance of their life [3]. All of these reactions can be defined as secondary metabolism, the products of which provide advantages for both survival and species perpetuation in the plant's ecosystem [4]. However, this protection has a cost for the plant because metabolic resources that could increase its biomass are used to produce these compounds. In addition to protection, secondary metabolites perform important ecological functions such as inhibiting the germination and growth of other plants, attracting both pollinators and seeddispersing animals and providing chemical defences against microorganisms [5].

Phenolic compounds, which have one or more hydroxyl groups linked to an aromatic ring, stand out from other classes of plant secondary metabolites because they are widely distributed and have various ecological functions that are scientifically proven to have numerous pharmacological activities and are well represented by tannins, flavonoids and coumarins.

\subsection{Tannins}

Tannins are water soluble phenolic compounds with a molecular weight between 500 and 3000 Daltons and may be chemically classified into two groups: hydrolysable tannins and condensed tannins [6,7]. Hydrolysable tannins are connected by ester-carboxyl linkages, which undergo hydrolysis under acidic and basic conditions [8]. Figure 1 presents an example of hydrolysable tannin (gallotannin), connected through a polyol (usually $\beta$-D-glucose) with the hydroxyl group esterified by gallic acid. Polyphenols connected with ellagic acid are called ellagitannins [9].<smiles>O=C(O)c1cc(O)c(O)c(O)c1</smiles>

Gallic acid<smiles>O=c1oc2c(O)c(O)cc3c(=O)oc4c(O)c(O)cc1c4c23</smiles>

Ellagic acid<smiles>O=C(OCC1OC(OC(=O)c2cc(O)c(O)c(O)c2)C(O)C(OC(=O)c2cc(O)c(O)c(O)c2)C1O)c1cc(O)c(O)c(O)c1</smiles>

1,3,6-tri-O-galloyl- $\beta$-D-glucose

Figure 1. Structure of gallic acid, ellagic acid and 1,3,6-tri-O-galloyl- $\beta$-D-glucose, a hydrolysable tannin. 
Condensed tannins, also known as proanthocyanidins (Figure 2), can contain dozens of units of flavan-3-ols (catechin) or flavan-3,4-diols (leucoanthocyanidins). These units have a complex structure and are resistant to hydrolysis; however, they can be soluble in aqueous organic solvents because of their structure [7].

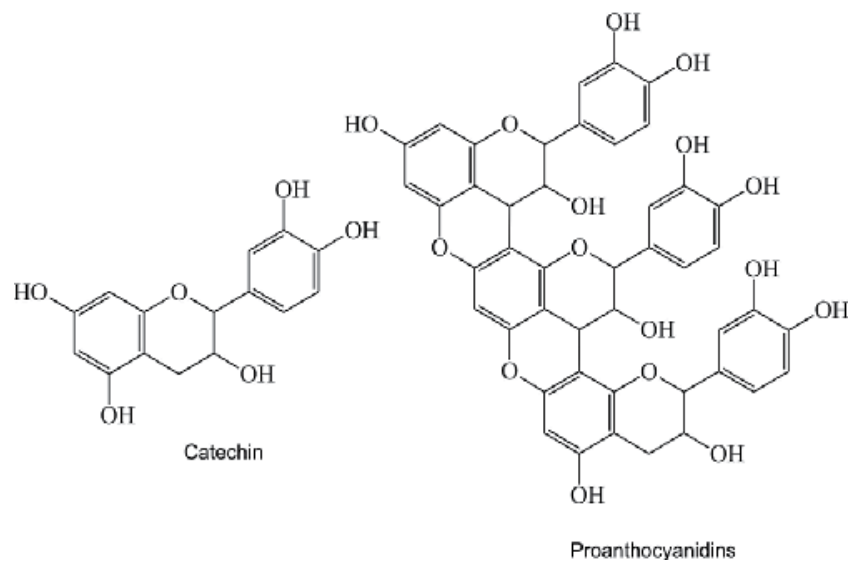

Figure 2. Basic structures of catechin and proanthocyanidins (condensed tannin).

Hydrolysable and condensed tannins may occur in the same plant simultaneously. However, the hydrolysable tannins are characteristic of Magnoliopsida herbaceous and woody plants and are restricted to certain taxonomic families. Ellagitannins have been used as taxonomic markers, particularly for Hemamelidaceae, Dilenidaceaa and Rosaceae. Condensed tannins have been identified in all plant groups, including Gymnosperms and Pteridophytes [10].

These secondary metabolites were initially identified by their astringent taste and capacity to bind proteins, which allows for the precipitation and formation of complexes with collagen skin fibres to increase their resistance to water and heat. Chemically speaking, hydrophobic interactions and hydrogen bonds between the phenolic groups in tannins and some macromolecules explain these features. However, the stability of the formed complexes only results after the formation of covalent bonds via the oxidation of tannins by quinones [11].

Since antiquity, plants containing tannins have been used medicinally as anti-inflammatory, antimicrobial, antitumor and antiviral agents and to treat both wound sand burns [7]. Tannins are also used to manufacture beverages and process animal skin into leather. Some researchers have shown that tannins protect plants against attack by herbivores and pathogens [12].

Although the use of tannin in the tanning industry has become restricted, interest in studying the ingestion of foods containing tannins to prevent diseases such as atherosclerosis or certain types of cancer has increased because of various epidemiological studies. Some studies report that the complexation of tannins with proteins gives them an important role in controlling bacteria, fungi and insects [13-15]. Other studies examined the inhibitory action of the enzyme reverse transcriptase [16] and the anticarcinogenic activity associated with 
green tea and diets rich in fruits containing tannins [17]. It is generally believed that the pharmacological activity of tannins occurs via their complexation with metal ions, antioxidant activity or the ability to complex with macromolecules.

Maytenus ilicifolia (Schrad.) Planch. (Celastraceae), popularly known as "espinheira-santa", is a species native to Brazil traditionally used to treat digestive disorders and the literature cites tannins as compounds that act to protect the stomach by helping to treat ulcers and gastritis [18-20]. In addition to these activities, M. ilicifolia has other pharmacological applications, such as anticancer, antimicrobial and antioxidant activity and treatment of the central nervous system [21]. For these reasons, M. ilicifolia was chosen as a reference species in this study.

\subsection{Flavonoids}

Flavonoids comprise a group of natural substances with great structural diversity and there are currently more than nine thousand known flavonoids that do not occur in humans but can be found in various plant parts such as the leaves, fruits, bark, roots, stems and flowers $[22,23]$. Flavonoids (Figure 3) are composed of a simple skeleton containing two phenol rings connected by a propionic chain; where ring $\mathrm{A}$ is the acetate derivative and both ring $\mathrm{B}$ and the three-carbon bridge are derived via a shikimate pathway, which may be associated with carbohydrates (heterosides), un associated (aglycones) or polymerised further (anthocyanins) [24].

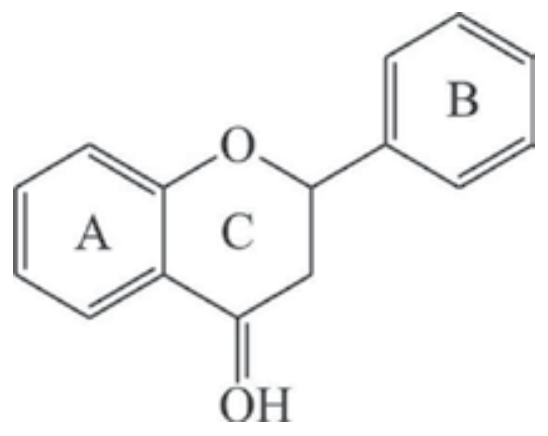

Figure 3. Basic structure of flavonoids.

This class of metabolites has several biological functions, such as defence against both herbivores and pathogens, the perpetuation of the species by attracting seed dispersing animals, protection from ultraviolet rays and allelopathy [24,25]. Flavonoids also possess important pharmacological properties, such as antioxidant, antiinflammatory, anti-thrombogenic, antimicrobial, anticancer, antidiabetic and hypocholesterolemic activities [23,26].

Studies show that flavonoids are chemical markers responsible for various pharmacological activities performed by the genus Bauhinia [27-29]. Bauhinia forficata Link (Fabaceae), popularly known as "pata-de-vaca", is a tree native to Brazil that prevails in the phytogeographical area of Mata Atlântica [30] and can also be found in Cerrado. Pata-de-vaca is used in folk 
medicine as a hypoglycemic, diuretic and antihypertensive agent [31,32]. Several plantbased products are sold in open markets, pharmacies and natural product stores [33]. Though not officially recommended, herbal products from the leaves of pata-de-vaca (Bauhinia L.) are popularly used for therapeutic purposes. This plant was used in our study as a reference species for flavonoids.

\subsection{Coumarins}

Coumarins are lactones of $O$-hydroxy-cinnamic acid derived from trans-cinnamic acid via oxidation-reduction and isomerisation to produce 1,2-benzopyrone. Coumarins are divided into simple coumarins, furanocoumarins, pyranocoumarins, dimeric coumarins and chromones (Figure 4) [34]. The difference between these classes is the position of the radical in the coumarin lactone ring, which varies between C-6, C-7 and C-8. Chromones represent a group of natural isomers to coumarin that may be linearly and angularly prenylated at C-6 and C-8, respectively, in furanochromones and pyranochromones, respectively [34].<smiles>O=C1CCc2ccccc2O1</smiles>

Simple coumarin<smiles>CC1(C)C=Cc2cc3ccc(=O)oc3cc2O1</smiles>

Pyranocoumarin<smiles>O=c1ccc2cc3ccoc3cc2o1</smiles>

Furanocoumarin<smiles>O=c1ccoc2ccccc12</smiles>

Chromone<smiles>O=c1oc2ccccc2c(O)c1Cc1c(O)c2ccccc2oc1=O</smiles>

Dimeric coumarin

Figure 4. Basic structure of coumarins and chromones.

Coumarins are used as antioxidants, anti-HIV drugs, antispasmodics, spasmolytics, hypolipidemics, hypotensives and vasodilating agents [34]; however, they are also used in food flavouring, perfumes, tobacco and cosmetic products [35]. It is estimated that the daily human exposure to coumarins from cosmetics and perfumes is $0.04 \mathrm{mg} / \mathrm{kg} / \mathrm{day}$ [36].

Coumarins are a class of secondary metabolites widely distributed throughout the Plantae kingdom and found in Fungi and Bacteria as well [37]. In plants, coumarins are found frequently in the families of Apiaceae, Rutaceae and Asteraceae, and less frequently in the families of Fabaceae, Oleaceae, Moraceae and Thymeleaceae [38]. 
Mikania glomerata Spreng. (Asteraceae), popularly known in Brazil as "guaco", has existed in the Brazilian Pharmacopoeia since 1929 and is used as an expectorant to treat respiratory problems. Most herbal products are marketed as some form of syrup; however, they are produced from fluid extracts and tinctures [39,40]. Chemical studies of this species show coumarins to be a major component that can be used as a chemical marker for the quality control of products based on guaco [41-43].

Coumarins have a characteristic UV spectrum due to the nature and position of their substituents, which facilitates both their identification and the development of analytical spectrophotometric techniques [11]. For these reasons, M. glomerata was chosen as the reference species to quantify the coumarin level.

\section{Problem statement}

Despite extensive literature presenting various analytical methods, the development of an SOP is often difficult for three reasons: 1 ) the work does not detail the difficulties and adjustments required to implement the methodology, 2) the steps are not clearly presented for reproduction and 3) the limits of interpretation are not discussed. One criterion recommended by the National Sanitary Surveillance Agency (Agência Nacional de Vigilância Sanitária ANVISA) in Brazil for the standardisation of herbal drugs is the active compound content or chemical class, which is the total concentration of tannins for products based on M. ilicifolia (espinheira-santa) [44], flavonoids for B. forficata (pata-de-vaca) $[45,46]$ and coumarins for $M$. glomerata (guaco) [44].

Thus, this paper presents research protocols adopted by our research group to study the levels of tannins, flavonoids and coumarins from plant extracts and the experimental application of these SOPs to analyse products sold in markets (pharmacies and natural product stores) as phytomedicines ${ }^{1}$ or plant drugs ${ }^{2}$ with high commercial value.

\section{Standard operating procedures (POP)}

The following SOPs describe the chemical classes to be analysed and the chemical basis of the methods. They provide a detailed list of all the reagents required for the preparation and describe the experimental procedure to be followed. Finally, there is a list of references used in the development of the SOP.

1 "All medicine is obtained using solely active raw vegetables. It is characterised by knowledge of the effectiveness and risks of their use, as well as the reproducibility and consistency of its quality. Its efficacy and safety are validated through ethnopharmacological surveys of use, documentation, technical and scientific publications or clinical trial phase 3"[44].

2 "Medicinal plant or their parts, after collection processes, stabilisation and drying and can be full, erasures, crushed or powdered" [44]. 


\subsection{Standard operating procedure for the quantification of tannins}

Description: Tannins are phenolic compounds with the ability to bind macromolecules, especially proteins. This class of compounds has traditionally been divided into two groups: hydrolysable and condensed tannins. Hydrolysable tannins are characterised by a central polyol, usually $\beta$-D-glucose, containing hydroxy groups esterified with gallic acid and ellagic acid. Condensed tannins are oligomers or polymers formed by the condensation of two or more molecules of flavan-3-ol or flavan-3,4-diol [3] [7].

Principle of the method: The phenolic compounds in the sample are oxidised using the FolinCiocalteu reagent. This reagent is a mixture of phosphotungstic and phosphomolybdic acids that are reduced by the oxidation of phenolic compounds in a mixture of tungsten and molybdenum oxides. The blue colour produced by the oxides has a maximum absorption at $760 \mathrm{~nm}$ and is proportional to the total phenolic concentration [47]. The tannin content is calculated as the difference between the total and waste phenol content.

\section{Reagents:}

(1) Tannic acid $(0.1 \mathrm{mg} / \mathrm{mL}, \mathrm{w} / \mathrm{v})$ : Dissolve $10 \mathrm{mg}$ of tannic acid in $100 \mathrm{ml}$ of distilled water.

(2) Folin-Ciocalteu reagent $(10 \%, v / v)$ : Dilute $5 \mathrm{ml}$ of Folin-Ciocalteu reagent with $45 \mathrm{~mL}$ of distilled water.

(3) Sodium carbonate $\mathrm{Na}_{2} \mathrm{CO}_{3}\left(7.5 \%\right.$, w/v): Dissolve $7.5 \mathrm{~g}$ of $\mathrm{Na}_{2} \mathrm{CO}_{3}$ in $100 \mathrm{ml}$ of distilled water. If necessary, solubilising the solution on a heating plate and magnetic stirrer.

(4) Methanol $(80 \%, \mathrm{v} / \mathrm{v})$ : Dilute $800 \mathrm{ml}$ of methanol with $200 \mathrm{ml}$ of distilled water.

The reagent volume is sufficient to examine a maximum of 100 analyses.

Preparation of samples: The powdered sample $(500 \mathrm{mg})$ should be extracted with $50 \mathrm{~mL}$ of $80 \%$ methanol for 30 minutes on a hot plate. The extract should be filtered through filter paper into a $50 \mathrm{~mL}$ volumetric flask and the volume should be completed using the same solvent. The final extract concentration will be $10 \mathrm{mg} / \mathrm{mL}$. If the products are liquid, they must be evaporated. The dried extract should be dissolved in $80 \%$ methanol for a final concentration of $1 \mathrm{mg} / \mathrm{mL}$.

Calibration curve: The calibration curve must be prepared using 100-500 $\mu \mathrm{L}$ aliquots of the tannic acid solution, $500 \mu \mathrm{L}$ of the Folin-Ciocalteu solution and $1 \mathrm{~mL}$ of the sodium carbonate solution. The final volume should be adjusted to $10 \mathrm{~mL}$ with distilled water. The final tannic acid concentration will be $1-5 \mu \mathrm{g} / \mathrm{mL}$.

Measurement procedure: To quantify the total phenol concentration (that is, all of the phenols present in the sample), $500 \mu \mathrm{L}$ of the extract must be transferred to a test tube. Next, 500 $\mu \mathrm{L}$ of the Folin-Ciocalteu solution, $1 \mathrm{~mL}$ of the sodium carbonate solution and $8 \mathrm{ml}$ of distilled water are added. The samples remain at room temperature for 30 minutes. The spectrophotometer should be adjusted to a wavelength of $760 \mathrm{~nm}$ and the equipment must be rinsed with distilled water. To quantify the phenol waste, (i.e., the phenols present in the sample except for the proteins precipitated with the tannin) $500 \mathrm{mg}$ of casein is weighed and 
transferred into a $25 \mathrm{ml}$ Erlenmeyer flask before adding $5 \mathrm{ml}$ of the extract and $5 \mathrm{ml}$ of water distilled. After two hours (time required for the complexing of the tannins to the total protein), the extracts are filtered into a $10 \mathrm{~mL}$ volumetric flasks and its volume is adjusted with distilled water. The phenols are considered to be equal to the residue from the total phenol. These assays are performed at least in triplicate and the total phenolic content is expressed as milligrams of tannic acid equivalents per gram of sample or extract (mg TAE/g).

\section{References:}

Amorim E. L. C, Nascimento J. E., Monteiro J. M., Peixoto Sobrinho T. J. S, Araújo T. A. S., Albuquerque U. P. A simple and accurate procedure for the determination of tannin and flavonoid levels and some applications in ethnobotany and ethnopharmacology. Functional Ecosystems and Communities 2008; 2(1) 88-94 [47].

Santos S. C., Mello J. C. P. Taninos. In: Simões C. M. O., Schenkel E. P., Gosmanm, G., Mello J. C. P., Mentz L. A., Petrovick P. R. (ed.) Farmacognosia: da planta ao medicamento. Porto Alegre: Universidade Federal do Rio Grande do Sul; 2004. p.615-656 [3].

\subsection{Standard operating procedure for the quantification of flavonoids}

Description: Flavonoids are the most important and diverse phenolic compounds. Most flavonoids have 15 carbon atoms and consist of two phenol rings connected by a chain of three carbons to form a tricyclic compound [24].

Principle of the method: The aluminium ion $\left(\mathrm{Al}^{3+}\right)$ is reacted with the flavonoids in the sample to form the stable flavonoid-Al ${ }^{3+}$ complex, which has a yellow colour and intensity proportional to the flavonoid concentration. This reaction causes a bathochromic shift and intensification in the absorption, which can be measured without influence from other phenolic compounds present in the sample [48].

\section{Reagents:}

(1) Rutin $(0.1 \mathrm{mg} / \mathrm{mL}, \mathrm{w} / \mathrm{v})$ : Dissolve $10 \mathrm{mg}$ of rutin in $100 \mathrm{ml}$ of methanol.

(2) Acetic acid solution $(60 \%, \mathrm{v} / \mathrm{v})$ : Dilute $30 \mathrm{ml}$ of acetic acid with $20 \mathrm{ml}$ of methanol.

(3) Pyridine Solution $(20 \%, \mathrm{v} / \mathrm{v})$ : Dilute $40 \mathrm{ml}$ of pyridine with $160 \mathrm{ml}$ of methanol.

(4) Aluminium chloride solution $\mathrm{AlCl}_{3}(5 \%, \mathrm{w} / \mathrm{v})$ : Dissolve $5 \mathrm{~g} \mathrm{AlCl}_{3}$ in $100 \mathrm{~mL}$ of methanol. If necessary, complete dissolution via magnetic stirring.

(5) Methanol $(80 \%, \mathrm{v} / \mathrm{v})$. Dilute $80 \mathrm{ml}$ of methanol with $20 \mathrm{ml}$ of distilled water.

The reagent volume is sufficient to examine a maximum of 100 analyses.

Preparation of samples: The powdered sample $(500 \mathrm{mg})$ should be extracted with $50 \mathrm{~mL}$ of $80 \%$ methanol on a hot plate for 30 minutes. The extract should be filtered through filter paper into a $50 \mathrm{~mL}$ volumetric flask and the volume should be adjusted with the same solvent. The final extract concentration will be $10 \mathrm{mg} / \mathrm{mL}$. Liquid products must be evaporated. The dried extract should be dissolved in $80 \%$ methanol to obtain a final concentration of $1 \mathrm{mg} / \mathrm{mL}$. 
Calibration curve: The calibration curve must be prepared using 100-1000 $\mu \mathrm{L}$ aliquots of the rutin solution, $500 \mu \mathrm{L}$ of the acetic acid solution, $2 \mathrm{~mL}$ of the pyridine solution and $1 \mathrm{ml}$ of the reagent aluminium chloride solution. The final volume should be adjusted to $10 \mathrm{~mL}$ with $80 \%$ methanol. The final rutin concentration will be $1-10 \mu \mathrm{g} / \mathrm{mL}$.

Measurement procedure: To quantify the flavonoids, $500 \mu \mathrm{L}$ of the extract should be transferred to a test tube. Next, $500 \mu \mathrm{L}$ of the acetic acid solution, $2 \mathrm{~mL}$ of the pyridine solution, 1 $\mathrm{ml}$ of the reagent aluminium chloride solution and $6 \mathrm{ml}$ of $80 \%$ methanol will be added. The samples remain at room temperature for 30 minutes. The spectrophotometer should be adjusted to a wavelength of $420 \mathrm{~nm}$ and the equipment must be rinsed with distilled water. The test shall be performed at least in triplicate and the flavonoid content is expressed as milligrams of rutin equivalents per gram of sample or extract (mg RE/g).

\section{References:}

Peixoto Sobrinho T. J. S, Silva C. H. T. P., Nascimento J. E., Monteiro J. M., Albuquerque U. P., Amorim E. L. C. Validação de metodologia espectrofotométrica para quantificação dos flavonóides de Bauhinia cheilantha (Bongard) Steudel. Brazilian Journal of Pharmaceutical Sciences 2008; 44 (4) 683-689 [48].

Zuanazzi, J. A. S.; Montanha, J. A. Flavonóides. In: Simões C. M. O., Schenkel E. P., Gosmanm, G, Mello J. C. P., Mentz L. A., Petrovick P. R. (ed.) Farmacognosia: da planta ao medicamento. Porto Alegre: Universidade Federal do Rio Grande do Sul; 2004, p.577-614 [24].

\subsection{Standard operating procedure for the quantification of coumarins}

Description: Coumarins are lactones of $O$-hydroxy-cinnamic acid and are metabolites of phenylalanine. 1,2-benzopyrone is the simplest coumarin representative, others being furocoumarins, pyranocoumarins, dimeric coumarins and chromones [34].

Principle of the method: The Borntrager reaction is based on the solubility of free coumarin derivatives in polar organic solvents and the solubility of their soluble alkali phenolates. Coumarin absorbs at $280 \mathrm{~nm}$; however, ionisation of phenolic hydroxyls in the molecule by alkaline hydroxide causes a bathochromic deviation to $320 \mathrm{~nm}$, which is proportional to the coumarin concentration [40].

\section{Reagents:}

(1) 1,2-benzopyrone (1 mg/mL, w/v): Dissolve $10 \mathrm{mg}$ of coumarin in $10 \mathrm{ml}$ of distilled water.

(2) Lead acetate $(5 \%, \mathrm{w} / \mathrm{v})$ : Dissolve $2,5 \mathrm{~g}$ of lead acetate in $50 \mathrm{ml}$ of distilled water.

(3) Hydrochloric acid solution, $\mathrm{HCl}(0.1 \mathrm{M}, \mathrm{v} / \mathrm{v})$ : Dilute $10 \mathrm{ml}$ of concentrated hydrochloric acid with $1000 \mathrm{ml}$ of distilled water.

(4) Methanol (80\%, v/v): Dilute $80 \mathrm{ml}$ of methanol with $20 \mathrm{ml}$ of distilled water.

The reagent volume is sufficient to examine a maximum of 100 analyses.

Preparation of samples: The powdered sample $(500 \mathrm{mg})$ should be extracted with $50 \mathrm{~mL}$ of $80 \%$ methanol for 30 minutes on a hot plate. The extract should be filtered through filter paper 
into a $50 \mathrm{~mL}$ volumetric flask and the final volume should be adjusted with the same solvent. The final extract concentration will be $10 \mathrm{mg} / \mathrm{mL}$. Liquid products must be evaporated. The dried extract should be dissolved in $80 \%$ methanol for a final concentration of $1 \mathrm{mg} / \mathrm{mL}$.

Calibration curve: The calibration curve must be prepared using 50-500 $\mu \mathrm{L}$ aliquots of the coumarin solution, $2 \mathrm{ml}$ of distilled water and $500 \mu \mathrm{L}$ of the lead acetate solution. The sample should be shaken and the final volume should be adjusted to $10 \mathrm{~mL}$ with distilled water before transferring $2 \mathrm{~mL}$ of this solution to a new test tube and adding $8 \mathrm{~mL}$ of hydrochloric acid solution. The final concentration of rutin will be $1-10 \mu \mathrm{g} / \mathrm{mL}$.

Measurement procedure: To quantify the coumarins, $500 \mu \mathrm{L}$ of the extract should be transferred to a test tube. Next, $2 \mathrm{ml}$ of distilled water and $500 \mu \mathrm{L}$ of lead acetate solution will be added. The sample is shaken and then $7 \mathrm{ml}$ of distilled water are added before transferring 2 $\mathrm{mL}$ of this solution to a new test tube and adding $8 \mathrm{~mL}$ of hydrochloric acid solution. The samples remain at room temperature for 30 minutes. The spectrophotometer should be adjusted to a $320 \mathrm{~nm}$ wavelength and the equipment must be rinsed with distilled water. The test should be performed in at least triplicate and the total coumarin content is expressed as milligrams of coumarin equivalents per gram of the sample extract (mg CE/g).

\section{References:}

Kuster R. A. M., Rocha L. A. M. A. Cumarinas, coronas e cantinas. In: Simões CMO, Schenkel EP, Gosmanm, G, Mello JCP, Mentz LA, Petrovick PR. (ed.) Farmacognosia: da planta ao medicamento. Porto Alegre: Universidade Federal do Rio Grande do Sul; 2004, p. 537-556 [34].

Osório O. K., Martins J. L. S. Determinação de cumarina em extrato fluido e tintura de guaco por espectrofotometria derivada de primeira ordem. Brazilian Journal of Pharmaceutical Sciences 2004; 40 (4) 481-486 [40].

\section{Results}

Analysis of the active component levels in raw plant materials and phytomedicines is essential for the safety and efficacy of pharmaceutical products [49]. The quantification of active compounds in herbals is still only incidentally performed due to the presence of active phytocomplexes plants and their extracts [50], which complicates their analysis. Through this framework, the use of standardised extracts focusing on specific groups of active components ensures the chemical homogeneity of the product, which improves product quality [51]. The compounds selected for this quality adjustment process should be the same as the assets in the product [52].

In this way, five products containing M. ilicifolia (all plant drugs), four containing B. forficata (all plant drugs) and five containing $M$. glomerata (three plant drugs and two fluid extract) sold in pharmacies and health food stores in Recife/PE, Northeast Brazil were obtained. 
Analysis of the active component concentrations were conducted as listed in the described standard operating protocols (SOPs) and the results are presented below.

\subsection{Calibration curves}

To quantify the active components, calibration curves with increasing concentrations proportional to their absorbance were constructed. A correlation equation was obtained from these curves (generally linear) of the type $y=a x+b$, where $y$ corresponds to the absorbance of the sample and $x$ the concentration. To convert the absorbance values (nm) to sample concentration $(\mu \mathrm{g} / \mathrm{mL})$, it is necessary to place the sample absorbance into the equation as $\mathrm{y}$. The correlation coefficient $\left(\mathrm{R}^{2}\right)$ shows the ability of the method to provide directly proportional results between the analyte concentration and the device response. The interval between the lowest and highest scalar values, which is also called the linearity range, should be determined with both precision and accuracy. The correlation coefficient must be equal to or greater than 0.98 .

The calibration curve constructed for tannic acid and tannins and used to quantify M. ilicifolia demonstrated a correlation equation of $y=0.067 x+0.01$ and a correlation coefficient of $R^{2}$ $=0.996$ (Figure 5).

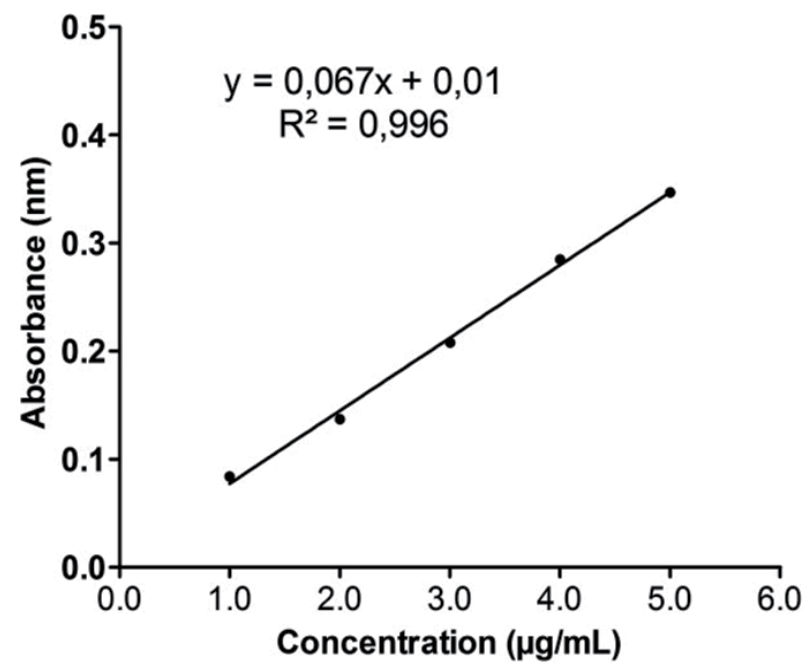

Figure 5. Calibration curve constructed using tannic acid concentrations of $1-5 \mu \mathrm{g} / \mathrm{mL}$ at $760 \mathrm{~nm}$ used to quantify the tannin content of Maytenus ilicifolia (Schrad.) Planch.

A calibration curve was constructed from rutin to quantify flavonoids in products from $B$. forficat $a$ and yielded the correlation equation $\mathrm{y}=0.022+0.0039 \mathrm{x}$ and correlation coefficient $\mathrm{R}^{2}=0.991$ (Figure 6). 


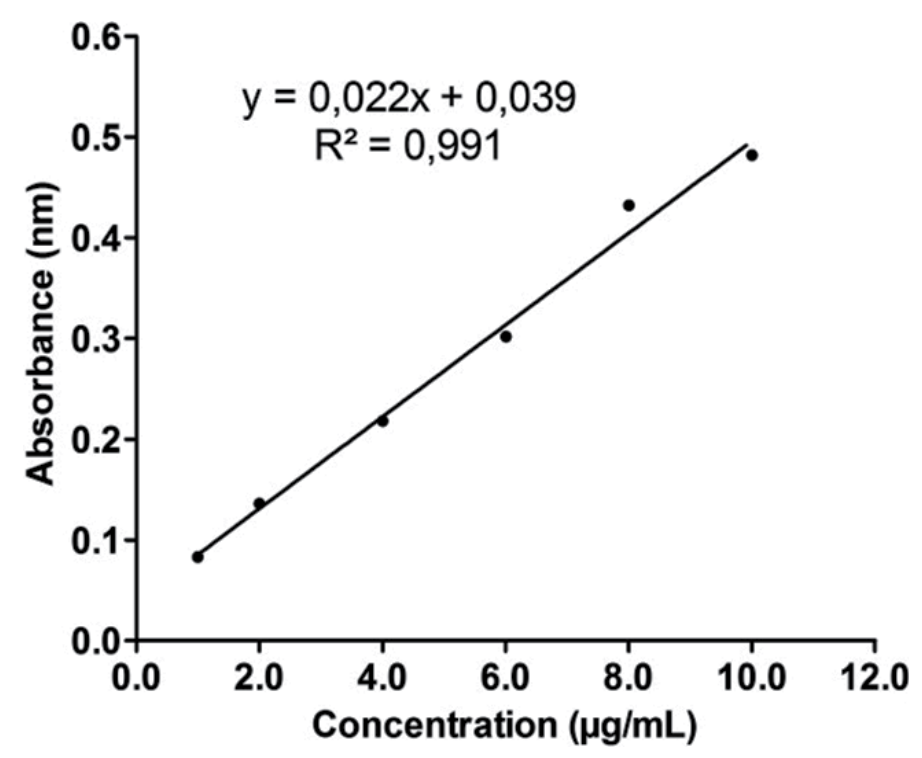

Figure 6. Calibration curve constructed from rutin for concentrations of $1-10 \mu \mathrm{g} / \mathrm{mL}$ at $420 \mathrm{~nm}$ used to quantify the flavonoids content in Bauhinia forficata Link.

The correlation equation and coefficient obtained from the calibration curve used to analyse coumarins in products containing M. glomerata were $\mathrm{y}=0.049 \mathrm{x}+0.031$ and $\mathrm{R}^{2}=0.994$ (Figure 7), respectively.

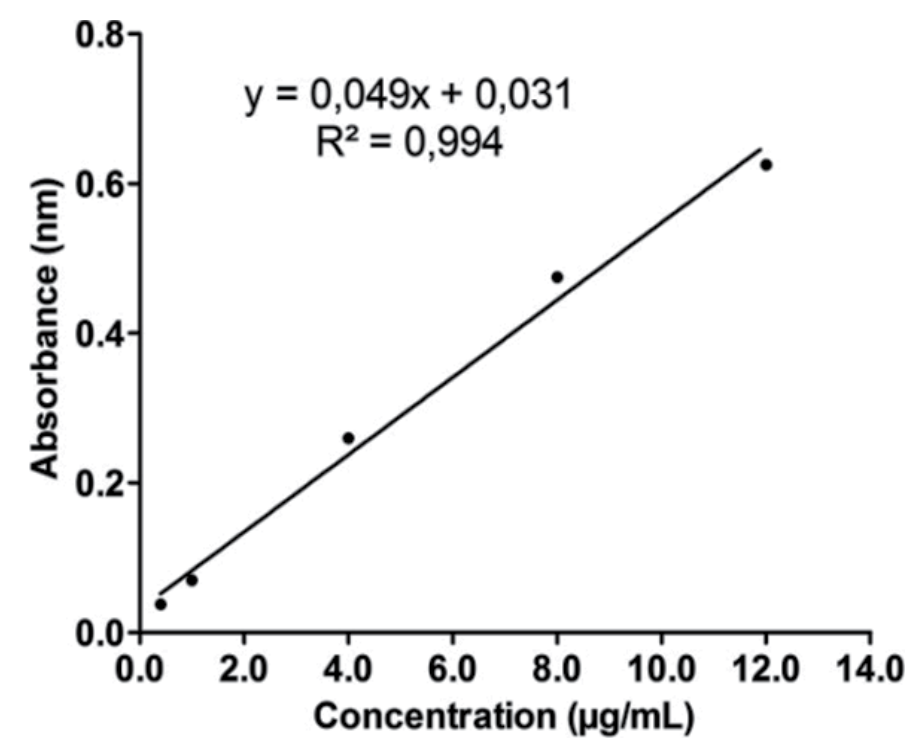

Figure 7. The calibration curve constructed using 1,2-benzopyrone in concentrations of $1-12 \mu \mathrm{g} / \mathrm{mL}$ using $320 \mathrm{~nm}$ excitation to quantify the coumarin content in Mikania glomerata Spreng. 


\subsection{Content of active principles}

The results of quality control of tannins of M. Ilicifolia, flavonoids of B. forficata and coumarins of M. glomerata are shown in Table 1.

\begin{tabular}{|c|c|c|c|}
\hline Specie / Marker & Sample & Concentration $\pm \mathrm{SD}(\mathrm{mg} / \mathrm{g})$ & CV (\%) \\
\hline \multirow{5}{*}{$\begin{array}{c}\text { Maytenus ilicifolia } \\
\text { Tannins }\end{array}$} & MI1 & $12.57 \pm 2.15 a$ & $17.12 \%$ \\
\hline & MI2 & $4.04 \pm 0.23 b$ & $5.75 \%$ \\
\hline & MI3 & $5.61 \pm 0.55 b c$ & $9.76 \%$ \\
\hline & MI4 & $7.72 \pm 0.84 c$ & $10.84 \%$ \\
\hline & MI5 & $11.81 \pm 1.00 \mathrm{a}$ & $8.44 \%$ \\
\hline \multirow{4}{*}{$\begin{array}{c}\text { Bauhinia forficata } \\
\text { Flavonoids }\end{array}$} & BF1 & $4.89 \pm 0.11 a$ & $2.33 \%$ \\
\hline & BF2 & $7.27 \pm 0.39 a$ & $5.41 \%$ \\
\hline & BF3 & $50.38 \pm 5.36 b$ & $10.64 \%$ \\
\hline & BF4 & $65.98 \pm 3.62 c$ & $5.49 \%$ \\
\hline \multirow{5}{*}{$\begin{array}{c}\text { Mikania glomerata } \\
\text { Coumarins }\end{array}$} & MG1 & $3.06 \pm 0.20 a$ & $6.67 \%$ \\
\hline & MG2 & $5.17 \pm 0.59 b$ & $11.40 \%$ \\
\hline & MG3 & $6.80 \pm 0.24 c$ & $3.46 \%$ \\
\hline & MG4 & $1.63 \pm 0.20 d$ & $12.50 \%$ \\
\hline & MG5 & $4.49 \pm 0.20 b$ & $4.55 \%$ \\
\hline
\end{tabular}

Table 1. Results of quality control of tannins, flavonoids and coumarins contained in products based on Maytenus ilicifolia (Schrad.) Planch., Bauhinia forficata Link and Mikania glomerata Spreng. respectively, sold in pharmacies in Recife/PE, Northeast of Brazil.

Values are mean \pm standard deviation. Values followed by the same letter in column are not statistically different $(n=6, p<0.05)$.

Analysis of variance (ANOVA) is one way to indicate significant differences $(p<0.01)$ for the drugs of $M$. ilicifolia, which is made from five plants. Samples Mi1 $(12.57 \pm 2.15 \mathrm{mg}$ TAE/g) and Mi5 (11.81 $\pm 1.0 \mathrm{mg} \mathrm{TAE} / \mathrm{g})$ both had higher concentrations of tannins and were not significantly different, whereas at least three of the other samples showed tannins (Figure 8). Comparing the average tannin concentration from different samples showed a low coefficient of variation $(\mathrm{CV}=37.61 \%)$.

Analysis of four products containing $B$. forficata showed a significant difference $(\mathrm{p}<0.01)$. The sample Bf4 presented the highest flavonoid concentration (65.98 $\pm 3.62 \mathrm{mg} \mathrm{RE} / \mathrm{g})$, whereas samples Bf1 and Bf2 had the lowest concentrations ( $4.89 \pm 0.11$ and $7.27 \pm 0.39 \mathrm{mg} \mathrm{RE} / \mathrm{g}$, respectively) with a content approximately 13 times lower. These results indicate that there is no standardisation regarding the flavonoid concentrations in products (Figure 9). 


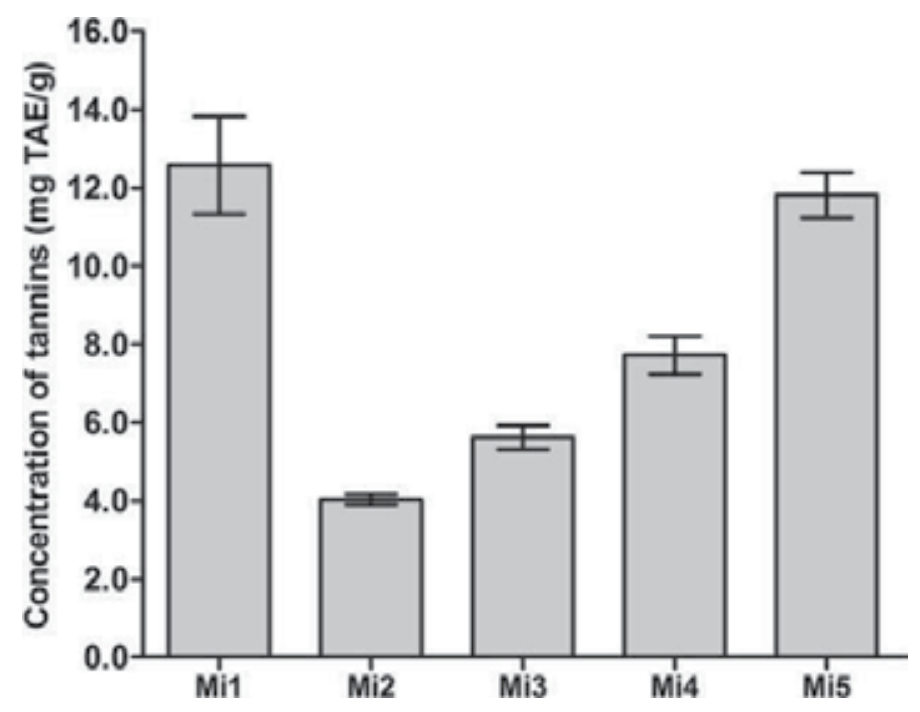

Figure 8. Concentration of tannin (mg TAE/g) contained in products from Maytenus ilicifolia (Schrad.) Planch. Sold in Recife/PE, Brazil.

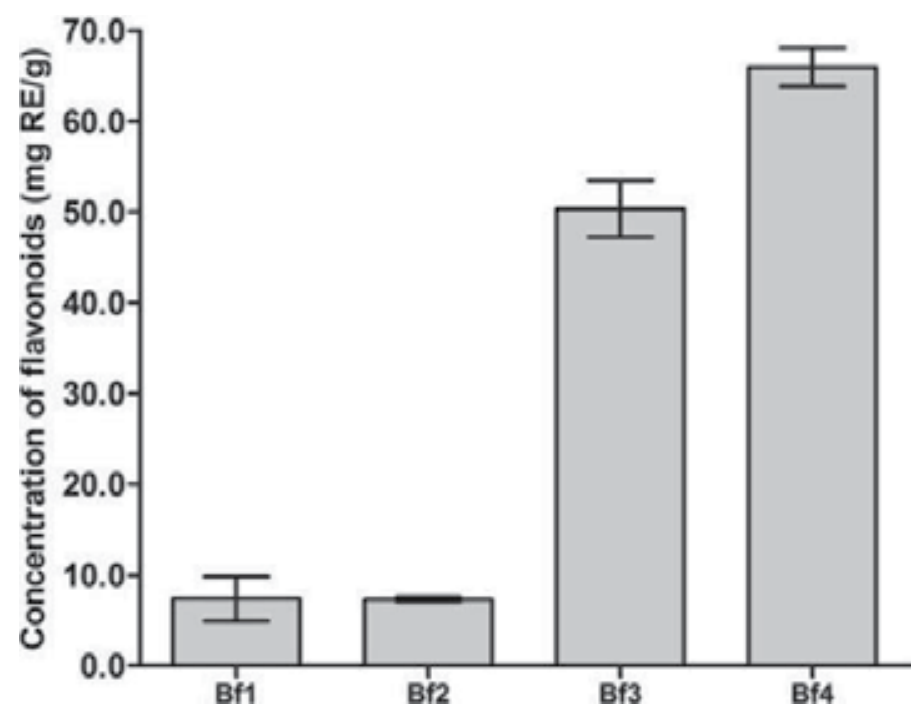

Figure 9. Concentration of flavonoids (mg RE/g) contained within Bauhinia forficata link. sold in Recife/PE, Brazil.

Of the five products from M. glomerata, three were plant drugs and two were fluid extracts. The plant drugs were extracted with ethanol as recommended by the Brazilian Pharmacopoeia 4th Edition to remove discrepancies from the results [53]. Both the extracts and liquids were evaporated to dryness. The one way ANOVA showed significant differences between the products $(\mathrm{p}<0.01)$ and sample $\mathrm{Mg} 3$ had the highest level $(6.80 \pm 0.24 \mathrm{mg} \mathrm{CE} / \mathrm{g})$, whereas 
sample $\mathrm{Mg} 4$ had the lowest level $(1.63 \pm 0.20 \mathrm{mg} \mathrm{CE} / \mathrm{g})$, with an approximately four times lower coumarin concentration.

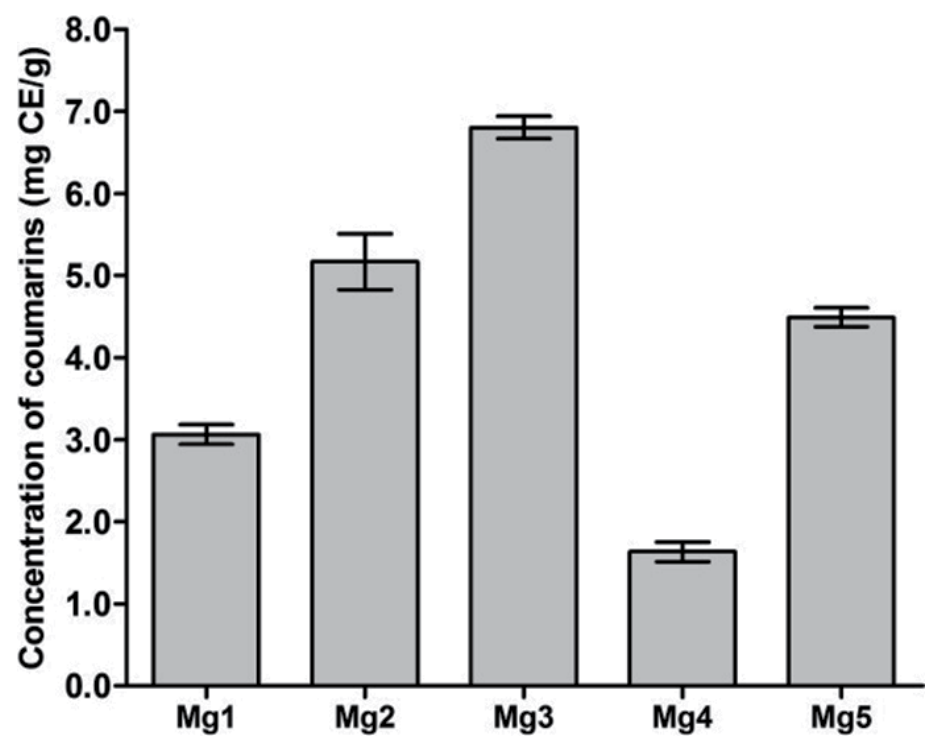

Figure 10. Concentration of coumarins ( $\mathrm{mg} \mathrm{CE} / \mathrm{g}$ ) contained in products from Mikania glomerata Spreng. sold in Recife/PE, Brazil.

The quantitative analysis of raw vegetables and phytomedicines is a fundamental quality control process that leads to security, stability, consistency and effectiveness in the produced phytomedicines [49]. It is important to emphasise the need for standardisation in analysing herbal medicines to determine the concentration of their active components in raw vegetable materials as well as for species identification.

\section{Conclusion}

This chapter provides easily reproducible standard operating procedures (SOPs) for the quality control of raw materials and herbal plants to ensure a minimal standard of quality in products sold. The implementation of these SOPs allows for the analysis of samples sold in establishments in Recife/PE and reveals an inconsistency in the concentration of tannins, flavonoids and coumarins within these products.

The low level of these metabolites may alter their effectiveness and more rigorous quality control and standardisation of these products is required to prevent compromising their therapeutic activity. 


\section{Author details}

Elba Lúcia Cavalcanti de Amorim ${ }^{1 *}$, Valérium Thijan Nobre de Almeida de Castro ${ }^{1}$, Joabe Gomes de Melo르. Allan Jonathan Chernichiarro Corrêa ${ }^{1}$ and Tadeu José da Silva Peixoto Sobrinho ${ }^{3}$

*Address all correspondence to: elba@ufpe.br

1 Department of Pharmacy, Federal University Pernambuco, Recife/PE, Brazil

2 Department of Biology, Federal Rural University of Pernambuco, Brazil

3 School of Medicine, Federal University of Tocantins, Palmas/TO, Brazil

\section{References}

[1] Aguilar-Sánchez, R., Ahuatl-García, F., Dávila-Jiménez, M. M., Elizalde-González, M. P., \& Guevara-Villa, M. R. (2005). Chromatographic and electrochemical determination of quercetin and kaempferol in phytopharmaceuticals. Journal of Pharmaceutical and Biomedical Analysis, 38(2), 239-249.

[2] Matysik, G., \& Wójciak-Kosior, M. (2005). Optimized Method for the determination of Flavonoid Glycosides and Aglycones. Chromatographia, 61(1-2), 89-92.

[3] Santos, R.I. (2004). Metabolismo básico e origem dos metabólitos secundários. In: Simões CMO, Schenkel E.P., Gosmanm, G., Mello J.C.P, Mentz L.A., Petrovick P.R. (ed.) Farmacognosia: da planta ao medicamento. Porto Alegre: Universidade Federal do Rio Grande do Sul;, 403-434.

[4] Edwards, P. J., \& Wratten, S. D. (1981). Ecologia das interações entre insetos e plantas. $1^{a}$ ed. São Paulo: Ed Pedagógica e Universitária; 78p.

[5] Harborne, J. B. (1993). Introduction to Ecological Biochemistry. 4 $4^{\underline{a}}$ ed. London Harcourt Brace and Company , 318p.

[6] Queiroz, C. R. A. A., Morais, S. A. L., \& Nascimento, E. A. (2002). Caracterização dos taninos da aroeira-preta (Myracrodruon urundeuva). Revista Árvore, 26(4), 485.

[7] Santos, S. C., \& Mello, J. C. P. (2004). Taninos. In: Simões C.M.O, Schenkel E.P., Gosmanm, G, Mello J.C.P., Mentz LA, Petrovick P.R. (ed.) Farmacognosia: da planta ao medicamento. Porto Alegre: Universidade Federal do Rio Grande do Sul, 615-656.

[8] Hagerman, A. E., \& Butler, L. G. (1981). The specificity of proanthocyanidin-protein interaction. Journal of Biological Chemistry, 256(9), 4494-4497. 
[9] Lewis, N. G., \& Yamamoto, E. (1989). Tannins: their place in plant metabolism. In: Hemingway RW, Karchesy JJ. (Ed) Chemistry and significance of condensed tannins. New York: Plenum Press, 23-46.

[10] Heil, M., Baumann, B., Andary, C., Linsenmair, K. E., \& Mckey, D. (2002). Extraction and quantification of "condensed tannins" as a measure of plant anti-herbivore defence? Revisiting an old problem. Naturwissenschaften, 89(11), 519-524.

[11] Bruneton, J. (1991). Elementos de Fitoquimica y Farmacognosia. 1 $1^{\underline{a}}$ ed. Zaragoza: Ed. Acribia Espanha , 594p.

[12] Harborne, J. B., Palo, R. T., \& Robbins, C. T. (1991). Plant defenses against mammalian herbivore. C R C Press LLC , 192.

[13] Gobbo-Neto, L., \& Lopes, N. P. (1991). Plantas medicinais: fatores de influência no conteúdo de metabólitos secundários., Química Nova x, 30(2), 374-381.

[14] Verpoorte, R. (1998). Exploration of nature's chemodiversity: the role of secondary metabolites as leads in drug development. Drug Development Trends, 3(5), 232-238.

[15] Wills, R. B. H., Bone, K., \& Morgan, M. (2000). Herbal products: active constituents modes of action and quality control. Nutrition Research Reviews, 13-47.

[16] Kilkuskie, R. E., Kashiwada, Y., Nonaka, G., Nishioka, I., Bodner, A., Cheng, Y., \& Lee, K. (1992). HIV and reverse transcriptase inhibition by tannins. Bioorganic $\mathcal{E} M e^{-}$ dicinal Chemistry Letters, 2(12), 1529-1534.

[17] Chung, K., Wei, C., \& Johnson, M. G. (1998). Are tannins a double-edged sword in biology and health. Trends in Food Science and Technology, 9(4), 168-175.

[18] Ferreira, P. M., Oliveira, C. N., Oliveira, A. B., Lopes, M. J., Alzamora, F., \& Vieira, M. A. R. (2004). A lyophilized aqueous extract of Maytenus ilicifolia leaves inhibits histamine-mediated acid secretion in isolated frog gastric mucosa. Planta, 219(2), 319-324.

[19] Ming, L. C., Castro, D. M., \& Delachiave, M. E. (1998). Plantas medicinais aromáticas e condimentares. Botucatu: Universidade Estadual Paulista.

[20] Pereira, A. M. S., Rodrigues, D. C., Cerdeira, R. M., \& França, S. C. (1993). Isolamento de metabólitos de maytenus associadas à ação anti-úlceragástrica. 12. Simpósio de Plantas Medicinais do Brasil, Curitiba.

[21] Santos-Oliveira, R., Coulaud-Cunha, S., \& Colaço, W. (2009). Revisão da Maytenus ilicifolia Mart. ex Reissek, Celastraceae. Contribuição ao estudo das propriedades farmacológicas. Brazilian Journal of Pharmacognosy, 19(2B), 650-659.

[22] Martens, S., \& Mithöfer, A. (2005). Flavones and flavone synthases. Phytochemistry, 66(20), 2399-2407. 
[23] Nijveldt, R. J., Nood, E., Hoorn, D. E. C., Boelens, P. G., Norren, K., \& Leeuwen, P. A. M. (2001). Flavonoids: a review of probable mechanisms of action and potential applications. The American Journal of Clinical Nutrition, 74(4), 418-25.

[24] Zuanazzi, J. A. S., \& Montanha, J. A. (2004). Flavonóides In: Simões CMO, Schenkel EP, Gosmanm, G, Mello JCP, Mentz LA, Petrovick PR. (ed.) Farmacognosia: da planta ao medicamento. Porto Alegre: Universidade Federal do Rio Grande do Sul; 577-614.

[25] Treutter, D. (2006). Significance of flavonoids in plant resistance: a review. Environmental Chemistry Letters, 4(3), 147-157.

[26] Harborne, J. B., \& Williams, C. A. (2000). Advances in flavonoids in research since 1992. Phytochemistry, 55(6), 481-204.

[27] Argolo, A. C. C., Sant', Ana. A. E. G., Pletsch, M., \& Coelho, C. B. B. (2004). Antioxidant activity of leaf extracts from Bauhinia monandra. Bioresource Technology, 95(2), 229-233.

[28] Silva, F. R. M. B., Szpoganicz, B., Pizzolatti, M. G., Willrich, M. A. V., \& Sousa, E. (2002). Acute effect of Bauhinia forficata on serum glucose levels in normal and alloxan-induced diabetic rats. Journal of Ethnopharmacology., 83(1-2), 33-37.

[29] Sousa, E., Zanatta, L., Seifriz, I., Creczynski-Pasa, T. B., Pizzolatti, M. G., Szpoganicz, B., \& Silva, F. R. M. B. (2004). Hypoglycemic effect and antioxidant potential of kaempferol-3,7-O- $(\alpha)$-dirhammnoside from Bauhinia forficata leaves. Journal of the Natural Products, 67(5), 829-832.

[30] Vaz, A.M.S.F. (2012). Bauhinia in Lista de Espécies da Flora do Brasil. Jardim Botânico do Rio de Janeiro., http://floradobrasil.jbrj.gov.br/2012/FB082666, accessed 27 June 2012).

[31] Damasceno, D. C., Volpato, G. T., Calderon, I. M. P., Aguilar, R., \& Rudge, M. V. C. (2004). Effect of Bauhinia forficata extract in diabetic pregnant rats: maternal repercussions. Phytomedicine, 11(2-3), 196-201.

[32] Di Stasi, L. C., Oliveira, G. P., Carvalhaes, M. A., Queiroz-Junior, M., Tien, O. S., Kakinami, S. H., \& Reis, M. S. (2002). Medicinal plants popularly used in the Brazilian Tropical Atlantic Forest. Fitoterapia, 73(1), 69-91.

[33] Melo, J. G., Nascimento, V. T., Amorim, E. L. C., Andrade-Lima, C. S., \& Albuquerque, U. P. (2004). Avaliação da qualidade de amostras comerciais de boldo (Peumus boldus Molina), pata-de-vaca (Bauhinia spp.) e ginco (Ginkgo biloba L.). Revista brasileira de farmacognosia, 14(2), 111-120.

[34] Kuster, R. M., \& Rocha, L. M. (2004). Cumarinas, cromonas e xantonas. In: Simões C M O, Schenkel E P, Gosmanm G, Mello JCP, Mentz LA, Petrovick PR. Farmacognosia: da planta ao medicamento. 5 $5^{\mathbf{a}}$ ed. Porto Alegre: Universidade Federal do Rio Grande do Sul,, 537-556. 
[35] Mello, M.M. (2009). Desenvolvimento de uma metodologia por espectroscopia de fluorescência para quantificação de cumarina e 7hidroxicumarina em Drágeas e sorosintético. Dissertação Universidade do Vale do Paraíba, São José dos Campos.

[36] Lake, B.G. (1999). Coumarin Metabolism, Toxicity and Carcinogenicity: relevance for human risk assessment. Food Chemistry Toxicology, 37(4), 423.

[37] Celeghini, R. M., Vilegas, J. H. Y., \& Lanças, M. (2001). Extraction and quantitative HPLC analysis of coumarin in hydroalcoholic extracts of Mikania glomerata Spreng. ("guaco") leaves. Journal Brazilian of Chemical Society, 12(6), 706-709.

[38] Ribeiro, C. V. C., \& Kaplan, M. A. C. (2002). Tendências evolutivas de famílias produtoras de Cumarinas em Angiospermae. Química Nova, 25(4), 533-538.

[39] Dias da Silva, J. L. S. (1926). Pharmacopéia dos Estados Unidos do Brasil. 1.ed., São Paulo:, Editora Nacional.

[40] Osório, A. C., \& Martins, J. L. S. (2004). Determinação de cumarina em extrato fluido e tintura de guaco por espectrofotometria derivada de primeira ordem. Brazilian Journal of Pharmaceutical Sciences, 40(4), 481-486.

[41] Bolina, R. C., Garcia, E. F., \& Duarte, M. G. R. (2009). Estudo comparativo da composição química das espécies vegetais Mikania glomerata Sprengel e Mikania laevigata Schultz Bip. ex Baker. Revista Brasileira de Farmacognosia, 19(1B), 294-298.

[42] Rocha, L., Lucio, E. M. A., França, H. S., \& Sharapin, N. (2008). Mikania glomerata Spreng: Desenvolvimento de um produto fitoterápico. Revista Brasileira de Farmacognosia, (18), 744-747.

[43] Silva, C. R., Gomes, V. S., Kulkamp, I. C., \& Kanis, L. A. (2008). Metodo espectroscópico para determinação de cumarina em xarope de Mikania glomerata Sprengel. Revista Brasileira de Farmacognosia, 18(4), 594-599.

[44] Brasil. Ministério da saúde. (2004). Agência Nacional de Vigilância Sanitária. Resolução da Diretoria Colegiada (RDC) $N^{\circ} 48$, de 16 de março de, Dispõe sobre o registro de medicamentos fitoterápicos. Diário Oficial da União de 18.03.2004.

[45] Engel, I. C., Ferreira, R. A., Cechinel-Filho, V., \& Meyre-Silva, C. (2008). Controle de qualidade de drogas vegetais a base de Bauhinia forficata Link (Fabaceae). Brazilian Journal of Pharmacognosy, 18(2), 258-264.

[46] Marques, G. S., Monteiro, R. P. M., Leão, W. F., Lyra, M. A. M., Peixoto, M. S., RolimNeto, P. J., Xavier, H. S., \& Soares, L. A. L. (2012). Avaliação de procedimento para quantificação espectrofotomêtrica de flavonóides totais em folhas de Bauhinia forficata Link. Química Nova, 35(3), 517-522.

[47] Amorim, E. L. C., Nascimento, J. E., Monteiro, J. M., Peixoto, Sobrinho. T. J. S., Araújo, T. A. S., \& Albuquerque, U. P. (2008). A simple and accurate procedure for the determination of tannin and flavonoid levels and some applications in ethnobotany and ethnopharmacology. Functional Ecosystems and Communities, 2(1), 88-94. 
[48] Peixoto, Sobrinho. T. J. S., Silva, C. H. T. P., Nascimento, J. E., Monteiro, J. M., Albuquerque, U. P., \& Amorim, E. L. C. (2008). Validação de metodologia espectrofotométrica para quantificação dos flavonóides de Bauhinia cheilantha (Bongard) Steudel. Brazilian Journal of Pharmaceutical Sciences, 44(4), 683-689.

[49] Bara, M. T. F., Cirilo, H. N., \& Oliveira, V. (2004). Determinação de ginkgoflavonóides por cromatografia líquida de alta eficiência em matérias-primas e produtos acabados. Revista Eletrônica de Farmácia, 1(1), 1-7.

[50] Williamson, E.M. (2001). Synergy and other interactions in phytomedicines. Phytomedicine, 8(5), 401-409.

[51] Capasso, R., Izzo, A. A., Pinto, L., Bifulco, T., Vitobello, C., \& Mascolo, N. (2000). Phytotherapy and quality of herbal medicines. Fitoterapia, 71-58.

[52] Calixto, J. B. S. (2000). Efficacy, Safety, quality control, marketing and regulatory guidelines for herbal medicines (Phytotherapeutic agents). Brazilian Journal of Medical and Biological Research, 33(2), 179-189.

[53] (2005). Farmacopéia Brasileira. 4. ed. São Paulo: Editora Atheneu, parte II, sexto fascículo. 
Chapter 4

\title{
Microbial Quality of Medicinal Plant Materials
}

\author{
Marcelo Gonzaga de Freitas Araújo and \\ Taís Maria Bauab \\ Additional information is available at the end of the chapter \\ http://dx.doi.org/10.5772/51072
}

\section{Introduction}

The use of medicinal plants is continually expanding worldwide. The increasing search for therapeutic agents derived from plant species is justified by the emergence of diseases, yet without proper treatment, and the growth of scientific knowledge about the herbal medicines as important treatment alternatives. Therefore, the quality and safety of herbal preparations are also of great concern [1]. The reference [2] explained that quality is the basis of reproducible efficacy and safety of herbal drugs, and to ensure the standard of research on herbal medicines, the quality of the plant materials or preparations is of utmost importance. With the ever increasing use of herbal medicines and the global expansion of the herbal medicines market, safety has become a concern for both health authorities and the public in many countries. This is because many contaminants and residues that may cause harm to the consumers have been reported [3].

The microbial load of plants is the result of a series of influences (Figure 1). By their origin, herbal drugs are subject to contamination by microorganisms from soil, air and water may be present potentially pathogenic microorganisms to man. Microbial contamination of medicinal herbal can be influenced by environmental factors such as temperature, humidity and extent of rainfall during pre-harvesting and post-harvesting periods, handling practices and the storage conditions of crude and processed medicinal-plant materials. In order to improve the purity and safety of the products, observation of basic hygiene during preparation, standardization of some physical characteristic such as moisture content, $\mathrm{pH}$ and microbiological contamination levels are desirable [1,4,5].

The presence of microbial contaminant in non sterile pharmaceutical products can reduce or even inactivate the therapeutic activity of the products and has the potential to adversely affect patients taking the medicines. As herbal medicinal products are complex mixtures 
which originate from biological sources, great efforts are necessary to guarantee a constant and adequate quality. Manipulation and processing factors largely determine the microbiological quality of the final products [6]. Previous studies have confirmed the presence of potential contaminants in herbal preparations [7-10]. Thus, manufacturers should ensure the lowest possible level of microorganisms in the raw material, finished dosage forms and the packaging components to maintain appropriate quality, safety and efficacy of the natural products [9].

This review intends to contribute to knowledge regarding the microbial contamination of medicinal plants by considering the influence of different commonly used pharmaceutical preparation techniques on the microbiological status of the products. Finally, quality standards will be discussed, considering the main guidelines of microbial quality control and through quality assurance measures such as good manufacturing practices (GMP) for herbal medicines.

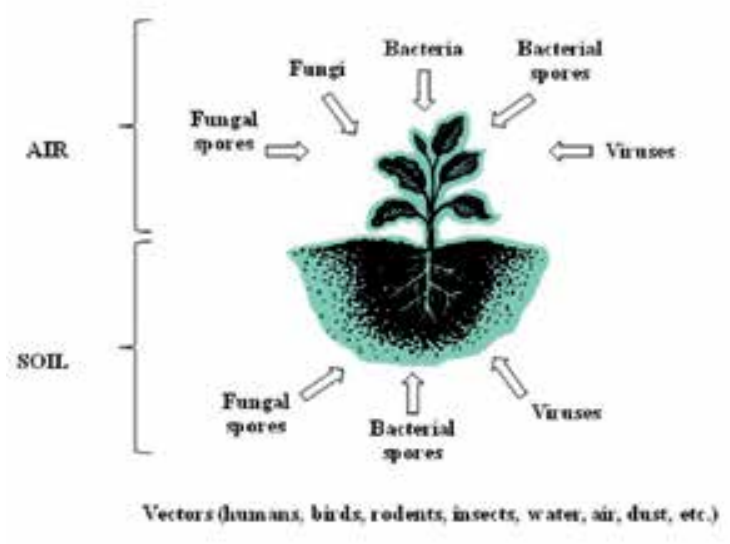

Figure 1. Influence of environmental factors and possible pathways of microbial contamination of medicinal herbs (adapted from [4]).

\section{Common microbial contaminants associated with medicinal plants}

The growing, harvesting and manipulation methods usually applied cannot avoid microbial contamination of the plant material which therefore reflects the environmental conditions as well as the specific hygiene during the diverse treatments [4]. Biological contamination refers to impurities in medicinal herbs and their preparations and products, and may involve living microbes such as bacteria and their spores, yeasts and moulds, viruses, protozoa, insects (their eggs and larvae), and other organisms. However, products of microbial metabolism such as toxic, low-molecular-weight metabolites from moulds are important chemical 
contaminants [11]. The main microbial contamination of plant materials, in general, are attributed to total aerobic mesophilic, enterobacterial, yeast and mould [4].

The presence of higher numbers of spores bacteria could be explained by the fact that some of these organisms (e.g. Bacillus and Clostridium spp.) produce spores which are resistant to harsh processing, elevated heat and dry conditions. Therefore, they can survive for a long time on the product in a dormant state. Bacillus cereus and Clostridium perfringens are recognized as having potential pathogenicity and have been incriminated in food poisoning [12]. Although bacterial endospores and fungal spores can be regarded as the two dominating groups of contaminants associated with medicinal plants, a broad diversity of bacterial, fungal cells and viruses can be found either in or on the plant material [4]. B. cereus and C. perfrigens were isolated from chamomile and other herbs by [13].

Although enterobacteria can be found in nature, this family possesses some indicative value towards faecal contamination. The presence of enterobacteria and E. coli reflect the situation regarding faecal contamination [7]. Together with the group of coliforms, it can be taken as an indicator for undesirable hygiene conditions, although this conclusion has to be related to the magnitude of viable count measured [4]. Staphylococcus aureus is not common contaminant of this type of plant material and relatively rarely found. However, contamination could provide amount of enterotoxin produced by $S$. aureus, depending on the specific nature of the individual [11].

Herbal medications are likely to be contaminated with a wide variety of others potentially pathogenic bacteria. In a study whose was evaluated the bacterial contamination of powdered herbal medicinal preparations sourced from identified herbal retail outlets in different parts of Kaduna, Nigeria, the results showed that a number of herbal remedies were contaminated with Salmonella typhi and Shigella spp., besides E. coli and S. aureus [1]. In addiction, the presence of pathogenic bacteria like B. cereus, Aeromonas hydrophila, Shigella spp., Enterobacter agglomerans, E. cloacae, Vibrio fluvialis, Pasteurella multocida, S. epidermidis, Acinetobacter iwoffii, Klebsiella spp., B. subtilis and Pseudomonas aeruginosa, and fungi Rhizopus stolonifer also were observed to be present in plant samples analyzed recently [14,15].

Because they are widespread in the atmosphere, moulds are common natural contaminants of medicinal herbs. It is known that, under favourable conditions, some fungi can synthesize toxical metabolites - mycotoxins. Among the known mycotoxins, the most toxic one is aflatoxin synthesised by species of $A$. flavus and A. parasiticus, and a minor number of other fungi [10]. Contamination by $A$. flavus, the most famous aflatoxin producer, is common in medicinal plant and herbal tea [16]. A. flavus colonization does not necessarily reduce yield, but causes economic losses by contaminating with aflatoxin [17]. In a study of 91 medicinal herb samples in Brazil [18], were found that $50 \%$ of aerial part samples were contaminated with fungi. Samples of medicinal plants were evaluated by [5] for the fungal contamination, and results indicated that predominant mycoflora (89.9\% of the isolates) corresponded to genera Aspergillus and Penicillium, which are extremely important from the mycotoxicological standpoint. The fungal contamination of powdered herbal medicinal preparations sourced from some herbal retail outlets in some parts of Nigeria was evaluated by [19] and 
the results showed that all of the herbal preparations had the presence of fungal contaminants with predominance of Aspergillus spp. and Penicillium spp., but Mucor spp., Candida spp., Trichosporium spp., also were found. The fungal deterioration adversely affects the chemical composition of the raw materials and thereby decreases the medicinal potency of herbal drugs [20].

The risk of the presence of microorganisms in a plant product depends on this finality of the use, its nature and its potential damage that may be caused to the consumers. Considering natural flora, current production conditions and the need to warrant the quality and the safety of these products, monographs establish a maximum fungal contamination limit for products that contain raw material of natural origin [5]. Although high fungal loads may be accepted due to the natural origin of those products, they indicate the potential for spoilage and mycotoxigenesis.

\section{Influence of different preparation techniques on the microbiological quality}

The production of an herbal medicine generally involves the steps in which a vegetable is subjected to unfavorable conditions to survival of microorganisms. Next, we introduce some of these processes and their influence on the microbial load.

\subsection{Drying process}

Drying is basically defined as the decreasing of plant moisture content, aimed at preventing enzymatic and microbial activity, and consequently preserving the product for extend shelf life [21]. Drying is the most common and fundamental method for post-harvest preservation of medicinal plants because it allows for the quick conservation of the medicinal qualities of the plant material in an uncomplicated manner. This process may also contribute to facilitate the marketing of plants, because drying results in reduction of the weight and volume of the plant with positive consequences for transport and storage [21,22].

The optimization of the drying process contributes to physical, chemical and microbiological stability of the medicinal herbs. The choice of drying conditions depend on the moisture content of tissue at harvest, the plant parts used, and the temperature best suited for preservation of the requested ingredients. For this reason, adequate dryers are needed, using temperature, velocity and humidity values for drying air that provides a rapid reduction in the moisture content without affecting the quality of the active ingredients of medicinal plants [21].

Medicinal plants can be dried in a number of ways: in the open air (shaded from direct sunlight); placed in thin layers on drying frames, wire-screened rooms or buildings; by direct sunlight, if appropriate; in drying ovens/rooms and solar dryers; by indirect fire; baking; 
lyophilization; microwave; or infrared devices. When possible, temperature and humidity should be controlled to avoid damage to the active chemical constituents. In the case of natural drying in the open air, efforts should be made to achieve uniform drying of medicinal plant materials and so avoid mould formation [23].

Spray drying technique has been widely used to obtain dried extracts presenting better technological characteristics and greater concentration of biological active constituents. This method is widely used in the pharmaceutical industry, despite the high temperature drying $\left(100^{\circ} \mathrm{C}\right.$ to $\left.200^{\circ} \mathrm{C}\right)$, the contact time between the material to be dry and hot air is extremely fast, less than 1 minute, theoretically is not enough to remove the microorganisms [24]. Comparative microbiological analysis of drug pulverized, extraction in liquid phase and the the spray drying extraction, using Phyllanthus niruri L., revealed that there is a significant reduction in microbial load, caused by the extraction in liquid process, while the spray dryer, despite the high temperature, did not affect the microbial load [25].

Drying at high temperature decreases the total aerobic microbial count in herbs. Water is a significant component of biological materials. Drying methods can lower the water activity to the level required for preventing growth of Aspergillus species and also for ensuring quality of medicinal herbs which may get destroyed upon over drying [10]. Exposure of herbs to microwaves and warm-air ovens can be efficient to reduce the microbial load, but they are not recommend to medicinal herbs containing volatile oils. The reference [26] evaluated both method of drying of plant, and reduction the microbial load present on the plants was observed but the effect on the volatile oil profile was profound by microwave drying, and warm drying air revealed that at temperatures $>60^{\circ} \mathrm{C}$, most of the volatile constituents were lost.

Other methods such as freeze-drying, oven drying and tray drying have been previously used to preserve medicinal herbs but to date there is little information in the literature on the effect of these drying conditions on the decrease of microbial loads [27].

Once drying is complete, plants are packaged in preparation for shipping or other further processing.

\subsection{Extraction methods}

Water is almost universally the solvent used to extract activity. At home, dried plants can be ingested as teas (plants steeped in hot water) or, rarely, tinctures (plants in alcoholic solutions) or inhaled via steam from boiling suspensions of the parts. Dried plant parts can be added to oils or petroleum jelly and applied externally. Poultices can also be made from concentrated teas or tinctures [28]. These kinds of preparations are usually called medicinal teas and are prepared using natural plants collected, dried and packaged without an effective hygienic and sanitary control. In addition, there can be microbiological contamination and controlling microbial contamination can be difficult in aqueous extracts [13]. 
Environmental dust settled on different parts of the plant and other contaminations can carry very significant amounts of bacterial and moulds spores [13]. However, those drugs which are subjected to cold water extraction (herbal maceration) may host a considerable amount of microbes, and the extraction procedure carried out at ambient temperature usually enables microbial multiplication [4]. The application of hot water extraction usually compensates for microbiological contaminations, since it can be expected that boiling water markedly reduces the viable counts by several log units and also inactivates possible pathogens [4]. However, bacterial spores of the Bacillaceae family are resistant to thermal treatment usually applied in infusion preparation, and this thermal shock may stimulate spore germination. Some of these bacteria like B. cereus and C. perfringens are recognized as having potential pathogenicity and have been incriminated in food poisoning [12]. Thus, in extractions using only water, hot or cold, as extractor liquid, the stability of the extract becomes compromised and the risk of microbiological contamination increases significantly. This contamination can compromise the quality and integrity of the plant material itself, as well as products arising from its use [29-30].

In addition to extraction temperature, the choice of extraction solvent is another important factor to prevent microbial contamination. The aim of an extraction process should be, of course, to provide for the maximum yield of substances and of the highest quality (concentration of target compounds and pharmacological power of the extracts). For extraction of active phytochemicals, the most commonly used solvents are methanol, ethanol, hexane, chloroform and diethyl ether [31]. Herbal extraction which made by ethanol or methanol extraction should, in general, provide good hygiene conditions, but the result depends on the alcoholic concentration applied [4].

\section{3. $\mathrm{pH}$ influence}

The $\mathrm{pH}$ value is one of the main factors influencing the quality of medicine. It always controls many chemical and microbiological reactions [32]. When the $\mathrm{pH}$ value is low (presence of acidic substances), the bacterial count could be low, but at neutral or higher $\mathrm{pH}$ the level of contamination of the herbal preparations could observed to be higher. This suggests that a neutral or alkaline $\mathrm{pH}$ favoured high contamination levels of the herbal preparations. This agrees with the observation that bacterial growth is optimal at more or less neutral $\mathrm{pH}$, around $\mathrm{pH}$ 5-8.5 [1].

\subsection{Storage}

Most pre-storage processing of plant material, such as that involving drying, heat, cooling and packaging, can prevent the degradation of plant material during storage [33]. Storage of medicinal herbs is an important part in the process production. During storage, due the factors in the outside world and their own physical and chemical properties of the interaction, gradually occurring physical, chemical and biological changes. Prolonged storage in poorly 
ventilated storehouse usually increases sample moisture content in the bulk due to heat exchange capacity, rendering herbs more susceptible to molds growth and toxin production. Fungi are the predominant contaminants of herbs, but most such microbial populations are probably regarded as commensal residents on the plant that survived drying and storage. Most fungi are present on plants, which develop after harvest if relative humidity is not controlled during storage [34-35].

Moulds are responsible for biodeterioration of a number of substrates including raw materials of some medicinal plants. These moulds reduce raw herbal drugs shelf life and market value. The fungal deterioration adversely affects the chemical composition of the raw materials and thereby decreases the medicinal potency of herbal drugs [20]. Samples of herbal parts stored for sale in markets located in Ibadan, Nigeria were analysed for mycoflora associated with their storage and twenty eight fungal species were isolated, showing that herbal drug plant pieces are hazardous for human health [36]. Some samples of herbal raw materials have been reported to contain aflatoxin. The reference [37] determined the incidence of toxigenic fungi and their mycotoxins on 152 dried medicinal and aromatic herbs from Argentina, which are used as raw material for drugs. A. flavus and A. parasiticus were the predominant species isolated, and high aflatoxin concentrations were detected. There is a potential risk for mycotoxins contamination, especially during prolonged storage in poorly conditions without temperature and moisture control that usually render medicinal plants more susceptible to moulds growth and mycotoxins production $[5,10,20]$.

The reduction of plant enzyme activity and inactivation of microorganisms is achieved by drying. Dried plant materials tend to be hygroscopic (readily absorbing moisture) and must be stored under controlled humidity. Rehydration can lead to the decomposition of the bioactive metabolites by enzymes from microorganisms or the plant itself. Significant contamination by bacteria and fungi suggest inadequate storage facilities and poor hygienic practice during preparation of these medicinal plants. The storage processes of such products are stages during which it is important to avoid even further contamination [38].

Studies on long-term stability of dried herbal teas and preparations are rare. In a study of [20] was examined the deterioration of herbal drug samples which were stored for 6-9 months by traders after collection. Some of the contaminated materials were found to be deteriorated by toxigenic strains of A. flavus and contain aflatoxin B1 which was above the permissible limit. In a study of [38], dried $P$. lanceolata leaves were exposed to atmospheres of different relative humidity $(75,45$ and $0 \%$ ) for 24 weeks and was evaluated the chemical changes of the compounds of interest. It was shown that exposure to water results in loss of bioactive molecules of $P$. lanceolata dried leaves, and that colonising fungi are the key contributors to this loss. The fungal deterioration adversely affects the chemical composition of the raw materials and thereby decreases the medicinal potency of herbal drugs. Biodeterioration of herbal products samples by associated fungi during storage has drawn attention regarding quality maintenance of these products [35,38-40].

It is common practice for herbalists to prepare herbal medicines and store them in a refrigerator. However, in previous study the effect of microbial contaminants on active com- 
pounds of African plant extracts was assessed and indicated that after 25 days of storage in low temperature there may be little or no active compounds due to spontaneous biodegradation by naturally-occurring microbes [41]. The World Health Organization (WHO) recommends that whenever required and when possible, fresh medicinal plant materials should be stored at appropriate low temperatures, ideally at $2-8^{\circ} \mathrm{C}$; frozen products should be stored at less than $-20^{\circ} \mathrm{C}$.

Processed medicinal plant materials should be packaged as quickly as possible to prevent deterioration of the product and to protect against unnecessary exposure to potential pest attacks and other sources of contamination.

\section{Decontamination of plant materials}

Attempts have always been made to decontaminate and preserve these medicinal plants so as to get more safe, natural and potent medicines. The number of methods has been tried for decontamination such as heat treatment, UV irradiation and fumigation. However, volatility and heat sensitivity of the delicate flavor and aroma components of the medicinal plants do not permit the use of heat treatment [42].

Low penetration power of UV radiations makes this irradiation method unsuitable [42]. Fumigation with gaseous ethylene oxide brings down the microbial burden but this method is now prohibited or restricted in many countries due to the carcinogenic nature of one of its residue in treated medicinal plants $[43,44]$. Various disinfectant technologies have been suggested which include electromagnetic radiations, photodynamic pulsing, ultrahigh pressure and $\mathrm{CO}_{2}$ treatment [42].

Gamma irradiation is now getting recognition throughout the world as a phytosanitary treatment of herbal materials. It improves the hygienic quality of various herbal materials and reduces the losses due to microbial contamination and insect damage [45]. Besides, it is a fast, safe, convenient, eco-friendly method which reduces the reliance on chemical fumigants and preservatives currently used by industries. The chances of recontamination are also reduced, as it can be done after packaging [46]. Some studies showed that the exposition of plant samples to different doses of gamma radiation can result in reduction in total bacterial counts and also indicated that the microbial load could be decreased by increasing the radiation-absorbed dose. These studies indicate that gamma irradiation is an effective treatment for microbial decontamination of medicinal plants $[42,47,48]$.

Certain plants contain natural barriers and antimicrobial substances which exert typical inhibitory effects on microbial growth and stability. It has been estimated that around 1400 herbs and spices may possess antimicrobial agents of different chemical nature as oils, peptides, liquid and organic extracts [4]. Some medicinal herbs contain essential oils which act as natural antimicrobials and may inhibit mould development and mycotoxin production [11]. Different studies have demonstrated the effectiveness of antimicrobials and their effec- 
tive compounds to control or inhibit the growth of pathogenic and spoilage microorganisms [49-51].

\section{Microbial quality parameters}

The most widely accepted and used technique is that recommended by WHO for total count of microorganisms in plant materials. According to the methodology of the WHO, $10 \mathrm{~g}$ of sample should be suspended in $90 \mathrm{ml}$ of buffer sodium chloride-peptone, adjusting the $\mathrm{pH}$ to 7.0. To count total aerobic bacteria, sample should be plated in duplicate, using the official technique of sowing depth on casein-soybean digest agar, and then incubated at $30-35^{\circ} \mathrm{C}$ for $48 \mathrm{~h}$. To count yeast and mold, the technique employed is the sowing depth in Sabourauddextrose plus a solution of $10 \%$ tartaric acid to obtain $\mathrm{pH} 3.0$ to 3.5. The dilution is plated in duplicate and incubated at $20-25^{\circ} \mathrm{C}$ for 5 days [52]. Analysis of specific pathogens, Enterobacteriaceae and other Gram negative bacteria (E. coli, Salmonella sp., P. aeruginosa and S. aureus) consists of specific methods of cultivation and through biochemical and serological tests. The specification of WHO for total aerobic microorganisms is not more than $10^{7} \mathrm{CFU} / \mathrm{g}$ for the plant material for use as teas and infusions and at most $10^{5} \mathrm{CFU} / \mathrm{g}$ for internal use. The specification of $\mathrm{WHO}$ for yeasts and molds are at most $10^{4} \mathrm{CFU} / \mathrm{g}$ for the plant material for use as teas and infusions and at most $10^{3} \mathrm{UFC} / \mathrm{g}$ for internal use. High counts of fungi are a risk because of the possibility to produce mycotoxin, such as aflatoxin, which is a carcinogen toxin. The WHO also recommends a test to detect the possible presence of aflatoxins, which are highly dangerous contaminants in any material of plant origin.

In Brazil, despite the large consumption of products derived from plants, products sold and consumed were not subject to any kind of quality control. In 1995, the Ministry of Health instituted the ordinance MS/SNVS No. 6, January 31, 1995 [53] that regulated the registration of herbal products for commercial purposes. Then came the Resolution RDC No. 17 [54] and, more recently, the RDC No. 48 [55] which confirms definitely that are herbal medicines and thereby rescues the need for the existence of safety studies, efficacy and quality, prior to the registration of these products. The Resolution RDC No. 48 of March 16 of 2004 [55] recommends that the contamination analysis on herbal medicines must be in accordance with pharmacopoeial specifications.

Both the Brazilian Pharmacopeia [56], as the United States Pharmacopeia [57] draw the following specifications for products for oral use: 104 aerobic bacteria/g or $\mathrm{mL}, 10^{2}$ fungi/g and absence of Salmonella spp, E. coli and S. aureus. However, the Brazilian Pharmacopeia also indicates the detection of other indicators of increased risk for oral administration, such as $P$. aeruginosa, B. cereus, Enterobacter spp, C. albicans, A. flavus and A. parasiticus. High microbial loads are indicative of the possibility of potentially pathogenic microorganisms.

In Europe the evaluation of microbial contamination of medicinal plants has increasingly become an integral part of Good Agricultural Practice (GAP) and Hazard Analysis and Critical Control Point (HACCP) concepts [33]. The limits of microbial contamination given in European Pharmacopoeia [58] for herbal medicinal products to which boiling water is added before use are: total aerobic bacteria $\left(10^{7} \mathrm{CFU} / \mathrm{g}\right)$, fungi $\left(10^{5} \mathrm{CFU} / \mathrm{g}\right)$; for herbal medicinal products to which boiling water is not added before use are: total aerobic bacteria $\left(10^{5}\right.$ 
$\mathrm{CFU} / \mathrm{g})$, fungi $\left(10^{4} \mathrm{CFU} / \mathrm{g}\right)$; Enterobacteria and other Gram-negative organisms $\left(10^{3} \mathrm{CFU} / \mathrm{g}\right)$; E. coli and Salmonella sp. should be absent. In general, the tests used to verify the presence of microorganisms in plant drugs and microbial limits show no significant variation and follow the recommendations used for non-sterile pharmaceutical products (Table 1).

\begin{tabular}{|c|c|c|c|c|}
\hline & $\begin{array}{c}\text { United States } \\
\text { Pharmacopoeia }\end{array}$ & $\begin{array}{c}\text { European } \\
\text { Pharmacopoeiab }\end{array}$ & WHOc & $\begin{array}{c}\text { Brazilian } \\
\text { Pharmacopoeia }^{d}\end{array}$ \\
\hline Aerobic bacteria & $10^{5} / 10^{4} / 10^{2}$ & $10^{7} / 10^{5}$ & $* / 10^{7} / 10^{5}$ & $10^{7} / 10^{5} / 10^{4}$ \\
\hline Mold and yeast & $10^{3} / 10^{2} / 10$ & $10^{5} / 10^{4}$ & $10^{5} / 10^{4} / 10^{3}$ & $10^{4} / 10^{3} / 10^{2}$ \\
\hline $\begin{array}{c}\text { Enterobacteria and } \\
\text { other Gram negative } \\
\text { bacteria }\end{array}$ & $10^{3} / * / *$ & $* / 10^{3}$ & $* / 10^{4} / 10^{3}$ & $10^{4} / 10^{3} / 10^{2}$ \\
\hline E. coli & absent & $10^{3} /$ absent & $10^{4} / 10^{2} / 10$ & absent \\
\hline Salmonella & absent & * / absent & * / absent /absent & absent \\
\hline \multicolumn{5}{|c|}{ 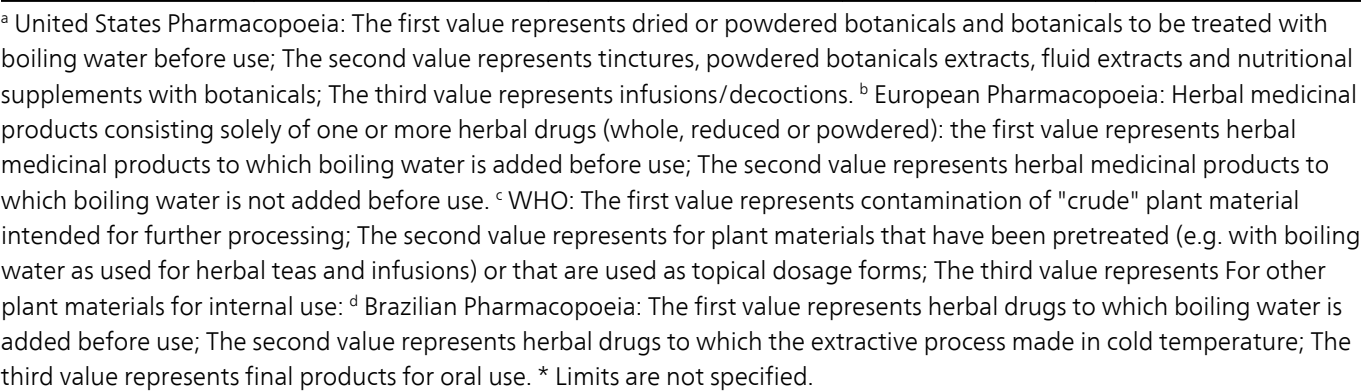 } \\
\hline
\end{tabular}

Table 1. Recommended microbial limits for herbal drugs (values in CFU/g).

\section{Conclusion}

Microbial contamination can lead to impaired performance of the product due to disruption of the stability of the formulation, modification of physical characteristics and appearance and lead to inactivation of the active ingredients and excipients in the formulation and also cause loss of confidence in the company. Herbalists should be trained to apply Good Manufacturing Practices, good harvesting practices and the safe handling and storage of herbal medicinal products. Further studies are recommended for herbal products to establish other contaminants and ways in which the contaminants can be reduced to recommended levels. The microbial loads should be established and the contaminants isolated and identified. In addition, alternative methods such as treatment with ethylene oxide or radiation with ionic rays lead to decontamination effects. These methods can be seen as a compromise between ensuring the microbiological safety of the product and avoiding consumer's risk and special 
legal permissions are required in many countries. It is evident that more detailed studies of plant species popularly used are needed in order to ensure the quality, an important concept for providing the wished security and reliability for its use.

\section{Author details}

Marcelo Gonzaga de Freitas Araújo* and Taís Maria Bauab

*Address all correspondence to: mgfaraujo@yahoo.com.br

Biological Sciences Department, Faculty of Pharmaceutical Sciences, São Paulo State University - UNESP, Araraquara, Brazil

\section{References}

[1] Abba, D., Inabo, H. I., Yakubu, S. E., \& Olonitola, O. S. (2009). Contamination of herbal medicinal products marketed in Kaduna Metropolis with selected pathogenic bacteria. African Journal of Traditional, Complementary and Alternative Medicines, 6, 70-77.

[2] Zhang, X. (1998). Regulatory situation of herbal medicines: a worldwide review. 2003: World Health Organization. Available at:, http://apps.who.int/ medicinedocs/en/d/Jwhozip57e/, (accessed 8 June 2012).

[3] Kunle, O. F., Egharevba, H. O., \& Ahmadu, P. O. (2012). Standardization of herbal medicines- A review. International Journal of Biodiversity and Conservation, 4, 101-112.

[4] Kneifel, W., Czech, E., \& Kopp, B. (2002). Microbial contamination of medicinal plants- A review. Planta Medica, 5-15, 68.

[5] Bugno, A., Almodovar, A. A. B., Pereira, T. C., Pinto, T. J. A., \& Sabino, M. (2006). Occurrence of toxigenic fungi in herbal drugs. Brazilian Journal of Microbiology, 37, 47-51.

[6] Busse, W. (2000). The significance of quality for efficacy and safety of herbal medicinal products. Drug Information Journal, 34, 15-23.

[7] Czech, E., Kneifel, W., \& Kopp, B. (2001). Microbiological status of commercially available medicinal herbal drugs- A screening study. Planta Medica, 67, 263-269.

[8] Tassaneeyakul, W., Razzazi-Fazeli, E., Porasuphatana, S., \& Bohm, J. (2004). Contamination of aflatoxins in herbal medicinal products in Thailand. Mycopathologia, 158, 239-244. 
[9] Okunlola, A., Adewoyin, B. A., \& Odeku, A. O. (2007). Evaluation of pharmaceutical and microbial qualities of some herbal medicinal products in South Western Nigeria. Tropical Journal of Pharmaceutical Research, 6, 661-670.

[10] Kulshrestha, R., Gupta, C. P., Shukla, G., Kundu, M. G., Bhatnagar, S. P., \& Katiyar, C. K. (2008). The effect of water activity and storage temperature on the growth of Aspergillus flavus in medicinal herbs. Planta Medica, 74, 1308-1315.

[11] Kosalec, I., Cvek, J., \& Tomic, S. (2009). Contaminants of medicinal herbs and herbal products. Archives of Industrial Hygiene and Toxicology, 60, 485-501.

[12] Kunene, N. F., Hastings, J. W., \& von Holy, A. (1999). Bacterial populations associated with a sorghum-based fermented weaning cereal. International Journal of Food Microbiology, 49, 75-83.

[13] Martins, H. M., Martins, M. L., Dias, M. I., \& Bernardo, F. (2001). Evaluation of microbiological quality of medicinal plants used in natural infusions. International Journal of Food Microbiology, 68, 149-153.

[14] Alwakeel, S. S. (2008). Microbial and heavy metals contamination of herbal medicines. Research Journal of Microbiology, 3(12), 683-691.

[15] Idu, M., Erhabor, J. O., \& Idele, S. O. (2011). Microbial load of some medicinal plants sold in local markets of Benin City, Nigeria. International Journal of Medicinal and Aromatic Plants, 1(3), 272-277.

[16] Halt, M. (1998). Moulds and mycotoxins in herb tea and medicinal plants. European Journal of Epidemiology, 14, 269-274.

[17] Amaike, S., \& Keller, N. P. (2011). Aspergillus flavus . Annual Review of Phytopathology, 49, 107-133.

[18] Bugno, A., Buzzo, A. A., Nakamura, C. T., Pereira, T. C., Matos, D., \& Pinto, T. J. A. (2005). Avaliação da contaminação microbiana em drogas vegetais. Revista Brasileira de Ciências Farmacêuticas, 41(4), 491-497.

[19] Anyanwu, C. U. (2010). Fungal contaminants of powdered herbal drugs sold in parts of Enugu State, Southeast, Nigeria. Plant Product Research Journal, 14, 46-50.

[20] Kumar, A., Shukla, R., Singh, P., \& Dubey, N. K. (2009). Biodeterioration of some herbal raw materials by storage fungi and aflatoxin and assessment of Cymbopogon flexuosus essential oil and its components as antifungal. International Biodeterioration $\mathcal{E}$ Biodegradation, 63, 712-716.

[21] Rocha, R. P., Melo, E. C., \& Radünz, L. L. (2011). Influence of drying process on the quality of medicinal plants: A review. Journal of Medicinal Plants Research, 5(33), 7076-7084.

[22] Müller, J., \& Heindl, A. (2006). Drying of medicinal plants. In: Bogers RJ, Craker LE, Lange D. (eds.), Medicinal and Aromatic Plants, Dordrecht: Springer, 237-252. 
[23] World Health Organization (WHO). (2003). WHO guidelines on good agricultural and collection practices [GACP] for medicinal plants. Geneva, World Health Organization.

[24] Oliveira, O. W., \& Petrovick, P. R. (2010). Secagem por aspersão (spray drying) de extratos vegetais: bases e aplicações. Revista Brasileira de Farmacognosia, 20, 641-650.

[25] Souza, T. P., Lionzo, M. I. Z., \& Petrovick, P. R. (2006). Avaliação da redução da carga microbiana de droga vegetal através do processamento tecnológico: decocção e secagem por aspersão. Revista Brasileira de Farmacognosia, 16, 94-98.

[26] Deans, S. G., Svoboda, K. P., \& Bartlett, M. C. (1991). Effect of microwave oven and warm-air drying on the microflora and volatile oil profile of culinary herbs. Journal of Essential Oil Research, 3, 341-347.

[27] Harbourne, N., Marete, E., Jacquier, J.C., \& O'Riordan, D. (2009). Effect of drying methods on the phenolic constituents of meadowsweet (Filipendula ulmaria) and willow (Salix alba). Food Science and Technology, 42, 1468-1473.

[28] Cowan, M. M. (1999). Plant products as antimicrobial agents. Clinical Microbiology Reviews, 12, 564-582.

[29] Migliato, K. F., Moreira, R. R. D., \& Mello, J. C. P. (2007). Sacramento LVS, Correa MA, Salgado HRN. Controle de qualidade do fruto de Syzygium cumini (L.) Skells. Revista Brasileira de Farmacognosia, 17, 94-101.

[30] Araújo, M. G. F., Galeane, M. C., Castro, A. D., Salgado, H. R. N., Almeida, A. E., Cunha, W. R., Veneziani, R. C. S., \& Moreira, R. R. D. (2010). Pharmacognostical evaluation of fruits of Solanum lycocarpum A. St.-Hill. (Solanaceae). Pharmacognosy Journal, 2(9), 248-253.

[31] Castillo, F., Hernández, D., Gallegos, G., Mendez, M., Rodríguez, R., Reyes, A., \& Aguilar, C. N. (2010). In vitro antifungal activity of plant extracts obtained with alternative organic solvents against Rhizoctonia solani Kühn. Industrial Crops and Products, 32, 324-328.

[32] Liu, X., Qiu, Z., Wang, L., \& Chen, Y. (2011). Quality evaluation of Panax notoginseng extract dried by different drying methods. Food and Bioproducts Processing, 89, 10-14.

[33] Fennel, C. W., Light, M. E., Sparg, S. G., Stafford, G. I., \& van Staden, J. (2004). Assessing African medicinal plants for efficacy and safety: agricultural and storage practices. Journal of Ethnopharmacology, 95, 113-121.

[34] Aziz, N. H., Youssef, Y. A., El -Fouly, M. Z., \& Moussa, L. A. (1998). Contamination of some common medicinal plant samples and spices by fungi and their mycotoxins. Botanical Bulletin of Academia Sinica, 39, 279-285.

[35] Mandeel, Q. A. (2005). Fungal contamination of some imported spices. Mycopathologia, 159, 291-298. 
[36] Efunyoye, M. O. (1996). Fungi associated with herbal drug plants during storage. Mycopathologia, 136, 115-118.

[37] Rizzo, I., Vedoya, G., Maurutto, S., Haidukowski, M., \& Varsavsky, E. (2004). Assessment of toxigenic fungi on Argentinean medicinal herbs. Microbiological Research, 159, 113-120.

[38] Gonda, S., Tóth, L., Gyémánt, G., Braun, M., Emrid, T., \& Vasas, G. (2012). Effect of high relative humidity on dried Plantago lanceolata L. leaves during long-term storage: Effects on chemical composition, colour and microbiological quality. Phytochemical Analysis, 23, 88-93.

[39] Singh, P., Srivastava, B., Kumar, A., \& Dubey, N. K. (2008). Fungal contamination of raw materials of some herbal drugs and recommendation of Cinnamomum camphora oil as herbal fungitoxicant. Microbial Ecology, 56, 555-560.

[40] Shukla, R., Kumar, A., Prasad, C. S., Srivastava, B., \& Dubey, N. K. (2008). Antimycotic and antiaflatoxigenic potency of Adenocalymma alliaceum Miers. on fungi causing biodeterioration of food commodities and raw herbal drugs. International Biodeterioration \& Biodegradation, 62, 348-351.

[41] Du Plessis-Stoman, D., Downing, T. G., van de Venter, M., \& Govender, S. (2009). Traditional herbal medicines: potential degradation of sterols and sterolins by microbial contaminants. South African Journal of Science, 105, 147-150.

[42] Gupta, P. C., Garg, N., \& Joshi, P. (2011). Effect of gamma irradiation on the extraction yield and microbial contamination of medicinal plants. Internet Journal of Food Safety, 13, 351-354.

[43] Kim, M. J., Yook, H. S., \& Byun, M. W. (2000). Effects of gamma irradiation on microbial contamination and extraction yields of Korean medicinal herbs. [2]. Radiation Physics and Chemistry, 57, 55-58.

[44] Satomi, L. C., Soriani, R. R., \& Pinto, T. J. A. (2005). Descontaminação de drogas vegetais empregando irradiação gama e óxido de etileno: aspectos microbianos e químicos. Revista Brasileira de Ciências Farmacêuticas, 41(4), 445-450.

[45] Farkas, J. (1998). Irradiation as a method for decontaminating food: A review. International Journal of Food Microbiology, 44, 189-204.

[46] Khattak, K. F., Simpson, T. J., \& Ihasnullah, . (2009). Effect of gamma irradiation on the microbial load, nutrient composition and free radical scavenging activity of $\mathrm{Ne}$ lumbo nucifera rhizome. Radiation Physics and Chemistry, 78, 206-212.

[47] Khattak, K. F. (2012). Evaluation of microbial loads, physical characteristics, chemical constituents and biological properties of radiation processed Fagonia arabica. Radiation Physics and Chemistry, 81, 679-685. 
[48] Aquino, S., Gonçales, E., Rossi, M. H., Nogueira, J. H. C., Reis, T. A., \& Corrêa, B. (2010). Evaluation of fungal burden and aflatoxin presence in packed medicinal plants treated by gamma radiation. Journal of Food Protection, 73(5), 932-937.

[49] Tiwari, B. K., Valdramidi, V. P., O’Donnell, C. P., Muthukumarappan, K., Bourke, P., \& Cullen, P. J. (2009). Application of natural antimicrobials for food preservation. Journal of Agricultural and Food Chemistry, 57, 5987-6000.

[50] Wang, Y., Lu, Z., Wu, H., \& Lv, F. (2009). Study on the antibiotic activity of microcapsule curcumin against foodborne pathogens. International Journal of Food Microbiology, $30,71-74$.

[51] Negi, P. S. (2012). Plant extracts for the control of bacterial growth: Efficacy, stability and safety issues for food application. International Journal of Food Microbiology, 156, 7-17.

[52] World Health Organization (WHO). (1998). Quality control methods for medicinal plant materials. Geneva, World Health Organization.

[53] Brasil, Ministério da Saúde, Secretaria Nacional de Vigilância Sanitária. (1995). Portaria no 6., Diário Oficial da Repúplica Federativa do Brasil, 31 jan. Institui e normaliza o registro de produtos fitoterápicos.

[54] Brasil, Ministério da Saúde, Agência Nacional de Vigilância Sanitária. (2000). RDC no 17., Diário Oficial da República Federativa do Brasil, 24 abr. Aprova regulamento técnico, normatizando o registro de medicamentos fitoterápicos junto ao Sistema de Vigilância Sanitária.

[55] Brasil. Resolução RDC no 48. (2004). de 16 de março de 2004. Dispõe sobre o registro de medicamentos fitoterápicos. Diário Oficial [da] República Federativa do Brasil, Brasília, DF, 18 mar. 2004. Disponível em:, http://www.anvisa.gov.br/legis/resol/ 2004/48_04rdc.htm, Acesso em: 8 june 2012.

[56] Farmacopéia Brasileira. (1988). 4. ed. São Paulo: Atheneu, Parte 1. pV.5.1.6.-1- V. 5.1.7.-6.

[57] The United States Pharmacopeia. (2005). 28 ed. Rockville: United States Pharmacopeial Convention, 3013.

[58] European Pharmacopoeia. (2007). Microbiological quality of pharmaceutical preparations. Chapter 5.1.4, 6. ed. Strasbourg: EDQM., 4451. 

Chapter 5

\title{
Application of ISO 9001 Industrial Standard to Herbal Drug Regulation
}

\author{
Sunday Ameh, Florence Tarfa, Magaji Garba and \\ Karniyus Gamaniel
}

Additional information is available at the end of the chapter

http://dx.doi.org/10.5772/50814

\section{Introduction}

We noted earlier [1] that 1978 was the turning point in current public perception of traditional medicine (TM) following the famous WHO declaration at Alma-Ata. That declaration ushered in a positive attitude that paved the way for the present global popularity of TM, especially herbal medicine. We noted earlier also [2,3] that whereas herbal remedies are called dietary supplements in the US, thereby shifting emphasis away from their medicinal attributes, the Dietary Supplement Health Education Act of 1994 [4], which occasioned the shift, actually helped to promote herbal medicine in the US, albeit indirectly, through the innovative provision it made for user information [5,6]. A similar situation obtained in Europe, where the net effect of the laws and rules passed in 2004 on herbal remedies had been to promote their production and use $[7,8]$. In terms of trade and economics of herbal drugs, the following fact is notable: Although, Asia contributed only US $\$ 7.3$ billion to herbal world trade in 1999 [9], by 2005, a mere 6 years, China's contribution alone rose to US\$ 14 billion [10]. This stupendous growth was due to policies and programmes that favoured herbal medicine - the cornerstone of Traditional Chinese Medicine (TCM). Similar situations as in China held sway in Japan, South Korea and the Indian sub-continent, where government policies also favoured herbal medicine. However, in many developing countries like Nigeria, a totally different picture obtained, not because policies were expressly against herbal medicine, but in these countries there had been a lingering absence of proper policies and laws supportive of traditional remedies. Another key fact on the political economy of herbal drugs is that: Although, about $80 \%$ of people in developing countries depended on herbs, these countries contributed only $7.2 \%$ to herbal drug trade in 1999. By contrast, the developed nations, where people relied less on herbs, contributed $55.2 \%$. Asia, less Japan and 
South Korea, contributed 37.6\%. Equally interesting is the comparison of Brazil with Nigeria. Both are rich in medicinal plants and have high populations that depend substantially on herbs. But, while herbs contributed an unknown amount to the Nigerian economy in 2007, in Brazil it contributed US\$ 160 million. By contrast, Nigeria's entire federal budget for health in 2007 was a mere US\$ 800 million [2]. These findings earlier led us [11,12] to conclude that developing countries need strategies that will enhance the regulation of herbal drugs and promote their trade. The present article is an attempt to enunciate one of such strategies. It is particularly of note that the superior performance of Brazil in comparison with Nigeria indicates that with proper policies and strategies, herbs can indeed contribute substantially to any economy.

\section{Methodology: Determinative Review of ISO 9001 and the Mandates of Nigeria's and Europe's DRAs}

\subsection{ISO 9001:2008 industrial standard - A synopsis}

ISO 9001:2008 industrial standard or quality management system (QMS) is a document of about 30 pages with 8 clauses, published by and obtainable from the International Organization for Standardization (ISO), Basle, Switzerland, or from any of its national affiliates. The standard is designed to be met by any organization that i) needs to demonstrate its ability to consistently provide product or service that meets both customer and applicable statutory and regulatory requirements (collectively legal requirements); ii) aims to enhance customer satisfaction by effectively and continually improving its QMS; and iii) plans to provide continual assurance of conformity to customer and applicable legal requirements. These aims/ approaches (often called "QMS requirements" or "quality procedures") are generic and intended to be applicable to all organizations regardless of type, size and product provided. Wherever any requirement cannot be applied due to the nature of an organization and its product, such can be considered for exclusion. But wherever exclusions are made, claims of conformity to the standard are not acceptable unless such exclusions are limited to requirements within clause 7 of the standard, and such exclusions do not affect the organization's ability, or responsibility, to provide product that meets customer and applicable legal requirements. ISO 9001:2008 defines the minimum requirements for a well managed organization. In other words, noncompliance to an ISO 9001:2008 requirement puts at risk an organization's ability to consistently and efficiently satisfy the expectations of its customers/ stakeholders.

\subsection{The six QMS requirements or "The Six Quality Procedures"}

These procedures or requirements, as one may choose to call them, actually refer to subclause 4.1 (General requirements) under clause 4 (Quality Management System) of ISO 9001:2008. The sub-clause prescribes that organizations shall establish, document, implement, and maintain a QMS, and continually improve its effectiveness. To do so means that the organization shall operate its QMS with a view to carrying out (or meeting) the following six procedures (or requirements): determine the processes needed for the QMS, and their 
application throughout the organization; determine the sequence of the processes and their interactions; determine the criteria and methods for operating and controlling the processes; determine and ensure the availability needed resources and supporting information; check, measure and analyze the processes, where applicable; and implement actions to achieve planned results and continual improvement of the processes.

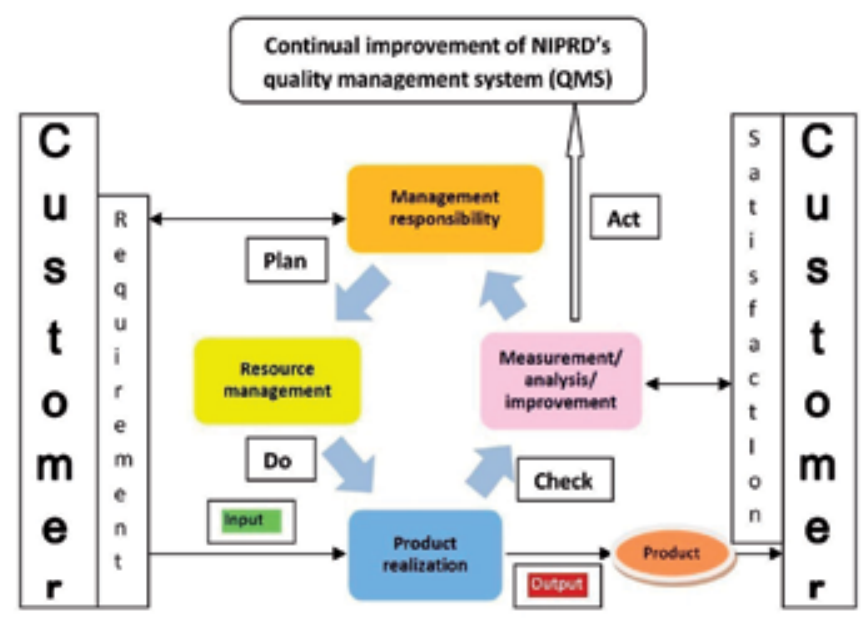

Management responsibility: NIPRD's CEO is accountable to the customerl stakeholder. The onus is upon the CEO to engage the right calibre of personnel and system in every unit to enable the QMS function optimally so that the customer/ stakeholder can derive satisfaction

Resource management: Administration and Finance are critical to the CEO's resource management functions, since they are respectively responsible for personnel administration and material management

Product realization: R\&D units are critical to the CEO's functions in product realization/ service provision since they are responsible for delivering on the Instifute's Mandate

Measurement/ analysis/ improvement: All unts have measureable objectives and functions. Thus, a major function of the CEO is to motivate and manage the various workers and systems to enable them satisfy the customer/ stakeholder and to improve the QMS on a continual basis

Figure 1. NIPRD's core business in the context of Plan-Do-Check-Act process-based QMS. ${ }^{1}$

The organization shall manage the processes above in accordance with ISO 9001:2008 requirements. It shall also define the type and extent of control to be applied to any outsourced process that can affect product conformity to requirements. ISO 9001:2008 specifically notes as follows:

1 Management responsibility corresponds to clause 5 of ISO 9001; while Resource management, Product realization and Measurement/ analysis/improvement correspond to clauses 6, 7 and 8 respectively. 
1. Processes needed for the QMS include the processes for management activities (clause 5), provision of resources (clause 6), product realization (clause 7), and measurement, analysis, and improvement (clause 8).

2. An outsourced process is a process the organization needs for its QMS, and which the organization chooses to have performed by an external party.

3. Ensuring control over outsourced processes does not absolve the organization of the responsibility to conform to customer and legal requirements.

The type and extent of control applied to an outsourced process can be influenced by factors such as: the potential impact of the outsourced process on the organization's capability to provide product that conforms to requirements; the degree to which the control over the process is shared; and the capability of the organization in achieving the necessary control via the application of sub-clause 7.4 (Purchasing). Philosophically, ISO 9001:2008 is formulated on the basis of management by objectives (MBO) and draws upon eight quality management principles. Ideally therefore, quality assurance covers activities in research, development, production and documentation. It embraces the rule: "do it right the first time". It involves regulating the quality of raw materials, the state of production line and works-in-progress, the product and related management processes. One of the most widely used paradigms for quality assurance management (QAM) is the "Shewhart cycle", also called "PDCA approach", meaning, "Plan-Do-Check-Act" [13,14]. The foregoing is illustrated in Figure 1 using NIPRD QMS processes as an example.

\subsection{The eight quality management principles that underlie ISO 9001:2008}

Like other ISO standards (Example: ISO 9004 - Managing for Sustained Success), ISO 9001:2008 is based on 8 quality management principles that are aligned with the philosophy and objectives of most quality award programmes in the world's most industrialized nations. The 8 principles are associated with the following themes:

1. Customer focus.

2. Leadership.

3. Involvement of people.

4. Process approach to management.

5. System approach to management.

6. Continual improvement.

7. Factual approach to decision making.

8. Mutually beneficial supplier relationships. 


\subsection{Key terminologies of ISO 9001:2008}

\subsubsection{Process approach to management}

A process is an activity or operation that receives inputs and converts them to outputs. Practically all activities or operations involved in generating a product or providing a service are processes. For an organization to function, it must define and manage several inter-linked processes. Most often, the output of one process becomes the input into the next process. The systematic identification and control of the various processes employed within an organization, and the interactions between such processes, is termed "process approach" to management. Thus process approach to management is a way of obtaining a desired result, by controlling activities and related resources as a process. Process approach is a key element of all ISO 9000 standards, including ISO 9001:2008.

\subsubsection{System approach to management}

System approach to management is based on the premise that the efficiency and effectiveness with which an organization achieves its quality objectives are contributed and enhanced by identifying, understanding and managing all the interrelated processes within the organization as a system

\subsubsection{Quality policy}

Quality policy is a formal statement from the management of an organization that is linked to the nature of its business and its plans to meet the needs of its customers/ stakeholders. The policy is designed to be understood and followed at all levels and by all staff.

\subsubsection{Quality objective}

Quality objective is the factual or tangible basis upon which quality policy and plans for implementing the quality programmes of an organization are built. Quality objective should be SMART (ie: specific, measurable, achievable, realistic and time-bound). Each staff of the organization is expected to work towards measurable objectives.

\subsubsection{Decision}

Decision simply means the selection of one or more options from a multitude of options in tackling a given organizational task. As far as the QMS is concerned, an organization should make SMART decisions based on recorded data. An example of a SMART decision is: The QMS must be audited and evaluated regularly for conformance and effectiveness, so as to assure quality and continual improvement. 


\subsubsection{Traceability}

Traceability is concerned with and refers to the fact that typically, recorded data are meant to show how and where raw materials and products were processed, in order to allow products and problems to be traced to their sources.

\subsubsection{Product realization}

Product realization refers to the scenario in which, when developing a new product, an organization plans the stages of development, with appropriate testing at each stage. The organization tests and documents whether the product meets design requirements, legal requirements, and user or customer needs.

\subsubsection{Quality plan}

Quality plan refers to a document specifying the QMS processes (including the product realization processes), and the resources to be applied to a specific product or project.

\subsubsection{Monitoring and measurement}

Monitoring and measurement refer to the scenario in which an organization must regularly review its performance through meetings and internal audits, and determine whether the QMS is working and what improvements can be made. The organization must have a documented procedure for internal audits and a procedure for dealing with past problems and potential problems. It must keep records of these activities and the resulting decisions, and monitor their effectiveness. It must have documented procedures for dealing with actual and potential nonconformances (problems involving suppliers, customers, or internal problems).

\subsubsection{Continual improvement}

Continual Improvement refers to the scenario in which an organization 1) makes sure no customer uses a bad product, 2) determines what to do with a bad product, 3) deals with the root cause of problems, and 4) keeps records to use as a tool to improve the QMS.

\subsubsection{Customer requirements}

Customer requirements refer to the attributes that the buyer of a product (or user of a service) wants. The core business of an organization is to determine customer requirements and to meet them, in accordance with sub-clause 5.2 (Customer focus).

\subsubsection{Drug Regulatory Agencies (DRAs)}

Drug regulatory agencies (DRAs) are organizations set up by the State on behalf of the general public with a Mandate to regulate drugs and related products and services. The Mandate of some DRAs may include production and distribution of certain goods like vaccines and orphan drugs. Either the State or the general public can be regarded as customer, stakeholder or shareholder. DRAs like all other organizations must have a system for communi- 
cating with customers or stakeholders about product information, inquiries, contracts, orders, feedback, and complaints. All DRAs are "service providers" but some produce and even distribute certain specific items, as mentioned above. Nigeria's National Agency for Food and Drug Administration and Control (NAFDAC) is a national DRA, while the European Medicines Evaluation Agency is a regional DRA.

\subsubsection{Mandate}

Mandate is a piece of legislation or instruction from a constituted authority to another constituted authority or body to carry out a named task. DRAs are mandated by the State to regulate drugs and health related products.

\subsection{The new industrial revolution and the aim of this chapter}

It is well established that the high state of development in the chemical/ pharmaceutical industrial sector in the US, Japan, South Korea, Britain, Germany and other European countries owes much to the powerful synergy between regulatory legislations, industrial standards and a focused political will. It is also manifest that the rapid, all-round industrial revolution in China in the past decade or so owes much to China's embrace of ISO standards, especially ISO 9001, as shown in Table 1 after a recent [15].

\begin{tabular}{llll}
\hline Country & Ranking & No. certificates & Pertinent remark \\
\hline China & 1 & 257,076 & Relies mostly on ISO standard. \\
\hline Italy & 2 & 130,066 & Relies mostly on ISO standard \\
\hline Japan & 3 & 68,484 & Relies only partly on ISO standard \\
\hline Spain & 4 & 59,576 & Relies substantially on ISO standard \\
\hline Russia & 5 & 53,152 & Relies substantially on ISO standard \\
\hline Germany & 6 & 47,156 & Relies only partly on ISO standard \\
\hline UK & 7 & 41,193 & Relies only partly on ISO standard \\
\hline India & 8 & 37,493 & Relies substantially on ISO standard \\
\hline South Korea & 9 & 28,935 & Relies substantially on ISO standard \\
\hline US & 10 & 23,400 & Relies only partly on ISO standard \\
\hline
\end{tabular}

*Source ISO Survey 2009 [15]. Most countries have their own national standards in addition to ISO standards. For example the UK is well known for its industrial standards pre-sufixed by BSI (British Standards Institution).

Table 1. The top 10 countries in ISO certification in 2009.

It must be stated that countries like Japan, Germany, Britain and US use their own national standards in addition to those of ISO. Based on the foregoing, we state that the specific aim of this article is: To examine the QMS requirements of ISO 9001:2008 and the requirements 
for regulating herbal drugs in Nigeria (a developing economy) and Europe (a developed economy), with a view to devising a framework that will better regulate herbal drugs and facilitate their trade worldwide. Such a framework will greatly benefit developing countries like Nigeria that are yet to benefit optimally from their comparative advantage in the abundance of spices, herbs and medicinal plants. In addition, marketers and users of herbs in consumer nations like the US, Canada, Germany, UK and France, where consumption now runs in to billions of US dollars, will also profit greatly from an improved and regularized world trade in herbs.

\section{Results \& discussion: A Framework for Efficient Herbal Drug Regulation (HDR)}

\subsection{Justification for establishing national or regional DRAs}

Most or all countries have a national or regional agency that regulates the production, distribution and use of drug products. The process of regulation commences with the registration of the producer, the product, the distributor and in some cases the user. In some countries drugs, foods and dietary supplements are regulated by the same body (eg: Nigeria's NAFDAC and US-FDA). The EU's EMEA however regulates only drug products. States or regions need to have DRAs in order to ensure order in the production, distribution and use of drugs. Without DRAs utter chaos and pandemonium will result in production (eg: manufacturers will do as they please without a uniform control), distribution (distributors and suppliers will do as they choose without a uniform order) and use (prescribers and users will do as they think without a uniform regime), which would allow incidences of counterfeit and expired drugs in drug distribution chain, drug abuse and emergence of drug resistant disorders, especially infective conditions like malaria and TB.

\subsection{Comparative analysis of Nigeria's and EU's requirements for herbal drug regulation}

A careful scrutiny of the requirements for registering and regulating herbal drugs in Europe and in Nigeria reveals their basic similarity, as shown in Table 2.

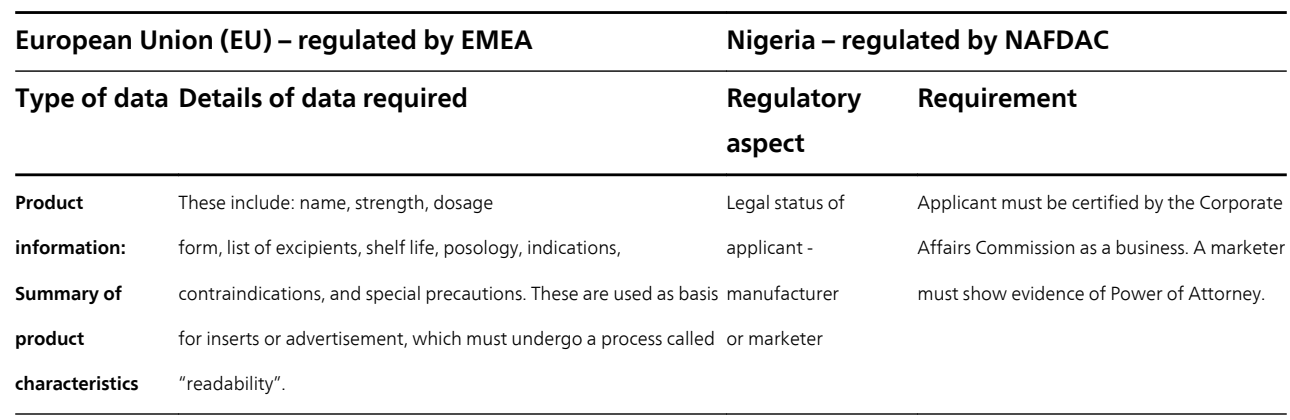




\begin{tabular}{|c|c|c|c|}
\hline \multirow{2}{*}{\multicolumn{2}{|c|}{$\begin{array}{l}\text { European Union (EU) - regulated by EMEA } \\
\text { Type of data Details of data required }\end{array}$}} & \multicolumn{2}{|c|}{ Nigeria - regulated by NAFDAC } \\
\hline & & $\begin{array}{l}\text { Regulatory } \\
\text { aspect }\end{array}$ & Requirement \\
\hline $\begin{array}{l}\text { Quality control } \\
\text { data: } \\
\text { Refer to GMP } \\
\text { requirements } \\
\text { for production. }\end{array}$ & $\begin{array}{l}\text { These include: production must be in a GMP compliant, product } \\
\text { must be produced with validated formula and method, there must } \\
\text { be a product specification, stability studies must be carried out in } \\
\text { the container proposed for marketing for purposes storage/ shelf- } \\
\text { life, and dossiers must be provided for starting materials and } \\
\text { finished product. }\end{array}$ & $\begin{array}{l}\text { Analytical status of the } \\
\text { t product for } \\
\text { registration. }\end{array}$ & $\begin{array}{l}\text { e The product must have: certificate of } \\
\text { analysis, dossier containing data on } \\
\text { ingredients, method of analysis, stability, } \\
\text { dosage and safety precautions. }\end{array}$ \\
\hline $\begin{array}{l}\text { Safety data } \\
\text { requirements }\end{array}$ & \multicolumn{3}{|c|}{$\begin{array}{l}\text { or human studies, review of potential drug-drug interactions, side inspection of premises. premises must be GXP compliant. Marketers } \\
\text { effects and contraindications. Others include: } \quad \text { must provide convincing evidence of GXP } \\
\text { recognized monographs, data special }\end{array}$} \\
\hline $\begin{array}{l}\text { Traditional use } \\
\text { evidence }\end{array}$ & $\begin{array}{l}\text { Evidence that the product has been in use } \\
\text { as medicine for } 30 \text { years or more (the last } \\
15 \text { must be in the EU. Notably, there is no requirement to prove } \\
\text { efficacy (De Smet, 2005). }\end{array}$ & $\begin{array}{l}\text { Post marketing } \\
\text { surveillance plan/ } \\
\text { report }\end{array}$ & $\begin{array}{l}\text { Applicant may be required to provide a plan } \\
\text { for reporting on the use of the product and } \\
\text { of any adverse reactions. }\end{array}$ \\
\hline 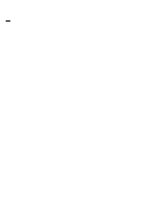 & - & $\begin{array}{l}\text { Others, such as fees } \\
\text { and } \\
\text { waivers. }\end{array}$ & $\begin{array}{l}\text { Fees are required at several stages of the } \\
\text { registration but waivers are not expressly } \\
\text { stated, thereby negating the concept and } \\
\text { need for transparency (see Table } 3 \text { for extra } \\
\text { requirements). }\end{array}$ \\
\hline \multicolumn{4}{|c|}{$\begin{array}{l}\text { *The Table was drawn based on data gathered from references including Goldman [5]; De Smet [7, 8]; Ann } \\
\text { Godsell Regulatory [16]; and various NAFDAC leaflets, including Akunyili [17]. Note that the requirements for } \\
\text { registration in Nigeria are not necessarily less tasking, but their lack of explicitness can be a booby trap and a } \\
\text { leeway for non-transparency. The necessity for explicitness and transparency is very important because some } \\
\text { years ago the Director General of China's drug regulatory agency was sentenced to death for alleged corrupt } \\
\text { practice [18]. In } 2000 \text { the entire Management of NAFDAC was sacked in similar grey circumstances. }\end{array}$} \\
\hline
\end{tabular}

Table 2. Requirements for herbal registration compared between EU and Nigeria.

But, while the EMEA approach is technically more explicit, though not necessarily more exerting than NAFDAC's, the latter is administratively much more cumbersome, and therefore more liable to inefficiency and abuse. Table 3 shows the extra bureaucratic demands of NAFDAC. We stated earlier that, although, $80 \%$ of people in developing countries like Nigeria depended on herbs, these countries contributed only $7.2 \%$ to herbal drug trade in 1999 . By contrast, the developed nations, where people relied less on herbs, contributed $55.2 \%$. This scenario is explained by the fact herbal drugs are better regulated in developed regions like the China, India, Japan and South East Asia, the EU and North America [2]. 


\subsection{Justification for selecting ISO $9001: 2008$ for this study}

Although most of the OECD countries and other highly industrialized economies, where herbal drugs are well regulated, have their own national standards, all do embrace ISO standards, especially ISO 9001:2008. For example, the British Standards Institution (BSI) is well known and widely adopted in many other countries worldwide, and although international in application, such national standards do not bear the tag "international". By contrast, the ISO family of standards bear the tag "international". ISO 9001:2008 is an international standard designed to address systemic change (ie: a change that affects an organization as a whole). The global popularity of ISO 9001:2000 - the predecessor of ISO 9001:2008, is attributable to the following factors: a) major purchasers require their suppliers to hold ISO 9001 certification [15, 19]; b) studies indicate significant financial benefits for organizations certified to ISO 9001 [19,20]; and c) similar superior operational performance of ISO certified firms has been severally confirmed [21-24]. As just noted, ISO 9001:2008 is an update of ISO 9001:2000, and we have selected it for this study by reason of its popularity and versatility, and because it is a process-based QMS that addresses systemic change affecting whole organizations like a national or regional drug DRA, like Nigeria's National Agency for Food and drug Administration and Control (NAFDAC), the US Food and Drug Administration (US-FDA) and the European Medicines Evaluation Agency (EMEA).

\begin{tabular}{|c|c|c|}
\hline S/No & Extra requirement & Remark \\
\hline 1 & Five (5) copies of the product dossier. & Probably unreasonable \\
\hline 2 & Three (3) packs of the products samples. & Probably reasonable \\
\hline 3 & $\begin{array}{l}\text { Notarized original copy of the duly executed Power of } \\
\text { Attorney from the product manufacturer. }\end{array}$ & $\begin{array}{l}\text { Clearly unreasonable for all } \\
\text { categories of applicants }\end{array}$ \\
\hline 4 & $\begin{array}{l}\text { Certificate of Manufacture issued by the competent } \\
\text { health or regulatory authority in country of origin and } \\
\text { authenticated by the Nigerian Mission in that country. } \\
\text { Where there is no Nigerian mission, The British High } \\
\text { Commission or an ECOWAS country Mission will } \\
\text { authenticate. }\end{array}$ & $\begin{array}{l}\text { Probably unreasonable for all } \\
\text { categories of applicants }\end{array}$ \\
\hline 5 & $\begin{array}{l}\text { If contract-manufactured, Contract Manufacturing } \\
\text { Agreement, properly executed and notarized by a } \\
\text { Notary Public in the country of manufacture. }\end{array}$ & $\begin{array}{l}\text { Clearly unreasonable for all } \\
\text { categories of applicants }\end{array}$ \\
\hline 6 & $\begin{array}{l}\text { Current World Health Organization Good } \\
\text { Manufacturing Practice Certificate for the } \\
\text { manufacturer, authenticated by the Nigerian Mission. }\end{array}$ & $\begin{array}{l}\text { Clearly unreasonable for all } \\
\text { categories of applicants }\end{array}$ \\
\hline 7 & $\begin{array}{l}\text { Certificate of Pharmaceutical Products (COOP) duly } \\
\text { issued and authenticated. }\end{array}$ & $\begin{array}{l}\text { Clearly unreasonable for all } \\
\text { categories of applicants }\end{array}$ \\
\hline 8 & $\begin{array}{l}\text { Current Superintendent Pharmacists license to } \\
\text { practice issued by the Pharmacists Council of Nigeria (PCN). }\end{array}$ & Only probably reasonable \\
\hline
\end{tabular}




\begin{tabular}{|c|c|c|}
\hline S/No & Extra requirement & Remark \\
\hline 9 & Premises Registration License from PCN & Only probably reasonable \\
\hline 10 & $\begin{array}{l}\text { Certificate of Registration of brand name with } \\
\text { trademark registry in the Ministry of Commerce here } \\
\text { in Nigeria; Letter of invitation from manufacturer to } \\
\text { inspect factory abroad, stating full name and location } \\
\text { of plant. }\end{array}$ & $\begin{array}{l}\text { Probably unreasonable for all } \\
\text { categories of applicants }\end{array}$ \\
\hline 11 & $\begin{array}{l}\text { The applicable fee payable only if documents are } \\
\text { confirmed to be satisfactory }\end{array}$ & $\begin{array}{l}\text { Likely to be abused if the } \\
\text { amount is high. The fee should } \\
\text { be a token amount paid by all } \\
\text { applicants }\end{array}$ \\
\hline 12 & $\begin{array}{l}\text { Nutraceuticals, medical devices and other regulated } \\
\text { drug products have similar requirements, with minor } \\
\text { variations. Specific details can be obtained from } \\
\text { NAFDAC. }\end{array}$ & $\begin{array}{l}\text { A sketch of the minor variations } \\
\text { should be provided in print no } \\
\text { matter how brief. Any } \\
\text { information provided by } \\
\text { NAFDAC should be printable } \\
\text { for sake of transparency }\end{array}$ \\
\hline
\end{tabular}

Table 3. NAFDAC's extra requirements for registering herbal medicines.

\subsection{A systematic review of the eight clauses of ISO 9001:2008 in relation to DRAs}

\subsubsection{A synopsis of the Mandate of DRAs and the eight clauses of ISO 9001:2008}

ISO 9001:2008 is the most widely used QMS standard, with over a million certificates issued worldwide. Alas, it was revealed at the SON-NIPRD course in 2011, that only two public institutions in Nigeria have ISO 9001 certification! Yet, as stated earlier, ISO 9001:2008 defines the minimum requirements for a well managed organization. The standard is published by the International Organization for Standardization (ISO), Basle, Switzerland. National accreditation bodies like the Standards Organization of Nigeria (SON) provide accreditation to registrars who issue the ISO 9001 certificates to those they audit. ISO 9001:2008 is set out in eight clauses designated clauses 1 to 8 . The structure and salient points/ directing principles of the clauses are tabulated below. A copy of ISO 9001:2008 is a prerequisite for this study. Similarly required, is a grasp of the requirements for registering and regulation herbal drugs in a developed economy like Europe; and in a developing country like Nigeria, as depicted in Table 2. It is well known that herbal drugs are better regulated in the developed than in developing countries. Table 3 suggests that undue bureaucracy or needlessly cumbersome requirements can hinder efficient regulation. Tables $4-13$ show the structure and salient points/ directing principles of the 8 clauses. 


\begin{tabular}{|c|c|c|}
\hline Clause & Title and subtitles, with remarks & Salient points/ directing principles/ application to DRAs \\
\hline 1 & $\begin{array}{l}\text { 1. Scope } \\
1.1 \text { General } \\
1.2 \text { Application }\end{array}$ & $\begin{array}{l}\text { ISO 9001:2008 can be used to establish, and to update a DRA's QMS. A DRA, like other parastatals or } \\
\text { private organizations must consider its unique operational environment and the dynamics and risks } \\
\text { associated therewith. }\end{array}$ \\
\hline 2 & $\begin{array}{l}\text { 2. Normative references } \\
\text { (eg: ISO 9000:2005 is devoted to QMS } \\
\text { Fundamentals and Vocabulary; and ISO } \\
\text { 9004:2009 is devoted to Managing for } \\
\text { Sustained Success) }\end{array}$ & $\begin{array}{l}\text { A normative reference implies, unless otherwise stated, that the most recent versions of the separate } \\
\text { documents should be referenced. DRAs would benefit immensely from such key references and } \\
\text { compendia such as the International Pharmacopoeia and others like the BP and USP, and the WHO } \\
\text { manual on Quality Control Methods for Medicinal Plant Materials. }\end{array}$ \\
\hline 3 & $\begin{array}{l}\text { 3. Terms and definitions } \\
\text { (see section } 2.4 \text { of this article on "Key } \\
\text { terminologies of ISO 9001:2008) }\end{array}$ & $\begin{array}{l}\text { The term "product" may also mean "service". "Legal requirements" means "statutory and regulatory } \\
\text { requirements". Most DRAs are service providers only, while others may produce and distribute certain } \\
\text { specialized health products. }\end{array}$ \\
\hline
\end{tabular}

*The Table is to be studied side by side with the contents of ISO 9001:2008 and Table 2, which is on regulatory requirements of DRAs.

Table 4. Clauses 1-3 of ISO 9001:2008 in relation to DRAs.

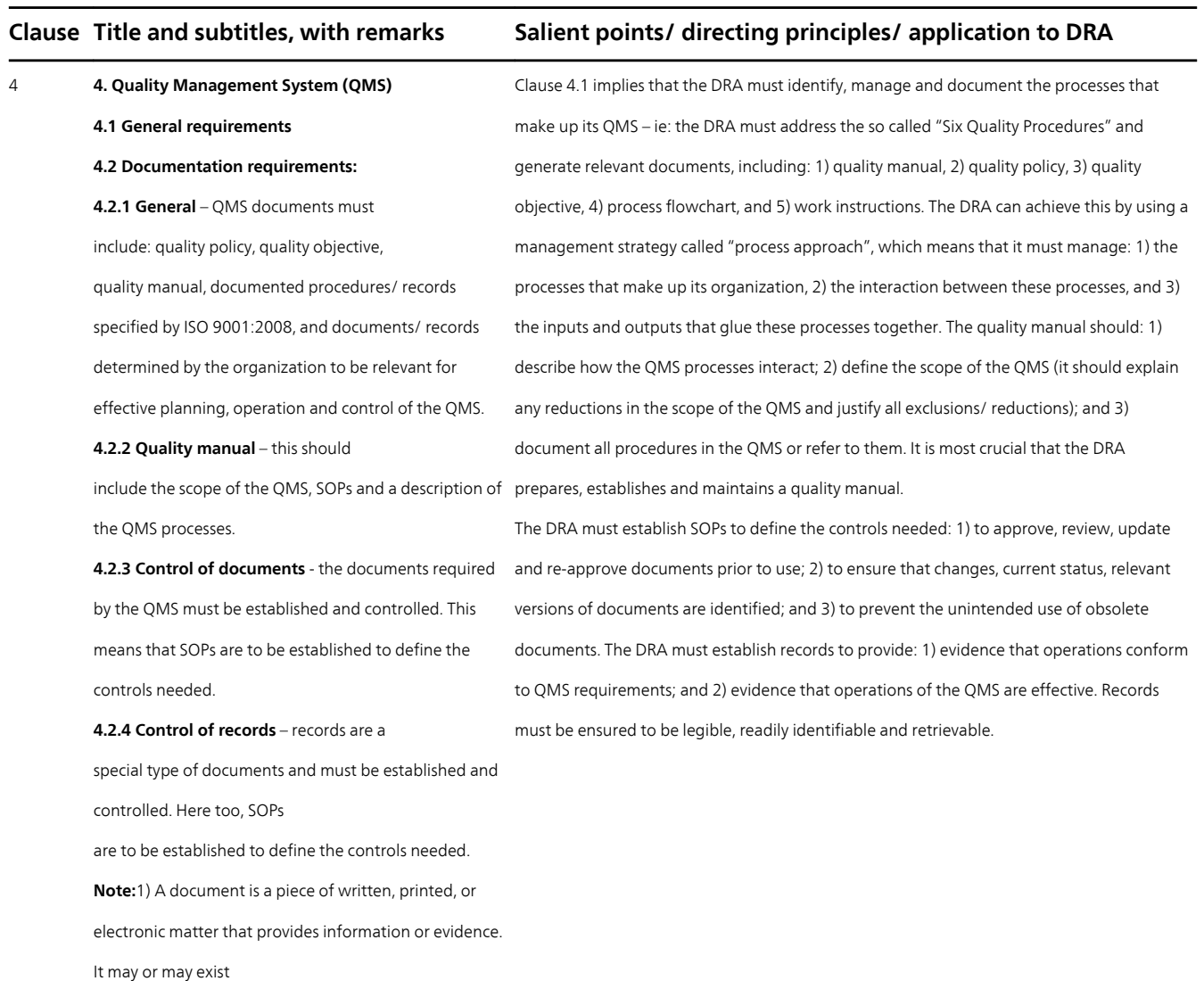




\begin{tabular}{l}
\hline Clause \\
\hline ititle and subtitles, with remarks Salient points/ directing principles/ application to DRA \\
in a permanent form. 2) A record is a permanent \\
document of something that is \\
kept for evidence or information. It specifically bears the \\
history of events or arrangements, and is preserved in a \\
lasting form.
\end{tabular}

*The Table is to be studied side by side with the contents of ISO 9001:2008 and Table 2, which is on regulatory requirements of DRAs.

Table 5. Clause 4 of ISO 9001:2008 in relation to DRAs.

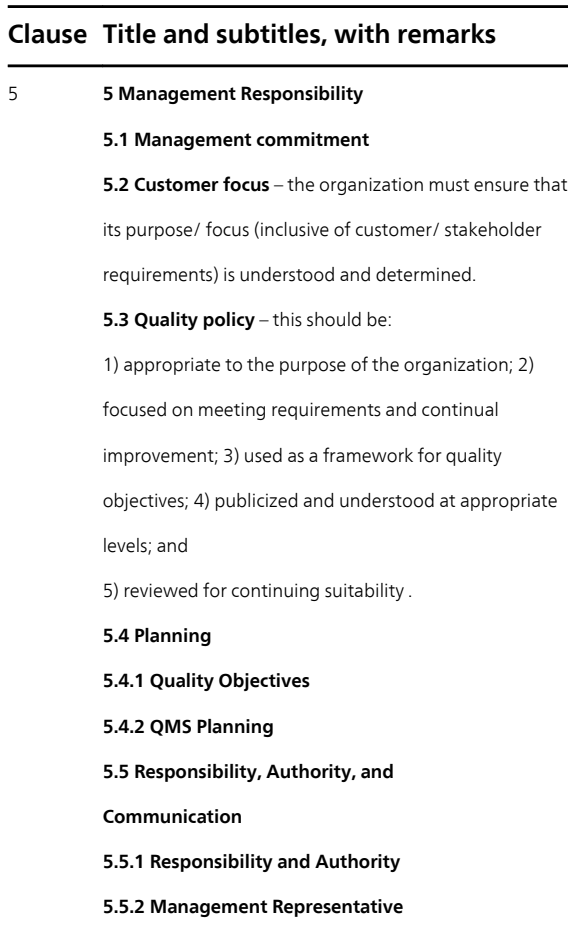

5.5.3 Internal Communication - it is crucial that the organization ensures that appropriate communication processes regarding the effectiveness of the QMS are established and implemented.

\subsection{Management Review}

5.6.1 General

5.6.2 Review Input - includes audit results, public feedback, process performance, status of preventive/ corrective action, follow-up from previous management review,

\section{Salient points/ directing principles/ application to DRA}

The DRA must be committed to developing and implementing a QMS, as well as, a commitment to continually improve the effectiveness of the QMS. The DRA can do this by 1) communicating and quality objectives; 3) conducting management reviews; and 4) by ensuring the availability of necessary resources. The "legal and customer requirements" of a DRA are implicit in its Mandate - which may be an act, law or decree. In planning, the DRA must 1) ensure that quality objectives are established at the relevant functions and levels within the Agency; 2) ensure that quality objectives are measurable and consistent with the quality policy; and 3) ensure that planning for the QMS meets the general requirements (clause 4.1) and quality objectives (clause 5.4.1), as well as, maintains the integrity of the QMS. In as much as operations must be carried out the DRA must ensure that the responsibilities and authorities for such are defined and communicated appropriately. It is essential that a member of top management, irrespective of other duties, be appointed (as Quality Manager) and given the responsibility to: 1) ensure that the needed processes are established, implemented, and maintained; 2) report to top management on the performance of the QMS; 3) report to top management on any need for improvement; and 4) ensure the promotion of awareness of Agency's Mandate. Most DRAs have a public relation office. For a DRA to be effective it must review its QMS at planned intervals to: 1) ensure an effective QMS; 2) assess possible opportunities for improvement; 3) evaluate the need for any changes; and 4) consider the need for changes to the policy and objectives. The DRA must of course maintain records of reviews as per clause 4.2.4. For a DRA, the inputs for review must include information on: 1) results of audits; 2) feedback from government and the public, eg - incidences of counterfeit drugs; 3) status of preventive and corrective actions, eg - incidences of drug abuse; 4) follow-up actions from earlier reviews; 5) changes that can affect the QMS; and 6) recommendations for improvement. 


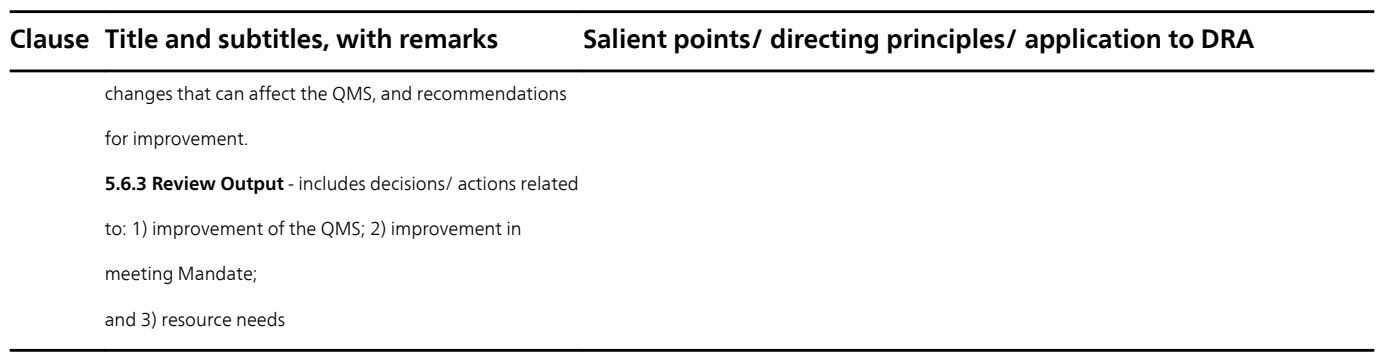

*The Table is to be studied side by side with contents of ISO 9001:2008 and Table 2, which is on regulatory requirements of DRAs.

Table 6. Clause 5 of ISO 9001:2008 in relation to DRAs.

\subsubsection{The immediate historical antecedent of NAFDAC and the continuing relevance of ISO 9001}

Nigeria's NAFDAC was created by decree in 1992/93 following the ethylene glycol disaster of 1991/92 in Langtang General Hospital, Plateau State, where ethylene glycol was used in the place of propylene glycol in preparing paracetamol elixir. The glycol had been purchased from a hitherto popular pharmacy shop located at Masalachin-Jumai Street, Jos. Prior to 1992/93, a department in the Federal Ministry of Health handled food and drug administration in Nigeria. Alas, in 2009 another ethylene glycol disaster occurred in Lagos. In this latter disaster a hitherto popular brand of paediatric mixture ("My Pikin") was found to contain ethylene glycol that had been purchased from an unregulated source. It is important to note that whereas the glycol implicated in the Lantang disaster was purchased from pharmaceutically regulated source, the glycol in the case of the Lagos disaster was purchased from a company that dealt in industrial chemicals associated with automobiles and cooling systems. The occurrence of this kind of disaster within less than two decades is matter of concern that calls for a more efficient programme for regulating drugs and industrial chemicals in developing countries. The US-FDA after whose image and likeness NAFDAC was created is known for efficiency mostly because it is supported by proper laws and strong institutions. Better laws and stronger institutions, including DRAs, are required to avoid or minimize this kind of disaster as seen in Nigeria. It seems instructive to mention the "Tylenol case" in the US, and how that case led to a new legislation. Between late September and early October 1982, seven persons in Chicago died after taking capsules of Tylenol (a brand of paracetamol), to which cyanide crystals had been added. The crystals had apparently been introduced into the capsules by someone who had removed bottles of Tylenol from several drugstores and then replaced them on the shelves. It took an intensive investigation by a team of over 100 agents, including FDA staff, to discover the mischief, which led to a 1982-legislation that required all over-the-counter drugs and medicines sold in the county (and later elsewhere in the US and beyond) carry manufacturers' seals which broken would be obvious. The rapid conclusion of the investigation led by the Illinois Attorney General himself (Tyrone C. Fahner) and the dispatch with which the new law was issued collectively testify to the inner workings of strong institutions - which developing nations lack. 


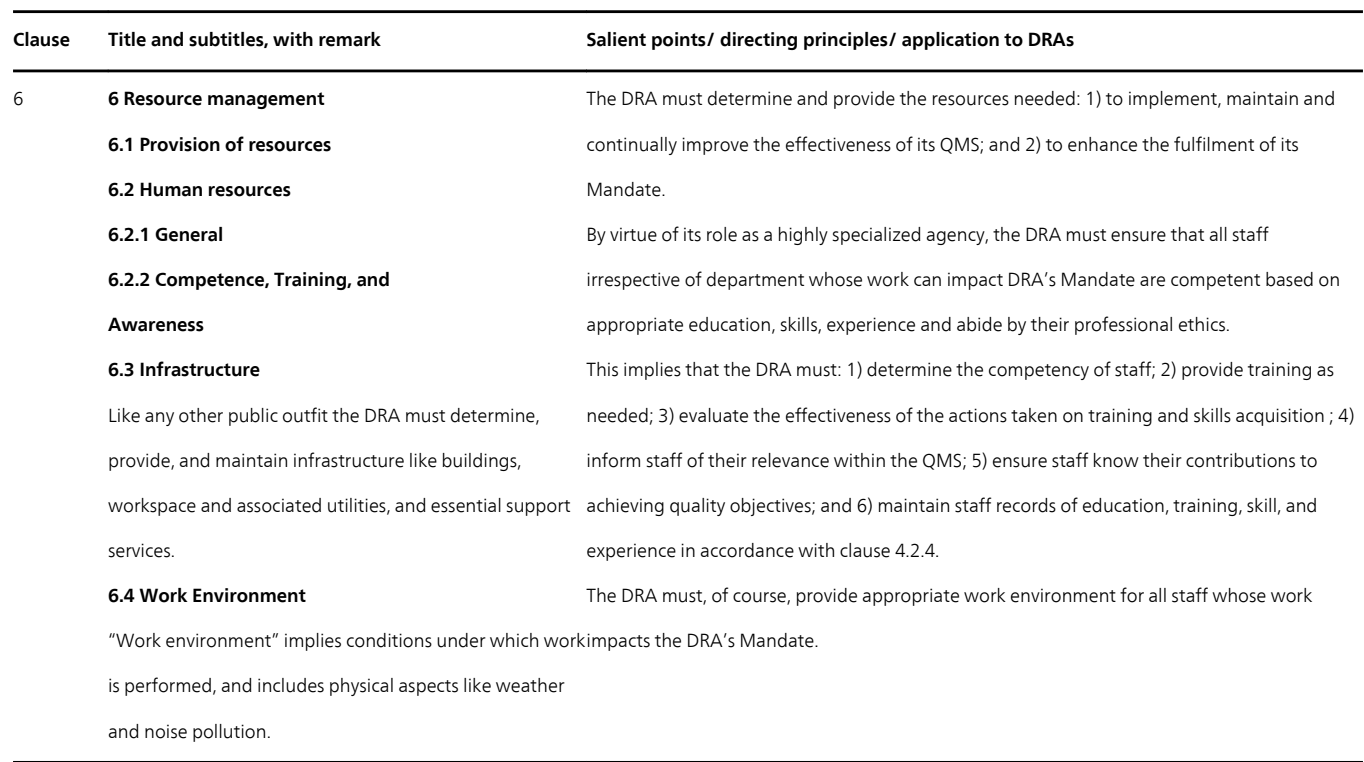

*The Table is to be studied side by side with contents of ISO 9001:2008 and Table 2, which is on regulatory requirements of DRAs.

Table 7. Clause 6 of ISO 9001:2008 in relation to DRAs.

\subsubsection{The making of stronger DRAs and the need for clearer demarcation of responsibilities}

In most countries where DRAs are not a department of the Ministry of Health, they exist as a parastatal or as a special department within the Ministry (as in Japan), with conditions of service being slightly more favourable than in the rest of the Ministry. The idea is to give special incentives to the staff on account of hazards perceived to be peculiar to the job. In Nigeria, NAFDAC is well housed both at the federal and state levels and the staff earn about the same remuneration as the universities and research institutes. In most countries the DRAs have well equipped offices and laboratories, and those DRAs that produce and distribute goods are equipped with the necessary plant and storage facilities.

\subsubsection{The inevitability of confusion in the absence of regulatory standardization}

In Nigeria, NAFDAC previously handled certain aspects of manufacture/ distribution of vaccines until certain developments (or rather controversies over quality/ effectiveness of polio vaccines during the late 1990s/ early 2000s) led, first to the creation of a National Programme on Immunization (NPI); and latter to the transfer of the same functions from NPI back to the Federal Ministry of Health. It is obvious from the foregoing that institutions like NAFDAC and NPI would have performed better had they been certified. It seems also that one of the keys to ending the cycle of poverty and underdevelopment in some countries is to ensure that elite institutions like the DRAs are certified to appropriate ISO standards. Certifications of agencies like the Health Insurance Scheme and the Pension Commission will definitely reduce perceive current levels of corruption in such institution. 


\section{Clause Title and subtitles, with remark}

\section{Product realization}

\subsection{Planning of product}

realization Product realization typically implies that manufacturers 1) plan and develop the QMS processes needed for product realization; 2) keep the planning consistent with other requirements of the QMS; 3 ) document the plan

in a suitable form; and 4) determine through the planning the following: a) quality objectives and product requirements; b) need for processes, documents, and resources; c) verification (establishment of truth confirmatory evidence), validation (formal registration/ obtainment of official sanction), monitoring, measurement, inspection, and test activities; d) criteria for product acceptance; and e) records providing evidence that the processes and resulting product diazepam over-the-counter in Europe but not in Nigeria? To what extent do political, social meet requirements. Since DRAs regulate manufacturers they and economic factors affect "planning of service realization" in different social too must be acquainted with clause 7. NOTE 1: Recall that environments? It well known that regulatory strategies that work in Europe often fail to "quality plan" (2.4.8 of this article) is a document specifying work outside despite obvious legislative similarities between nations. the processes, and the resources to be applied to a specific product, project, or contract.

The customers/ stakeholders of a typical DRA are the general public, manufacturers, suppliers and the government. DRAs that engage in production and distribution must

\section{Salient points/ directing principles / application to DRAs}

Some DRAs produce/ store/ distribute specialized and non-profit products like vaccines and orphan drugs. For such, all aspects of clause 7 apply. The DRAs of developed economies concentrate on regulating manufacturers, distributors and use of products. Different processes are involved in drug regulation but these often have some aspects in common. For example, the process of registering a manufacturer and that of registering a product are essentially the same, but they differ in their aims, point of action, who by, and so on. Some of the processes involved in "planning of service realization", which is the core business of a typical DRA, require a wide range of differing concepts, technicalities, approaches, specializations, and so on. For example, although the technical aspects of producing tablets of aspirin, diazepam, B-complex, erythromycin, and orphan drugs may be similar, the modes of their regulation and distribution are different. Given the involved Mandate of DRAs, different strategies must be developed to grapple with the differing nuances and intricacies associated with the regulation of the five products. Typically, questions that have answer buried in culture/ society rather than the lab do arise in drug regulation. Why, for example, despite the similarities between NAFDAC and EMEA, it is impossible to buy erythromycin or clause 7.3 (vide infra) to the development of product realization processes 7.2 Customer-Related Processes

\subsubsection{Determination of \\ Requirements Related to the \\ Product}

7.2.2 Review of Requirements

Related to the Product

7.2.3 Customer Communication

The intensity and scope of communication depends on the product and the associated

mandate. Thus the DRA must determine and implement the documented, they must be confirmed before acceptance. But if product requirements are necessary arrangements for communicating with stakeholders

on aspects like 1) product information; 2) inquiries and contracts; 3) customer/ stakeholder feedbacks - positive or negative desirable attributes. Such DRAs must also determine the legal requirements applicable to the product. Other desirable requirements, including post-delivery activities like maintenance services, may be considered. DRAs that produce or distribute would normally review the product requirements before committing to supply in order to: 1) ensure that product requirements are defined; 2) resolve any requirements differing from those previously expressed; and 3) ensure its ability to meet the requirements. In the same vein when a DRA plans a regulatory strategy or legislation the plan should be graduated and made reasonable to its purpose and scope and with reference to the operating socioeconomic environment. The DRA must maintain the results of reviews, and any subsequent follow-up actions in accordance with 4.2.4. When the requirements are not changed, the DRA must ensure relevant documents are amended and relevant personnel are made aware of the changed requirements.

NOTE: In some situations a formal review is impractical for each order. In such cases reviews can cover relevant product information such as catalogues or adverts. - determine customer requirements, which invariably include specified and unspecified but ${ }^{*}$ The Table is to be studied side by side with contents of ISO 9001:2008 and Table 2, which is on the regulatory re-
quirements of DRAs.

Table 8. Clause 7 of ISO 9001 in relation to DRAs (Product planning and Customer-Related processes). 


\section{Clause Title and subtitles, with remarks}

\subsection{Design and Development}

7.3.1 Design/ Development Planning

DRAs that produce or distribute must plan and control product design/development: 2) appropriate testing, review and validation for each stage; and 3) responsibility/authority for design/ development.

\subsubsection{Design /Development Inputs}

In designing/ developing a physical good or a service, the DRA must determine the needed inputs and keep records as per 4.2.4. The inputs must include: 1) functional and performance requirements; 2) applicable legal requirements; 3) applicable information derived from similar designs; and 4) requirements essential for design and development.

\subsubsection{Design/ Development Outputs}

Where applicable, DRAs must

document the outputs of the design/ development process in a form suitable for verification against the inputs to the process. The outputs must 1) meet or match design and development input requirements; 2 ) provide information

for purchasing, production and service; 3) contain or reference product acceptance criteria; 4) define essential characteristics for safe and proper use; 5) be approved before their release

\subsubsection{Design/ Development Review}

7.3.5 Design/ Dev. Verification

\subsubsection{Design/ Dev. Validation}

Validation activities are performed in accordance with 7.3.1 to confirm that the resulting product is capable of meeting the requirements for its specified application or intended use.

\subsubsection{Control of Des./ Dev Changes}

For either physical goods or policy,

DRAs must 1) identify design and development changes and maintain records as per $4.2 .4 ; 2$ ) review, verify, validate and approve changes before implementation; 3 ) evaluate the changes in terms of their effect on constituent parts (raw material) and products (or policies) already delivered (or implemented). design/ development. They must determine 1) the stages of

\section{Salient points/ directing principles/ application to DRAs}

The same principle followed in planning a physical product is followed in planning a service. The interfaces between the different groups involved must be managed to ensure effective communication/ clear assignment of responsibility. Design and development review, verification and validation have distinct purposes. They can be conducted and recorded separately or in any combination, as the DRA deems suitable for the product or the type of service.

A DRA would review the selected inputs for adequacy and resolve any incomplete, ambiguous, or conflicting requirements. Examples of application inputs include: (1 applicable information derived from similar designs; and (2 requirements essential for design and development. If a DRA is designing a policy to curb drug abuse in a particular locality, useful inputs for the design would include statistics like 1) the age, gender and occupation of abusers; 2) the type of drugs abused; and 3) the success rate of similar policies elsewhere. NOTE: Information for production and service can include details for product preservation

A DRA must perform systematic reviews of design and development at suitable stages in accordance with planned arrangements (7.3.1) so as to: 1) evaluate the ability of the results to meet requirements; and 2) identify problems and propose necessary actions. Reviews must include representatives of the functions concerned. Results of reviews and subsequent follow-up actions must be maintained as per 4.2.4. A DRA would perform design and development verification in accordance with 7.3.1 to ensure that output meets the design and development input requirements; and maintain the results of such verification and subsequent follow-up actions. When practical and desirable, validation must be completed before delivery or implementation of the product. Results of the validation and of subsequent follow-up actions must be maintained as per 4.2.4. Just as some DRAs produce or distribute physical products, some DRA have their own testing facilities while others contract out such tests. Thus the purchase needs of DRAs differ with their Mandate. However, whenever purchasing is indicated the DRA must 1) ensure that purchased items conform to specified purchase requirements (Note: The type and extent of control applied to the supplier and purchased product depends upon the effect of the product on the subsequent realization processes or the final product); 2) evaluate and select suppliers based on their ability to supply goods in accordance with requirements; 3) establish the criteria for selection, evaluation, and re-evaluation; and 4) maintain the results of such evaluations and subsequent follow-up actions in accordance with sub-clauses 4.2 .3 and 4.2 .4

*The Table is to be studied side by side with contents of ISO 9001:2008 and Table 2, which is on the regulatory requirements of DRAs.

Table 9. Clause 7 of ISO 9001:2008 in relation to DRAs (Design and Development). 


\subsubsection{Some causes and signs of a malfunctioning $D R A$}

Once the staff recruitment system can be skewed to favour persons, a serious non-compliance exists. Once the purchase processes can be demonstrated to have vested interest, a serious flaw exists in the QMS. Once there is a convincing evidence of maladministration, arbitrary treatment of personnel or executive high handed, a serious condition against performance exists.

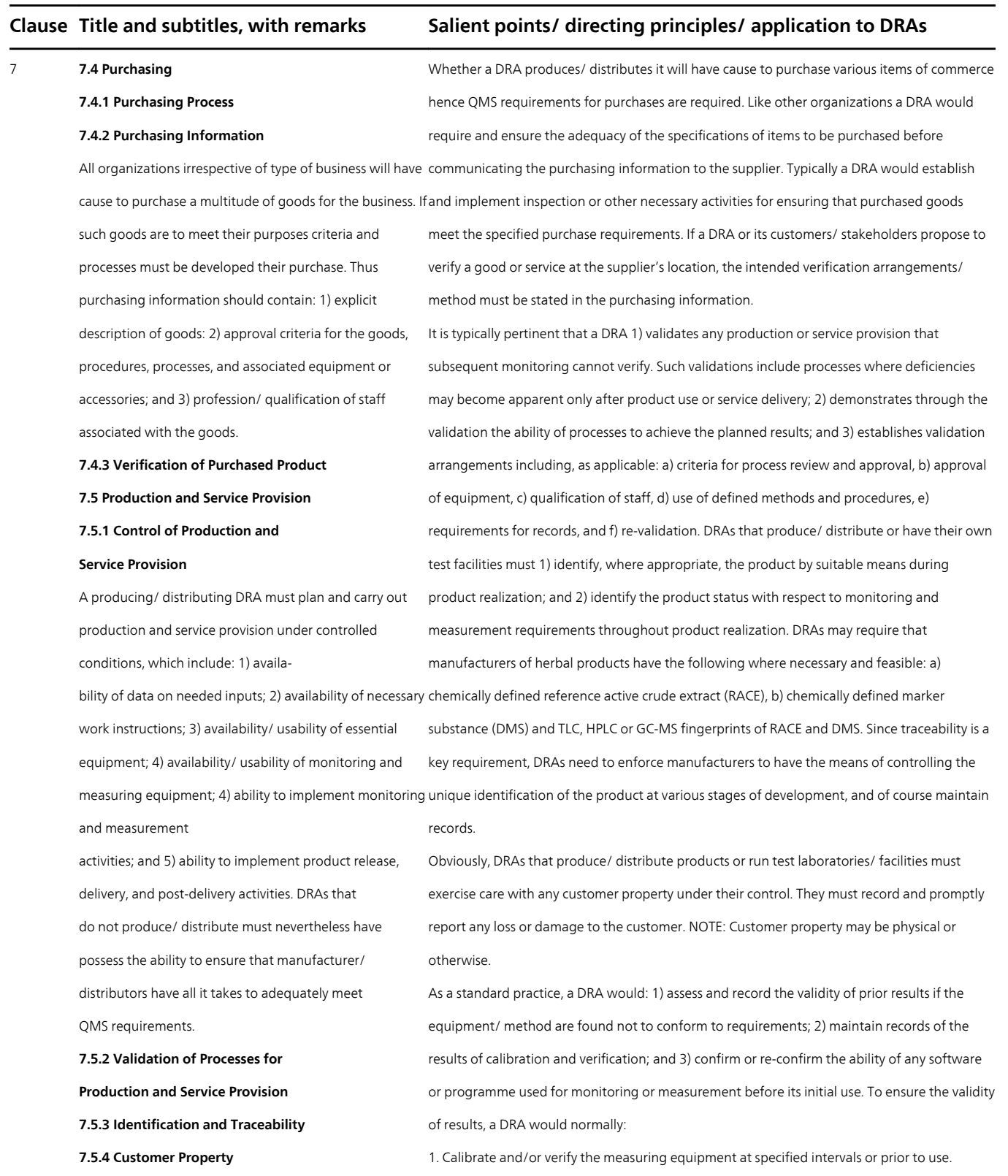




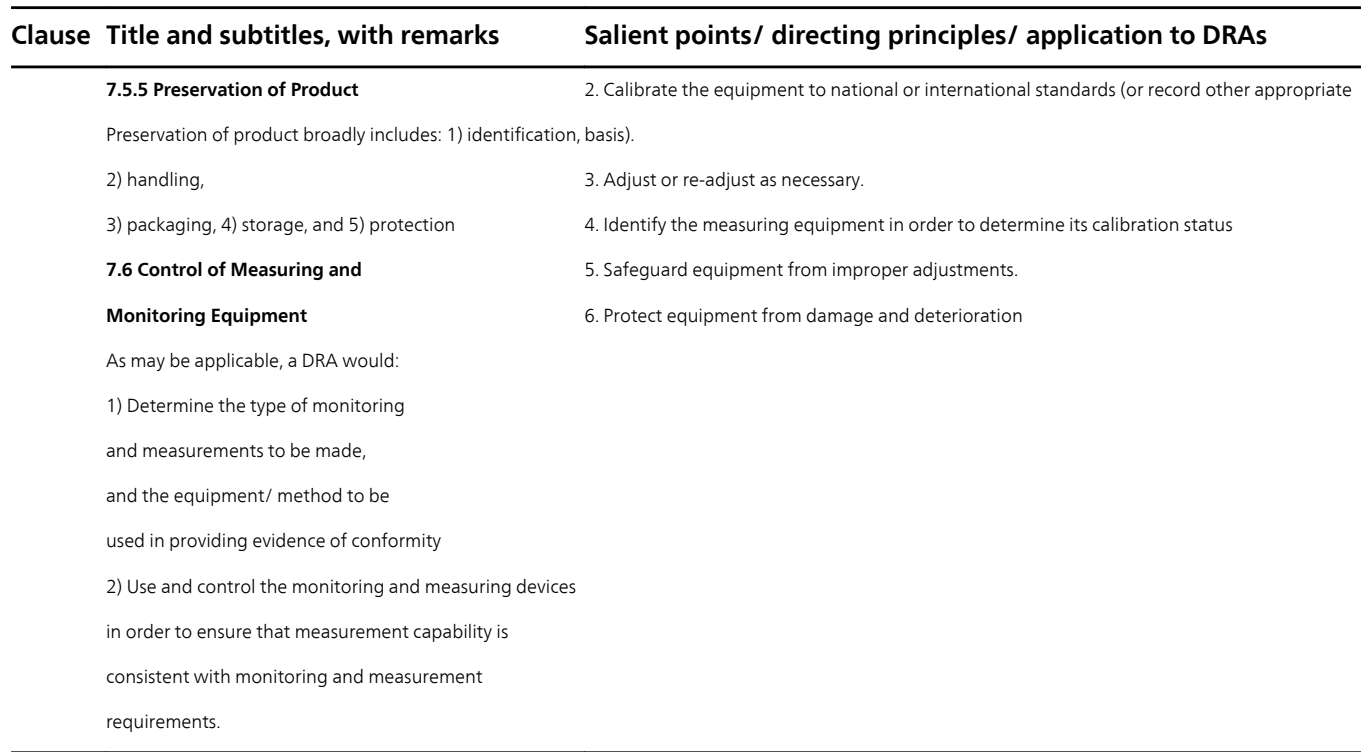

*The Table is to be studied side by side with contents of ISO 9001:2008 and Table 2, which is on the regulatory requirements of DRAs.

Table 10. Clause 7 of ISO 9001:2008 in relation to DRAs (Purchasing/ Production/ Control of Equipment).

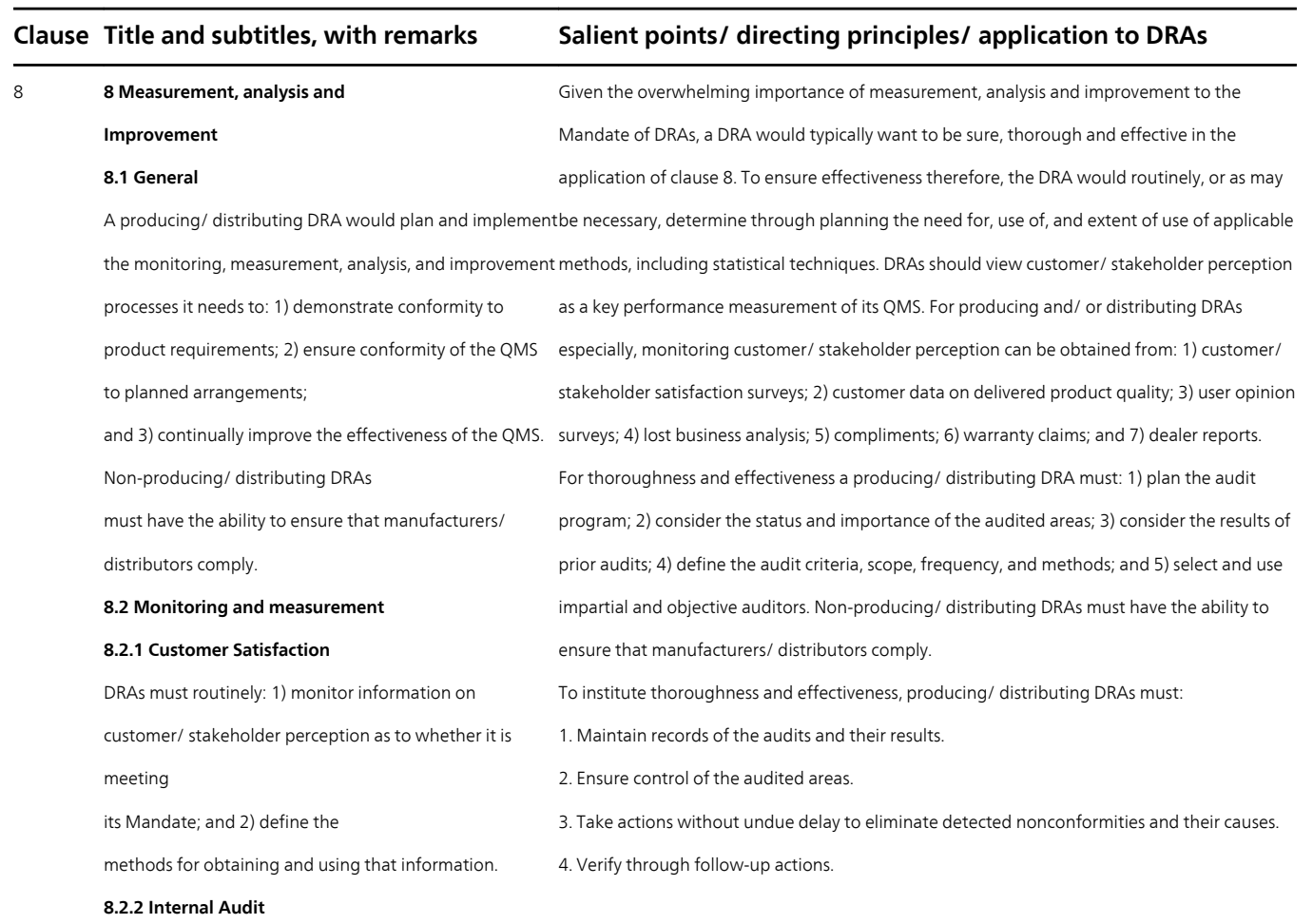




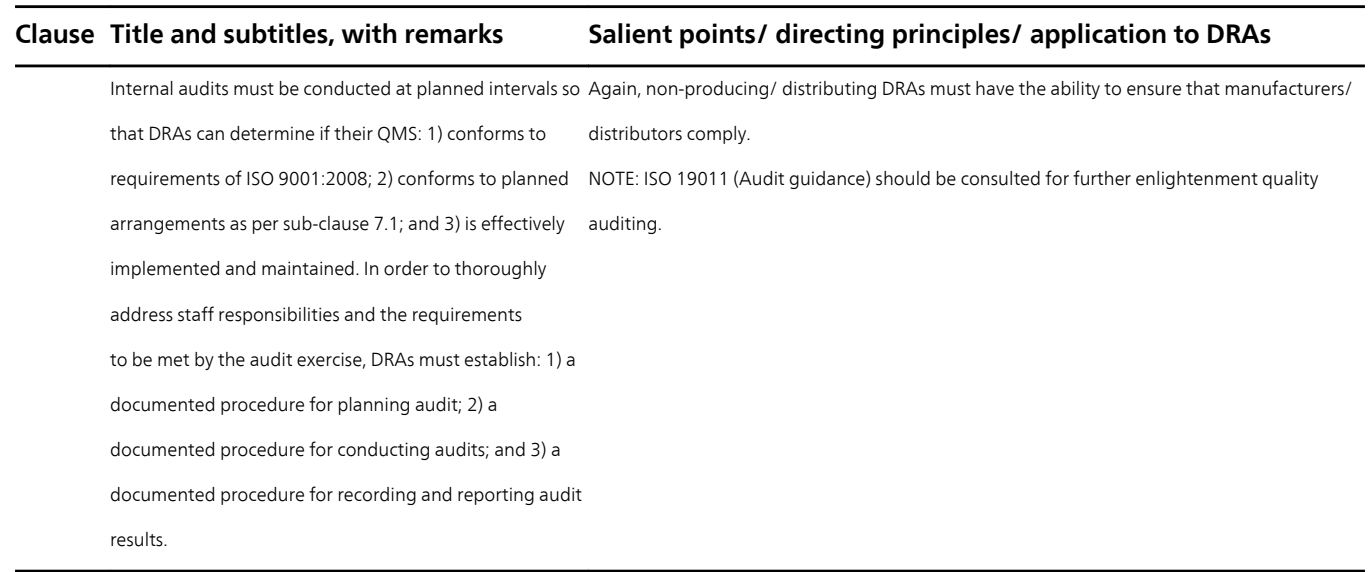

*The Table is to be studied side by side with contents of ISO 9001:2008 and Table 2, which is on the regulatory requirements of DRAs.

Table 11. Clause 8 of ISO 9001:2008 in relation to DRAs (Monitoring and measurement).

\subsubsection{Remediation of a malfunctioning DRA}

A national or regional DRA is a critical factor in socioeconomic development and wellbeing in at least two ways: i) by "guaranteeing the health of the nation" (as trumpeted in NAFDAC's adverts); and by supporting the emergence of responsible manufacturers of regulated products. It is well known that the US-FDA more than any US organization has made the US the world leader in manufacture of health products. The prominence of India and China in world drug trade owes much to the vibrancy and relative efficiency of their DRAs. There is therefore a critical need for DRAs to be vibrant and responsible. The gravity with which China views the role of her DRA can be gauged by the death sentence passed on the Director General in 2007 for accepting a bribe [15].

\begin{tabular}{|c|c|c|}
\hline Clause & Title and subtitles, with remarks & Salient points/ directing principles/ application to DRAs \\
\hline \multirow[t]{11}{*}{8} & 8.2.3 Monitoring and & Producing/ distributing DRAs need to 1) apply suitable methods for monitoring and measuring \\
\hline & Measurement of Processes & QMS processes; and 2) confirm through these methods the continuing ability of each process to \\
\hline & 1) Apply suitable methods to monitor and, where & satisfy its intended purpose. \\
\hline & applicable, measure the QMS processes. & Non-producing/ distributing DRAs must have the ability to ensure that manufacturers/ \\
\hline & 2) Confirm through these methods & distributors comply. \\
\hline & the continuing ability of each process to satisfy its & NOTE: When determining "suitable" methods, consideration is given to the type and extent of \\
\hline & intended purpose. & monitoring or measurement for each process in relation to its impact on product conformity and \\
\hline & 3) When the planned results are not achieved, take & on the effectiveness of the QMS. \\
\hline & correction and corrective action, as appropriate. & To better fulfil their Mandate producing/ distributing DRAs must 1) monitor and measure product \\
\hline & 8.2.4 Monitoring and & characteristics so as to verify if product requirements are being met; 2) carry out the monitoring \\
\hline & Measurement of Product & and measurements at the appropriate stages of product realization in accordance with planned \\
\hline
\end{tabular}




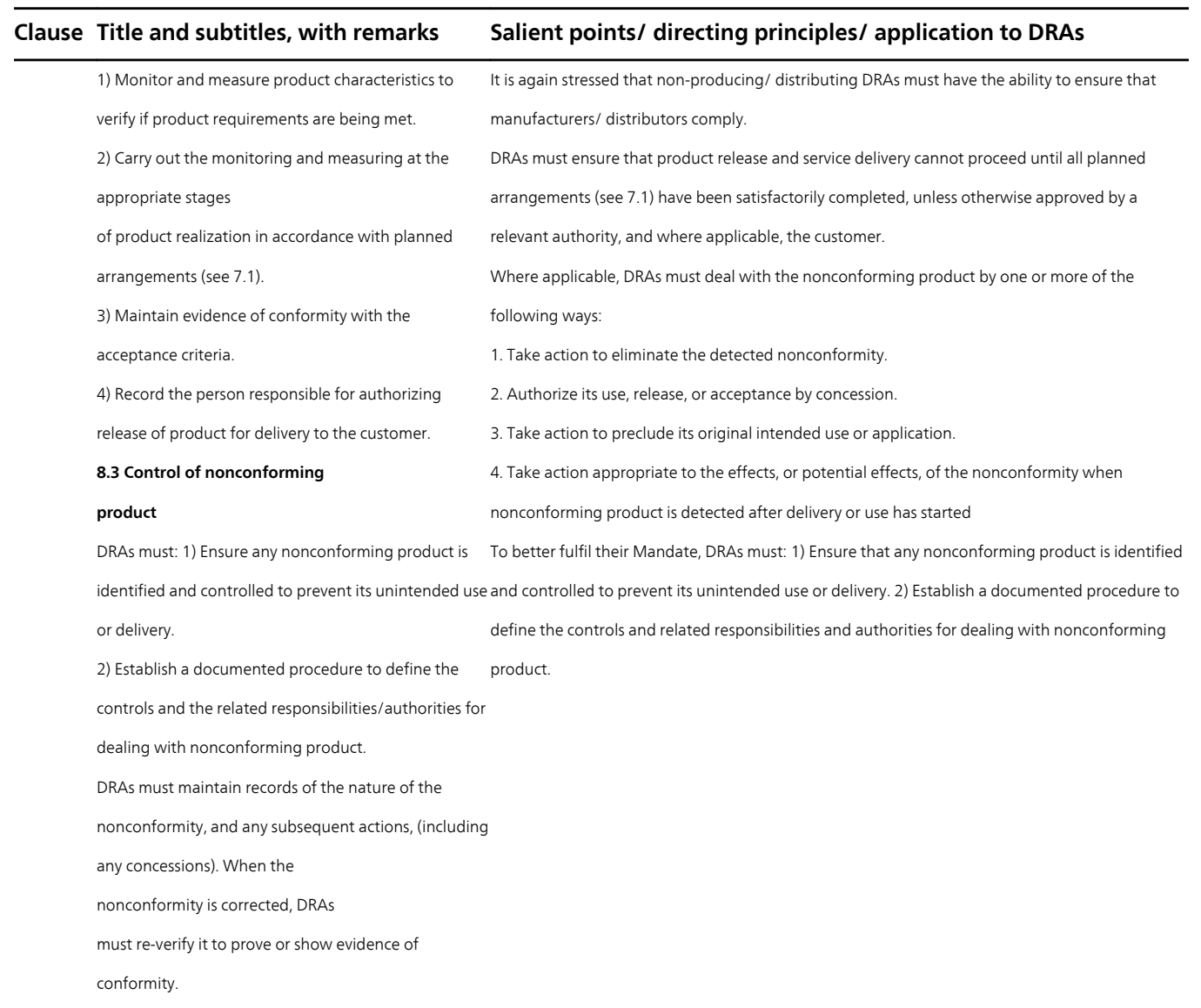

*The Table is to be studied side by side with contents of ISO 9001:2008 and Table 2, which is on the regulatory requirements of DRAs. Although a DRA may not possess certain facilities for measurements and monitoring, it should possess the ability or the means necessary to ensure that manufacturers/ distributors possess and use them in accordance with approved QMS guidelines.

Table 12. Clause 8 of ISO 9001 in relation to DRAs (Product characteristics/ Control of nonconformities).

In Nigeria, the entire NAFDAC Management was sacked on alleged acts of corruption in 2000. It seems to us that the following are essential for a DRA to perform optimally:

1. The laws creating/ amending a DRA should be well articulated as is the case with the USFDA.

2. DRAs should be so well funded as not to rely on a plethora of frivolous fees as with NAFDAC.

3. Staffing of DRAs must be transparent - competence and integrity must be the decisive criteria.

4. DRAs should be audited frequently, at least yearly or twice yearly.

5. DRAs should have a Board of Governors to whom the Management reports. 
6. Parliamentary health committees should view DRAs as critical to socioeconomic wellbeing of the nation.

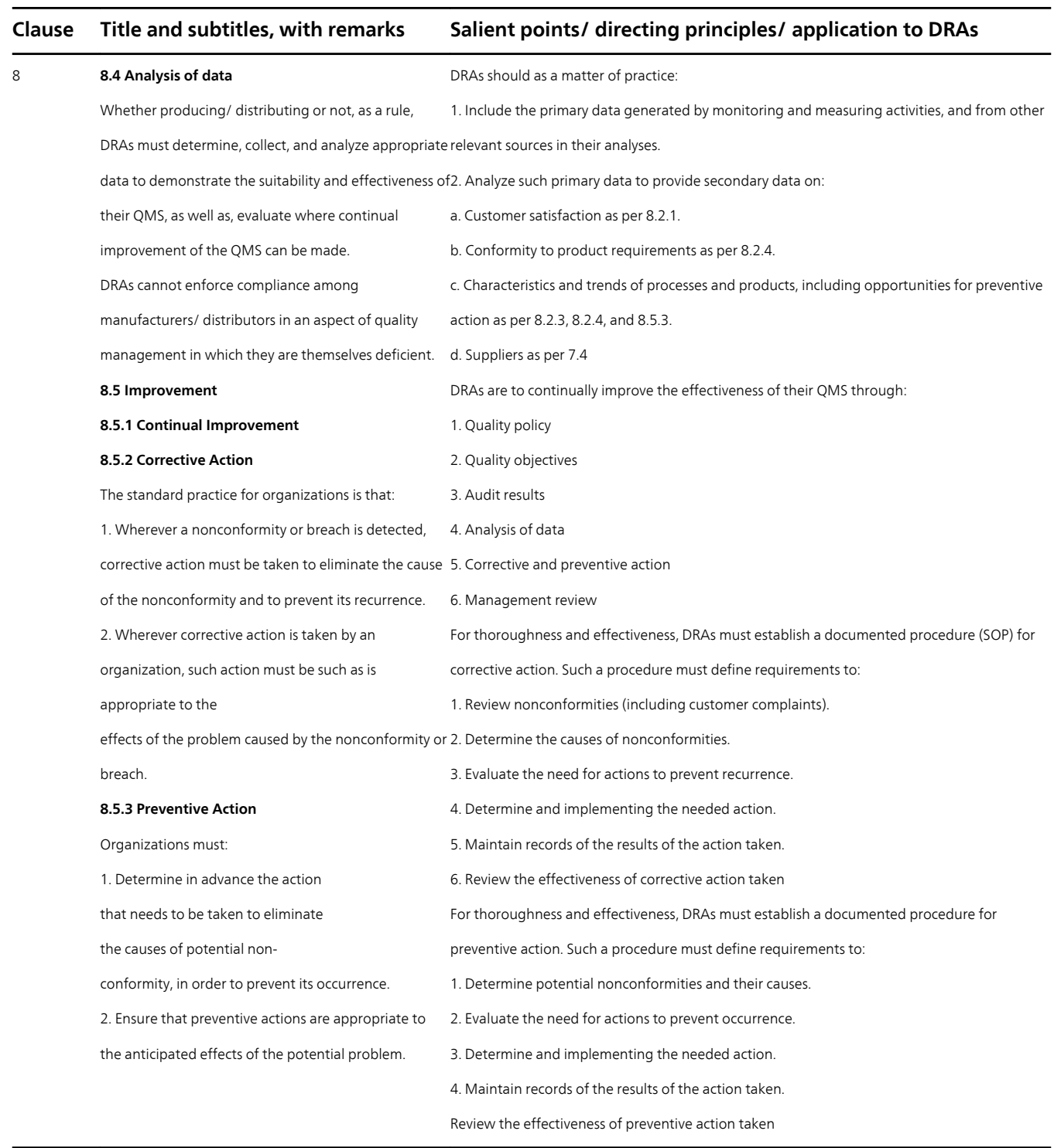

*The Table is to be studied side by side with contents of ISO 9001:2008 and Table 2, which is on the regulatory requirements of DRAs. It must be stressed once again that if DRAs are to persuade manufacturers/ distributors to comply with the provisions of this and other clauses of ISO 9001:2008 industrial standard, they too must be conversant with and adept in them.

Table 13. Clause 8 of ISO 9001:2008 in relation to DRAs (Analysis of data/ Improvement). 


\subsubsection{Further remarks on clauses 7 and 8 of ISO 9001:2008}

Although the principles of clauses 7 and 8 apply to all organizations, they are strictly speaking, the deeds and stuff intended for high profile institutions with elaborate concern and facilities for design and R\&D, and with tall entrepreneurial ambition. Such organizations include the most successful pharmaceutical and biotechnology companies of the US, Europe, Japan and India; NASA, aircraft manufacturers, international airlines and 5-star hospitality concerns. However, in as much as DRAs must regulate the work and product of advanced pharmaceutical manufacturers, the onus is upon the DRAs themselves to be conversant with the entire provisions of these clauses and be as intellectually equipped as the manufacturer. This explains why it is often desirable that regulators have a stint in both academia and industry. In many countries, especially the US and India, top rate biomedical facilities/ institutions and personnel are to be found in the following four circles: i) the DRAs (eg: US-FDA); ii) health research institutions (eg: NIH); iii) the universities/ R\&D institutions patronized by the DRAs; and iv) big transnational drug manufacturers (eg: Pfizer). We once again refer to the Nigeria polio vaccine controversy of the late 1990s/ early 2000s mentioned earlier, and ask the following question: When a DRA produces or distributes product as is the case in many developing economies, who regulates the DRA? Can subsequent revisions of ISO 9001or some other ISO standard provide an answer?

\section{Conclusions}

It is evident from the foregoing that all the eight clauses of ISO 9001:2008 apply to the Mandate of DRAs. However, most of what appears in clauses 7 and 8, the lengthiest of the clauses, relates more pertinently to high stake pharmaceutical manufacturers that have elaborate R\&D than they do to the average DRA, which nevertheless should be thoroughly acquainted with the clauses. Some DRAs like the US-FDA and EMEA that have advance laboratories or access to such or that heavily fund R\&D must be guided by the rigorous provisions of clauses 7 and 8 . Needless to say, those DRAs that produce/ distribute products must be similarly guided to the extent of their relevance to the scope and size of their operations. The USFDA, Japan's Ministry of Health and Social Services and EMEA are certified to appropriate performance standards and are known for their efficiency. By contrast NAFDAC and other developing national DRAs are not similarly certified and are less well known for efficiency, considering the rampancy of counterfeit drugs and other ills in their drug delivery systems. The DRAs of China, India and Southeast Asian countries compare quite well in many aspects with those of Europe, Canada and the US, and are by far more efficient than those of many African and South American countries. From the foregoing, and in view of the historical and international dimensions of phytotherapy, especially its galloping global patronage in recent times $[1,2,11,12]$, it is necessary that there to be a minimum global standard to which DRAs should be certified. We propose ISO 9001 because of its global popularity, applicability and suitability. The standard provides the general climate for DRAs to efficiently discharge their Mandate. We project that a carefully planned application of ISO 9001 to herbal drug regulation will improve the production, distribution and usage of herbal drugs. 
It will also boost the economy of developing economies that rely to a large extent on herbal drugs. But since the DRAs of many developing economies produce/ distribute certain products, there is a need for subsequent revisions of ISO 9001 to take cognisance of the question of who regulates the regulator that produces/ distributes? In the meantime we recommend that the Minister/ Secretary of Health and/ or the Parliamentary Committees of Health take note of this significant lacuna.

\section{Acknowledgements}

We gratefully acknowledge a copy of ISO 9001:2008 kindly furnished by the SON and the enlightenment offered by Engineer Timothy N. Abner, Dr. Justin B. Nickaf and Engineer. Shehu I. Maik during the NIPRD-SON workshop on ISO 9001:2008 held at Bolton White Apartments, Abuja, in November-December 2011.

\section{Author details}

Sunday Ameh $^{1 *}$, Florence Tarfa ${ }^{1}$, Magaji Garba ${ }^{2}$ and Karniyus Gamaniel ${ }^{3}$

*Address all correspondence to: sjitodo@yahoo.com

1 Department of Medicinal Chemistry \& Quality Control, National Institute for Pharmaceutical Research and Development (NIPRD), Idu Indusrial Area, Nigeria

2 Department of Pharmaceutical \& Medicinal Chemistry, Ahmadu Bello University, Nigeria 3 Office of the Director General, NIPRD, Idu Indusrial Area, Nigeria

\section{References}

[1] Ameh, S. J., Obodozie, O., Inyang, U., Abubakar, M., \& Garba, M. (2010). Current phytotherapy- a perspective on the science and regulation of herbal medicine. Journal of Medicinal Plants Research, 4(2), 072-081.

[2] Ameh, S. J., Obodozie, O., Inyang, U., Abubakar, M., \& Garba, M. (2010). Current phytotherapy- an inter-regional perspective on policy, research and development of herbal medicine. Journal of Medicinal Plants Research, 4(15), 1508-1516.

[3] Ameh, S. J., Obodozie, O., Gamaniel, K., Abubakar, M., \& Garba, M. (2011). Herbal Drug Regulation Illustrated with Niprifan ${ }^{\circledR}$ Antifungal Phytomedicine. 
In: Eldin AB, editor. Modern Approaches to Quality Control, Rijeka, InTech, 367-382.

[4] DSHEA (1994). Dietary Supplements Health Education Act of 1994. Available: http://fda/Food/DietarySupplements/ucm109764.htm Accessed 2012 April 25.

[5] Goldman, P. (2001). Herbal medicines today and the roots of modern pharmacology. Ann. Int. Med., 135(8), Pt 1, 594-600.

[6] Chineseherbsdirect.com (2010). What are Chinese Herbs? Available: http:// www.chineseherbsdirect.com Accessed 2012 April 25.

[7] EDTHMP (2004). European Directive on Traditional Herbal Medicinal Products. Available: http://eur-lex.europa.eu/LexUriserv/LexUriserv.do?uri=CELEX: 32004L0024:EN:NOT Accessed 2012 April 25.

[8] De Smet, P. (2005). Herbal medicine in Europe- relaxing regulatory standards. N. Eng. J. Med., 352(12), 1176-78.

[9] Mapdb.com (2003). Current status of medicinal plants. Available: http:// www.mapbd.com/cstatus.htm Accessed 2012 April 25.

[10] WHO. (2008). Traditional Medicine. WHO Fact sheet [134], Revised: December World Health Organization, Geneva.

[11] Ameh, S. J., Obodozie, O. O., Chindo, B. A., Babalola, P. C., \& Gamaniel, K. S. (2012). Herbal clinical trials - historical development and application in the $21^{\text {st }}$ Century. Pharmacologia . DOI: 10.5567/pharmacologia.2012.121.131 Accessed 2012 April 25., 3, 121-131.

[12] Ameh, S. J., Obodozie, O. O., Babalola, P. C., \& Gamaniel, K. S. (2011). Medical Herbalism and Herbal Clinical Research - A Global Perspective. British Journal of Pharmaceutical Research, 1(4), 99-123.

[13] Juran, J. M., \& Godfrey, A. B. (1999). Juran's Quality Handbook: The Complete Guide to Performance Excellence (5 ${ }^{\text {th }}$ Edition), McGraw-Hill Professional, New York, USA, 1-1872.

[14] Pyzdek, T. (2003). Quality Engineering Handbook. $2^{\text {nd }}$ Edition (Ed. Keller, P.), CRC Press, New York, USA, 1-744.

[15] ISO Survey (2009). Available: http://www.iso.org/iso/survey2009.pdf Accessed 2012 April 25.

[16] Ann Godsell Regulatory (2008). Pharmaceutical Good Manufacturing Practice for Herbal Drug Substances 2008 [cited 2010 April 8]. Available: http:// www.pharmaceutical-int.com/article/category/treatment-herbal-medicines Accessed 2012 April 25. 
[17] Akunyili, D. (2002). Herbal Preparations. NAFDAC Consumer Safety Bulletin, $1(2), 5-6$.

[18] Gross, A., \& Minot, J. (2007). Chinese Manufacturing: Scandals and Opportunities. Published in MX, November/ December, Pacific Bridge Medicals. Available: http://www.pacificbridgemedicals.com/ Accessed 2012 April 25.

[19] Corbett, C. J., Montes-sancho, M. J., \& Kirsch, D. A. (2005). The financial impact of ISO 9000 certification in the United States: An empirical analysis. Management Science Available: http://personal.anderson.ucla.edu/charles.corbett/ paper/does_iso_pay.pdf Accessed 2012 April 25., 51(7), 1046-1059.

[20] Heras, I., Dick, G. P., \& Casadesus, M. (2002). ISO 9000 registration's impact on sales and profitability - A longitudinal analysis of performance before and after accreditation. International Journal of Quality and Reliability Management Available: http://eps.udg.es/oe/webmarti/p774.pdf Accessed 2012 April 25., 19(6), 774-791.

[21] Naveh, E., \& Marcus, A. (2007). Financial performance, ISO 9000 standard and safe driving practices effects on accident rate in the U.S. motor carrier industry. Accident Analysis \& Prevention PMID 17166474Accessed 2012 April 25., 39(4), 731-742.

[22] Sharma, D. S. (2005). The association between ISO 9000 certification and financial performance. The international Journal of Accounting Available: http://masp.bus.ku.ac.th/files/ISO\%209000\%20and\%20performamce.pdf Accessed 2012 April 25., 40, 151-172.

[23] Chow-chua, C., Goh, M., \& Wan, T. B. (2002). Does ISO 9000 certification improve business performance? The International Journal of Quality \& Reliability Management Available: http://www.emeraldinsight.com/journals.htm? articleid=840633\&show=abstract Accessed 2012 April 25., 20(8), 936-953.

[24] Rajan, M., \& Tamimi, N. (2003). Payoff to ISO 9000 registration. Available: http://www.iijournals.com/doi/abs/10.3905/joi.2003.319536Accessed 2012 April 25. 
Quality Control in Food Science 

Chapter 6

\title{
QA: Fraud Control for Foods and Other Biomaterials by Product Fingerprinting
}

\author{
Edoardo Capuano and Saskia M. van Ruth \\ Additional information is available at the end of the chapter \\ http://dx.doi.org/10.5772/51109
}

\section{Introduction}

Fraud can be generally defined as "the intentional deception made for personal gain or to damage another individual". In particular, food fraud consists in the deliberate misdescription in order to deceive the consumers about the real nature of the product or of any of its ingredients. It results in the mismatch between what a food product is and what it is claimed to be. Food fraud is a broad term that also involve criminal acts such as tax-avoidance and smuggling. In the following we will mainly discuss about economically motivated adulteration and mislabelling i.e. food fraud issues falling in one of the following categories: 1) the substitution of an ingredient with a cheaper alternative (e.g., substitution of ethanol with methanol in wine or proteins with melamine in milk powders), 2) misdescription of the real nature of the product or one of its ingredients (e.g. counterfeiting, conventional products that are sold as added value products such as organic, fair trade, biodynamic), 3) incorrect quantitative ingredient declaration and 4) implementation of non-acceptable process practices such as irradiation, heating or freezing (e.g. thawed fish sold as fresh).

Food fraud can be implemented in any step of the food chain but it is mainly a food industry issue. In criminology, there are 3 elements of fraud opportunity (the crime triangle): victim, fraudster and guardian [1]. The typical set-up is that where final consumers play the role of victims, food industry (but in general food producers, processors, traders or retailers) plays the role of the fraudsters and governmental control authorities, non-governmental and certification organizations play the role of the guardian. However, food producers can also be victim or guardian. They are victims when, for example, their products are counterfeited or simulated and guardian when they implements QA systems for the assessment of the authenticity of the raw materials. Nowadays food fraud represents a major problem that costs the EU food industry and governments hundreds of millions of euros every year. But the 
problem that food fraud poses is not merely an economic one: It is also a problem of public health because the adulteration can pose toxicological and hygienic risks to purchasers and consumers. In 2008, for example, Chinese milk was adulterated with melamine, an hazardous chemical, to increase milk nitrogen content causing 900 infants to be hospitalized with six deaths. Several other such examples can be given.

Since no one likes to be swindled, neither producers, traders, importers, retailers, and consumers, fraud prevention and detection is an important issue. Nowadays, authenticity of ingredients or products is mainly warranted by paper trailing. Analytical tests which can help to confirm the authenticity of ingredients/products compose a very useful complementary approach to paper trailing.

\section{Fingerprinting approach: generalities and tools}

Traditional strategies for the food fraud control have relied on the determination of the amount of a marker compound or compounds and the comparison of the obtained values for the test material with those established for the genuine material. The presence of an undesired adulterant can be uncovered by checking for its presence in the food material whereas the compliance of the food composition with the established legislative standards or with the amount of an ingredient as declared on the label can be simply proved by measuring the target compound or compounds. However, some aspects of food authenticity such as the geographical origin, the farming management systems (organic, free range..), or the application of some specific processes cannot be dealt with those traditional approaches. No single marker exists for the unequivocal authentication of an organic egg or a Dutch specialty cheese. Furthermore, based on conventional target analyses, an adulteration can be detected only if the adulterant is known beforehand and explicitly searched for by the analyst. Traditional quality control strategies are not designed to look for a near infinite number of potential contaminants so that new adulterants will not be unveiled until their presence in food is first acknowledged. For those reasons a more holistic approach is needed that is based on the measurement and the evaluation of several compounds at once, i.e. a fingerprinting approach. Moreover, in industrial and laboratory settings, there is always the need of implement screening methods that are able to reliably identify, in large numbers of samples, those that are potentially non-compliant before more detailed and accurate analysis with confirmatory methods are performed. A fingerprinting approach may, in many cases, provide rapid and high-throughput analyses well suited for screening purposes.

Fingerprint refers to the characteristic spectrum or image of a test material which can be related to its properties and thus to its authenticity in the same way as a human fingerprint is specific of a certain person and unequivocally identify him/her. The term thus recalls a comprehensive description of a test material that is carried out in a non-selective (or untargeted) way. Fingerprints can be generated through many analytical techniques. They can be obtained from chromatograms, spectroscopic measurements, spectral measurements or any other specific signal of complete spectra (Figure 1). 

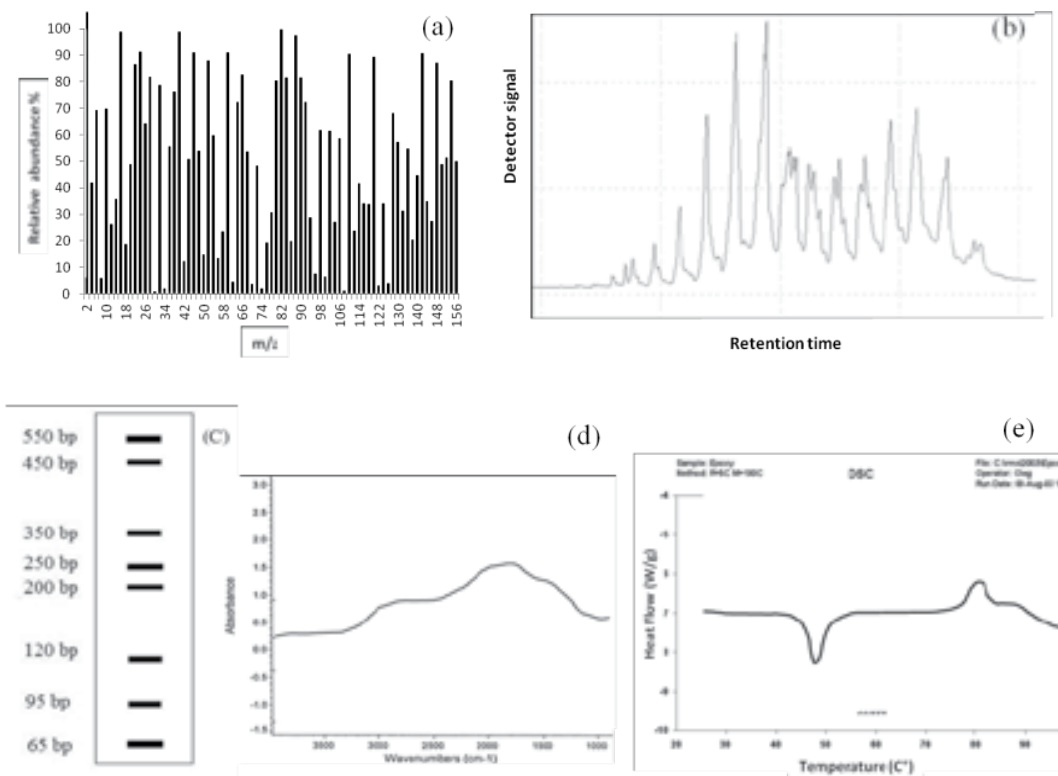

(d)

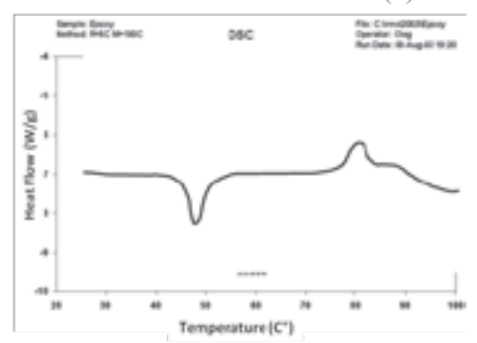

Figure 1. Analytical fingerprints: A mass spectrum (a), a chromatogram (b), a schematic representation of a DNA fingerprint on gel electrophoresis (c), an infrared spectrum (d) and a thermogram (e).

In a mass spectrum (Figure $1 \mathrm{a}$ ), a collection of $\mathrm{m} / \mathrm{z}$ and relative signal intensities is a chemical fingerprint of the material. Similarly, a chromatographic profile (Figure $1 \mathrm{~b}$ ) is a fingerprint of a more or less broad class of constituents of the material. The specific distribution of the restriction fragments of a selected DNA sequence on the electrophoretic gel (Figure 1c) is a genetic fingerprint of the test material. A NIR spectrum (Figure 1d) is a representation of the interaction of a test material with the infrared radiation whereas a thermogram (Figure 1e) is a representation of its interaction with thermal energy. (a) and (b) can be referred to as chemical fingerprints. They may be composed of as many groups of compounds as possible or alternatively of a specific group of compounds which requires higher level of purification and a selective extraction from the sample. (d) and (e) can be referred to as physical fingerprints even though chemical information can be obtained as well.

A fingerprinting approach implies that the whole information contained in the fingerprint (or a selected part of it) is used to infer about the properties of the system under study. To do that, a special statistical tool is necessary, i.e. chemometrics. Chemometrics can be defined as the science of extracting chemically relevant information from multivariate data by using statistical techniques to reduce the dimensionality of the dataset. It offers a tool to graphically summarise the analytical data to reveal relationships between samples and to detect characteristic patterns that can be used to identify a certain material. As a first step, an exploratory analysis is carried out in order to investigate the natural relations between the samples. This is carried out by so called unsupervised pattern recognition techniques because they do not require any prior knowledge of the properties of the samples. Examples of such techniques 
are: Hierarchical cluster analysis (HCA), cluster analysis (CA) and principal component analysis (PCA). PCA is the most widespread of those explorative tools. In a PCA model the original variables are transformed in new uncorrelated variables that arise from the linear combination of the original variables: the principal components (PCs). A number of PCs are extracted in sequence with each principal component accounting for the maximum of the residual variance in the data. The PCs extraction stops when most of the variance in the original data (typically around 90\%) is explained. The new set of PCs define therefore a new space where the contribution of each original variables to each PC can be easily represented and the relationships between the original samples highlighted (Figure 2).

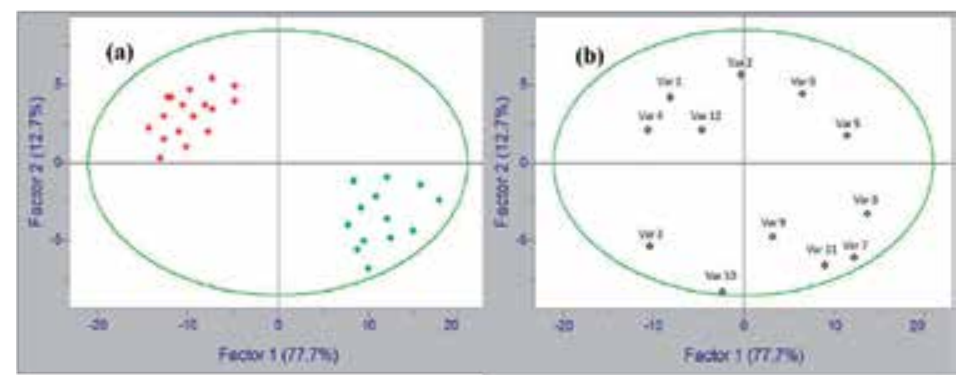

Figure 2. Plot of the first two dimensions in a typical PCA model. In a scores plot (panel a), samples are plotted in the space defined by the PCs. Similarities and differences between samples can be highlighted (in this case, two well separated groups of samples, red and green are apparent). In a loading plot (panel b) each variable (analytical response) is plotted on the new space defined by PCs. When score plot and loading plots are superimposed, information on the effect of the variable on samples properties can be obtained. When a variable is plotted close to a sample, this indicates that this variable shows relatively high concentration in this particular sample compared to the other samples. In the figure, variables 1,4,12 are higher in the samples of the red group and variables 6,7 and 11 are higher in the green group.

As a further step, multivariate methods are applied to either classify a certain product or quantify a certain property of the product. In the classification models, information about the class membership of the samples to a certain group (class or category) is used to classify new unknown samples in one of the known classes on the basis of its pattern of measurements. Classification models are useful, for instance, for the authentication of organic products or of geographical origin. Supervised pattern recognition techniques are used such: $k$ nearest neighbours $(\mathrm{kNN})$, soft independent modelling of class analogy (SIMCA), partial least square regression discriminant analysis (PLS-DA), linear discriminant analysis (LDA), support vector machine (SVM) and artificial neural network (ANN). Classification models may either build a delimiter between the classes so that they always assign a new object to the class to which it most probably belongs (suitable for limited and defined number of possible classes) or build a model for each class studied and then evaluate the fitting of new objects to each model (suitable for unlimited number of classes). In the regression models, a functional relationship is established between some quantitative sample property, the dependent variable, and a multivariate independent variables such as a raw chromatogram or a IR spectrum and the model is used to predict the property of interest in the unknown sample. Regression model are used, for instance, to quantify the level of adulteration in a food sample. For the build- 
ing of regression models, multivariate regression methods such as principal component regression (PCR) and partial-least square regression (PLSR) are used.

The building of a classification (or regression) model comprises four essential steps: 1) Selection of a training set, which consist of objects of known class membership (or known quantitative values of a sample property) for which variables are measured. (2) Variable selection. Variables that contain information for the aimed classification are retained, whereas those encoding noise and/or with no discriminating power are eliminated. (3) Building of a model using the training set. A mathematical model is derived between the selected variables measured on the training set and their known categories (or quantitative values of the sample property). (4) Validation of the model. The model is validated in order to evaluate the reliability of the classification achieved either using an independent test set of samples (external validation) or the training set (cross validation).

The fingerprinting techniques are gaining more and more popularity over the past years thanks to advancements in the analytical instruments that are able to generate enormous amount of data at once and the application of chemometrics techniques. Herewith, fingerprinting techniques are classified in five broad categories according to the kind of fingerprint that can be obtained: Mass spectrometry (MS) fingerprinting, chromatographic fingerprinting, electrophoretic fingerprinting, spectroscopic fingerprinting, and other fingerprinting. This classification is shown in Table 1.

\begin{tabular}{lllll}
\hline $\begin{array}{l}\text { MS } \\
\text { fingerprinting }\end{array}$ & $\begin{array}{l}\text { Chromatographic Electrophoretic } \\
\text { fingerprinting }\end{array}$ & $\begin{array}{l}\text { Spectroscopic Other } \\
\text { fingerprinting }\end{array}$ & fingerprinting fingerprinting \\
\hline PTR-MS & LC (HPLC, LC-MS..) & CE (CZE, CIF...) & NMR & DSC \\
\hline ICP-MS & GC (GC-FID, GC- & Gel electrophoresis & Fluorescence & Electronic nose \\
& MS..) & $\begin{array}{l}\text { (isoelectric focusing, spectroscopy } \\
\text { DNA } \\
\end{array}$ & electrophoresis...) & \\
\hline IR-MS & & IR (NIR, MIR, & Microarray \\
& & FTIR) & technologies, \\
& & & reverse PCR \\
\hline Direct infusion & & & \\
and ambient MS & & & \\
(ESI, MALDI-TOF, & & & \\
DART...) & & & & \\
\hline
\end{tabular}

Table 1. Classification of fingerprinting techniques. For abbreviations, see text.

\section{MS fingerprinting}

MS is a powerful analytical technique that measures the mass-to-charge ratio of ions. The samples are first ionised, the ions are separated and their relative abundance assessed based 
on the intensity of the ions flux. MS produces therefore a mass spectrum representing the fingerprint of the sample components (Figure 1a). A number of different MS set-ups are possible based on the ionisation technique and the mass analyser used. MS can be used alone or they can be coupled with separation techniques. In this section, the stand-alone MS techniques will be dealt with in details whereas the application of MS as coupled with separation techniques will be dealt with in the next sections.

Stand-alone MS fingerprinting techniques that proved to be very useful for the fraud control and prevention are: proton transfer reaction MS (PTR-MS), inductively coupled MS (ICPMS), isotope ratio mass spectrometry (IRMS), and direct infusion MS techniques.

\section{Proton Transfer Reaction Mass Spectrometry (PTR-MS)}

PTR-MS is a relatively new technique that is rapidly gaining popularity in the food analysis. PTR-MS allows quantitative on-line monitoring of volatile organic compounds. The volatile compounds are softly ionized by means of hydroxonium ions that are generated in an external ion source operating in pure water vapour. Only the volatile compounds that have a higher affinity for the ions are protonated and then accelerated by an electric field to the reaction chamber where they are separated and quantified. Because of this soft chemical ionisation the fragmentation of the parent compounds is limited and the interpretation of the spectra are much easier. Other major advantages of this technique are the great sensitivity with detection limits as low as few part per trillion, volume (pptv) and the possibility to monitor the food samples in real time, without any work up procedure. As a result, a fingerprint of all the volatile compounds comprised in a well definite mass range is obtained. The main disadvantage of this technique is that compounds are characterized only via their masses which is insufficient for their unequivocal identification.

PTR-MS has been extensively used in several aspects of food fraud control. It proved, for example, very successful for the geographical authentication of foods. The EU has long recognized the importance of differentiating food products on a regional basis. The normative framework introduced by the EU comprises the EU Regulations 509/2006 and 510/2006 and the EU Regulation 1898/2006. The EU Regulation introduced three geographical indications to a food product: protected designation of origin (PDO), protected geographical indication (PGI) and traditional specialities (TSG). In a study of 2008, the geographical origin (country) of butter samples was successfully predicted in $88 \%$ of the cases based on PTR-MS fingerprint and PLS-DA [2]. Recently, volatile fingerprint generated by PTR-MS has been used to discriminate between the Boeren Leidse specialty cumin cheeses with EU PDO from other 29 cumin cheese manufactured in the Netherlands [3]. The volatile fingerprint coupled with a PLS-DA model allowed the correct classification of $96 \%$ of the traditional boeren leidse cheese samples and $100 \%$ of the other commercial cheese samples. Another typical added value that is protected by the EU regulations is represented by the monovarietal extra virgin olive oil (EVOO), i.e. oil that is produced out of just one variety of olive trees. Frauds can be committed by mixing the more valuable monovarietal virgin olive oil with cheaper oils or by mixing different monovarietal olive oils. Volatile fingerprint of virgin olive oil obtained by PTR-MS and subjected to PLS-DA proved successful in discriminating among 5 different monovarietal EVOO from Spain with $100 \%$ sensitivity (\% of objects of the modelled class 
correctly accepted by the model) and specificity (\% of object, extraneous from the modelled class, correctly refused by the model) close to $100 \%$ [4].

In conclusion, PTR-MS is a rapid and low cost analytical technique that can be also fully automated and implemented on-line. Recently, the coupling of the time of flight (TOF, see below in this section) mass analyser to PTR-MS instruments has generated PTR-TOF-MS which is characterized by a high sensitivity with limits of detection down to few pptv and a high time resolution. The technique has been recently applied to discriminate among PDO labelled hams from Spain and Italy [5].

\section{Inductively coupled plasma mass spectrometry (ICP-MS)}

ICP-MS provides quantitative measurements of a wide range of metals and non-metals (inorganic elements) at trace and ultratrace concentration level (ppt). In this technique, the sample (even solid or liquid) is decomposed to neutral elements in a high-temperature argon plasma and the single elements are separated based on their mass/charge ratio and analysed. The great advantage of this technique compared to others (e.g. atomic spectroscopy) is that more than one element can be analysed at once so that a multi-elemental fingerprint is obtained in a very fast and sensitive way. The multi-elemental composition of animal and vegetal tissues can provide valuable information on the characteristics of the soil where a crop has been cultivated and on plants composition of the animal diet. The multi-element fingerprint is thus a valuable marker of the geographical origin of food. For instance, the authenticity of Tropea red onion, an onion Italian variety that achieved the PGI certification by the European Union as "Cipolla Rossa di Tropea Calabria" can be proved by means of multi-elemental analysis by ICP-MS and multivariate statistics [6]. All the statistical models applied (LDA, stepwise LDA, SIMCA, ANN), allowed a success rate of prediction $>90 \%$ for the genuine samples. Moreover, the availability of nutrients from the soil strictly depends on the fertilization strategies and the pest and weed control management systems. In organic farming synthetic fertilizers are not permitted and the pest and weed control is based exclusively on natural products. It has been thus proposed that the multi-elemental fingerprint might be a marker for organically cultivated crops as compared to conventionally cultivated ones. Laursen et al. managed to discriminate between organic and conventional wheat, barley and faba beans (but not potatoes) based on the profile of 25 elements measured by ICP-MS [7].

Isotope ratio mass spectrometry (IRMS)

IRMS is a technique that can measure the ratio of the stable isotopes of the constituents of a biological material. Light elements like carbon, nitrogen, hydrogen, oxygen and sulphur stable isotopes ratios are most frequently assessed with this technique. Those ratios vary according to specific food production factors and geo-climatic conditions. Carbon stable isotope ratio depends, for example, from the plant composition of ruminant diets and can then be used to authenticate feeding regime or the farming management system (organic, free-range). Nitrogen stable isotope ratio is on the other hand depending on the type of fertilizers used in agriculture and is thus much useful for the authentication of farming practices for vegetal products and crops. Oxygen isotope ratio is instead highly dependent on the 
distance from the ocean and the altitude above sea level and could then be used for the authentication of the geographical origin of a food product.

The stable isotope fingerprint has been successfully used for the authentication of geographical origin and the farming practice. Normally the data are measured for many different elements and analysed with multivariate statistics. As an example, Fontina PDO cheese can be discriminated with good success from other cheeses based on stable isotopes ${ }^{2} \mathrm{H} /{ }^{1} \mathrm{H},{ }^{13} \mathrm{C} /{ }^{12} \mathrm{C}$, ${ }^{15} \mathrm{~N} /{ }^{14} \mathrm{~N}$ and ${ }^{34} \mathrm{~S} /{ }^{32} \mathrm{~S}$ and PCA analysis [8]. However, frequently the isotope ratios (or a selection of them) are combined with other markers (elements) to improve the accuracy of the classification models. IRMS is often combined with ICP-MS for simultaneous elemental analysis. Stable isotope analysis combined with multi-elemental analysis has proven ideally suited to determine geographical origin of foods. The most accurate measurements of the isotope ratios is obtained by dual inlet (DI) IRMS. However, the purchasing and operating costs of a DI-IRMS instrument coupled with the time-consuming sample preparation are major disadvantages for the diffusion of this technique. The introduction of continuous flow (CF) IRMS instruments offers on-line, rapid and automated sample preparation, greater cost-effectiveness and easier interfacing with other preparation techniques.

\section{Direct infusion mass spectrometry}

Direct infusion MS techniques are based on the direct injection of the sample in the ion source without or with small sample pre-treatment. This allows for rapid analysis suited for high-throughput screenings. Electrospray ionisation (ESI), matrix assisted laser desorption ionization (MALDI) and direct analysis in real time (DART) are typical ionization techniques used for direct infusion MS. They are coupled with a variety of mass analysers, e.g. time of flight (TOF), Fourier Transform Ion Cyclotron Resonance (FT-ICR), single quadrupole (Q) and ion trap (IT) in many different set-ups.

ESI is a typical soft ionisation technique that is particularly suited for the determination of the molecular mass of large molecules (proteins, peptides, polysaccharides, triglycerides), because the ionisation does not bring about the fragmentation of the molecule. The liquid in which the analyte is contained is dispersed by electrospray to a fine aerosol. The droplets shrink as the solvent evaporates till solvated ions desorb from their surface. ESI-MS has proven to be very helpful in the authentication of vegetable oils. Lipid composition of vegetable oils depends on their botanical origin and the way they are processed. Fatty acids (FAs) and/or triglycerides (TGs) profile can thus help authenticate the type of oil, its origin, its quality grade and potential adulteration. Direct infusion ESI-MS has been for example used to predict the olive oil quality according to European Union marketing standards based on fatty acids and LDA analysis [9]. In the same research, the percentage of either EVOO and VOO in binary mixture with other lower grade oils was predicted with $5-11 \%$ average prediction errors by using PLS and multilinear regression (MLR). Samples were 1:50 diluted in an alkaline 85:15 (v/v) propanol/methanol mixture and directly infused into the MS system. Triglycerides analysis has some advantage over the analysis of the fatty acids profile for authentication or fraud control. Indeed, different oils can have specific TG fingerprint despite showing the same fatty acids composition. The triglyceride profiles, obtained using Q-TOF-ESI-MS was used to predict adulteration of olive oils with other vegetable oils. 
The adulteration with hazelnut oil was predicted at a level of $10 \% \mathrm{v} / \mathrm{v}$ [10]. This adulteration is difficult to detect at levels below $20 \%$ by conventional methods due to the compositional similarity between the two oils. Similarly, PCA and HCA methodologies, applied to the ESI(+)-MS data were able to readily detect adulteration of EVOO with ordinary olive oils, at levels as low as 1\% w/w [11]. Mono-, di- and triglycerides together with vitamins and antioxidants were detected and quantified with this method. Direct infusion ESI-MS has been used to authenticate other food commodities. The chemical fingerprint generated by direct infusion ESI-Q-TOF-MS in the negative mode can be used to discriminate between genuine whisky from Scotland and US, from counterfeited whisky produced in Brazil [12] and between alembic (the most valuable) and industrial cachaças (Brazilian sugarcane spirit) as well as the fraudulent addition of sucrose to the spirit [13]. Finally, direct-infusion ESI-QqQ-TOF-MS and atmospheric pressure photoionization (APPI)-QqQ-TOF-MS have been used for Iberian ham typification. APPI is a soft ionization technique based on a photoionisation mechanism. Five types of Iberian hams were successfully classified. Applying a PLSDA model [14].

MALDI is another soft ionisation technique that proved very useful in the analysis of macromolecules, especially proteins. In MALDI the molecules are desorbed from the support matrix and ionised by means of a UV laser beam in a complex process mediated by the support matrix itself. MALDI is mainly coupled with a time-of-flight (TOF) mass analyser which separate the ions based on their flying time to the detector, which on turns depends on their $\mathrm{m} / \mathrm{z}$ ratio. An example of application of MALDI-TOF-MS for authentication issues is represented by the fast method developed by Wang et al. for the fingerprinting of honey proteins [15]. The mass spectra were used to build up a database library to be used for authentication purpose. The protein fingerprint was thus successfully used to authenticate the geographical origin of commercial honeys produced in the US and other countries. In a similar fashion, peptide fingerprinting obtained by MALDI-TOF has been converted in a biological bar code for the authentication of high quality Campania white wines [16].

An innovative technique for food fingerprinting is represented by the direct analysis in real time (DART)-MS. DART is an ambient ionisation technique i.e. in which ions are formed outside the mass spectrometer without sample preparation or separation. The samples, either gaseous, liquid or solid are ionised in open air under ambient conditions. This means that organic compounds can be directly, and in real time, determined without time-consuming analytical protocols and thus with high sample throughput. DART coupled with TOFMS has been used to obtain the fingerprint of the triglycerides from olive oil [17]. This method, coupled with LDA allowed the discrimination between EVOO, olive oil and olive oil pomace and the detection of hazelnut oil in EVOO at $6 \% \mathrm{v} / \mathrm{v}$. DART-TOF-MS with solid phase micro extraction (SPME) pre-concentration of the analytes has been also reported to allow discrimination between trappist and non-trappist beers based on volatiles and phenolic compounds [18]. A combination of DART-TOF-MS and chemometrics was used for animal fat (lard and beef tallow) authentication [19]. TGs and polar compounds were extracted and analyzed. Mass spectral records were processed by PCA and stepwise LDA. The LDA model developed using TAG data enabled the classification of lard and beef tallow samples but also detection of admixed lard and tallow at adulteration levels of 5 and $10 \% \mathrm{w} / \mathrm{w}$. 
Additional ambient ionisation techniques have been recently proposed for authentication and fraud control by product fingerprinting. For instance, easy sonic spray ionisation (EASI)MS fingerprinting of fatty acids and phenolic compounds have been used for the authentication of olive oil geographical origin [20].

\section{Chromatographic fingerprinting}

Chromatographic techniques aim at resolving complex mixtures in well separated compound. Based on the detection system, each single compounds generates a signal that can be used for the qualitative and quantitative analysis of the mixture. The graphical representation of such signal as a function of time is referred to as a chromatogram and can be thought of as the fingerprint of one or more classes of compounds occurring in the sample. Different strategies are available to obtained multivariate data matrices from chromatographic analyses (Figure 3). The fingerprint can be composed by the set of concentrations of the separated compounds based on an identification/calibration/quantification procedure as depicted in the path (a) of Figure 3. Alternatively, the fingerprint can be represented by the set of peak areas/heights (b). In this case the identification of each single peak is not necessary. Finally, it can be represented by the whole chromatogram that is handled as a continuous signal (c). In this case, the multivariate dataset is composed by as many variables as the sampling points the chromatogram is made up of (each data point of the chromatogram represents an individual variable). However, the application of chemometrics on raw chromatographic data requires specific data pre-processing techniques. In fact, problems related to the peak alignment or baseline shifts are particularly critical when a raw chromatogram is used as a data set.

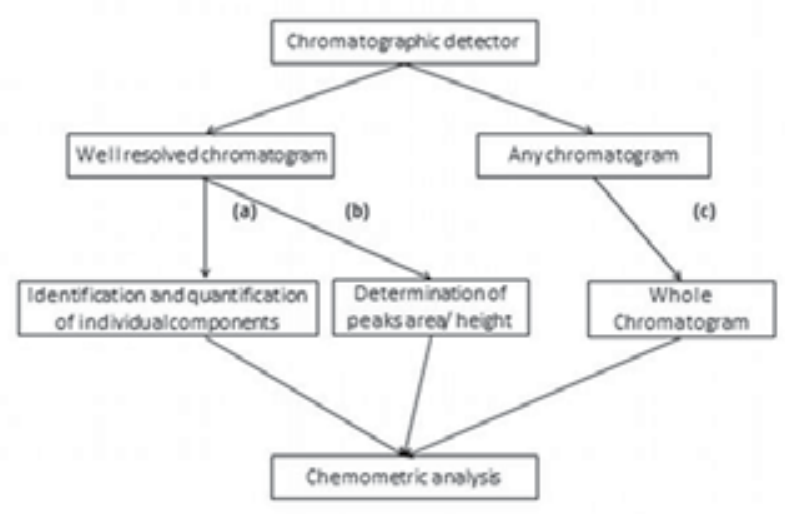

Figure 3. Schematic representation of the strategies to obtain multivariate dataset from a chromatogram for chemometric analysis. 


\section{Liquid chromatography}

In liquid chromatography, the mixture components are separated as they pass through a column based on their selective partition behaviour between a stationary phase (column material) and a mobile liquid phase. Depending on the type of stationary phase, compounds can be separated based on their size, charge, molecular mass, hydrophobicity etc. The most popular LC technique is high performance liquid chromatography (HPLC) that is a straightforward, robust and reproducible technique. HPLC has been used for the analysis of a wide range of food compounds such as vitamins, proteins, carbohydrates, TGs, additives, secondary plant metabolites. A typical example of the application of HPLC based fingerprint for fraud control is the authentication of the organic eggs by means of the carotenoids profile [21]. Carotenoids are a group of fat-soluble pigments that occur in the egg yolk in concentrations of about $10 \mathrm{mg} \mathrm{kg}^{-1}$. In animals carotenoids are entirely of dietary origin. Since the feeding regime of organic hens is clearly different from that of conventionally reared hens, the carotenoids fingerprint in eggs were used to discriminate between production systems. The carotenoids profile was determined by HPLC with UV detection, the single carotenoids quantified and the resulting concentrations used to build up a classification model by kNN. Almost all the conventional eggs and all the organic ones were correctly classified in external validation. The robustness of the method has been recently improved by testing eggs produced in several EU and non-EU countries. An example of HPLC fingerprint based on raw chromatographic data is in [22]. The authors applied PLS to the full TGs chromatogram profiles of vegetable oils to predict the \% of olive oil in the mixtures with errors not exceeding $10 \%$. Liquid chromatography can also be coupled with MS which allows higher resolution and higher sensitivity for metabolites occurring in relatively low amount. As an example, an untargeted method for proteins analysis based on LC-QTOF-MS has been developed which allowed to detect the fraudulent addition of cheaper vegetal proteins (from soy and pea) to skimmed milk powders based on the different peptides profile [23].

\section{Gas Chromatography}

In gas chromatography, the mixture is first vaporised in a heated chamber and then the mixture components are separated as they travel through the column transported by the flow of an inert gas (helium, nitrogen or hydrogen) based on their selective interaction with the column material. GC is a very popular separation technique mainly used for the analysis of volatile compounds. However a wide spectrum of compounds can be rendered volatile by proper derivatisation and thus analysed by GC. The analysis of fatty acids and triglycerides is usually carried out by GC with flame ionisation detector (FID) previous derivatisation in fatty acids methyl esters (FAME) and TG trimethylethers, respectively. FID is a general detector capable of high sensitivity and robustness. Fatty acids composition of animal tissues and animal products strongly depends on the feeding regime. FAs composition of fish muscle fat is affected by animal diet/feeding, the geographical area of catch and the marine conditions and is thus different between farmed and wild fishes. The discrimination between wild and farmed Atlantic salmon (Salmo salar L.) and Wild Turbot (Psetta maxima) has been reported based on FAs analysis and chemometrics [24-25]. The fatty acid fingerprint obtained by GC-FID followed by PLS-DA analysis has been also reported for the authentica- 
tion of organic eggs and of organic feeds [26-27]. In the last case $90 \%$ of the analysed samples were correctly classified in their proper group in external validation. GC-FID can also be used for the TG profiling. TG fingerprinting by GC-FID has been for example reported for the authentication of three fat classes (animal fats, fish oils, recycled cooking oils) [28]. The TGs fingerprint was subjected to multivariate analysis (PLS-DA) and allowed the correct classification of $96 \%$ of the fat samples.

GC coupled with MS represents the method of choice for the analysis of volatile compounds because of its high reproducibility. On the other hand, GC-MS analysis requires careful sample cleaning and is quite expensive and time-consuming. The volatile fingerprint of coffee obtained by GC-TOF-MS after SPME has been reported for the geographical authentication of coffee [29]. SPME preconcentration of volatiles followed by GC-MS analysis coupled with PCA analysis allowed the detection of adulteration of ground roasted coffee with roasted barley [30]. The adulteration is detectable at level of $1 \% \mathrm{w} / \mathrm{w}$ in mixtures of dark roasted barley and coffees. Metabolomics studies can be also fruitfully performed by GC-MS. The fingerprint of a large range of metabolites obtained by GC-MS has been used to discriminate between mechanical separated meat (MSM) from hand-deboned meat [31]. MSM could be detected in raw meat mixtures down to a level of $10 \%$.

\section{Electrophoretic fingerprinting}

Electrophoretic techniques are able to separate a complex mixture under a spatially uniform electric field, based on electrophoretic mobility of its components that depends, in turn, from their hydrodynamic properties and charge. Positively charged molecules move towards the anode and negatively charged molecules towards the cathode at a different rate based mainly on their mass to charge ratio. Smaller molecules move faster than larger ones.

\section{Gel electrophoresis}

In a gel electrophoresis, a gel is used as a medium for the movement of the charged particles under the applied electric field. Agar and polyacrylamide are typical medium used in gel electrophoresis. Proteins and nucleic acid fragments are usually separated by gel electrophoresis. Gel electrophoresis is of major importance for the genomic fingerprint of a sample material. Genomic fingerprints are obtained when properly amplified targeted or untargeted DNA or RNA fragments are separated by electrophoresis thus providing patterns that can be associated to sample properties (specie, variety and the like). Unlike the fingerprints discussed in the previous (and the next) sections, DNA fingerprint shows somehow different characteristics. The single features of the fingerprint are not quantitative variables (physical or chemical variables allowed to take on quantitative values, e.g. area of a peak in a chromatogram, signal intensity for a $\mathrm{m} / \mathrm{z}$ or absorbance at a fixed wavelength in a IR spectra) but rather categorical variables, i.e. electrophoretic bands that can be either present or absent (see Figure 1 (c)). The sample identification is thus mainly carried out by checking for the presence (or absence) of one or more target bands. Multivariate analysis of the DNA fragments patterns is rarely performed. 
Genomic fingerprinting mainly relies on polymerase chain reaction (PCR) based techniques. PCR is based on the amplification of a target DNA sequence by means of a thermostable DNA polymerase. The process consists of several cycles where the DNA molecule is denaturated, specific primers (small DNA sequences) anneal to the target DNA sequence and the DNA polymerase synthetizes a new DNA fragment delimited by the two primers. In each cycle the number of DNA molecules increases exponentially. A PCR-derived fingerprint can be obtained in different ways. In PCR-RFLP (restriction fragment length polymorphism) the amplified region is digested with an endonuclease and the resulting DNA fragments are separated by electrophoresis and properly visualised. The pattern of fragment represents a fingerprint of the DNA sequence that has been amplified. In multiplex PCR, two or more DNA fragments are simultaneously amplified by means of different target primer pairs, separated by electrophoresis and visualised. In RAPD (random amplification of polymorphic DNA) random DNA fragments are amplified by means of arbitrarily created primers. After separation, the DNA fragments will give rise specific patterns on the gel. Finally, in singlestrand conformation polymorphism (SSCP), DNA sequences are amplified, denatured and the resulting single strand DNA molecules separated by electrophoresis based on their specific secondary structures.

PCR-based fingerprinting techniques have been widely used for species identification. For instance, the identification of ten species of salmon genus in a wide range of commercial products can be accomplished by PCR-RFLP based on the amplification of a specific region of the mitochondrial cytochrome $b$ gene followed by polyacrylamide gel electrophoresis (PAGE) [32]. Similarly, PCR-RFLP has been used to identify 15 species of gadoid fishes based on the amplification of a small region of the cytochrome $b$ gene and three restriction enzymes [33]. Gadidae family is one of the most commercially important fish family comprising species as Atlantic cod (Gadus morhua), the pollack (Pollachius pollachius) and the haddock (Melanogrammus aeglenus). Duplex PCR targeting the cytochrome $b$ gene can be used to detect cow milk in buffalo mozzarella at a level of $1 \%$. Buffalo mozzarella is labelled with PDO and can be produced only with pure water buffalo milk (Bubalus bubalis) [34]. In an original approach, the multiplex PCR fingerprint of the $16 \mathrm{~S}$ and $23 \mathrm{~S}$ rDNA genes of the lactic bacteria naturally occurring in milk has been used to discriminate the geographical origin of PDO mozzarella cheese [35]. The PCR fingerprint was subjected to cluster analysis (neighbour-joining algorithm) which allowed a fair discrimination of the samples.

Genomic fingerprinting shows a unique potential for the species or variety authentication in food products. The introduction of PCR has notably increased the potential of this approach. However, compared to other fingerprinting techniques, genomic fingerprinting is relatively time-consuming and labour-intensive. Its applicability to fraud issues other than genetic identification is limited. Furthermore, food processing may degrade the DNA molecule and lower its recovery thus negatively affecting the results of a analysis when applied to heavily processed foods.

\section{Capillary electrophoresis}

Capillary electrophoresis (CE) is the electrophoretic technique that shows a notable potential for food fraud detection based on product fingerprint. CE is a family of separation techni- 
ques that separate charged analytes based on their electrophoretic mobility: capillary zone electrophoresis (CZE), capillary isoelectric focusing (CIF), capillary gel electrophoresis, capillary electrochromatography. An electric field is applied to the ends of a capillary column. The ions migrate through the column in the same direction pulled by the electrosmotic flow and are separated based on their electrophoretic mobility. The signal that is generated when the mixture components are detected as they are eluted from the column is referred to as a capillary electropherogram. Multivariate dataset can be obtained from electropherogram in the same way as depicted in Figure 3. However, only strategy (a) has been used so far for authentication and fraud control purposes. CE are capable of rapid, low cost and high resolution analysis with little consumption of mobile phase. Main disadvantages of the technique are the low reproducibility (compared to other separation techniques) and low sensitivity that makes $\mathrm{CE}$ not suitable for the analysis of compounds occurring in trace amounts. CE represents a good alternative for the multiple detection of inorganic and organic acids. Many fruits and vegetables is rich in organic acids occurring in varying quantities in different fruits types, giving each fruit a unique organic acid profile. These profiles can be thus used to authenticate a vegetable product or identify the addition of another fruit type. For example the organic acids content measured by CE and LDA has been used to classify Spanish white wines [36].

\section{Spectroscopic fingerprinting}

Spectroscopy is the study of the interaction between a material and radiated energy. The graphical representation of such interaction is what is referred to as a spectrum i.e. a plot of the response of interest as a function of the wavelength or the frequency of the radiation used (see Figure 1d). Such a spectrum is by its very nature a fingerprint of the target material and contains information that are multivariate in nature. The extraction of the chemically relevant information from such a fingerprint requires the application of multivariate statistical techniques. The whole spectrum is used (or part of it) to obtain a multivariate dataset for further chemometric analysis in the same way as described for raw chromatograms (see Figure 3c). Spectral fingerprinting can be used either to classify and discriminate between samples or to quantify a certain compounds. According to the nature of the radiating energy (infrared, visible, ultraviolet, $x$-rays) and the nature of the interaction between energy and matter (absorbance, emission, scattering, resonance) different kind of spectra can be obtained. In the following we will mainly focus on nuclear magnetic resonance (NMR), fluorescence spectra and infrared (IR) spectra.

$N M R$

NMR spectra are generated by the absorption of radiofrequency radiation by atomic nuclei with non-zero spin in a strong magnetic field. Such absorption is affected by the surroundings of the atomic nucleus so that precise information about the molecular structure of a sample can be obtained. The atomic nuclei with non-zero spin that are most frequently used in NMR are ${ }^{1} \mathrm{H},{ }^{13} \mathrm{C}$ even though ${ }^{15} \mathrm{~N},{ }^{17} \mathrm{O},{ }^{19} \mathrm{~F}$ and ${ }^{31} \mathrm{P}$ can be also employed. 
Generally, NMR is superior to other spectroscopic technique because of the much richer and more detailed information that can be gathered from the NMR spectra, at least with high resolution instruments that use frequencies above $100 \mathrm{MHz}$. Those information can be used for the simultaneous quantitative determination of a number of compounds without any prior separation. Furthermore the NMR spectrum can be considered a molecular fingerprint of the test material and subjected to multivariate analysis. The main disadvantage of this technique is the elevated costs of the instruments and the running costs. Nowadays, low resolution NMR instruments are available that use frequency ranging from 10 to $40 \mathrm{MHz}$. Those instruments are much cheaper and easy to use but do not provide the same detailed information as the high resolution instruments. NMR instruments are also capable of good accuracy but the sensitivity is lower compared to MS.

A recent study on the quality control of cola beverages using NMR is exemplar of the potential application of this technique for food authentication and fraud control [37]. ${ }^{1} \mathrm{H}$ NMR spectroscopy was used to discriminate with high success between premium and discount cola brands. This is important in the light of possible counterfeiting. The whole NMR spectra were used in combination with PCA. In addition, the information contained in specific regions of the NMR spectra combined with multivariate calibration (PLS) allowed the quantification of several cola ingredients (caffeine, aspartame, acesulfame-K, and benzoate) which concentration must comply with regulatory limits. NMR has been also used for the authentication of the geographical origin of olive oils. ${ }^{1} \mathrm{H}$ NMR spectra of the bulk olive oil, its corresponding unsaponifiable fraction, and a subfractions of the unsaponifiable fraction (alcohol, sterol, hydrocarbon, and tocopherol fractions) were used to classify olive oils according to their origin [38]. The unsaponifiable fraction had to be extracted to avoid the signal to be masked by that from the TGs in the bulk oil. The adulteration of virgin olive oil with a wide range of seed oils can be detected at level as low as $5 \%$ by means of combined ${ }^{1} \mathrm{H}$ and ${ }^{31} \mathrm{P}$ NMR spectra and discriminant analysis provided that the virgin olive oil are fresh (as reflected by their high 1,2-diglycerides to total diglycerides ratio) [39]. In this case the multivariate analysis was performed on 13 compositional parameters derived from the spectra rather than on the whole NMR spectral fingerprint. ${ }^{13} \mathrm{C}$ NMR spectra have been used for the authentication of fish and fish products. Discrimination between farmed and wild salmon is possible based on the NMR spectra of the muscle lipids and neural networks (PNN) and support vector machines (SVM) multivariate analysis [40]. Using the peak intensities of 12 selected chemical shifts an excellent discrimination is obtained by using PNN and SVM (98.5 and 100.0\%, respectively). The authentication of different gadoid species was also achieved based on the NMR spectra of muscle lipids and Bayesian belief networks (BBN) with successful classification of $100 \%$ [41]. However, ${ }^{1} \mathrm{H}$ NMR spectroscopy can also provide useful information for the authentication of wild fish. In Figure 4, the PLS-DA scores plot for the ${ }^{1} \mathrm{H}$ NMR data measured in the authors' group on frozen, smoked and canned salmons both wild and farmed is presented. The score plot shows a clear separation of the two groups in distinct regions of the three dimensional plot. The results of the classification model (leave 5 out internal validation) were extremely positive with $100 \%$ of the wild samples (29 samples) and almost $100 \%$ of the farmed samples (60 out of 62 samples) correctly classified. 


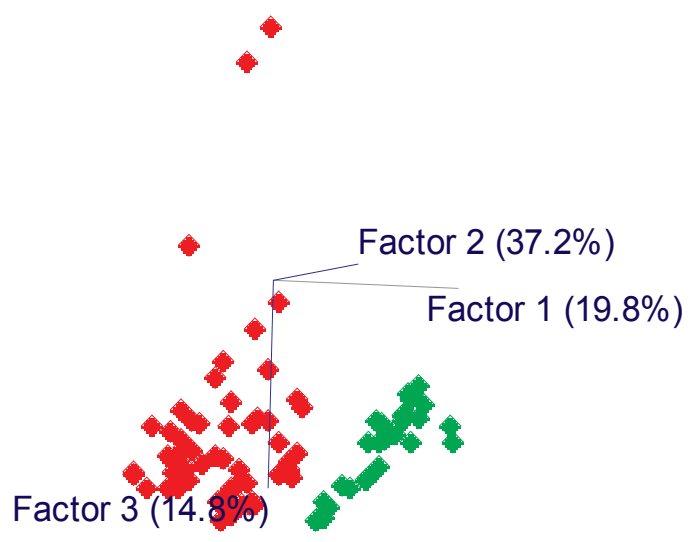

Figure 4. PLS-DA scores plot for the ${ }^{1} \mathrm{H}$ NMR data on wild and farmed salmon (frozen, canned and smoked). Red=farmed salmons; green=wild salmons

\section{Fluorescence spectroscopy}

Fluorescence spectra are normally obtained by exciting the test material. With radiations at a fixed wavelength and recording the intensity of the emitted radiation over a range of wavelengths. However, a 3D spectrum can be obtained by recording the emission spectra at different excitation wavelengths: the so called fluorescence excitation-emission matrix (EEM) which provides more information about the fluorescent compounds occurring in the sample. In synchronous fluorescence scan (SFS) the whole fluorescence landscape can also be achieved by scanning the excitation and the emission wavelengths simultaneously keeping a fixed wavelength interval (the so-called offset). Products that contains natural (or added) fluorophores are suitable for Fluorescence spectroscopy. Tryptophan, tyrosine and phenylalanine residues are fluorophores typically present in a variety of foods along with vitamin A, riboflavin (vit B2), NAD, NADH and compounds originating from Maillard reaction/ lipid oxidation. Fluorescence spectra provide information about the amount of those compounds and on the way the fluorophore environment interacts with them. Traditionally, the fluorescence spectra have been treated by means of univariate approaches i.e. taking advantage of one specific wavelength or the derived fluorescence peak features. Nowadays the multivariate information contained in the fluorescence spectra is processed by chemometric techniques. When EEMs or SFS spectra are produced, decomposition methods such as twoway PCA, TUCKER and parallel factorial analysis (PARAFAC) are necessary to extract information from such a multi-way dataset (the data can be arranged in a cube instead of a 
matrix as in standard multivariate data sets). In the right-angle fluorescence spectroscopy the incidence angle between the excitation and the emission radiation is $90^{\circ}$. Only liquids and diluted solutions can be analysed and an attenuation of the signal intensity at high absorbance $(>0.1)$ is observed. To overcome this problem the front-face fluorescence (FFF) has been developed where only the surface of the material is analysed and the incidence angle is around $56^{\circ}$ to minimise the artefacts from the excitation radiation reflected or scattered by the sample. Solid and powdered samples as well as bulk liquids can be analysed by FFF.

Fluorescence spectra are a promising tool to verify the egg freshness. Albumen samples stored for 1,2,3 and 4 weeks can be discriminated by means of the Maillard reaction products fluorescence and factorial discriminant analysis (FDA) with high success rate [42]. Vitamin A fluorescence together with FDA allows discrimination among egg yolk samples stored for different times [43]. Similarly, the freshness of fish can be predicted based on NADH and tryptophan fluorescence spectra. NADH fluorescence spectra can be also considered as a promising tool for the discrimination between frozen-thawed fish and fresh fish. The NADH emission spectra show a typical maximum at $455 \mathrm{~nm}$ in fresh fish and at $379 \mathrm{~nm}$ in frozenthawed fish. The multivariate analysis (FDA) of the NADH spectrum allowed the correct classification of $100 \%$ of the analysed samples [44]. The authenticity of edible oils has also been extensively investigated by fluorescence spectroscopy. Chlorophyll and vitamin E are important fluorophores in olive oils and contribute greatly to oil colour and stability during storage. Refining processes decreases the content of chlorophyll and vitamin E with a corresponding change in the fluorescence spectrum. However, the discrimination between virgin and refined olive oil is mainly based on the fluorescence of lipid oxidation products (more abundant in the less resistant refined oils). A fast screening method has been also developed to detect adulteration of EVOO with olive-pomace oil [45]. It is based on the EEMs and it is able to detect adulteration at a level of $5 \%$. Similarly, the discrimination between olive oils according to their overall acidity are also possible with fluorescence spectroscopy [46]. In this case, the maximum differentiation between the oils was obtained in the region 429-545 $\mathrm{nm}$ of the spectrum and allowed 100\% correct classification of lampante olive oil (acidity $>3.3 \%$, not edible) from virgin olive oil with lower acidity $(<3.3 \%)$. Finally, SFS with multiple regression analysis has been reported to for the detection of adulteration of EVOO with olive oil to a level as low as $8.4 \%$ when a $80 \mathrm{~nm}$ wavelength interval is used [47].

The great advantage of fluorescence spectroscopy is the rapidity, the limited costs and the non-destructive nature of the analysis. The sensitivity is also much greater than that of other techniques because the fluorescence signal has in principle no background.

\section{Infrared spectroscopy}

Infrared spectra are produced by measuring the intensity of the absorbance of infrared light by a sample as function of the wavelength. The absorption of infrared light is ascribed to transitions in the vibrational energies of the molecules contained in a sample. Each functional group of a molecule shows characteristic IR absorption at specific frequency ranges regardless of the interaction of the functional group with the rest of the molecule. However, interaction between atoms within a molecule may sometimes affect the position of characteristic bands in a IR spectra depending on the surroundings of the functional group. IR 
spectra can thus provide qualitative information about the nature of the functional group present in a food sample and quantitative information on their amount. When the effect of all the functional groups is taken together, the whole spectrum represents a molecular fingerprint that can be used to verify the nature of the sample. The IR region of the electromagnetic spectrum can be divided in 3 portion: The far IR (FIR, 400-10 $\mathrm{cm}^{-1}$ ) has the lower energy and induces rotational transitions in the molecules. The mid IR (MIR, 4000-400 $\mathrm{cm}^{-1}$ ) induces fundamental vibrational transitions in the molecules. The near IR region (NIR, $14000-4000 \mathrm{~cm}^{-1}$ ) also induces transitions in the vibrational energies of the molecules. However, the transitions of the vibrational energy induced by the NIR portion of the spectrum are more complex than those induced by the MIR region. Overtones (transitions from the fundamental vibrational level over two or higher energy levels) and combination modes (arising from the interaction of two or more vibrations taking place simultaneously in different functional groups) give rise to very complex bands in the NIR spectrum that can give more complex structural information than MIR. On the other hand, NIR spectra are less selective than MIR spectra because of the superposition of different overtones and combination bands. A raw spectrum contains background information and noise beside valuable information. To remove those interferences as well as those coming from scattering, to normalise the effect of particle size and light distance, pre-processing methods such as smoothing, derivative, standard normal variate transformation (SNC), multiplicative scatter correction (MSC) or wavelet transforms (WT) are required. Recently, the introduction of the Fourier transform technique in IR (FTIR) has further increased the application range of the IR spectroscopy in the food field. In such a case the spectrum is obtained by mean of an interferogram in which multiple frequencies are measured simultaneously. The resulting interferogram is then deconvoluted using proper algorithms in order to have the original spectrum. The advantages of that technology is a faster analysis and a higher throughput and a better alignment of spectrum obtained by repetitive scans.

IR spectroscopy have been successfully applied to detect adulteration of juices, purees and syrups with cheaper juice concentrates. Adulteration of orange juice with orange pulpwash, grapefruit juice or synthetic sugars/acids mixture can be detected at a level as low as $50 \mathrm{~g} / \mathrm{kg}$ by NIR [48]. Similarly, the adulteration of strawberry or raspberry juice with apple juice can be detected at level> $10 \%$ by transmittance NIR coupled with PLS [49]. MIR spectra have been used to detect adulteration of pure pomegranate juice with grape juice $(2 \%-14 \% \mathrm{v} / \mathrm{v})$ [50] and to predict the percent fruit content in strawberry jam [51]. Adulteration of honey and maple syrup can also be detected by NIR and MIR spectroscopy. NIR and FTIR have been successfully applied for the detection and quantification of cane and beet sugars in maple syrup [52]. Attenuated total reflectance (ATR)-FTIR coupled with LDA and PLS was used to discriminate the type of adulterant in three different honey varieties. A success rate of prediction of $100 \%$ was achieved for honey samples adulterated with $7-25 \% \mathrm{w} / \mathrm{w}$ of simple (glucose, fructose, sucrose) and complex (beet and cane invert) sugars [53]. NIR and MIR have also been employed for the authentication of lard and fats. Lard adulteration can be detected in cake [54] and in chocolate [55]. The adulteration of shortening with lard can be detected at levels ranging from 0 to $100 \%$ and a standard error of calibration (SEC) of 1.75 by using the regions $1.117-1.097 \mathrm{~cm}^{-1}$ and $990-950 \mathrm{~cm}^{-1}$ of the NIR spectrum. Adulteration of 
olive oils has also attracted much attention due to the economic value of the product. Adulteration of EVOO with palm oil can be detected by FTIR and PLS in concentration varying from 1.0 to $50.0 \% \mathrm{w} / \mathrm{w}$ [56]. The region $1500-1000 \mathrm{~cm}^{-1}$ of the MIR spectra was used for the regression model. The adulteration of EVOO with sunflower, corn, soyabean and hazelnut oil can be detected at level as low as 5\% by using FTIR and LDA [57]. In this latter case, the normalized absorbance of peaks and shoulders areas were used in the model as predictors. The standard of identity for butter require that no vegetal oil nor margarine is added to the product. The presence of margarine can be detected by NIR coupled with PLS in the range $0-100 \%$ with a standard error of calibration after validation (SECV) $<1.2 \%$ [58]. IR spectroscopy has been also widely used to predict and control meat quality. The discrimination between fresh and frozen-thawed beef can be accomplished by IR spectroscopy due to modification of the myofibrillar proteins and the corresponding change in their water holding capacity [59].

Infrared spectroscopy is a well-established technique for fast, high-throughput and non-destructive analysis of food and other biological samples. The analysis can be easily implemented on-line, can be automated and does not requires trained personnel to be carried out. It is little expensive and environmental friendly since does not require solvents, chemicals and does not produce waste. However, even though the analysis per se (collection of the spectrum) is fast, post-processing (pre-processing of the spectra and model building) of the input data can be laborious and time-consuming. The calibration models are usually built against reference analytical methods so that the measurement errors accumulated and the total predictive error increases. Finally, the classification or regression models are theoretically valid only on the instruments with which the training and the calibration has been carried out. The transfer of a multivariate model to other instruments affects its precision and accuracy compared to the original ones.

\section{A case study on NIRS and adulteration.}

As an example of the potential of the NIR Spectroscopy for the detection of adulteration, the results of an investigation that has been carried out in the authors' research group will be shown. NIR spectra were used to detect the presence of nitrogen replacers in milk powders. The compositional standards for milk powders require that the amount of milk proteins in milk solids-not-fat should be at least equal to $34 \% \mathrm{~m} / \mathrm{m}$, unless declared. The low prices of some nitrogen containing compounds make them attractive as potential adulterants to increase the level of apparent proteins in milk powders. The Kjeldahl method (official reference method for proteins content) measures the total amount of $\mathrm{N}$ irrespective of whether it comes from proteins or not. Expensive and time-consuming analytical methods such as enzyme-linked immunosorbent assay (ELISA), LC-MS/MS and GC-MS/MS are necessary for confirmatory analysis of melamine and its analogues in milk powders. To prove the potential of NIR spectroscopy for the detection of such adulteration, 33 skim milk powders were randomly adulterated with adulterants ammonium chloride, caprolactam, diammonium phosphate and polyvinylpyrrolidone (PVP) in order to produce an increment of $0.10,0.50$, 1.00 and $2.00 \%$ in the (apparent) proteins content of the milk powder. The samples were measured by NIR spectroscopy and the spectra subjected to PLS-DA analysis. A few milk 
powders were randomly selected and adulterated by melamine, ammelide and urea to test the robustness of the predictive models.

A PLS-DA model was first developed to predict the type of adulterant. The training set consisted of $80 \%$ genuine milk powders ( 26 samples) and $80 \%$ adulterated samples (19 samples from each adulterant) which were randomly selected. The remaining $20 \%$ of the samples, and those adulterated with the non-modelled adulterants melamine, ammelide and urea were used for external validation. The success rate of prediction was $100 \%$ in cross-validation and $78 \%$ for the external validation set (Table 2). Three out of 6 samples adulterated with melamine, ammelide and urea were correctly predicted as adulterated.

\begin{tabular}{|c|c|c|c|c|c|c|}
\hline $\begin{array}{l}\text { Class } \\
\text { item }\end{array}$ & $\begin{array}{l}\text { Genuine } \\
\text { powder }\end{array}$ & $+\mathrm{NH}_{4} \mathrm{Cl}$ & + caprolactam & $+\left(\mathrm{NH}_{4}\right)_{2} \mathrm{HPO}_{4}$ & + PVP & $\begin{array}{c}\text { No } \\
\text { match }\end{array}$ \\
\hline & \multicolumn{6}{|c|}{ PLS-DA model based on training set } \\
\hline Genuine powder & 26 & - & - & - & - & - \\
\hline$+\mathrm{NH}_{4} \mathrm{Cl}$ & - & 19 & - & - & - & - \\
\hline +caprolactam & - & - & 19 & - & - & - \\
\hline$+\left(\mathrm{NH}_{4}\right)_{2} \mathrm{HPO}_{4}$ & - & - & - & 19 & - & - \\
\hline \multirow[t]{2}{*}{$+P V P$} & - & - & - & - & 19 & - \\
\hline & \multicolumn{5}{|c|}{ External validation } & \\
\hline Genuine powder & 7 & - & - & - & - & - \\
\hline$+\mathrm{NH}_{4} \mathrm{Cl}$ & - & 4 & - & - & 1 & - \\
\hline +caprolactam & - & - & 3 & - & 1 & 1 \\
\hline$+\left(\mathrm{NH}_{4}\right)_{2} \mathrm{HPO}_{4}$ & - & - & - & 4 & - & 1 \\
\hline \multirow[t]{2}{*}{$+P V P$} & - & - & - & - & 3 & 2 \\
\hline & \multicolumn{6}{|c|}{ External validation based on melamine, ammelide and urea } \\
\hline +melamine & - & - & - & - & - & 2 \\
\hline tammelide & 2 & - & - & - & - & - \\
\hline+ urea & 1 & - & - & - & - & 1 \\
\hline
\end{tabular}

Table 2. Prediction results of PLS-DA model for the type of adulterant in milk powders

A PLS-DA model was then developed to discriminate generally between genuine and adulterated samples. The training set and the validation set were built in the same way as previously described. The PLS-DA scores plot is presented in Figure 4. The prediction results are reported in Table 3.

In external validation only one genuine sample was wrongly predicted as adulterated. One adulterated sample was incorrectly predicted as genuine (PVP added at its lowest concen- 
tration) and 3 adulterated samples could not be classified. Samples adulterated with melamine, ammelide and urea were all correctly predicted as adulterated.

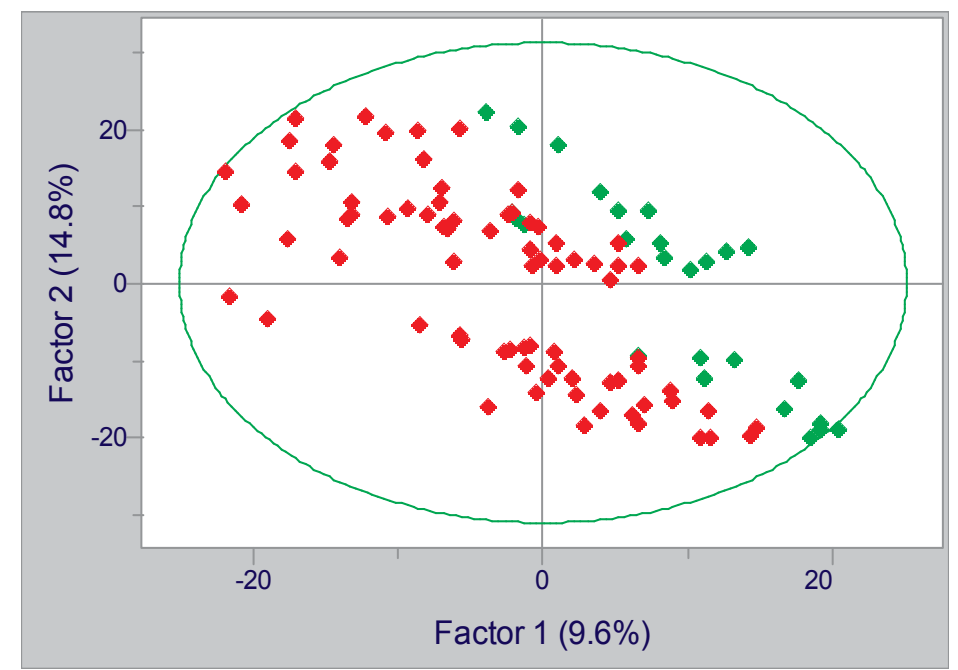

Figure 5. PLS-DA scores plot of IR spectra for genuine and adulterated milk powders. Red = adulterated powders; green=genuine powders.

\begin{tabular}{lccc}
\hline $\begin{array}{l}\text { Class } \\
\text { Item }\end{array}$ & $\begin{array}{c}\text { Genuine } \\
\text { powder }\end{array}$ & $\begin{array}{c}\text { Adulterated } \\
\text { powder }\end{array}$ & No match \\
\hline \multicolumn{3}{c}{ Internal validation } \\
\hline $\begin{array}{l}\text { Genuine powder } \\
\text { pdulterated }\end{array}$ & 24 & 2 & - \\
\hline $\begin{array}{l}\text { powder } \\
\text { Adulterated } \\
\text { powder }\end{array}$ & 0 & 68 & 8 \\
\hline $\begin{array}{l}\text { Genuine powder } \\
\text { A }\end{array}$ & 6 & 1 & 0 \\
\hline
\end{tabular}

Table 3. Prediction results of PLS-DA model for genuine versus adulterated milk powders.

NIRS combined with chemometrics proved to be a promising tool for the cost-effective detection of adulteration of milk powders with a range of nitrogen replacers. The reliability of the classification models can be improved by the careful selection of those regions of the NIR-visible spectrum which showed the best discrimination power between genuine and adulterated samples. 


\section{Other fingerprintings}

\section{Differential scanning calorimetry (DSC)}

Differential scanning calorimetry is a themoanalytical technique that measures the amount of heat required to increase the temperature of a sample relative to a reference material. When the amount of heat absorbed/released is plotted as a function of temperature a thermal spectrum of the sample is obtained from which kinetic and thermodynamic information such as the heat capacity and the enthalpy of any phase transition (fusion, evaporation, glass transition etc) the sample undergoes in the temperature span of the experiments can be determined. Those physical properties depends in turn on compositional and structural properties of the samples.

DSC has been mainly applied for the authentication of fats/oils. The level of adulteration of canola oil with lard, beef tallow and chicken fat as well as that of virgin coconut oil with palm kernel oil (PKO) and soybean oil can be predicted by the analysis of the DSC thermogram and stepwise multilinear regression (SMLR) [60]. The melting profiles of cow, goat, sheep, camel, horse and water buffalo milk fat samples were also determined by DSC. Differences in the DSC profiles for the fat fraction of milk of different species can be attributed to their fatty acid/TAG composition. In general, the melting point of the fats decreases with decreasing chain length and increasing degree of unsaturation of the fatty acids in the milk. The thermograms were subjected to PCA analysis which showed a clear distinction of the four milk samples analysed. The loadings plots of the heat flow data showed that the data are most influenced between the temperatures of $13^{\circ}$ and $24^{\circ} \mathrm{C}$ [61].

Sensor technology

Sensor technology is often referred to as electronic nose technology. In this technology the volatile compounds present in the headspace of a sample are detected by an array of semiselective sensors. Normally each sensor is sensitive to all the volatile but each in a peculiar way. There are many sensors for EN instruments such as metal oxide sensors, conducting polymer sensors, quartz crystal membrane sensors, or mass spectrometers (MS-EN) available on the market. The sensor's response is then transformed in a spectrum that represents a fingerprint of the volatile compounds of the tested sample. Electronic nose analysis are often cheaper and faster than GC analysis and the sample preparation is usually quite simple. The technique, however, does not have the same sensitivity of other techniques. Furthermore, single volatile compounds cannot be identified and the signal is sensitive to water vapour.

Electronic nose has been used mainly for the detection of adulteration in fats/oils. Rapid detection of pork and lard in food samples for halal authentication (compliance with Islamic dietary rules) are reported with electronic nose [62]. Under Jewish and Islamic dietary laws, foods containing porcine-based ingredients such as lard are strictly prohibited from consumption. Electronic nose and chemometric analysis was applied for the detection of adulteration of olive oil samples with sunflower and olive-pomace oil at levels as low as 5\% [63]. Application are also reported for the authentication of the geographical origin of Italian wines [64], and emmenthal cheese [65]. 


\section{Transcriptomics}

Transcriptomics is a post-genomic technique that consists in the simultaneous measurements of all the transcripts (mRNA molecules) in a given organism, or of a specific subset of transcripts present in a particular cell type. Unlike genome, transcriptome varies according to the environmental conditions and represents the genes that are actively expressed in a certain cell at a certain time. Transcriptome is usually obtained by DNA microarray technologies and reverse PCR. The set of all the mRNA produced (and hence of the genes actively expressed) represents a fingerprint of that target cell/organism and can thus use for fraud detection and authentication purposes. At the present the potential of transcriptomics in this respect is still underexplored.

\section{Fingerprinting options for other biomaterials}

Biofuels are an important environmental-friendly alternative to fossil fuels. The term biodiesel refers primarily to FAME obtained after transesterification of triglycerides with methanol. The methyl esters can be produced from many different triglyceride sources, primarily rapeseed oil but also sunflower oil, soybean oil, palm oil, linseed oil, tallow, and used frying oil. Blends of biodiesel with conventional petrodiesel fuel represent a common utilization of biodiesel. The ability to predict retail biodiesel blend percent composition is important to detect adulterations. It has been reported that biodiesel from different sources can be discriminated by direct infusion ESI-MS and multivariate statistics [66]. In addition, the \% of rapeseed or salmon biodiesel in petrodiesel in concentrations ranging from $0.5 \%$ to $10 \%$ can be predicted with good accuracy by applying a PLS model. Similarly, the feedstock source of blends of biodiesel and conventional diesel, as well as the \% composition of the blend can be predicted applying a kNN and a PLS model respectively to the total ion current chromatograms from gas chromatography-quadrupole mass spectrometry (GC-qMS) using a polar column [67]. The precision of the prediction was between $4-5 \%$. Furthermore, the addition of residual oil (non-transesterified residual vegetable oil) is one of the easiest ways of adulterating biofuels. Synchronous fluorescence combined to LDA can be used to discriminate between diesel oils, biodiesels and biodiesel adulterated with residual oil and the \% of residual oil can be predicted with good accuracy when a PLS model is used [68].

Perfume counterfeiting is an illegal practice that causes huge economical loss to the perfume industry and pose potential health risk to consumers who might be exposed to harmful chemical from counterfeited products. Traditionally, the quality control analyses for perfume focuses on volatile and semi-volatile compounds and are performed by GC based techniques. Recently, more straightforward methods have been proposed for the fast authentication of premium perfumes and detection of adulteration/counterfeiting. A fingerprint of the polar compounds can be achieved by direct infusion EASI-MS (see section 3) [69]. The samples are sprayed onto a glass rod and directly exposed to the ionisation source of the MS system. An almost instantaneous, simple and reproducible fingerprint of the polar compounds in the product is obtained that allows a complete discrimination between au- 
thentic and counterfeited products. A fast discrimination between authentic and counterfeited products can be also obtained by fingerprinting of the polar compounds by ESI-MS in the positive ion mode and chemometric analysis previous extraction of few $\mu \mathrm{L}$ of the sample in a 1:1 methanol/water solution [70]. Even more recently, a fast, simple and low-cost method for the authentication of perfume based on a commercial electronic olfactory system (EOS) equipped with thin film metal oxide semiconductors has been proposed [71]. The PCA analysis of the $R / R_{0}$ values (resistance of the sensor in the presence of the volatile compounds relative to that of the sensor balanced in air) generated by 6 sensors based on different metal oxide semiconductors can unequivocally discriminate between authentic and counterfeited perfumes. The prior removal of ethanol from the samples is necessary for the correct discrimination of the samples.

Essential oils are also widely employed for their fragrance in perfumery but also in cosmetics and household products. One of the most common fraud is the blend of valuable essential oils with other less valuable alternatives. As an example, the valuable Rosewood essential oil, obtained from the trees of Aniba rosaeodora Ducke and employed in fine perfumery, can be blended with the far cheaper synthetic linaool, obtained by re-distillation of Rosewood leaf oil. The ESI-MS fingerprint in the positive ions mode of the polar compounds extracted by an acidified 1:1 water methanol solution can easily detect adulteration of Rosewood oil with synthetic linaool at concentration as low as $10 \% \mathrm{v} / \mathrm{v}$ [72]. A PCA model is necessary to extract the relevant information from the fingerprint.

Direct infusion MS can be used to authenticate wood as well. Venturi easy ambient sonic spray ionization (V-EASI)-MS fingerprint of a very simple methanolic extract of wood chips or directly acquired from the freshly scratched wood surface may help to control the illegal logging and trade of the noble Mahogany tropical wood and its falsification [73]. V-EASIMS is a novel ambient ionization MS technique characterized by sonic spray ionization and a self-pumping system based on the Venturi effect. It allows the direct analysis of solid or liquid samples. Ionization is assisted by compressed nitrogen and the apparatus is thus free of electrical discharge, thermal interferences since no heating, voltage or radiation is used. The introduction of fast and high-throughput analytical techniques for wood authentication is especially valuable since the classical controls are based on time-consuming morphological evaluations. Recently an original and fast approach for the authentication of wood species has been proposed. It is based on the analysis of the volatile compounds measured by a low-cost conductive polymer-based portable electronic nose formed by an array of only three gas sensors and/or the elemental fingerprint measured by laser-induced breakdown spectrometry (LIBS) which performs a multielemental and direct analysis even in solid samples [74].

The assurance of quality of herbal supplements and medicines is a major concern for the phytopharmaceutical and the food industry. The identification of the herbal drug and the presence of adulterant is a mandatory test to ensure the quality, the efficacy and the safety of a medical preparation or an herbal supplement. Among a variety of quality control methods, chromatographic fingerprinting has gained more and more attention recently and have been used to authenticate a large number of herbal products. They are accepted by many international organization for the quality control of herbal medicine but are relatively timeconsuming. More recently, spectroscopic techniques have been explored to rapidly authenti- 
cate herbal products. For instance, different species of Echinacea, e.g., E. purpurea, E. angustifolia, and E. pallida are used for commercial preparations to prevent or cure the common cold, flu, and several other diseases due to their nonspecific stimulating effect of the immune system. It is also well known that Echinacea preparation are commonly adulterated with roots of Parthenium integrifolium L. This adulteration can be detected by NIRS at a minimum of $10 \%$ of adulteration [75]. The method requires just the milling of the sample and can be carried out within 1 minute.

The list of potential applications of products fingerprinting is not limited to the cases discussed above and many other examples may be provided. In the authors' research group for instance, PTR-MS and ICP-MS are used for the authentication of the geographical origin of flower bulbs. Counterfeiting of pharmaceuticals is another area where the application of fingerprinting techniques has proved of great help. The topic is so huge that we would address the interested readers to specialized publications.

\section{Conclusions}

Product fingerprinting combined with chemometrics represents a valuable tool for fraud detection and control for food products and other biomaterials. A fingerprinting approach is particularly useful:

- For the authentication of products for which target analyses based on specific markers are not available

- For the detection of adulteration based of yet unknown adulterants

- For a fast and high-throughput screening of the samples before more elaborated confirmatory analysis are applied.

At the same time a fingerprinting approach may substantiate nutritional, sensory or other product qualities.

LC, GC, and IR spectroscopy are already common instrumental platforms available in most QA laboratories and they will continue to provide valuable support for food fraud prevention. IR and other spectroscopic techniques have the great advantage of providing fast, highthroughput and non-destructive analyses with limited costs. They can be easily automated and adapted for in-line or in-situ analysis which makes these techniques well suited for implementation in the industrial setting. MS and NMR are not as common in QA laboratories, principally because of their high costs but they may become more important in routine QA testing because of their superior performances. However, even though the costs of MS and NMR instruments is still very high, the cost per sample can be very low if a high samples turnover can be achieved.

From an analytical point of view, a further improvement is expected in the future from the broader application of multi-dimensional separation techniques such as GCXGC or LCxLC which provide enhanced resolution and an higher number of peaks. Ultra-performance liq- 
uid chromatography (UPLC) and CE may also provide rapid separation with limited costs. The application of novel (or still underexploited) ambient ionisation MS techniques that allow the rapid analysis of liquid and solid samples with little, if any, preparation will be particularly valuable in the future.

The applicability and the reliability of a fingerprint approach also depends from the correct and tailored usage of the relevant and appropriate chemometric tools. For the development of regression and/or classification models, special care should be devoted to ensure:

- The representativeness of the classes considered, in order to cover all the possible source of variability for the class at stake.

- A robust validation of the model (external validation to be preferred over internal validation).

- The use of the appropriate chemometric tools depending on the problem at stake. Whereas a few pattern recognition techniques are frequently used (PLS-DA, SIMCA, LDA..), some other such as classification and regression trees (CART), quadratic discriminant analysis (QDA) are still underexploited despite the good results that they can provide.

Another key aspect is represented by the validation of methods based on fingerprinting and chemometrics that is essential for their application in a commercial context. Whereas standards exist for the validation of regular analytical methods (see for instance, Commission Decision 2002/657/EC and ISO 17025), internationally accepted protocols for the validation of methods based on fingerprinting techniques and chemometric classification models are lacking at the present. Such protocols should indicate the performance characteristics that have to be checked and the criteria to be met in order to verify the compliance of the method with the performance characteristics.

\section{Acknowledgements}

We acknowledge Martin Alewijn, Grishja van der Veer and Pan Weijing for the work on NIR spectroscopy and milk powder and Angela Dura de Miguel, Arjen Lommen and Maikel Rozijn for the work on ${ }^{1} \mathrm{H}$ NMR and fish. We also acknowledge the Dutch ministry of agriculture, economic affair and innovation for the funding of this book chapter.

\section{Author details}

Edoardo Capuano ${ }^{1^{*}}$ and Saskia M. van Ruth ${ }^{1}$

*Address all correspondence to: edoardo.capuano@wur.nl

1 RIKILT - Institute of Food Safety, Wageningen University and Research Centre,, The Netherlands 


\section{References}

[1] Felson, M. (1998). Crime and everyday life. 2nd ed. Thousand Oaks, Calif. Pine Forge Press.

[2] Macatelli, M., Akkermans, W., Koot, A., Buchgraber, M., Paterson, A., \& van Ruth, S. (2009). Verification of the geographical origin of European butters using PTR-MS. DOI: 10.1016/j.jfca.2008.10.009.

[3] Galle, S. A., Koot, A., Soukoulis, C., Cappellin, L., Biasioli, F., Alewijn, M., \& van Ruth, S. (2011). Typicality and Geographical Origin Markers of Protected Origin Cheese from The Netherlands Revealed by PTR-MS. Journal of Agricultural and Food Chemistry, 59(6), 2554-2563.

[4] Ruiz-Samblas, C., Tres, A., Koot, A., van Ruth, S., Gonzales-Casado, A., \& QuadrosRodriguez, L. (2012). Proton transfer reaction-mass spectrometry volatile organic compound fingerprinting for monovarietal extra virgin olive oil identification. Food Chemistry, 134(1), 589-596.

[5] Del Pulgar, J. S., Soukoulis, C., Biasioli, F., Cappellin, L., Garcia, C., Gasperi, F., Granitto, P., Mark, T. D., Piasentir, E., \& Schuhfried, E. (2011). Rapid characterization of dry cured ham produced following different PDOs by proton transfer reaction time of flight mass spectrometry (PTR-ToF-MS). Talanta, 85(1), 386-393.

[6] Furia, E., Naccarato, A., Sindona, G., Stabile, G., \& Tagarelli, A. (2011). Multielement Fingerprinting as a Tool in Origin Authentication of PGI Food Products: Tropea Red Onion. DOI: 10.1021/jf201556e.

[7] Laursen, K. H., Schjoerring, J. K., Olesen, J. E., Askegaard, M., Halekoh, U., \& Husted, S. (2011). Multielemental Fingerprinting as a Tool for Authentication of Organic Wheat, Barley, Faba Bean, and Potato. Journal of Agricultural and Food Chemistry, 59(9), 4385-4396.

[8] Pillonel, L., Bütikofer, U., Rossmann, A., Tabacchi, R., \& Bosset, J. O. (2004). Analytical methods for the detection of adulteration and mislabeling of Raclette Suisse and Fontina PDO cheese. Mitteilungen aus Lebensmittel Untersuchung und Hygiene;, 95-489.

[9] Lerma-Garcia, M. J., Herrero-Martinez, J. M., Ramis-Ramos, G., \& Simo-Alfonso, E. F. (2008). Evaluation of the quality of olive oil using fatty acid profiles by direct infusion electrospray ionization mass spectrometry. Food Chemistry, 107(3), 1307-1313.

[10] Gomez-Ariza, J. L., Arias-Borrego, A., Garcia-Barrera, T., \& Beltran, R. (2006). Comparative study of electrospray and photospray ionization sources coupled to quadrupole time-of-flight mass spectrometer for olive oil authentication. Talanta, 70(4), 859-869.

[11] Alves , J. de O., Neto, W. B., Mitsutake, H., Alves, P. S., \& Augusti, R. (2010). Extra virgin $(\mathrm{EV})$ and ordinary $(\mathrm{ON})$ olive oils: distinction and detection of adulteration 
(EV with $\mathrm{ON}$ ) as determined by direct infusion electrospray ionization mass spectrometry and chemometric approaches. DOI: 10.1002/rcm.4590.

[12] Moller, J. K. S., Catharino, R. R., \& Eberlin, M. N. (2005). Electrospray ionization mass spectrometry fingerprinting of whisky: immediate proof of origin and authenticity. Analyst, 130-890.

[13] De Souza, P. P., de Oliveira, L. C. A., Catharino, R. R., Eberlin, M. N., Augusti, D. V., Siebald, H. G. L., \& Augusti, R. (2009). Brazilian cachaça:“Single shot typification of fresh alembic and industrial samples via electrospray ionization mass spectrometry fingerprinting. Food Chemistry, 115(3), 1064-1068.

[14] Gonzalez-Dominguez, R., Garcia-Barrera, T., \& Gomez-Ariza, J. L. (2012). Iberian ham typification by direct infusion electrospray and photospray ionization mass spectrometry fingerprinting. Rapid Communications in Mass Spectrometry, 26(7), 835-844.

[15] Wang, J., Kliks, M. M., Quw, Jun. S., Shi, G., \& Li, Q. X. (2009). Rapid determination of the geographical origin of honey based on protein fingerprinting and barcoding using MALDI TOF MS. Journal of Agricultural and Food Chemistry, 57-10081.

[16] Chambery, A., del Monaco, G., di Maro, A., \& Parente, A. (2008). Peptide fingerprint of high quality Campania white wines by MALDI-TOF mass spectrometry. DOI: 10.1016/j.foodchem.2008.08.031.

[17] Vaclavik, L., Cajka, T., Hrbek, V., \& Hajslova, J. (2009). Ambient mass spectrometry employing direct analysis in real time (DART) ion source for olive oil quality and authenticity assessment. Analytica Chimica Acta, 645(1-2), 56-63.

[18] Cajka, T., Riddellova, K., Tomaniova, M., \& Hajslova, J. (2011). Ambient mass spectrometry employing a DART ion source for metabolomic fingerprinting/profiling: a powerful tool for beer origin recognition. Metabolomics, 7-500.

[19] Vaclavik, L., Hrbek, V., Cajka, T., Rohlik, B. A., Pipek, P., \& Hajslova, J. (2011). Authentication of Animal Fats Using Direct Analysis in Real Time (DART) Ionization -Mass Spectrometry and Chemometric Tools. Journal of Agricultural and Food Chemistry, 59(11), 5919-5926.

[20] Riccio, M. F., Sawaya, A. C. H. F., Abdelnur, P. V., Saraiva, S. A., Hadda, R., Eberlin, M. N., \& Catharino, R. (2011). Easy Ambient Sonic-Spray Ionization Mass Spectrometric of Olive Oils: Quality Control and Certification of Geographical Origin. Analytical Letters, 44(8), 1489-1497.

[21] van Ruth, S. M., Alewijn, M., Rogers, K., Newton-Smith, E., Tena, N., Bollen, M., \& Koot, A. (2011). Authentication of organic and conventional eggs by carotenoid profiling. Food Chemistry, 129-1299.

[22] de la Mata-Espinoza, P., Bosque-Sendra, J. M., Bro, R., \& Cuadros-Rodriguez, L. (2011). Olive oil quantification of edible vegetable oil blends using triacylglycerols chromatographic fingerprints and chemometric tools. Talanta, 85-177. 
[23] Cordawener, J. H. G., Luykx, D. M. A. M., Frankhuizen, R., Bremer, M. G. E. G., Hooijerink, H., \& America, A. H. P. (2009). Untargeted LC-Q-TOF mass spectrometry method for the detection of adulterations in skimmed-milk powders. Journal of Separation Science, 32-1216.

[24] Axelson, D. E., Standal, I. B., Martinez, I., \& Aursand, M. (2009). Classification of Wild and Farmed Salmon Using Bayesian Belief Networks and Gas Chromatography-Derived Fatty Acid Distributions. Journal of Agricultural and Food Chemistry., 57-7634.

[25] Busetto, M. L., Moretti, V. M., Moreno-Rojas, J. M., Caprino, F., Giani, I., Malandra, R., Bellagamba, F., \& Guillou, C. (2011). Authentication of Farmed and Wild Turbot (Psetta maxima) by Fatty Acid and Isotopic Analyses Combined with Chemometrics. DOI: $10.1021 /$ jf0734267.

[26] Tres, A., O'Neil, R., \& van Ruth, S. M. (2011). Fingerprinting of fatty acid composition for the verification of the identity of organic eggs. Lipid technology, 23-40.

[27] Tres, A., \& van Ruth, S. M. (2011). Verification of Organic Feed Identity by Fatty Acid Fingerprinting. Journal of Agricultural and Food Chemistry, 59(16), 8816-8821.

[28] van Ruth, S. M., Rozijn, M., Koot, A., Perez-Garcia, R., van der Kamp, H., \& Codony, R. (2010). Authentication of feeding fats: Classification of animal fats, fish oils and recycled cooking oils. Animal Feed Science and Technology, 155(1), 65-73.

[29] Risticevic, S., Carasek, E., \& Pawliszyn, J. (2008). Headspace solid-phase microextraction-gas chromatography-time-of-flight mass spectrometric methodology for geographical origin verification of coffee. Analytical Chimica Acta, 617-72.

[30] Oliveira, R. C. S., Oliveira, L. S., Franca, A. S., \& Augusti, R. (2009). Evaluation of the potential of SPME-GC-MS and chemometrics to detect adulteration of ground roasted coffee with roasted barley. Journal of Food Composition and analysis, 22(3), 257-261.

[31] Jiye, A., Surowiec, I., Fraser, P., Patel, R., Halket, J., \& Bramley, P. (2010). Metabolomic approach to the identification of robust markers for the detection of mechanically separated meat (MSM) in meat products. http://www.foodbase.org.uk/ results.php?f_report., FSA final technical report.

[32] Russell, V. J., Hold, G. L., Pryde, S. E., Rehbein, H., Quinteiro, J., Rey-Mendez, M., Sotelo, C. G., Perez-Martin, R. I., Santos, A. T., \& Rosa, C. (2000). Use of Restriction Fragment Length Polymorphism To Distinguish between Salmon Species. Journal of Agricultural and Food Chemistry, 48-2184.

[33] Calo-Mata, P., Sotelo, C. G., Pérez-Martín, R. I., Rehbein, H., Hold, G. L., Russel, V. J., Pryde, S., Quinteiro, J., Rey-Méndez, M., Rosa, C., \& Santos, A. T. (2003). Identification of gadoid fish species using DNA-based techniques. European Food Research and Technology, 217-259. 
[34] Bottero, M. T., Civera, T., Anastasio, A., Turi, R., \& Rosati, S. (2002). Identification of cow's milk in "buffalo" cheese by duplex polymerase chain reaction. Journal of Food Proteins, 65-362.

[35] Bonizzi, I., Feligini, M., Aleandri, R., \& Enne, G. (2006). Genetic traceability of the geographical origin of typical Italian water buffalo Mozzarella cheese: A preliminary approach. Journal of Applied Microbiology, 102(3), 667-673.

[36] Garrido-Delgado, R., Lopez-Vidal, S., Arce, L., \& Valcarcel, M. (2009). Differentiation and identification of white wine varieties by using electropherogram fingerprints obtained with CE. Journal of Separation Science, 32(21), 3809-3816.

[37] Maes, P., Monakhova, Y. B., Kuballa, T., Reusch, H., \& Lachenmeyer, D. W. (2012). Qualitative and Quantitative Control of Carbonated Cola Beverages Using ${ }^{1} \mathrm{H}$ NMR Spectroscopy. DOI: 10.1021/jf204777m.

[38] Alonso-Salces, R. M., Heberger, K., Moreno-Rojas, G. M., Bellan, M. G., Reniero, F., \& Guillou, C. (2010). Multivariate analysis of NMR fingerprint of the unsaponifiable fraction of virgin oliveoils for authentication purposes. Food Chemistry, 118(4), 956-965.

[39] Vigli, G., Philippidis, A., Spyros, C., \& Dais, P. (2003). Classification of Edible Oils by Employing ${ }^{31} \mathrm{P}$ and ${ }^{1} \mathrm{H}$ NMR Spectroscopy in Combination with Multivariate Statistical Analysis. A Proposal for the Detection of Seed Oil Adulteration in Virgin Olive Oils. DOI: 10.1021/jf030100z.

[40] Aursand, M., Standal, I. B., Prael, A., Mc Evoy, L., Irvine, J., \& Axelson, D. E. (2009). 13C NMR Pattern Recognition Techniques for the Classification of Atlantic Salmon (Salmo salar L.) According to Their Wild, Farmed, and Geographical Origin. DOI: $10.1021 /$ jf8039268.

[41] Standal, G. B., Axelson, D. E., \& Aursand, M. (2010). ${ }^{13} \mathrm{C}$ NMR as a tool for authentication of different gadoid fish species with emphasis on phospholipid profiles. Food Chemistry, 121(2), 608-615.

[42] Karoui, R., Kemps, B., Bamelis, F., De Ketelaere, B., Decuypere, E., \& De Baerdemaeker, J. (2000). Development of a rapid method based on front face fluorescence spectroscopy for the monitoring of egg freshness: evolution of thick and thin egg albumens. DOI: 10.1007/s00217-005-0204-x.

[43] Karoui, R., Kemps, B., Bamelis, F., De Ketelaere, B., Merten, K., Schoonheydt, R., Decuypere, E., \& De Baerdemaeker, J. (2006). Development of a rapid method based on front-face fluorescence spectroscopy for the monitoring of egg freshness: 2 -evolution of egg yolk. DOI: 10.1007/s00217-005-0179-7.

[44] Karoui, R., Thomas, E., \& Dufour, E. (2006). Utilisation of a rapid technique based on front-face fluorescence spectroscopy for differentiating between fresh and frozenthawed fish fillets. . Food Research International; ., 39-349. 
[45] Guimet, F., Ferre, J., \& Boque, R. (2005). Rapid detection of olive-pomace oil adulteration in extra virgin olive oils from the protected denomination of origin"Siurana using excitation-emission fluorescence spectroscopy and three-way methods of analysis. Analytica Chimica Acta, 544-143.

[46] Poulli, K. I., Mousdis, G. A., \& Georgiou, C. A. (2005). Classification of edible and lampante virgin olive oil based on synchronous fluorescence and total luminescence spectroscopy. DOI: 10.1016/j.aca.2005.03.061.

[47] Dankowska, A., \& Malecka, M. (2009). Application of synchronous fluorescence spectroscopy for determination of extra virgin olive oil adulteration. DOI: 10.1002/ejlt. 200800295.

[48] Twomey, M., Downey, G., \& Mc Nulty, P. B. (1995). The potential of NIR spectroscopy for the detection of the adulteration of orange juice. .Journal of the Science of Food and Agriculture , 67-77.

[49] Contal, L., Leon, V., \& Downey, G. (2002). Detection and quantification of apple adulteration in strawberry and raspberry purees using visible and near infrared spectroscopy. DOI: 10.1255/jnirs.345.

[50] Vardin, H., Tay, A., Ozen, B., \& Mauer, L. (2008). Authentication of pomegranate juice concentrate using FTIR spectroscopy and chemometrics. Food Chemistry, 108-742.

[51] Fugel, R., Carle, R., \& Schieber, A. (2005). Quality and authenticity control of fruit purees, fruit preparations and jams: a review. Trends in Food Science and Technology, 16-433.

[52] Paradkar, M. M., Sivakesava, S., \& Irudayaraj, J. (2002). Discrimination and classification of adulterants in maple syrup with the use of infrared spectroscopic techniques. DOI: $10.1002 /$ jsfa.1332.

[53] Sivakesava, S., \& Irudayaraj, J. (2002). Classification of simple and complex sugar adulterants in honey by midinfrared spectroscopy. International Journal of Food Science and Technology, 37-351.

[54] Syahariza, Z. A., Che Man, Y. B., Selamat, J., \& Bakar, J. (2005). Detection of lard adulteration in cake formulation by Fourier transform infared (FTIR) spectroscopy. Food Chemistry, 92-365.

[55] Che Man, Y. B., Syahariza, Z. A., Mirghani, M. E. S., Jinap, S., \& Bakar, J. (2005). Analysis of potential lard adulteration in chocolate and chocolate products using Fourier transform infrared spectroscopy. Food Chemistry, 90-815.

[56] Rohman, A., \& Che Man, Y. B. (2010). Fourier transform infrared (FTIR) spectroscopy for analysis of extra virgin olive oil adulterated with palm oil. Food Research International, 43(3), 886-892. 
[57] Lerma-Garcia, M. J., Ramis-Ramos, G., Herreo-Martinez, J. M., \& Simo-Alfonso, J. M. (2010). Authentication of extravirginoliveoils by Fourier-transform infrared spectroscopy. Food Chemistry, 118(1), 78-83.

[58] Koca, N., Kocaoglu-Vurma, N. A., Harper, W. J., \& Rodriguez-Saona, L. E. (2010). Application of temperature controlled attenuated total reflectance-mid-infared (ATRMIR) spectroscopy for rapid estimation of butter adulteration. Food Chemistry, 121-778.

[59] Downey, G., \& Beauchene, D. (1997). Discrimination between fresh and frozen-thenthawed beef $\mathrm{m}$. Longissimus dorsi by combined visible-near infrared reflectance spectroscopy: A feasibility study. Meat Science, 45-353.

[60] Marina, A. M., Che Man, Y. B., Nazimah, S. A. H., \& Amin, I. (2009). Monitoring the adulteration of virgin coconut oil by selected vegetable oils using differential scanning calorimetry. Journal of Lipid Science, 16(1), 50-61.

[61] Smiddy, M. A., Huppertz, T., \& van Ruth, S. (2012). Triacylglycerol and melting profiles of milk fat from several species. International Dairy journal, 24(2), 64-69.

[62] Nurjuliana, N., Che Man, Y. B., \& Mat Hashim, D. (2011). Analysis of Lard's Aroma by an Electronic Nose for Rapid Halal Authentication. Journal of the American Oil Chemical Society, 88-75.

[63] Oliveros, M. C. C., Pavon, J. L. P., Pinto, C. G., Laespada, M. E. F., Cordero, B. M., \& Forina, M. (2002). Electronic nose based on metal oxide semiconductor sensors as a fast alternative for the detection of adulteration of virgin olive oils. Analytica Chimica Acta, 459-219.

[64] Penza, M., \& Cassano, G. (2004). Chemometric characterization of Italian wines by thin-film multisensors array and artificial neural networks. Food Chemistry, 86-283.

[65] Pillonel, L., Ampuero, S., Tabacchi, R., \& Bosset, J. O. (2003). Analytical methods for the determination of the geographic origin of Emmental cheese: Volatile compounds by GC/MS-FID and electronic nose. European Food Research and Technology, 216-179.

[66] Eide, I., \& Zahlsen, K. (2007). Chemical Fingerprinting of Biodiesel Using Electrospray Mass Spectrometry and Chemometrics: Characterization, Discrimination, Identification, and Quantification in Petrodiesel. Energy \& Fuels, 21-3702.

[67] Schale, S. P., Le , T. M., \& Pierce, K. M. (2012). Predicting feedstock and percent composition for blends of biodiesel with conventional diesel using chemometrics and gas chromatography-mass spectrometry. Talanta, http://dx.doi.org/10.1016/j.talanta. 2012.03.050.

[68] Corgozinho, C. N. C., Pasa, V. N. D., \& Barbeira, P. J. S. (2008). Determination of residual oil in diesel oil by spectrofluorimetric and chemometric analysis. Talanta, 76(2), 479-484.

[69] Haddad, R., Catharino, R. R., Marques, L. A., \& Eberlin, M. N. (2008). Perfume fingerprinting by easy ambient sonic-spray ionization mass spectrometry: nearly instan- 
taneous typification and counterfeit detection. Rapid Communication in Mass Spectrometry, 22-3662.

[70] Marques, L. A., Catharino, R. R., Bruns, R. E., \& Eberlin, M. N. (2006). Electrospray ionization mass spectrometry fingerprinting of perfumes: rapid classification and counterfeit detection. Rapid Communication in Mass Spectrometry, 20(24), 3654-3658.

[71] Cano, M., Borrego, V., Roales, J., Idigoras, J., Lopes-Costa, T., Mendoza, P., \& Pedrosa, J. M. (2011). Rapid discrimination and counterfeit detection of perfumes by an electronic olfactory system. Sensors and Actuators B: Chemical, 156(1), 319-324.

[72] Souza, R. C. Z., Eiras, M. M., Cabral, E. C., Barata, L. E. S., Eberlin, M. N., \& Catharino, R. R. (2011). The Famous Amazonian Rosewood Essential Oil: Characterization and Adulteration Monitoring by Electrospray Ionization Mass Spectrometry Fingerprinting. Analytical Letters, 44-2417.

[73] Cabral, E.C., Simas, R.C., Santos, V.G., Queiroga, C.L., da Cunha, V.S., de Sa, G.F., Daroda, M.J., \& Eberlin, M.N. (2012). Wood typification by Venturi easy ambient sonic spray ionization mass spectrometry: the case of the endangered Mahogany tree. Journal of Mass Spectrometry, DOI 10.1002/jms.2016.

[74] Cordeiro, J. R., Martinez, M. I. V., Li, R. W. C., Cardoso, A. P., Nunes, L. C., Krug, F. J., Taixao, T. R. L. C., Nomura, C. S., \& Gruber, J. (2012). Identification of Four Wood Species by an Electronic Nose and by LIBS International. Journal of Electrochemistry, doi:10.1155/2012/563939.

[75] Laasonen, M., Harmia-Pulkkinen, T., Simard, C. L., Michiels, E., Rasanen, M., \& Vuorela, H. (2002). Fast identification of Echinacea purpurea dried roots using nearinfrared spectroscopy. Analytical Chemistry, 74-2493. 

Chapter 7

\title{
Principle of Meat Aroma Flavors and Future Prospect
}

\author{
Hoa Van Ba, Inho Hwang, Dawoon Jeong and \\ Amna Touseef \\ Additional information is available at the end of the chapter \\ http://dx.doi.org/10.5772/51110
}

\section{Introduction}

The population growth fact of the world has been much quickly increasing through the years. As reported by the United Nations Population Fund (UNFPA) the estimated world population of 6.1 billion in the year 2000 and reached to 7 billion in the year 2011, increased 0.9 billion people only after 10 years. The population increases always proportionally accompany to the consumption demands in which including foods. Calculating the global meat consumption only and based on the data collected from IFPRI/ FAO/ILRI by Delgado et al (1999) [1] suggested that global production and consumption of meat will continue to raise from 233 million metric tons in the year 2000 to 300 million metric tons in 2020. On the other hand, income growth of people in most of the countries especially in the developed countries has been significantly increasing in the recent years. Combination of the large populations together with a high-income that will give a big pressure for the food producers in general and meat producers in particular. As a consequence, higher income growth in countries has led to an increase in living standards and changes in consumer diets to include a higher proportion of meat and meat products. While, productivity and provision of meats on the markets has been limited and rising costs of production resulting in not keeping pace with the strong growth in demand, that has caused a rise in meat prices.

Although, a strong demand for meat amounts but consumers are getting quite fastidious to choose meat and meat products since consumer's preference for meat buying is strongly based on quality, freshness and hygiene. Quality factors are very important in the meat purchasing behavior of consumers including marbling (intramuscular fat tissues), texture, color, tenderness and especially flavor characteristics.

Aroma flavor characteristics of cooked meat in particular play the most important level in eating quality of meat, acceptance and preference by consumers. The aroma flavor charac- 
teristics of cooked meats are derived from volatile flavor components which derive from thermally induced reactions occurring during heating via the four pathways including (1) Maillard reaction of amino acid or peptides with reducing sugars, (2) Lipid oxidation, (3) interaction between Maillard reaction products with lipid-oxidized products and (4) vitamin degradation during cooking [2]. Aroma flavor is perceived through the nostrils (orthonasal aroma) it gives the first impression of a certain food. When the food is placed in the mouth, the volatile flavor compounds will be transferred through the pharynx to the olfactory receptors (retronasal aroma). It has been reported that flavors together with other sensory attributes such as tenderness and juiciness are specially considered the most important criterion of acceptability and the palatability of meat that affects consumer's purchasing decisions [3,4]. It has been well known that all volatile flavor components are organic and they have low molecular weight [5]. The chemical structures of volatile flavor classes are varied widely including aldehydes, ketones, hydrocarbons, pyrazines, acids, esters, alcohols, nitrogen and sulfur-containing compounds and other heterocyclic compounds as well. Due to the differences in chemical structures therefore their volatility is also quite different.

Many factors have been found to be as influences on the aroma flavors of cooked meat. Rabe et al (2003) [6] found that among all food constituents, lipids generally have the greatest influence on production of aroma flavor components, as they not only reduce the vapour pressure of most flavor compounds. Otherwise, Kinsella (1990) [7] showed that aroma compounds are more lipophilic than hydrophilic therefore fats act as a solvents for aroma compounds reducing their volatility. In addition to these effects, other factors such as diets, breed, sex, chiller ageing, meat $\mathrm{pH}$, cooking conditions which all also affect the flavor $[8,9,40,11]$

With the crucial importance of aroma flavor of meat for the acceptance and preference of consumers and as well as the factors influencing the generation of aroma flavor compounds as mentioned above, the present chapter aims to highlight the basic information regarding aroma flavor components in terms of mechanisms of formation pathways; current techniques being used for detection; factors that affect aroma flavors; and final ideas and as well as suggestions are also given out to improve flavor quality attributes according to criterion of acceptability, satisfaction and the palatability for consumer.

\section{Meat aroma flavor}

\subsection{The importance of volatile flavor compounds in contributing to the flavor characteristics of cooked meat}

Flavor characteristics of cooked meat are directly detected by the nose (i.e., olfactory receptors) before and during chewing. Raw meat has little aroma and only blood-like taste, meat develops its aroma flavor characteristics during cooking as the result of complex interaction of precursors derived from both the lean and fat compositions of meat generating volatile flavor compounds that contribute to meat flavor [12]. To date, approximately thousands of volatile flavor compounds have been detected and identified in cooked meat. There is a large number of these compounds contributing to the flavor characteristics of cooked meat have been identified in previous works [13, 14, 15, 16, 17, 18, 19]. 
As mentioned above, regarding the chemical structures of volatile flavor classes, among that the heterocyclic compounds especially those containing sulfur are the important flavor compounds produced in the Maillard reaction providing savory, meaty, roasty and boiled flavor characteristics. While, lipid-degraded- compounds which give 'fatty' aromas to cooked meat and compounds which determine some of the aroma flavor differences between meats from different species [20]. The individual volatile compounds have been found to determine distinct aroma flavors of cooked meat represent; dimethylsulfide, 2-butanone, ethyl acetate, 2- and 3-methylbutanal, 2-heptanone, dimethyl trisulphide and nonanal were detected as key flavor compounds of cooked Irish Angus beef, while methional, 2,4-nonadienal and bezothiazole were characterized as meaty, oily notes in cooked Belgian Blue, Limousin and Aberdeen Angus beefs [21]. Kerscher \& Grosch, (1997) [22] reported that 2-furfurylthiol, 4hydroxy-2,5-dimethyl-3(2H)-furanone and 2-methyl-3-furanthiol were the most important odorants of boiled beef. 2-ethyl-3,5-dimethyl pyrazine and 2,3-diethyl-5-methylpyrazine possess roasty, caramel-like, burnt and earthy notes of roasted beef [23]. Other carbonyl compounds such as methional, E-2-undecenal, E-2-dodecenal, decanal, heptanal and 2methylbutanal also were found to be associated with roasty, sweet, fruity and fatty odor notes of cooked beef [14,17]. Also, a great number of studies considered on the objective volatile flavor components in cooked pork, chicken, lamb, ham and etc... have been documented over the last years [24, 25, 26]. In fact, although thousands of volatile compounds identified but not all of them are important because their high odor detection threshold, only some of them play a significant role in the overall aroma flavor characteristics of cooked meat. An aroma flavor compound with its distinct odor note can be defined as its flavor dilution factor indicating that at the lowest concentration at which the compound still can be detected by the sense of smell. Some represent volatile flavors active-compounds have been detected in cooked meats by using gas chromatography-olfactometry technique (GC-O) are showed in Table 1.

\begin{tabular}{ll}
\hline Compound name & Aroma flavor characteristics \\
\hline Aldehydes & Cooked potato, meaty \\
\hline Methional & Fatty \\
\hline E,2-nonenal & Fatty \\
\hline E,E,2,4-decadienal & Sweet, honey \\
\hline Benzenacetaldehyde & Fatty \\
\hline Decanal & Sweet, fruity, like aldehydes, roasty \\
\hline Heptanal & Fruity, fatty, sweet, oil \\
\hline Nonanal & Sweet, fatty, green \\
\hline Undecanal & Sweet, pungent, green \\
\hline E,2-heptenal & Fatty \\
\hline E,2-heptenal & Fatty
\end{tabular}




\begin{tabular}{|c|c|}
\hline Hexanal & Green, fatty \\
\hline E,2-hexenal & Green \\
\hline E,Z,2,6-nonadienal & Cucumber \\
\hline Undecanal & Sweet, pungent, green \\
\hline 2-methylbutanal & Pungent, sweet, roasty \\
\hline E,2-undecenal & Sweet, fruity, fatty \\
\hline 2,E-dodecenal & Sweet, fruity, roasty, pungent \\
\hline Ethanol & Grilled (weak), acetaldehyde-like \\
\hline 3-methylbutanal & Meaty, fish, rotten, aldehyde,valeric acid, fatty \\
\hline Octanal & Green, lemon, citrus, aldehyde \\
\hline $\mathrm{E}, \mathrm{E}, 2,4$-heptadienal & Aldehyde, green, broth, spicy \\
\hline Propanal & caramel, sweet, alcoholic, "cooked", broth, spicy \\
\hline Butanal & smoky, fish, amylic, aldehyde-enal or dienal \\
\hline \multicolumn{2}{|l|}{ Ketones } \\
\hline 2-octanone & Fruity, musty \\
\hline 2-decanone & Fruity, musty \\
\hline 2-dodecanone & Fruity, musty \\
\hline 1-octen-3-one & fresh, mushrooms, pungent, rubbery \\
\hline 3-octanone & Fruity, nutty, moldy, fatty, earthy \\
\hline $\begin{array}{l}\text { 2,5-dimethyl-4-hydroxy-3(2H)- } \\
\text { furanone }\end{array}$ & Roasted almonds, sweet \\
\hline \multicolumn{2}{|c|}{ 4,5-dihydro-5-propyl-2(3H)-furanone Fruity, fatty, sweet, pungent, roasty } \\
\hline 2,3-butanedione & Sweet, buttery \\
\hline 2-heptanone & Citrus grapefruit, limonene, floral, cheese \\
\hline 2,3-pentanedione & buttery, lemon-like, sweet, fruity \\
\hline 2-nonanone & Hot milk, soap, green, fruity, floral \\
\hline 3-octen-2-one & Nut, crushed bug, earthy, spicy, sweet, mushroom, \\
\hline 6-Methyl 2-heptanone & Cloves, menthol \\
\hline 2-undecanone & Fruity \\
\hline 2,2,6-Trimethylcyclohexanone & Mint, acetone \\
\hline \multicolumn{2}{|l|}{ Alcohols } \\
\hline 1-octen-3-ol & Mushroom \\
\hline Cyclobutanol & Roasted \\
\hline
\end{tabular}




\begin{tabular}{|c|c|}
\hline 1-heptanol & Fragrant, woody, oily, green, fatty, winey, sap \\
\hline 1-hexanol & Woody, cut grass, chemical-winey, fatty, fruity \\
\hline 2-Ethyl 1-hexanol & Resin, flower, green \\
\hline 1-octanol & Penetrating aromatic odor, fatty, waxy, citrus, oily, \\
\hline 2-Octen-1-ol & Green citrus \\
\hline 1-pentanol & Mild odor, fuel oil, fruit, balsamic \\
\hline Propanol & Alcoholic \\
\hline \multicolumn{2}{|l|}{ Hydrocarbons } \\
\hline Ethenylbenzene & Pungent, aromatic, fragrant, roasty \\
\hline 1-undecen & Fatty, burnt, nutty, rubbery \\
\hline Hexane & Faint peculiar odor \\
\hline (Z)-3-Octene & Fruity, old apples \\
\hline Pentane & Very slight warmed-over flavor, oxidized \\
\hline Styrene & Penetrating odor, sweet smell \\
\hline Tridecane & Alkane \\
\hline Tetradecane & Alkane \\
\hline Ethenylbenzene & Aromatic, fragrant, roasty \\
\hline \multicolumn{2}{|l|}{ Pyrazines } \\
\hline 2-ethyl-3,5-dimethylpyrazin & Burnt, fragrant, meaty, green \\
\hline 2-ethenyl-3,6(5)-dimethylpyrazine & Sweet, cooked rice, fatty \\
\hline 2-ethyl-3,6-dimethylpyrazine & Burnt, roasty \\
\hline 2,3-diethyl-5-methylpyrazine & meaty, roasty, fragrant, sweet \\
\hline 2,5-dimethylpyrazine & Fried rice, popcorn, pungent, green \\
\hline 2-ethenyl-5(6)-methylpyrazine & Roasty break-like, cooked rice, coffee-like \\
\hline 2,5-dimethylpyrazine & Fried rice, popcorn, pungent, green \\
\hline 2-ethyl-5-methylpyrazine & Fruity, sweet, pungent \\
\hline 2-ethenyl-5(6)-methylpyrazine & Smoky, roasty, break-like, cooked rice, popcorn \\
\hline 2-ethyl-3,6-dimethylpyrazine & Burnt, pungent, roasty \\
\hline 2-ethenyl-3,6(5)-dimethylpyrazine & Pungent, sweet, cooked rice, fatty \\
\hline 2,3-diethyl-5-methylpyrazine & Meaty, roasty, fragrant, sweet \\
\hline 2-isopentyl-3,6-dimethylpyrazine & Sweet, fragrant, fatty, fruity, pungent \\
\hline
\end{tabular}




\begin{tabular}{|c|c|}
\hline 2-acetyl-1-pyrroline & Roasted, sweet \\
\hline 2-formyl-5-methylthiophene & Sulfurous \\
\hline 2-methyl-3-furanthiol & Meaty, sweet, sulfurous \\
\hline Benzylthiol & Sulphurous \\
\hline 2,4-dimethylthiazole & Rubber y, moldy, fruity, pungent \\
\hline 2-acetylthiazole & Roasted \\
\hline Dimethyltrisulfide & Fragrant, musty, roasty, rubbery \\
\hline 2-acethylthiophene & Sulphurous, sweet \\
\hline Bis(2-methyl-3-furyl)disulfide & Meaty-like \\
\hline Benzothiazole & Metallic \\
\hline Dimethyldisulfide & Moldy, pungent, rubbery, onion-like \\
\hline 2,4-dimethylthiazole & Rubbery, moldy, fruity, pungent \\
\hline 4,5-dimethylthiazole & Smoky, roasty, fragrant, nutty \\
\hline 2-methylchinoxaline & Aromatic, roasted, nutty, sweet, fruity, fatty \\
\hline 3-mercapto-2-butanone & Fried onion, sulfury, cooked meat \\
\hline 2-mercapto-3-pentanone & Brothy, mashed potatoes meaty, roast meat \\
\hline 2-[(methyldithio)methyl]furan & Brothy, spices, roast, fatty \\
\hline
\end{tabular}

Table 1. The representative volatile flavor compounds with their aroma flavor characteristics found in cooked meat. [References: 13, 14, 20, 15, 27]

\subsection{Precursors of meat flavor}

Earlier studies on meat flavor, researchers recognized that the low molecular weight, watersoluble compounds and fats in meat constituents are the most important precursor of aroma flavor characteristics of cooked meat [28, 29]. The flavor precursor of meat namely, free sugars, free amino acids, peptides, vitamin, sugar phosphate, nucleotide-bound sugars and nucleotides [30,31,32,33], all of them are able to either participate the Maillard reaction or oxidation/ degradation and interaction on heating to generate volatile flavor compounds then create the final aroma flavor characteristics of cooked meat. It is suggested that these precursor components found to contribute to the development of meaty flavor, while the adipose tissues and intramuscular fat not only occupy an important role in development of flavor characteristics of cooked meat but also contribute to the characteristic-specific species flavors. This means that the distinct flavor characteristics between the meats from different species are due to the intramuscular fat content and not from water-soluble precursor compounds. The details on flavor precursors of meat found in the past years are showed in Table 2. However, researchers found that the roles of these flavor precursors in the development of flavor characteristics 
of cooked meat are not similar. Macey et al (1964) [28] found some sugars present in beef such as glucose, fructose, mannose and ribose, in that ribose was the most heat-labile sugar among these whereas fructose was the most stable. Among the amino acids present in meat, systein and systine are two sulfur-containing amino acids, the reaction of these with other sugars lead to formation of many sulfur-containing flavor compounds [34], while the reaction of other nonsulfur containing amino acids with sugars dominated by the nitrogen-containing products such as pyrazines [72]. In the recent years, researchers have found that the flavor precursor components in meats are influenced by several factors. Koutsidis et al (2008) [31] indicated that diets significantly affected the reducing sugars in beef longissimus lumborum muscle, higher total reducing sugars was obtained in beef from concentrate feeding group compared to the grass silage feeding group whereas beef from cattle fed with grass silage had higher level of free amino acids. When the beef was chiller aged for several days at chilling condition resulted in several times increase in free sugars such as ribose, free amino acids also increased with conditioning especially phenylalanine, methionine, lysine, leucine and isoleucine were the amino acids showing the greatest increase with conditioning time [32]. Meinert et al (2009) [35] have found that feeding, fasting and post-mortem ageing factors significantly influenced the concentration of flavor precursors of beef longissimus dorsi muscle. Additionally, the recent works also showed that fat-supplemented diets had large effect on the fatty acid compositions, for instance, dietary linseed oil and soybean oil significantly increased the contents of C18:3 and C18:2 in the neutral lipids and phospholipids in both longissimus and biceps brachii muscles [36], and subsequently influence the volatile flavor compounds of cooked beef $[19,25,37]$.

\begin{tabular}{|c|c|c|}
\hline Flavor precursors & Names in detail & Reference \\
\hline Free amino acids & $\begin{array}{l}\text { Systine; systeine; glycine; lysine; alanine; valine; isoleucine } \\
\text { leucine; threonine; serine; proline; asparagines; aspartic } \\
\text { acid; methionine; glutamic acid; phenylalanine; } \\
\text { glutamine; ornithine; histidine; tyrosine; tryptophan; } \\
\text { arginine. }\end{array}$ & $\begin{array}{c}; 38,39,40, \\
31,3232]\end{array}$ \\
\hline Reducing sugars & $\begin{array}{l}\text { Ribose; glucose; xylose; starch; mannose; fructose; } \\
\text { maltose; mannose 6-phosphate, glucose 6-phosphate; } \\
\text { fructose 6-phosphate; ribose 6-phosphate. }\end{array}$ & $\begin{array}{l}{[38,39,72,} \\
41,31,32]\end{array}$ \\
\hline Fats/ lipids & $\begin{array}{l}\text { Triglycerides and phospholipids } \\
\text { Oleic acid (C18:1n-9) } \\
\text { Linoleic acid (C18:2n-6) } \\
\text { Linolenic acid (C18:3n-3) and etc. }\end{array}$ & $\begin{array}{l}{[42,19,} \\
43,34]\end{array}$ \\
\hline Vitamin & Thiamin & {$[33,44]$} \\
\hline $\begin{array}{l}\text { Nucleotides and } \\
\text { peptides }\end{array}$ & $\begin{array}{l}\text { Glutathione; carnosine inosine; inosine monophosphate; } \\
\text { inosine 5'-monophosphate; guanosine 5- } \\
\text { monophosphate; creatine; creatinine; Hypoxanthine and } \\
\text { etc. }\end{array}$ & $\begin{array}{l}{[45,44,} \\
31,32]\end{array}$ \\
\hline
\end{tabular}

Table 2. The representative precursors of meat flavor. 


\subsection{Pathways for the formation of volatile flavor compounds}

\subsubsection{Maillard reaction}

Maillard reaction, a non-enzymatic browning which plays an important role in generation of volatile flavor compounds and appearances of the cooked foods, it is due to most of important volatile flavor compounds found in cooked foods are originated from this reaction. Otherwise, Maillard reaction also can produce antioxidative components and toxicological implications as well. However, in the present chapter we are focusing on the Maillard reaction in relation to aroma flavor characteristics, particularly the formation of volatile flavor compounds in cooked meat. Maillard reaction was firstly mentioned in the early time, 1912 by Maillard [46] since he wanted to investigate the browning reaction between glucose and glycine. After that many studies focused on determining the fundaments and mechanisms of this reaction $[47,48,49]$.

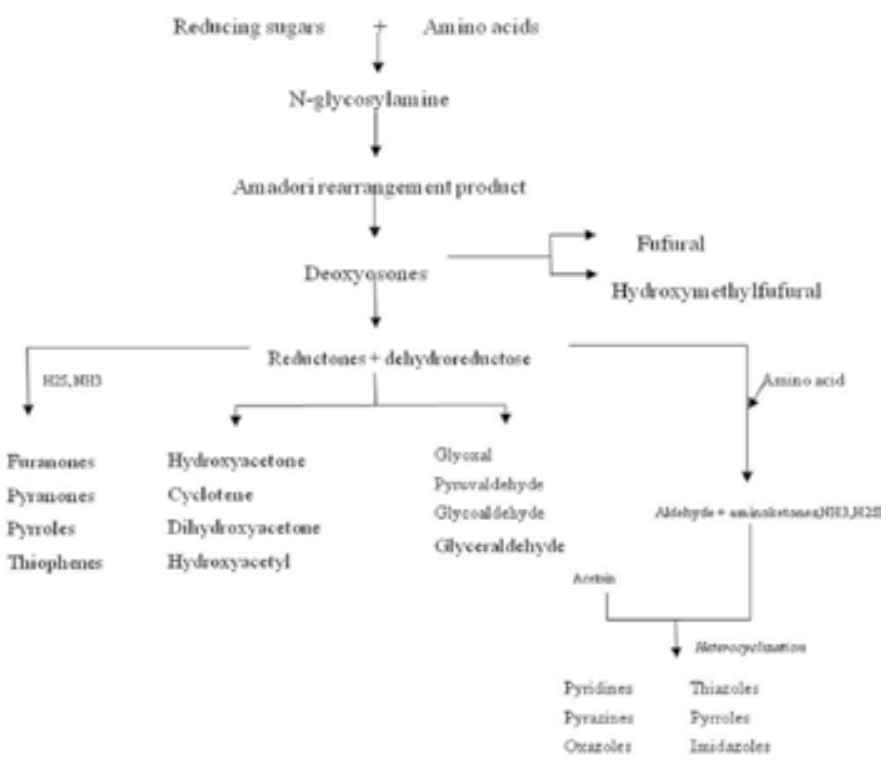

Figure 1. General stages of Maillard reaction showing the formations of flavor compounds (based on van Boekel, 2006) [51].

The Maillard reaction is taken placed with the participation of reducing sugars (e.g., ribose, glucose) and free amino compounds (e.g., amino acids, amines, peptides, proteins, ammonia) at certain heating condition to produce the Maillard products, and usually this reaction is divided into three main stages. In which the firstly initial stage starts with a condensation between a reducing sugar and an amino group, the loss of water from this molecule produces an amine that is able to cyclise resulting in formation of an N-glycosylamine (a sugar attached to $\mathrm{NR}_{2}$ group) or called Amadori product. The next intermediate stage involves the rearrangement and decomposition of the Amadori product to release amino group and sugar fragmentation. The final stage of Maillard reaction is leading to dehydration, fragmenta- 
tion, polymeration and cyclization reactions. A general scheme of the Maillard reaction is given in Figure 1.

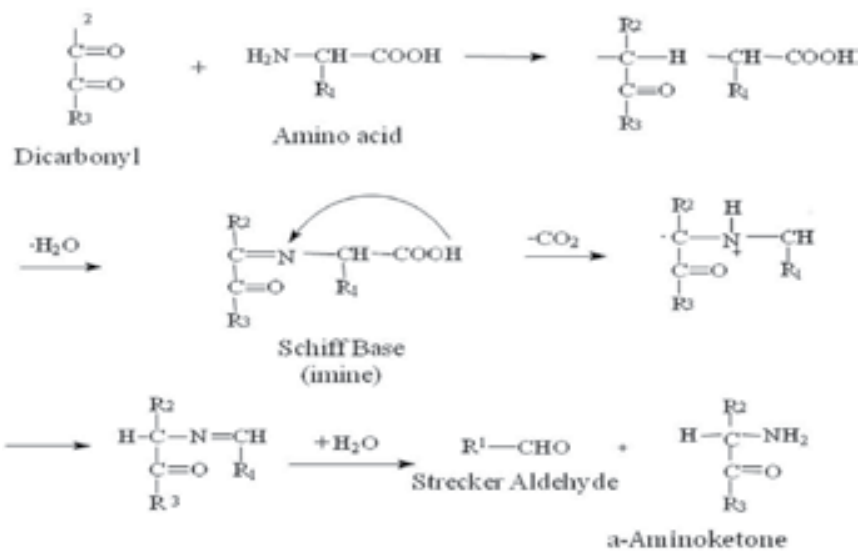

Figure 2. Strecker degradation mechanisms, a part of Maillard reaction

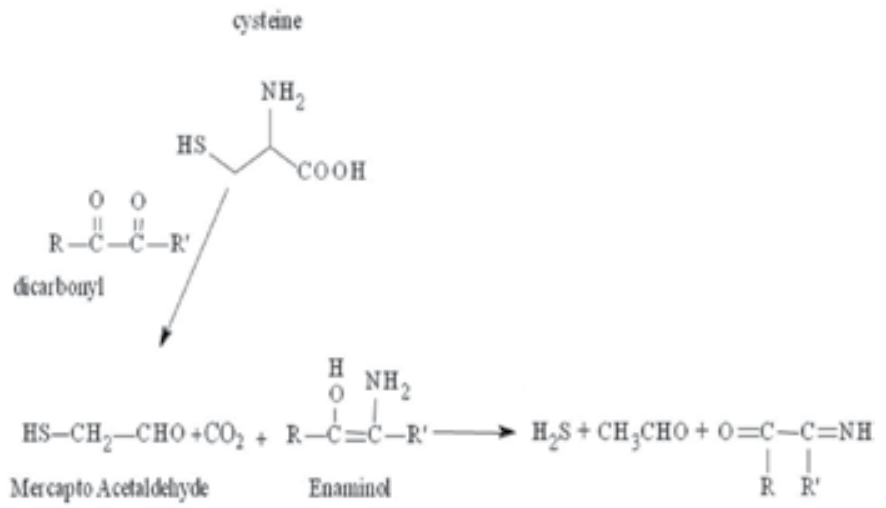

Figure 3. The formation of $\mathrm{H}_{2} \mathrm{~S}$ from the Strecker degradation of cysteine

Among events occurring in the Maillard reaction, Strecker degradation is one of the quite important events, in which amino acids are undergone degradation processes (oxidative deamination and decarboxylation) in the presence of a dicarbonyls compound formed from Maillard reaction. The Strecker degradation processes lead to formation of aldehydes (e.g., fufural) and aminoketone (Figure 2). Especially the other important intermediate products such as $\mathrm{H}_{2} \mathrm{~S}, \mathrm{NH}_{3}$, etc are also formed from the Strecker degradation by sulphur-containing amino acids such as cystein and systine (Figure 3); all of these intermediate products can further react with other compounds or with each other to produce low and high molecular weight end flavor compounds. 


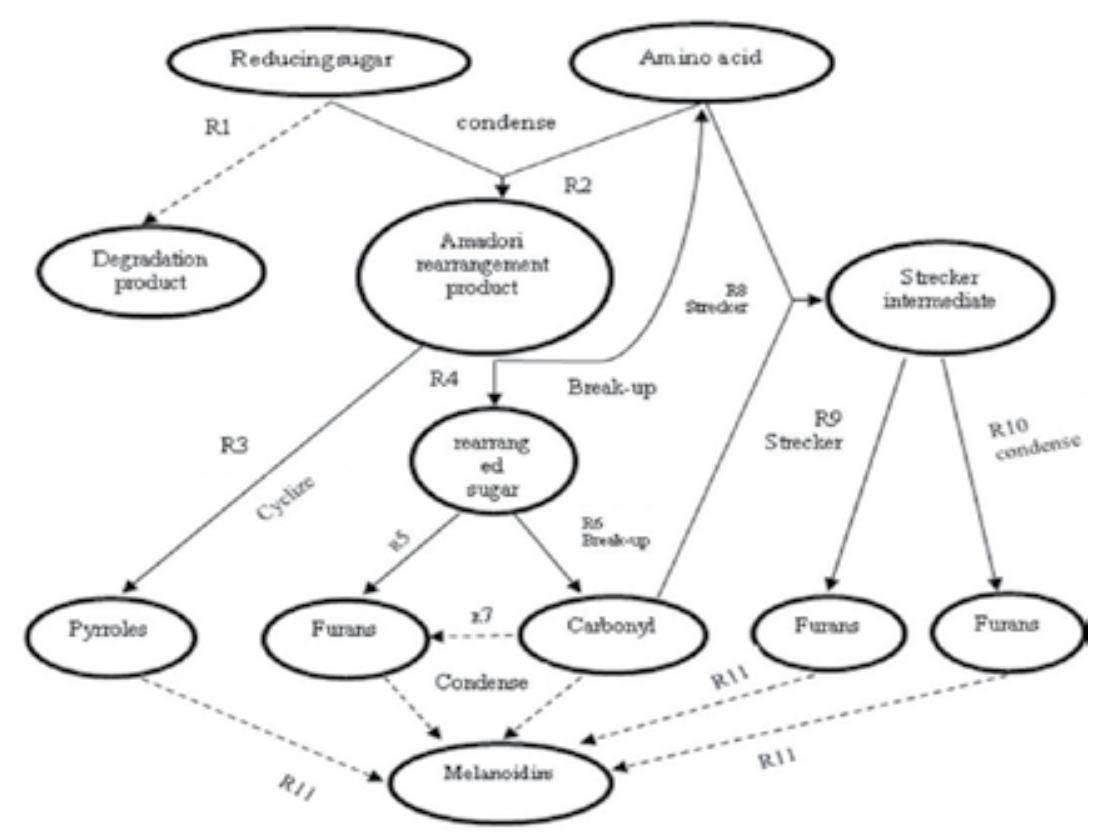

Figure 4. Kinetic scheme of flavor formation by Maillard reaction (Jousse et al., 2002) [50]

\begin{tabular}{lll}
\hline Flavor class & Characterized Flavor/aroma notes & Remark \\
\hline Pyrazines & $\begin{array}{l}\text { Cooked, roasted, toasted, baked } \\
\text { cereals }\end{array}$ & \\
\hline Alkylpyrazines & Nutty, roasted & Unpleasant flavor \\
\hline Alkylpyridines & Green, bitter, astringent, burnt & \\
\hline Acetylpyridines & Caracker-like & \\
\hline Pyrroles & Cereal -like & \\
\hline Furan, furanones, & Sweet, burnt, pungent, caramel-like & \\
pyranone & & Formed from heated \\
\hline Oxazoles & Green, nutty, sweet & meat by the reaction of \\
\hline Thiophenes & Meaty & systein and ribose
\end{tabular}

Table 3. Some representative classes of flavor compounds formed from the Maillard reaction (based on van Boekel, 2006) [51].

The formation of volatile flavor compounds in the Maillard reaction largely depend on the reactants (e.g., the nature of reducing sugars and amino acids participated) and also the cat- 
alytic condition (e.g., heating temperature, moisture, $\mathrm{pH}$ ). For the type of reducing sugars and amino acids which determine the kinds of flavor compounds generated for instance, many sulfur-containing flavor compounds are formed from the Maillard reaction between systeine and ribose [34] whereas, the nitrogen-containing compounds (e.g., pyrazines) dominated in the Maillard reaction containing glucose and lysine [72]. Therefore, it should be noted that nature of reactants will require the kinds of Maillard products. For the catalytic condition of Maillard reaction, it usually influences the kinetics of flavor compound generation by Maillard reaction in that depending on each catalytic condition (temperature, $\mathrm{pH}$ and etc) will determine the yields and also kinds of Maillard products. The kinetic of flavor compound formation resembles the scheme in Figure 4 with 11 determining steps [50]. Based on the kinetic scheme it shows that there are many chemical classes of flavors are formed via the Maillard reaction, some of the representative classes associated with odor notes are showed in Table 3.

\subsubsection{Lipid oxidation and degradation}

Lipids and fatty acids play an important role in direct and indirect generating the volatile flavor compounds and some of them contributing to the aroma flavor characteristics of cooked meat. Therefore, the levels of fat contents and as well as fatty acids of meats should be concerned, and it has been reported that the fatty acids of meat are influenced by several factors but almost are the pre-harvest factors such as diets, feed regimes and breeds [52, 53, 54]. Based on our surveillance it seems that the fatty acid profiles significantly vary across the breeds even these breeds are fed with the same diets [55, 56, 57]. Both adipose tissue and intramuscular fat contents are constituted by fatty acids including saturated and unsaturated fatty acids which all are capable to get oxidized and degraded under a certain condition to create a prolific number of volatile flavor compounds [2]. Hundreds of volatile flavor compounds derived from lipid degradation have been found in cooked meat including aliphatic hydrocarbons, aldehydes, ketones, alcohols, carboxylic acids and esters. In general, the odor detection threshold values for the lipid-derived compounds are much higher than those for the sulfur and nitrogen-containing heterocyclic compounds which are formed from the water-soluble precursors via the Maillard reaction. Therefore, the aroma significance of many of these lipid-derived compounds is not as great as that for relatively low concentrations of the heterocyclic compounds. However, certain classes of compounds such as particular aldehydes included saturated and unsaturated aldehydes which containing from 6 to 10 carbons in the structures are major volatile components of all cooked meats and, therefore, they probably play an important part in meat aroma [20]. The oxidation of subcutaneous fat, adipose tissues and intramuscular fat occur in raw meat and continues under the catalysis of many factors such as metals, oxygen, light, heating and etc.

Among the oxidation-induced factors for instance, lights (e.g., ultraviolet) is thought to be thermodynamically capable of production of free radicals directly in lipids, the principles of light-absorbing groups of lipids are double bonds, peroxide bonds and carbonyls which subsequently under the other steps to generate volatiles. And other factors such as oxygen, lypoxygenase, metals and etc which all also affect the lipid oxidation however that is anoth- 
er concern, in the present work we only consider on the heat effect that similar to cooking condition to induce the oxidation and degradation of fatty acids in producing volatile flavor compounds of cooked meat. The degrees of heating temperatures have been reported to affect variously lipid oxidation, in that high heating temperatures (e.g., frying, roasting) can have highly sufficient energies to break the single bonds (e.g., $\mathrm{C}-\mathrm{C}$ or $\mathrm{C}-\mathrm{H}$ ) in the acyl back bonds to generate a lot of lipid alkyl radicals that participate the radical chain formation of oxidation [58]. Lower heating temperatures have lower energies which can break O-O bonds in traces of ROOH. Mottram (1985) [59] also stated that meat is cooked under boiled and lightly roasted conditions, lipid oxidation products dominated the detected compounds, and many of among them such as aldehydes, alcohols, ketones and lactones which have sufficiently low odor threshold to be contributors of meat aroma flavors.

Early work of Mottram et al (1982) [60] found that lipid has a considerable role in meat flavor, when the adipose tissue is added to lean meat does not affect the lipid-derived flavor compounds. A later study by Mottram and Edwards (1983) [42] found that the removal of intramuscular fats and phospholipids from beef caused marked differences in flavor compounds and sensory characteristics as well. So that the intramuscular fat contents (marbling fats) and membrane lipids are the main source of volatile flavor components and make species-specific flavors. However, it has been demonstrated that high levels of lipids especially polyunsaturated fatty acid contents (PUFA) cause undesirable aroma flavors due to their PUFA-derived products lower or inhibit the formation of some heterocyclic Maillard products [42]. This phenomenon has recently been elucidated by researchers when they used model systems. In the model systems containing systeine, ribose and lipid (e.g., lecithin or individual fatty acids) the concentrations of heterocyclic compounds and especially sulfurcontaining compounds were lower several times compared with the model system without lipid content [43, 38, 61, 34]. However, the interaction between the lipid-derived products with Maillard products to form volatile flavor components has been much considered in the previous studies and thought as the important pathway for formation of flavor compounds.

\subsubsection{Thiamin degradation}

Thiamin is considered as a source of meat flavor generated on heating. Researchers found that the thermal degradation of thiamin produces some ended and intermediate flavor compounds $[62,63]$. It was assumed that thermal degradation of thiamin is a quite complex reaction including various degradation pathways to produce interesting flavor compounds in which most of them contain one or more sulfur and/or nitrogen atoms, and many of them are heterocyclic structures. The thermal degradation of thiamin under the basic condition to produce several flavor compounds is illustrated in Figure 5.

It was reported that the primary products of thermally-degraded thiamin including 4-methyl-5-(2-hydroxyethyl)thiazole which subsequently responds for formation of thiazoles and other sulfur compounds such as 5-hydroxy-3-mercaptopentan-2-one which then gives some sulfur-containing compounds such as thiophenes and furans as well [62]. Heating temperature and $\mathrm{pH}$ conditions have been showed to affect the degradation products of thiamin. At pH 5.0 and 7.0 the 2-methyl-3furanthiol and bis (2-methyl3-furyl) disulfide (meaty aroma) 
and thiophenes were the dominant aroma volatile compounds. But the levels of these meaty compounds decrease when increasing $\mathrm{pH}$ to 9.0 [64]. Similarly, a recent study by Dreher et al (2003) [65] also showed that the most significant thiamin thermal degradation products in the model reaction of orange juice containing $0.024 \mathrm{mM}$ thiamin are 2-methyl-3-furanthiol and bis(2-methyl-3-furyl) disulfide produce intense meaty aromas. Otherwise, some other aroma-active compounds also were found such as 4, 5-dimethylthiazole (skunky, earthy), 3thiophenethiol (meaty, cooked), 2-methyl-4, 5-dihydro-3(2H)-thiophenone (sour-fruity, musty, green), 2-acethylthiophene (burnt), 2-formyl-5-methylthiophene (meaty), and 2-methyl-3(methyldithio) furan (meaty).

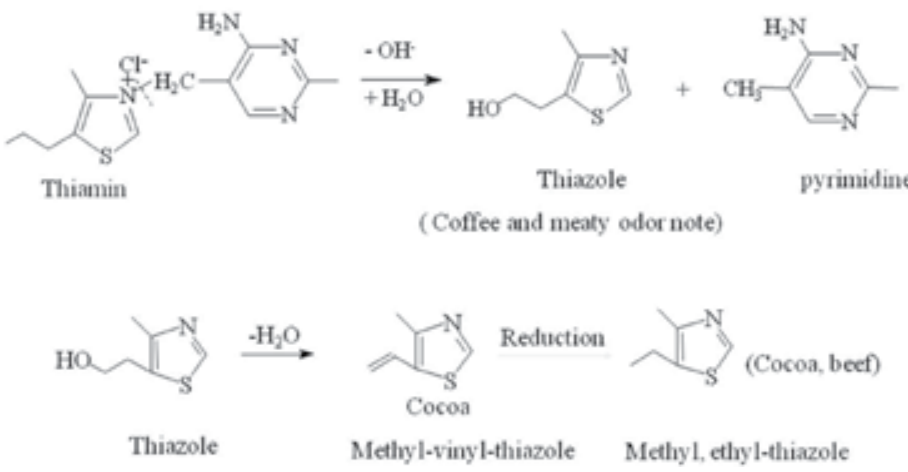

Figure 5. The thermal degradation of thiamin under basic condition

\subsubsection{Interaction between lipid-oxidized products with Maillard products}

The interaction between oxidized lipids and amino acids or proteins is very complex, in term of a consequence of the contribution of both lipid hydroperoxide and its secondaryoxidized products. This interaction may imply both the formation of physical complexes between the oxidized lipids and the amino acids or protein and the formation of various types of covalent bonds. Protein polymerizarion produced by reaction with peroxy free radicals generated during lipid peroxidation is known to occur during nonenzymatic browning [66, $67,68]$. However, in term of flavor study, the interaction between lipid-oxidized products (secondary products) with amino acids or proteins is the most concerned. Lipid-oxidized products are generic terms used to describe a mixture of aldehydes, alcohols, ketones and other products obtained by the decomposition of lipid hydroperoxides. Although it is not widely recognized, this decomposition does not necessarily imply the breakage of the lipid chain, and the formation of covalent bonds in the reaction between long chain oxidized lipids and amino acids and proteins has been described $[69,70]$. This is a consequence of the existence of fatty acids that produce a complex and diverse mixture of lipid oxidation products that are able to react with the different reactive protein residues.

In the Maillard reaction, amino acids can undergo the Strecker degradation process that subsequently generates some reactive radicals such as ammonia, hydrosulfide and etc which al- 
so are able to further react with the secondary oxidized products of lipid to produce volatile flavor compounds such as thiols, thiophenes, thiazoles and etc as showed in Figure 6.

The interaction between lipid and Maillard reaction have extensively been studied in a number of studies using model systems containing amino acids and sugars in the presence of lipid $[43,61,39,34]$. In these studies, systeine and ribose were used for Maillard reaction and in the presence of phospholipids from various sources including egg-yolk and beef. The reaction mixtures produced a lot of aroma volatiles which dominated by sulfur-containing components especially heterocyclics such as thiols and thiophenes. These studies also observed that the presence of phospholipids made a great reduction in amounts of these compounds. Famer and Mottram (1990) [61] also noted that beef-originated triglyceride has much less influence on amounts of heterocyclics than the phospholipids from beef do. The study also found that the addition of beef triglyceride to the Maillard reaction did not influence the sulfurous and rubbery aroma but when beef phospholipids were added resulting in higher meaty aroma note whereas the sulfurous notes were less. However, the Maillard reaction systems containing phospholipids usually had lower level of some meaty compounds especially 2-methyl-3-furanthiol this is due to the lipid limits generation of these compounds and only maintain theme at an optimum level in the reaction mixture.

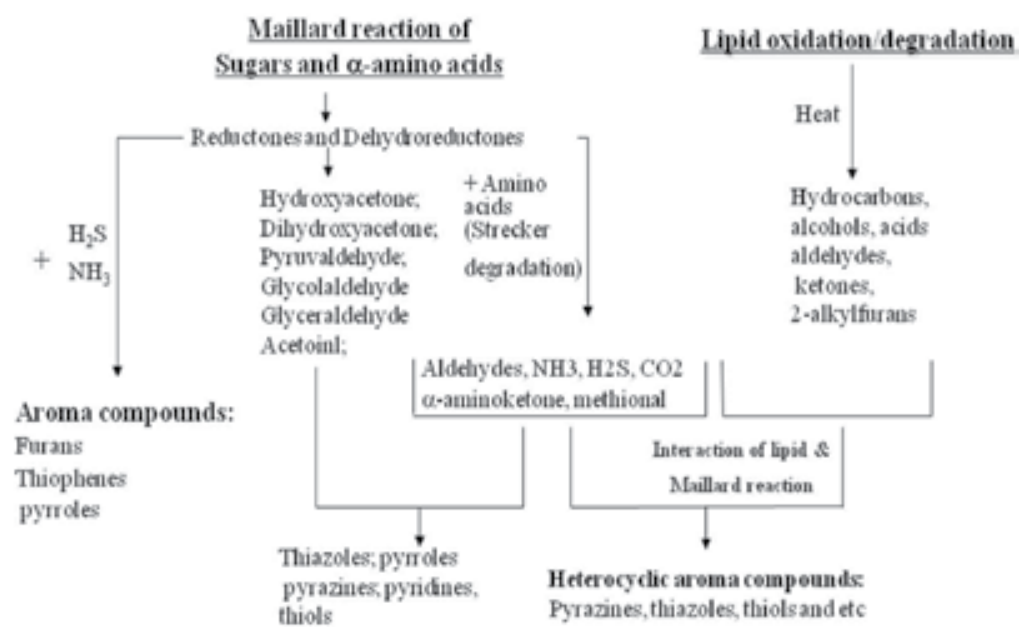

Figure 6. The interaction between lipid-oxidized products with Maillard products.

In general, in Maillard reaction mixtures containing lipids produce a lot of lipids-derived volatile compounds such as aldehydes, furans, hydrocarbons, alcohols and ketones. Furthermore, the reaction mixtures also containing the Maillard products such as $\mathrm{H}_{2} \mathrm{~S}, \mathrm{NH}_{3}$, etc. Which all are able to interact with each other to form new heterocyclic aroma volatile compounds as the consequences of the interactions between lipids with Maillard products. The most abundant compounds have been detected as results of the interactions are thiophene class such as 2-pentylthiophene, 2-hexylthiophene and thiol class such as 2-thiophenethiol, 2-furylmethanethiol, 2-methyl-3-furanthiol and etc [38, 61]. A recent study by Elmore and 
colleagues (2002) [34] concluded that breakdown products of polyunsaturated fatty acids especially are $n-3$ acids have a shorter chain length have lower odor thresholds will also be present at relatively high concentrations and are more reactive. These breakdown products will affect meat flavor by interacting with the Maillard reaction reducing levels of meaty aroma compounds, such as sulfur-substituted thiophenes and furans. As n-3 PUFAs are readily oxidized, they could initiate the free radical oxidation of more saturated acids, increasing levels of breakdown products of $n-6$ and $n-9$ fatty acids, which may also alter the aroma compounds of the cooked meat.

\section{The factors affect aroma flavors}

\subsection{Effect of diets}

Diet is as an important indicator to show the growth rate, performance, reproducibility effects and as well as meat quality of cattle. There has been an existed hypothesis of meat flavor changes due to feeding diets in which some works stated a large difference in meat flavor characteristics of the same cattle breed but fed on different diets. Early work by Melton (1983) [71] stated that steers fed with corn-based diets had more intense beef flavor (desirable flavor) than the same age steers fed based pasture or Bermuda pellets. A later study of Melton (1990) [10] found that the less desirable flavor of meat from cattle is mainly caused by several grass species. Conversely, no significant difference in flavors existed between the grass and grain diets-fed animals [73]. The less desirable flavors were also seen on meats from the hay diets-fed animals compared to corn silage diets [74], while Oltjen et al (1971) [75] showed the opposite results. It has been hypothesized that majority of flavor effects due to feeding of forages is mainly due to changes in fatty acid compositions. Fishy off-flavor was significantly higher in meat from grass-finished cattle with increasing unsaturated fatty acids [76]. Recently, researchers have attempted to higher level of PUFA in meat aiming to increase the health benefits by using the fat supplemented-diets (e.g., linseed, sunflower oil and fish oil) to cattle [77, 78], although these works have achieved an increase in several benefit fatty acids however, the detrimental effects on meat flavor characteristics appeared due to higher levels of PUFA [79]. A large number of studies regarding the effect of diets on volatile flavor compounds of cooked meat have been performed. Melton (1983) [71] also noted that the greatest difference in the flavors of meat from cattle fed on grass and grain-based diets is due to fatty acid concentration and type as fatty acids are the primary source of carbonyl. Suzuki and Bailey (1985) [80] indicated that higher concentrations of pentanoic,heptanoic, octanoic, nonanoic, decanoic, and dodecanoic acids were formed in the meat fat from grass-fed animals while heptanal, 2,3-octanedione, 3-hydroxyoctan-2-one, 2-decenal, 2-tridecanone, hexadecane, heptadecane, octodecane, d-dodecalactone, phyt-1-ene, neophytadiene, phyt-2-ene, an isomer of neophytadiene, 2-heptadecanone, dihydrophytol, and phytol with the terpenoids in much higher concentration due to rumen-fermented chlorophyll. Individual volatile flavor compounds like 4-heptanal, 2, 4-heptadienal and 2, 6-nonadienal (derived 
from C18:3n-3) and hexanal, 2-heptanal and 2, 4-decadienal (derived from C18:2n-6) found to be higher concentration in meats from grass and grain-fed animals, respectively [81]. Elmore et al (1997) [82] also reported that cooked meat from the animals that had been fed fish oil had considerably higher concentrations of saturated and unsaturated aldehydes than meat from the control. While, Descalzo et al (2005) [83] found that some classes of volatile flavor components affected by diets in which aldehydes increased in meat from concentrate diets-fed animals. In general, we can see that diets have a large influence on meat flavors due to directly affect the meat contents especially the intramuscular fat contents which play an important role in interaction and generation of volatile flavor compounds. On the other hand, it is worth noting that the uses of fat -supplemented diets to feed cattle may result in increases of important polyunsaturated fatty acids (e.g., n-3 fatty acids, DHA, EPA) which known to positively affect on consumers health however, a negative effect on meat flavors may appear due to these fatty acids not only produce some unexpected volatile compounds but also inhibit production of other Maillard products.

\subsection{Effect of breeds and sex}

Researchers have reported that breed also affects volatile flavor components and then influence overall flavor notes of cooked meat. Elmore et al (2000) [25] stated that fifty-four compounds were affected by breed, $75 \%$ of which was Maillard reaction products. Over 40 compounds were present at higher levels in the Soay breed than in the Suffolk breed. Other sulfur-containing compounds present at higher levels in the Suffolks than the Soays were bis-(2-furylmethyl) disulfide and 2-methyl-4,5-dihydro-thiophene and the differences in sulfur and nitrogen-containing compounds could contribute to flavor differences between the two breeds. A study on pork flavors as affected by breeds also have found that twenty-three among the detected flavor compounds were significantly affected by breed. Based on sensory analysis indicated that cooked longissimus muscle from hybrid breed (Duroc $x$ Landrace $\mathrm{x}$ Large White) had the lowest pork flavor intensity and flavor-liking compared with the Chinese indigenous breeds. Laiwu and Dahuabai breeds showed the highest pork flavor intensity and flavor-liking in cooked longissimus muscle [36]. In beef, Insausti and colleagues (2005) [84] also found the differences in volatile flavor compounds among the breeds were considerable and may contributed to the perception of flavor differences in the cooked beef. Particularly, level of dimethyl sulfide probably related to cauliflower notes, was highest for meat from the Pirenaica breed. While, levels of the sulfur-compounds in cooked beef from the Asturiana breed were low-intermediate and potentially related to blood and liver notes and unpleasant flavors.

For the sex effect, it has been reported that meat from bulls has a strong livery and blood flavors while meat from heifers has a strong characteristic flavor. The differences could be associated with the differences in amounts of certain volatile compounds such as hydrocarbons, aldehydes, alcohols and ketones [85]. On the other hand, the differences in meat flavors between bulls and heifers could be explained by the genetic control of animal development and production of sex hormones and their influence the lipid composition which affects the kinds of volatile flavor compounds [86]. Overall, it may be assumed that 
the differences in meat flavors existing between breeds or sexes are probably due to the differences in the levels of flavor precursors especially the fat contents which large affect the formation of aroma flavor compounds and also interact with other contents in determining flavor characteristics of cooked meat.

\subsection{Effect of chiller ageing}

Ageing has been become a universal method widely used to improve eating quality of meat (e.g. tenderness, juiciness, flavor). Un-aged beef has a weak, bland odor while aged beef has a strong, savory, roasted odor. Ageing of meat makes an increase in fatty flavor characteristics however; long term ageing (e.g., $>3$ weeks) could cause a decrease in positive flavor notes and increase liver-like aroma, bloody, bitter and off-flavor [87, 85, 88]. Ismail et al (2008) [89] stated that ethanol was responsible for the increase in alcohols caused by the microbial growth in beef during storage furthermore, the levels of aldehydes significantly increased after 7 days of storage. Beef from various muscles including gluteus medius, rectus femoris, vastus lateralis, vatsus medialis, teres major, complexus, serratus ventralis, psoas major and longissimus dorsi of heifer carcasses were chiller aged for 7 or 14 days the results showed that flavor-active volatiles included nonanal, 2,3-octanedione, pentanal, 3-hydroxy-2-butanone, 2-pentyl furan, 1-octen-3-ol, butanoic acid, pentanal and hexanoic acid which all often associated with lipid oxidation were affected by enhancement and ageing in the various muscles [90]. Additionally, ageing of beef achieved an increase in characteristic flavor and also aftertaste intensity, making an appreciable improvement of its flavor. After slaughter, loss of circulatory competency results in the accumulation of metabolic by-products, including lactic acid, in the muscle, that induces $\mathrm{pH}$ decline. The endogenous enzymes (e.g., cathespins B and L) are activated at near pH 5.4. Spanier and Miller (1993) and Spanier et al (1990) [91, 92] suggested that these thiol proteinases can hydrolyze more peptide bonds than any other group of enzymes, are redistributed during ageing period. Proteolytic enzyme activity is temperature-dependent; some enzymes retain high activity levels even at cooking temperatures. The combined effect of postmortem ageing and cooking, via enzyme redistribution and activity can influence the production of aroma flavor compounds. Toldrá and Flores (2000) [93] stated that enzymes known primarily for textural changes (e.g., $\mu$ - and $\mathrm{m}$-calpain) during the postmortem period affect flavors by producing peptides, but it was observed that these enzymes correlate with increases in rancid, sour and salty flavors. The ageing conditions (e.g., oxygen availability, temperature, humidity and aging time) under which beef is aged influences the ultimate flavors of the meat particularly ageing in a higher oxygen environment cause a burnt, toasted off-odor. In addition, dry-ageing increases beef flavor attributes more than ageing in vacuum or in carbon dioxide $[94,95]$. Based on the results reported in the previous studies it could be concluded that chiller ageing of meat resulted in increases of most of flavor compounds however a long ageing period (e.g., $>3$ weeks) may negatively influences the flavor quality of cooked meat due to increase in amounts of some unexpected compounds which associated with undesirable flavors and decrease in the some important compounds which associated with desirable flavors. 


\subsection{Effect of cooking temperature and $\mathrm{pH}$ conditions}

Cooking temperature is one of the important factors impacting the development of flavors through the Maillard reactions and lipid oxidation. Amino acids can undergo Strecker degradation to produce Strecker products. Degradation of sulfur-containing amino acids (e.g., cysteine, cystine and methionine) generates sulfur that contributes to subsequent processes of Maillard reaction. These compounds can react with amines and amino acids to produce a number of flavor-contributing compounds and potent cooked meat odorants such as pyrazines, oxazoles, thiophenes, thiazoles and other heterocyclic sulfur containing compounds [20]. It was well seen that cooking temperatures affect these reactions and then determine flavor characteristics, for instance the stewed meat lacks flavors of the roasted products because of stewed meat has a water activity of approximately 1.0 and not exceed temperature of $100^{\circ} \mathrm{C}$ while roasted meat has dried surfaces and temperature may exceed $100^{\circ} \mathrm{C}$ therefore, the conditions like low water activity and high surface temperature will increase production of flavor compounds which give roasted odor notes rather than meat is stewed. Ames et al (2001) [40] concluded that the amounts of most volatile flavor compounds increased with cooking temperature. Cooking at lower temperatures $(<165$ $\left.{ }^{\circ} \mathrm{C}\right)$ versus higher temperatures $\left(>180{ }^{\circ} \mathrm{C}\right)$ results in differences in the concentrations of a number of compounds such as 2, 4, 5-trimethyl- 3-oxazoline; 2, 4-dimethyl-5-ethyl-3-oxazoline; 2, 5- dimethyl-4-ethyl-3-oxazoline; 2, 4-dimethyl-3-thiazoline; 2, 4, 5-trimethyl-3-thiazoline [96]. Previous works found that a strong relationship existing between cooking temperature, concentration of free amino acids, carnosine, pyrazines and hexanol, and roasted, burnt and beefy flavor intensity $[97,98]$. Cooking beef generates urea content which can also reduce sulfur-containing compounds generating important nitrogen-containing compounds like pyrazines and thiazoles in which pyrazines are formed mostly on the surface of meat and having nutty and roasty odor notes [99]. In general, the higher degree of heating, the higher the concentration of aliphatic aldehydes, benzenoids, polysulfides, heterocyclic compounds and lipid-derived volatiles. Ketones, alcohols sulfur-containing components make smaller contributions.

$\mathrm{pH}$ is one of the important factors that influence the kind of volatile flavor compounds formed in the Maillard reaction, and then determine the final flavor characteristics of cooked food. Madruga and Mottram (1995) [8] showed that as $\mathrm{pH}$ increases, color and polymeric compounds increase and nitrogen-containing compounds like pyrazines are favored, therefore it was assumed that higher ultimate $\mathrm{pH}$ in meat from grass-fed animals may favor the formation of thiazoles and thiophenones due to the availability of amino acid degradation products while decreasing other sulfur volatiles that favor lower $\mathrm{pH}$. A number of early studies have been performed to investigate the effect of $\mathrm{pH}$ changes on volatile flavor compounds using model systems (El'Gde et al., 1966; Shu et al., 1985; Meynier and Mottram, 1995) [100, 101, 102]. These studies found that high $\mathrm{pH}$ values also favor the formation of many volatile compounds but other compounds are only favored at low $\mathrm{pH}$ condition. Meynier and Mottram (1995) [102] used meat-like model systems containing amino acids and ribose on different $\mathrm{pH} 4.5$ and 6.5, results showed that nitrogen-containing compounds such as pyrazines were detected at higher pHs. While, dimethyldisulphide and methional 
showed decrease as the $\mathrm{pH}$ increased, and an increase in the disulphide was observed. It was observed that a large number of sulphur-containing compounds such as 2-methyl-3-furanthiol a strong meaty aroma, whose formation was greatly favored by lower $\mathrm{pH}$ condition. Ames et al (2001) [40] used model reactions containing cysteine and reducing sugar at varied $\mathrm{pH}$ conditions 5.5, 6.5 and 7.5, results showed that amounts of most of compounds increased with pH especially are pyrazines. Cerny and Biffod (2007) [103] recently found that $\mathrm{pH}$ determined strongly which volatile flavors were formed and to what extent. In general, based on the results of the previous studies which all found that $\mathrm{pH}$ condition strongly influence the formation of flavor components.

\subsection{Effect of irradiation on meat flavors}

Irradiation is a food safety technology designed to eliminate disease-causing germs from foods. Depending on the dose levels of irradiation applying on the raw meat and poultry, or ready-to-eat meats that can eliminate bacteria commonly found such as E. coli, Salmonella and Listeria; virus; or parasites. However, irradiation may result in off-odors and flavors. The odors vary with the type of meat, temperature during irradiation, oxygen exposure during and/or after the irradiation process, packaging and presence of antioxidative substances [104]. Most of studies have reported that the aroma flavors of irradiated meat associated with rotten egg, sweet, bloody, cooked meat, barbecued corn, burnt, sulfur, metallic, alcohol, acetic acid, liver-like serumy and bloody [105, 106, 107]. Irradiation can initiate or promote lipid oxidation resulting in undesirable off-odors and flavors [108, 109]. Jo and Ahn, (2000) [110] showed that reactions of sulfur-containing amino acids with radiolytic products of water appear to be the source of hydrogen sulfide and other volatile sulfur-containing compounds which contribute to off-flavor. On the other hand, irradiation may result in the formation of free radicals from unsaturated fatty acids at double bond positions [109]. An increase in lipid peroxidation products such as hexanal and (E)-4,5-epoxy-(E)-2-decenal in combination with a loss of desirable meaty odorants (4-hydroxy-2,5-dimethyl-3(2H)-furanone and 3-hydroxy-4,5-dimethyl-2(5H)-furanone) result in development of warmed over flavor of cooked, refrigerated beef [16]. However, the effects of irradiation on aroma flavors are also depended on: (1) Dose levels of irradiation, it has been demonstrated that the dose levels of irradiation influence variedly on volatile flavor components of cooked meat, as reported by Jo and Ahn (2000) [110] who indicated some of hydrocarbons included 1-heptene and 1-nonene increased with irradiation dose immediately after irradiation of beef. A similar observation also was reported by Yong et al (2000) [111] who indicated that among the 150 flavor compounds indentified in beef the cyclodecene, (E)-2-hexenal, nonene and 2nonenal showed an increase in a dose-dependent fashion. For the effect of irradiation on chicken flavors, Yong e al (2000) [112] showed that among the 129 identified volatile flavor compounds the cyclotetradecene, 2-methylpentanal and 4-methylcyclohexene were formed specifically in response to irradiation, and level of cyclotetradecene increased in a dose-dependent fashion; (2) Oxygen presence, the presence of oxygen around meats during irradiating can diffuse into the meats, and then results in radiolytic changes which precipitate oxidation and unacceptable secondary breakdown products. As well known, lipid oxidation needs oxygen presence to produce oxidized-products such as aldehydes, Nam and Ahn 
(2003) [113] indicated that irradiation of meat in aerobic packaging promoted production of aldehydes such as propanal and hexanal which is assumed as a good indicator of lipid oxidation. The similar observation also was reported by Nam et al (2001) [114] who also showed that irradiation increased TBARS values and off-flavor in aerobically-packaged pork (3) Temperature effect, temperature during irradiating meats has a large effect on aroma flavors of irradiated meat because temperature affects what radiolytic products are formed and what ratios [104]. Using lower temperature during irradiation of meat by freezing meat before irradiation can reduce detrimental effects via retarding autoxidation and extending shelf life; (4) $\mathrm{pH}$ effect, it has also been demonstrated that the ultimate $\mathrm{pH}$ of meat at the time of irradiation influences lipid oxidation. Nam et al (2001) [114] recently showed that irradiation increased lipid oxidation of normal and pale-soft-exudative (low $\mathrm{pH}$ group) muscles, whereas dark-firm-dry (high $\mathrm{pH}$ group) muscle was very stable and resistant to oxidative changes. Therefore, to minimize the detrimental effects of irradiation on aroma flavor characteristics we can modify atmosphere packaging by using vacuum packaging (anaerobic packaging) or replacement with inert gases (i.e. nitrogen, helium, hydrogen, carbon dioxide) to eliminate oxygen. Reducing the temperature (freezing) prior to irradiation and addition of antioxidants. Vacuum packaging retains irradiation-generated sulfur-containing compounds, however re-packaging meat in oxygen-permeable materials allows for dissipation of these flavor compounds.

\section{Warm-off flavor and liver-like off flavor in cooked meat}

Warm-off flavor and liver-like off flavor are undesirable flavors that result from the flavor changes and deterioration in meats that have been pre-cooked, chilled-stored and reheated. The warm-off flavor includes odors and tastes commonly described as stale, cardboardlike, painty, rancid, bitter and sour [115], and together with liver-like off flavor they both are the main factors that negatively affect eating sensory quality, purchase, economic impact of meat industry, and consumer complaint. Researchers have found that warm-off flavor appearing in cooked meat is mainly caused by oxidation of membrane phospholipids [116,115]. A recent report of Byrne et al (2001) [117], which also demonstrated that warm-off flavor associated with the development of lipid oxidation derived nuance offflavor and odor notes such as rancid-like flavor and linseed oil-like odor, in association with a concurrent decrease in cooked pork meat-like flavor. The development of warmoff flavor usually results in loss of meaty flavor due to mask by lipid-oxidized products. Additionally, processes which involve any action that disrupts the muscle fiber membrane, such as chopping, restructuring, or heating which all can enhance warm-off flavor of meat product [118]. Previous works also suggested that reactions involving sulphydryldisulfide interchanges in proteins and the degradation of sulfur-containing heteroatomic compounds, leading to a decrease in the "meatiness" of freshly cooked meat may also be an integral part of warm-off flavor [119,120]. For the liver-like off flavor of cooked meat, it was hypothesized that since foodservice preparation traditionally cooked the meat quickly and then held the product in warming ovens until the food was presented to the consum- 
er these conditions might promote the liver-like flavor [27]. James and Calkins, (2005) [121] also hypothesized that the slower cooking and longer hold time allow the undesirable volatile flavor compounds to dissipate.

\section{The current techniques used for extraction and detection of aroma flavor components}

Up to present time, various techniques have been designed, combined with gas chromatography and mass spectrometry (GC/MS) or Flame ionizing detector (GC/FID) and applied to evaluate volatile flavor components in cooked meat. Of which, simultaneous steam distillation-extraction (SDE), dynamic headspace entrainment on Tenax TA, and solid-phase microextraction (SPME) are the techniques widely used for the extraction of volatile compounds in cooked meat $[19,122,123,85,90,124,18,125]$. SDE is a simple technique which involves small volumes of solvent, efficient stripping of volatiles and quantitative recovery of many compounds. The sample is dispersed in water which is heated to boiling. The steam that is generated carries volatiles with it into a section of the apparatus where the steam condenses in the presence of extracting solvent vapor. The co-condensation of volatile-laden steam and extracting solvent results in an effective extraction of volatiles [123]. The Dynamic headspace entrainment on Tenax has been used in the studies regarding cooked meat volatile flavor compounds since the 1980s. This technique probably has been used more than any other aroma extraction technique for the analysis of meat aroma and continues to be widely used. The action mechanism of this technique involving purging the headspace of a sample with a purified inert gas (e.g., nitrogen or helium), followed by collection of the volatiles onto a trap containing a suitable adsorbent, which will retain the volatile analytes carried there by the purge gas. Finally, the volatiles of meat samples collected on this trap are desorbed onto a GC or GC-MS column using a modified injection port. In the recent years, SPME technique has been widely adopted and considered as an alternative to isolate volatile flavor components in cooked meat. In SPME, the needle is coated with an absorbent material (e.g. CAR/ PDMS), is placed above the cooked meat samples. Volatiles will migrate from the sample matrix to the needle coating and be absorbed. Volatile components will then be desorbed from the needle coating by inserting the needle in GC injection port.

The extraction techniques as mentioned above in combination with GC/MS or GSC/FID can help researchers to tentatively detect the volatile flavor compounds in experimented meat samples but it could not identify the aroma flavors or odor characteristics of detected compounds. It would be advantageous to combine two or more different techniques, such as gas chromatography (GC) and olfactometry, the combination of measuring odor notes is called gas chromatography-olfactometry (GC/O). Gas chromatography-olfactometry (GC-O) is a bioassay that measures human response to odorants separated by gas chromatography. The superior sensitivity and selectivity of human olfaction make GC-O a powerful and meaningful tool for flavor chemistry. In the recent year, GC-O is one of the main techniques which have been used to determine intensity of aroma (odor) characteristics of volatile compounds in cooked meat $[15,21,125]$. 


\section{Conclusion and Implication}

In order to have a cooked meat product with its desirable aroma flavors as expectation of consumer, it is important to understand how aroma flavors are derived, the mechanisms by which flavor components are generated, and the factors affect formation of flavor compounds then determine the final aroma flavor characteristics of cooked meat. Regarding the effects of factors on aroma flavors of cooked meat and to minimize the detrimental effects it is suggested if increasing the polyunsaturated fatty acids (e.g. C18:3n-3, DHA, EPA) to increase nutritional benefits to the consumer by using fat-supplemented diets however the undesirable flavors may result. Because the breakdown products of these fatty acids have a shorter chain length therefore are more volatile and they affect meat flavors by interacting with the Maillard reaction results in reducing levels of meaty aroma compounds such as sulfur-substituted thiophenes. Therefore, diets, feeding regimes, welfare and management of animals should be taken into account. Cooking conditions such as temperature, holding time and cooking methods play an important role in determining the formation volatile flavor compounds. In general, it has been demonstrated that cooking meat at high temperature (by roasting, grilling) will produce better aroma flavor characteristics due to the important Maillard products are formed. In addition to the cooking effect, it is suggested that a slow cooking and longer hold time can allow the undesirable volatile flavor compounds to dissipate, thus reduce warm-off flavor. Irradiation of meat can eliminate pathogens however, offflavor may result therefore, and to minimize the detrimental effect of this method we can lower temperature during irradiation of meat by freezing meat before irradiation. Modifying atmosphere packaging by using vacuum packaging (anaerobic packaging) or replacement with inert gases (i.e. nitrogen, helium, hydrogen, carbon dioxide) to eliminate oxygen in meat during irradiation are also the alternatives. Chiller ageing of meat should be applied to improve eating quality however should not age for a long time ( 3 week period in maximum is encouraged) because chiller ageing meat for a too long period may result in flavor deterioration and decreasing desirable flavors.

\section{Author details}

Hoa Van $\mathrm{Ba}^{1^{*}}$, Inho Hwang ${ }^{1}$, Dawoon Jeong ${ }^{2}$ and Amna Touseef ${ }^{2}$

*Address all correspondence to: ba_cnu1981@yahoo.com

1 Department of Animal Science and Biotechnology, Lab of Muscle Biology and Meat Science, Chonbuk National University, South Korea

2 Department of Animal Science and Biotechnology, Lab of Muscle Biology and Meat Science, Chonbuk National University, South Korea 


\section{References}

[1] Elgado, C., Rosegrant, M., Steinfeld, H., Ehui, S, \& Courbois, C. (1999). Paper presented at Livestock to 2020. The Next Food Revolution. Food, Agriculture, and the Environment. Discussion Paper 28. International Food Policy Research Institute, Food and Agriculture Organization of the United Nations and the International Livestock Research Institute., IFPRI, Washington, D.C.

[2] Mac, Leod. G. (1994). The flavor of beef. In Shahidi F (ed) Flavor of meat and meat products. Glasgow Chapman and Hall , 4-37.

[3] Savell, J. W., Branson, R. E., Cross, H. R., Stiffler, D. M., Wise, J. W., Griffin, D. B., \& Smith, G. C. (1987). National consumer retail beef study: palatability evaluations of beef loin steaks that differed in marbling. Journal of Food Science, 52-517.

[4] Robbins, K., Jensen, J., Ryan, K. J., Homco-Ryan, C., Mc Keith, F. K., \& Brewer, M. S. (2003). Consumer attitudes towards beef and acceptability of enhanced beef. Meat Science, 65-721.

[5] Landy, P., Courthaudon, J. L., Dubois, C., \& Voilley, A. (1996). Effect of interface in model food emulsions on the volatility of aroma compounds. Journal of Agriculture and Food Chemistry, 44-526.

[6] Rabe, S., Krings, U., \& Berger, R. G. (2003). Influence of oil-in-water emulsion properties on the initial dynamic flavor release. Journal of the Science of Food and Agriculture, 83-1124.

[7] Kinsella, J. E. (1990). Flavor perception and binding. INFORM, 1(3), 215-226.

[8] Madruga, M. S., \& Mottram, D. S. (1995). The effect of $\mathrm{pH}$ on the formation of Maillard-derived aroma volatiles using a cooked meat system. Journal of the Science of Food and Agriculture, 68-305.

[9] Bredie, W. L. P., Mottram, D. S., \& Guy, R. C. E. (1998). Aroma volatiles generated during extrusion cooking of maize flour. Journal of Agriculture and Food Chemistry, 46-1497.

[10] Melton, S. L. (1990). Effects of feeds on flavor of red meat: A review. Journal of Animal Science, 68-4421.

[11] Mottram, D. S., \& Whitefield, F. B. (1994). Aroma volatiles from meat-like Maillard systems. In Thomas H (ed) Thermally generated flavors. ACS Symposium series. American chemical society, Washington, D.C , 543, 180-191.

[12] Mottram, D. S. (1991). Meat: In Hank Maarse (ed) Volatile compounds in foods and Beverages. Marcell Dekker Inc., New York; , 107-177.

[13] Gasser, U., \& Grosch, W. (1988). Identification of flavor volatile compounds in high aroma values from cooked beef. Z. Lebensm. Unters. Forsh, 186-489. 
[14] Specht, K., \& Baltes, W. (1994). Identification of volatile flavor compounds with high aroma values from shallow-fried beef. Journal of Agriculture and Food Chemistry, 42, 2246-2253.

[15] Rochat, S., \& Chaintreau, A. (2005). Carbonyl odorants contributing to the in-oven roast beef top note. Journal of Agriculture and Food Chemistry, 53-9578.

[16] Kerler, J., \& Grosch, W. (1996). Odorants contributing to warmed-over flavor (WOF) of refrigerated cooked beef. Journal Food Science, 61(6), 1271-1274.

[17] Machiels, D., Istasse, L., \& van Ruth, S. M. (2004). Gas chromatography-olfactometry analysis of beef meat originating from differently fed Belgian Blue, Limousin and Aberdeen Angus bulls. Food Chemistry, 86-377.

[18] , H. V., Oliveros, M. C., Ryu, K. S., \& Hwang, I. H. (2010). Development of analysis condition and detection of volatile compounds from cooked Hanwoo beef by SPMEGC/MS analysis. Journal of Food Science of Animal Resource, 30-73.

[19] Elmore, J. S., Mottram, D. S., Enser, M., \& Wood, J. D. (1999). Effect of the polyunsaturated fatty acid composition of beef muscle on the profile of aroma volatiles. Journal of Agriculture and Food Chemistry, 47-1619.

[20] Mottram, D. S. (1998). Flavor formation in meat and meat a review. Food Chemistry, 62-415.

[21] Machiels, D., van Ruth, S. M., Posthumus, M. A., \& Istasse, L. (2003). Gas chromatography-olfactometry analysis of the volatile compounds of two commercial Irish beef meats. Talanta, 60-755.

[22] Kerscher, R., \& Grosch, W. (1997). Comparative evaluation of potent odorants of boiled beef by aroma extracts dilution and concentration analysis. Zeitschrift für Lebensmitteluntersuchung und Forschung A, 204, 3-6.

[23] Cerny, C., \& Grosch, W. (1992). Evaluations of potent odorants in roasted beef by aroma extract dilution analysis. Zeitschrift für Lebensmitteluntersuchung und Forschung $A$, 194, 323-325.

[24] Cañedo, A. R., Juez-Ojeda, C., Nuñez, M., \& Fernández-García, E. (2011). Effects of high-pressure processing on the volatile compounds of sliced cooked pork shoulder during refrigerated storage. Food chemistry, 124, 749-758.

[25] Elmore, J. D., Mottram, D. S., Enser, M., \& Wood, J. D. (2000). The effects of diet and breed on the volatile compounds of cooked lamb. Meat Science, 55, 149-159.

[26] Wettasinghe, M., Vasanthan, T., Temelli, F., \& Swallow, K. (2001). Volatile flavor composition of cooked by-product blends of chicken, beef and pork: a quantitative GC-MS investigation. Food research international, 34, 149-158.

[27] Calkins, C. R., \& Hodgen, J. M. (2007). A fresh look at meat flavor. Meat Science, 77, 63-80. 
[28] Macey, R. L., Naumann, H. D., \& Bailey, M. E. (1964). Water-soluble flavor and odor precursors of meat. (II) Effects of heating on amino nitrogen constituents and carbohydrates in lyophilized diffusates from aqueous extracts of beef, pork and lamb. Journal of Food Science, 29, 142-148.

[29] Macey, R. L., Naumann, H. D., \& Bailey, M. E. (1970). Water-soluble flavor and odor precursors of meat. 5 . Influence of heating on acidextractable non-nucleotide chemical constituents of beef, lamb and pork. Journal of Food Science, 35, 83-87.

[30] Mottram, D. S. (1994). Some aspects of the chemistry of meat flavor. In Shahidi, F (ed) the flavor of meat and meat products: Blackie Glasgow , 210-230.

[31] Koutsidis, G., Elmore, J. S., Oruna-Concha, M. J., Campo, M. M., Wood, J. D., \& Mottram, D. S. (2008). Water-soluble precursors of beef flavor: I. Effect of diet and breed. Meat Science, 79, 124-130.

[32] Koutsidis, G., Elmore, J. S., Oruna-Concha, M. J., Campo, M. M., Wood, J. D., \& Mottram, D. S. (2008). Water-soluble precursors of beef flavor: part II. Effect of diet postmortem conditioning. Meat Science, 79, 270-277.

[33] Dwivedi, B. K., \& Arnold, R. G. (1973). Chemistry of thiamine degradation in food products and model systems: a review. Journal of Agriculture and Food Chemistry, 21, 54-60.

[34] Elmore, J. S., Campo, M. M., Enser, M., \& Mottram, D. S. (2002). Effect of Lipid composition on Meat-like model systems containing cystein, ribose and polyunsaturated fatty acids. Journal of Food chemistry, 50, 1126-1132.

[35] Meinert, L., Tikk, K., Tikk, M., Brockhoff, P. B., Bredie, W. L. P., Bjergegaard, C., \& Aaslyng, M. D. (2009). Flavor development in pork. Influence of flavor precursor concentrations in longissimus dorsi from pigs with different raw meat qualities. Meat Science, 81, 255-262.

[36] Lu, P., Zhang, L. Y., Yin, J. D., Everts, A. K. R., \& Li, D. F. (2008). Effects of soybean oil and linseed oil on fatty acid compositions of muscle lipids and cooked pork flavor. Meat Science, 80, 910-918.

[37] Elmore, J. S., Cooper, S. L., Enser, M., Mottram, D. S., Sinclair, L. A., \& Wilkinson, R. G. (2005). Dietary manipulation of fatty acid composition in lamb meat and its effect on the volatile aroma compounds of grilled lamb. Meat Science, 69, 233-242.

[38] Farmer, L. J., Mottram, D. S., \& Whitfield, F. B. (1989). Volatile compounds produced in Maillard reaction involving systeine, ribose and phospholipids. Journal of the Science of Food and Agriculture, 49, 347-369.

[39] Salter, L. J., Mottram, D. S., \& Whitfield, F. B. (1988). Volatile compounds produced in the Maillard reaction involving glycine, ribose and phospholipids. Journal of the Science of Food and Agriculture, 46, 227-242. 
[40] Ames, J. M., Guy, R. C. E., \& Kipping, G. J. (2001). Effect of pH and temperature on the formation of volatile compounds in cysteine/reducing sugar/starch mixtures during extrusion cooking. Journal of Agriculture and Food Chemistry, 49, 1885-1894.

[41] Tai, C. Y., \& Ho, C. T. (1997). Influence of cysteine oxidation on thermal formation of Maillard aromas. Journal of Agricultural and Food Chemistry, 45, 3586-3589.

[42] Mottram, D. S., \& Edwards, R. A. (1983). The role of triglycerides and phospholipids in the aroma of cooked beef. Journal of the Science of Food and Agriculture, 34, 517-522.

[43] Whitfield, F. B., Mottram, D. S., Brock, S., Puckey, D. J., \& Salter, L. J. (1988). Effect of phospholipid on the formation of volatile heterocyclic compounds in heated aqueous solutions of amino acids and ribose. Journal of the Science of Food and Agriculture, 42, 261-272.

[44] Huang, T. C., \& Ho, C. T. (2001). Flavors of meat products. In Hui. Y. H., Nip, W. K., Roger, R. W and Young, O. A (eds) Meat Science and Application Marcel Dekker, Inc , 71-102.

[45] Tai, C. C., \& Ho, C. T. (1998). Influence of glutathione oxidation and $\mathrm{pH}$ on thermal formation of Maillard-type volatile compounds. Journal of Agriculture and Food Chemistry, 46, 2260-2265.

[46] Maillard, L. C. (1912). Action des acides amines sur les sucres: formation des melanoidines par voie methodique. Compte-rendu de l'Academie des Sciences, tome, 154, 66-68.

[47] van den, Ouweland. G. A. M., Peer, H. G., \& Tjan, S. B. (1978). Occurrence of Amadori and Heyns rearrangement products in processed foods and their role in flavor formation. In Charalambous, G and Inglett, G. E (eds) flavor in foods and Beverages Academic press New York, 131-143.

[48] Mouron, J. (1981). The Maillard reaction in food: a critical review from the nutritional standpoint. In Erickson, C (ed) Maillard reaction in food Pergamon Press Oxford, 3-35.

[49] Hurrent, R. F. (1982). Maillard reaction in flavor. In Morton, I. D and Macleod, A. J (eds) food flavor Elsevier Amsterdam , 399-437.

[50] Jousse, F., Jongen, W., Agterof, W., Russell, S., \& Braat, P. (2002). Simplified kinetic scheme of flavor formation by the Maillard reaction. Journal of Food Science, 7, 2534-42.

[51] van Boekel, M. A. J. S. (2006). Formation of flavor compounds in the Maillard reaction. Biotechnology Advances, 24, 230-233.

[52] Realini, C. E., Duckett, S. K., Brito, B. M., Dalla, Rizza. M., \& De Mattos, D. (2004). Effect of pasture vs. concentrate feeding with or without antioxidants on carcass characteristics, fatty acid composition, and quality of Uruguayan beef. Meat Science, 66, 567-577. 
[53] Warren, H. E., Scollan, N. D., Enser, M., Hughes, S. I., Richardson, R. I., \& Wood, J. D. (2008). Effects of breed and a concentrate or grass silage diet on beef quality in cattle of 3 ages. I: Animal performance, carcass quality and muscle fatty acid composition. Meat Science, 78, 256-269.

[54] Cristina, P. M. A., Susana, P. A., Susana, I. V. M., Ana, S. H. C., Carlos, M. G. A. F., Jose, P. C. L., Rui, J. B. B., \& Jose, A. M. P. (2009). Effect of the feeding system on intramuscular fatty acids and conjugated linoleic acid isomers of beef cattle, with emphasis on their nutritional value and discriminatory ability. Food Chemistry; , 114, 939-946.

[55] Cuvelier, C., Clinquart, A., Hocquette, J. F., Cabaraux, J. F., Dufrasne, I., Istasse, L., \& Hornick, J. L. (2006). Comparison of composition and quality traits of meat from young finishing bulls from Belgian Blue, Limousin and Aberdeen Angus breeds. Meat Science, 74, 522-531.

[56] Muchenje, V., Hugo, A., Dzama, K., Chimonyo, M., Strydom, P. E., \& Raats, J. G. (2009). Cholesterol levels and fatty acid profiles of beef from three cattle breeds raised on natural pasture. Journal of Food Composition and Analysis, 22, 354-358.

[57] Moreno, T., Keane, M. G., Noci, F., \& Moloney, A. P. (2008). Fatty acid composition of M. Longissimus dorsi from Holstein-Friesian steers of New Zealand and European/ American descent and from Belgian Blue Holstein-Friesian steers, slaughtered at two weights/ages. Meat Science, 78, 157-169.

[58] Nawar, W. W. (1969). Thermal degradation of lipids-a review. Journal of Agriculture and Food Chemistry, 17, 1-9.

[59] Mottram, D. S. (1985). Effect of cooking conditions on the formation of volatile heterocyclic compounds in pork. Journal of Science and Food Agriculture, 36, 377-382.

[60] Mottram, D. S., Edwards, R. A., \& Macfie, H. J. H. (1982). A comparison of flavor volatiles from cooked beef and pork meat systems. Journal of the Science of Food and Agriculture, 33, 934-944.

[61] Farmer, J. J., \& Mottram, D. S. (1990). Recent study on the formation of meat-like aroma compounds. In Bessere, Y and Thomas, A. F (eds) Flavor Science and technology Wiley, Chichester, UK, 113-116.

[62] van der Lide, L. M., van Dort, J. M., de valois, P., Boelens, H., \& de Rijike, D. (1979). Volatile components from thermally degraded thiamin. In Land, D. G and Nursten, H. E (eds) progress in flavor research. Applied science London, 219-224.

[63] Guntert, M., Bruning, J., Emberger, R., Kopsel, M., Kuhn, W., Thielmann, T., \& Werkhoff, P. (1990). Identification of formation of some selected sulfur-containing flavor compounds in various food model systems. Journal of Agriculture and Food Chemistry, 38, 2027-2041.

[64] Reineccius, G. A., \& Liardon, R. (1985). The use of charcoal traps and microwave desorption for the analysis of headspace volatiles above heated thiamin solutions. In R. 
G, Berger, S. Nitz and P, Schreier (eds)Topics in flavor research Eichhorn, MarzlingHangenhan , 125-136.

[65] Dreher, J. G., Rouseff, R. L., \& Naim, M. (2003). GC-Olfactometric Characterization of Aroma Volatiles from the Thermal Degradation of Thiamin in Model Orange Juice. Journal of Agriculture and Food Chemistry, 51, 3097-3102.

[66] Karel, M., Schaich, K., \& Roy, R. B. (1975). Interaction of Peroxidizing Methyl Linoleate with Some Proteins and Amino Acids. Journal of Agriculture and Food Chemistry, 23, 159-163.

[67] Kikugawa, K., Kato, T., Beppu, M., \& Hayasaka, A. (1990). Fluorescent and Crosslinked Proteins Formed by Free Radical and Aldehyde Species Generated During Lipid Oxidation in Lipofuscin and Ceroid Pigments. E. A. Porta, Plenum Press New York., 345-357.

[68] Neukom, H. (1980). Oxidative Crosslinking of Proteins and Other Biopolymers. In Simic, M. G. and Karel, M (eds.) Autoxidation in Food and Biological Systems Plenum Press New York, 249-259.

[69] Gardner, H. W., Kleiman, R., Weisleder, D., \& Inglett, G. E. (1977). Cysteine adds to lipid hydroperoxide. Lipids, 12, 655-660.

[70] Hidalgo, F. J., \& Zamora, R. (1995). In Vitro Production of Long Chain Pyrrole Fatty Esters from Carbonyl-Amine Reactions. Journal of Lipid Research, 36, 725-735.

[71] Melton, S. L. (1983). Effect of forage feeding on beef flavor. Food Technology, 37, 239-248.

[72] Ames, J. M., Defaye, A. B., \& Bates, L. (1997). The effect of $\mathrm{pH}$ on the volatiles formed in an extruded starch-glucose-lysine model system. Food chemistry, 58, 323-327.

[73] French, P., O’Riordan, E. G., Monahan, F. J., Caffrey, P. J., Mooney, M. T., \& Troy, D. J. (2001). The eating quality of meat of steers fed grass and/or concentrates. Meat Science, 57, 379-386.

[74] Dube, G., Bramblett, V. D., Howard, R. D., Homler, B. E., Johnson, H. R., \& Harrington, R. B. (1979). Dietary effects on beef composition.4. Processing and palatability attributes. Journal of Food Science, 36, 147-154.

[75] Oltjen, R. R., Rumsey, T. S., \& Putnam, P. A. (1971). All-forage diets for finishing beef cattle. Journal of Animal Science, 32(2), 327-333.

[76] Nuernberg, K., Wood, J. D., Scollan, N. D., Richardson, R. I., Nute, G. R., \& Nuernberg, G. (2005). Effect of a grass-based and a concentrate feeding system on meat quality characteristics and fatty acid composition of longissimus muscle in different cattle breeds. Livestock Production Science, 94, 137-147.

[77] Mandell, I. B., Buchanan-Smith, J. G., Holub, B. J., \& Campbell, C. P. (1997). Effects of fish meal in beef cattle diets on growth performance, carcass characteristics, and fatty acid composition of longissimus muscle. Journal of Animal Science, 75(4), 910-919. 
[78] Scollan, N., Richardson, I., Moloney, A., Dannenberger, D., Hocquette, J. F., \& Nuernberg, K. (2006). Innovations in beef production systems that enhance the nutritional and health value of beef lipids and their relationship with meat quality. Meat science, 74(1), 17-33.

[79] Miller, R. K. (2001). Beef flavor: A white paper. Centennial, CO, National Cattlemens' Beef Association.

[80] Suzuki, J., \& Bailey, M. E. (1985). Direct sampling capillary GLC analysis of flavor volatiles from ovine. Food Chemistry, 33(3), 343-347.

[81] Larick, D. K., Hedrick, H. B., Bailey, M. E., Williams, J. E., Hancock, D. L., \& Garner, G. B. (1987). Flavor constituents of beef as influenced by forage- and grain-feeding. Journal of Food Science, 52(2), 245-251.

[82] Elmore, J. S., Mottram, D. S., Enser, M., \& Wood, J. D. (1997). Novel if thiazoles and 3-thiazolines in cooked beef aroma. Journal of Agriculture and Food Chemistry, 45, 3603-3607.

[83] Descalzo, A. M., Garcia, P. T., Pensel, N. A., Josifovich, J. A., Insani, E. M., \& Biolatto, A. (2005). Influence of pasture or grain-based diets supplemented with vitamin $E$ on antioxidant/oxidative balance of Argentine beef. Meat Science, 70(1), 35-44.

[84] Insausti, K., Goni, V., Petri, E., Gorraiz, C., \& Beriain, M. J. (2005). Effect of weight at slaughter on the volatile compounds of cooked beef from Spanish cattle breeds. Meat Science, 70, 83-90.

[85] Gorraiz, C., Beriain, M. J., Chasco, J., \& Insausti, K. (2002). Effect of aging time on volatile compounds, odor, and flavor of cooked beef from Pirenaica and Friesian bulls and heifers. Journal of Food Science, 67, 916-922.

[86] Sink, J. D. (1979). Symposium on meat flavor factors influencing the flavor of muscle foods. Journal of Food Science, 44, 1-5.

[87] Spanier, A. M., Flores, M., Mc Millin, K. W., \& Bidner, T. D. (1997). The effect of post mortem aging on meat flavor quality in Brangus beef. Correlation of treatments, sensory, instrumental and chemical descriptors. Food Chemistry, 59, 531-538.

[88] Yancey, E. J., Grobbel, J. P., Dikeman, M. E., Smith, J. S., Hachmeister, K. A., \& Chambers, E. C. (2006). Effects of total iron, myoglobin, hemoglobin, and lipid oxidation of uncooked muscles on livery flavor development and volatiles of cooked beef steaks. Meat Science, 73, 680-686.

[89] Ismail, H. A., Lee, E. J., Ko, K. Y., \& Ahn, D. U. (2008). Effects of aging time and natural antioxidants on the color, lipid oxidation and volatiles of irradiated ground beef. Meat Science, 80, 582-591.

[90] Stetzer, A. J., Cadwallader, K., Singh, T. K., Mckeith, F. K., \& Brewer, M. S. (2008). Effect of enhancement and ageing on flavor and volatile compounds in various beef muscles. Meat Science, 79, 13-19. 
[91] Spanier, A. M., \& Miller, J. A. (1993). Role of Proteins and Peptides in Meat Flavor. In Spanier, A.M., Okai, H. and Tamura, M (eds) Food Flavor and Safety. ACS Symposium Series Am. Chem. Soc. Washington, D.C (528), 78-97.

[92] Spanier, A. M., Mc Millin, K. W., \& Miller, J. A. (1990). Enzyme activity levels in beef: effect of postmortem aging and end point cooking temperature. Journal of Food Science, 55, 318-322.

[93] Toldrá, F., \& Flores, M. (2000). The use of muscle enzymes as predictors of pork meat quality. Food Chemistry, 69, 387-395.

[94] Campbell, R. E., Hunt, M. C., Levis, P., \& Chambers, E. I. V. (2001). Dry-aging effects on palatability of beef longissimus muscle. Journal of Food Science, 66, 196-199.

[95] Jeremiah, L. E., \& Gibson, L. L. (2003). The effects of postmortem product handling and aging time on beef palatability. Food Research International, 36, 929-941.

[96] Mussinan, C. J., Wilson, R. A., Katz, I., Sanderson, A., \& Vock, M. H. (1975). Identification and flavor properties of some 3oxazolines and 3-thiazolines isolated from cooked beef. Abstract, Journal of American Chemical Society AGFD , 170, 22.

[97] Lorenzen, C. L., Davuluri, V. K., Adhikari, K., \& Grün, I. U. (2005). Effect of end point temperature and degree of doneness on sensory and instrumental flavor profile of beefsteaks. Journal of Food Science, 70, 113-118.

[98] Cambero, M. I., Seuss, I., \& Honikel, K. O. (1992). Flavor compounds of beef broth as affected by cooking temperature. Journal of Food Science, 57, 1285-1290.

[99] Hogan, B. (2002, July). Putting punch in meat flavor profiles. Food Product Design.

[100] El'Ode, K. E., Domseifer, T. P., Keith, K. S., \& Powers, J. J. (1966). Effect of pH and temperature on the carbonyls and aromas produced in heated amino acid-sugar mixtures. Journal of Food Science, 31, 351-8.

[101] Shu, C. K., Hagedom, M. L., Mookherjee, B. D., \& Ho, C. T. (1985). pH effect on the volatile components in the thermal degradation of cysteine. Journal of Agriculture and Food Chemistry, 33, 442-6.

[102] Meynier, A., \& Mottram, D. S. (1995). The effect of $\mathrm{pH}$ on the formation of volatile compounds in meat-related model systems. Food Chemistry, 52, 361-366.

[103] Cerny, C., \& Briffod, M. (2007). Effect of pH on the Maillard Reaction of [13C5] Xylose, Cysteine and Thiamin. Journal of Agriculture and Food Chemistry, 55, 1552-1556.

[104] Brewer, M. S. (2009). Irradiation effects on meat flavor: A review. Meat Science, 81, 1-14.

[105] Hampson, J. W., Fox, J. B., Lakritz, L., \& Thayer, D. W. (1996). Effect of low dose gamma radiation on lipids in five different meats. Meat Science, 42, 271-276. 
[106] Jo, C., Lee, J. I., \& Ahn, D. U. (1999). Lipid oxidation, color changes and volatile production in irradiated pork sausage with different fat content and packaging during storage. Meat Science, 51(4), 355-361.

[107] Lee, M., Sebranek, J. G., Olson, D. G., \& Dickson, J. S. (1996). Irradiation and packaging of fresh meat and poultry. Journal of Food Protection, 59(1), 62-72.

[108] Lescano, G., Narvaiz, P., Kairiyama, E., \& Kaupert, N. (1991). Effect of chicken breast irradiation on microbiological, chemical and organoleptic quality. Lebensmittal Wissenund Technology, 24, 130-134.

[109] Thakur, B. R., \& Singh, R. K. (1994). Food irradiation Chemistry and applications. Food Reviews International, 10(4), 437-473.

[110] Jo, C., \& Ahn, D. U. (2000). Volatiles and oxidative changes in irradiated pork sausage with different fatty acid composition and tocopherol content. Journal of Food Science, 65(2), 270-275.

[111] Yong, J., Hun, K., Sung, Y. P., So, J. K., \& Young, J. Y. (2000). Identification of irradiation- induced volatile flavor compounds in beef. Journal of Korean Society of Food Science and Nutrition, 29(6), 1042-1049.

[112] Yong, J., Hun, K., Sung, Y. P., Wo, J. C., Seong, S. Y., \& Young, J. Y. (2000). Identification of irradiation-induced volatile flavor compounds in chicken. Journal of Korean Society of Food Science and Nutrition, 29(6), 1050-1056.

[113] Nam, K. C., \& Ahn, D. U. (2003). Double-packaging is effective in reducing lipid oxidation and off-odor volatiles of irradiated raw turkey meat. Poultry Science, 82(9), 1468-1474.

[114] Nam, K. C., Ahn, D. U., Du, M., \& Jo, C. (2001). Lipid oxidation, color, volatiles, and sensory characteristics of aerobically packaged and irradiated pork with different ultimate pH. Journal of Food Science, 66, 1220-1225.

[115] St, Angelo. A. J., Vercellotti, J. R., Legendre, M. G., Vinnett, C. H., Kuan, J. W., James Jr, C., Jr, \& Dupuy, H. P. (1987). Chemical and instrumental analysis of warmed-over flavor in beef. Journal of Food Science, 52, 1163-1168.

[116] Ingene, J. O., \& Pearson, A. M. (1979). Role of phospholipids and triglycerides in warmed-over flavor development in meat model systems. Journal of Food Science, 44, 1285-1290.

[117] Byrne, D. V., Bredie, W. L. P., Bak, V., Bertelsen, G., Martens, H., \& Martens, M. (2001). Sensory and chemical analysis of cooked porcine meat patties in relation to warmed-over flavor and pre-slaughter stress. Meat Science, 59, 229-249.

[118] St Angelo, A. J. (1996). Lipid oxidation in foods. Critical Reviews in Food Science and Nutrition, 36(3), 175-224.

[119] St, Angelo. A. J., Vercellotti, J. R., Dupuy, H. P., \& Spanier, A. M. (1988). Assessment of beef flavor quality: a multidisciplinary approach. Food Technology, 42, 133-138. 
[120] St, Angelo. A. J., Crippen, K. L., Dupuy, H. P., \& James Jr, C., Jr. (1990). Chemical and sensory studies of antioxidant-treated beef. Journal of Food Science, 55, 1501-1539.

[121] James, J. M., \& Calkins, C. R. (2005). Cooking rate and holding time effect on the flavor of muscles from the beef chuck and the round. In Proceedings of the international congress of meat science and technology Baltimore, MD.

[122] Elmore, J. S. (2008). Aroma. In Nollet L. M. L and Toldra. F (eds.) Handbook of muscle foods analysis Boca Raton CRC , 242-262.

[123] Madruga, M. S., Elmore, J. S., Dodson, A. T., \& Mottram, D. S. (2009). Volatile flavor profile of goat meat extracted by three widely used techniques. Food Chemistry, 115, 1081-1087.

[124] Moon, S. Y., Cliff, M. A., \& Li-Chan, E. C. Y. (2006). Odour-active components of simulated beef flavor analyzed by solid phase microextraction and gas chromatography-mass spectrometry and-olfactometry. Food Research International, 39, 294-308.

[125] Xie, J. C., Sun, B. G., \& Wang, S. B. (2008). Aromatic constituents from Chinese traditional smoke-cured bacon of mini-pig. Food science and technology international, 14, 329-340. 
Chapter 8

\title{
Novel Analytical Tools for Quality Control in Food Science
}

\author{
Christian W. Huck \\ Additional information is available at the end of the chapter \\ http://dx.doi.org/10.5772/51915
}

\section{Introduction}

Due to the fast technological and data treatment advancements new insights into food can be considered. The application of these novel analytical techniques belongs to the responsibility of food chemists and analysts. Thereby, an increase in efficiency is based on an improved lower limit of detection (LOD), selectivity to separate analytes of interest and speed of analysis.

High-performance liquid chromatography (HPLC) belongs to the traditional separation techniques applied to a broad range of hydrophilic and hydrophobic ingredients in both the reversed-phase (RP) [1] as well as normal-phase (NP) [2] mode. In a conventional HPLC system the inner diameter of the separation column, which is the core of the separation unit, is $4.6 \mathrm{~mm}$. During the last decade miniaturization down to $20 \mu \mathrm{m}$ allowed to increase on one side the sensitivity and on the other side speed of analysis could be enhanced dramatically. Therefore, novel stationary phases mainly based on polymers have been designed and brought to the market to enable both the separation of low and highmolecular weight analytes [3]. As an alternative separation technique capillary electrophoresis (CE), which separates analytes due to their different ion mobility based on charge and molecular weight in an electric field within a fused silica capillary having an inner diameter of approximately $200 \mu \mathrm{m}$ can be applied [4]. Thereby, the appearance of the electroosmotic flow (EOF) can influence the separation efficiency by either speeding up the separation process or by improving the resolution. Capillary electrochromatography (CEC) is a hybrid technique of both HPLC and CE in which both pressure and an electrical field are applied and enables extreme high resolution. The drawback of this separation method is the fact, that real samples can hardly be analysed due to the disturbance by the matrix [5]. In many cases the analyte of interest is only available in very low con- 
centrations. Therefore, selective enrichment and purification steps are the method of choice, which can be accomplished by solid-phase extraction (SPE). Therefore, a material designed for a special analytical question is filled into a cartridge or pipette tip and the sample of interest is put onto the material in liquid form [6]. In the following, analytes of interest can interact with the functional groups of the stationary phase and compounds being not of interest can simply be washed away. In the final elution step, only some micro liters of liquid are required to elute the analytes of interest from the stationary phase being available in relatively high concentrations for the following analytical steps. The following analytical procedure can be either a separation or spectroscopic method. Spectroscopic methods at this stage of the analytical procedure either include mass spectrometry (MS) and/or vibrational spectroscopy, respectively. In MS most of the samples are analysed applying electrospray ionization (ESI) as an interface with different types of mass detectors including e.g. time of flight (TOF), ion trap, ion cyclotron and quadrupoles. As an alternative, matrix assisted laser desorption ionization time of flight mass spectrometry (MALDI-TOF/MS) can be applied for the determination of high molecular weight compounds including proteins, peptides and lipids. For the analysis of low molecular ingredients < $1000 \mathrm{Da}$ the so called matrix-free laser desorption ionization (mf-LDI) MS technique must be applied [7].Vibrational spectroscopy in the field of food analysis is mainly applied in the mid $\left(400-4000 \mathrm{~cm}^{-1}\right)$ as well as in the near infrared $(4000-12000$ $\mathrm{cm}^{-1}$ ) of the electromagnetic spectrum. In combination with chemo metrical algorithms these methods can be used for the authentication of the material on one hand, on the other hand quantitative analysis allows to control selected quality parameters [8].

In the following a systematic analytical approach is introduced, which allows combining the different analytical techniques in a synergistic manner to get deeper insights into the composition and origin of food samples.

\section{Systematic analytical approach}

The key technologies described in the above chapter can be combined according to the scheme depicted in Figure 1. In this approach extraction of the material for the further analytical steps and individual procedures can be linked to sample enrichment/purification, separation, vibrational spectroscopy and mass spectroscopy followed by database analysis. The different parts are described in the following sub-chapters.

\subsection{Sample enrichment/purification}

In many cases interesting analytes are only available in extremely low concentrations and/or in very complex matrices, respectively. Therefore, pre-concentration steps based on solid-phase extraction (SPE) can be very helpful. Nano-materials such as nanotubes, fullerenes, diamond offer excellent physiochemical properties due to a high ratio of surface to size, which results in a high capacity and allows analyte detection with high sensitivity down to the femtomole range in the case when mass spectrometry is applied for 
detection. Especially carbon nano materials can be easily further derivatised with a number of different functional groups including reversed-phase (RP), normal-phase (NP), ion exchange (IEX), immobilized affinity (IMAC) and so on depending on the specific demand. As an alternative they can be incorporated into a polymer matrix for highly selective extraction by certain compound characteristics. For the practical handling pipette tips have been tested to be most suitable and this special type of SPE is called "hollow monolithic incorporated tip" as it has an open flow channel in the middle enabling an easy pipetting procedure. For the highly efficient pre-concentration of phosphopeptides nano particular $\mathrm{TiO}_{2}, \mathrm{ZrO}_{2}$ and mixtures thereof are incorporated into a polymer matrix as depicted in Figure 2 [9]. By this technique hundreds of microliters can be flushed over the system and finally elution of the desired compounds to be analysed is carried out with only a few microliters causing a dramatic increase in concentration from which further analytical investigations can benefit due to the easier handling of the systematic investigation.

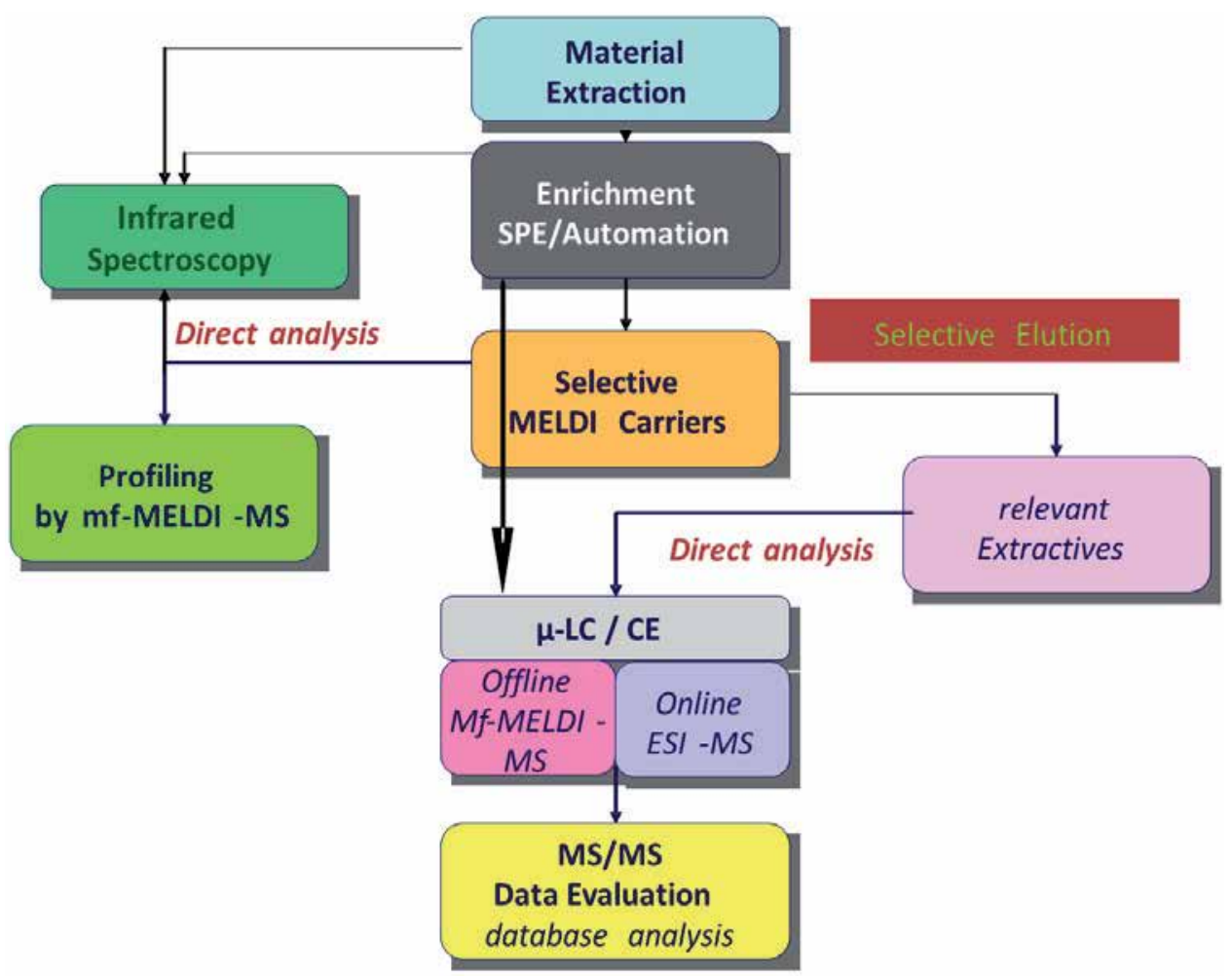

Figure 1. Multidimensional analytical approach 


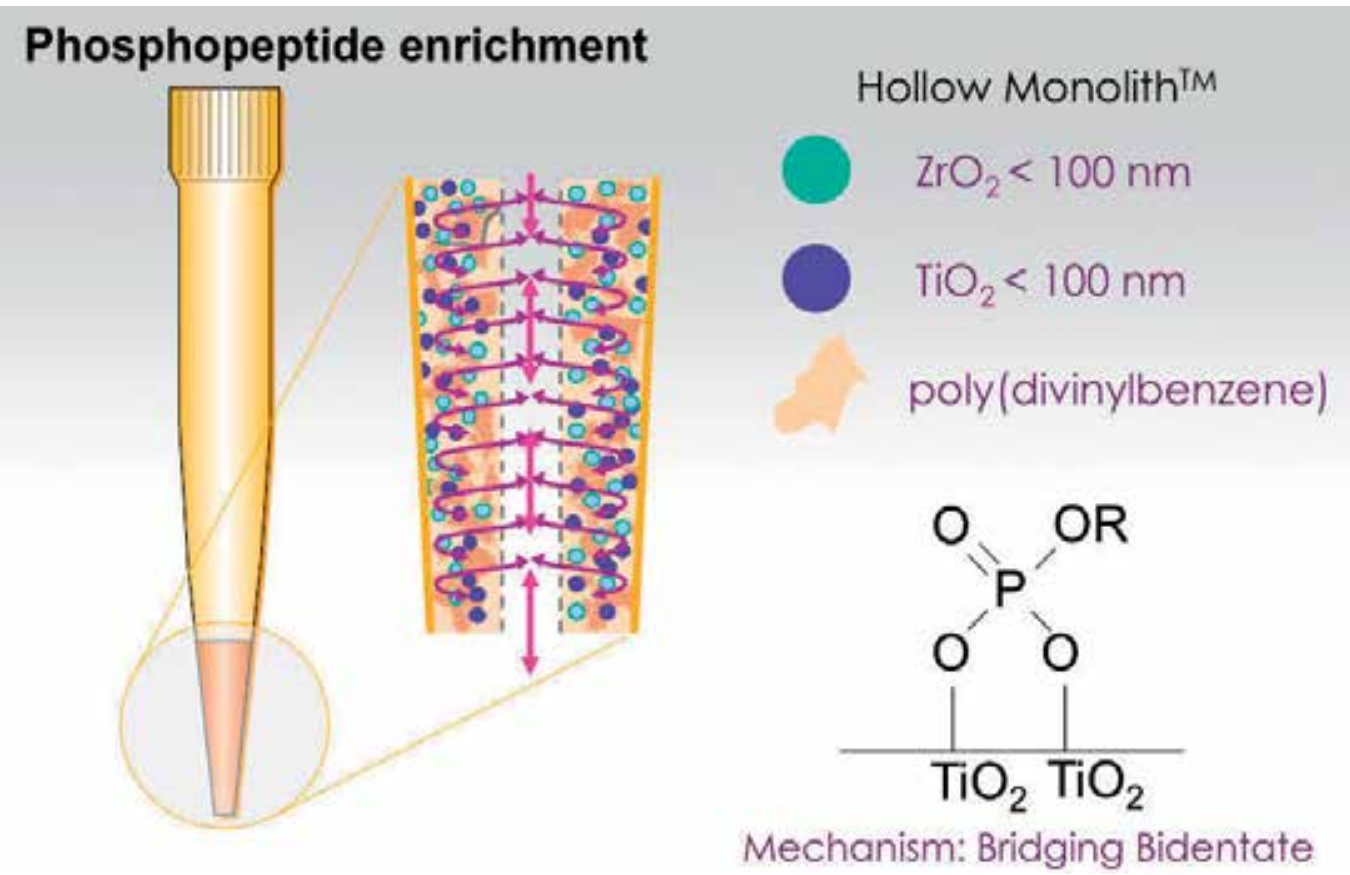

Figure 2. $\mathrm{TiO}_{2}, \mathrm{ZrO}_{2}$ incorporated into a polymer matrix for phosphopeptide enrichment

Immobilisation of such polymers into pipette tips with trypsin can be used for fast digestion of peptides and proteins within only a few minutes ensuring high capacity and sequence coverage (Figure 3) even in the high-throughput mode using robotic pipetting systems [10]. In comparison to this quite young approach the conventional digestion procedure lasts approximately 24 hours and doesn't show in any case better results by higher sequence coverages. For this reason this approach is of high interest for the routine analysis and/or diagnostics, respectively. As a carrier glycidylmethacrylate-co-divinylbenzene (GMA/DVB) polymerized in pipette tips was chosen. The major advantages of in-tip digestion are easy handling and small sample amount required for analysis. Microwave-assisted digestion was applied for highly efficient and time saving proteolysis. Adaption to an automated robotic system allowed fast and reproducible sample treatment. Investigations with matrix-assisted laser desorption/ionization time-of-flight mass spectrometry (MALDI-TOF/MS) and liquid chromatography coupled to electrospray-ionization mass spectrometry (LC-ESI/MS) attested high sequence coverages (SCs) for the three standard proteins, myoglobin (Myo, 89\%), bovine serum albumin (BSA, 78\%) and alpha-casein ( $\alpha$-Cas, $83 \%$ ). Compared to commercially available trypsin tips clear predominance concerning the digestion performance was achieved. Storability was tested over a period of several weeks and results showed only less decrease $(<5 \%)$ of protein sequence coverages. The application of microwave-assisted in-tip digestion (2 minutes) with full automation by a robotic system allows high-throughput analysis (96 samples within 80 minutes) and highly effective proteolysis. 


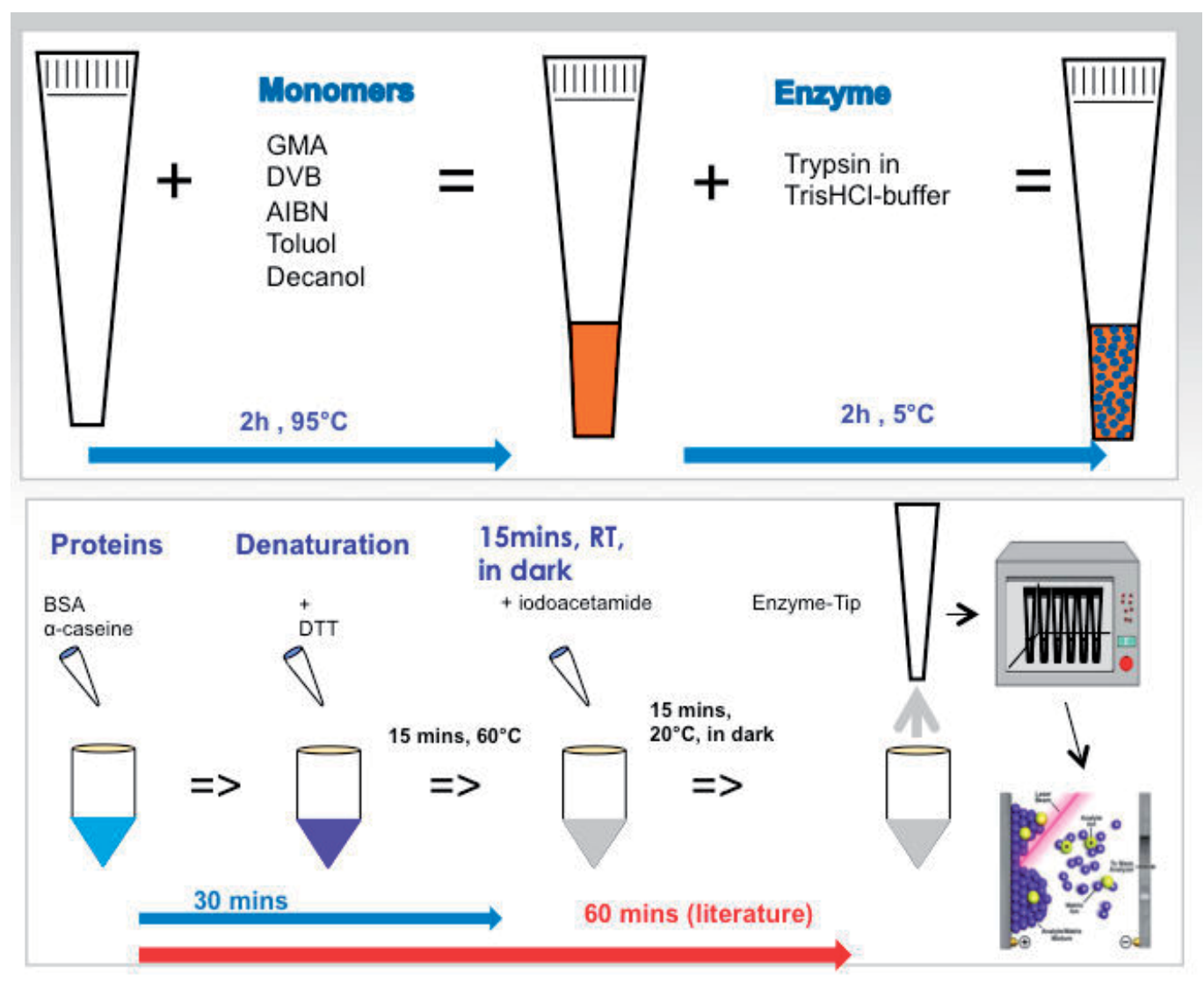

Figure 3. Trypsin immobilized pipette tips for high-throughput analysis of peptides

\subsection{Selective MELDI-carriers}

Material enhanced laser desorption ionisation (MELDI) is a method, which is based on the conventional matrix assisted laser desorption ionisation time of flight mass spectrometric (MALDI-TOF/MS) detection with the significant difference that before LDI MS step a selective enrichment procedure is carried out for the distinct analysis of a certain compound class. Compared to other similar techniques in this field, this approach benefits from the physical properties of the material itself (pore size, surface area, capacity, etc.) and its chemical derivatisation/functionalisation. In the past this technique was proven to be highly efficient for the analysis of biomarkers following an optimised strategy (Figure 4). In the first step a selected material including e.g., nanotubes, fullerenes, nano-crystalline diamond, polymers, cellulose, etc., which are derivatised with functional groups (C18, IMAC (immobilised metal affinity chromatography), IEX and others) is activated and the serum sample of interest is incubated. During this step, selective binding of molecules according to their functional group is achieved and finally undesired components can be washed away applying an optimised protocol. In the next step the incubated material is put onto a conventional 
steel target used in MALDI-TOF/MS, a matrix substance is added (e.g., sinapinic acid) and finally the mass spectrum is generated by the laser desorption ionisation process. The result is a mass spectrum being characteristic for a patient and/or the nutrition profile. Multivariate analysis (MVA) can be applied for further data analysis and interpretation, a clustering into certain stages of an illness can be achieved, respectively. From the mass spectrum potent biomarker molecules can be selected and identified by further analytical steps. The biomarker itself and/or the profile of the corresponding mass spectrum can be used for the screening of certain diseases, stages therefrom, allergies, nutrition effects and so on [7].

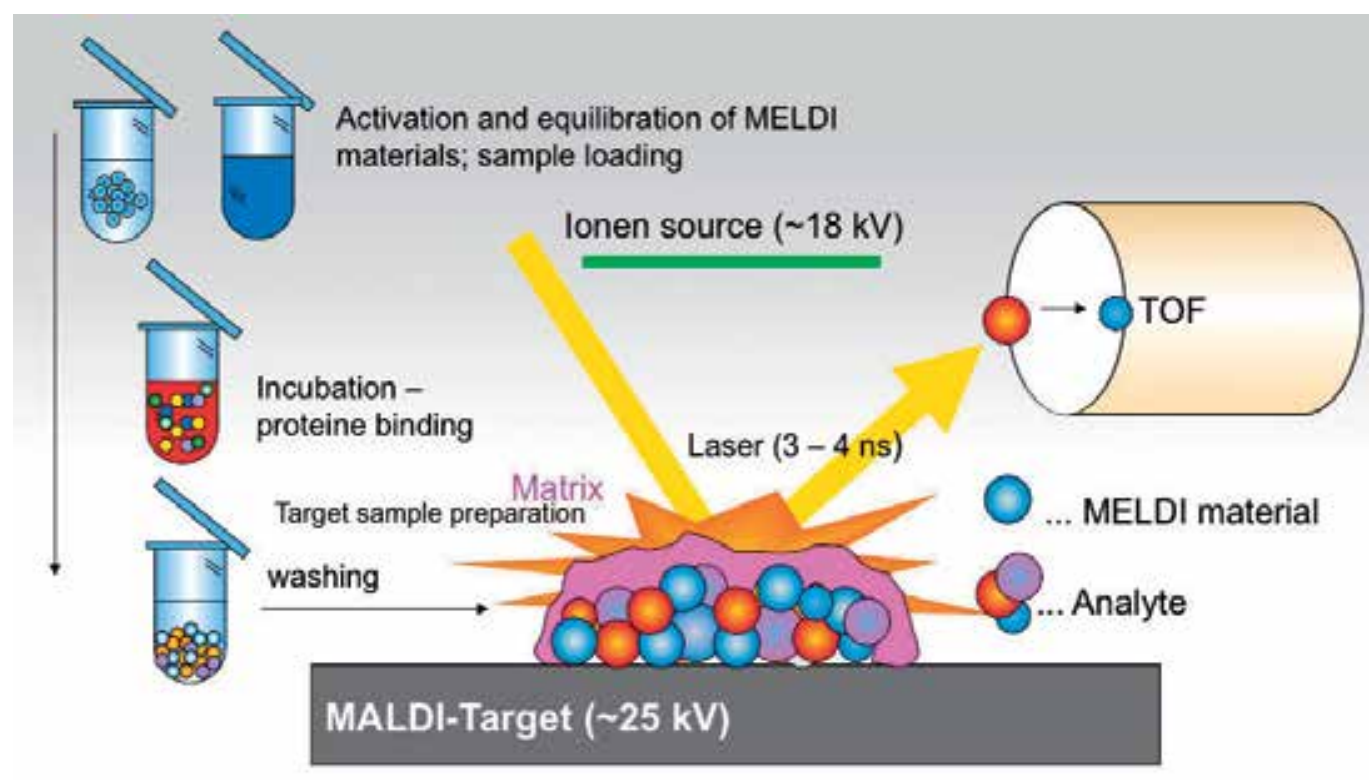

Figure 4. Principle of material enhanced laser desorption ionization (MELDI)

For the analysis of low-molecular weight compounds (MW $<1000 \mathrm{Da})$ the conventional MELDI approach is replaced by the matrix-free ( $\mathrm{mf}$ ) MELDI approach for which the addition of a matrix substance is not required so that no disturbing peaks appear. In this approach a conventional steel target with a $50 \mathrm{~nm}$ thick titanium oxide layer can be applied fulfilling all requirements for a successful laser desorption ionization process [11].

As an alternative the incubated analytes of interest can be selectively eluted from the functinalised carrier material and further analysed by liquid chromatography (LC) or capillary electrophoresis (CE).

\subsection{Liquid chromatography, capillary electrophoresis and electrochromatography}

Novel materials used in miniaturised liquid chromatography ( $\mu$-LC) are mainly polymer based, e.g. poly(1,2-bis(p-vinylphenyl)ethane). These polymers possess the huge advantage that chemical (composition of the polymer) and physical parameters including mainly po- 
rosity can be adjusted [12]. Extensive investigations on polymerisation time and temperature have been carried out enabling a tailored design of micro-, meso- and macro-pore distribution $[13,14]$. This results in the applicability of such capillaries with an inner diameter between 20 and $200 \mu \mathrm{m}$ for even the separation of high- and low-molecular weight compounds. These capillaries can be highly successfully applied analysing peptides, proteins, oligonucleotides, DNA fragments as well as "small molecules" such as phenols, flavonoids, catechins, acids etc. Figure 5 shows as an example the separation of olive oil ingredients. This separation is characterised by a very high ratio of flow to back pressure, which is of high interest to perform extremely rapid Coupling to mass spectrometry enables a highly efficient analysis even of crude samplesoffering all the possibilities of collision induced dissociation (CID) and database search [15].

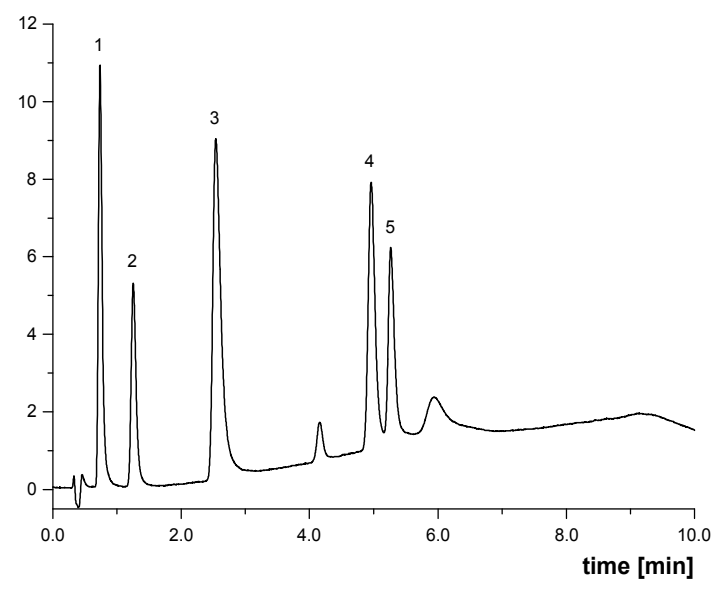

Column, PA/PDA (Kap. 155, polymerised for $10 \mathrm{~min}$ ), $80 \times 0.2 \mathrm{~mm}$; chromatographic conditions: mobile phase, A: $0.1 \%$ TFA, B: ACN, $0.1 \%$ TFA; gradient, $5-45 \%$ B in $10 \mathrm{~min}$; flow rate, $8 \mu \mathrm{\mu l} / \mathrm{min}$; temperature, RT; detection, UV $210 \mathrm{~nm}$;

$10 \mathrm{~min}$; flow rate, $8 \mu \mathrm{l} / \mathrm{min}$; temperature, RT; detection, UV $210 \mathrm{~nm}$;
Peak identification: (1) Hydroxytyrosol, (2) Tyrosol, (3) Caffeic acid, (4) Vanillin and

(5) Oleuropein.

Figure 5. Separation of olive oil ingredients using a monolithic capillary column. Conditions: capillary $80 \times 0.2 \mathrm{~mm}$; mobile phase, A: 0.1\% TFA; B: CAN; gradient, 5-45\%B in $10 \mathrm{~min}$; Flow rate $8 \mu \mathrm{l} / \mathrm{min}$; temperature, RT; detection, UV $210 \mathrm{~nm}$. Peak assignment, (1) hydroxytyrosol, (2) tyrosol, (3) caffeic acid, (4) vanillin, (5) oleuropein.

As an alternative separation method capillary electrophoresis (CE) and /or electrochromatography (CEC) can be applied. In CE separation of analytes is achieved due to their different ion mobility based on charge and molecular weight in an electric field within a fused silica capillary having an inner diameter of approximately $200 \mu \mathrm{m}$ [4]. As has already been remarked the electroosmotic flow (EOF) has a main influence on the separation and can be used for speeding up. In CEC both an electrical field and high pressure are applied resulting in high resolution. This technique can be applied to check the identification and purity of standards compounds with very high efficiency. For the reproducible separation and analysis of food ingredients such as phenols, acids, peptides, lipids, coating of the capillary's inner wall was shown being advantageous as irreversible analyte adsorption by free hydroxyl-groups from the silanole of the fused silica capillary can be avoided. Latex-diol 
and fullerene coated capillaries were successfully introduced and as a detection system online hyphenation to MALDI-TOF/MS was shown to be highly efficient not only for the investigation of flavonoids but also for peptides, especially phosphorylated (Figure 6) [16, 17]. This system can be used for the investigation of the casein profile in milk offering the advantage over all other more classical analysis tools that in this case also higher phosphorylated species can be separated and detected. From the ratio of different phosphorylation degrees several interpretations concerning the quality but also the origin of the milk can be carried out.

fused silica Polymicro capillary (uncoated)

fused silica Polymicro capillary (latex diol coated)

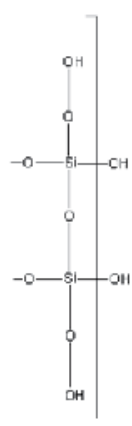

fused silica Polymicro capillary (fullerenol coated)

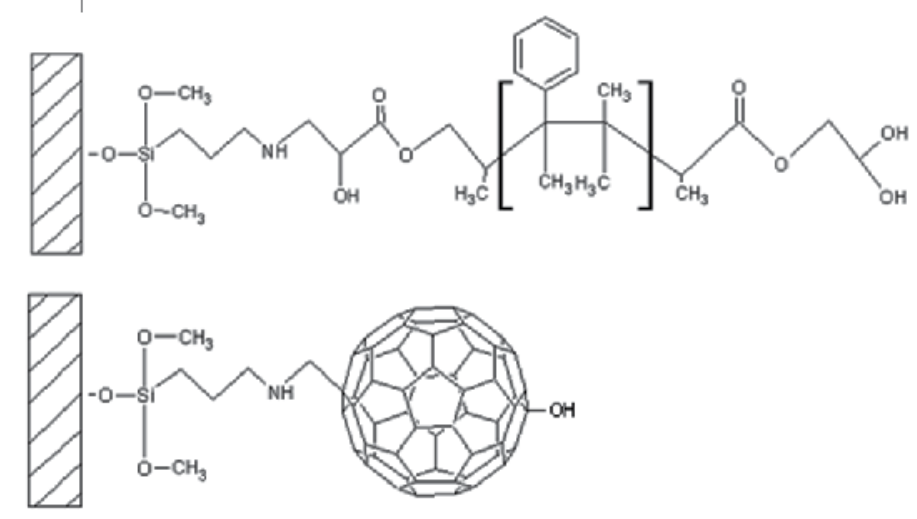

Figure 6. Inner capillary wall coatings applied in CE and CEC

\subsection{Vibrational spectroscopy}

For quality control both mid- (MIR, $400-4000 \mathrm{~cm}^{-1}$ ) and near-infrared (NIR, $4000-12000$ $\mathrm{cm}^{-1}$ ) can be conducted. In MIR fundamental stretching and bending vibrations occur, in NIR the corresponding overtones and combination vibrations are detected. This means that NIR-spectra can contain a lot of more vibrational information, which is an advantage for the analysis of highly complex samples. Therefore, during the last decade several applications in the field of food analysis were developed in the NIR region. Samples can be analysed either in transmission, reflectance and interactance mode (Figure 7) so that liquid as well as solid samples can be investigated. Due to the quite broad bands compared to MIR, chemometrical spectra treatment is required for establishing adequate calibration models and to 
analyse data. These are mainly multivariate (MVA) methods allowing to correct baseline, atmospheric noise etc. For qualitative analysis in most cased principal component analysis (PCA), for quantitative partial least square regression (PLSR) are applied [18].

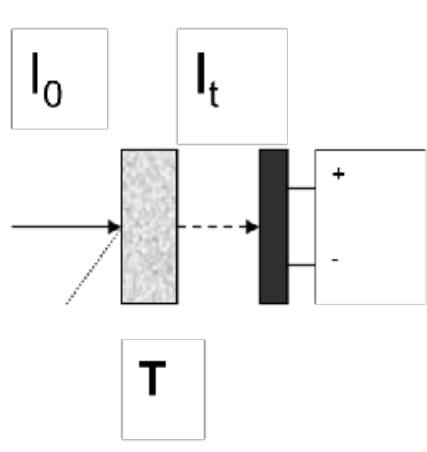

TRANSMISSION

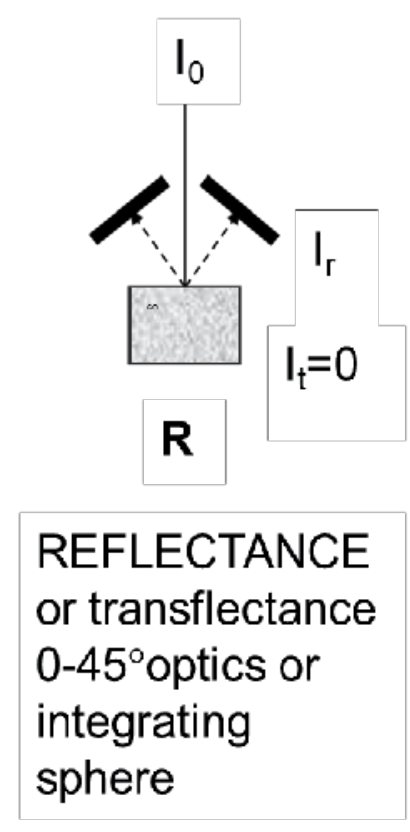

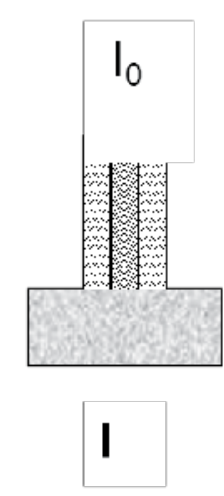

INTERACTANCE Fibre optic probe

Figure 7. Sample measurement modes in NIR

An impressive example for the successful implementation in the food related production is the quality control of wine. It has been shown that NIR can be used to identify grapes, vines, age by qualitative (Figure 8) and its ingredients (acids, carbohydrates, $\mathrm{pH}$ etc.) simultaneously, non-invasively within a few seconds by quantitative analysis [19]. Another big advantage of this method can be found by the fact that the sample is not destroyed and can therefore be used for further purposes including following analytical steps.

Quantitative NIRS methods, which allow determining the carbohydrate, total acid, tartaric acid, malic acid, $\mathrm{pH}$ in grape variety and the polyphenol content in grapes were established [19]. The method can control the quality already at a very early stage during the wine production and allows improvement of its quality by this. Grapes of 12 different vines (Weißburgunder, Chardonnay, Ruländer, Silvaner, Müller Thurgau, Gewürztraminer, Sauvignon, Lagrein, Grossvernatsch, Blauburgunder, Cabernet, Merlot) were harvested in autumn 2000 and squeezed. The obtained grape variety was thermo stated at $23^{\circ} \mathrm{C}$ and analyzed quantitatively by NIRS in the transflection mode using an optical thin layer thickness of $1 \mathrm{~mm}$. In order to establish a calibration model 252 spectra of samples with lower and upper concentration as a reference were recorded. $76 \%$ of all spectra were randomly used for calibration, $24 \%$ for validation. Data preparation was carried out in order to minimize technical influences, 
which mainly cause a drift in baseline. Quantitative analysis was carried out by partial least square regression (PLSR).

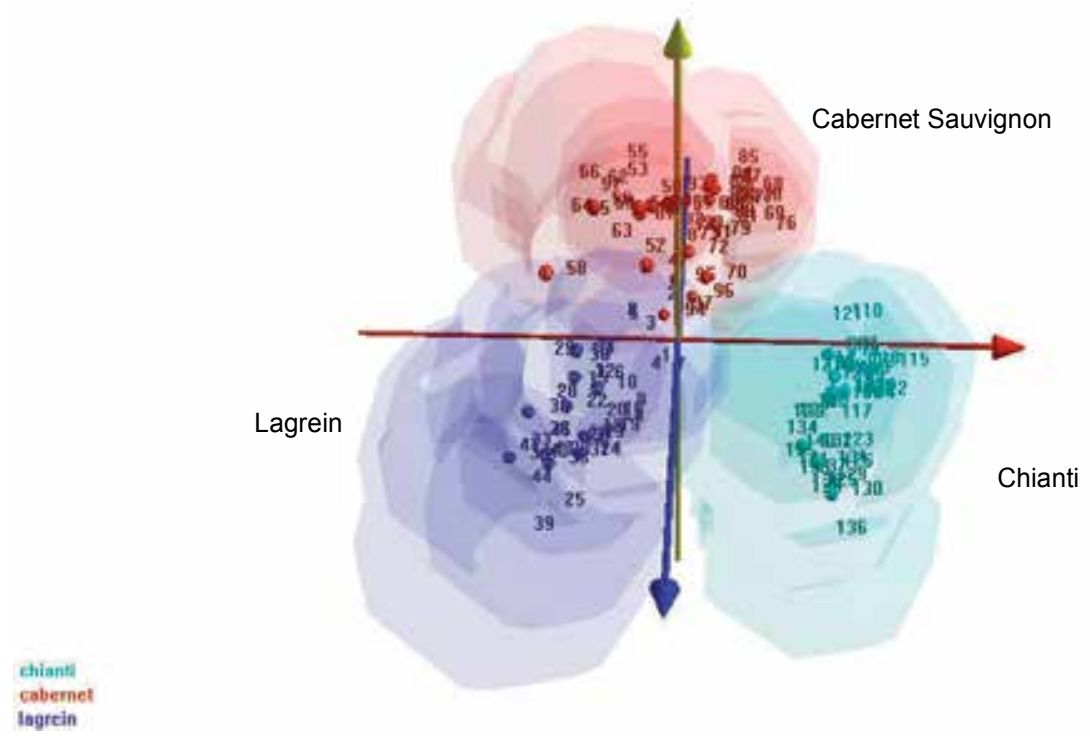

Figure 8. Factor plot of 141 specta of different wines (Lagrein, Chianti, Cabernet Sauvignon). Conditions: Normalisation, 1. derivative; wavenumber range, $4500-10000 \mathrm{~cm}^{-1}$; thickness $3 \mathrm{~mm}$; scans, 10 ; temperature, $23^{\circ} \mathrm{C}$.

Carbohydrates. Data preparation comprised normalization between 0 and 1 and following calculation of the first derivative using a wavenumber range from $4500-7548 \mathrm{~cm}^{-1}$. The PRESS function showed that 3 factors were needed for the calculation of the model. Calculation with 3 factors resulted in a good conformity between SEE and SEP. Linear regression between true and predicted values resulted in a value for the correlation coefficient of $R^{2}=0.99$ for calibration and $R^{2}=0.99$ for validation. Results for SEE and SEP: $0.13^{\circ} \mathrm{KMW}$ and $0.11^{\circ} \mathrm{KMW}$, the BIAS value is $2.30 \times 10^{-15}$.

Total acids. PLSR in a concentration range between 5 and $11 \mathrm{~g} / \mathrm{l}$ included normalization between 0 and 1, full multiplicative scatter correction (MSC) and calculation of the $1^{\text {st }}$ derivative (Taylor 3 points) between 4500 and $7548 \mathrm{~cm}^{-1} .3$ factors were necessary to obtain a minimum for PRESS and an agreement between SEE $(0.60 \mathrm{~g} / \mathrm{l})$ and SEP $(0.61 \mathrm{~g} / \mathrm{l})$ of nearly $100 \%$.The highly linear model allows determining the total acid content with a prediction error of $0.61 \mathrm{~g} / \mathrm{l}$.

Tartaric acid. After normalization, performing of the $1^{\text {st }}$ derivative over between 4500 and $7308 \mathrm{~cm}^{-1}$, four factors were used for creation of the highly linear model depicted in Figure $6 c$ with $R^{2}=0.91$ for calibration and $R^{2}=0.87$ for validation). Despite the small concentration range between 3.1 and $6.7 \mathrm{~g} / \mathrm{l}$ used for calibration this system allows to determine the tarta- 
ric acid content in grape variety with an absolute error of estimation of $0.40 \mathrm{~g} / \mathrm{l}$ and prediction of $0.54 \mathrm{~g} / 1$.

Malic acid. Malic acid often shows 2-5 times higher values compared to tartaric acid. Calibration between 2.9 and $7.0 \mathrm{~g} / \mathrm{l}$ after normalization between 0 and 1 and calculation of a second smoothened derivative was carried out using three factors, SEE and SEP showing acceptable agreement.Absolute values for SEE, and BIAS were $0.43 \mathrm{~g} / 1$ and $-4.25 \times 10^{-15}$. Straight line for calibration showed a linearity of $\mathrm{R}^{2}=0.89$ and allowed a prediction of the malic acid content with an absolute error of $0.55 \mathrm{~g} / \mathrm{l}$.

$p H$. Normalization and calculation of the smoothed $2^{\text {nd }}$ derivative between 4500 and 7308 $\mathrm{cm}^{-1}$ showed an optimum for BIAS at five factors. Despite the narrow calibration range of $\mathrm{pH} 3.09$ - 3.74 the calibration equation shows a $\mathrm{R}^{2}$ of 0.82 .

In order to enable the determination of these parameters with only one single measurement, simultaneous analysis of the carbohydrate, total acid, tartaric acid, malic acid content and $\mathrm{pH}$ was achieved by performing normalization (between 0 and 1 ) and calculating its $2^{\text {nd }}$ derivative (Taylor 3 points). Four factors over a wavenumber range from 4500 to $7308 \mathrm{~cm}^{-1}$ showed $73-100 \%$ agreement between SEE and SEP. Linear regression showed high linearity for each investigated parameter with slightly lower values for $\mathrm{R}^{2}$. Compared to the abovedescribed single analysis this method allows a quantitative analysis of all parameters at once within a few seconds. Values for SEP are slightly increased (Table 1).

Polyphenols mainly influence taste, sensory properties and color of a wine. Therefore, a rapid method to analyze its quantity is important. The method according to Folin - Ciocalteu was used as a reference method (see Materials and Methods). Gallic acid-1-hydrate was used as reference standard in a concentration range from 0 to $4.93 \mu \mathrm{g} / \mathrm{ml}$ with equidistant steps. 24 gallic acid-1-hydrate solutions in a concentration range between 0.442 and 7.08 $\mathrm{mg} / \mathrm{ml}$ were measured in the transmission mode threefold and in random order by NIRS. Evaluation using PLSR was achieved by dividing 72-recorded spectra randomly into a calibration (54 spectra) and validation (18 spectra) set. Data pretreatment comprised normalization between 0 and 1 and calculation of the $1^{\text {st }}$ derivative (Savitzky-Golay) between 4008-7512 $\mathrm{cm}^{-1}$. Using three factors, the PRESS function showed a minimum and a good agreement between SEE $(0.45 \mathrm{mg} / \mathrm{ml})$ and SEP $(0.46 \mathrm{mg} / \mathrm{ml})$. Linear regression between predicted and true values allowed to predict the gallic acid-1-hydrate concentration between 0 and $7 \mathrm{mg} / \mathrm{ml}$ with $\mathrm{R}^{2}=0.98$.

In order to determine the total polyphenol concentration 30 must samples were measured in the transmission mode threefold and in random order. 90 spectra were divided into 72 calibration and 18 validation spectra. Normalization and performing of the $1^{\text {st }}$ derivative allowed minimizing shifts in the baseline. 4 factors were necessary to obtain a minimum for the PRESS function and to get a maximum agreement of SEE and SEP. Linear regression allowed correlating true and predicted values with a $\mathrm{R}^{2}$ of 0.97 . Compared to the traditionally used Folin - Ciocalteu method in a winery, which is very time-consuming and expensive due to the usage of different chemicals, the NIRS method is very simple, precise and incomparably fast. 


\begin{tabular}{lccccccc}
\hline \multicolumn{1}{c}{ Parameter } & Unit & \multicolumn{2}{c}{ SEE } & & SEP & \multicolumn{2}{c}{ BIAS } \\
\hline Carbohydrates & $\mathrm{KMW}$ & 0.13 & 0.21 & 0.11 & 0.19 & $2.30 \times 10^{-15}$ & $3.33 \times 10^{-16}$ \\
\hline Total acids & $\mathrm{g} / \mathrm{l}$ & 0.60 & 0.43 & 0.61 & 0.53 & $7.17 \times 10^{-15}$ & $-1.08 \times 10^{-14}$ \\
\hline Tartaric acid & $\mathrm{g} / \mathrm{l}$ & 0.40 & 0.41 & 0.54 & 0.55 & $-1.08 \times 10^{-14}$ & $-3.43 \times 10^{-15}$ \\
\hline Malic acid & $\mathrm{g} / \mathrm{l}$ & 0.43 & 0.49 & 0.55 & 0.65 & $-4.25 \times 10^{-15}$ & $-2.44 \times 10^{-15}$ \\
\hline $\mathrm{pH}$ & & 0.07 & 0.09 & 0.06 & 0.09 & $-1.26 \times 10^{-15}$ & $-7.15 \times 10^{-15}$ \\
\hline
\end{tabular}

Note. a Single analysis; b Simultaneous analysis

Table 1. Prediction results for the determination of the carbohydrate, total acid, tartaric acid, malic acid content and $\mathrm{pH}$

Quality control of coffee ingredients including caffeine, theobromine and theophylline [20] and of food additives deriving from the highly interesting field of Traditional Chinese Medicine (TCM) [21] can be carried out in a similar way. Thereby, emphasis must be put onto the calibration method for which the above mentioned techniques can be applied as a reference. A new analytical method based on near infrared spectroscopy (NIRS) for the quantitation of the three main alkaloids caffeine (Caf), theobromine (Tbr) and theophylline (Tph) in roasted coffee after discrimination of the rough green beans into Arabic and Robusta was established. This validated method was compared to the most commonly used liquid chromatography (LC) connected to UV and mass spectrometric (MS) detection. As analysis time plays an important role in choosing a reference method for the calibration of the NIR-spectrometer, the non-porous silica-C18 phase offers a very fast method. Coupling of the optimised LC method to a mass spectrometer (MS) via an electrospray ionisation (ESI) interface not only allowed to identify Caf, Tbr and Tph by their characteristic fragmentation pattern using collisionally induced dissociation (CID), but also to quantitate the content of the three analytes, which was found to be $6 \%$ higher compared to UV-detection. The validated LC-UV method was chosen as a reference method for the calibration of the NIRS system. Analysis of 83 liquid coffee extracts in random order resulted for Caf and Tbr in values for S.E.E. (standard error of estimation) of $0.34,0.40 \mathrm{~g} / 100 \mathrm{~g}$, S.E.P. (standard error of prediction) of 0.07 and 0.10 $\mathrm{g} / 100 \mathrm{~g}$ with correlation coefficients of 0.86 and 0.85 in a concentration range between 0.10 and $4.13 \mathrm{~g} / 100 \mathrm{~g}$. Compared to LC the lower limit of detection (LOD) of the NIRS-method is found at $0.05 \mathrm{~g} / 100 \mathrm{~g}$ compared to $0.244-0.60 \mathrm{ng} / 100 \mathrm{~g}$ in LC, which makes it impossible to analyse Tph by NIRS.

The possibility to hyphenate a MIR/NIR spectrometer to a microscope unit allows determining the distribution of active ingredients within a tissue sample down to a resolution of $1.2 \mu \mathrm{m}$ [22]. A "hyperspectral cube" is recorded with the dimensions of the sample on the $x$ - and y-axis and the absorbance on the z-axis from which the image can be extracted (Figure9).

Fourier Transform Infrared (FTIR) spectroscopic imaging and mapping techniques have become essential tools for the detection and characterization of the molecular components of 
biological tissues and the modern analytical techniques enabling molecular imaging of complex samples. These techniques are based on the absorption of IR radiations by vibrational transitions in covalent bonds and their major advantage is the acquisition of local molecular expression profiles, while maintaining the topographic integrity of the tissue by avoiding time-consuming extraction, purification and separation steps. These new techniques enable global analysis of biological samples with high spatial resolution and provide unique chemical-morphological information about the tissue status. With these non-destructive examination methods it is possible to get qualitative and quantitative information of heterogeneous samples.

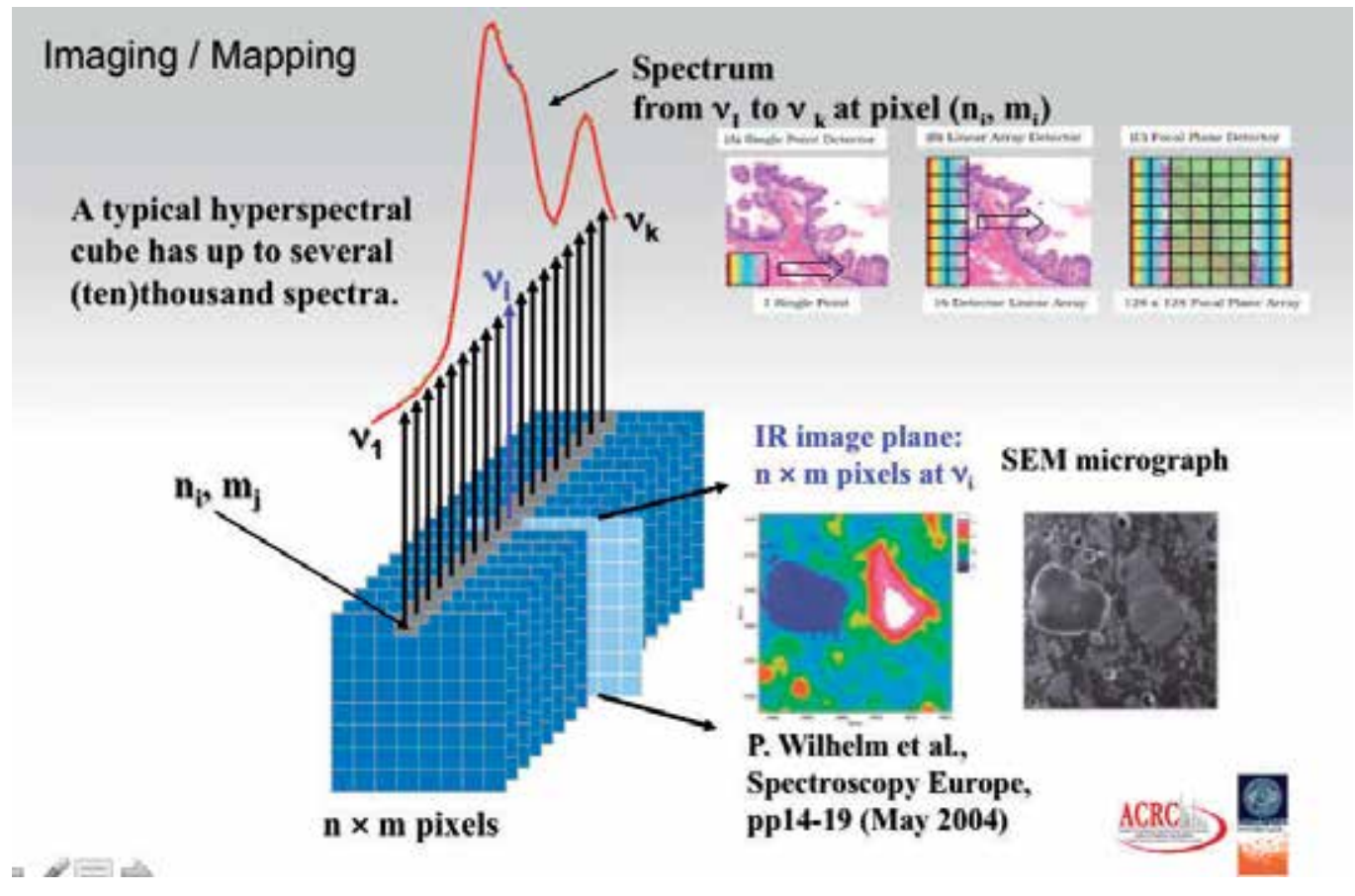

Figure 9. Principle of hyperspectral cube

Additionally, MALDI-TOF/MS imaging can be applied from the same sample of interest to get knowledge concerning the molecular weight distribution. This method is also suitable for studying the effect of nutrition onto different kinds of diseases, e.g. prostate cancer.

\section{Conclusions}

The techniques described can be applied according to the scheme depicted in Figure 1. This systematic analytical strategy allows getting multifacial knowledge and insights into food and samples derived therefrom. 


\section{Acknowledgements}

This work was financially supported by the Efre-project "Originalp" (EU) and Agrarmarketing Austria (AMA, Vienna, Austria).

\section{Author details}

Christian W. Huck

Institute of Analytical Chemistry and Radiochemistry, CCB - Center for Chemistry and Biomedicine, Innsbruck, Austria

\section{References}

[1] Huck C.W., Popp M., Scherz H., Bonn G.K.Development and Evaluation of a New Method for the Determination of the Carotenoid Content in Selected Vegetables by HPLC and HPLC-MS/MS.J. Chromatogr. Sci. (2000). , 38-441.

[2] Stöggl, W. M., Huck, C. W., Scherz, H., Bonn, G. K., Analysis, of., Vitamin, E., in, Food., Phytopharmaceutical, Preparations., by, H. P. L. C., -A, H. P. L. C., -M, P. C. I., \& , S. M. S. Chromatographia (2001). , 54-179.

[3] JakschitzT., HuckC.W., LubbadS., Bonn G.K.Monolithic Poly(TMSM-DMSBMS) Stationary Phases for the fast Separation of Oligonucleotides and Proteins.J. Chromatogr. A (2007). , 1147-53.

[4] Huck C.W., Bakry R., Bonn G.K.Progress in capillary electrophoresis of biomarkers and metabolites between 2002 and 2005.Electrophoresis (2006). , 27-111.

[5] Stöggl W.M., Huck C.W., Stecher G., Bonn G.K., Capillary Electrochromatography of Biologically Relevant Flavonoids. (2006). Electrophoresis, 27-787.

[6] Huck C.W., Bonn G.K.Review: Polymer based sorbents for solid-phase extraction.J. Chromatogr. A (2000). , 885-51.

[7] Feuerstein I., Najam-ul-Haq M., Rainer M., Trojer L., Bakry R., Hidayat Aprilita N., Stecher G., Huck C.W., Klocker H., Bartsch G., Guttman A., BonnG.K.Material Enhanced Laser Desorption/Ionization (MELDI)- a new protein profiling tool utilizing specific carrier materials for TOF-MS Analysis.J. Am. Soc. Mass Spectrom. (2006). , 17-1203.

[8] Petter C.H., Heigl N., Bachmann S., Huck-Pezzei V.A.C., Najam-ul-Haq M., Bakry R., Bernkop-Schnürch A., Bonn G.K., Huck*C.W.Near Infrared Spectroscopy Compared to Liquid Chromatography Coupled to Mass Spectrometry and Capillary Electrophoresis as a Detection Tool for Peptide Reaction Monitoring.Amino Acids (2008). 
[9] Rainer, M., Sonderegger, H., Bakry, R., Huck, C. W., Morandell, S., Huber, L. A., Gjerde, D. T., \& Bonn, G. K. Analysis of protein phosphorylation by monolithic extraction columns based on poly(divinylbenzene) containing embedded titanium dioxide and zirconium dioxide nano-powders. Proteomics (2008). , 8(21), 4593-4602.

[10] Hahn, H., Rainer, M., Ringer, T., Gjerde, D., Huck, C. W., \& Bonn, G. K. Ultra-fast Microwave-Assisted In-Tip Digestion of Proteins. J. Proteom. Res. (2009). , 8(9), 4225-4230.

[11] Bonn, G. K., Bakry, R., Huck, C. W., Vallant, R., \& Szabo, Z. Analysis of low molecular weight molecules by maldi-ms. Eur. Pat. Appl. ((2008). pp. CODEN: EPXXDW EP 1973142 A1 20080924 AN 2008:1151584

[12] Greiderer A., Clark Ligon S.Jr., Huck C.W., Bonn G.K.Monolithic poly( -bis(p-vinylphenyl)ethane capillary columns for simultaneous separation of low- and high-molecular weight compounds.J.Sep. Sci. (2009).

[13] Greiderer A., Trojer L., Huck C.W., Bonn G.K.Influence of the Polymerisation time on the Porous and Chromatographic Properties of Monolithic Poly(1,2-bis(p-vinylphenyl)ethane Capillary Columns.J.Chromatogr. A (2009). , 1216(45), 7747-7754.

[14] Heigl, N., Greiderer, A., Petter, C. H., Siesler, H. W., Bonn, G. K., \& Huck, C. W. Simultaneous Determination of Physical and Chemical Parameters of Monolithic Porous Polymers with a Combined Use of Fourier-Transform Near Infrared Diffuse Reflection Spectroscopy and Multivariate Techniques. Anal. Chem. (2008). , 80(22), 8493-8500.

[15] Trojer, L., Greiderer, A., Bisjak, C. P., Wieder, W., Heigl, N., Huck, C. W., Bonn, G. K., in, Handbook., of, H. P. L. C., Second, Edition., edited, by., Corradini, D., \& Press, . (2010). Print 978-1-57444-554-1eBook ISBN: 978-1-4200-1694-9, 3-45.

[16] Bachmann S., Vallant R., Bakry R., Huck C.W., Corradini D., Bonn G.K.Capillary electrophoresis coupled to matrix assisted laser desorption ionization with novel covalently coated capillaries.Electrophoresis, (2010). , 31, 618-629.

[17] Stöggl W.M., Huck C.W., Stecher G., Bonn G.K.Capillary Electrochromatography of Biologically Relevant Flavonoids.Electrophoresis, (2006). , 27-787.

[18] Petter C.H., Heigl N., Bachmann S., Huck-Pezzei V.A.C., Najam-ul-Haq M., Bakry R., Bernkop-Schnürch A., Bonn G.K., Huck C.W.Near Infrared Spectroscopy Compared to Liquid Chromatography Coupled to Mass Spectrometry and Capillary Electrophoresis as a Detection Tool for Peptide Reaction Monitoring.Amino Acids, (2008). , 34(4), 605-616.

[19] Guggenbichler, W., Huck, C. W., Kobler, A., Bonn, G. K., Near, Infrared., Reflectance, Spectroscopy., Tool, A., for, Quality., Control, In., \& Wine, Production. J. Food Agric. Environm., (2006). , 4(2), 98-106.

[20] Huck C.W., Guggenbichler W., Bonn G.K., Analysis of Caffeine, Theobromine and Theophylline in Coffee by Near Infrared Reflectance Spectroscopy (NIRS) Compared 
to High Performance Liquid Chromatography (HPLC) Coupled to Mass Spectrometry.Anal. Chim. Acta, (2005). , 538-195.

[21] Mattle, C., Heigl, N., Bonn, G. K., \& Huck, C. W. Near Infrared Diffuse Reflection Spectroscopy and Multivariate Calibration Hyphenated to Thin Layer Chromatography for Quality Control and Simultaneous Quantification of Methoxylated Flavones in a Phytomedicine. J. Planar Chromatogr., (2010). , 23(5), 348-352.

[22] Pezzei, C., Pallua, J. D., Schaefer, G., Seifarth, C., Huck-Pezzei, V., Bittner, L. K., Klocker, H., Bartsch, G., Bonn, G. K., Huck, C. W., Prostate, cancer., characterization, by., Fourier, Transform., \& infrared, microspectroscopy. Mol. Biosys., (2010). , 6-2287. 


\section{Section 3}

\section{Quality Control in Pharmaceutics}



Chapter 9

\title{
Microbial Quality \\ Concerns for Biopharmaceuticals
}

\author{
Farzaneh Lotfipour and Somayeh Hallaj-Nezhadi \\ Additional information is available at the end of the chapter \\ http://dx.doi.org/10.5772/52114
}

\section{Introduction}

Finding an appropriate definition or a clear classification for biologically occurring pharmaceutical products is a complicated task because of overlapping borders and consequent misconceptions in this area. Indeed, numerous definitions and classifications for this category of products have been proposed so far, and different points of view for this concept can be found in research literature, business, industry, and even the general public [1, 2].

To obtain a better view of biopharmaceutical concept, first, it is necessary to know the present definitions for the main constituents of the word, that is, pharmaceutical product and biological product.

According to the $\mathrm{WHO}$, a finished pharmaceutical product (FPP) is "A finished dosage form of a pharmaceutical product, which has undergone all stages of manufacture, including packaging in its final container and labeling." [3]

An active pharmaceutical ingredient can be defined as "A substance used in a finished pharmaceutical product (FPP), intended to furnish pharmacological activity or to otherwise have direct effect in the diagnosis, cure, mitigation, treatment or prevention of disease, or to have direct effect in restoring, correcting or modifying physiological functions in human beings." [3] Hence, in brief, it can be said that any material, regardless of its origin or structure, with treatment, diagnosis, or prevention applications and passing regulatory requirements, is a pharmaceutical product.

On the other hand, the FDA definition for biological products is as follows: "Biological products or biologics are medical products made from a variety of natural sources (human, animal or microorganism). Like drugs, some biologics are intended to treat diseases and 
medical conditions or to prevent or diagnose diseases." [4] Consequently, any product of biological origin with treatment, diagnosis, or prevention applications is a biological product.

A biotechnology-derived product is another concept that should be taken into consideration. It is defined by Walsh as "any pharmaceutical product used for a therapeutic or in vivo diagnostic purpose, which is produced in full or in part by either traditional or modern biotechnological means." [5]

On comparing the definitions for a biotechnology-derived product and a biological product, both of which should be of biological origin, it is obvious that the key element in the former definition is the application of biotechnological means for production.

In practice, the regulatory requirements needed for a biotechnology-derived product and a biological product are methodologically different from the pharmaceutical product due to their biological essence. For example, the determination of adventitious agents such as viruses, transmitting spongiform encephalopathy (TSE), and mycoplasma are included in most of the related guidelines and pharmacopeias for a biotechnology-derived product and a biological product.

Finally, a biopharmaceutical is defined by Walsh as "A protein or nucleic acid based pharmaceutical substance used for therapeutic or in vivo diagnostic purposes, which is produced by means other than direct extraction from a native (non-engineered) biological source." This definition that will be used in the present chapter for biopharmaceuticals includes all pharmaceutical products produced by modern biotechnology techniques as well as nucleic acid (DNA or RNA) based pharmaceutical products for gene therapy. Hence, the overlapping area between biotechnology-derived products and biopharmaceuticals is the application of modern biotechnological means in their production. However, the differentiating area can be the application of traditional biotechnological means for the production of biotechnology-derived products. In addition, nucleic acid-based pharmaceutical products that are categorized as biopharmaceuticals are not biotechnology-derived products [6]. Figure 1 illustrates these overlapping and differentiating areas. Moreover, some examples of products in these categories are shown in Table1.

\begin{tabular}{|c|c|c|c|}
\hline & \multicolumn{2}{|c|}{ Biotechnology-derived product by: } & Biopharmaceuticals \\
\hline Biological products & Traditional technology & Modern technology & Recombinant proteins \\
\hline Blood and blood products & $\begin{array}{c}\text { Therapeutic proteins from } \\
\text { natural sources }\end{array}$ & Recombinant proteins & $\begin{array}{c}\text { Nucleic acid-based } \\
\text { Human cells and tissues } \\
\text { from microorganisms }\end{array}$ \\
\hline
\end{tabular}

Table 1. Some examples of products related to biological, biotechnology-derived, and biopharmaceutical products. 


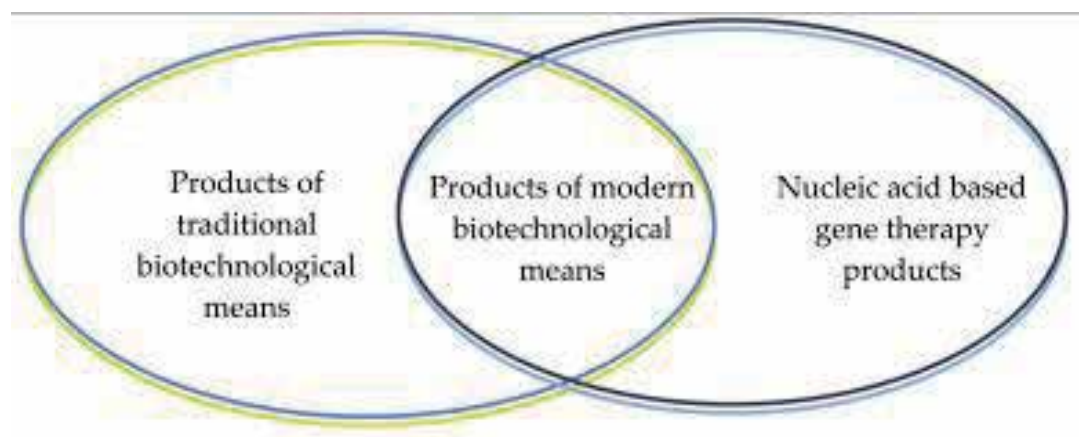

Biotechnology derived product

Biopharmaceutical

Figure 1. The schematic representation for biotechnology-derived products and biopharmaceutical categories and the overlapping areas between them.

\section{Methods for biopharmaceutical production}

\subsection{Production of an original recombinant system}

\subsubsection{Recombinant DNA technology}

The main category of biopharmaceuticals is manufactured via recombinant DNA technology. Indeed, recombinant DNA technologies are enabling techniques that manipulate and engineer different gene fragments and which have been introduced less than 50 years ago by the revolutionary invention of Polymerase Chain Reaction (PCR) by Kary Mullis [7].

DNA and RNA extraction from different cell types, cutting DNA fragments using restriction endonucleases, joining DNA fragments by DNA ligases, PCR to amplify gene fragments, cloning of the gene fragments into different vectors, introduction of recombinant constructs into proper hosts, protein expression, extraction, and purification are some of the most widely used means in recombinant protein production.

Figure 2 schematically represents the summarized process of production of a recombinant protein. As can be seen from the chart, first, the gene of interest should be isolated and amplified from the original cell. According to the type of the cell, it can be done through direct total DNA extraction followed by a PCR using proper primers to obtain the gene in prokaryotes. On the other hand, in eukaryotes, due to the existence of introns and some modifications that occur in the transcribed mRNA, the process is considerably complicated. Introns are non-coding sequences which are removed after transcription versus coding sequences 
that are called exons. In addition, mRNA is more modified by the addition of a methylated guanine (CAP) on its 5'end and a poly-adenine tail on its 3'end. After these modifications, mature mRNA is exported to the cytoplasm in order to start the translation process. Consequently, to obtain a gene of interest in eukaryotes, the mature mRNA should be extracted from the cell, and the complementary DNA should be synthesized followed by amplification of the gene by PCR using proper primers. However, in both cases (prokaryotes and eukaryotes), the short genes can be obtained by a solid-phase synthesis process.

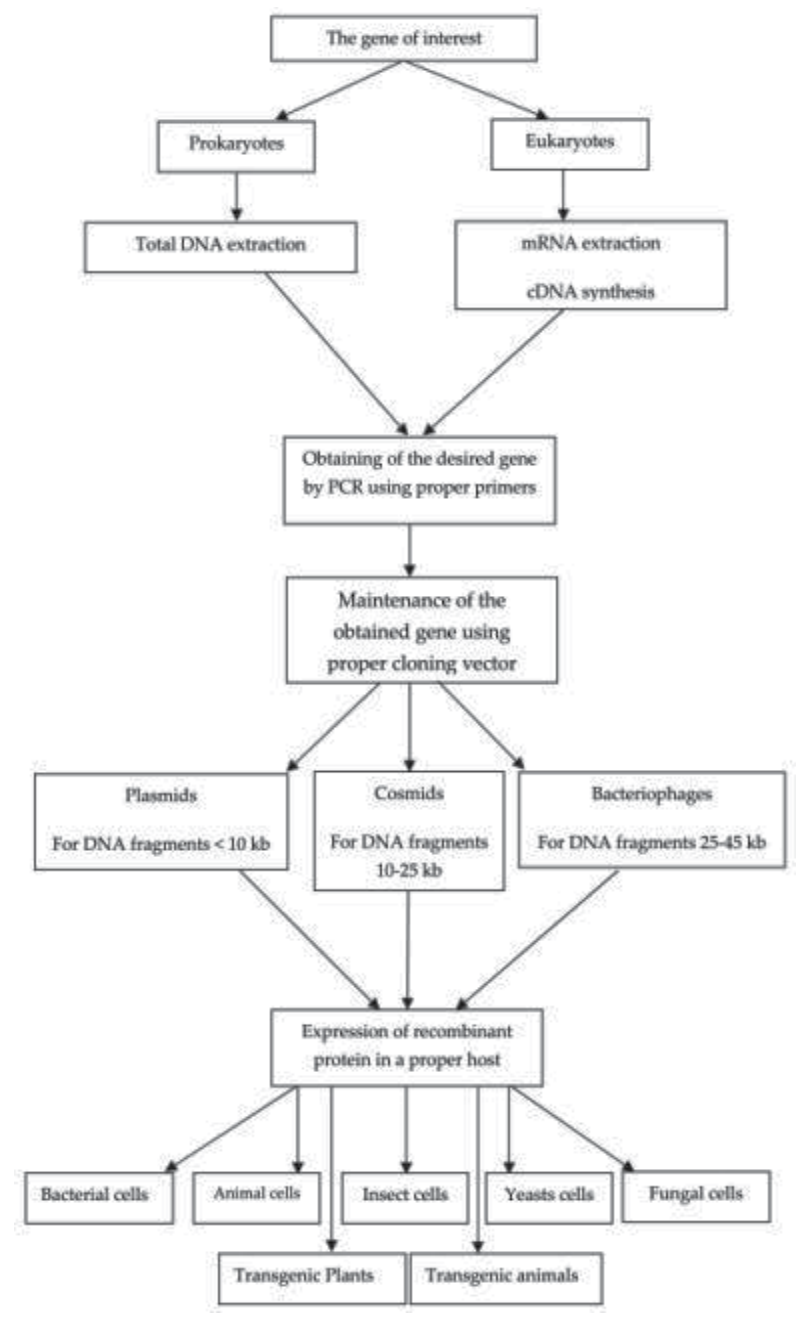

Figure 2. Schematic representation of recombinant protein production process

Based on their sizes, the obtained genes can now be introduced into a proper vector for maintenance, replication, or expression purposes. Plasmids, cosmids, and bacteriophages are the most important cloning vectors that are classified according to the size of the DNA 
fragment that can be inserted into them. Ultimately, the new recombinant construct should be introduced into an expression system for production. Different classes of expression systems with their certain merits and disadvantages are available and range from cell systems such as bacterial, animal, fungal, and yeast cells to transgenic systems such as transgenic plants and animals $[7,8]$.

\subsubsection{Monoclonal antibody production}

\subsubsection{Classical hybridoma technology}

Each specific antibody is secreted by a specific B cell and could recognize a specific region on the antigen that is called epitope. Each antibody-secreting B cell could be used as a source of an antibody of interest if it is isolated and cultured in vitro. Nevertheless, B cells are not considered a satisfactory source, as they are not immortalized and cannot survive for a long time. The main approach for overcoming this problem is cell hybridization, which includes the fusion of antibody-secreting B cells with tumor cells (such as mouse myeloma cells) proliferating ever more. The classical hybridoma technology was first introduced by Georges Kohler and Cesar Milstein in the mid 1970s for the generation of immortalized hybridoma cells that could grow in cell culture for a long time and produce the desired monoclonal antibodies $[9,10]$. The basic process (Figure 3) includes the immunization of a mouse with the desired antigen. The mouse was then sacrificed, and B lymphocytes secreting antibodies that were selective for the specific epitope on the antigen were isolated from the spleen. The spleen is considered the most ready source for antigen-specific lymphocytes that provides access to a large number of antibody-secreting cells [1]. The isolated B cells were subsequently fused with immortal mouse myeloma cells. The resultant hybridoma cells were then separated from the unfused cells by culturing in specific cell culture media. The cell culture media for the hybridoma growth and production of monoclonal antibodies have been reviewed in detail by Bols et al. [11].

In general, for the successful fusion of hybridomas, the cells are grown in HAT selection medium. The selection medium is called HAT, as it has Hypoxanthine, Aminopterin, and Thymidine. This is because a mutation in either the hypoxanthine-guanine phosphoribosyltransferase (HGPRT) or the thymidine kinase (TK) gene of the cells would cause their death in the HAT medium. Generally, a TK-deficient cell (TK-negative mutant) is resistant to bromodeoxyuridine (BrdU), and an HGPRT-deficient cell (HGPRT-negative mutant) is resistant to 6-thioguanine (6-TG) and 8-azaguanine. Hence, in order to make myeloma cells sensitive to HAT (unable to grow in HAT media), they are treated with one of these drugs before their passage to HAT media.

Normal cells can synthesize the required nucleotides in two pathways: (1) the main one or de novo biosynthetic pathway, and (2) the alternative one or the salvage pathway (when the main pathway is blocked).

Aminopterin (a folic acid analog that inhibits dihydrofolate reductase) blocks the activation of tetrahydrofolate, which is required for the synthesis of nucleotides via the de novo synthetic pathway, and, therefore, the main pathway is blocked. Thus, in aminopterin-treated 
cells $\left(\mathrm{HGPRT}^{+}\right.$and $\mathrm{TK}^{+}$), the synthesis of nucleotides shifts to the salvage pathway only if hypoxanthine and thymidine are supplied in the medium. HGPRT and TK, the two enzymes, are required for the salvage pathway, and they catalyze the synthesis of purine and thymidylate from hypoxanthine and thymidine substrates, respectively.

Since unfused myeloma cells lack HGPRT or TK, they cannot use the salvage pathway. Thus, the unfused myeloma cells get killed in the HAT medium, as both biosynthetic pathways are blocked. Normal unfused B cells die in the HAT medium, as they are not immortalized and cannot grow for a long time. Nevertheless, the fusion of normal B cells with the HGPRT'or TK'myeloma cells allows the hybridoma cells to grow in HAT medium, as the B cells provide the necessary enzymes for growth of the hybridoma cells.

Hence, the HAT selection medium offers an ideal environment for the isolation of fused myeloma and B cells (hybridoma cells) from unfused myeloma cells and unfused B cells, as this medium allows only the hybridoma cells to survive in the culture.

The production of monoclonal antibodies could be accomplished by ascites (ascitic fluid) production (in vivo) or by cell culture (in vitro) methods. In the in vivo method, hybridoma cells are injected intraperitoneally into mice. The peritoneum serves as a growth chamber for the injected cells. These cells could secrete a high-titered solution of desired antibodies as they grow in the cavity. Finally, the produced antibodies are extracted from the ascitic fluid accumulated in the peritoneal cavity [6]. The antibody concentrations typically range between 1 and $15 \mathrm{mg} / \mathrm{ml}$. The in vivo method offers a very high concentration of monoclonal antibody that often does not need more concentration procedures. Nevertheless, monoclonal antibodies produced by this technique may be contaminated by considerable levels of mouse proteins and other contaminants that might require more complicated, subsequent downstream purifications. The other disadvantage of the ascites production is related to animal welfare issues, as these could cause distress in mice.

Currently, more than $90 \%$ of monoclonal antibodies are produced by in vitro techniques [12] that use large-scale manufacturing plants containing several 10,000-L or larger culture bioreactors [13]. The in vitro method of monoclonal antibody production decreases the use of mice and also avoids the need for experienced personnel for animal handling. Regardless of the privileges and importance of the in vitro methods of antibody production, there are some situations in which this method is not applicable; for instance:

1. Hybridoma cells do not adapt well to in vitro conditions.

2. Downstream purification methods cause protein denaturation or decreased antibody activity.

3. The cell line cannot maintain the production of monoclonal antibodies.

4. When hybridoma cells are contaminated with infectious agents (such as yeasts or fungi), the cells must often be passed through mice. Since removal of the organisms cannot be accomplished by current antimicrobial drugs, thus the in vivo method may save a valuable hybridoma. 
5. When in vitro methods result in monoclonal antibodies that are glycosylated at positions different from those harvested from mouse ascites, they affect antigen-binding capacity as well as biological functions [12].

Taken together, the cell culture technique is a method of choice for large-scale monoclonal antibody production due to the simplicity of the cell culture and financial considerations without ethical concerns that are related to animal use.

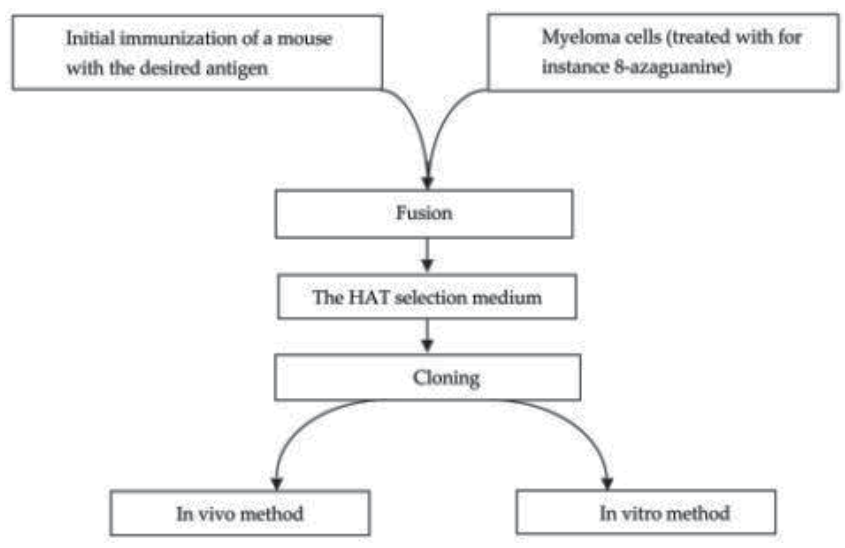

Figure 3. The diagram of the monoclonal antibody production via classical hybridoma technology

\subsubsection{Chimeric and humanized antibodies}

In 1986, about 10 years after the conception of monoclonal antibody technology, Orthoclone OKT3 was approved by the FDA for use in patients with acute rejection of a transplanted kidney [14]. Unfortunately, early clinical applications of murine monoclonal antibodies were disappointing. This was due to the fact that monoclonal antibodies produced via the classical method are of murine origin and are, therefore, immunogenic to human subjects. In general, patients receiving an antibody exhibit HAMA responses (human anti-mouse antibodies) within two weeks. Multiple infusions of murine monoclonal antibodies significantly enhance the HAMA reactions [6]. In addition, the immune system eliminates the murine monoclonal antibody molecule. Thus, murine monoclonal antibodies demonstrate short serum half lives after administration to humans. Furthermore, the other main difficulty related to murine monoclonal antibodies is the poor recognition of the Fc region by human effector systems of complement and Fc receptors.

Thus, new strategies that are used for producing humanized mouse antibodies that are less immunogenic have been discovered. The first strategy includes the production of functional specific recombinant IgG molecules consisting of mouse variable regions and human constant regions; these are known as chimeric antibodies. Taken together, in the chimeric antibody, 8 out of 12 domains are of human origin (constant regions of the heavy and light chains) $\left(C_{H}\right.$ and $\left.C_{L}\right)$ [15]. Chimeric antibodies exhibit reduced HAMA responses compared 
with mouse antibodies, but the affinity and the selectivity are the same. Furthermore, since the $\mathrm{Fc}$ region contains human sequences, the activation of Fc-mediated immune effector functions is allowed.

To further minimize the antigenicity of murine antibodies, humanized antibodies were developed. For their generation, hyper-variable complementarity-determining regions (CDRs) of the specific murine antibody are transferred to a fully human framework. In comparison with the mouse antibodies, humanized antibodies suggest a lower occurrence of HAMA responses.

Further efforts have been invested in the development of technologies that generate fully human monoclonal antibodies. One of the approaches entails the development of transgenic mice, in which a repertoire of human immunoglobulin germline gene segments is inserted into the mouse genome. After the immunization of these mice, they produce fully human antibodies, which can subsequently be separated with the classical hybridoma technology [15].

Figure 4 illustrates the schematic structures of mouse, chimeric, humanized, and human antibodies.

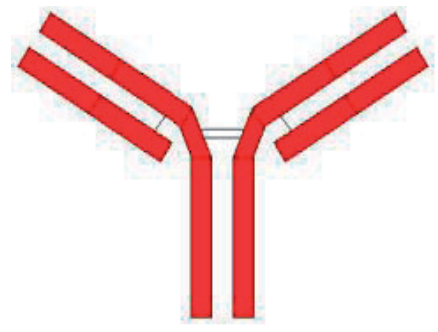

Mouse

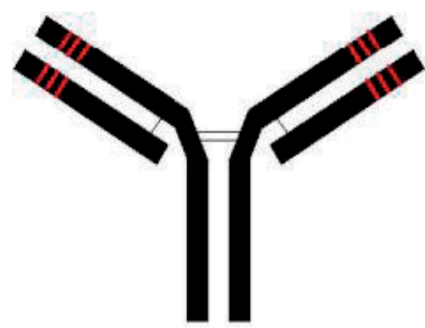

Humanized

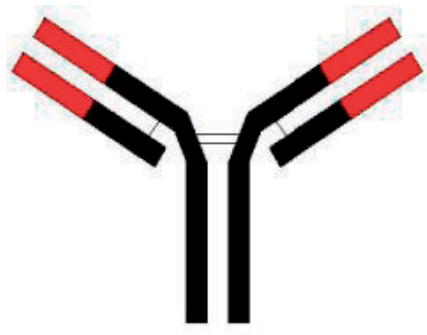

Chimeric

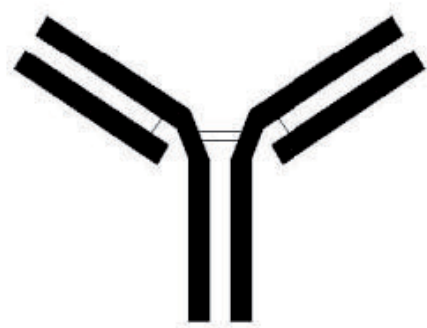

Human

Mouse CDRs

Figure 4. The structure of mouse (shown in red color), chimeric, humanized (shown in black color), and human antibodies. Chimeric antibodies comprise mouse variable regions and human constant regions. Humanized antibodies consist of murine hyper-variable complementarity-determining regions (CDRs) that are grafted to fully human framework. 


\subsection{Mass production of a recombinant product}

Mass production of recombinant products can be achieved in a process that is divided into two main sections called upstream and downstream processing, as schematically depicted in Figure 5 .

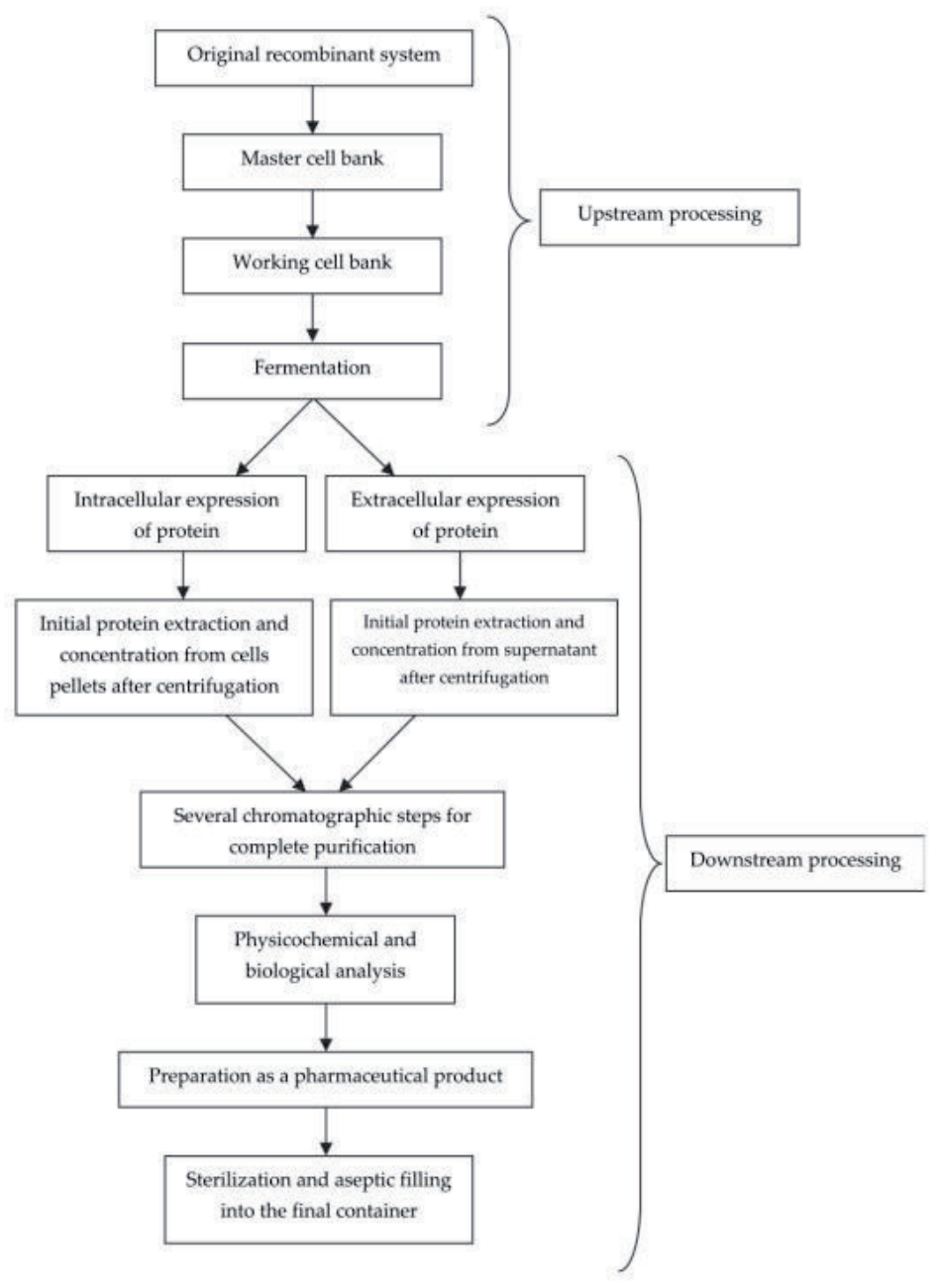

Figure 5. Mass production of a recombinant product.

The first step, the upstream processing step, is the mass production of a recombinant protein via the fermentation process. The original recombinant system that is used for the expression of the recombinant protein (i.e. in the form of a recombinant plasmid maintained in a suitable host cell) forms the cell deposit in a cell banking system. There are two levels of cell deposits in the cell banking system: The first line is called master cell bank, which is prepared 
directly from a culture of the original recombinant system that includes several hundred stored ampoules.

The second line that is referred to as the working cell bank is produced from a single master cell bank ampoule. Each ampoule from the working cell bank is thawed and used to seed the fermentor for the production of a batch. Obviously, the fermentation process for various recombinant systems, such as bacterial fermenting systems or animal cell systems, is quite different and needs its own certain requirements.

On the other hand, downstream processing, which is the next step, refers to the purification of the mass produced protein. The first step in downstream processing is the initial extraction and concentration of the product, which depends on the situation of the expressed protein; that is, it should be extracted from the pellet cells for intracellular proteins or from the supernatant for extracellular proteins after centrifugation.

The second stage entails (1) several chromatographic steps that complete the purification of the product; (2) the potency test; (3) the addition of suitable excipients; (4) sterilization; (5) filling of the product in its final form (liquid or solid) into the final container before sealing; and labeling [6].

\section{Sources of biological contamination of biopharmaceuticals}

In line with conventional pharmaceutical products, the main sources of biological contamination in biopharmaceuticals can be related to raw materials and the production environment. Indeed, the biological contaminant content of any pharmaceutical product is a representative of their starting materials and the production environment flora.

\subsection{Raw materials}

Animal origin materials, such as cell culture media, sera, and supplements that are extensively used in biopharmaceutical production, are of high contamination risk. These materials can be considered the main source for the contamination of biopharmaceuticals with adventitious agents such as TSEs, viruses, and mycoplasmas. Therefore, they should be supplied from reliable resources, and special attention should be paid to their quality control procedure. It should be ensured that all raw materials, especially those of high risk, gain quality specifications for current good manufacturing practice.

Standard methods for sterilization of cell culture media, sera, and supplements should be established according to the properties of the materials. Due to the heat-labile nature of the majority of materials used in biopharmaceutical production, autoclaving is usually replaced with alternative strategies such as filter-sterilization or less frequently high-temperature, short-time treatment strategies. In spite of the routine filter-sterilization procedure that uses $0.22 \mu \mathrm{m}$, it is usually performed with $0.1-\mu \mathrm{m}$ membrane filters due to the risk of contamination with adventitious agents. 
Furthermore, high-temperature, short-time treatment strategies are sometimes employed for the elimination of biological contaminants from small solutes such as vitamins and amino acids [16].

Another important raw material that is used in the production of any pharmaceutical product, including biopharmaceuticals, is water, which can be considered an important source for contamination, with water-borne bacteria such as Pseudomonas spp., Alcaligenes spp., Flavobacterium spp., Chromobacter spp., and Serratia spp. Water for pharmaceutical purposes is discussed in detail in the USP [17]. Due to the fact that the intended administration of biopharmaceuticals in the majority of cases is via injection, Water for Injection (WFI) which is sterile and apyrogen is routinely used in this area.

\subsection{Production environment}

Pharmaceutical products' contamination may occur from the transformation of microorganisms from the production environment to the product. The production environment includes air, surfaces, instruments, equipments, and personnel.

The main groups of microorganisms that are isolated from air are the spore-forming bacteria (Bacillus spp. and Clostridium spp., the non-sporing bacteria Staphylococcus spp., Streptococcus spp., and Corynebacterium spp.), the molds (Penicillium spp., Cladosporium spp., Aspergillus spp., and Mucor spp.), and the yeast (Rhodotorula spp.). These contaminants may be air borne or can be initiated from process equipment or personnel [16]. Consequently, environmental monitoring programs in a production environment are essential actions. Furthermore, the critical operations in biopharmaceutical production should be performed in controlled environments or clean rooms. A clean room is a place with high control of the entrance of particles via the establishment of some air filters called high-efficiency particulate air (HEPA) filters. HEPA filters made from a microglass material with a pleated construction system provide a large surface area that efficiently filters the incoming air and generates a constant air motion. Based on the permitted quantity of viable microorganisms and particulates, various classes of clean rooms can be established using HEPA filters with required efficiencies.

According to the EC Guide to Good Manufacturing Practice for Medicinal Products (EC GGMP), four grades for clean rooms are available, such as grade A, B, C, or D, based on the number of viable microorganisms and particulates (Table 2).

\begin{tabular}{|c|c|c|}
\hline Clean room grade & $\begin{array}{c}\text { Maximum permitted number of } \\
\text { particles } / \mathbf{m}_{3}\end{array}$ & $\begin{array}{c}\text { Maximum permitted CFU of viable } \\
\text { microorganisms } \mathbf{m}_{3} \text { in air sample }\end{array}$ \\
\hline A & 3500 & $<1$ \\
\hline B & 3500 & 10 \\
\hline C & 350000 & 100 \\
\hline D & 3500000 & 200 \\
\hline
\end{tabular}

Table 2. Clean room grades according to the number of viable microorganisms and the number of particulates 
Critical operations such as inoculum preparation and aseptic filling are generally performed in the highest air grade (A); however, less critical operations can be performed in lower grades or even non-classified air.

In addition to the establishment of suitable filters in the clean rooms, special attention should be paid to the position, type, and texture of surfaces, floors, and fixtures. They should be made from smooth and chemically stable materials. In addition, a distinct transfer lock area should exist before entry to the clean room for sanitization of materials and personnel or garment changing. Furthermore, all doors should be interlocking [6].

\section{Hazards of biological contamination of biopharmaceuticals}

Similar to other pharmaceuticals, biological contamination of biopharmaceuticals may perhaps cause product spoilage. It may result in product metabolization by microorganisms, and, therefore, lead to a decrease in biopharmaceutical potency. The product spoilage may also provide a potential health hazard to patients and lead to outbreaks of infections that may cause additional complications. In addition, microbial-derived agents secreted in products such as endotoxins can be hazardous to a patient's health.

\section{Determination of biological contaminants}

\subsection{Bacteria and fungi}

Bacteria and fungi can be considered important contamination sources for all kinds ofpharmaceutical products, including biopharmaceuticals; hence, the control of them is of critical importance. The control of both bacteria and fungi is considered to be worthy of mandatory tests for nearly all kinds of pharmaceuticals in pharmacopoeias. All the related tests and procedures are covered in detail in the major pharmacopoeias such as USP and EP $[17,18]$.

Since almost all the biopharmaceuticals are administered intravenously, general sterility testing must be carried out for these products. Basically, sterility testing can be defined as "a test that evaluates whether a sterilized pharmaceutical product is free of contaminating microorganisms." The European Pharmacopoeia (2002) proposes two media for sterility testing:

(1) fluid mercaptoacetate medium (also known as fluid thioglycollate medium), which is mainly appropriate for the culture of anaerobic organisms at $30-35^{\circ} \mathrm{C}$; and (2) soyabean casein digest medium, which is used for the culture of both aerobic bacteria at $30-35^{\circ} \mathrm{C}$ and fungi at $20-25^{\circ} \mathrm{C}$.

Two main methods are used for sterility tests: (1) direct inoculation of the test samples in the media mentioned earlier; or (2) filtration of the test material through a sterile membrane filter with a pore size of $0.45 \mu \mathrm{m}$; then, the filter containing any microorganism present in the fluids is divided aseptically, and portions are transferred to the media. 
The eradication of bacteria and fungi from the products is generally carried out via inactivation and sterile filtration.

\subsection{Endotoxins}

Since most of the biopharmaceuticals are administered intravenously, finished-product biopharmaceuticals must be sterile and free from pyrogenic substances. The endotoxin limit for the intravenous administration of pharmaceutical and biological products is 5 endotoxin units (EU)/kg of body weight/hour by all pharmacopoeias [19]. Hence, the detection and removal of pyrogenic substances, especially endotoxins (lipopolysaccharides in the cell wall of gram-negative bacteria), are necessary to ensure safety of biopharmaceutical products. Currently available methods for endotoxin detection include the U.S. Pharmacopeia rabbit test and the Limulus amebocyte lysate (LAL) test [7].

The rabbit pyrogen test entails measurements of the rise in body temperature of rabbits after an intravenous injection of a test substance. The presence of pyrogens of all kinds can be tested using this method. However, this method suffers from a number of disadvantages and limitations: (1) Endotoxin tolerance occurs after repeated use of rabbits; (2) variations in the response depending on sex, age, and species; (3) differences between the responses of rabbits and humans to various pyrogen types; and (4) the rabbit pyrogen test is inadequate for sera, radiopharmaceuticals, chemotherapeutics, analgesics, cytokines, immunosuppressive agents, and others [20].

Accordingly, the use of the rabbit pyrogen test has been reduced. Nowadays, the most widely used endotoxin detection systems are based on the highly sensitive LAL test. It is based on the coagulation cascade of the blood of a horseshoe crab, Limulus polyphemus, which is induced by lipopolysaccharide. The currently known methods for lipopolysaccharide detection entail (1) gel-clot assay, (2) turbidimetric LAL technique, and (3) the chromogenic LAL technique.

The gel-clot assay is a limit test that provides simple positive or negative results. The LAL reagent is introduced to a sample, and the test material is considered endotoxin positive if a gel is formed via a clotting reaction.

The turbidimetric and the chromogenic LAL techniques are quantitative tests. The former is based on the fact that turbidity increases as a result of the precipitation of the clottable protein that is related to endotoxin concentration in the sample. The optical density is read by a spectrophotometer at either a fixed time (for the end-point method) or progressively (for the kinetic assay) as turbidity develops.

The chromogenic LAL technique makes use of a synthetic substrate which contains an amino acid sequence similar to that of the clottable protein, coagulogen, in order to detect endotoxin. The enzyme cleaves a yellow-colored substance from the chromogenic substrate, and the color intensity produced is proportional to the amount of endotoxin present in the sample. 
Endotoxins are temperature and $\mathrm{pH}$ stable, and, therefore, their removal is one of the most challenging issues. Numerous techniques are used to reduce endotoxin contamination of biopharmaceuticals, including ion-exchange chromatography, sucrose gradient centrifugation, gel filtration chromatography [19], affinity adsorption [21], charged membrane/depth filtration, and ultrafiltration [22].

\subsection{Viruses}

Owing to the risks of transmission of adventitious agents to patients, the different cell levels should be studied for the absence of these agents. Among the adventitious agents, special attention should be paid to viruses that are capable of contaminating the original species. Generally, the virological safety of biopharmaceuticals includes several levels of control at various manufacturing stages, including 1 - rigorous screening of cell banks (both master cell bank and working cell bank) for viruses; 2 - screening of each cell culture harvest for adventitious agents; and 3 - a demonstration that the purification process can clear potential adventitious agents [15].

The detection of viruses in cell lines can be carried out via various techniques. The commonly used methods of detecting viral infections include

- co-cultivation assays (specific in vitro tests),

- in vivo assays,

- antibody production in animals (MAPs, RAPs, or HAPs),

- immunoassays for viral specific proteins,

- Transmission Electron Microscopy (TEM),

- Polymerase Chain Reaction (PCR).

For the co-cultivation assays (specific in vitro tests), the cells used for production, or culture supernatant, or the final product are incubated with the detector cells. The detector cell lines are susceptible to different viruses and are used to detect desired viruses via monitoring subsequent cytopathic effects, hemadsorption, morphological changes, or other signs of viral infection. The detector cells usually contain humans, primates, and cells from the same species.

The in vivo assay can be performed by the inoculation of cells or cell lysates into animals, including newborn and adult mice, guinea pigs, rabbits, or embryonated chicken eggs to detect viruses. The animals are consequently monitored for any abnormality.

Species-specific viruses potentially present in rodent cell lines can be examined using assays for antibody production in the animals. The MAP, RAP, and HAP (mouse, rat, and hamster antibody production assays, respectively) tests involve an injection of the test article into the animals. The inoculated animals are bled after four weeks, and the sera are tested for the presence of the antibodies against the specific viral antigens. For instance, Hantaan virus, Lactic Dehydrogenase virus, and Sendai virus have been screened using MAP. 
An immunoassay for viral-specific proteins can be undertaken through production of the relevant antibodies after an injection of a virus of interest into animals. Currently commercially available immunoassays are able to detect various viruses.

Another method that is used for virus detection is TEM (Transmission Electron Microscopy). TEM is a quantitative assay that is based on the visualization and morphological identification of virus particles in samples [23].

Nevertheless, more sensitive methods, such as the PCR identification methods, can be employed for the detection of sequences of the viruses [16].

Since the biopharmaceuticals can be originated from mammalian cell lines with a high risk of endogenous retroviruses, on one hand, and these products may be infected with adventitious viruses through processing, on the other hand, virus inactivation and removal steps in the purification process are required [24]. These entail gamma irradiation, low $\mathrm{pH}$ treatment, or virus filtration.

Indeed, ensuring the absence of virus contamination in biopharmaceuticals is challenging. For instance, a limited number of commercial poultry vaccines were contaminated by avian leukosis virus even after routine quality assurance procedures. In addition, reovirus was found as a contaminant in urokinase. On the whole, sourcing and testing alone cannot guarantee the virological safety of biopharmaceuticals owing to some limitations: the limit of sensitivity for cell culture and PCR tests and also due to the fact that cell culture or in vivo tests are not able to detect all known kinds of potential contaminants [25]. Thus, practical methods are required for the virological safety of biopharmaceuticals, which involve the inclusion of risk assessment as well as management policies.

\subsection{Mycoplasma}

Mycoplasmas are the smallest free-living and self-replicating organisms in nature that are sized between 50 and 500nm. They lack a rigid cell wall and, consequently, are highly pleomorphic from round to filamentous. They are filterable and penicillin-resistant forms. Furthermore, their membrane contains sterol and due to this, mycoplasmas require the addition of serum or cholesterol to the growth medium. They grow on special media in aerobic or anaerobic conditions with optimum growth at $37^{\circ} \mathrm{C}$ and $\mathrm{pH} 7.0$ and form with a "fried egg" morphology on agar media (Figure 6).

Mycoplasma contamination of cell culture systems for the production of mycoplasmas is a critical problem due to its effect on various parameters within the cell culture system. Mycoplasma contaminates cell cultures approximately without any sign, and it persists for a long time. Indeed, mycoplasma-positive cell cultures can be considered the major source of biopharmaceutical infection, and they should be discarded or effectively decontaminated. Taken together, mycoplasma-positive cell cultures pose a serious problem and should be effectively detected and eradicated [26]. 


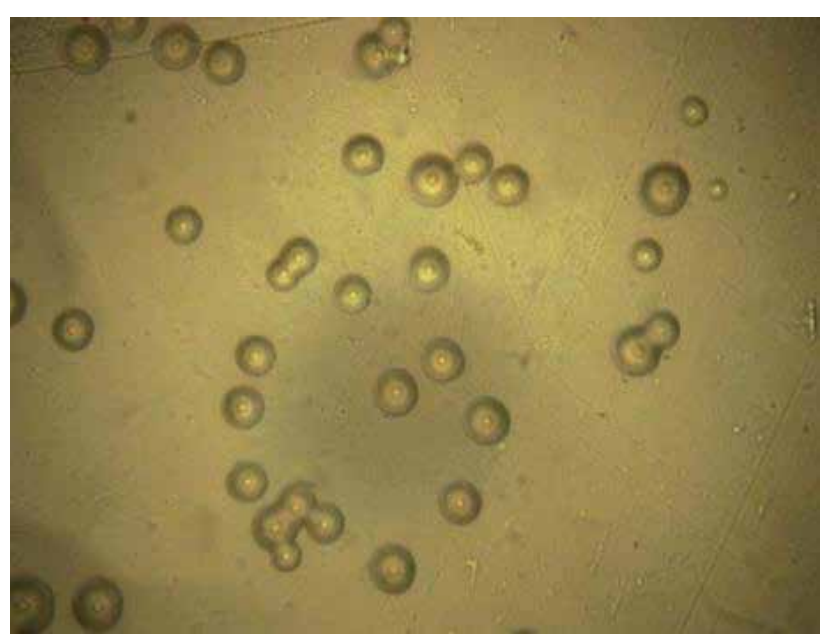

Figure 6. Mycoplasma colonies with fried egg morphology on mycoplasma agar medium. Picture was taken at Mycoplasma Reference laboratory, Razi vaccine and serum research institute, Iran

Different methods are used in international pharmacopoeias and guidance for detecting mycoplasma in biological test samples, mainly categorizing them as direct assay by microbiological culture, indirect assay by indicator mammalian cell culture, and PCR.

Direct assay by microbiological culture: The principle of detection is based on the growth of mycoplasma on supporting agar and liquid media (broth). First, the test sample is introduced into a special broth culture, is incubated for an appropriate time, and, consequently, it is sub-passaged to plate agar. After the required incubation period, the presence of mycoplasma colonies is observed microscopically in the agar plates (Figure 6).

Indirect assay by indicator mammalian cell culture: The indirect method requires the co-cultivation of the test sample with an indicator cell line for two to three days. Typically, VERO cells with a large cytoplasm area around the nucleus were used. Consequently, the cells were stained using a DNA binding stain (such as Hoechst stain) that binds specifically to DNA and is observed via fluorescent microscopy. Due to the affinity of mycoplasmas for the mammalian cell membrane, mycoplasmas appear as granules surrounding the nucleus.

Mycoplasma PCR: In this method, detection is carried out using specific oligonucleotide primers for the amplification of mycoplasma DNA. This method is specially recommended for detecting contamination with the non-cultivable strains of M. hyorhinis [27].

On the whole, it is advisable to use two different methods in the detection of mycoplasmas in order to allow for the differentiation between false-positive and false-negative results.

\subsection{DNA}

The importance of DNA contamination detection in biopharmaceuticals is related to the fact that the DNA from some sources such as hybridoma cell lines in monoclonal antibody production may act as active oncogenes. These kinds of DNA contaminants can be introduced 
and expressed in human cells and result in the initiation of cancer cells. According to guidelines, the acceptable level of residual DNA in recombinant products is 10 pg per therapeutic dose. DNA hybridization studies that use radiolabeled DNA probes with a specific nucleic acid sequence constitute one of the most widely used methods for the detection of DNA contaminants in the product to a nanogram (ng) range [6]. The important steps involved in DNA hybridization are shown in Figure 7.

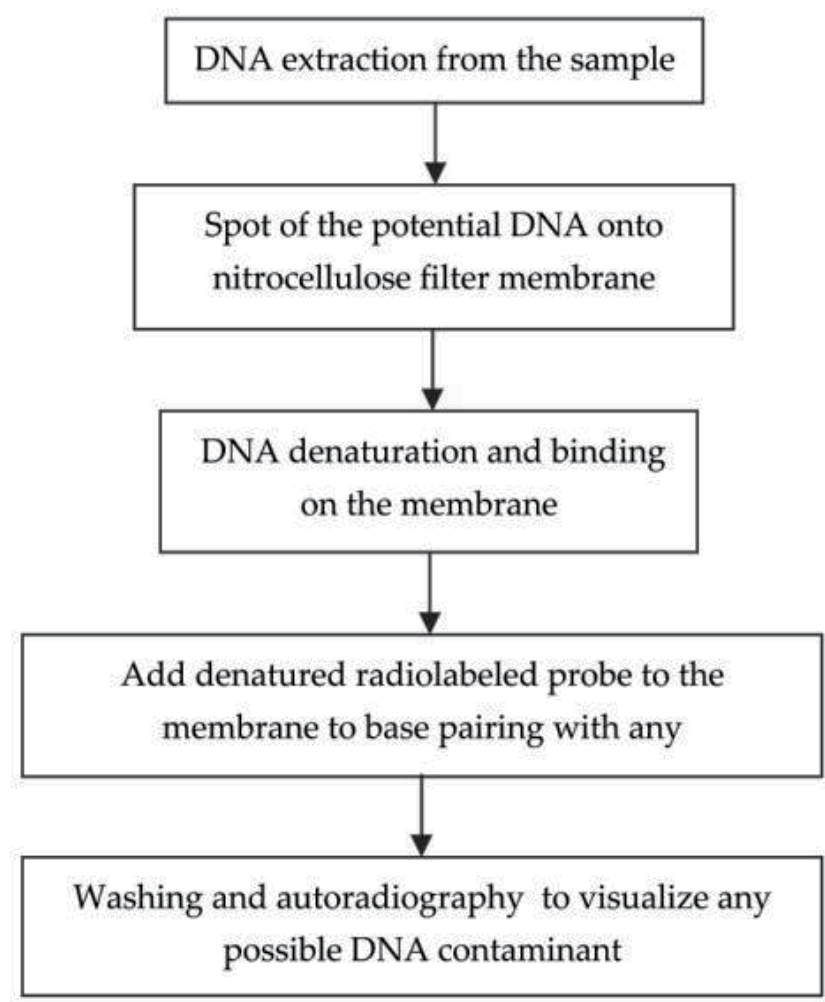

Figure 7. The main steps of the DNA hybridization procedure

\subsection{Cross-contamination}

Cell cultures may be infected with other cell types due to the use of contaminated items or operator mistakes. Also fail of the sterilization process can be another reason. The detection of cross-contamination is very challenging due to the fact that macroscopic and microscopic properties of the original and contaminant cells are commonly the same. Cross-contamination in the production of biopharmaceuticals would prove to be disastrous and terrible.

Various tests for detecting cross-contaminations can be applied; however, a product-specific identity test will be the best choice [16]. 


\section{Summary}

Microbial quality control plays a prominent role in the manufacture of safe and effective biopharmaceuticals. The main sources of microbial contamination can be related to raw materials and the production environment. The main categories of raw materials that are involved in the manufacturing of biopharmaceuticals with a high risk of contamination are those of animal origin such as cell culture media, sera, and supplements. The production environment includes air, surfaces, instruments, equipments, and personnel. All these can be considered the main source for the contamination of biopharmaceuticals with adventitious agents such as viruses, bacteria, fungi, transmitting spongiform encephalopathy, and mycoplasma. The use of contaminated biopharmaceuticals causes product spoilage, which may lead to (i) metabolization of the therapeutic agents by microorganisms, thus bringing about a decrease in the potency of the therapeutic agent; (ii) a potential health hazard to patients as a result of either infectious diseases or microbial-derived agents such as endotoxins that are secreted into products.

Various methods are used for detecting and eliminating different biological contaminants that are used in the manufacturing of biopharmaceuticals. Generally, bacteria and fungi can be detected by standard sterility testing or macroscopic and microscopic characteristics, as well as biochemical tests. In addition, viruses can be detected via a number of methods such as co-cultivation assays (specific in vitro tests), in vivo assays, antibody production in animals (MAPs, RAPs, or HAPs), immunoassays for viral specific proteins, TEM, or PCR. The detection of endotoxin can be carried out using the pharmacopeial rabbit test or LAL test. The available approaches for the detection of mycoplasma include direct assay using special culture media, indirect assay by mammalian cells, and DNA staining, as well as PCR. Furthermore, DNA hybridization is a widely used approach for the detection of DNA contaminants in biopharmaceuticals. The best method for cross-contamination detection includes a product-specific identity test.

With regard to the collection of tests for biological quality control of biopharmaceuticals summarized in this chapter, it is obvious that various sets of methods are available in different guidelines and pharmacopeias which are complicated and problematic. The development and compilation of harmonized guidelines for biological quality control of biopharmaceuticals is a critical necessity that can facilitate the control of the safety of these ever-increasing products.

\section{Author details}

Farzaneh Lotfipour and Somayeh Hallaj-Nezhadi

Faculty of Pharmacy, Immunology Research Center, Tabriz University of Medical Sciences, Tabriz, Iran 


\section{References}

[1] Rader, R. A. (2005). What is a Biopharmaceutical, Part 1: (Bio)Technology-Based Definitions BioExecutive. March and May

[2] Rader, R. A. (2005). What is a Biopharmaceutical, Part 2: Company and Industry Definitions. BioExecutive. March and May

[3] WHO. Definition of active pharmaceutical ingredient.(2011). July Available from: http://www.who.int/medicines/areas/quality_safety/quality_assurance/DefinitionAPI-QAS Rev1-08082011.pdf.

[4] FDA. What is a biological product? ; Available from: http://www.fda.gov/AboutFDA/Transparency/Basics/ucm194516.htm.

[5] Walsh, G. (2002). Biopharmaceuticals and biotechnology medicines: an issue of nomenclature. Eur J Pharm Sci. 15(2):, 135-138.

[6] Walsh, G. (2003). Biopharmaceuticals Biochemistry And Biotechnology. Second ed, England: John Wiley \& Sons, Ltd.

[7] Denyer, S. P., Hodges, N. A., \& Gorman, S. P. (2004). Hugo and Russell's Pharmaceutical Microbiology. SEVENTH ed: Blackwell Science.

[8] Brown, T.A., Gene Cloning and DNA Analysis: An Introduction.th ed(2010). Wiley Blackwell.

[9] Kohler, G., \& Milstein, C. (2005). Continuous cultures of fused cells secreting antibody of predefined specificity. 1975. J Immunol. 174(5):, 2453-2455.

[10] Kohler, G., \& Milstein, C. (1975). Continuous cultures of fused cells secreting antibody of predefined specificity. Nature. 256(5517):, 495-497.

[11] Bols, N. C., et al. (1988). Media for hybridoma growth and monoclonal antibody production. Biotechnol Adv. 6(2):, 169-182.

[12] ANTIBODIES, C.O.M.O.P.M., Monoclonal Antibody Production A Report of the Committee on Methods of Producing Monoclonal Antibodies Institute for Laboratory Animal Research National Research Council., (1999). : Washington, DC.

[13] Li, F., et al. (2010). Cell culture processes for monoclonal antibody production. MAbs. 2(5):, 466-479.

[14] Ho, R. J. Y., \& Gibaldi, M. (2003). Biotechnology and Biopharmaceuticals: Transforming Proteins and Genes into Drugs, New Jersey.: A John Wiley \& Sons, Inc.

[15] Knäblein, J. (2005). Modern Biopharmaceuticals: Design, Development and Optimization: WILEY-VCH Verlag GmbH \& Co. KGaA, Weinheim.

[16] Denyer, S. P., \& Baird, R. M. (2007). Guide to Microbiological Control in Pharmaceuticals and Medical Devices. second ed: CRC Press. 
[17] United States Pharmacopocia 29, National Formulary 24. USP Convention,Rockville (2006).

[18] European Pharmacopoeia, 4th ed. EPSecretariat, Strasbourg.(2002).

[19] Magalhaes, P. O., et al. (2007). Methods of endotoxin removal from biological preparations: a review. J Pharm Pharm Sci. 10(3):, 388-404.

[20] Daneshian, M., et al. (2006). In vitro pyrogen test for toxic or immunomodulatory drugs. J Immunol Methods. 313(1-2):, 169-175.

[21] Petsch, D., \& Anspach, F. B. (2000). Endotoxin removal from protein solutions. J Biotechnol. 76(2-3):, 97-119.

[22] Salema, V., Saxena, L., \& Pattnaik, P. (2009). Removing endotoxin from biopharmaceutical solutions. Pharmaceutical Technology Europe. 21(10):, 36.

[23] Vale, F. F., et al. (2010). Applications of transmission electron microscopy to virus detection and identification Microscopy: Science, Technology, Applications and Education.

[24] Liu, H. F., et al. (2010). Recovery and purification process development for monoclonal antibody production. MAbs,. 2(5):, 480-499.

[25] Bailey, A. (2011). Learning from Experience European Biopharmaceutical Review, Spring.

[26] Fleckenstein, E., \& Drexler, H.G. Iochemica n 19961.

[27] Edition, T. J. P. F. Mycoplasma Testing for Cell Substrates used for the Production of Biotechnological/Biological Products. Available from: http://db.yaozh.com/foreign/ JP15/jp5/F5_14.pdf. 
Chapter 10

\title{
New Approachs in Drug Quality Control: Matrices and Chemometrics
}

\author{
Sigrid Mennickent, M. de Diego, B. Schulz, M. Vega \\ and C. G. Godoy
}

Additional information is available at the end of the chapter

http://dx.doi.org/10.5772/50825

\section{Quality control}

Quality control refers to the process of quality evaluation that focuses on the internal measurement of the quality of a process, institution, product, service, or other. Often used interchangeably with quality management and quality assurance [1-3].

\section{Drug quality control}

Quality Assurance plays a very important role in making sure that the GMP standards are met and products comply with the international quality standards. The main functions carried out by drug quality control are:

- Approval of raw materials

- Monitoring of manufacturing processes

- Approval of finished products

- Documentation of technical information

- Implementation of cGMP

Manufacturing processes are monitored and controlled by testing of raw materials, in-process parameters. Final active pharmaceutical ingredients and dosage forms are tested for specified parameters before release. Analytical testing is carried out with highly sophisticated instruments: viz. HPLC, GC, IR, UV spectrophotometer mettler titrators, particle size analyzer etc. 
All the analytical test procedures and manufacturing procedures are well documented and revision is undertaken as per specified protocol. Analytical methods are validated to give the reproducible results. Stability study as per stability protocol is considered to be very important area of Quality Assurance.

Automated systems are becoming increasingly important tools for appropriate monitoring and controlling of the pharmaceutical packaging process. Solutions for comprehensive quality assurance or production data acquisition and evaluation are just as important as applications that meet the legislative requirements of different countries in terms of serial numbering and the unique marking of products.

Quality control involves many phases, such as sample collection, measuring, analysis of results, and the approval/rejection of the batch. Nonetheless, the most important thing is the continuity and systematization of the quality control.

Effective process validation contributes significantly to assuring drug quality. The basic principle of quality assurance is that a drug should be produced that is fit for its intended use. This principle incorporates the understanding that the following conditions exist: Quality, safety, and efficacy are designed or built into the product.

Quality cannot be adequately assured merely by in-process and finished-product inspection or testing.

Process validation is defined as the collection and evaluation of data, from the process design stage through commercial production, which establishes scientific evidence that a process is capable of consistently delivering quality product. Process validation involves a series of activities taking place over the lifecycle of the product and process. Usually, process validation includes three stages:

- Process Design: The commercial manufacturing process is defined during this stage based on knowledge gained through development and scale-up activities.

- Process Qualification: During this stage, the process design is evaluated to determine if the process is capable of reproducible commercial manufacturing.

- Process Verification: Ongoing assurance is gained during routine production that the process remains in a state of control [1-3].

\section{New approaches in drug quality control}

\subsection{Matrices}

\subsubsection{Residue analysis of pharmaceuticals in the aquatic environment}

Residue analysis of pharmaceuticals in the aquatic environment has attracted considerable interest during the last few years. 
Traces of such compounds have been detected in surface water samples from all countries where pharmaceuticals are widely in use.

Pharmaceutically active compounds have captured the attention of the scientific community because such pollutants result not primarily from manufacturing but from widespread, continual use in human and veterinary clinical practice. The biological activity of these compounds can lead to adverse effects in aquatic ecosystems and potentially have an impact on drinking-water supplies [4].

In the human body, pharmaceuticals can be transformed to one or more metabolites and excreted as a mixture of parent compound and metabolites, in which the parent compound is often the minor component. However, some drugs are poorly metabolized and are excreted unchanged. The degree of metabolism depends on a number of parameters, including age, gender and ethnicity, the constitution of the patient and the time of administration. Drugdrug interactions caused by enzyme induction or inhibition, as well as enhanced metabolism due to previous exposure, can also influence the pharmacokinetics of drugs [5].

Both the parent compound and the metabolites enter the aquatic environment once they are excreted from the human body. Monitoring studies in the environment have demonstrated the discharge of pharmaceuticals and their metabolites through municipal wastewater-treatment plants (WWTPs). Although unchanged drugs can undergo biochemical transformations during sewage treatment, some studies indicate that the absence of pharmaceutical compounds in treated water does not necessarily imply their complete removal. In most instances, human drugs are metabolized in the body to more polar compounds that are more likely to pass through the WWTP. In some cases, pharmaceuticals and their human metabolites can be microbially degraded in the activated sludge treatment.

Knowledge of the formation of stable metabolites in WWTPs is also important in order to understand the environmental fate of the parent compound. Once in the environment, these compounds can be transported and distributed in rivers, streams, and possibly further biodegraded. For most pharmaceuticals and their biotransformation products, these pathways in the aquatic environment are largely unknown, and investigations into their occurrence in environmental compartments are still rare.

Studies have been carried out to investigate their fate not only in surface waters, but also in sediment and soil environments. By nature, most pharmaceuticals are designed to be at least moderately water-soluble and to possess half-lives in the human body in the range of hours. Because human and microbial degradates will generally coexist with their parent compounds in the environment, indicators that summarize all the information on parent substances and degradates would be important instruments for decision-making and assessment [6].

Progress in instrumental analytical chemistry has resulted in the availability of methods that allow a monitoring of these pollutants at ng levels.

Improvements in detection limits over the past years have mainly been due to sophisticated mass spectrometric detection techniques. Furthermore, robust sample preparation and preconcentration protocols have contributed significantly to the achievements observed so far. 
Nowadays it is a well-established fact that pharmaceutical drugs used during medical treatment may partly be excreted in an un-metabolized form, enter municipal sewage systems, and can even survive the passage through the sewage treatment plant. Therefore, sewage treatment plant effluents are the major source for introduction of pharmaceuticals into the aquatic environment. Furthermore, pharmaceuticals employed in veterinary medicine may be introduced into soil (and eventually into water) via manure, or may find a direct way into the aquatic system when used in fish farms.

Unfortunately, the consequences of continuous presence of low concentrations of pharmaceuticals for the ecosystem are still not fully known.

In many cases, the analytical procedures for residue analysis of pharmaceutical drugs nowadays available includes a pre-concentration and clean-up step by solid-phase extraction or related techniques, followed by chromatography in combination with mass spectrometry (MS) as detector.

Although GC-MS may still be the perfect technique for certain classes of pharmaceuticals, high-performance liquid chromatography (HPLC) hyphenated with atmospheric pressure ionization-MS has established itself as the better choice for simultaneous determination of pharmaceuticals of widely differing structures.

The concentration levels of pharmaceuticals found in environmental water samples are generally too low to allow a direct injection into a chromatographic system. Therefore, efficient pre-concentration steps are necessary which should also result in some sample clean-up. One of the most widely used sample treatment technique for residue analysis of pharmaceuticals in water is the extraction of the analytes by means of a solid sorbent.

This extraction procedure can be based on multiple equilibria between the liquid phase and the sorbent filled into a small cartridge (solid-phase extraction, SPE), or on a single equilibrium (sorptive extraction) [7-23].

\subsubsection{Solid-phase extraction}

Pharmaceuticals of adequate hydrophobicity can easily be pre-concentrated using any reversed-phase material such as alkyl-modified silica or polymer-based materials. Deprotonation of acidic compounds and protonation of basic compounds should be suppressed to ensure sufficient hydrophobicity of the analytes. Therefore, acidic pharmaceuticals should be pre-concentrated under acidic conditions, whereas basic analytes should be pre-concentrated at an alkaline $\mathrm{pH}$. Alternatively, mixed-mode SPE materials can be used which exhibit both reversed-phase and cation-exchange properties due to the presence of sulfonic acid groups on the hydrophobic surface of the particles. Using acidified sample solutions, acidic and neutral analytes would be extracted by hydrophobic interactions, whereas protonated basic analytes would interact via ion exchange mechanisms.

A recent review has summarized new SPE materials that can improve the recoveries for polar analytes. These materials are mainly polymeric sorbents that improve the retention of polar compounds either by novel functional groups in the polymeric structure (resulting in a 
hydrophilic-hydrophobic balance material) or by considerably increased surface area. Some of these new materials have turned out to be well suited for multi-class analysis of pharmaceuticals in water samples. Nowadays, one of the most widely used sorbent is a copolymer of divinylbenzene and vinylpyrrolidone [7-23].

\subsubsection{Sorptive extraction}

Sorptive extraction based on a single partitioning equilibrium of analytes between the aqueous sample and a solid sorbent includes solid-phase microextraction (SPME), stir-bar sorptive extraction (SBSE), and several related variants. Originally, these techniques were based on polydimethylsiloxane (PDMS) as material for trapping trace analytes from a water sample due to partitioning between the aqueous matrix and the PDMS phase. Besides PDMS, some alternative sorptive materials have become commercially available recently, such as polyacrylates, copolymers of PDMS with divinylbenzene, copolymers of polyethylene glycol with divinylbenzene, and mixtures of carboxen (an inorganic adsorbent) with PDMS or divinylbenzene [7-23].

\subsubsection{Sample pre-concentration procedures for sediment and sludge samples}

Extraction of pharmaceuticals from sediment and sludge is generally done by blending the sample with an organic solvent or with mixtures of aqueous buffers and organic solvents.

Ultrasonication is frequently applied to assist the extraction process.

Additional clean-up steps for the extract may be necessary employing SPE or liquid-liquid extraction. Somewhat more advanced procedures are based on pressurized liquid extraction (accelerated solvent extraction) which may need less time and less solvent consumption [7-23].

\subsubsection{Derivatization of the compounds}

Various groups of pharmaceuticals can be derivatized to make them suited for GC analysis. Typical derivatization reagents for acidic pharmaceuticals include pentafluorobenzylbromide, methyl chloromethanoate, methanol/BF3, or tetrabutylammonium salts (for derivatization during injection). Phenazone-type drugs have been derivatized by silylation using $\mathrm{N}$ tert-butyldimethylsilyl- $N$-methyltrifluoroacetamide (MTBSTFA). Silylation procedures are also commonly used for synthetic estrogens [7-23].

\subsubsection{Some latest researches in this area}

3.2.1.1. Pharmaceuticals in the aquatic environment: a critical review of the evidence for health effects in fish

The authors review the current data on the presence and reported biological effects in fish of some of the most commonly detected pharmaceuticals in the aquatic environment; namely nonsteroidal anti-inflammatory drugs (NSAIDs), fibrates, beta-blockers, selective serotonin 
reuptake inhibitors (SSRIs), azoles, and antibiotics. Reported biological effects in fish in the laboratory have often been shown to be in accordance with known effects of pharmaceuticals in mammals. Water concentrations at which such effects have been reported, however, are generally, between microg L(-1) and mg L(-1), typically at least 1 order of magnitude higher than concentrations normally found in surface waters (ng L(-1)). There are exceptions to this, however, as for the case of synthetic oestrogens, which can induce biological effects in the low ng L(-1) range. Although generally effect levels for pharmaceuticals are higher than those found in the environment, the risks to wild fish populations have not been thoroughly characterised, and there has been a lack of consideration given to the likely chronic nature of the exposures, or the potential for mixture effects. As global consumption of pharmaceuticals rises, an inevitable consequence is an increased level of contamination of surface and ground waters with these biologically active drugs, and thus in turn a greater potential for adverse effects in aquatic wildlife [24].

\subsubsection{Human Pharmaceuticals, Hormones and Fragrances: The Challenge of Micropollutants in Urban Water Management}

The observed concentrations of pharmaceuticals and personal care products (PPCPs) in raw wastewater confirm that municipal wastewater represents the main disposal pathway for the PPCPs consumed in households, hospitals and industry. In sewage treatment plant effluents most PPCPs are still present, since many of these polar and persistent compounds are being removed only partially or, in some cases, not at all. Treated wastewater therefore represents an important point source for PPCPs into the environment. After passing a sewage treatment plant the treated wastewater is mostly discharged into rivers and streams or sometimes used to irrigate fields. If drinking water is produced using resources containing a substantial proportion of treated wastewater (e.g. from river water downstream of communities) the water cycle is closed and indirect potable reuse occurs. Human Pharmaceuticals, Hormones and Fragrances provides an overview of the occurrence, analytics, removal and environmental risk of pharmaceuticals and personal care products in wastewater, surface water and drinking water. [25].

\subsubsection{Factors affecting the concentrations of pharmaceuticals released to the aquatic environment}

Although recent research has demonstrated that pharmaceuticals are widely distributed in the aquatic environment, it is difficult to assess the threat that they

pose to drinking water supplies or their rate of attenuation in natural systems without an adequate understanding of the sources of contamination. To identify pharmaceutical compounds of significance to water supplies in the United States, the authors have reviewed available data on the use of prescription drugs. Results of our analysis indicate that approximately 40 compounds could be present in municipal wastewater effluent at concentrations above $1,000 \mathrm{ng} / \mathrm{L}$ and at least 120 compounds could be present at concentrations above 1 ng/L. Important classes of prescription drugs include analgesics, beta-blockers, and antibiotics. Analysis of a group of the most commonly used pharmaceuticals in the United States indicates that they are ubiquitous in wastewater effluents. Authors have detected concentra- 
tions ranging from approximately 10-3,000 ng/L for high use pharmaceuticals such as betablockers (e.g., metoprolol, propranolol) and acidic drugs (e.g., gemfibrozil, ibuprofen). The concentration of pharmaceuticals in effluent from conventional wastewater treatment plants is similar. Advanced wastewater treatment plants equipped with reverse osmosis systems reduce concentrations of pharmaceuticals below detection limits. In addition to removal during biological wastewater treatment, pharmaceuticals also are attenuated in engineered natural systems (i.e., treatment wetlands, ground water infiltration basins). Preliminary evidence suggests limited removal of pharmaceuticals in engineered treatment wetlands and nearly complete removal of pharmaceuticals during ground water infiltration [26].

\subsubsection{A preliminary ecotoxicity study of pharmaceuticals in the marine environment}

Environmental fates and effects of pharmaceuticals in the aquatic environment have been the focus of recent research in environmental ecotoxicology. Worldwide studies of common over-the-counter pharmaceuticals have reported detectable levels in the aquatic environment, but there are few studies examining impacts on marine habitats. These drugs can affect the functions of various vertebrates and invertebrates. The stability of two pharmaceuticals, cyclizine (CYC) and prochlorperazine (PCZ), in seawater was examined under light and dark conditions, as well as the toxicity of these compounds to larvae of the barnacle Balanus amphitrite, which is a cosmopolitan marine organism found in most of the world's oceans. CYC was very stable under all the tested conditions. On the other hand, PCZ degraded in light but not in the dark, and was more stable in seawater than fresh water. For the barnacle larvae, the LC50 of prochlorperazine was $0.93 \mathrm{microg} / \mathrm{mL}$ and the LC50 for CYC was approximately $0.04 \mathrm{microg} / \mathrm{mL}$ [27].

\subsubsection{Estrogenic activity of pharmaceuticals in the aquatic environment}

In the last years pharmaceuticals have aroused great interest as environmental pollutants for their toxic effects towards non target organisms. This study wants to draw attention to a further adverse effect of drugs, the endocrine interference. The most representative drugs of the widespread classes in environment were investigated. The YES-test and the E-screen assay were performed to detect the capability of these substances to bind the human estrogenic receptor alpha (hER alpha) in comparison with 17beta-estradiol. Out of 14 tested pharmaceuticals, 9 were positive to YES-assay and 11 were positive to Escreen assay; in particular, Furosemide and the fibrates (Bezafibrate, Fenofibrate and Gemfibrozil) gave the maximal estrogenic response. Tamoxifen showed its dual activity as agonist and antagonist of hER alpha [28].

\subsubsection{Colloids as a sink for certain pharmaceuticals in the aquatic environment}

The occurrence and fate of pharmaceuticals in the aquatic environment is recognized as one of the emerging issues in environmental chemistry and as a matter of public concern. Existing data tend to focus on the concentrations of pharmaceuticals in the aqueous phase, with limited studies on their concentrations in particulate phase such as sediments. Furthermore, current water quality monitoring does not differentiate between soluble and colloidal phas- 
es in water samples, hindering our understanding of the bioavailability and bioaccumulation of pharmaceuticals in aquatic organisms. In this study, an investigation was conducted into the concentrations and phase association (soluble, colloidal, suspended particulate matter or SPM) of selected pharmaceuticals (propranolol, sulfamethoxazole, meberverine, thioridazine, carbamazepine, tamoxifen, indomethacine, diclofenac, and meclofenamic acid) in river water, effluents from sewage treatment works (STW), and groundwater in the UK. Colloids were isolated by cross-flow ultrafiltration (CFUF). Water samples were extracted by solid-phase extraction (SPE), while SPM was extracted by microwave. All sample extracts were analyzed by liquid chromatography-tandem mass spectrometry (LC-MS/MS) in the multiple reaction monitoring.

Five compounds propranolol, sulfamethoxazole, carbamazepine, indomethacine, and diclofenac were detected in all samples, with carbamazepine showing the highest concentrations in all phases. The highest concentrations of these compounds were detected in STW effluents, confirming STW as a key source of these compounds in the aquatic environments. The calculation of partition coefficients of pharmaceuticals between SPM and filtrate, between SPM and soluble phase, and between colloids and soluble phase showed that intrinsic partition coefficients are between $25 \%$ and $96 \%$, and between $18 \%$ and $82 \%$ higher than relevant observed partition coefficients values, and are much less variable. Secondly, $K_{\text {coc }}$ values are 3-4 orders of magnitude greater than Kocint values, indicating that aquatic colloids are substantially more powerful sorbents for accumulating pharmaceuticals than sediments. Furthermore, mass balance calculations of pharmaceutical concentrations demonstrate that between $23 \%$ and $70 \%$ of propranolol, $17-62 \%$ of sulfamethoxazole, $7-58 \%$ of carbamazepine, $19-84 \%$ of indomethacine, and $9-74 \%$ of diclofenac are present in the colloidal phase.

The results provide direct evidence that sorption to colloids provides an important sink for the pharmaceuticals in the aquatic environment. Such strong pharmaceutical/colloid interactions may provide a long-term storage of pharmaceuticals, hence, increasing their persistence while reducing their bioavailability in the environment.

Recommendations and perspectives from this study:

Pharmaceutical compounds have been detected not only in the aqueous phase but also in suspended particles; it is important, therefore, to have a holistic approach in future environmental fate investigation of pharmaceuticals. For example, more research is needed to assess the storage and long-term record of pharmaceutical residues in aquatic sediments by which benthic organisms will be most affected. Aquatic colloids have been shown to account for the accumulation of major fractions of total pharmaceutical concentrations in the aquatic environment, demonstrating unequivocally the importance of aquatic colloids as a sink for such residues in the aquatic systems. As aquatic colloids are abundant, ubiquitous, and highly powerful sorbents, they are expected to influence the bioavailability and bioaccumulation of such chemicals by aquatic organisms. It is therefore critical for colloids to be incorporated into water quality models for prediction and risk assessment purposes [29]. 


\section{Chemometrics}

Chemometrics is the science of extracting information from chemical systems by data-driven means. It is a highly interfacial discipline, using methods frequently employed in core dataanalytic disciplines such as multivariate statistics, applied mathematics, and computer science, in order to address problems in chemistry, biochemistry, medicine, biology and chemical engineering.

Chemometrics is applied to solve both descriptive and predictive problems in experimental life sciences, especially in chemistry. In descriptive applications, properties of chemical systems are modeled with the intent of learning the underlying relationships and structure of the system (i.e., model understanding and identification). In predictive applications, properties of chemical systems are modeled with the intent of predicting new properties or behavior of interest. In both cases, the datasets can be small but are often very large and highly complex, involving hundreds to thousands of variables, and hundreds to thousands of cases or observations.

Chemometric techniques are particularly heavily used in analytical chemistry and metabolomics, and the development of improved chemometric methods of analysis also continues to advance the state of the art in analytical instrumentation and methodology. It is an application driven discipline, and thus while the standard chemometric methodologies are very widely used industrially, academic groups are dedicated to the continued development of chemometric theory, method and application development [30-33].

\section{Author details}

Sigrid Mennickent ${ }^{*}$, M. de Diego ${ }^{1}$, B. Schulz ${ }^{1}$, M. Vega ${ }^{2}$ and C. G. Godoy ${ }^{1}$

*Address all correspondence to: smennick@udec.cl

1 Department of Pharmacy, Faculty of Pharmacy, University of Concepción, Concepción, Chile

2 Department of Bromatology, Nutrition and Dietetic, Faculty of Pharmacy, University of Concepción, Concepción, , Chile

\section{References}

[1] USP (1999). The United States Pharmacopeia/ The National Formulary (USP 24/NF 19), United States Pharmacopeial Convection, Inc., Rockville

[2] ICH. (2003). The Sixth ICH International Conference on Armonization of Technical Requirements for Registration of Pharmaceuticals for Human Use, Osaka,. 
[3] Buchberger, W. W. (2007). Analytica Chimica Acta, 593, 129-139.

[4] Jones, O., Lester, J. N., \& Voulvoulis, N. (2005). Trends in Biotechonology, 23(4), 163-167.

[5] Lemmer, B. (1996). Chronopharmacology- Cellular and Biochemical Interactions. Birkhäuser,, New York, USA.

[6] Boxall, A., Fenner, K., Kolpin, D. W., \& Maund, S. (2004). Environ. Sci. Technol., 38, 369A.

[7] Moldovan, Z. (2006). Chemosphere, 64, 1808.

[8] Cunningham, V. L., Buzby, M., Hutchinson, T., Mastrocco, F., Parke, N., \& Roden, N. (2006). Env. Sci. Technol, 40, 3457.

[9] Fent, K., Weston, A. A., \& Caminada, D. (2006). Aquatic Toxicol, 76, 122.

[10] Crane, M., Watts, C., \& Boucard, T. (2006). Sci. Tot. Environ, 367, 23.

[11] Hernando, M. D., Mezcua, M., Fernandez-Alba, A. R., \& Barcelo, D. (2006). Talanta, $69,334$.

[12] Benito-Peña, E., Partal-Rodera, A. I., Leon-Gonzalez, M. E., \& Moreno-Bondi, M. C. (2006). Anal. Chim. Acta, 556, 415.

[13] Fontanals, N., Marce, R. M., \& Borrull, F. (2005). Trends Anal. Chem ., 24, 394.

[14] Gomez, M. J., Petrovic, M., Fernandez-Alba, A. R., \& Barcelo, D. (2006). J. Chromatogr. A, 1114, 224.

[15] Gros, M., Petrovic, M., \& Barcelo, D. (2006). Talanta, 70, 678.

[16] Petrovic, M., Gros, M., \& Barcelo, D. (2006). J. Chromatogr. A, 1124, 68.

[17] Trenholm, R. A., Vanderford, B. J., Holady, J. C., Rexing, D. J., \& Snyder, S. A. (2006). Chemosphere, 65, 1990.

[18] Roberts, P. H., \& Bersuder, P. (2006). J. Chromatogr. A, 1134, 143.

[19] Himmelsbach, M., Buchberger, W., \& Klampfl, C. (2006). Electrophoresis, 27, 1220.

[20] Seitz, W., Weber, W. H., Jiang, J. Q., Lloyd, B. J., Maier, M., Maier, D., \& Schulz, W. (2006). Chemosphere, 64, 1318.

[21] Pozo, O. J., Guerrero, C., Sancho, J. V., Ibañez, M., Pitarch, E., Hogendoorn, E., \& Hernandez, F. (2006). J. Chromatogr. A, 1103, 83.

[22] Rodriguez-Mozaz, S., Lopez de Alda, D., \& Barcelo, D. (2007). J. Chromatogr. A, 1152, 97.

[23] Quintana, J. B., Miro, M., Estela, J. M., \& Cerda, V. (2006). Anal. Chem., 78, 2832.

[24] Corcoran, J., Winter, M. J., \& Tyler, C. R. (2010). Crit, Rev. Toxicol, 40(4), 287-304. 
[25] Ternes, T. (2006). Human Pharmaceuticals, Hormones and Fragrances: The Challenge of Micropollutants in Urban Water Management. Iwa Publishing.

[26] Sedlak, D., \& Pinkston, K. (2011). Factors affecting the concentrations of pharmaceuticals released to the aquatic environment, University of California.

[27] Choong, A. M., Teo, S. L., Leow, J. L., \& Ho, P. C. (2006). J. Toxicol. Environ. Health A., 69(21), 1959-1970.

[28] Isidori, M., Bellota, M., Cangiano, M., \& Parrella, A. (2009). Environ. Int, 35(5), 826-829.

[29] Maskaoui, K., \& Zhou, J. (2010). Environmental Science and Pollution Research, 17(4), 898-907.

[30] Verenitch, S. S., Lowe, C. J., \& Mazumder, A. (2006). J. Chromatogr. A, 1116.

[31] Gemperline, P. J. (2006). Practical guide to chemometrics. 2nd Edition, CRC Press $10.1201 / 9781420018301$

[32] Mark, H., \& Workman, J. (2007). Chemometrics in spectroscopy. Academic PressElsevier.

[33] Maeder, M., \& Neuhold, Y. M. (2007). Practical Data Analysis in Chemistry. Elsevier. 



\title{
Quality Control of Formulated Medicines
}

\author{
Alexandre S. Leal, Maria Ângela de B. C. Menezes, \\ Ilza Dalmázio, Fernanda P. Sepe, \\ Tatiana C. B. Gomes, Amalia S. Santana, \\ Luzia H. da Cunha and Radojko Jaćimović \\ Additional information is available at the end of the chapter \\ http://dx.doi.org/10.5772/51459
}

\section{Introduction}

A pharmaceutical drug is technically obtained or prepared for prophylactic, curative, palliative or diagnostic purposes. The final product must meet quality standard, be safe and effective. In Brazil, there is a high demand for formulated drugs. This is mainly due to their lower price compared to manufactured drugs, evidenced by the rapid growth - an increase of $350 \%$ from 1998 to 2010.

Even after the ANVISA's (Agency National Health Surveillance) establishment of the new handling standards to be followed by the magistral pharmacies, several serious cases, including death reports, caused by the consumption of formulated drugs have recently become public [1-5].

Other problems related to this subject occurred in 2004, when deaths caused by manipulated medicines of low therapeutic index (clonidine and levotiroxine) led ANVISA to modify the regulation for manipulated medicines. The concentration of each compound was not totally assured and the contamination by impurities, not included in the original formula, were present in the final product.

In a previous work, we have also observed the presence of impurities - such as metals - in different kinds of medicines. The ingestion of metals, even at low levels, can be very harmful to humans. Besides this, the long-term uptake of some drugs is also risky. This should require attention and surveillance from the public health-related agencies [6-10].

The quality and safety of drugs must follow the specifications described in the official compendia - among them, the pharmacopoeias. Medicines cannot contain impurities or 
other substances that endanger the patient's health. According to the second edition of the Brazilian Pharmacopoeia National Formulary [1], to ensure safety, efficacy and quality of the handled products it is necessary correct calculations, exact measurements, and adequate conditions and procedures of preparation. The prudent judgment of the pharmacist, who must be a qualified professional for this purpose, is another fundamental aspect. Additionally, an appropriate profile with a proven stability must be sought in the literature [11-14].

The requirements of sanitary legislation and quality control of raw materials for magistral solid preparations are:

- raw material: character sensory, solubility, pH determination, melting point, density, weight and volume; analysis report of manufacturer/supplier;

- raw material of vegetal origin: organoleptic characters, solubility, $\mathrm{pH}$ determination, melting point, density, weight and volume, evaluation of vendor analysis report;

- manipulated product: solid dosage forms: description, appearance and organoleptic characteristics, determination of average weight

The legislation also determines that all pharmacies must perform analyzes every two months of at least one of the formulas containing $\operatorname{drug}(\mathrm{s}) \leq 25 \mathrm{mg}$ of drugs. The priority is to those that contain $\leq 5 \mathrm{mg}$ of drugs. The legislation establishes special quality control requirements for preparations of substances with low therapeutic index, like hormones, antibiotics and cytotoxic drugs, homeopathic products, and sterile products. The raw materials used in sterile preparations must also be analyzed [15-17].

The Legislation on Good Practices for Handling does not require impurity tests for the raw materials received by the pharmacies. It is only necessary to check the certificate of a qualified supplier - issued in accordance with methods described in the pharmacopoeia, which are only suitable for the detection of some elements $(\mathrm{Ag}, \mathrm{As}, \mathrm{Bi}, \mathrm{Cd}, \mathrm{Hg}$, $\mathrm{Mo}, \mathrm{Pb}, \mathrm{Sb}$, and $\mathrm{Sn})$ [18].

Quality control tests for the products handled do not include detection and quantification of impurities. Moreover, the analysis required for formulated preparations allow limited conclusions about the quality of the process, since they do not testify the homogeneity of the active principle directly, but only as to the uniformity of filling of the capsules. So, a particular formulation can have the acceptance criteria for average mass, standard deviation and coefficient of variation but not the uniformity of this active content in the capsules [19].

Periodic reviews performed every two months for formulated drugs do not statistically have significant value, so that a reliable conclusion about the quality of formulated drugs can not be reached [20]. The analysis of thirty batches of $20 \mathrm{mg}$ of Sinvastatin medicine manipulated in pharmacies of Belo Horizonte, showed that only fourteen of them, met the quality standard required by pharmacopeia. Thus, the therapeutic efficacy of $53 \%$ of the analyzed products can not be totally dependable [21]. 
In this study, the quality of medicines Omeprazole and Enalapril Maleate from five (5) different magistral pharmacies was evaluated according to the methodology described in pharmacopoeia. The analyses for mass determination, identification of active principle, content, content uniformity and related compounds were performed.

The target drugs, Omeprazole and Enalapril Maleate, were chosen because of their representativeness of consumption and availability of related reference data in the pharmacopoeias.

In order to evaluate the presence and concentration of chemical elements, the technique used in this study was neutron activation analysis (NAA), applying the $\mathrm{k}_{0}$-standardization method [23-25]. The neutron activation analysis is a very sensitive and reliable multielemental technique, suitable for determination of the elements such as: $\mathrm{As}, \mathrm{Ba}, \mathrm{Br}, \mathrm{Ca}, \mathrm{Ce}, \mathrm{Cl}, \mathrm{Co}$, $\mathrm{Cr}, \mathrm{Eu}, \mathrm{Fe}, \mathrm{Hf}, \mathrm{Mg}, \mathrm{Mn}, \mathrm{Na}, \mathrm{Sb}, \mathrm{Sc}, \mathrm{Sm}, \mathrm{Ti}$ and $\mathrm{Zn}$, in different drugs [14]. The technique is based on the principle that when the material is irradiated by neutrons, some elements with suitable nuclear characteristics become radioactive isotopes. Thus, the concentration of each element can be determined by counting the respective radiation emitted by the corresponding radionuclide [26].

The results described here are part of a wider project which also includes the analyses of Fluoxetin and Sinvastatin medicines and will be published briefly.

\section{Quality control of formulated drugs}

\subsection{The pharmacopeia}

The 5th edition of the Brazilian Pharmacopoeia [27] defines quality control as: "The set of measures to ensure, at any time, the batch production of medicines and other products that meet the standards of identity, activity, content, purity, efficacy and safety." According to Resolution RDC №. 67, October 8, 2007 [15], which provides the Technical Regulation establishing the Good Handling Practices in Pharmacies (Good Compounding Practices) quality control of magistral and officinal preparations, is given by the completion of at minimum, the tests described in Table 1, according to the Brazilian Pharmacopoeia or other Official Compendium recognized by the National Health Surveillance Agency (ANVISA).

Results of tests must be recorded in the same order of handling, in addition to other relevant information. The pharmacist must evaluate the results to approve or not the preparation for dispensing. Each pharmacy is responsible for the quality of magistral preparations that handles, keeps, transports and dispenses. Raw materials should be checked in its receipt and moved to quarantine soon after, until the release of the reports of quality control. In the absence of pharmacopoeia monograph, the scientific literature should be used as a reference, and only with the lack of literature, the specification provided by the supplier may be used. All results must be written and stored [15]. 


\begin{tabular}{cr}
\hline Preparation & Test \\
\hline Solid & Description, appearance, organoleptic characteristics, average mass \\
\hline Semi-solid & Description, appearance, organoleptic characteristics, pH (where applicable), mass \\
\hline Non-sterile liquid & Description, appearance, organoleptic characteristics, pH, mass or volume before filling \\
\hline
\end{tabular}

Table 1. Tests for quality control of magistral drugs

However, some studies also show that the rule of Good Practices on Handling does not answer and does not guarantee the quality of compounded drugs [20].

\subsection{Analyses performed}

The analyses of quality control were performed at the Laboratory for Quality Control of Chemical Physics Drug, and Cosmetic Sanitizing of the Ezequiel Dias Foundation (FUNED). The following tests were performed $[15,27,28]$ :

- Aspect;

- Identification ;

- Labeling;

- Content;

- Related compounds;

- Dosage uniformity;

- Unit Change in mass;

The test of aspect is just a visual description of the product to be analyzed, coloration of the capsule and its content.

The test of identification allows determining the presence of the active principle in the product analyzed. It is performed through the high performance liquid chromatography (HPLC) [28].

The analysis of content aims to verify whether the drug has a dose of active ingredient on the label provided and used to quantify the active ingredient in the product analyzed. This test is performed according to the pharmacopoeia for each product, and may be performed in the ultraviolet and visible spectrophotometry, by high performance liquid chromatography, among other methods. The test uses usually ten to twenty capsules and each capsule analyzed separately, but the "pool" of these. There are limits specified in the monograph, which should be within the active drug, usually 90 to $110 \%$. Results below the limit can result in ineffective therapy and above, intoxication, depending on the drug analized [28].

The analysis of related compounds determines the amount of by-products of synthesis of the substance and / or its degradation products and / or contaminants from the process of 
obtaining the substance which can be normally found within a specified limit. This test is done only when specified in the pharmacopoeia.

The variation of the mass allows checking the uniformity of mass between units within a batch. For products in hard capsules should be weighed individually, twenty units, the contents of each one should be removed, properly cleaned and reweighed. The mass content of each capsule is determined by mass difference between the full and the empty capsule. Then the average mass of the contents can be determined. For hard capsules, the limit of variation is $\pm 10 \%$ of the mass corresponding to less than $300 \mathrm{mg}$. If the mass corresponds to $300 \mathrm{mg}$ or more, the maximum range is $\pm 7.5 \%$. It cannot be tolerated more than two units outside the limits specified in the official compendia, but none can be above or below twice the percentages indicated [27].

The uniformity of dosage units evaluate the uniformity of distribution of active component units in a single batch can be determined by two methods: mass variation and content uniformity. The mass variation test is only applicable in specific cases. The test for content uniformity is based on the content of each active ingredients in a number of unit doses in order to determine whether the content is within specified limits, being applicable in all cases [28].

\section{Methodology}

\subsection{High performance liquid chromatography}

In this study the identification tests, content, related compounds and content uniformity was performed by high performance liquid chromatography (HPLC) according to the specifications of literature [28]. The chromatograph Shimadzu detector was coupled to molecular absorption spectrophotometry in the ultraviolet-visible Perkin Elmer Lambda 25 model, Class-VP software. All chemical reference substances (SQR) were purchased from USP (The United States Pharmacopeia).

To analyze the Enalapril Maleate, $\mathrm{L} 7 \mathrm{C}_{8}$ column $(4.6 \mathrm{~mm} \times 25 \mathrm{~cm} \times 5 \mathrm{~mm})$ was used. Isocratic elution was performed with a buffer monobasic sodium phosphate $\mathrm{pH} 2.2$ /acetonitrile at a ratio of 75:25. Solvents and solutions were degassed in ultrasonic bath (Elma Transsonic Digitals) and filtered through a Millipore membrane of 0.45 micrometers. Chromatography was performed at $50^{\circ} \mathrm{C}$, flow rate of $2 \mathrm{~mL} \cdot \mathrm{min}^{-1}$, with injections of $50 \mu \mathrm{L}$, detection at $215 \mathrm{~nm}$ and running time of $30 \mathrm{~min}$. The calculations were based on the content of the samples obtained areas of the areas of the SQR of Enalapril Maleate. For related compounds the content of diketopiperazine compounds and enalaprilat was also calculated.

For omeprazole, $\mathrm{L}_{7} \mathrm{C}_{8}$ column $(4.6 \mathrm{~mm} \times 15 \mathrm{~cm} \times 5 \mathrm{~mm})$ was used. Elution was performed by mixing two solutions - solution A (6 g of glycine in $1500 \mathrm{~mL}$ water, $\mathrm{pH}$ 9) and solution $\mathrm{B}$ (acetonitrile and methanol, 85:15 ratio) - as shown in Table 3. Solvents and solutions were degassed in ultrasonic bath (Elma Transsonic Digitals) and filtered through a Millipore membrane of 0.45 micrometers. Chromatography was performed with a flow of 1,2 mL.min ${ }^{-1}$, with injections of $10 \mu \mathrm{L}$, detection at $305 \mathrm{~nm}$ and running time of $30 \mathrm{~min}$. The cal- 
culations were based on the content of the samples obtained areas of the areas of the SQR of omeprazole.

\begin{tabular}{cccc}
\hline Time (minutes) & Solution A (\%) & Solution B (\%) & Elution \\
\hline $0-20$ & $88 \rightarrow 40$ & $12 \rightarrow 60$ & Linear gradient \\
\hline $20-21$ & $40 \rightarrow 88$ & $60 \rightarrow 12$ & Linear gradient \\
\hline $21-25$ & 88 & 12 & Isocratic \\
\hline
\end{tabular}

Table 2. Parameters of elution of the HPLC analysis of Omeprazole

\subsubsection{Results and discussion}

a. Appearance, Identification and Labeling

Both, Omeprazole and Enalapril Maleate samples, showed similar aspects as their samples: hard capsule containing white pellets for Omeprazole, and hard capsule containing white powder varying only the color of the hard capsule used by each pharmacy. All samples, Omeprazole and Enalapril Maleate, were satisfactory for labeling and identification, confirming that the identity of the material was in accordance with the label from its packaging. In addition, all labels contain information provided by RDC Resolution № 67, October 8, 2007 [15]:

1. Name of the prescriber;

2. Name of the patient;

3. Registration Number of the formulation;

4. Data handling and shelf life;

5. Formulation components and their quantities;

6. Number of units;

7. Dosage;

8. Identification of pharmacy, full address and federal registration;

9. Name and professional register of the responsible person.

b. Related substances (Enalapril Maleate)

The test for related substances is performed only when described in the pharmacopeia of the compound to be analyzed, or another official compendium regulated by ANVISA [28]. For the Enalapril Maleate it is specified that no more than 5\% of diketopiperazine and enalaprilat can be found in the final product. All samples of Enalapril Maleate were satisfactory for this analysis and the results are shown in Table 5. 


\begin{tabular}{cccc}
\hline Pharmacy & Enalaprilat (\%) & Diketopiperazine (\%) & $\begin{array}{c}\text { Enalaprilat + } \\
\text { Diketopiperazine (\%) }\end{array}$ \\
\hline A & 0.66 & 0.82 & 1.48 \\
\hline B & 0.16 & 0.07 & 0.23 \\
\hline C & 0.53 & 0.87 & 1.40 \\
\hline D & 0.05 & 0.001 & 0.055 \\
\hline E & 1.18 & 2.77 & 3.95 \\
\hline
\end{tabular}

Table 3. Content of related compounds of Enalapril Maleate

\section{c. Content}

The reference values for the content of both drugs should not be less than $90 \%$ nor exceed $110 \%$ of the declared value, $10 \mathrm{mg}$ and $20 \mathrm{mg}$ for omeprazole and enalapril, respectively. The results for content of active ingredient are described in Table 6.

It can be observed that four from the five samples of omeprazole were unsatisfactory; two of them with content above the permissible and the other two with the content below. For the samples of enalapril, two were unsatisfactory, one exceeding the limit and the other with recommended content lower than expected, as showed in Table 6.

\begin{tabular}{ccc}
\hline Pharmacy & Omeprazole (10 mg/caps) & Enalapril Maleate (20 mg/caps) \\
\hline A & $(11.3 \pm 0.4) \mathrm{mg} /$ caps or & $(18.5 \pm 0.2) \mathrm{mg} / \mathrm{caps}$ or \\
& $112.7 \%$ declared & $92.6 \%$ of declared \\
\hline B & $(8.4 \pm 2.2) \mathrm{mg} /$ caps or & $(20.5 \pm 0.1) \mathrm{mg} / \mathrm{caps}$ or \\
& $84.4 \%$ declared & $102.7 \%$ declared \\
\hline C & $(6.9 \pm 2.6) \mathrm{mg} /$ caps or & $(18.5 \pm 0.1) \mathrm{mg} / \mathrm{caps}$ or \\
& $68.7 \%$ declared & $92.5 \%$ declared \\
\hline D & $(11.2 \pm 0.1)$ mg/caps or & $(11.2 \pm 4.3) \mathrm{mg} / \mathrm{caps}$ or \\
& $111.5 \%$ declared & $56.0 \%$ declared \\
\hline \multirow{2}{*}{ E } & $(11.0 \pm 0.2)$ mg/caps or & $(16.9 \pm 1.2) \mathrm{mg} /$ caps or \\
& $109.6 \%$ declared & $84.7 \%$ do declared
\end{tabular}

Table 4. Final content of Omeprazole and Enalapril Maleate

d. Uniformity of the dosage unit

All Omeprazole samples were considered unsatisfactory for uniformity of the dosage unit. Three samples were satisfactory for Enalapril Maleate. The results for uniformity of dosage unit are described in Table 7 as contained in the final analysis report issued by FUNED. Variations in dose uniformity should not exceed $15 \%$ [28]. 


\section{e. Mass Variation}

The acceptable limit for the analysis of variation in mass of capsules, weighing less than 300 $\mathrm{mg}$ is $\pm 10 \%$ above the average mass, and it is tolerable no more than two units outside the specified limit and any unit may be above or below twice the percentages indicated. Thus, only a sample of Omeprazole was considered unsatisfactory. The results for the samples of Omeprazole and Enalapril Maleate are presented in Tables 8 and 9, respectively [27].

\begin{tabular}{ccc}
\hline Pharmacy & Omeprazole (10 $\mathbf{~ g / c a p s )}$ & $\begin{array}{c}\text { Enalapril Maleate } \\
\text { (20 } \mathbf{~ m g / c a p s )}\end{array}$ \\
\hline A & $18.7 \%$ & $7.8 \%$ \\
\hline B & $24.0 \%$ & $14.2 \%$ \\
\hline C & $47.9 \%$ & $10.6 \%$ \\
\hline D & $15.6 \%$ & $14.0 \%$ \\
\hline E & $15.8 \%$ & $46.5 \%$ \\
\hline
\end{tabular}

Table 5. Dose uniformity of the capsules of Omeprazole and Enalapril Maleate

\begin{tabular}{cccc}
\hline Pharmacy & average weight (mg/caps.) & Lower & Higher \\
\hline${ }^{*} \mathrm{~A}$ & $220.4 \pm 1.2$ & 2.4 & 1.6 \\
\hline $\mathrm{B}$ & $226.8 \pm 5.8$ & 9.5 & 8.7 \\
\hline $\mathrm{C}$ & $210.0 \pm 4.9$ & 9.7 & 7.5 \\
\hline${ }^{*} \mathrm{D}$ & $119.0 \pm 2.1$ & 6.5 & 10.9 \\
\hline${ }^{*} \mathrm{E}$ & $120.7 \pm 2.9$ & 10.9 & 7.9 \\
\hline
\end{tabular}

*One unit above the limit. ${ }^{* *}$ Two units above the limit. Caps, capsules

Table 6. Variation (\%) in mass of the capsules of Enalapril Maleate (20 mg/caps)

\begin{tabular}{cccc}
\hline Pharmacy & average mass (mg/caps.) & Lower & Higher \\
\hline${ }^{*} \mathrm{~A}$ & $108.3 \pm 3.0$ & 7.1 & 15.1 \\
\hline $\mathrm{B}$ & $195.4 \pm 1.8$ & 4.6 & 3.0 \\
\hline $\mathrm{C}$ & $175.9 \pm 3.0$ & 9.1 & 4.7 \\
\hline${ }^{* *} \mathrm{D}$ & $107.0 \pm 7.0$ & 15.3 & 40.8 \\
\hline${ }^{* \star} \mathrm{E}$ & $105.0 \pm 2.1$ & 10.6 & 7.2 \\
\hline
\end{tabular}

*Two units above the limit. ${ }^{* *}$ Four units above the limit. Unsatisfactory. ${ }^{* * *}$ One unit above the limit.

Table 7. Variation (\%) in mass of the capsules of Enalapril Maleate (20 mg/caps) 
From the ten samples analyzed, seven were rated as unsatisfactory, considering the analysis of aspect, mass variation, identification, related substances, uniformity of dosage units, content and labeling.

It was observed that, if only the official established procedures (description, appearance, organoleptic characteristics and average mass) were considered from the seven samples rated as unsatisfactory, just one would be classified in this status. The remaining six samples would erroneously be rated satisfactory, meaning that would be approved for human consumption [18].

Some factors may cause deviations, inherent to the handling process of drugs in capsules, such as the loss of substance during the grinding, mixing and filling the capsules. Miscalculations and weight of the formulation components, errors inherent to the operator and the use of damaged equipment may also compromise the process and therefore the quality of the final product [29].

The results of Omerazole and Enalapril Maleate were analyzed by ANOVA followed by Tukey's test for uniformity of content and unit dose. Results were considered significantly different at $\mathrm{p}<0.05$.

\subsection{Neutron activation analysis}

\subsubsection{Material and methods}

All samples of Omeprazole and Enalapril Maleate were purchased in the market of the Belo Horizonte, state of Minas Gerais, Brazil from five (5) different magistral pharmacies.

Due to operational reasons, Jožef Stefan Institute (JSI) performed analyses only from three (3) different pharmacies from the sampling group. Due to same operational reasons, the JSI did not analyze the short half-lives radionuclides of elements like $\mathrm{Al}, \mathrm{Cl}, \mathrm{Mg}, \mathrm{Mn}$ and Ti.

The samples of Omeprazole and Enalapril Maleate performed by the JSI were packed in polyethylene capsules in plastic bottle containing 20 capsules each. Whole powder mass from 20 capsules was taken to prepare homogenized samples, which was transferred in clean polyethylene bottle. In the samples of Omeprazole performed by CDTN, just one the mass of one capsule taken randomly was considered by each sample. The difference of procedures carried out by both Institutes was due to operational reasons.

Both institutes CDTN/CNEN and JSI followed the same procedure to prepare the samples. The aliquots of each sample were manually crushed or ground using an agate mortar with pestle, whenever necessary, to avoid any contamination. In most cases, unless the amount of material did not allow it, two replicates were taken and weighed in polyethylene vials. It is relevant to emphasize that no additional chemical sample preparation was performed. At CDTN/CNEN and IJS, the samples were irradiated together with several Al-0.1\% Au disks as neutron flux monitors, according to the $\mathrm{k}_{0}$-standardisation method procedure $[23,24,30]$.

Table 10 shows the characteristics of the applied technique such as the parameters $f$ (thermal to epithermal fluxes ratio) and the $\alpha$ (parameter which measures the epithermal flux devia- 
tion from the ideal (1/E) distribution), needed for the $\mathrm{k}_{0}$-method, the irradiation times and gamma spectrometry systems at each Institute.

\subsubsection{Results and discussion}

The obtained results of NAA from the medicines Omeprazole and Enalapril Maleate are showed in the Tables 11 to 13 .

The technique applied was suitable for determining 20 chemical elements - $\mathrm{Al}, \mathrm{Br}, \mathrm{Ca}, \mathrm{Cl}$, $\mathrm{Co}, \mathrm{Cr}, \mathrm{Fe}, \mathrm{Mg}, \mathrm{Mn}, \mathrm{Na}, \mathrm{Sb}, \mathrm{Sc}, \mathrm{Sm}, \mathrm{Sr}, \mathrm{Ta}, \mathrm{Th}, \mathrm{Ti}, \mathrm{U}$ and $\mathrm{Zn}$ - in a large range of concentration, without any chemical process. The elements $\mathrm{Cl}, \mathrm{Fe}, \mathrm{K}, \mathrm{Mg}, \mathrm{Mn}, \mathrm{Na}$, and $\mathrm{Zn}$ could be expected in this kind of samples. Other elements, not considered essential, for the human being such as As and $\mathrm{Sr}$, found in lower concentration compared to $\mathrm{Cl}, \mathrm{Fe}, \mathrm{K}, \mathrm{Mg}, \mathrm{Mn}, \mathrm{Na}$, and $\mathrm{Zn}$ can also represent a health problem in a long term consumption. Even essential elements were determined but in high concentrations, like Fe may be toxic.

High concentration of elements such as $\mathrm{Cl}, \mathrm{Ca}, \mathrm{Mg}$, $\mathrm{Na}$ and $\mathrm{Ti}$ are expected because they are frequently components of excipients in the preparation of pellets. The presence of $\mathrm{Mg}$ is due to the excipients usually used: magnesium is a component of magnesium estearate $\left(\mathrm{Mg}\left[\mathrm{C}_{18} \mathrm{H}_{35} \mathrm{O}_{2}\right]\right)$, a lubricant for tablets and capsules and opadry, coloring agent, respectively [26]. $\mathrm{Mg}$ also is present in magnesium silicate $\left(\mathrm{Mg}_{3} \mathrm{SiO}_{4}(\mathrm{OH})_{2}\right.$ ] $\mathrm{Na}$ is a component of sodium laurilsulfate, $\left(\left[\mathrm{CH}_{3}\left(\mathrm{CH}_{2}\right)_{10}\left(\mathrm{CH}_{2} \mathrm{O}\right)\left(\mathrm{SO}_{3}\right) \mathrm{Na}\right]\right.$ and sodium bicarbonate $\mathrm{NaHCO}_{3}$. Ca is added as excipient as calcium phosphate and $\mathrm{Ti}$ as titanium dioxide, $\mathrm{TiO}_{2}$. Fe comes from red iron oxide, used as excipient as well [10,13]. The impurities such as $\mathrm{Br}, \mathrm{Co}, \mathrm{Cr}, \mathrm{Hf}, \mathrm{La}, \mathrm{Sb}, \mathrm{Sc}, \mathrm{Sm}, \mathrm{Sr}$, $\mathrm{Ta}$, Th and $\mathrm{U}$, are probably original from the raw material and/or from the process of production and manipulation of the medicine. All elements determined not foreseen in the original formula can be considered as impurities.

\begin{tabular}{ccc}
\hline & & INSTITUTE \\
\hline & CDTN/CNEN & JSI \\
\hline $\begin{array}{c}\text { Thermal Flux } \\
\left.\text { (neutrons } \mathrm{cm}^{-2} \mathrm{~s}^{-1}\right)\end{array}$ & $6.4 \times 10^{11}$ & $1.1 \times 10^{12}$ \\
\hline $\mathrm{k}_{0}$-standardisation parameters & & \\
$\mathrm{f}$ & 20.4 & 28.6 \\
\hline Detector nominal efficiency (\%) & 0.197 & -0.011 \\
\hline Software used for: & 8 & 13 \\
\hline Acquisition spectra & 50 & 40 \\
Spectra Analysis & Genie 2000 & Genie 2000 \\
Concentration calculation & $($ CANBERRA) & HyperLab \\
\hline Sample mass (mg) & HyperLab & Kayzero for Windows, V.2.42 \\
\hline
\end{tabular}

Table 8. Experimental information of neutron activation analysis 
The data presented in Tables 12, 13 and 14 cannot be compared directly because the samples analyzed are not from the batch, but the results are, in general, very similar. Most results determined by the CDTN in one capsule of Omeprazole taken randomly were also determined by the JSI in the homogenized samples, except for the elements $\mathrm{Br}, \mathrm{Cr}$ and La. The concentrations of the elements determined by both institutes have, in general, the same magnitude.

The discussion about toxicity levels and possible consequences for humans being is very difficult, due to the low concentration of the elements and limitations on the studies available in the literature. For most trace elements, there are just some available data on acute and chronic toxicity in experimental animals, not sufficient data to assess the risks to the human health on a long term daily intake [22].

\section{Conclusion}

The obtained results of samples of omeprazole and enalapril from five different magistral pharmacies of Belo Horizonte, Brazil, confirm the concern about the quality and safety for consumption of formulated medicines. They represent a preliminary part of a more complete investigation, still under way.

From the ten samples analyzed, seven were considered unsatisfactory. Most of the problems found through analyses Omeprazole and Enalapril Maleate medicines, like the variation of active principle mass, mass variation and dosage unit, come from the inadequacy of procedures for handling the ingredients in the pharmacy.

Problems can also be caused by the quality of the raw material used and inefficient or inexistence of test for checking the material. Diversified impurities reinforce the hypothesis that these elements are not controlled by the quality system. It also suggests that quality control over the purity of medicines in general should be established, as well as the concentration limits for the impurities, at least for some elements like $\mathrm{As}, \mathrm{Cd}, \mathrm{Cu}, \mathrm{Hg}, \mathrm{Pb}$ and $\mathrm{Sn}$, already foreseen for food in the Brazilian legislation.

The possible harmful and/or toxicological effects for the human health as a consequence of long term use of the formulated medicines represent an important concern for the authorities of the public health system. Recent cases of contamination and death in Brazil due to the consumption of inadequate formulated medicines has been enhancing the debate about the quality of the magistral pharmacy.

In conclusion, the results point out the necessity of prompt and efficient actions by the authorities of the health public system to assure the quality of formulated medicines. The aim of this work is just to provide evidences in order to contribute with this initiative. 


\begin{tabular}{|c|c|c|c|c|c|c|c|c|c|c|c|c|c|c|c|}
\hline \multirow{3}{*}{$\frac{\text { Element }}{\mathrm{Al}}$} & \multicolumn{15}{|c|}{ Pharmacy } \\
\hline & \multicolumn{3}{|c|}{ A } & \multicolumn{3}{|c|}{ B } & \multicolumn{3}{|c|}{ C } & \multicolumn{3}{|c|}{ D } & \multicolumn{3}{|c|}{$E$} \\
\hline & 532 & \pm & 20 & 260 & \pm & 10 & 305 & \pm & 11 & 452 & \pm & 17 & 335 & \pm & 12 \\
\hline $\mathrm{Br}$ & $\mathrm{DL}$ & & & 0.60 & \pm & 0.03 & $\mathrm{DL}$ & & & $\mathrm{DL}$ & & & $\mathrm{DL}$ & & \\
\hline $\mathrm{Ca}$ & 17740 & \pm & 793 & 9379 & \pm & 446 & 11230 & \pm & 512 & 19600 & \pm & 880 & 14260 & \pm & 190 \\
\hline $\mathrm{Cl}$ & 806 & \pm & 48 & 313 & \pm & 18 & 262 & \pm & 18 & 490 & \pm & 30 & 514 & \pm & 31 \\
\hline $\mathrm{Co}$ & 0.5 & \pm & 0.1 & 0.3 & \pm & 0.1 & 0.3 & \pm & 0.1 & $\mathrm{DL}$ & \pm & & 0.10 & \pm & 0.01 \\
\hline $\mathrm{Cr}$ & 11.7 & \pm & 0.5 & 7.9 & \pm & 0.3 & 6.7 & \pm & 0.3 & 11.6 & \pm & 0.5 & 13 & \pm & 1 \\
\hline $\mathrm{Fe}$ & 65 & \pm & 5 & 64 & \pm & 11 & 47 & \pm & 10 & 49 & \pm & 11 & 51 & \pm & 5 \\
\hline $\mathrm{Mg}$ & 4643 & \pm & 192 & 449 & \pm & 28 & 390 & \pm & 28 & 998 & \pm & 57 & 697 & \pm & 47 \\
\hline $\mathrm{Mn}$ & 2.8 & \pm & 0.3 & 1.4 & \pm & 0.1 & $\mathrm{DL}$ & & & 1.8 & & 0.2 & 2.2 & & 0.2 \\
\hline $\mathrm{Na}$ & 8134 & \pm & 326 & 4003 & \pm & 144 & 3918 & \pm & 140 & 6838 & \pm & 241 & 6718 & \pm & 247 \\
\hline $\mathrm{Sb}$ & 0.09 & \pm & 0.01 & 0.41 & \pm & 0.02 & 0.04 & \pm & 0.01 & 0.06 & \pm & 0.01 & 0.13 & \pm & 0.01 \\
\hline
\end{tabular}

Table 9. Elemental concentration (mg. $\left.\mathrm{kg}^{-1}\right)$ for Omeprazole (CDTN/CNEN)

\begin{tabular}{|c|c|c|c|c|c|c|c|c|c|c|c|c|c|c|c|}
\hline \multirow{3}{*}{$\frac{\text { Element }}{\mathrm{SC}}$} & \multicolumn{15}{|c|}{ Pharmacy } \\
\hline & \multicolumn{3}{|c|}{ A } & \multicolumn{3}{|c|}{ B } & \multicolumn{3}{|c|}{ C } & \multicolumn{3}{|c|}{ D } & \multicolumn{3}{|c|}{$\mathbf{E}$} \\
\hline & 0.03 & \pm & 0.01 & 0.03 & \pm & 0.01 & 0.01 & \pm & 0.01 & 0.02 & \pm & 0.01 & 0.02 & \pm & 0.01 \\
\hline $\mathrm{Sm}$ & $\mathrm{DL}$ & & & 0.02 & \pm & & $\mathrm{DL}$ & & & 0.02 & & 0.01 & $\mathrm{DL}$ & & \\
\hline $\mathrm{Sr}$ & $\mathrm{DL}$ & & & $\mathrm{DL}$ & & & $\mathrm{DL}$ & & & 21 & \pm & 4 & $\mathrm{DL}$ & & \\
\hline Ta & 0.02 & \pm & 0.01 & 0.14 & \pm & 0.01 & $\mathrm{DL}$ & & & 0.15 & \pm & 0.01 & 0.09 & \pm & 0.01 \\
\hline $\mathrm{Ti}$ & 2748 & \pm & 105 & 1483 & \pm & 55 & 897 & \pm & 34 & 2124 & \pm & 79 & 1907 & \pm & 71 \\
\hline U & $\mathrm{DL}$ & & & 0.22 & \pm & 0.01 & $\mathrm{DL}$ & & & $\mathrm{DL}$ & & & 0.4 & \pm & 0.1 \\
\hline $\mathrm{Zn}$ & 3.4 & \pm & 0.4 & 1.8 & \pm & 0.3 & $\mathrm{DL}$ & & & 2.9 & \pm & 0.4 & 2.5 & \pm & 0.3 \\
\hline
\end{tabular}

* DL - Lower than the Detection Limit

Table 10. Elemental concentration (mg. $\left.\mathrm{kg}^{-1}\right)$ for Omeprazole (CDTN/CNEN)

\begin{tabular}{cccccccccc}
\hline \multicolumn{10}{c}{ Pharmacy } \\
\hline Element & & A & \multicolumn{10}{c}{ B } & & C \\
\hline $\mathrm{Br}$ & 0.19 & \pm & 0.01 & 0.17 & \pm & 0.01 & 0.22 & \pm & 0.01 \\
\hline $\mathrm{Ca}$ & 16453 & \pm & 592 & 14908 & \pm & 540 & 12817 & \pm & 467 \\
\hline $\mathrm{Ce}$ & $\mathrm{DL}$ & & & $\mathrm{DL}$ & \pm & & 0.09 & \pm & 0.01 \\
\hline
\end{tabular}




\begin{tabular}{|c|c|c|c|c|c|c|c|c|c|}
\hline \multirow[b]{2}{*}{ Element } & \multicolumn{9}{|c|}{ Pharmacy } \\
\hline & & A & & & B & & & $\mathrm{C}$ & \\
\hline $\mathrm{Co}$ & 0.18 & \pm & 0.01 & 0.016 & \pm & 0.001 & 0.51 & \pm & 0.02 \\
\hline $\mathrm{Cr}$ & 0.52 & \pm & 0.03 & 0.26 & \pm & 0.02 & 0.59 & \pm & 0.04 \\
\hline $\mathrm{Fe}$ & 102 & \pm & 4 & 53 & \pm & 2 & 265 & \pm & 9 \\
\hline $\mathrm{Hf}$ & 0.020 & \pm & 0.001 & 0.009 & \pm & 0.001 & 0.021 & \pm & 0.002 \\
\hline La & 0.019 & \pm & 0.002 & 0.030 & \pm & 0.004 & 0.040 & \pm & 0.002 \\
\hline Mo & 0.34 & \pm & 0.05 & $\mathrm{DL}$ & \pm & & $\mathrm{DL}$ & & \\
\hline $\mathrm{Na}$ & 6616 & \pm & 232 & 5988 & \pm & 210 & 4836 & \pm & 169 \\
\hline $\mathrm{Sb}$ & 0.014 & \pm & 0.001 & 0.012 & \pm & 0.001 & 0.011 & \pm & 0.001 \\
\hline Sc & 0.013 & \pm & 0.005 & 0.010 & \pm & 0.001 & 0.022 & \pm & 0.001 \\
\hline $\mathrm{Sm}$ & $\mathrm{DL}$ & & & $\mathrm{DL}$ & & & 0.0052 & \pm & 0.0003 \\
\hline $\mathrm{Sr}$ & 22.3 & \pm & 1.1 & 17.4 & \pm & 1.0 & 9.0 & \pm & 1.0 \\
\hline $\mathrm{Ta}$ & 0.34 & \pm & 0.01 & 0.076 & \pm & 0.003 & 0.42 & \pm & 0.02 \\
\hline Th & 0.012 & \pm & 0.002 & $\mathrm{DL}$ & & & 0.027 & \pm & 0.002 \\
\hline$U$ & 0.09 & \pm & 0.01 & 0.14 & \pm & 0.01 & 0.053 & \pm & 0.004 \\
\hline $\mathrm{Zn}$ & 0.60 & \pm & 0.1 & 0.4 & \pm & 0.1 & 0.7 & \pm & 0.1 \\
\hline
\end{tabular}

Table 11. Elemental concentration $\left(\mathrm{mg}^{\mathrm{kg}}{ }^{-1}\right)$ for Omeprazole (JSI)

\begin{tabular}{cccccccccc}
\hline & \multicolumn{1}{c}{ Pharmacy } \\
\hline Element & & A & \multicolumn{7}{c}{ B } \\
\hline $\mathrm{Br}$ & 0.13 & \pm & 0.01 & 0.47 & \pm & 0.02 & 0.24 & \pm & 0.02 \\
\hline $\mathrm{Cr}$ & 0.07 & \pm & 0.01 & 0.15 & \pm & 0.01 & 0.10 & \pm & 0.01 \\
\hline $\mathrm{Na}$ & 12540 & \pm & 439 & 271 & \pm & 10 & 72480 & \pm & 2538 \\
\hline $\mathrm{Sc}$ & 0.0009 & \pm & 0.0001 & 0.0007 & \pm & 0.0001 & 0.0054 & \pm & 0.0002 \\
\hline $\mathrm{Sb}$ & 0.09 & \pm & 0.01 & 0.41 & \pm & 0.02 & 0.04 & \pm & 0.01 \\
\hline $\mathrm{Th}$ & $\mathrm{DL}$ & & & $\mathrm{DL}$ & & & 0.015 & \pm & 0.001 \\
$\mathrm{Zn}$ & 0.49 & \pm & 0.04 & $\mathrm{DL}$ & & & 0.015 & \pm & 0.001 \\
\hline
\end{tabular}

* DL - Lower than the Detection Limit

Table 12. Elemental concentration (mg. $\left.\mathrm{kg}^{-1}\right)$ for Enalapril Maleate (JSI) 


\section{Acknowledgements}

The authors would like to thank Dr. Adailton Pereira de Paiva and Dr. Marco Antonio Franzero for their kind and helpful collaboration.

\section{Author details}

Alexandre S. Leal ${ }^{1 *}$, Maria Ângela de B. C. Menezes ${ }^{1}$, Ilza Dalmázio ${ }^{1}$, Fernanda P. Sepe ${ }^{1}$, Tatiana C. B. Gomes ${ }^{1}$, Amalia S. Santana ${ }^{2}$, Luzia H. da Cunha ${ }^{2}$ and Radojko Jaćimović ${ }^{3}$

*Address all correspondence to: asleal@cdtn.br

1 Centro de Desenvolvimento da Tecnologia Nuclear/Comissão Nacional de Energia Nuclear (CDTN/CNEN), Belo Horizonte, Brazil

2 Fundação Estadual Ezequiel Dias (FUNED), Belo Horizonte, Brazil

3 Jožef Stefan Institute (JSI), Ljubljana, Slovenia

\section{References}

[1] Formulário Nacional Da Farmacopeia BrasileiraEd. 2. (2011). http://www.anvisa.gov.br/farmacopeiabrasileira/arquivos/FNFB\%202\%20Vers\%C3\%A3o\%20DICOL \%2009\%20Dez\%202012.pdfAcessed 23 May 2012)., 37.

[2] BrasilLei no 5991, de 17 de dezembro de (1973). Dispõe sobre o controle sanitário do comércio de drogas, medicamentos, insumos farmacêuticos e correlatos, e dá outras providências. Diário Oficial da República Federativa do Brasil, Brasília, 19 Dec. 1973.

[3] Szatkowski, L. T. D, \& Oliveira, C. L. O uso de medicamentos manipulados no município de Toledo. Infarma, Brasília, DF, n. 1-2, (2004). , 16, 77-80.

[4] Conselho Federal De FarmáciaHttp://Www.Cff.Org.Br/Acessed 01 Oct. (2011).

[5] Agência Nacional De Vigilância Sanitária (ANVISA)Boletim Informativo. Ed. 56. Jun. 2005. http://www.anvisa.gov.br/divulga/public/boletim/56_05.pdfAcessed (26 Sep. 2011).

[6] Gisele, H. Comunicação na Oficina: “Medicamentos Manipulados: um Desafio para o Sistema Nacional de Vigilância Sanitária". Rio de Janeiro, 08 and 09 May (2006).

[7] UOLFarmácia assume culpa por contaminação que causou doping de Cielo. http:// esporte.uol.com.br/natacao/ultimas-noticias/2011/07/01/farmacia-assume-culpa-porcontaminacao-que-causou-doping-de-cesar-cielo.htmAcessed (10 Oct. 2011). 
[8] Notícia, A. Remédio pode ter matado duas pessoas em Corupá, Norte de SC. http:// www.clicrbs.com.br/anoticia/jsp/default.jsp?uf=2\&local=18\&section=Geral\&newsID=a3497429.xmlAcessed 10 Oct. (2011).

[9] G1MG. Análise parcial comprova erro em produção de remédio, diz secretaria. http://g1.globo.com/minas-gerais/noticia/2011/12/analise-parcial-comprova-erro-emproducao-de-remedio-diz-secretaria.html (Acessed 23 Dec. 2011).

[10] Remington, J. P, \& Gennaro, A. R. The Science and Practice of Pharmacy. Philadelphia College of Pharmacy and Science, Philadelphia, ed. 20, (2000). , 2077.

[11] Fergusson, J. E. The Heavy Elements: Chemistry, Environmental Impact and Health Effects. Pergamon, Oxford, (1990). , 614.

[12] World Health Organization (WHO)www.who.intAcessed 03 Mar. (2010).

[13] Leal, A. S, Menezes, M. Â. B. C, Vermaercke, R. R, \& Rodrigues, O. Andonie; Sneyers, L. Investigation of chemical impurities in formulations, phytotherapics and polyvitaminic medicines by $\mathrm{k} 0$-instrumental neutron activation analysis. Nucl. Instrum. Methods Phys. Res. A 564, (2006). , 729-732.

[14] Leal, A. S, Menezes, M. Â. B. C, Vermaercke, P, Sneyers, L, \& Jensen, C. A comparative neutron activation analysis study of common generic manipulated and reference medicines commercialized in Brazil. App. Rad. Isotop. 66, (2008). , 1307-1312.

[15] BrasilResolução RDC n 67, de 8 de outubro de (2007). Diário Oficial da União, Poder Executivo, Brasília, DF, 09 Oct. 2007. Seção I, , 29-58.

[16] BrasilResolução RDC n 87, de 21 de novembro de (2008). Diário Oficial da União, Poder Executivo, Brasília, DF, 24 Nov. 2008, Seção I, , 58-59.

[17] BrasilResolução RDC n 21, de 20 de maio de (2009). Diário Oficial da União, Poder Executivo, Brasília, DF, 21 May 2009, Seção I, , 53.

[18] Wang, T, Wu, J, Hartman, R, Jia, X, \& Egan, R. S. A multi-element ICP-MS survey method as an alternative to the heavy metals limit test for pharmaceutical materials. Journal of Pharmaceutical and Biomedical Analysis. 23, (2000). , 867-890.

[19] Buurma, H, Smet, P. A. G. M, Hoff, O. P, Sysling, H, Storimans, M, \& Egberts, A. C. G. Frequency, nature and determinants of pharmacy compounded medicines in Dutch community pharmacies. Pharmacy World \& Science, Netherlands, n. 6, (2003). , 25, 280-287.

[20] Almeida, M. L, \& Filho, A. P. N. Análise das cápsulas manipuladas segundo a RDC 67/2007 da ANVISA/MS para a garantia da qualidade. Rev. Bras. Farm., n. 3, (2010). , 91, 119-125.

[21] Marinho, F. D. M. Zanon, J.C.C; Sakurai, E. Reis, I.A.; Lima, A.A.; Soares, C.D.V. Quality evaluation of simvastatin compounded capsules. Brazilian Journal of Pharmaceutical Sciences, n. 3, (2011). , 47, 495-502. 
[22] Oskarsson, A, \& Sandstrom, B. A Nordic Project-risk evaluation of essencial trace elements: esencial versus toxic levels of intake. Analyst., (1995). , 120, 911-912.

[23] De Corte, F, \& The, k. standardisation method; a move to the optimisation of neutron activation analysis. Ed. Ryksuniversiteit Gent, Faculteit Van de Wetenschappen, (1986). , 464.

[24] De Wispelaere, F, De Corte, F, Bossus, D. A. W, Swagten, J. J. M. G, \& Vermaercke, P. Re-determination and re-evaluation of the $\mathrm{f}$ and $\alpha$ parameters in channels $\mathrm{Y} 4$ and S84 of the BR1 reactor for use in K0- NAA at DSM research. Nuclear Instruments and Methods in Physics Research Section A, (2005). , 564, 636-640.

[25] Vermaercke, P, Robouch, P, Eguskiza, M, De Corte, F, Kennedy, G, Smodis, B, Yonezawa, C, Matsue, H, Lin, X, Blaauw, M, \& Kucêra, J. Characterization of synthetic multi-element standards SMELS used for validation of K0- NAA. Nuclear Instruments and Methods in Physics Research Section A, (2006). , 564, 675-682.

[26] Nordberg, G. F, et al. Handbook on the Toxicology of Metals. (2007). http:// pt.scribd.com/doc/59673422/Toxic-Metals-HandbookAcessed 01 Oct. 2011).

[27] Farmacopéia BrasileiraAgência Nacional de Vigilância Sanitária, Ed. 5, Brasília, (2010).

[28] USP 32 (The United States Pharmacopeia 32)United States Pharmacopeial Convention, (2009).

[29] Futuro, D. O, \& Silva, R. F. Uso de ferramentas de controle estatístico para o entendimento do processo de produção de cápsulas em farmácias magistrais. XXVIII Encontro Nacional de Engenharia de Produção Foz do Iguaçu, Paraná, Brasil, Oct. (2007). , 09-11.

[30] Menezes, M. Â. B. C, Jacimovic, R, \& Optimised, k. instrumental neutron activation method using the TRIGA MARK I IPR-R1 reactor at CDTN/CNEN, Belo Horizonte, Brazil. Nuclear Instruments and Methods in Physics Research Section A, (2006). , 564, 707-715. 


\section{Section 4}

Quality Control in Radiology and Clinical Imaging 

Chapter 12

\title{
Quality Assurance in Diagnostic Medical Exposures in Ghana - A Medical Physicist's Perspective
}

\author{
Stephen Inkoom \\ Additional information is available at the end of the chapter \\ http://dx.doi.org/10.5772/51445
}

\section{Introduction}

It is well known that medical expsoure procedures such as diagnostic radiology, nuclear medicine and radiotherapy remains the largest source of man made exposure to ionising radiation and continues to grow substantially. This makes the role of quality assurance (QA), an important tool in medical exposure procedures. This paper reviews the future of quality assurance in diagnostic medical exposures in Ghana from the perspective of a Medical Physicist, since a viable QA programme must be developed under the guidance and supervision of a medical physicist who is qualified in this area of expertise by education, training and experience. The Medical Physicist is expected to give guidance and supervision to the Technologists and other staff to execute the programme but should be prepared to perform higher level QA procedures as required. The focus of this review is on diagnostic radiology since it is the dominant mode of medical exposure as compared to nuclear medicine and radiotherapy procedures in Ghana as per the database of the Regulatory Authority Information System "(RAIS)" of the Radiation Protection Institute. It is also worth noting that most of the issues under consideration for discussion mirrors similar conditions in many developing countries. The main goal of a diagnostic quality assurance programme is to make sure that radiation doses to patients, staff and public are as low as reasonable achievable (ALARA) consistent with high quality diagnostic images of patients. An adequate diagnostic QA program involves periodic checks of all major components in the respective diagnostic imaging modalities. On the other hand, an optimum QA programme for any individual diagnostic facility will depend on some items such as the type of procedures performed, type of equipment utilized, patient workload, etc. The current scope of diagnostic imaging procedures in Ghana covers conventional, fluoroscopy, dental, computed tomography, interventional procedures and nuclear medicine scans. Interventional radiology procedures performed are 
quite few but the future looks promising in this field. The performance of QA practices are done on three fronts; namely at the hospital, equipment engineers and the Regulatory Authority (RA). The hospital based QA are done mainly by the Radiographic Technologist through their routine equipment warm ups and minor quality checks. Equipment Engineers perform engineer related QA checks through installation and acceptance testing, performance tests and periodic preventative maintenance procedures. On the other hand, the RA is largely in charge of major QA procedures through it's on site safety assessment inspections by assessing the compliance of the equipment within regulatory requirements. This is largely so because the RA has the technical expertise and equipment. Due to the expansion of diagnostic imaging procedures in medicine coupled with rapid technological advances, the availability of qualified and trained personnel is crucial if the desired quality is to be achieved. Some measures have been put in place for human resource development, but there is room for improvement. On the way forward, there is a strong need for the establishment of National Quality Control Centre for Diagnostic Radiology. This body must be equipped with the requisite state of the art equipment, highly qualified and trained personnel in order to coordinate all QA activities in the country. Such a body can initiate some guidelines on the minimum instrumentation requirements for all imaging modalities. Nevertheless, a good QA programmme is not a guarantee for the assurance of the radiation safety of patients, staff and public. What is also needed is a separate radiation safety programme, which is very essential in every diagnostic imaging facility and must also be under the direction of a qualified expert in radiation protection.

\section{Overview of quality assurance}

The 2008 United Nations Scientific Committee on the Effects of Atomic Radiation (UNSCEAR) report on medical exposures from the assessment of the global population dose from medical exposures for the period 1997-2007 indicates that medical exposure remains the largest source of man made exposure to ionizing radiation and continues to grow substantially. (UNSCEAR, 2008). A summary of the annual per caput effective dose to the global population due to all sources of ionizing radiation is illustrated in Table 1.

It is evident that diagnostic examinations result in a per caput effective dose of $0.66 \mathrm{mSv}$, while medical exposures now contribute around $20 \%$ of the average annual per caput dose to the global population. Medical exposures are defined as; (i) exposure of patients as part of their medical diagnosis or treatment; (ii) exposure of individuals as part of health screening programmes; and (iii) exposure of healthy individuals or patients voluntarily participating in medical, biomedical, diagnostic or therapeutic research programmes. These exposures include diagnostic radiology, nuclear medicine and radiation therapy (Fig. 1), out of which diagnostic radiology accounts for the largest contribution. Diagnostic radiology generally refers to the analysis of images obtained using x-rays. In nuclear medicine, a radiopharmaceutical is administered to the patient and concentrates primarily in a specific region of the body which allows: (i) external imaging of the body to evaluate structure and/or function, and (ii) or delivery of a large radiation dose to control a specific disease. Radiation therapy 
involves the use of intense radiation beams and high-activity sources for the treatment of many types of cancer.

\begin{tabular}{lll}
\hline Source & Annual per caput effective dose (mSv) & Contribution (\%) \\
\hline Natural background & 2.4 & 79 \\
\hline Diagnostic medical radiology & 0.62 & 20 \\
\hline Diagnostic dental radiology & 0.0018 & $<0.1$ \\
\hline Nuclear medicine & 0.031 & 1.1 \\
\hline Fallout & 0.005 & $<0.2$ \\
\hline Total & 3.1 & 100 \\
\hline
\end{tabular}

Source: UNSCEAR 2008 report on medical radiation exposures. (UNSCEAR, 2008).

Table 1. Sources of ionizing radiation and the annual per caput effective dose to the global population.

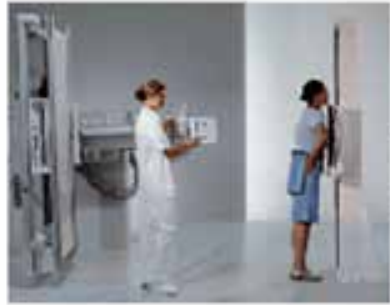

a

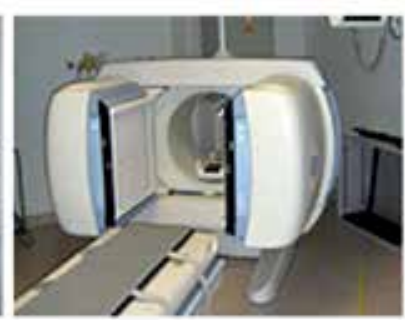

b

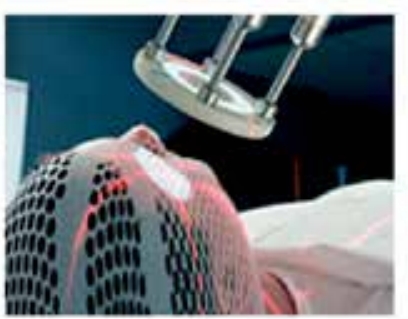

$\mathrm{c}$

Figure 1. An illustration of (a) diagnostic radiology, (b) nuclear medicine and (c) radiation therapy procedures.

This makes the role of (QA), an important tool in medical exposure procedures. Quality assurance procedures must aim at to produce images of optimal diagnostic quality while ensuring that the radiation exposures to patients, staff and the public are kept as low as practicable. In achieving this goal, QA procedures if well implemented would ensure that any problem in the imaging chain will be dealt without a compromise in the diagnostic quality of the images. The World Health Organization (WHO), (WHO, 1982), indicates that achieving adequate diagnostic information and least possible exposure of the patient to radiation should be done at the lowest possible cost. An adequate diagnostic QA program involves periodic checks of all major components in the respective diagnostic imaging modalities. On the other hand, an optimum QA programme for any individual diagnostic facility will depend on some items such as the type of procedures performed, type of equipment utilized, patient workload, etc. Any QA procedure must be in agreement with the relevant national and international legislation or regulations on the protection and safe uses of ionising radiation. Another form of QA is to establish clinical audit, which is an essential 
tool for quality improvement in any diagnostic centre. For instance, the European Council directive (Commission of the European Communities. 1997) defines clinical audit as: "a systematic examination or review of medical radiological procedures which seeks to improve the quality and the outcome of patient care, through structured review whereby radiological practices, procedures, and results are examined against agreed standards for good medical radiological procedures, with modifications of the practices where indicated and the application of new standards if necessary."

This paper reviews the future of quality assurance in diagnostic medical exposures in Ghana from the perspective of a Medical Physicist, since a viable QA programme must be developed under the guidance and supervision of a medical physicist who is qualified in this area of expertise by education, training and experience. The Medical Physicist is expected to give guidance and supervision to the Technologists and other staff to executive the programme but should be prepared to perform higher level QA procedures as required.

\section{Current status of quality assurance in diagnostic medical exposures}

The types of diagnostic medical exposure procedures in Ghana are;

- Plain radiography

- Mammography

- Fluoroscopy

- Computed Tomography

- Dental

In addition to the above procedures for diagnosis, some hospitals perform interventional or invasive procedures on a limited scale. On the other hand, the types of medical exposure in nuclear medicine procedures are the use of gamma camera and single photon emission computed tomography (SPECT/CT) for imaging various organs. Plain radiography (screen-film and digital systems) is the dominant mode of all the diagnostic medical procedures, accounting for more than $80 \%$ of the total contribution of all the imaging modalities. (RAIS, 2011).

The performance of QA practices are done on three fronts; namely at the hospital, equipment/service engineers and the (RA). The hospital based QA are done mainly by the Radiographers/Radiologic Technologist through their routine equipment warm ups and minor quality checks. In this scenario, a qualified Medical Physicist with the requisite expertise must supervise such QA procedures. Unfortunately, there are not many Medical Physicists in diagnostic departments as compared to radiation therapy centres. Equipment/service engineers perform engineer related QA checks through installation and acceptance testing, performance tests and periodic preventative maintenance procedures as well as when there is equipment down time. On the other hand, the RA is largely in charge of major QA procedures through it's on site safety assessment inspections by assessing the compliance of the 
equipment within regulatory requirements. This is largely so because the RA has the requisite technical expertise and equipment.

In Ghana, Medical Physicists are engaged in service, teaching, research and administration activities. They perform some of the tasks outlined by the International Organisation for Medical Physics (IOMP) in its definition of who a Medical Physicist is and the roles and responsibilities (International Organisation for Medical Physics, 2010) such as:

- teaching principles of medical physics to physicians, residents, graduate students, medical students, technologists, and other health care professionals by means of lectures, problem solving, and laboratory sessions.

- conducting research into various human disorders, illnesses and disabilities, develop instrumentation, mathematical analysis and applications of computers in medicine; investigating biophysical techniques associated with any branch of medicine. Research is very important for advancement of medical physics as a profession and science.

- responsible for ensuring the quality, safety testing and correct maintenance of all radiation emitting devices in order to get an accurate diagnosis of illnesses. Medical Physicists also involved in the formulation of radiation protection guides and procedures specific to clinical environment and producing protocols to minimize radiation exposure of patients, staff and the general public.

- in administration, they supervise and manage radiation workers and other health professional workers.

- participating in and contributing to the development and implementation of national and

- prepares guidance on education and training drawing-up standards and guidance relating to medical devices.

- preparing, publishing and presenting scientific papers and reports

Ghana is involved in several International Atomic Energy Agency (IAEA) Technical and Research Projects. Some of the Projects in which Medical Physicists are involved are:

- RAF/9/033 - Strengthening Radiological Protection of Patients and Medical Exposure Control.

- RAF/9/034 - Establishment of National Capabilities for Response to a Radiological and Nuclear Emergency.

- RAF/9/035 - Education and Training in Support of Radiation Protection Infrastructure.

- RAF/9/032 - Development of Technical Capabilities for the Protection of Health and Safety of Workers Exposed to Ionizing Radiation.

- RAF/9/027 - National Regulatory Control and Occupational Radiation Protection Programmes.

- RAF/9/031 - Strengthening National Regulatory Infrastructure for the Control of Radiation Sources. 
- GHA/6/015 - Upgrading and Expansion of Radiotherapy and Nuclear Medicine Services

- INT/6/054 - Strengthening Medical Physics in Radiation Medicine

- RAF/2/008 - Strengthening and Expanding Radiopharmacy Services in Africa (AFRA)

- RAF/6/032 - Promoting Regional and National Quality Assurance Programmes for Medical Physics in Nuclear Medicine (AFRA II-7)

- RAF/6/041 - Supporting the Development of Comprehensive National Cancer Control Programmes

- RAF/6/044 - Medical Physics in Support of Cancer Management (AFRA II-8)

- RAF/6/045 - Enhancing Accessibility and Quality in the Care of Cancer Patients (AFRA II-10)

The main objectives of some of the projects are discussed. For instance in RAF/9/033, the objectives are to upgrade / strengthen radiological protection of the patient in medical exposures due to:

i. Diagnostic Radiology and Interventional Radiological procedures

ii. Nuclear Medicine procedures

iii. Radiotherapy practice

The objectives of other projects are as follows:

- GHA/6/015 - To consolidate existing radiotherapy and nuclear medicine facilities at two leading Teaching Hospitals located in the southern part of the country, and establish a third one in the northern part to cater for the diagnosis, curative and palliative treatment of cancer patients and the efficient diagnosis and management of other diseases.

- INT/6/054 - To promote the recognition of medical physics in radiation medicine and to harmonize educational material in order to ensure safe and effective diagnosis and treatment of patients.

- $\mathrm{RAF} / 2 / 008$ - To strengthen radiopharmacy in support of in vivo and in vitro nuclear medicine in Africa.

- RAF/6/032 - To improve the effectiveness and safety of nuclear medicine procedures by providing support for design and implementation of quality assurance (QA) programmes and by establishing training and education programmes in medical radiation physics, focusing on aspects related to the application of nuclear medicine techniques.

- RAF/6/041 - To assist Member States in performing comprehensive cancer capacity need assessments and national cancer strategic planning via collaboration with IAEA, WHO, and other partners under the Programme of Action for Cancer Therapy (PACT) umbrella.

- RAF/6/044 - To strengthen national and regional medical physics capabilities to ensure efficient support of cancer management in AFRA Member States and to sustain quality as- 
surance/quality control (QA/QC) programmes, including the promotion of safety culture and innovative practices in dosimetry.

- RAF/6/045 - To establish national and regional networks in clinical radiation oncology. To interact with National Organizations with the aim to promote the comprehensive management of commonest cancers. Support academic education, training and accreditation, patients and personnel safety in radiotherapy improvement of documentation of clinical outcomes through regular patient assessment.

\section{Regulatory guidelines for quality assurance procedures}

The National Competent/Regulatory Authority in Ghana charged with the responsibility for authorization and inspection of practices using ionizing radiation sources and radioactive materials is the Radiation Protection Board (RPB) (Radiation Protection Instrument LI 1559, 1993). However, the operational functions of the RPB are carried out by RPI, which was established in 2000 to provide scientific and technical support for the enforcement of the provisions in LI 1559. Details about how the RA was established and the main activities have been described elsewhere (Inkoom et al, 2011). There are plans to establish a new Regulatory Body to regulate the peaceful uses of nuclear energy and technology which will be independent of any governmental agency. Currently, the RA is answerable to the Ghana Atomic Energy Commission (GAEC) which is a promoter for the peaceful uses of nuclear energy and technology and also plays the role of a regulator. However, the new RA is expected to be only a regulator and not a promoter of the application of nuclear technology.

\section{Human resource development}

The categories of Radiographic Staff available in Ghana are Radiologists, Medical Physicists, Biomedical Engineers and Radiographers/X-ray Technicians. Most of our Radiologists were trained overseas until the last few years when local training of Radiologists started and the accreditation is given by either the Ghana College of Surgeons or the West African College of Physicians and Surgeons. Similarly, the other professionals were also trained overseas. Currently, the School of Allied Health Sciences (SAHS), College of Health Sciences (CHS) of the University of Ghana (UG) is responsible for churning out medical and dental technical graduates in physiotherapy, medical laboratory science and radiography. There are plans to establish another Allied Health University and some private institutions are also running some of the programmes. A Post-Graduate School of Nuclear and Allied Sciences which was established jointly by the GAEC and UG, in co-operation with the IAEA is training the Medical Physicists, Radiation Protection Professionals, Nuclear Engineers, etc. at the National and Sub-Regional levels. 


\section{Recent trends in quality assurance}

The increasing expansion of diagnostic imaging procedures in medicine coupled with rapid technological advances makes the availability of qualified and trained personnel to be very crucial if the desired quality is to be achieved. This come with a lot challenges to the medical imaging community. This offers practitioners the opportunity to continually undergo retraining and other continuous professional development programmes in their respective fields. Also with the emergence of picture archiving and communication system (PACS) in many hospitals, there is the need for the development of appropriate on line QA procedures and in corporating them into hospital PACS systems. Special attention must also be given to the emergence of digital technology over the last decade as one of the greatest technological advances in medical imaging. This new technolgy poses a great challenge in medical imaging, requiring re-training of staff on the safe use of equipment and radiation protection issues. In Ghana for instance, the RPI of GAEC, in collaboration with the IAEA, has in the previous years developed a lot of expertise in the training of occupationally exposed workers in Ghana and the rest of Africa, spanning a period of almost two decades (Boadu et al. 2011). This local expertise in training can be tapped. In this regard, a critical review of all QA procedures that were developed for screen-film systems needs special attention.

\section{The way forward}

Various practitioners in the medical imaging community must brace themselves in order to face challenges of technological developments. With the advent of digital radiography: advances in computed radiography, direct digital radiography, digital subtraction angiography, new digital receivers, image processing techniques, computer applications in radiology and PACS offers enormous challenges. The advantages of digital technology:postprocessing capabilities, decreased costs, multiple viewing options, electronic transfer, possibilities of archiving, wide dynamic range of flat panel detectors and increased detection quantum efficiency has led to a high demand of this technology by the medical imaging community. Therefore, the development of the requisite human resource must be continued and sustained in order to deal with the challenges.

There is a strong need for the establishment of National Quality Control Centre for Diagnostic Radiology. This body must be equipped with the requisite state of the art equipment, highly qualified and trained personnel in order to coordinate all QA activities in the country. Such a body can initiate some guidelines on the minimum instrumentation requirements for all imaging modalities. With the training of more Medical Physicists sand Radiation Protection Professionals, it is expected that they would take up positions 
in all major hospitals which have a myriad of imaging modalities. The Ghana Society of Medical Physics, RA, Ministry of Health and other stakeholders must initiate procedures for the establishment of Medical Physics Departments in such hospitals. This would give the necessary recognition to the profession of Medical Physics in Ghana, which has been given recognition by the International Labour Organization (ILO) in its International Standard Classification of Occupations (ISCO) (ILO, 2008). With this recognition, Medical Physics has been accepted as modern applied branch of physics. Clinical audit should also be incorporated in the overall QA procedures in the country.

As the uses of ionizing radiation continue to increase in medicine, it is also expected that the services of Medical Physicist would increase. As such, more physicists would be required to be trained in subsequent years. Appropriate accreditation bodies charged with issuing accreditation certificates, for a period of years must be put in place to regulate the profession of Medical Physics and maintain international standards of practice.

\section{Conclusion}

The role of an effective QA programme in any diagnostic department cannot be overemphasized especially if the desired quality of producing good diagnostic images and the least radiation exposure are to be achieved. Nevertheless, a good QA programmme is not a guarantee for the assurance of the radiation safety of patients, staff and public. What is also needed is a separate radiation safety programme, which is very essential in every diagnostic imaging facility and must also be under the direction of a qualified expert in radiation protection or a Medical Physicist expert. With significant contributions in clinical service, education, and research, Medical Physics continues to grow in importance both as a profession and as science, driven by the technological developments of societies in general and medicine in particular.

\section{Acknowledgements}

The support received from the Radiation Protection Institute of Ghana Atomic Energy Commission is appreciated.

\section{Author details}

Stephen Inkoom

Radiation Protection Institute, Ghana Atomic Energy Commission, Legon, Accra, Ghana 


\section{References}

[1] Boadu, M., Schandorf, C., Emi-Reynolds, G., Faanu, A., Inkoom, S., Kwabena, Gyekye. P., \& Kaikor, M.C. (2011). Systematic approach to training of occupationally exposed workers in Ghana andthe rest of Africa. Health Phys, 101(2: S116YS120).

[2] Commission of the European Communities. (1997). Council Directive 97/43/ Euratom of 30 Juneon health protection of individuals against the dangers of ionizing radiation in relation to medical exposure, and repealing Directive 84/466 Euratom. Off. J. Eur. Commun. Rep. L., 180(1997), 22-27.

[3] International Labour Organization (ILO). International Standard Classification of Occupations (ISCO), Geneva, Switzerland, (ISCO-08), 2008.

[4] International Organisation for Medical Physics (IOMP). (2010). IOMP Policy Statement No.1. The Medical Physicist: Role and Responsibilities, IOMP Working Group on Policy Statement [1].

[5] Radiation Protection Instrument LI 1559. (1993). Provisional National Defence Council Law 308, Accra, Ghana. Date of Gazette Notification: 2nd April, 1993.

[6] Regulatory Authority Information System [RAIS]. (2011). Radiation Protection Institute, Ghana Atomic Energy Commission, Accra, Ghana.

[7] Inkoom, S., Schandorf, C., Reynolds, G. E., \& Fletcher, J. J. (2011). Quality Assurance and Quality Control of Equipment in Diagnostic Radiology Practice-The Ghanaian Experience. Wide Spectra of Quality Control, Isin Akyar (Ed.), 291-308, 978-9-53307-683-6, InTech, http://www.intechopen.com/articles/show/title/quality-assurance-and-quality-control-of-equipment-in-diagnostic-radiology-practice-the-ghanaian-exp.

[8] United Nations Scientific Committee on the Effects of Atomic Radiation. (2008). Sources and effects of ionizing radiation. Report to the General Assembly with scientific annexes (UNSCEAR).

[9] World Health Organization (1982). Quality Assurance in Diagnostic Radiology, Macmillan Procrom,9-24154-164-4 
Chapter 13

\title{
Quality by Design and Risk Assessment for Radiopharmaceutical Manufacturing and Clinical Imaging
}

\author{
Kung-Tien Liu, Jian-Hua Zhao, Lee-Chung Men and \\ Chien-Hsin Chen
}

Additional information is available at the end of the chapter

http://dx.doi.org/10.5772/51112

\section{Introduction}

Radiopharmaceuticals have been widely used in many clinical and nonclinical applications, such as in vivo and non-invasive diagnosis or treatment of human diseases. The quality of radiopharmaceuticals administered for a patient is primarily related for the radiation dose delivered to achieve optimizing diagnostic imaging or therapeutic efficacy. Radiopharmaceuticals with different half-lives (short, medium, and long), decay modes (alpha, beta, gamma, and electron capture), and biochemical properties (of ligands) can determine their utilities in medicine. Moreover, chemical and radiochemical impurities in a radiopharmaceutical can produce a serious trouble of diagnosis or treatment. Therefore, different requirements, regulations, and instrumentations for ensuring their high quality and high safety have been developed in many countries.

There are only few years for the progress of "Quality by Design (QbD)" in International Conference on Harmonisation (ICH) Guidelines, e.g. ICH Q8, ICH Q9, and ICH Q10 [1-3]. According to the requirement of ICH Q8, quality can not be tested into products; i.e., quality should be built in by design, i.e. QbD. Enhanced QbD approach to pharmaceutical development can improve the product and process knowledge.

In this chapter, we provide a harmonized framework of $\mathrm{QbD}$ for manufacturing and clinical applications of radiopharmaceuticals in accordance with the requirements and guidelines of U.S. Food and Drug Administration (FDA), International Atomic Energy Agency 
(IAEA), World Health Organization (WHO) and European Association of Nuclear Medicine (EANM). The attributes of the components in the quality system (QA/QC), including organization, staffing and personnel, facilities, instrumentation and equipment, operation procedure, radiopharmaceuticals, protocol and conduct of a study or a treatment, records and reports, and audit framework were further characterized. Assessments and comparisons of critical quality attributes (CQAs) for assuring accurate radioactive dosimetry calculation in the efficiency tracing of absolute activity measurement and patient- and technologistrelated risks for nuclear medicine imaging including Positron Emission Tomography (PET), Computed Tomography (CT), PET/CT, and Single Photon Emission Computed Tomography (SPECT) were identified.

\section{Quality system design based on the Requirements and Guidelines}

\subsection{Quality policy and system}

The quality system by design for radiopharmaceuticals and clinical imaging techniques is aimed to maintain and improve the qualified service for the patients, fulfill the regulatory requirements, optimize the safety and efficacy for patient care, demonstrate a proper equipment operating condition, and obtain a reliable quantitative performance in both diagnostic and therapeutic nuclear medicine procedures [4,5]. The pursuit of excellence in quality system is not a single action over a short period, instead, it is achieved through the whole life cycle of instruments, analytical methods or education for example, from planning and procurement to decommissioning based on advanced technology [6]. Continuous quality improvement implies a commitment to continuously struggle to advance based on state-ofthe-art information and techniques developed by the nuclear medicine and metrology community at large [5].

Implementation of a quality system must be in accordance with the quality police, i.e. the overall quality intentions and direction of an organization, as formally expressed by top management. And quality system includes the structure, responsibilities, and procedures for implementing quality management. An integrated infrastructure of quality policy and system design is demonstrated as in Figure 1, which is mainly developed from the European Standard EN 28402 proposed by Bergmann et al. [7]. The attributes of the components in the quality sub-system (QA/QC), e.g. organization, personnel, facilities, instrumentation, operation procedures, preparation of radiopharmaceuticals, protocol and conduct, records and reports, and audit or inspection, were further integrated and classified in this article. 


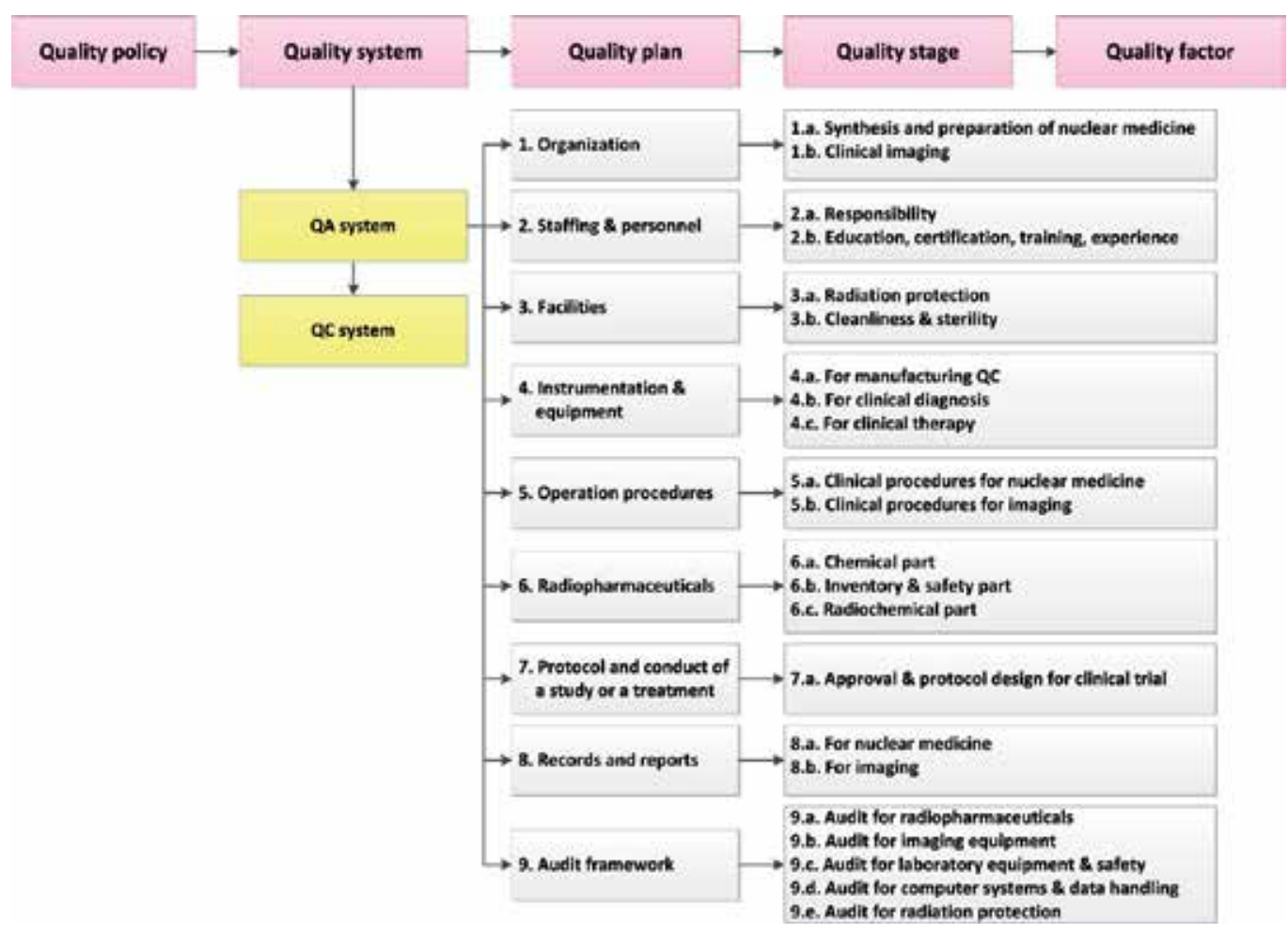

Figure 1. Quality policy and system for the radiopharmaceuticals [7].

\subsection{Quality plan and key factors}

\subsubsection{Organization}

The organization of quality system could be grouped into two categories: (a) synthesis and preparation of nuclear medicine and (b) clinical imaging as shown in Figure 2 [8-10]. For synthesis and preparation of nuclear medicine, three important guidelines were considered [11-13]. Basically, preparation of "classical" radiopharmaceuticals in "kit" procedures and in a "distinct chemical" procedures for PET radiopharmaceuticals are distinguished as two different parts [11].

For the clinical imaging, the major differences PET and SPECT in QbD are related to the properties and applications of a radiotracer. The most commonly used nuclides for PET imaging, such as carbon-11, oxygen-15, nitrogen-13, and fluorine-18, exhibit shorter half-life and more complicated labelling technology than that for SPECT imaging (Table 1)[14-31]. For example, the short half-lives of radionuclides used in PET modality allow for better de- 
tection sensitivity over a given period of time. This is because radiotracers with shorter halflives can be injected in higher activities to the patient without posing any additional radiation damage to the patient (since overall accumulation over time remains the same) leading to the increased detectable radiation over a shorter time. Moreover, arguments that the natural occurrence of PET isotopes in biologically active molecules (as opposed to heavy isotopes used in SPECT) results in a less challenging task of synthesizing physiologically useful tracers in PET modality [32,33]. In general, PET generally has a higher resolution, higher sensitivity, and a better quantitation capability than SPECT. However, SPECT is more practical as a routine procedure [18] and is more cost-effective for the system setting or maintain than a PET facility [8].

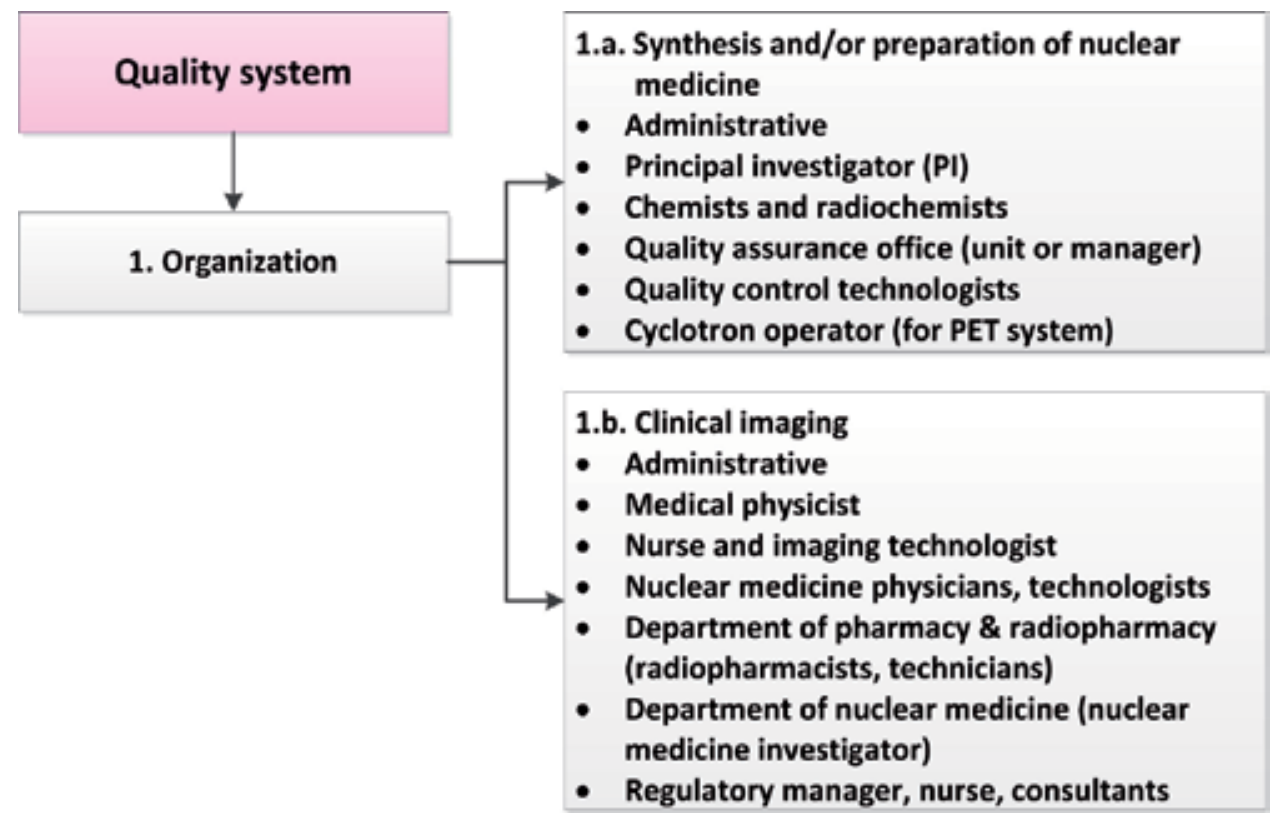

Figure 2. Quality system of organization [8-10].

\begin{tabular}{|c|c|c|c|c|}
\hline Agents & Isotope & $\begin{array}{l}\text { Half-life } \\
\left(t_{1 / 2}\right)\end{array}$ & Radiopharmaceutical & Applications \\
\hline \multirow{5}{*}{$\begin{array}{l}\text { PET imaging } \\
\text { agents }\end{array}$} & $C-11$ & $20.4 \min$ & C-11-raclopride & D2/D3 dopamine receptor \\
\hline & & & C-11-MADAM & Serotonin transporter \\
\hline & $\mathrm{N}-13$ & $9.96 \min$ & N-13-ammonia & Blood flow (ventricle) \\
\hline & O-15 & $2.07 \mathrm{~min}$ & 0-15 water & $\begin{array}{l}\text { Myocardium perfusion, brain } \\
\text { perfusion }\end{array}$ \\
\hline & Ga-68 & $68 \mathrm{~min}$ & Ga-68-DOTA & Neuroendocrine tumours \\
\hline
\end{tabular}




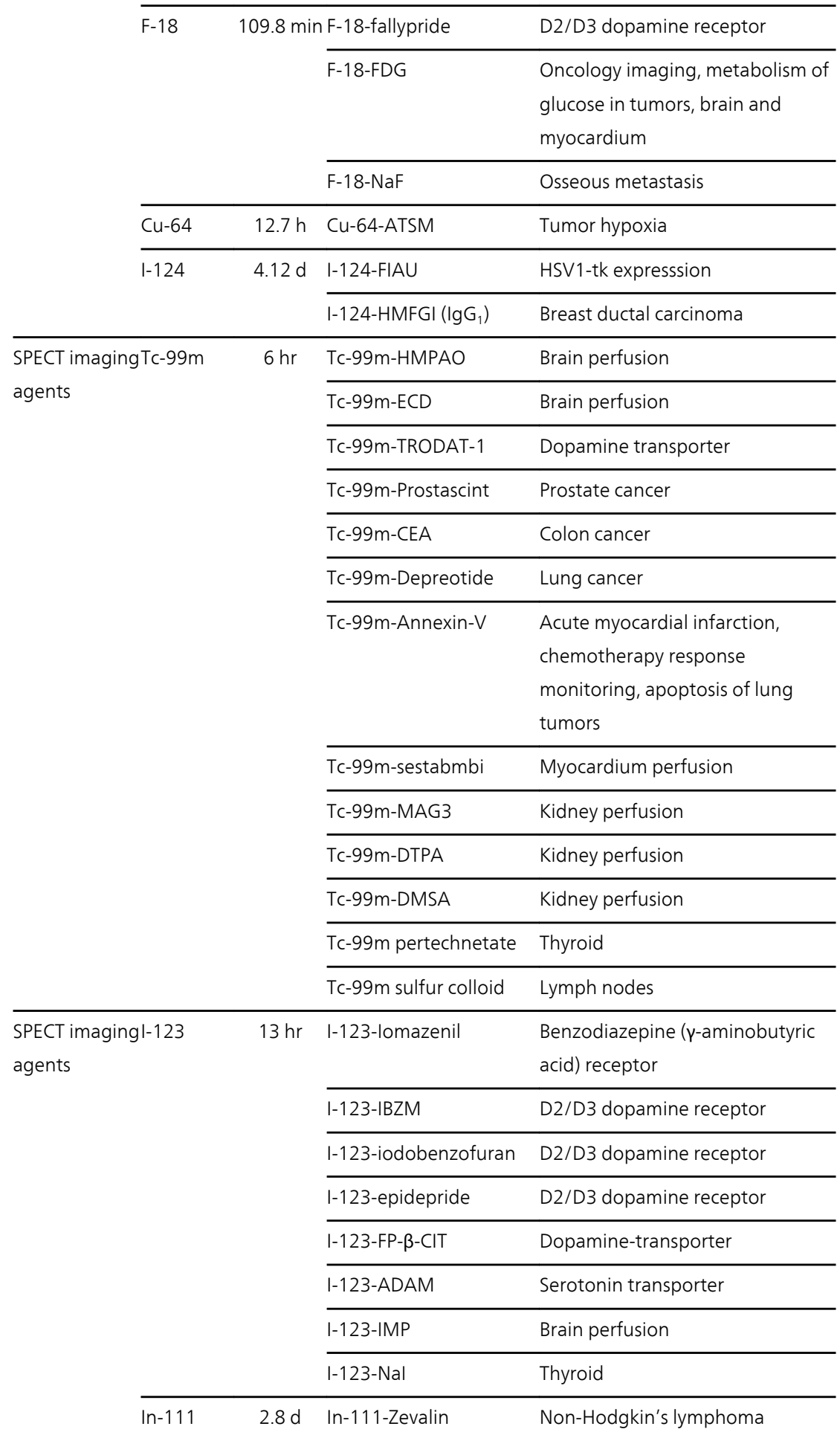




\begin{tabular}{|c|c|c|c|c|}
\hline & & & \\
\hline & & & In-111-Octreotide & $\begin{array}{l}\text { Somatostatin receptor } \\
\text { (Neuroendocrine tumors) }\end{array}$ \\
\hline & TI-201 & $3.04 \mathrm{~d}$ & Tl-201 & Myocardium perfusion \\
\hline & Ga-67 & $3.3 d$ & Ga-67 citrate & Non-Hodgkin's lymphoma \\
\hline \multirow{11}{*}{$\begin{array}{l}\text { Therapy } \\
\text { agents }\end{array}$} & Sm-153 & $1.95 d$ & Sm-153 EDTMP & Metastatic bone pain palliation \\
\hline & Sr-89 & $50.5 d$ & $\mathrm{SrCl}_{2}$ & $\begin{array}{l}\text { Palliative treatment of bone } \\
\text { cancers and for prostate cancer }\end{array}$ \\
\hline & P-32 & $14.28 d$ & Orthophosphate & Metastatic bone pain palliation \\
\hline & Re-186 & $3.78 d$ & Re-186-HEDP & Metastatic bone pain palliation \\
\hline & Re-188 & $17 \mathrm{~h}$ & \multicolumn{2}{|c|}{ Re-188-bisphosphonate Metastatic bone pain palliation } \\
\hline & Y-90 & $64.14 \mathrm{~h}$ & \multicolumn{2}{|l|}{ Tiuxetan } \\
\hline & $\mathrm{I}-131$ & $8 d$ & I-131 Tositumomab & B-cell non-Hodgkin's lymphoma \\
\hline & Lu-177 & $6.7 \mathrm{~d}$ & \multicolumn{2}{|l|}{ Octreotate } \\
\hline & Ho-166 & $1.1 \mathrm{~d}$ & Ho-166-DOTMP & Multiple myeloma \\
\hline & Sn-117m & $13.6 d$ & Sn-117m-DTPA & Metastatic bone pain palliation \\
\hline & At-211 & $7.2 \mathrm{~h}$ & At-211-81C6 & Glioblastoma multiforme tumors \\
\hline
\end{tabular}

Table 1. Some examples of radiopharmaceutical classification and applications [14-31]. ADAM: 2-((2-

((dimethylamino)-methyl) phenyl)thio)-5- iodophenylamine; DTPA: diethylenetriaminepentaacetic acid; ECD: ethyl cysteinate dimer; FDG: fluoro-deoxy-glucose; FIAU: 1-(2-fluoro-2-deoxy-ß-D-arabinofuranosyl)-5-[I-124]iodouracil; FP$\beta$-CIT: N-propyl-2-beta-carboxy-methoxy-3-beta(4-iodophenyl)-nortropane; HMPAO: hexamethyl propylene amine oxime; IBZM: iodobenzamide.

\subsubsection{Staffing and personnel}

Facilities should have written staff and personnel responsibilities and requirements. Two types of staff in the requirements for synthesis and preparation of nuclear medicine and clinical imaging are necessary [4]:

a. Personnel for synthesis and preparation of nuclear medicine may include such as facility management, administrative staff, study director (SD), principal investigator (PI), production chemists, QA manager or quality assurance unit (QAU), radiochemists, QC chemists, cyclotron operators, and technologists.

b. Personnel for PET and SPECT imaging examination may include such as facility management, administrative staff, medical physicists, nurses, referring physicians, nuclear medicine physicians, radiopharmacist, radiochemists, radiation protection॰ officer, engineers, QA manager or QAU, and technologists. 


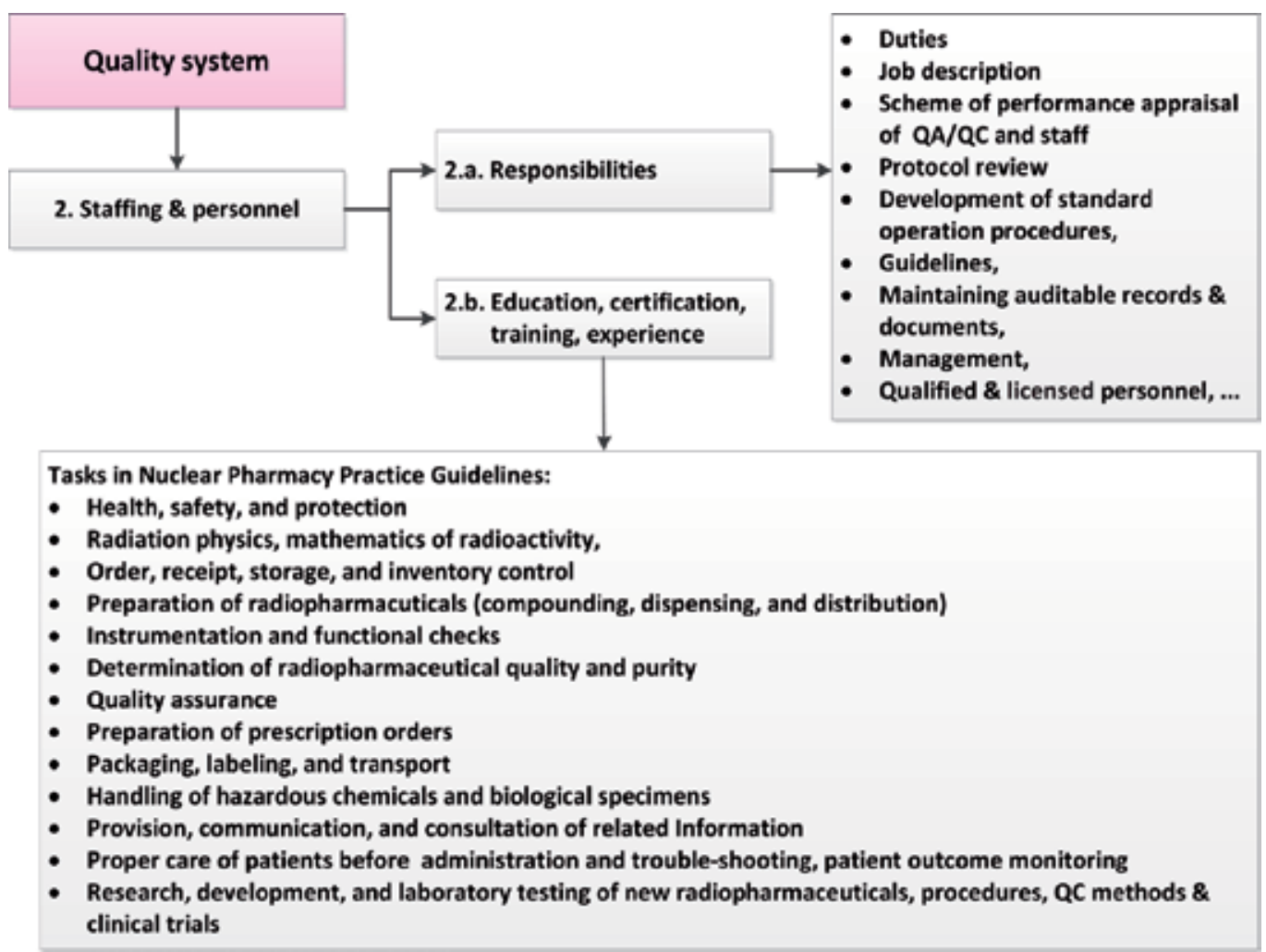

Figure 3. Quality system of staffing and personnel $[9,34,35]$.

The responsibilities for staffing and personnel in a quality system are classified in Figure 3 $[9,34,35]$ and briefly introduced below $[4,6,7,36-38]$ :

a. Facility management: ensure the requirements, guidelines, and practices are complied within facility, sufficient qualified personnel, appropriate facilities, equipment, and materials are available, ensure that personnel clearly understand the functions they are to perform and appropriate and technically valid Standard Operating Procedures (SOPs) are established and followed, ensure that there is a QA manager or QAU with designated personnel and their responsibility is being performed, ensure that for each study an individual with the appropriate qualifications, training, and experience is designated by the management as the SD and PI, ensure that an individual is identified as responsible for the management of the archive.

b. Administrative staff: represent the first encounter a patient has with the centre. They receive the patients according to the established protocols. In collaboration with the medical and technical staff, they are responsible of the application of the procedures for scheduling studies. 
c. SD and PI: they are responsible for approving, conducting, documenting, recording and archiving the overall of the study and for its final report.

d. Nuclear medicine physicians: responsible for quality encompasses the general services of the centre. In particular, supervises all patient care and management procedures and all clinical protocols. In addition, he/she supports and enforces the QA/QC of equipment, establish clinical review and auditing.

e. QA manager or QAU: all those planned and systematic actions necessary to provide adequate confidence that a product or service will satisfy given requirements for quality, express the closeness with which the outcome of a given procedure approaches some ideal, free from all errors and artefacts. Quality assurance embraces all efforts made to this end.

f. Radiopharmacist and Radiochemists (Nuclear pharmacy): they are responsible for compounding, dispensing, quality assessment, patient monitoring, drug use review, new drug development and evaluation, product selection and performance evaluation, pharmacokinetic modeling, drug information and educational services. They are also responsible for the performance of acceptance testing and organization/supervision of routine calibration and QC of all radiopharmacy equipment; QC of chemicals, enriched materials, precursors, and kits; QC of radiopharmaceuticals products and batch release.

g. Cyclotron operators: they are in charge of the daily operations, take part in the acceptance test of the cyclotron and related equipment, and are responsible for calibration and QC procedures for equipment.

h. Production chemists: synthesis and preparation of nuclear medicine.

i. QC chemists: the restriction of QC persons is independent of the production operations or must have independent oversight of these duties. The operational techniques and activities that are used to fulfill requirements for quality and are used in reference to the specific measures taken to ensure that one particular aspect of the procedure is satisfactory.

j. Medical physicists: specialized in nuclear medicine and responsible for the performance of acceptance testing and organization/supervision of routine calibration and QC of imaging and radiation measurement equipment, including radiation protection instrumentation.

k. Radiation protection officer: ensure the radiation safety for patient, staffing, and environmental.

1. Engineers and Technologists: contribute to the preparation of clinical examination protocols and the performance of patient examinations according to the established protocols, involved in the performance of routine calibration and QC of scanners. 
m. Nurses: manage and care of the patient, collaborate in preparing protocols of patient management and information material as well as in checking the operation of other institutional services.

In IAEA, quality manager is responsible for the entire quality management system supervision, the authority to enforce it and act on its findings, and should be involved in the evaluation and periodic review of the results [5,6]. But, in EANM, the responsibility for overseeing the preparation operations of a qualified radiopharmaceutical is called QAU [11,12].

\subsubsection{Facility}

In a PET facility, it should include the facility for (a) PET/CT scanner, (b) cyclotron, and (c) radiopharmacy. The location of the facility is a very important issue for the flow of patients, materials, and radiation protection. According to the risk of radiation exposure, two areas are planned [4]:

a. low risk area, cold area or uncontrolled area is the area of offices, reception, waiting room, consulting room, cleaning utilities room or store, and

b. high risk area, hot area or controlled area is the area of hot laboratory, preparation, injection and uptake room, toilet, control and scanning room, post-examination waiting room, reporting room, and waste disposal room.

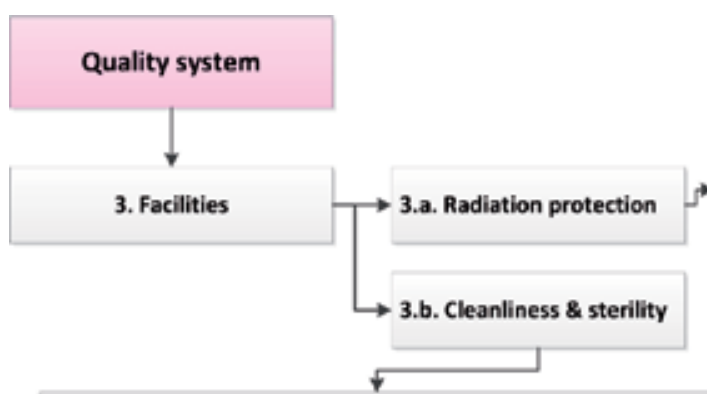

- Location and layout

- Interior surfaces, e.g., walls, floors and ceilings

- Sinks

- Air condition for temperature and relative humidity

- Ventilation

- Pressure (under-pressure) and airflow patterns

- Air systems for radioactive and non-radioactive areas

- Alarm system

- Shielding (lead, perspex) ....

- Glove box

- Decontamination system: shower

Controlled areas:

- Grade A: controlled area for extremely high-risk operations, e.g., filling of radiopharmaceuticals and making aseptic connections.

- Grade B: background environment for the Grade A zone.

- Grade C: for the synthesis and filling of products before terminal sterilization.

- Grade D: less critical stages in the manufacture of sterile products.

- Grade of air: high-efficiency particulate air (HEPA) filters \&

(1) A laminar flow cabinet (Grade A) placed in a

Grade B environment, e.g. for Tc-99m radiopharmaceuticals

(2) An airtight cabinet (a hot cell) connected to the outside environment by airlocks or in a Grade B environment, e.g. for pharmaceuticals without final sterilization process is performed (F-18-FDG)

Figure 4. Quality system of facilities [34].

More considerations for the requirements of radiation protection and cleanliness are summarized in Figure 4 [34]. 


\subsubsection{Instrumentation and equipment}

The instrumentation and equipment in the quality system are summarized in Figure 5 [34,38]. Apparatus and equipment for the purposes of manufacturing QC, diagnosis, and therapy, including validated computerized systems, used for the generation, storage and retrieval of data, and for controlling environmental factors relevant to the study should be suitably located and of appropriate design and adequate capacity. Apparatus used in a study should be periodically inspected, cleaned, maintained, and calibrated according to SOPs. Records of these activities should be maintained. Calibration should be traceable to national or international standards of measurement [39].

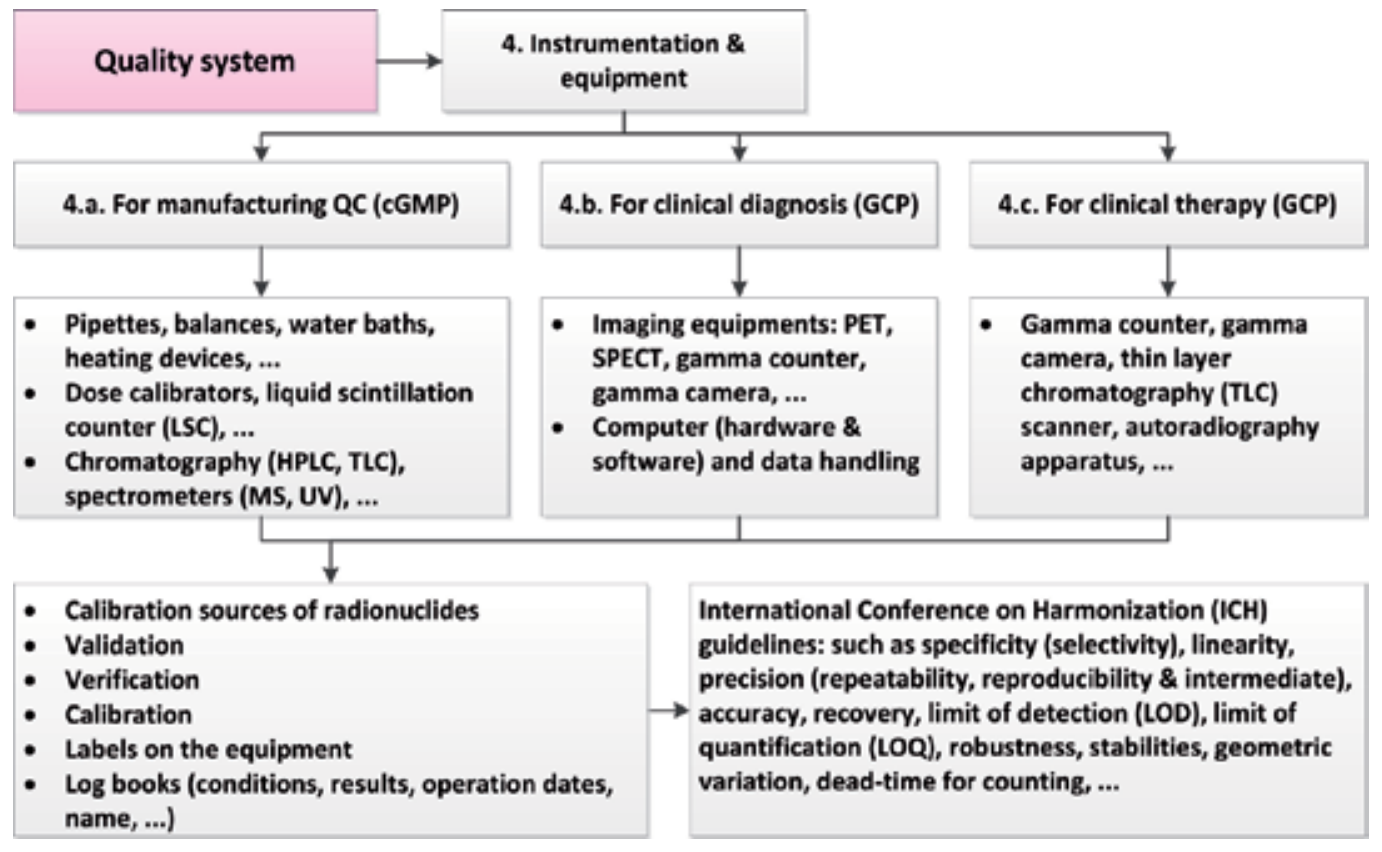

Figure 5. Quality system of instrumentation and equipment [34,38].

Performance tests and operation verification for the nuclear medicine units are achieved daily, weekly, monthly, quarterly, or annually by a qualified medical physicist, a qualified nuclear medicine technologist, or a medical physicist in training, with management by a qualified medical physicist. The tests results of intrinsic or system spatial resolution, uniformity, center of rotation, sensitivity, energy resolution, counting rate parameters, multiple-window spatial registration, formatter and video display, linearity, leak test, overall system performance for imaging systems, interlocks, dose calibrators, thyroid uptake and counting systems must be reviewed and documented in an annual survey report in accordance with the ACR Technical Standard for Medical Nuclear Physics Performance Monitoring of Nuclear Medicine Imaging Equipment [37]. 


\subsubsection{Operation procedures}

A test facility should have written SOPs approved by facility management for ensuring the quality and integrity of the data generation. Deviations from SOPs related to the manufacturing, study, or treatment should be documented and should be acknowledged by the study director, the principal investigator, the medical physician, quality assurance personnel and/or radiopharmacist. The historical file of different version of all SOPs should be well recorded and stored. The requirements of SOPs for nuclear medicine manufacturing and imaging are summarized in Figures 6 and 7 [11,12,40-44].

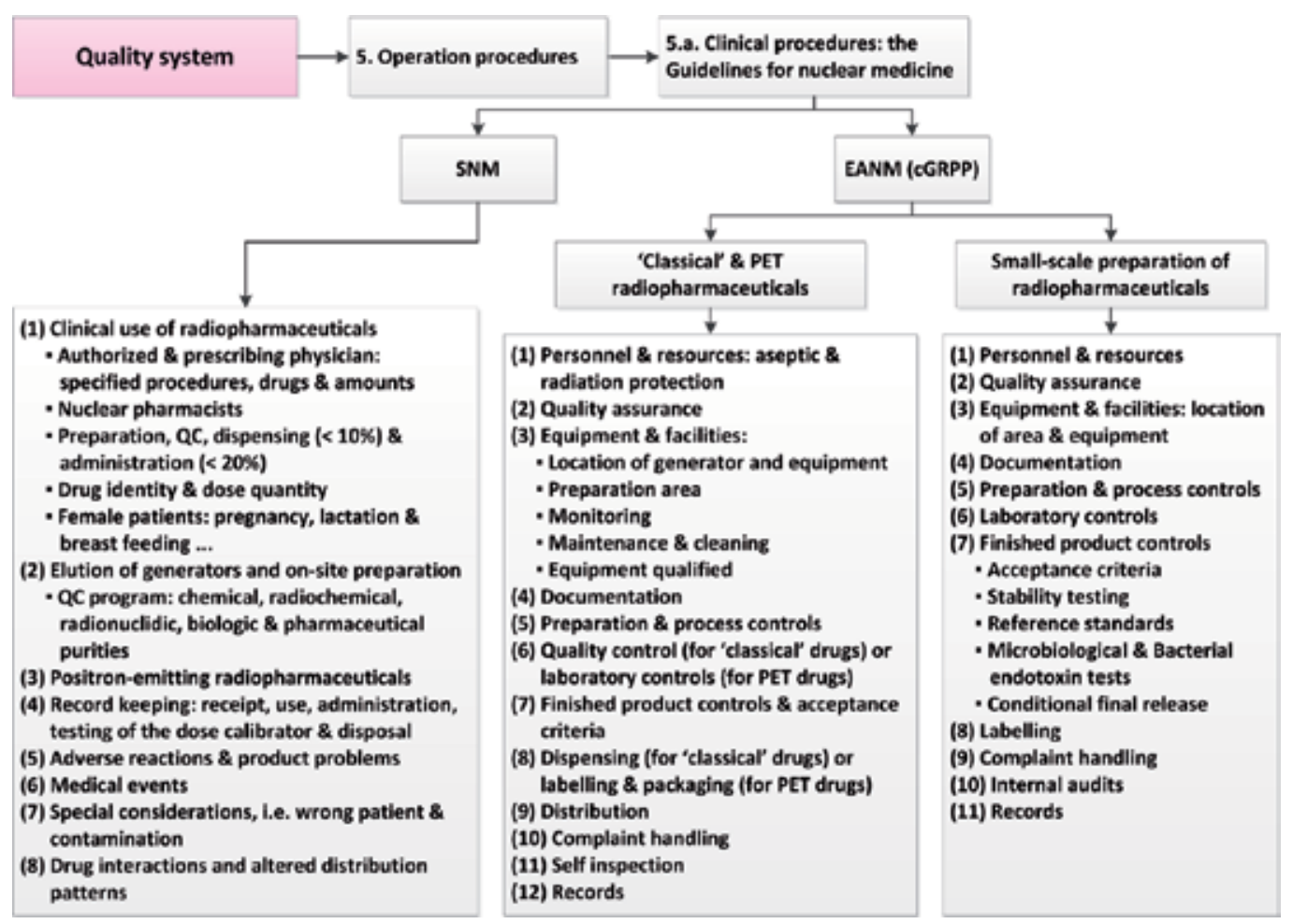

Figure 6. Quality system of clinical operation procedures for nuclear medicine $[11,12,40]$.

\subsubsection{Radiopharmaceuticals}

(a) Manufacturing of radiopharmaceuticals

Manufacturing and quality control plans for radiopharmaceuticals are indicated in Figure 8 $[34,40,45]$. Radiopharmaceuticals might be manufactured or prepared in hospital radiopharmacies, centralized radiopharmacies, nuclear centers, institutes, industrial manufacturers, or PET centers in accordance with the requirements of good manufacturing practices (GMP) or Current Good Radiopharmacy Practice (cGRPP) [11-13,34]. 
Two categories of radiopharmaceuticals are classified in EANM Radiopharmacy Committee according to the significant difference of preparation procedures, i.e. "kit" and PET radiopharmaceuticals. Also, significant consideration in the "Guidelines on Current Good Radiopharmacy Practice (cGRPP) in the Preparation of Radiopharmaceuticals" is proposed by EANM Radiopharmacy Committee. Two types of preparation methods, i.e. in "classical" procedure and in "synthetical" procedure, have been distinguished in cGRPP [11]. According to WHO guideline, radiopharmaceuticals are divided into four categories including ready-to-use, radionuclide generators, "kits" for the labelled with a radioactive component, and precursors used for radiolabelling other substances before administration (e.g. samples from patients) [13].

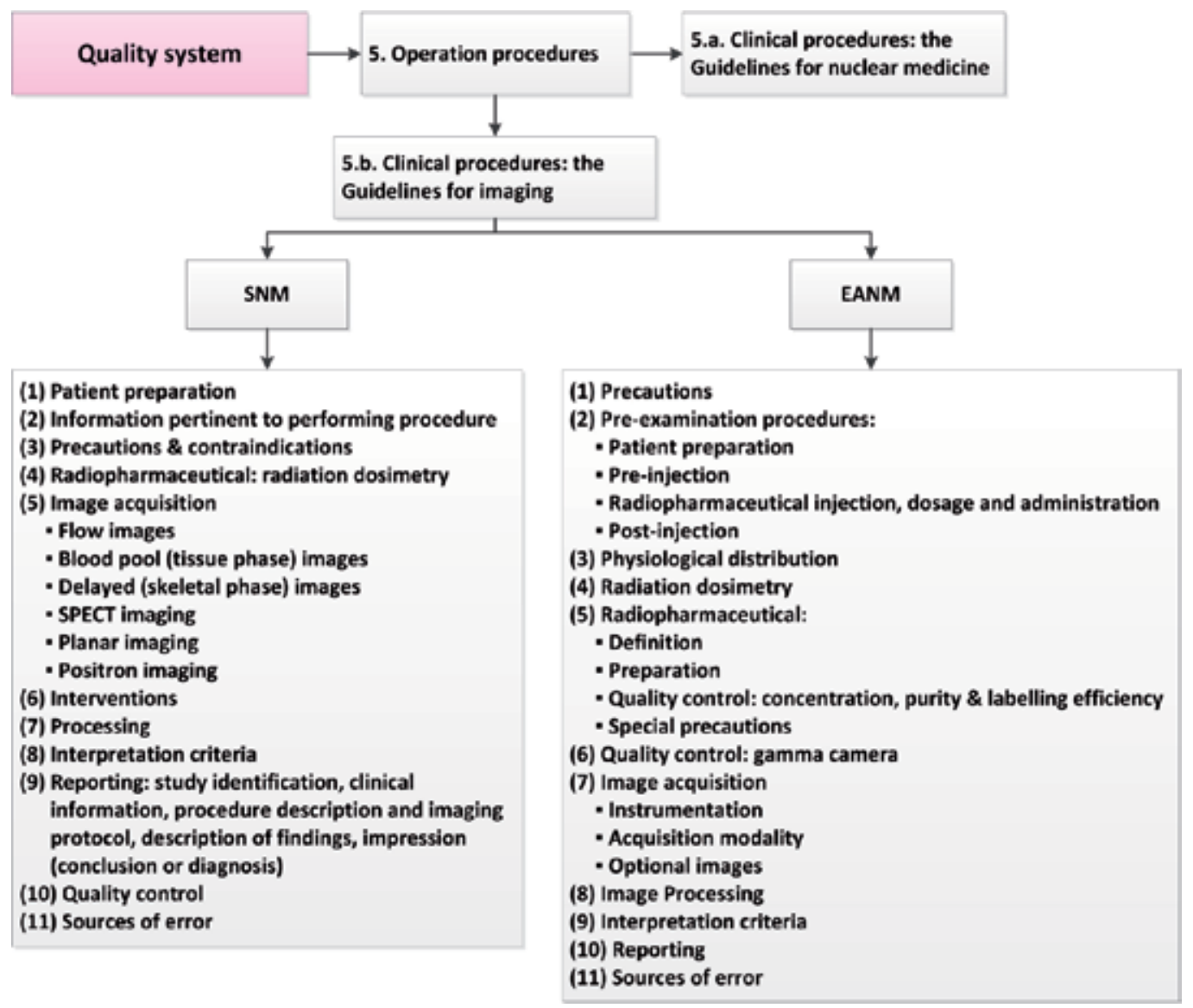

Figure 7. Quality system of clinical operation procedures for imaging [41-44].

Clinical investigations of radiopharmaceuticals can be approved by FDA as "legend drugs." The investigational radiopharmaceutical drug service (IRDS) is responsible for establishing study-specific procedures for radiopharmaceutical drug, including preparation, storage, dis- 
pensing and destruction of investigational drugs within the hospital [9]. Manufacturing or preparation of radiopharmaceuticals must follow the FDA 21CFR Part 212 "Current Good Manufacturing (cGMP) for PET drugs," USP Chapter <797> "Pharmaceutical Compounding-Sterile Preparations," USP Chapter $<823>$ "Radiopharmaceuticals for Positron Emission Tomography - Compounding," and U.S. FDA Guidance: PET Drugs - Current Good Manufacturing Practice (CGMP) [10].

(b) Quality control of radiopharmaceuticals

Three essential parts i.e. chemical, inventory, and radiochemical QC diagrams for radiopharmaceuticals are also indicated in Figure 8 [30,50,45].

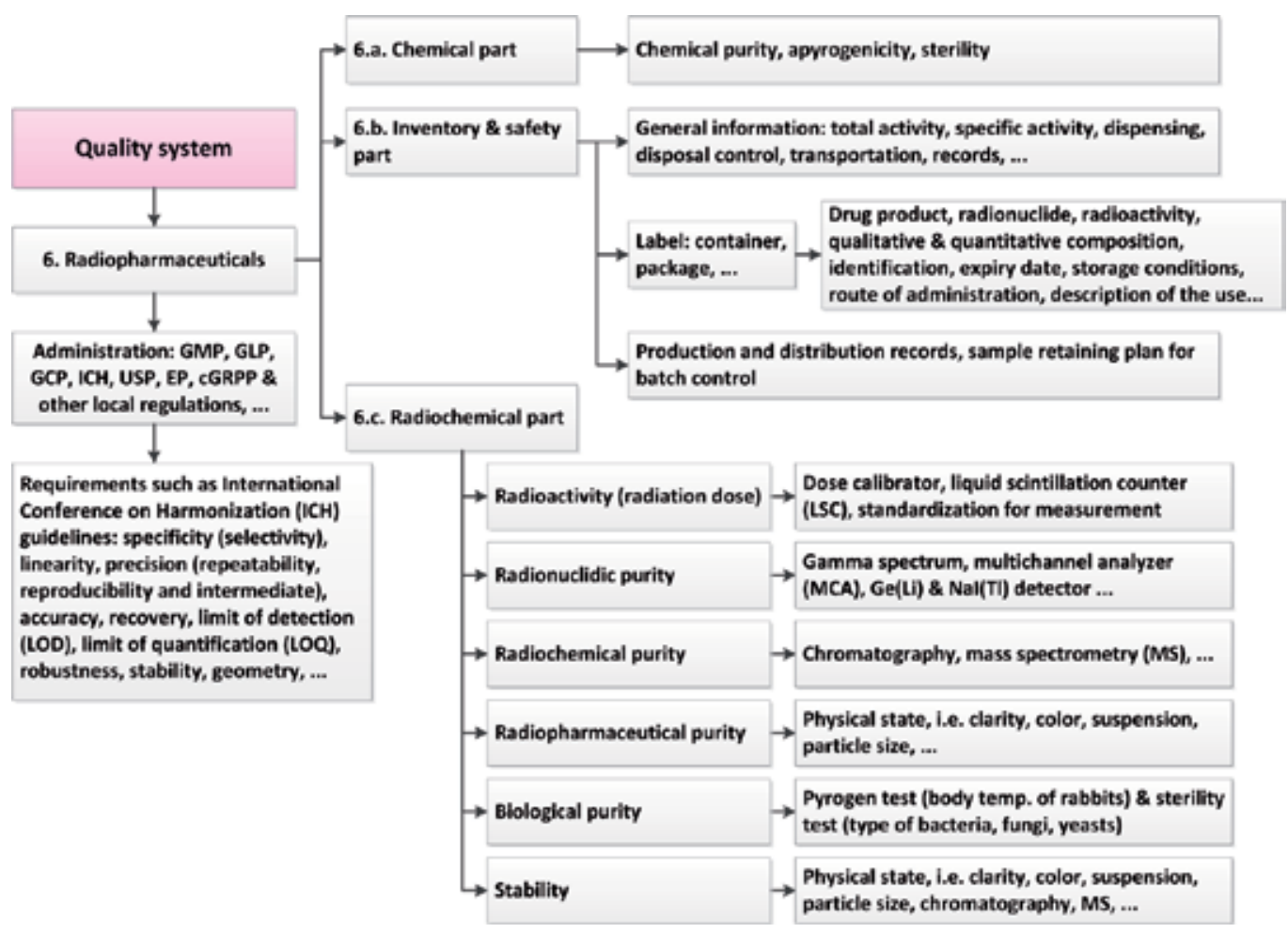

Figure 8. Quality system for radiopharmaceutical manufacturing and quality control $[34,40,45]$.

Method developments for the chemical and radiochemical analysis of starting material, intermediates, precursor used for the radiolabelling, active pharmaceutical ingredient (APIs or drug substance), and finished product (drug product or finished dosage form) are essential requirements of Chemistry, and Manufacturing and Controls (CMC). In the applications of investigational new drug (IND), New Drug Application (NDA), and Abbreviated New Drug Application (ANDA), information on the CMC has to be filed as per 21 CFR 312.23(a) for a drug substance and drug product. The contents for the CMC sections of the 
EU and U.S. are very much the same. However, the sequence and titles of the sections are quite different $[46,47]$.

According to International Conference on Harmonization (ICH) guidelines, the parameters for the validation of analytical methods should basically include specificity (selectivity), linearity, precision (repeatability, reproducibility and intermediate), accuracy, recovery, limit of detection (LOD), limit of quantification (LOQ), robustness, and stability. However, instrument validation parameters for the radioactivity measurement or isotopic analysis, such as dose calibrator or liquid scintillation spectrometry, are partially different.

Radiopharmaceuticals are usually used before all quality control testing has been completed. The implementation of and compliance with the quality assurance program are therefore essential. Principal responsibilities of QA/QC are detailed by WHO and De vos et al., including preparation of detailed instructions for each test and analysis, ensuring the adequate identification, ensuring equipment and process validation, release or rejection of materials, evaluation of the quality and stability of the finished products, expiry dates, storage conditions, control procedures, specifications, and records keeping [13,34].

\subsubsection{Protocol and conduct}

Protocol for a medicine manufacturing study or imaging examination should be evaluated according to the purposes of a study, a treatment, or a clinical trial. Safety issue, such as algorithm proposed by ASNC for maximal benefit in patient radiation exposure must be included [33].

For each study and treatment, a written plan or protocol should exist prior to the initiation of the study. The protocol should be approved by dated signature of the study director, principal investigator or medical physician, facility management, sponsor and verified by quality assurance personnel and/or radiopharmacist. The study and treatment should be conducted in accordance with the study plan or protocol by using a unique identification to each study.

Clinical protocol should be evaluated based on the patient characteristics (e.g. patient history of disease or ability to complete the examination) and complexity of clinical situation in accordance with the current statements and guidelines [33]. For instance, advantages and disadvantages of assessing myocardial perfusion with PET, as compared to SPECT imaging, was reported and concluded that use of very short half-life tracers injected at very high activities, as well as the introduction of increasingly fast scintillators technology, which in turn has allowed reduction of random coincidences and introduced the possibility of timeof-flight (TOF) PET are expected to further contribute to high sensitivity imaging capabilities of PET [32].

An example for approving of protocol design for a clinical trial is shown in Figure. 9 [10,47]. Two pathways for the clinical studies of investigational radiopharmaceuticals are called Ra- 
dioactive Drug Research Committee (RDRC) and IND. For an investigational medical product (IMP, investigation only), if there are adequate data from literature or original assessments that no pharmacologic effects are likely in humans, and the chosen radioactivity is small enough to result in the total radiation absorbed dose, clinical trial can be approved by National Competent Authority (NCA) and Ethical Committee (EC) in EU or approved by RDRC in U.S.. Otherwise, it is approved by EC in EU or approved by FDA in U.S., depending on the phase of drug development [47].

The FDA allows certain unique applications by the local RDRC, consisting of at least five individuals and three individual specialists in nuclear medicine, in formulate radioactive drugs, and in radiation safety, to approve and monitor for the use of radiopharmaceuticals in humans without IND approval. This is due to the low potential for toxicity of radiopharmaceuticals that are typically administered in tracer quantities. Requirements to establish a local RDRC at one's institution is outlined in regulation 21 CFR 361.1. And RDRC has to submit an annual report to the FDA as part of the procedures for maintaining an active and approved RDRC program [48].

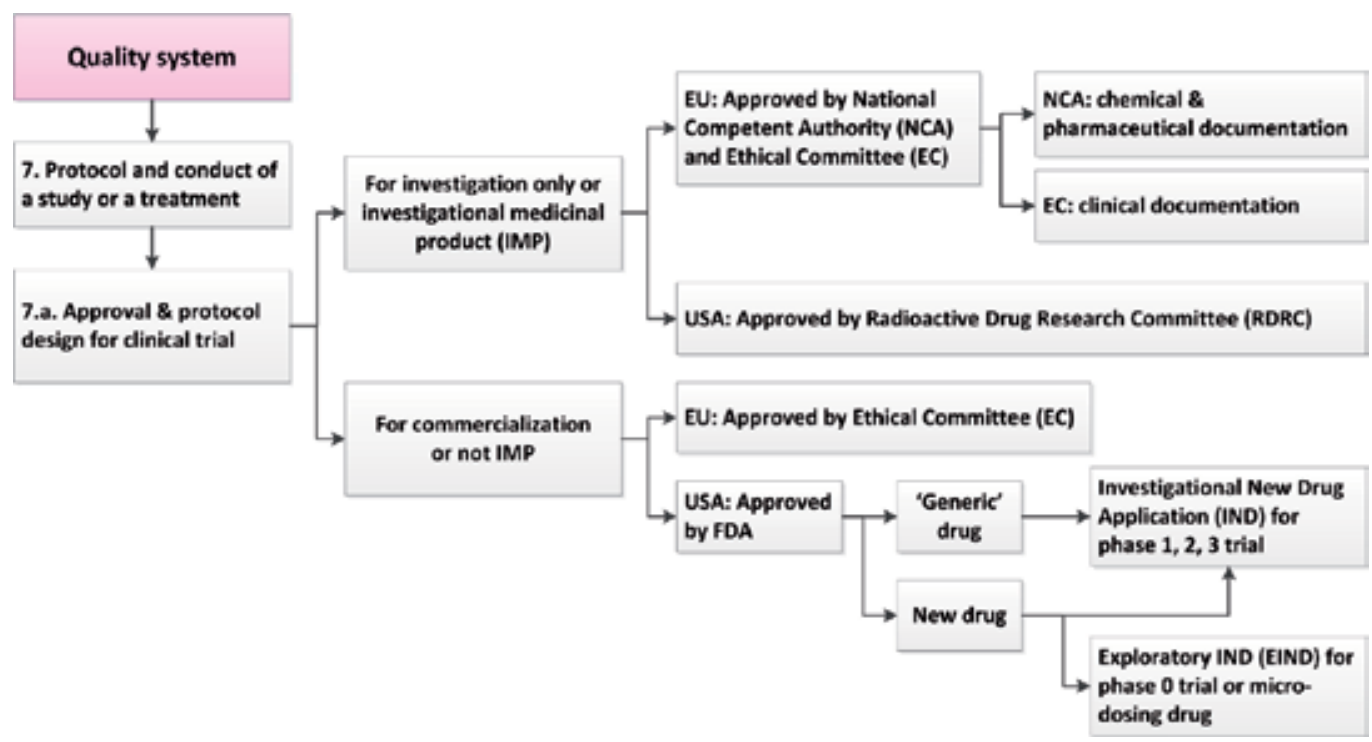

Figure 9. Quality system of protocol and conduct for a study or a treatment $[10,47]$.

\subsubsection{Records and reports}

Records and reports for the manufacturing of radiopharmaceuticals and imaging trial or testing are summarized in Figure 10 [7,9,40-44]. All records and reports should be maintained at the radiopharmaceutical laboratory or another location that is accessible to respon- 
sible officials and to government employees designated to perform inspections [11,12]. Storage of records must ensure safekeeping for many years. Archive facilities of independent locations should be provided for the secure storage and retrieval of study plans, raw data, final reports, samples of test items and specimens. Archive conditions, e.g. fireproof, waterproof, and insect prevention are designed for protecting contents from untimely deterioration [38].

\begin{tabular}{|c|c|}
\hline Quality system & $s$ and reports \\
\hline $\begin{array}{l}\text { 8.a. For nuclear medicine: } \\
\text { - Receipt, use, administration, and disposal } \\
\text { of all radiopharmaceuticals } \\
\text { - Receipt of packages, the results of } \\
\text { inspection for physical damage, } \\
\text { measurement of the radiation dose-rate } \\
\text { emanating from the package, and testing } \\
\text { for removable contamination, as required } \\
\text { by the regulatory agency. } \\
\text { - The date and time of preparation, the } \\
\text { quantity, volume, and concentration of } \\
\text { radioactivity used, reagent lot numbers, } \\
\text { QC data, expiration time, waste disposal } \\
\text { information } \\
\text { - Testing of the dose calibrator \& geometric } \\
\text { variation } \\
\text { - Aisposal }\end{array}$ & $\begin{array}{l}\text { 8.b. For imaging: } \\
\text { - Study identification, } \\
\text { - Clinical information, } \\
\text { - Procedure description and imaging protocol: } \\
\text { radiopharmaceuticals, administration (activity, route), } \\
\text { field of view and patient positioning, processing } \\
\text { protocol ... } \\
\text { - Description of findings: quality of the study, equipment } \\
\text { performance (quality control), data accuracy and } \\
\text { integrity (software quality assurance), data \& image } \\
\text { review, location, extent, and intensity of abnormal } \\
\text { radiopharmaceutical uptake, limitations, accuracy, } \\
\text { timeliness, confidentiality, distribution and comparative } \\
\text { data } \\
\text { - Impression (conclusion or diagnosis) } \\
\text { - Training and experience of nuclear medicine specialists \& } \\
\text { operators } \\
\text { Archiving }\end{array}$ \\
\hline
\end{tabular}

Figure 10. Quality system of records and reports [7,9,40-44].

\subsubsection{Audit framework}

Laboratory inspections and study audits should be established for periodical monitoring compliance with GLP, GCP, or GMP principles, study protocol, and SOPs [9,38]. Audits for radiopharmaceutical drug products typically begin by confirming the clinical site is appropriately licensed and authorized to receive, possess, store, handle, prepare and administer radiopharmaceuticals. The audit framework of quality system for radiopharmaceuticals, imaging equipment, laboratory equipment, safety, computer systems, data handling, and radiation protection are displayed in Figure 11 [9,39]. 
(a)

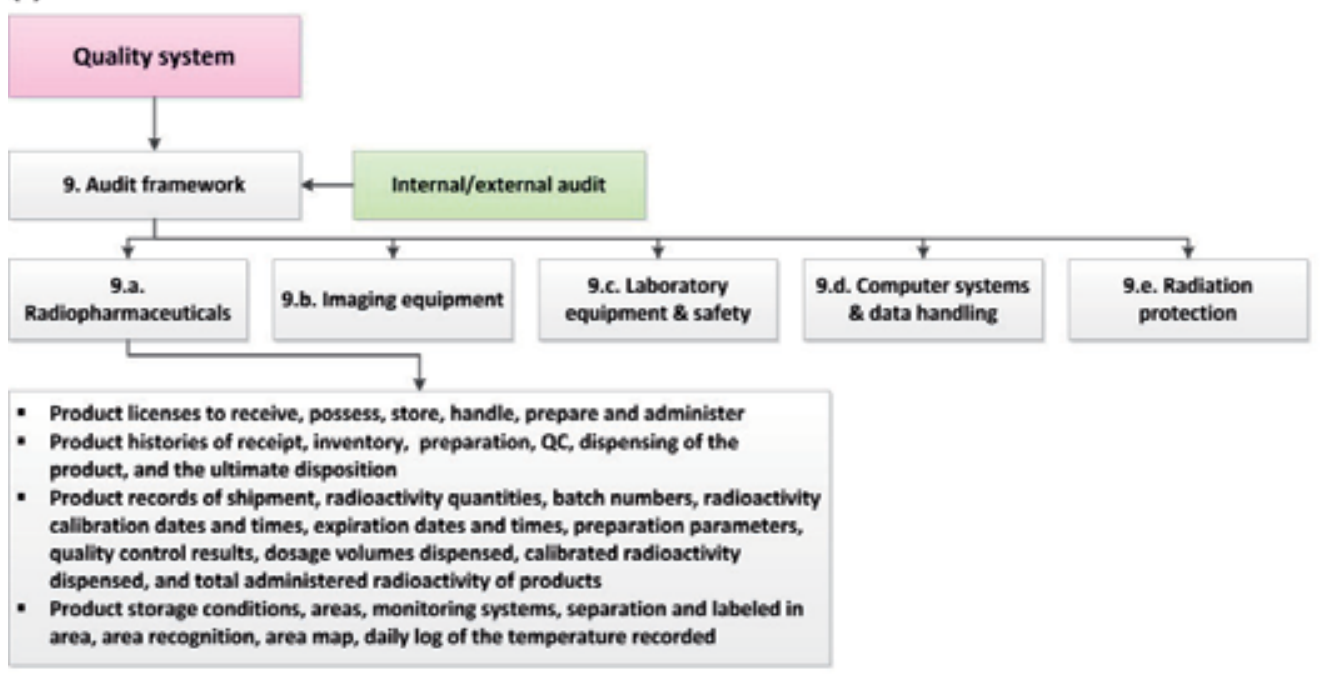

(b)

\begin{tabular}{|c|c|c|c|}
\hline Quality system & $\begin{array}{l}\text { 9. Audit framework } \\
\text { (The BNMS) }\end{array}$ & 9.a. Radiopharmaceuticals & \\
\hline 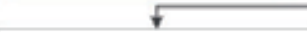 & 7 & $\downarrow$ & $\nabla$ \\
\hline 9.b. Imaging equipment & $\begin{array}{l}\text { 9.c. Laboratory equipment \& } \\
\text { safety }\end{array}$ & $\begin{array}{l}\text { 9.d. Computer systems \& } \\
\text { data handling }\end{array}$ & 9.e. Radiation protection \\
\hline$\downarrow$ & $\downarrow$ & $\downarrow$ & $\downarrow$ \\
\hline $\begin{array}{l}\text { Documented policies and } \\
\text { protocols for the operation, } \\
\text { QC \& QA for each clinical } \\
\text { gamma camera } \\
\text { - Documented procedures } \\
\text { and results of dally QA } \\
\text { program for the assessment } \\
\text { of gamma camera } \\
\text { functionality } \\
\text { - Documented procedures } \\
\text { and results of QC program } \\
\text { for the measurement of } \\
\text { clinical gamma camera } \\
\text { performance } \\
\text { - Documented policies and } \\
\text { testing of new gamma } \\
\text { cameras } \\
\text { - Reference results of gamma } \\
\text { camera } \\
\text { - History logs of gamma } \\
\text { camera }\end{array}$ & $\begin{array}{l}\text { Documented QA policies } \\
\text { and protocols with records } \\
\text { for equipment } \\
\text { - Departmental safety officer } \\
\text { - Written procedures and } \\
\text { records of radioactive goods } \\
\text { - Written procedures and } \\
\text { records for electrical \& } \\
\text { mechanical safety } \\
\text { - Written procedures and } \\
\text { records for compliance with } \\
\text { hazardous substances } \\
\text { - Written procedures and } \\
\text { training records for manual } \\
\text { handling of goods }\end{array}$ & $\begin{array}{l}\text { - Policy and records for } \\
\text { computer installation } \\
\text { (hardware \& software) } \\
\text { acceptance } \\
\text { - Policy and records for } \\
\text { computer upgrades } \\
\text { - Policy and records for } \\
\text { software QA (commercial \& } \\
\text { in-house) } \\
\text { - Procedure and records for } \\
\text { the assessment of the } \\
\text { integrity of data following } \\
\text { major software revisions } \\
\text { (i.e. count rate losses, data } \\
\text { framing, image } \\
\text { quantification, image } \\
\text { arithmetic, and activity-time } \\
\text { curve arithmetic) }\end{array}$ & $\begin{array}{l}\text { - Warning signs of entrances, } \\
\text { controlled, supervised, } \\
\text { reception, patients' waiting } \\
\text { area \& patients' toilet) } \\
\text { - Local rules for staff and } \\
\text { revised regularly } \\
\text { - Personnel monitored for } \\
\text { radiation exposure } \\
\text { - Syringes shielded } \\
\text { - Decontamination kits } \\
\text { - Contamination monitoring \& } \\
\text { - monitors calibrated } \\
\text { - Materials stored security } \\
\text { - Waste disposal policy, } \\
\text { records, and shielding } \\
\text { - Radioactive Substances Act } \\
\text { (RSA) rezistration and } \\
\text { - Acrtificates } \\
\text { - Activity checked prior to } \\
\text { - Diagninistration \& recorded } \\
\text { (DRL) } \\
\text { - Transportation system }\end{array}$ \\
\hline
\end{tabular}

Figure 11. Quality system of audit framework. Audit (a) for radiopharmaceuticals manufacturing, and (b) for imaging equipment, laboratory equipment, safety, computer systems, data handling, and radiation protection. [9,39] 


\section{Quality evaluation and sources of uncertainty}

\subsection{Radiopharmaceuticals}

\subsubsection{Standardization: principle and applications}

Quality control for the quantification of radiopharmaceutical activity is critical for accurate dosimetry calculations, from whole body to cell microscopy. Tumor uptake of radiopharmaceutical need to be correlated with tumor response and to be related to the tumor radiation absorbed dose. [14]

\begin{tabular}{|c|c|c|c|c|c|}
\hline Isotope & $\begin{array}{c}\text { Major decay } \\
\text { modes }\end{array}$ & $\begin{array}{c}\text { Method for } \\
\text { standardization }\end{array}$ & $\begin{array}{c}\text { Detection } \\
\text { efficiency }(\varepsilon)\end{array}$ & $\begin{array}{c}\text { Activity accuracy \& } \\
\text { uncertainty (U) }\end{array}$ & Ref. \\
\hline- & $\begin{array}{l}\beta, \gamma, \beta-\gamma, E C \text {, } \\
E C-\gamma, a, \text { and } \\
\text { mixed decay } \\
\text { nuclides }\end{array}$ & $\begin{array}{l}\text { LSC and C/N } \\
\text { method }\end{array}$ & $\begin{array}{l}\varepsilon_{\text {pure }}: \sim 100 \% \\
\varepsilon_{\text {pure EC }}:<75 \% \\
\varepsilon_{\mathrm{EC}-\mathrm{\gamma}}: \sim 100 \% \\
\varepsilon_{\mathrm{a}}: \sim 100 \%\end{array}$ & $\begin{array}{l}\text { U: } 0.2-0.5 \%(\text { pure } \beta) \\
U: 0.2-0.5 \%(\beta-\gamma)\end{array}$ & 51 \\
\hline Co-57 & $\begin{array}{l}\text { EC decay to } \\
\text { Fe- } 57\end{array}$ & $\begin{array}{l}4 \pi \beta-\gamma \text { coincidence } \\
\text { method }\end{array}$ & $\varepsilon: \sim 75 \%$ & $U: 2 \%$ & 50 \\
\hline $\mathrm{Ge}-68 / \mathrm{Ga}$ & -68 & ү spectrometer & \multicolumn{2}{|c|}{ To ground state: $\beta^{+} U: 1 \%$} & 52 \\
\hline 1. Ge-68 & $\begin{array}{l}\text { EC decay to } \\
\text { Zn- } 68\end{array}$ & $\begin{array}{l}x \text {-ray spectrometer } \\
4 \pi \beta-\gamma \text { coincidence }\end{array}$ & $\begin{array}{l}(87.85 \%), E C \\
(8.92 \%)\end{array}$ & & \\
\hline 2. Ga-68 & $\begin{array}{l}\beta^{+}, E C, \gamma \text { decay } \\
\text { to } Z n-68\end{array}$ & LSC & $\begin{array}{l}(1.29 \%), E C \\
(1.93 \%) \\
\text { Annihilation } \\
\text { radiation: } 178.29 \% \\
\text { Y-ray to } 1077 \mathrm{keV} \text { : } \\
3.22 \%\end{array}$ & & \\
\hline $\begin{array}{l}\text { Sn-117m- } \\
\text { DTPA }\end{array}$ & $\begin{array}{l}\text { Decay to } \\
\text { Sn-117 }\end{array}$ & $\begin{array}{l}4 \pi \beta \mathrm{LS} \text { and } 4 \pi \gamma \\
\text { methods }\end{array}$ & & $\begin{array}{l}\mathrm{U}_{c}: 0.60 \% \text { (for DTPA } \\
\text { by LS) } \\
\mathrm{U}_{c}: 2.43 \% \text { (for DTPA } \\
\text { by } \mathrm{Nal}(\mathrm{T} 1) \text { ) }\end{array}$ & 17 \\
\hline Cs-131 & $\begin{array}{l}\text { EC decay to } \\
\text { Xe-131 }\end{array}$ & $\begin{array}{l}\text { Coincidence } \\
\text { methods: L Auger } \\
\text { electrons plus L X- } \\
\text { rays and KX-rays }\end{array}$ & - & $\mathrm{U}: 1 \%$ & 53 \\
\hline Cs-134 & $\begin{array}{l}\beta \text { and } \gamma \text { decay } \\
\text { to } \mathrm{Ba}-134\end{array}$ & LSC & $\varepsilon: \sim 95 \%$ & $\begin{array}{l}3191 \pm 8 \mathrm{kBq} / \mathrm{g} \\
(0.54 \%)\end{array}$ & 54 \\
\hline
\end{tabular}




\begin{tabular}{lll}
\hline $\begin{array}{l}4 \pi \beta-\gamma \text { coincidence } \\
\text { method } 65-87 \%\end{array}$ & $\begin{array}{l}3194 \pm 12 \mathrm{kBq} / \mathrm{g} \\
(0.88 \%)\end{array}$ \\
\hline $4 \pi \gamma$ method & $\varepsilon: \sim 83 \%$ & $3174 \pm 25 \mathrm{kBq} / 9$ \\
& & $(2.09 \%)$
\end{tabular}

\begin{tabular}{|c|c|c|c|c|c|}
\hline & & & & & \\
\hline & & $\mathrm{C} / \mathrm{N}$ method & & & \\
\hline Tl-201 & $\gamma$ decays to & High-pressure IC & y ray (167.4 keV) & $7.207 \pm 0.033$ (NIST) & 55 \\
\hline & $\mathrm{Hg}-201$ & $4 \pi \gamma$ coincidence & probability: 0.1000 & $7.197 \pm 0.027$ (NPL) & \\
\hline & & method & \pm 0.0006 & $7.116 \pm 0.050$ (PTB) & \\
\hline TI-204 & $\beta$ decay & Windowless $4 \pi-$ & & & 56 \\
\hline & $(97.4 \%)$ to & CsI(TI) -sandwich & & & \\
\hline & $\mathrm{Pb}-204$ and $\mathrm{EC}$ & spectrometer, LSC, & & & \\
\hline & decay $(2.6 \%)$ & PPC & & & \\
\hline & to $\mathrm{Hg}-204$ & $\begin{array}{l}4 \pi \beta-\gamma \text { coincidence } \\
\text { method and Cs-134 }\end{array}$ & $\begin{array}{c}\varepsilon_{\beta}: 71-91 \% \\
4 \varepsilon_{\mathrm{EC}}\left(\sim \varepsilon_{\mathrm{AES}}\right): 50-\end{array}$ & $U_{c}: 0.76 \%$ & 57 \\
\hline & & tracer & $100 \%$ & & \\
\hline $\mathrm{Pb}-210$ & $\beta$ decay to & $4 \pi \beta-\gamma$ coincidence & & $U_{c}: 2.7 \%$ & 58 \\
\hline & $\mathrm{Bi}-210\left(\mathrm{t}_{1 / 2}\right.$ & method & & & \\
\hline & $5.103 \mathrm{~d})$ & Germanium $\gamma$ & & & \\
\hline & Po-210 ( $t_{1 / 2}$ & spectrometry & & & \\
\hline & 138.4 d), and & & & & \\
\hline & a decay to & & & & \\
\hline & Pb-206 & & & & \\
\hline
\end{tabular}

Table 2. Some examples of absolute standardization of radiopharmaceuicals and related radioisotopes [17,50-58]

The theoretical counting efficiency, i.e. counts/disintegration or counts per minute/disintegration per minute (cpm/dpm), for a radionuclide can be used to examine the absolute activity, in disintegration or disintegration per minute $(\mathrm{dpm})$ of the radionuclide. Different efficiency tracing methods has been developed for more than six decade by characterizing the effects of sample volume, medium composition (matrix), pulse discrimination conditions, photomultiplier voltage, amplifier gain, and luminophor concentration on counting efficiency of a radioactive species [49]. The use of $4 \pi \beta$ scintillation counting and $4 \pi \beta-\gamma$ coincidence counting for the standardization of certain electron capture (EC) nuclides with simple decay schemes is established since 1952 [49] and 1957 [50].

Some examples of absolute standardization of radiopharmaceuicals and related radioisotopes are shown in Table 2 [17,50-58]. Below, we introduce different tracing methods, including (a) efficiency tracing (and extrapolation) method using a non-H-3 standard solution, (b) CIEMAT-NIST $(\mathrm{C} / \mathrm{N})$ efficiency tracing method, (c) non-extrapolation tracer method, $(\mathrm{d})$ coincidence method by a $4 \pi \beta-\gamma$ system, (e) triple to double coincidence ratio (TDCR) method, and (f) $4 \pi \gamma$ counting method.

(a) The efficiency tracing (and extrapolation) method using a non-H-3 standard solution 
The efficiency tracer techniques, using Co-60, Cs-134, C-14, Cr-51, Mn-54 or Am-241 standard solution for the standardization of the $\beta-\gamma$ nucides were developed. The $4 \pi$ liquid scintillation (LS) consisted of the extrapolation of the $4 \pi$ counting rate to the zero discrimination level for the standardization of the Tl-204 (97.6\% $\beta$ emission and 2.4\% electron capture) solution was carried out for efficiency tracing using a Co-60 standard solution received in the framework of the 1997 BIPM comparison was carried out by Sahagia et al. [59]. A germanium spectrometer was calibrated for the standardization of Pb-210 using Am-241 as a normalizing agent has been proposed [58]. Instead, Dias et al. chose Cs-134 as an efficiency tracer to standardize Tl-204 as well as a $4 \pi \beta-\gamma$ coincidence system for the calibration [57]. This method can be also successfully used for the standardization of radionuclides such as Ir-192, Zn-65, Mn-54, with the detection of the $\beta$ rays, Auger electrons, $X$ rays, in the proportional counter (PC) [60]. Efficiency tracing with C-14 and zero detection threshold techniques with $\mathrm{H}-3$ as tracers was applied for standardization of various $\beta$-emitting radionuclides, e.g. C-14, Cl-36, and Tl-204 using LS spectrometer [61].

Recently, different methodologies were proposed. Koskinas et al. developed a "dual-tracers", e.g. Cr-51 and Mn-54 procedure followed by the Laboratório de Metrologia Nuclear (LMN) for the standardization of EC nuclide, i.e. Fe-55. The efficiency was obtained by selecting a $\gamma$-ray window set at $320 \mathrm{keV}(\mathrm{Cr}-51)$ and at $834 \mathrm{keV}(\mathrm{Mn}-54)$ [62]. The activity of EC radionuclides is usually determined by $4 \pi$ (proportional counter, PC) $-\gamma$ coincidence counting and by an efficiency extrapolation method. However, an alternative method, called "wet extrapolation method", utilizes an absorption change during the drying of a water droplet added onto the source surface, variation of the PC detection efficiency can be achieved. Slopes of extrapolation curves and resulting activity values obtained are compared for several radionuclides (Mn-54, Ce-139, Y-88, and Co-57) [63].

(b) The CIEMAT-NIST (C/N) efficiency tracing method

CIEMAT/NIST (C/N) method, developed by Centro de Investigationes Energéticas, Medioambientales y Tecnologicas (CIEMAT), Spain and the National Institute of Standards and Technology (NIST), U.S. is used for standardization of radionuclides with Liquid Scintillation (LS) Spectrometry by calculating the counting efficiency of the radionuclide to be assayed and using $\mathrm{H}-3$ as a tracer [61]. $\mathrm{C} / \mathrm{N}$ program is suitable used for the calculation of the efficiency of nuclides decayed by $\beta, \beta-\gamma, \mathrm{EC}, \mathrm{EC}-\gamma$ and nuclides with mixed decay [51]. The basic principle of $\mathrm{C} / \mathrm{N}$ LS efficiency tracing method is a combination of a theoretical calculation of the counting efficiency and an experimental determination of correction factors in three steps [61,64]:

- Count rates (cpm) and the quench-indicating parameters (QIPs, i.e. tSIE) are determined for a set of samples of the nuclide to be measured, and for a set of $\mathrm{H}-3$ standard samples, with a different quench. The tSIE values were calculated using the Ba- 133 source inside of the instrument. By combining these data, a corresponding H-3 efficiency is obtained for each sample of the nuclide.

- The universal curve of Figure of Merit (FOM) as a function of tSIE was plotted. The efficiency of the nuclide is theoretically calculated as a function of the efficiency of the tracer nuclide $\mathrm{H}-3$. 
- This relation is used in conjunction with the measured data to calculate the efficiency for the nuclide and an activity value in $\mathrm{dpm}$ for each single measurement.

The parameters of emitters in different decay modes used for the $\mathrm{C} / \mathrm{N}$ calculations are summarized as follows [51]:

- Pure $\beta$ emitters (Sr-89, Sr-90, Y-90, and K-40): atomic number Z of the radionuclide, the mass number $\mathrm{A}$, the endpoint energy EMax, and the shape parameters.

- Pure $\gamma$ emitters (Nb-93m): the efficiency is nearly $100 \%$.

- $\beta+\gamma$ emitters, if the radionuclide has significant levels with half-lives in the order of the coincidence resolving time or the dead time of the equipment, a $\mathrm{C} / \mathrm{N}$ calculation is not possible.

- Pure EC emitters: the input parameters are the capture probabilities, PK; PL; PM, and the atomic parameters for the rearrangement: the fluorescence yields $\omega \mathrm{K}$ and $\omega \mathrm{L}$ (averaged), the probabilities of the X-rays (PKL, PKX, and PLX) and their average energies (EKL, EKX, and ELX), the emission probabilities of the Auger electrons (PKLL, PKLX, PKXY, and PLXY) and their average energies (EKLL, EKLX, EKXY, and ELXY).

- EC+ $\gamma$ emitters (Co-57, Se-75, Sr-85, and Ba-133): the calculation method is the same as for $\beta+\gamma$ nuclides.

- The efficiency of LSC systems with respect to alpha radiation is in each case very close to unity. A tracer method is not necessary.

(c) The non-extrapolation tracer method

An alternative called "non-extrapolation tracer method" was proposed by Steyn et al. in 1979, where Fe-55 was used as a tracer to establish the figure-of-merit (FOM) of the detection system for the calculation of counting efficiency [65]. The liquid scintillation method, for the determination of absolute activity of Mn-54 and Zn-65 from $4 \pi$ (LS)e- $\gamma$ data by direct calculation without efficiency extrapolation was performed. The non-extrapolation LS method relies on determining the probability of the $\gamma$-ray interacting with the scintillator solution, is described and validated by measurements made on Co-60 [66].

(d) The coincidence method by a $4 \pi \beta-\gamma$ system

Coincidence method comes from the additional coincidence channel, which records a disintegration event when it is detected in both $\beta$ - and $\gamma$-channels. Typically, the system for absolute standardization is usually consisted of a gas-flow or pressurized proportional counter with $4 \pi$ geometry as the $\alpha, \beta$, electrons or X-ray detector and coupled to a pair of $\mathrm{NaI}(\mathrm{Tl})$ scintillation counters or a semiconductor detector, as $\gamma$ detectors. The $4 \pi \beta-\gamma$ coincidence technique has been considered a primary standardization method due to its high accuracy and because it can obtain the radionuclide activity depending only on observables quantities $[57,67]$.

Alternatively, solid or liquid scintillation counters (LSC) are used in place of gas-flow proportional counters. Advantages of using LSC counting in the $4 \pi$ channel are that self-absorption does not occur, leading to Auger electrons being detected with relatively high 
efficiency; source preparation is easy; and the source geometry is highly reproducible. The latter leads to good reproducibility of the counting efficiency of the X-rays and Auger electrons, which in turn gives rise to consistent results amongst the counting sources. The efficiency data can generally be fitted with a linear function, particularly in the high-efficiency region, or by a low-order polynomial expression, giving rise to reliable extrapolated activity values [68].

Several examples for the applications of the coincidence method by a $4 \pi \beta-\gamma$ system are such as standardization of Ho-166m using the normal gas flow $4 \pi \beta-\gamma$ coincidence method [69], standardization of Tl-204 using Cs-134 as tracer and a $4 \pi \beta-\gamma$ coincidence system was used for the calibration [57], directly measured of radionuclides with EC decay schemes, e.g. I-125, Ir-192, Zn-65, and Ce-139 by a LS coincidence extrapolation technique [68], and standardization of Fe-55 using a "dual-tracers" method coupled with a $4 \pi \beta-\gamma$ coincidence calibration system [62].

(e) The triple to double coincidence ratio (TDCR) method

The TDCR method was first developed at the R.C., Poland and at the LNHB, France. The equipment consists in a detection unit, provided with three photomultipliers (PMs), acted by the light emitted in the vial containing the radioactive solution dissolved in a liquid scintillator, and the electronic unit [60]. TDCR, allowing the observation of three kind of double coincidences (2-photodetectors) and triple coincidence (3-photodetectors) method in LSC, is a fundamental measurement method suitable to the standardization of pure-beta emitters, i.e. H-3, C-14, P-32, Ni-63, Tc-99, T1-204 and some low energy electron-capture emitters, i.e. Fe-55 [59,60,70,71]. Detection efficiency variation can be achieved using techniques of chemical quenching, coaxial grey filters and PM tubes defocusing. The two former processes reduce the mean quantity of light emitted and the later reduces the detection probability [71]. Basically, the specific experimental parameter $(\mathrm{K})$ is equal to the ratio of the triple coincidences counting rate $\left(\mathrm{N}_{T}\right)$ to the sum of double coincidences counting rate $\left(\mathrm{N}_{\mathrm{D}}\right)$. Determination of a counting efficiency $\left(\varepsilon_{\mathrm{D}}\right)$ for each counting point $\left(\mathrm{N}_{\mathrm{D}}\right)$ leads to the activity of the source $\left(\mathrm{N}_{0}\right)$. The efficiency functions $\varepsilon_{\mathrm{T}}$ and $\varepsilon_{\mathrm{D}}$ are nonlinear functions for a particular emitter and counting system [70].

Two innovative TDCR instrumentations were developed:

- The TDCR method of LSC is well established for measuring the activity of pure beta emitting and electron capture radionuclides. Recently, a new TDCR counting system was designed by the National Physical Laboratory (NPL) for activity assays of low-energy, pure $\beta$-emitting radionuclides and EC nuclides. Three photomultiplier tubes (PMT) were arranged in the optical chamber as well as a $\mathrm{NaI}(\mathrm{Tl})$ detector was mounted below the optical chamber. The detector allows $4 \pi \beta-\gamma$ coincidence measurements to be performed in parallel [72].

- Radionuclides such as P-32, Sr-89, Y-90, T1-204, and Rh-106 were successfully studied using an in-house built new TDCR-Čerenkov counter developed by Kossert. Since Čerenkov counting acts as natural discrimination for $\alpha$ emitters and low-energy $\beta$ emitters, some potential radioactive impurities or progenies will not disturb the measurements. Two standard sources, e.g. Cl-36 and P-32 were used to determine the free parameter and to 
calculate the Čerenkov counting efficiencies. Since Čerenkov counting is more sensitive to changes in the computed $\beta$ spectra, the method was extensively used to investigate $\beta$ shape factor functions [73].

(f) The $4 \pi \gamma$ counting method.

An ionization chamber system referring to a long living and stable standard source is very adequate for the comparison of $\gamma$-ray emitting radio-nuclides. In most cases Ra-226 sealed sources have been used as the reference because the Ra-226 sources were widely used in radiotherapy [69]. Zimmerman et al. standardized and compared solution of Sn-117m by $4 \pi \beta$ liquid scintillation (LS) spectrometry and $4 \pi \gamma$-ray spectrometry $(\mathrm{NaI}(\mathrm{Tl})$ and high-purity germanium detectors). Massic activities were measured for determining the dose calibrator factor settings [17].

\subsubsection{Uncertainty of measurement}

Examples for the evaluation of detection efficiency $(\varepsilon)$, activity accuracy, and measurement uncertainty $(\mathrm{U})$ of absolute activity of radiopharmaceuicals and related radioisotopes are shown in Table 2. Components of combined uncertainty were further summarized in this section.

(a) Uncertainty for the efficiency tracing (and extrapolation) method using a non-H-3 standard solution

Components of combined uncertainty in the activity determination include counting statistics, background, dead time, weighing, decay scheme parameter, half-life, and extrapolation of efficiency curve [57]. Source of uncertainty evaluated by Woods et al. in the absolute standardization of low energy $\beta$ emitter, i.e. Pb-210 are counting, background, half life, $\beta$ dead time, $\gamma$ dead time, resolving times, choice of fit, count rate dependence, dead time formula, weighing, separation time, extrapolation range, contaminants, and reproducibility [58].

(b) Uncertainty for the $\mathrm{C} / \mathrm{N}$ efficiency tracing method

Component of uncertainty in the standardization of Re-186 by the C/N method of LS efficiency tracing with H-3 include source preparation, scintillator stability, dead time, liquidscintillation measurements, uncertainty due to $\mathrm{H}-3$ reference standard, EC/ $\beta^{-}$branching ratio, spectral distributions for EC and $\beta^{-}$branches. [74]

The contributions to the uncertainty of the value of the specific activity are volatility of $\mathrm{H} 2$ [GeCl6] during the preparation of solid sources for coincidence measurements, drop masses, counting statistics, background variation, accidental coincidences and dead time losses, Compton continuum of the $1077 \mathrm{keV}$ peak included in the $\gamma$ window around the $511 \mathrm{keV}$ peak, decay scheme correct ion factor, correction factor for non-vanishing $\varepsilon E C$, impurities and halflife uncertainty, and detection of $511 \mathrm{keV}$ quanta in the $\beta$ detector due to its $\gamma$ sensitivity [52].

The components contributing to the uncertainty of $4 \pi \beta-\gamma$ coincidence method were estimated as follows: counting statistics and background variation, instrumental corrections, impurities, half-life uncertainty, decay scheme correction factor, and mass of droplet. Standard 
deviation of LSC composed of the following contributions: counting statistics, background variation, scintillator stability, comparison with H-3 tracer, instrumental corrections (dead time), dilution factor, droplet mass, radioactive impurities, half-life uncertainty, main decay data, uncertainty of the $\varepsilon$ calculation due to the K-L model, capture probabilities $\mathrm{P}_{\mathrm{K}}, \mathrm{P}_{\mathrm{L}}$, fluorescence yields, $\omega_{\mathrm{K}}, \omega_{\mathrm{L}}$, spectral distribution of $\beta$ particles, and average energy of weak Auger electrons [75].

Source of the uncertainty: counting statistics, mass, dead time, background, timing, chemical effects (adsorption, sample spread, impurities), input parameters and statistical model, quenching, kB influence, decay scheme parameters, and pulse shape discriminator setting. [76]

(c) Uncertainty for the non-extrapolation tracer method

The quoted total uncertainty $(1 \sigma)$ of $0.85 \%$ comprised mainly the components due to counting statistics $(0.28 \%)$, afterpulsing $(0.40 \%)$ and the evaluated decay-scheme data $(0.63 \%)$. $\varepsilon_{\mathrm{M}}$ : double tube detection efficiency of $\mathrm{Mn}-54, \varepsilon_{\mathrm{M}}{ }^{*}$ : reduced Mn-54 efficiency due to quenching caused by the addition of the Fe-55 aliquot [65].

(d) Uncertainty for the coincidence method by a $4 \pi \beta-\gamma$ system

Uncertainty components assayed by Koskinas et al. for the standardization of Eu-152 were counting statistics, weighing, dead time, impurities, half life, extrapolation of efficiency curve [77].

(e) Uncertainty for the TDCR method

The main source of uncertainty of TDCR method comes from the model describing the nonlinearity of the scintillator due to the ionization quenching phenomenon [71]. Type A standard uncertainty, i.e. counting statistics and type B standard uncertainty, i.e. extrapolation (interception uncertainty), spurious pulses, nonuniformity of sources, tracer activity, E. C. correction, dead-time, background, half-life, weighing were evaluated by Sahagia et al. [59].

(f) Uncertainty for the $4 \pi \gamma$ counting method.

Construction of an ionization chamber efficiency curve is not a straightforward process as the curve has to be extracted from experimental calibration points analytically. The efficiency curve is implicitly contained in individual radionuclide coefficients and these are obtained experimentally or by Monte Carlo modelling or calculated back from the efficiency curve. Due to this variety, the interpretation and intercomparison of different efficiency curves is often hard and transferring individual radionuclide calibration coefficients between ionization chambers of different constructions is not a simple process [78].

\subsubsection{International measurement program}

One of the most important components in the quality system of radiopharmaceuticals is to establish the measurement traceability to international standards for ensuring the accurate and consistent of measurement results [5]. Traceability of activity measurements is the critical part in the production and use of unsealed radioactive sources in nuclear medicine. The U.S. Nuclear Regulatory Commission (NRC) defines a medical event as a patient receiving 
an injected activity greater than $20 \%$ different from the prescribed dosage. Tthe Society of Nuclear Medicine (SNM) guidelines also recommend that the measurement be with $10 \%$ of the prescribed dosage. Moreover, the instruments being used are capable of accurate measurements to within 5\% [79]. Therefore, programs for the establishment and dissemination of activity measurement standards in nuclear medicine are held in many countries.

International comparison of standard sources and solutions, such as P-32, Mn-54, Zn-65, Ir-192, Tl-204, and Am-241, which is organized by the International Committee of Weights and Measures (CIPM), the EUROMET system, the former COMECOM, and bilateral comparisons, has been held since 1962 [60].

South Africa's national radioactivity measurement standard is maintained by the National Metrology Laboratory (NML) of the Council for Scientific and Industrial Research (CSIR). Standardizations are undertaken by a number of direct methods utilizing liquid scintillation counting (LSC) [80].

Comparisons of activity measurements for I-131, Tl-201 and Tc-99m with radionuclide calibrators were organized in Cuba since 2002. During 2002, the Radionuclide Metrology Department of the Isotope Center (CENTIS-DMR) has organized several comparisons with various radionuclides in order to obtain information on the quality of the activity measurements during production and administration of radiopharmaceuticals in Cuba [81].

The Australian Radiation Protection and Nuclear Safety Agency (ARPANSA) conducts a series of Radiopharmaceutical Quality Assurance Test Program under a Memorandum of Understanding (MOU) between ARPANSA and the Therapeutic Goods Administration (TGA). For example, in 2005, 46 batches of 24 different types of radiopharmaceuticals, e.g., ready to use radiopharmaceuticals and kits for the preparation of Tc-99m were tested. Two percent in 46 batches of radiopharmaceuticals tested was failure to meet full specifications [82].

International comparison program of national metrological institutes for the standardization of Fe-55, which is a suitable radionuclide standard for X-ray spectrometers, was held by the Comité Consultative pour les Etalons de Mesures des Rayonnements Ionisants (CCEMRI) of the Bureau International des Poids et Mesures (BIPM) [62]. National Metrology Institute of Japan - Advanced Industrial Science and Technology (NMIJ/AIST, Japan) and National Institute of Ionizing Radiation Metrology (ENEA-INMRI, Italy) have been involved in recent years, particularly those relevant in the frame of the international cooperation coordinated by the BIPM and the International Committee for Radionuclide Metrology (ICRM). Particular research activities are devoted on the field of the nuclear safety, nuclear medicine and environmental radionuclide measurements. [83]. International comparisons held by BIPM also can be traced by laboratories such as National Institute for Physics and Nuclear Engineering (Romania) [59], Laboratorio de Metrologia Nuclear (Brazil) in collaboration with the Laboratório Nacional de Metrologia das Radiações Ionizantes, from Rio de Janeiro [57], Radiation Safety Systems Division, Bhabha Atomic Research Centre (India) [61], and Electrotechnical Laboratory (ETL) (Japan) [69].

The Ce-139 measurements formed part of a regional comparison organized by the Asia Pacific Metrology Programme (APMP) [68]. 
The National Institute of Standards and Technology (NIST) maintains a program for the establishment and dissemination of activity measurement standards in nuclear medicine, i.e. Ga-67, Y-90, Tc-99m, Mo-99, In-111, I-125, I-131, and Tl-201 for more than ten years. These standards are disseminated through Standard Reference Materials (SRMs), Calibration Services, radionuclide calibrator settings, and the NIST Radioactivity Measurement Assurance Program (NRMAP, formerly the NEI/NIST MAP). For over 3600 comparisons, $96 \%$ of the participants' results differed from that of NIST by less than $10 \%$, with $98 \%$ being less than $20 \%$. The percentage of participants results within $10 \%$ of NIST ranges from $88 \%$ to $98 \%$ [79].

Measurements from a variety of types of detectors including, ionization chambers, radionuclide calibrators, solid state detectors, Ge detectors, NaI(Tl) detectors, liquid scintillation counters (LSC), Cherenkov counting, and proportional counter are reported [79].

\subsection{Nuclear medicine imaging}

\subsubsection{PET, CT, PET/CT, and SPECT imaging}

PET, CT, PET/CT, and SPECT are non-invasive imaging tools and applied for creating two dimensional (2D) cross section images of three dimensional (3D) objects. PET and SPECT can potentially provide functional or biochemical information by measuring distribution and kinetics of radiolabelled molecules, whereas CT visualizes X-ray density in tissues in the body. The PET imaging in oncology has been migrating from the use of dedicated PET scanners to the use of PET/CT tomographs. This is due to the advantages that PET/CT offers over dedicated PET. One of these advantages is that the integration of PET and CT imaging into a single scanning session allows excellent fusion of the acquired data. Although these nuclear medicine imaging tools provide many advantages and applications in diagnosing diseases clinically, they also poses some challenges and induce artifacts and quantitative errors that can affect the image quality.

\subsubsection{Risks of artifact in PET, CT, and SPECT imaging}

Artifacts and pitfalls can arise at any stage in the process of nuclear medicine imaging and can be grouped into issues related to the (i) patient, (ii) the equipment, or the technologist.

(a) Patient-related risks:

In PET/CT, the patient-related artifacts commonly found are due to metallic implants, truncation, and respiratory motion (or patient motion). These artifacts occur because the CT scan is used to replace a PET transmission scan for the purpose of attenuation correction of the PET data.

Metallic implants, such as dental fillings, hip prosthetics, or chemotherapy ports, cause high CT numbers and generate streaking artifacts on CT images due to their high photon absorption $[85,86]$. This increase CT numbers causes correspondingly high PET attenuation coefficients, resulting in an overestimation of the PET activity and thereby to a falsepositive PET finding. 
In PET/CT, truncation artifacts occur due to the difference in size of the field of view between the CT $(50 \mathrm{~cm})$ and PET $(70 \mathrm{~cm})$ tomographs $[87,88]$ and frequently seen in large patients or patients scanned with arms down, such as in the case of melanoma and head and neck indications. When a patient extends beyond the CT field of view, the extended part of the anatomy is truncated and consequently is not represented in the reconstructed CT image. Truncation also causes streaking artifacts at the edge of the CT image, leading to an overestimation of the attenuation coefficients used to correct the PET data. This increase in attenuation coefficients creates a rim of high activity at the truncation edge, resulting in the misinterpretation of the PET scan.

The most prevalent artifact in PET/CT imaging is respiratory motion during scanning. The artifact is due to the discrepancy between the chest position on the CT image and the chest position on the PET image. PET images are acquired over time periods (time frames) that can vary from a few seconds to tens of minutes. Therefore, during such time periods various motions may have significant effects on the PET images. Both respiratory and contraction induced heart motions have major effect (source of error) on PET imaging of cardiac and thoracic regions. Some equipment, e.g., dose calibrators for the measurements of quantitative measurements is calibrated against or traceable to a reference source of whole body tomographs [89]. Because of the long acquisition time of a PET scan, it is acquired while the patient is freely breathing. The final image is hence an average of many breathing cycles. On the other hand, a CT scan is usually acquired during a specific stage of the breathing cycle. This difference in respiratory motion between PET scans and CT scans results in breathing artifacts on PET/CT images. Several literatures have described this problem [90-91]. The artifacts resulted from respiratory motion or patient motion is also commonly found in myocardial perfusion SPECT. This is because that SPECT requires that the object of interest remains constant for the duration of the acquisition [92-93]. Visually detectable patient motion has been reported in $36 \%$ of clinical studies in one study [94] and $43 \%$ in another [95].

Source of clinical problems of the patients were also indicated by Hladik III, including (i) special patient populations, e.g., pregnant or breast-feeding women, pediatric and geriatric patients, patients requiring dialysis, incontinent, catheterized or miscellaneous patients, (ii) insufficient patient care, education, and preparation, e.g. insufficient patient instruction, shielding or protection in exposure and contamination problems, pregnancy testing, withholding xanthine-containing foods and drug-drug interaction prior to imaging, delay in the administration or imaging, metal implants of patient, (iii) improper behavior of patient, e.g., excessive movement, contamination from incontinence, attenuation from jewelry, prostheses, or implants, etc., and (iv) unexpected altered biodistributions may be undetectable, adverse reactions or untoward effects, [96]

(b) Equipment- or technologist-related risks:

There are several patient-related artifacts and interpretation pitfalls that can potentially compromise nuclear medicine imaging, as discussed above. In order to minimize or identify these artifacts, technologists play an important role in recognizing and correcting them. For example, technologists should ask patients to remove all metallic objects before imaging and should document the location of non-removable metallic objects to minimize or identify the 
artifacts from metallic implants. In PET/CT imaging, it is crucial for technologists to carefully position patients at the center of the field of view and with arms above head to reduce truncation artifacts. Moreover, in order to minimize the artifacts from respiratory motion and produce accurately quantifiable images, it is also essential that technologists instruct patients about breath-hold techniques before the scanning session.

Moreover, sources of clinical problems of error medication also include fail of (i) patient identification, (ii) dosage prescription and administration, (iii) radionuclide administration, (iv) radiopharmaceutical prescription and administration in kinetics or finished product purity testing, (v) interventional medications, (vi) injection technique, (vii) radiopharmaceutical labelled, (viii) preparation or execution of diagnostic or therapeutic procedure, and (ix) radiation protection $[7,96]$.

QC performed on nuclear medicine cameras provides the confidence to technologists and physicians that a scan supplies an accurate representation of the radioisotope distribution in the patient. The instrumentation for nuclear medicine imaging is more complex than that used for whole-body and planar imaging, and requires careful quality control to ensure optimum performance. According to the standards, the main performance parameters are divided into two groups. The first group includes basic intrinsic measurements: spatial resolution in axial and transaxial directions, sensitivity, count rate capabilities by measuring the system dead time and the generation of random events at different radioactivity levels, and scatter fraction of $\gamma$ rays emitted by the annihilation of positron. The second group includes measurements of the accuracy of corrections for physical effects, specifically: uniformity correction, scatter correction, attenuation correction, and count rate linearity correction. Other possible tests to be added to the list of acceptance or performance tests such as: noise equivalent count rate, partial volume and spillover, motion artefacts, image quality test, and PET/CT image co-registration [89].

Nuclear medicine imaging increases the accuracy of diagnosis by combining anatomic information with functional imaging. It is highly dependent on a host of technical considerations. Knowledgeable technologists can minimize or reduce artifacts and other potential problems with image acquisition and, in that way, produce better-quality images.

\section{Conclusion}

Implement of ICH QbD for the radiopharmaceutical manufacturing and imaging technology can be harmonized to a globalized framework in accordance with the regulations and requirements of U.S. FDA, IAEA, WHO and EANM. The attributes of the components in the quality unit (QA/QC), including the aspects of organization, staffing and personnel, facilities, instrumentation and equipment, operation procedure, radiopharmaceuticals, protocol and conduct of a study or a treatment, records and reports, and audit were reviewed and indentified. Critical quality attributes (CQAs) for assuring accurate radioactive dosimetry calculation in the efficiency tracing of absolute activity measurement and in the patient- and technologist-related risks for nuclear medicine imaging (PET, CT, and SPECT), i.e. potential 
sources of error or uncertainty, were elucidated. Although there still have many hard-tocontrolled quantitative errors and artifacts that can eventually affect the quality of imaging, therapeutic efficacy, or safety, it is important for the facility staffs to be aware and continual improvement of these quality factors. By reducing uncertainty and risk or increasing process knowledge and product understanding resulting from $\mathrm{QbD}$ can significantly improve the efficiency of manufacturing processes.

\section{Abbreviations}

API Active pharmaceutical ingredient

ARPANSA Australian Radiation Protection and Nuclear Safety Agency

BNMS British Nuclear Medicine Society

BIPM Bureau International des Poids et Mesures (France)

CT Computed Tomography

CFR Code of Federal Regulations (U.S.)

CMC Chemistry, and manufacturing and controls

CQAs Critical quality attributes

CSIR Council for Scientific and Industrial Research

CGMP Current Good Manufacturing Practice

CGRPP Current Good Radiopharmacy Practice (EU)

EC Ethical Committee (EU)

EANM European Association of Nuclear Medicine (EU)

EDQM European Directorate for the Quality of Medicines \& HealthCare

EIND Exploratory IND (FDA, U.S.)

FDA U.S. Food and Drug Administration

HPLC High-performance liquid chromatography

ICH International Conference on Harmonisation

IMP Investigational Medicinal Product (for drugs used in clinical trials of EU)

IND Investigational new drug

IAEA International Atomic Energy Agency

IRDS Investigational Radiopharmaceutical Drug Service

LSC Liquid scintillation counting 
MA Marketing authorization (EU)

MS Mass spectrometry

MOU Memorandum of Understanding

NRC Nuclear Regulatory Commission

NCA National Competent Authority (EU)

PET Positron emission tomography

QA Quality assurance

QC Quality control

QP Qualified persons who are professional responsible for the release of a drug in Europe

QbD Qulaity by design

RPR Responsible person for the small-scale preparation of radiopharmaceuticals

RDRC Radioactive Drug Research Committee (FDA, U.S.)

SPECT Single photon emission computed tomography

SSRP Small-scale "in-house" radiopharmaceutical

SOP Standard operating procedure

TGA Therapeutic Goods Administration

TLC Thin layer chromatography

USP United States Pharmacopeia

WHO World Health Organization

\section{Author details}

Kung-Tien Liu ${ }^{1 *}$, Jian-Hua Zhao ${ }^{2}$, Lee-Chung Men² and Chien-Hsin Chen ${ }^{1}$

*Address all correspondence to: ktliu@ecic.com.tw

1 Everlight Chemical Industrial Corporation,, Taiwan

2 Chemistry Division, Institute of Nuclear Energy Research,, Taiwan

\section{References}

[1] International Conference on Harmonisation. (2009). ICH Harmonised Tripartite Guideline, Pharmaceutical Development, Q8 (R2)., http://www.ich.org/fileadmin/ 
Public_Web_Site/ICH_Products/Guidelines/Quality/Q8_R1/Step4/Q8_R2_Guideline.pdf.

[2] International Conference on Harmonisation. (2005). ICH Harmonised Tripartite Guideline, Quality Risk Management, Q9., http://www.ich.org/fileadmin/Public_Web_Site/ ICH_Products/Guidelines/Quality/Q9/Step4/Q9_Guideline.pdf.

[3] International Conference on Harmonisation. (2008). ICH Harmonised Tripartite Guideline, Pharmaceutical Quality System, Q10., http:/www.ich.org/fileadmin/ Public_Web_Site/ICH_Products/Guidelines/Quality/Q10/Step4/Q10_Guideline.pdf.

[4] International Atomic Energy Association. (2010). Planning a Clinical PET Centre. http://www-pub.iaea.org/MTCD/publications/PDF/Pub1457_web.pdf.

[5] Zimmerman, B. E., Herbst, C., Norenberg, J. P., \& Woods, M. J. (2006). International Guidance on the Establishment of Quality Assurance Programmes for Radioactivity Measurement in Nuclear Medicine. Applied radiation and isotopes, 64(10), 1142-1146.

[6] International Atomic Energy Association. (2009). Quality Assurance for SPECT systems. http://www-pub.iaea.org/MTCD/publications/PDF/Pub1394_web.pdf.

[7] Bergmann, H., Busemann-Sokole, E., \& Horton, P. W. (1995). Quality Assurance and Harmonisation of Nuclear Medicine Investigations in Europe. European Journal of $\mathrm{Nu}$ clear Medicine and Molecular Imaging, 22(5), 477-480.

[8] Chuck, A., Jacobs, P., Logus, J. W., St, Hilaire. D., Chmielowiec, C., \& Mc Ewan, A. J. B. (2005). Marginal Cost of Operating a Positron Emission Tomography Center in a Regulatory Environment International. Journal of Technology Assessment in Health Care, 21(4), 442-451.

[9] Norenberg, J. P., Petry, N. A., \& Schwarz, S. (2010). Operation of a Radiopharmacy for a Clinical Trial. Seminars in Nuclear Medicine, 40, 347-356.

[10] Decristoforo, C., \& Schwarz, S. W. (2011). Radiopharmacy: Regulations and Legislations in Relation to Human Applications. Drug Discovery Today: Technologies, 8(2-4), e71-77.

[11] European Association of Nuclear Medicine. (2007). Guidelines on current good Radiopharmacy Practice (cGRPP) in the Preparation of Radiopharmaceuticals, Version 2., http:// www.eanm.org/publications/guidelines/gl_radioph_cgrpp.pdf.

[12] Elsinga, P., Todde, S., Penuelas, I., Meyer, G., Farstad, B., Faivre-Chauvet, A., et al. (2010). Guidance on Current Good Radiopharmacy Practice (CGRPP) for the SmallScale Preparation of Radiopharmaceuticals. European Journal of Nuclear Medicine and Molecular Imaging., 37(5), 1049-1062.

[13] World Health Organization. (2003). WHO Expert Committee on Specifications for Pharmaceutical Preparations- WHO Technical Report Series, 908-Thirtyseventh Report., http:// whqlibdoc.who.int/trs/who_trs_908.pdf. 
[14] Barbet, J., Kraeber-Bodéré, F., \& Chatal, J. F. (2008). Review: What Can Be Expected from Nuclear Medicine Tomorrow? Cancer biotherapy and radiopharmaceuticals, 23(4), 483-504.

[15] International Atomic Energy Agency. (2001, 18-22 January 1999). Paper presented at Therapeutic Applications of Radiopharmaceuticals, Proceedings of an International Seminar, Held in Hyderabad, India. http://www-pub.iaea.org/MTCD/ publications/PDF/te_1228_prn.pdf.

[16] Atkinson, B. J., \& Tu, S. M. (2011). Radiopharmaceuticals: Present and Future. Journal of Supportive Oncology, 9(6), 206-207.

[17] Zimmerman, B. E., Cessna, J. T., \& Schima, F. J. (1998). The Standardization of the Potential Bone Palliation Radiopharmaceutical 117mSn (+4) Dtpa. Applied radiation and isotopes., 49(4), 317-328.

[18] Kung, H. F., Kung, M. P., \& Choi, S. R. (2003). Radiopharmaceuticals for Single-Photon Emission Computed Tomography Brain Imaging. Seminars in Nuclear Medicine, 33(1), 2-13.

[19] Imam, S. K. (2005). Molecular Nuclear Imaging: The Radiopharmaceuticals (Review). Cancer biotherapy and radiopharmaceuticals, 20(2), 163-172.

[20] Wong, F. C., \& Kim, E. E. (2009). A Review of Molecular Imaging Studies Reaching the Clinical Stage. European Journal of Radiology, 70(2), 205-211.

[21] Glaser, M., Luthra, S. K., \& Brady, F. (2003). Applications of Positron-Emitting Halogens in Pet Oncology (Review). International Journal of Oncology, 22(2), 253-268.

[22] Elsinga, P. H. (2002). Radiopharmaceutical Chemistry for Positron Emission Tomography. Methods, 27(3), 208-217.

[23] Srivastava, S., \& Dadachova, E. (2001). Recent Advances in Radionuclide Therapy. Seminars in Nuclear Medicine, 31, 330-341.

[24] Jastrzebski, J. (2012). Radioactive Nuclei for Medical Applications. Acta Physica Polonica $B, 43(3), 49-70$.

[25] Miederer, M., Scheinberg, D. A., \& Mc Devitt, M. R. (2008). Realizing the Potential of the Actinium-225 Radionuclide Generator in Targeted Alpha Particle Therapy Applications. Advanced drug delivery reviews, 60(12), 1371-1382.

[26] International Atomic Energy Agency. (2009). Therapeutic Radionuclide Generators: Sr-90/Y-90 and W-188/Re-188 Generators.., http://www-pub.iaea.org/MTCD/publications/PDF/trs470_web.pdf.

[27] Al-Nahhas, A., Win, Z., Szyszko, T., Singh, A., Nanni, C., Fanti, S., et al. (2007). Gallium-68 PET: A New Frontier in Receptor Cancer Imaging. Anticancer research, 27(6B), 4087-4094. 
[28] Anderson, C. J., \& Ferdani, R. (2009). Copper-64 Radiopharmaceuticals for PET Imaging of Cancer: Advances in Preclinical and Clinical Research. Cancer Biotherapy and Radiopharmaceuticals., 24(4), 379-393.

[29] Farncombe, T. H., Gifford, H. C., Narayanan, M. V., Pretorius, P. H., Frey, E. C., \& King, M. A. (2004). Assessment of Scatter Compensation Strategies for 67Ga SPECT Using Numerical Observers and Human Lroc Studies. Journal of Nuclear Medicine, 45(5), 802-812.

[30] Rajendran, J. G., Eary, J. F., Bensinger, W., Durack, L. D., Vernon, C., \& Fritzberg, A. (2002). High-Dose 166Ho-Dotmp in Myeloablative Treatment of Multiple Myeloma: Pharmacokinetics, Biodistribution, and Absorbed Dose Estimation. Journal of Nuclear Medicine., 43(10), 1383-1390.

[31] Schmitt, A., Bernhardt, P., Nilsson, O., Ahlman, H., Kölby, L., Maecke, H. R., et al. (2004). Radiation Therapy of Small Cell Lung Cancer with 177Lu-Dota-Tyr3-Octreotate in an Animal Model. Journal of Nuclear Medicine.; , 45(9), 1542-1548.

[32] Rahmim, A., \& Zaidi, H. (2008). Pet Versus Spect: Strengths, Limitations and Challenges. Nuclear Medicine Communications, 193-207.

[33] Bateman, T. M. (2012). Advantages and Disadvantages of PET and SPECT in a Busy Clinical Practice. Journal of Nuclear Cardiology, 19, S3-S11.

[34] De Decker, M., \& Dierckx, R. A. (2005). The Good Laboratory Practice and Good Clinical Practice Requirements for the Production of Radiopharmaceuticals in Clinical Research. Nuclear Medicine Communications, 575-579.

[35] Patidar, A. K., Patidar, P., Tandel, T. S., Mobiya, A. K., Selvam, G., \& Jeyakandan, M. (2010). Current Trends in Nuclear Pharmacy Practice. International Journal of Pharmaceutical Sciences Review and Research, 5(2), 145-150.

[36] Laven, D. L., \& Martin, W. R. (1989). Justification for Hospital-Based Nuclear Pharmacy Services. Journal of Pharmacy Practice, 2(3), 152-161.

[37] Mac, Farlane. C. R. (2006). Acr Accreditation of Nuclear Medicine and PET Imaging Departments. Journal of Nuclear Medicine Technology, 34(1), 18-24.

[38] Jarritt, P. H., Perkins, A. C., \& Woods, S. D. (2004). Audit of Nuclear Medicine Scientific and Technical Standards. Nuclear Medicine Communications, 77-75.

[39] Organisation for Economic Co-operation and Development. (1998). OECD Series on Principles of Good Laboratory Practice and Compliance Monitoring. , OECD Principles on Good Laboratory Practice , 1

[40] Callahan, R. J., Chilton, H. M., Ponto, J. A., Swanson, D. P., Royal, H. D., \& Bruce, A. D. (2007). Procedure Guideline for the Use of Radiopharmaceuticals 4.0. Journal of Nuclear Medicine Technology, 35(4), 272-275. 
[41] Bombardieri, E., Aktolun, C., Baum, R. P., Bishof-Delaloye, A., Buscombe, J., Chatal, J. F., et al. (2003). Bone Scintigraphy: Procedure Guidelines for Tumour Imaging. European Journal of Nuclear Medicine and Molecular Imaging, 30(12), 99-106.

[42] Donohoe, K. J., Brown, M. L., \& Collier, B. D. ((2003). ). Society of Nuclear Medicine Procedure Guideline for Bone Scintigraphy. Bone Scintigraphy , 205-209.

[43] Delbeke, D., Coleman, R. E., Guiberteau, M. J., Brown, M. L., Royal, H. D., Siegel, B. A., et al. (2006). Procedure Guideline for SPECT/CT Imaging 1.0. Journal of Nuclear Medicine, 47(7), 1227-1234.

[44] Strauss, H. W., Miller, D. D., Wittry, M. D., Cerqueira, M. D., Garcia, E. V., Iskandrian, A. S., et al. (2008). Procedure Guideline for Myocardial Perfusion Imaging 3.3. Journal of Nuclear Medicine Technology, 36(3), 155-161.

[45] Stelmach, H., \& Quinn, J. L. (1974). Radiopharmaceutical Quality Control. Seminars in Nuclear Medicine, 4, 295-303.

[46] Ahuja, S., \& Scypinski, S. (2001). Handbook of Modern Pharmaceutical Analysis. Academic Press.

[47] Harapanhalli, R. S. (2010). Food and Drug Administration Requirements for Testing and Approval of New Radiopharmaceuticals. Seminars in Nuclear Medicine, 40, 364-384.

[48] Hoffman, J. M., Gambhir, S. S., \& Kelloff, G. J. (2007). Regulatory and Reimbursement Challenges for Molecular Imaging1. Radiology., 245(3), 645-660.

[49] Belcher, E. H. (1953). Scintillation Counters Using Liquid Luminescent Media for Absolute Standardization and Radioactive Assay. Journal of Scientific Instruments, 30, 286 -289 .

[50] Troughton, M. E. C. (1966). The Absolute Standardization of Cobalt-57. The International Journal of Applied Radiation and Isotopes, 17(3), 145-150.

[51] Günther, E. (2002). What Can We Expect from the Ciemat/Nist Method? Applied Radiation and Isotopes, 56(1-2), 357-360.

[52] Schönfeld, E., Schötzig, U., Günther, E., \& Schrader, H. (1994). Standardization and Decay Data of 68ge/68ga. Applied Radiation and Isotopes, 45(9), 955-961.

[53] Plch, J., Zderadicka, J., \& Kokta, L. (1974). Coincidence Methods of Standardization for 131cs and Measurement of Decay Parameters. The International Journal of Applied Radiation and Isotopes., 25(10), 433-444.

[54] Barquero, L., \& Roteta, M. (2002). Standardization of $134 \mathrm{cs}$ by Three Methods. Applied Radiation and Isotopes., 56(1-2), 211-214.

[55] Funck, E., Debertin, K., \& Walz, K. F. (1983). Standardization and Decay Data of 201tl. International Journal of Nuclear Medicine and Biology, 137-140. 
[56] Hult, M., Altzitzoglou, T., Denecke, B., Persson, L., Sibbens, G., \& Reher, D. F. G. (2000). Standardisation of 204tl at Irmm. Applied Radiation and Isotopes, 52(3), 493-498.

[57] Dias, M. S., \& Koskinas, M. F. (2003). Standardization of a 204tl Radioactive Solution. Applied Radiation and Isotopes, 58(2), 235-238.

[58] Woods, D. H., Bowles, N. E., Jerome, S. M., de Lavison, P., Lineham, S., Makepeace, J. L., et al. (2000). Standardisation of 210pb. Applied Radiation and Isotopes, 52(3), 381-385.

[59] Sahagia, M., Razdolescu, A., Grigorescu, E. L., Luca, A., \& Ivan, C. (2000). The Standardization of a 204tl Solution. Applied Radiation and Isotopes, 52(3), 487-491.

[60] Sahagia, M., Razdolescu, A. C., Grigorescu, E. L., Luca, A., \& Ivan, C. (2006). Results Obtained by the Radionuclide Metrology Laboratory of IFIN-HH, in the International Comparisons of Standard Solutions, During 2002-2004. Romanian Journal of Physics, $51,21-26$.

[61] Kulkarni, D. B., Reddy, P. J., Bhade, S. P. D., Narayan, K. K., Narayanan, A., Krishnamachari, G., et al. (2006). Comparison of Efficiency Tracing and Zero Detection Threshold Techniques with Ciemat/Nist Standardization Method under Different Quench Conditions with Liquid Scintillation Spectrometer. Current science, 90(1), 83-87.

[62] Koskinas, M. F., Pires, C. A., Yamazaki, I. M., et al. (2008). Standardization of 55Fe by Tracing Method. Kidlington, Royaume-Uni: Elsevier.

[63] Sochorová, J., Auerbach, P., \& Havelka, M. (2008). Application Of "Wet" Extrapolation Method for Activity Standardisation of Electron Capture Radionuclides. Applied Radiation and Isotopes., 66(6), 919-924.

[64] Gunther, E. W. (1994). Standardization of 59Fe and 131I by Liquid Scintillation Counting. Nuclear Instruments and Methods in Physics Research Section A: Accelerators, Spectrometers, Detectors and Associated Equipment, 339(1-2), 402-407.

[65] Simpson, B. R. S., \& Meyer, B. R. (1998). Activity Measurement of 55Fe by an Efficiency Calculation Method. Applied Radiation and Isotopes, 1073-1076.

[66] Simpson, B. R. S., \& Morris, W. M. (2004). Direct Activity Determination of 54Mn and $65 Z n$ by a Non-Extrapolation Liquid Scintillation Method. Applied Radiation and Isotopes, 60(2), 475-479.

[67] Dias, M. S., Silva, F. F. V., \& Koskinas, M. F. (2010). Standardization and Measurement of Gamma-Ray Probability Per Decay of 177Lu. Applied Radiation and Isotopes, 1349 -1353.

[68] Van Wyngaardt, W. M., \& Simpson, B. R. (2006). Absolute Activity Measurement of the Electron-Capture-Based Radionuclides 139Ce, 125I, 192Ir and 65Zn by Liquid Scintillation Coincidence Counting. Applied radiation and isotopes: including data, in- 
strumentation and methods for use in agriculture, industry and medicine, 64(10-11), 1454 -1458 .

[69] Hino, Y., Matui, S., Yamada, T., Takeuchi, N., Onoma, K., Iwamoto, S., et al. (2000). Absolute Measurement of $166 \mathrm{mHo}$ Radioactivity and Development of Sealed Sources for Standardization of $\gamma$-Ray Emitting Nuclides. Applied Radiation and Isotopes, 52(3), 545-549.

[70] Broda, R., Péron, M. N., Cassette, P., Terlikowska, T., \& Hainos, D. (1998). Standardization of 139 Ce by the Liquid Scintillation Counting Using the Triple to Double Coincidence Ratio Method. Applied Radiation and Isotopes, 1035-1040.

[71] Cassette, P., Broda, R., Hainos, D., \& Terlikowska, T. (2000). Analysis of DetectionEfficiency Variation Techniques for the Implementation of the TDCR Method in Liquid Scintillation Counting. Applied Radiation and Isotopes, 52(3), 643-648.

[72] Johansson, L. C., \& Sephton, J. P. (2010). Validation of a New TDCR System at NPL. Applied radiation and isotopes, $1537-1539$.

[73] Kossert, K. (2010). Activity Standardization by Means of a New Tdcr-Cerenkov Counting Technique. Applied radiation and isotopes, 68(6), 1116-1120.

[74] Coursey, B. M., Cessna, J., Garcia-Torano, E., Golas, D. B., Grau, Malonda. A., Gray, D. H., et al. (1991). The Standardization and Decay Scheme of Rhenium-186. International Journal of Radiation Applications and Instrumentation Part A Applied Radiation and Isotopes., 42(9), 865-869.

[75] Schönfeld, E., Janssen, H., Schotzig, U., Gunther, E., \& Schrader, H. (1994). Standardization and Decay Data of 186Re. Nuclear Instruments and Methods in Physics Research Section A: Accelerators, Spectrometers, Detectors and Associated Equipment, 339(1-2), 174-179.

[76] Günther, E. (2000). Standardization of 237np by the Ciemat/Nist Lsc Tracer Method. Applied Radiation and Isotopes., 52(3), 471-474.

[77] Koskinas, M. F., Fonseca, K. A., \& Dias, M. S. (2002). Disintegration Rate Measurement of a 152Eu Solution. Applied Radiation and Isotopes, 56, 1-2, 441-445.

[78] Švec, A. (2009). Interpretation of Ionization Chamber Efficiency Curves. Metrologia, 43-46.

[79] Cessna, J. T., \& Golas, D. B. (2012). The Nist Radioactivity Measurement Assurance Program for the Radiopharmaceutical Industry. Applied radiation and isotopes.

[80] Simpson, B.R.S. (2002). Radioactivity Standardization in South Africa. Applied Radiation and Isotopes, 56(1-2), 301-305.

[81] Oropesa, P., Hernández, A. T., Serra, R., Martinez, E., \& Varela, C. (2003). Comparisons of Activity Measurements with Radionuclide Calibrators. Applied Radiation and Isotopes, 59(5-6), 383-387. 
[82] Ivanov, Z. (2006). Results of the Quality Assurance Testing Program for Radiopharmaceuticals (2005), Version 3. Australian: Australian Radiation Protection and Nuclear Safety Agency, http://www.arpansa.gov.au/pubs/technicalreports/tr144.pdf.

[83] Capogni, M., De Felice, Y., Saito, N., \& De Felice, P. (2011). Ionising Radiation Metrology in the Field of Nuclear and Life Science Applications, http://www.enea.it/it/internazionali/eventi-internazionali/enea-in-japan-2011/radiation-metrology/capogni.pdf.

[84] Hino, Y., \& Ohgaki, H. (1998). Absolute Measurement of 192Ir. Applied Radiation and Isotopes, 1179-1183.

[85] Goerres, G. W., Hany, T. F., Kamel, E., von Schulthess, G. K., \& Buck, A. (2002). Head and Neck Imaging with PET and PET/CT: Artefacts from Dental Metallic Implants. European journal of nuclear medicine, 29(3), 367-370.

[86] Kamel, E. M., Burger, C., Buck, A., von Schulthess., G. K., \& Goerres, G. W. (2003). Impact of Metallic Dental Implants on CT-Based Attenuation Correction in a Combined PET/CT Scanner. European radiology, 13(4), 724-728.

[87] Carney, J., Townsend, D. W., Kinahan, P. E., Beyer, T., Kachelriess, M., Kalender, W. A., et al. (2001). CT-Based Attenuation Correction: The Effects of Imaging with the Arms in the Field of View. Journal of Nuclear Medicine (suppl) , 42, 56P-57P.

[88] Mawlawi, O., Pan, T., \& Cody, D. D. (2004). Evaluation of a New CT Truncation Correction Algorithm for Accurate Quantification of PET/CT Images. Journal of Nuclear Medicine (suppl): , 45, 413P.

[89] Teräs, M. (2008). Performance and Methodological Aspects in Positron Emission Tomography. Finland, University of Turku.

[90] Osman, M. M., Cohade, C., Nakamoto, Y., \& Wahl, R. L. (2003). Respiratory Motion Artifacts on PET Emission Images Obtained Using CT Attenuation Correction on PET-CT. European journal of Nuclear Medicine and Molecular Imaging, 30(4), 603-606.

[91] Cohade, C., Osman, M., Marshall, L. T., \& Wahl, R. L. (2003). PET-CT: Accuracy of PET and CT Spatial Registration of Lung Lesions. European Journal of Nuclear Medicine and Molecular Imaging, 30(5), 721-726.

[92] Botvinick, E.H., Zhu, Y.Y., O'Connell, W. J., \& Dae, M. W. (1993). A Quantitative Assessment of Patient Motion and Its Effect on Myocardial Perfusion SPECT Images. Journal of Nuclear Medicine, 34(2), 303-310.

[93] Cooper, J. A., Neumann, P. H., \& Mc Candless, B. K. (1992). Effect of Patient Motion on Tomographic Myocardial Perfusion Imaging. Journal of Nuclear Medicine, 33(8), 1566-1571.

[94] Wheat, J. M., Currie, G. M., \& Ramsay, B. (2006). Visual Quality Control of Gated Myocardial Perfusion SPECT. The Internet Journal of Cardiology, 4(1). 
[95] Wheat, J. M., \& Currie, G. M. (2004). Incidence and Characterization of Patient Motion in Myocardial Perfusion SPECT: Part 1. Journal of Nuclear Medicine Technology, $32(2), 60-65$.

[96] Hladik, W. B., \& Norenberg, J. P. (1996). Problems Associated with the Clinical Use of Radiopharmaceuticals: A Proposed Classification System and Troubleshooting Guide. European Journal of Nuclear Medicine and Molecular Imaging, 23(8), 997-1002. 
Chapter 14

\title{
Unified Procedures for Quality Controls in Analogue and Digital Mammography
}

\author{
Barbara Testagrossa, Giuseppe Acri, Federica Causa, \\ Raffaele Novario, Maria Giulia Tripepi and \\ Giuseppe Vermiglio
}

Additional information is available at the end of the chapter

http://dx.doi.org/10.5772/51349

\section{Introduction}

Breast cancer is the most commonly diagnosed cancer in women [1]. Current attempts to control breast cancer concentrate on early detection by means of massive screening campaign, via periodic mammography and physical examination, because ample evidence indicates that such screening indeed can be effective in lowering the death rate [2]. Early diagnosis of breast cancer plays a leading role in reducing the mortality and improving the prognosis of this disease [3].

Mammography consists in imaging the female breast using X-rays with low contrast (to keep the delivered dose low), but at the same time high resolution (especially used for early detection).

The goal of mammography is to achieve the image quality required for a given detection task, while ensuring that the patient-absorbed dose is kept as low as reasonably achievable [4]. As practised now, it normally requires a dedicated X-ray tube with special anode materials such as molybdenum or rhodium, small focal spots, operating at a tube voltage around 25 to $32 \mathrm{kV}$, and carefully chosen films and screens in dedicated cassettes. Stationary or moving grids are used as in other branches of plain film radiography. Present-day mammography can be described as a low-dose procedure [5]. In recent years, advances in screen-film technology and filmprocessing techniques have contributed to major improvements in the quality of mammographic images. At present, two distinct mammographic techniques exist:

- Analogue mammography in which the image is recorded on a film. 
- Digital mammography in which the image is digitalised.

The production of analogue or digital mammography images is based on two distinct concepts of image formation [6].

The analogue image is a continuous representation of spatial and intensity variations of the X-ray pattern transmitted by the tissue under analysis. Traditionally, the mammographic image is analogue, obtained using conventional screen-film image receptors as the standard detector [7]. The advantages of screen-film mammography are: high spatial resolution and low contrast sensitivity achieved through improvements in X-ray tube design, screen-film combinations, grids, and film processing [8]. Thus, analogue mammography permits high image quality, low patient dose, and most importantly, the ability to detect small, nonpalpable breast cancers.

In digital systems, image acquisition and display are two independent processes [4]. In such systems images are captured as a digital signal, making electronic transfer and storage of images possible. Digital systems offer a large dynamic range of operation, improving visualization of all areas of the breast and increasing exposure latitude. Also, the digital format allows grayscale adjustment to optimize contrast for any imaging task.

In addition, with the digitalization of the diagnostic image, new medical applications have now emerged, such as Computer-Aided Diagnosis (CAD), stereo mammography, tomosynthesis, contrast medium imaging and dual energy imaging [7].

For a successful screening function the mammograms should contain sufficient diagnostic information to be able to detect breast cancer, using a radiation dose as low as reasonably achievable (ALARA principle). In this context, it is necessary to establish and actively maintain regular and adequate Quality Assurance (QA) procedures that take into account medical, organisational and technical aspects. The QA procedure should include periodic tests to ensure accurate target and critical structure localization. Such tests are referred to as Quality Controls (QC). They are fundamental for the QA procedure because they help ascertain that the equipment performs consistently at a high quality level.

However, whilst the requirement for standardisation is impelling, the Italian legislation (D.L.vo 187/00) is not keeping pace with the advances in mammographic technology. Indeed, at present both analogue and digital formats are used in an un-regulated way, without introducing a proper regulation especially for digital mammography. As a consequence, the QA protocols have been adapted ad hoc to the new digital technology, thus resulting in multiple protocols, some of which valid only for specific machines, resulting in high costs of operation.

On the other hand, at the European level, QA procedures for both analogue and digital mammography systems have been properly addressed and defined, [European guidelines for quality assurance in mammography screening $-4^{\text {th }}$ Edition, Section 2]. In both cases, in fact, the QC of the physical and technical aspects must guarantee the best possible diagnostic information obtainable and image quality stability, within the limits imposed by the ALARA principle.

However, for the case of digital systems the imaging chain can be divided into three independent parts, as cited in [9]: 
a. Image acquisition, including $\mathrm{X}$-ray generation system, image receptor and (in some systems) image receptor corrections;

b. Image processing software;

c. Image presentation, including monitor, imaging presentation software, printer and viewing box.

To produce images with adequate quality, each part of the imaging chain must function within the limits dictated by the standards of screen-film mammography [9], although the definition of such limits for digital systems is still in progress.

In the EUREF protocol it is assumed that digital mammography should perform at least as screen-film mammography.

In this context, a unified protocol is proposed here that can be used with either analogue or digital mammography systems, with the view of reducing the volume of verification procedures to test the operation of such equipment. The advantage of the proposed protocol is that it can be applied as is to both analogue and digital mammography. The results obtained from the application of this protocol to analogue and digital mammography are presented in Section 3, with particular emphasis on image quality. The remaining part of this Section is dedicated to a review of mammographic techniques.

\subsection{Screen-film mammography}

In screen-film mammography, the film is used as the medium for both image acquisition and display. However, whilst providing excellent spatial resolution in high contrast structures, screen-film mammography has limited detection capability for low-contrast lesions in dense breasts [10]. On phantoms, the highest spatial resolution can be as high as 15-20 $\mathrm{lp} / \mathrm{mm}$ but with a very low associated contrast. In addition, noise can limit the reliability of detection, especially for the small or subtle structures [11]. Although considerable advances in film-screen mammography have occurred over the past 20 years, some inherent limitations to further technical improvement exist [12]. One such limitation results from the trade off between dynamic range (latitude) and contrast resolution (gradient) [13]. The relationship between X-ray exposure, image density, and contrast is illustrated by the Hurter and Driffield (H\&D) sigmoid curve (Fig. 1) which uniquely characterises a given type of screen-film system under specific conditions [14].

Because of the sigmoid shape of the characteristic curve, the range of $\mathrm{X}$-ray exposures over which the film display gradient is significant, i.e., the image latitude, is limited. The parts of the H\&D curve where the slope is flat indicate poor contrast (i.e. over- or underexposed images) $[12,16]$.

In screen-film mammography, the automatic exposure control (AEC) has the critical role of ensuring that the appropriate amount of radiation reaches the image receptor to produce a target optical density on the processed film [16]. In AEC systems, an ion chamber or other radiation detector is placed beneath the film cassette and connected electrically to the exposure time control circuit. When a pre-set amount of radiation has been detected, the expo- 
sure is automatically terminated. Other limitations of film-screen mammography include (a) noise caused by the random fluctuation of X-ray quantum absorption by the fluorescent screen and the film emulsion, which can limit the detection of subtle structures, (b) the trade-off between spatial resolution and detection efficiency of the film and screen, and (c) the inefficiency of rejection of scatter radiation by the mammographic grid [12].

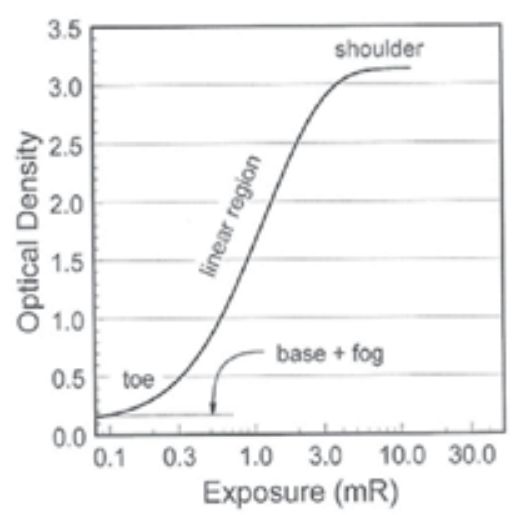

Figure 1. The Hurted \& Driffield (H\&D) curve describes optical density (OD) vs. the logarithm (base 10) of exposure [15].

\subsection{Digital mammography}

Digital mammography is an emerging technology, first approved in January 2000 [17], in which the image acquisition, display and storage functions can be performed independently, allowing for optimisation of each function. It offers several potential advantages including wider dynamic range, improved contrast, increased signal to noise ratio for overcoming the limitations of the film-screen combination (limited latitude, limited display contrast, low detection efficiency and noise), and therefore, increasing the sensitivity and specificity of breast cancer detection [18, 19]. Moreover, digital images offer a variety of new and improved applications. The digital image will provide image archiving and retrieval advantages over film, and will facilitate the use of computer-aided diagnosis [11, 20]. Other advanced applications made possible through digital imaging, such as dual energy and 3D tomosynthesis are expected to further improve diagnostic sensitivity and specificity.

In particular, Full Field Digital Mammography (FFDM) offers the promise of revolutionizing the practice of mammography through its superior dose and contrast performance [20]. In FFDM the screen-film is substituted by a fixed or removable digital detector. The digital image is obtained by sampling the X-ray pattern at discrete increments of spatial position and image signal intensity. Any digital image is a 2-dimensional grid of picture elements (pixels), which is defined by its size and bit depth. The size of an image is given by the length by width (in pixels) product. The bit depth is the number of shades of gray that can be displayed [1].

In a digital imager a detector absorbs the $\mathrm{X}$-rays and produces an electronic signal at each pixel. The signal is then translated into a digital value by an analog-to-digital converter 
(ADC). Once the digital image is stored in the computer memory it can be displayed with contrast independent of the detector properties [12].

Digital mammography systems, unlike screen-film mammography systems, allow manipulation of fine differences in image contrast by means of image processing algorithms [10]. The physical properties of the digital image (contrast, resolution and noise) can vary noticeably according to the detection technology used. There are two methods of image capture used in digital mammography that represent different generations of technology: indirect conversion and direct conversion [20].

Indirect conversion digital detectors uses a two step process for $\mathrm{X}$-rays detection, similar to screen-film [1].

Direct conversion should not be confused with "direct readout", which is a capability of all electronic detectors.

Fully digital mammography (FDM) detectors are the final class of detectors. These detectors are sealed units that are permanently mounted to a mammography system. FDM detectors are electronic devices that directly capture X-ray images. In general, such devices require that a new mammography system be installed [8].

\subsubsection{Photostimulable phosphors (Computed Radiography systems)}

Computed Radiography (CR) is at this moment the most common digital radiography modality in radiology departments, in place of conventional screen film systems [21].

CR for mammography system employ as the X-ray absorber a storage photostimulable phosphor imaging plate (typically BaFBr:Eu ${ }^{+2}$, where the atomic energy levels of the europium activator determine the characteristics of light emission), that replaces the traditional screen-film combination [22]. In this case, the removable detector or the Imaging Plate (IP) is inserted as a cassette in a conventional mammography unit. So, the IP can be used in a standard mammography machine without modification [7].

X-ray absorption mechanisms are identical to those of conventional phosphors. The peculiarity here is that the useful optical signal is not derived from the light that is emitted in prompt response to the incident radiation, but rather from the subsequent emission.

$\mathrm{CR}$ digital phosphor plates have shown promise in mammographic imaging because of the wide exposure latitude and linear response [23].

The potential advantages of this technology are the small detector-element size, the fact that the plates can be used also in conventional mammography units, the ease of having multiple plate sizes, and the relatively low cost. In addition the plates are reusable since they can be readily erased optically [8].

However scattering of the light within the phosphor causes the release of traps over a greater area of the image than the size of the incident laser beam. This results in loss of spatial resolution [24]. 


\subsubsection{Optical detector}

The detector consists of a phosphor screen, a charged coupled device (CCD) camera, and a fiberoptic taper to couple the light from the screen to the camera. It now represents the most widely used digital mammography technique for cassette-free imaging [8].

The imaging performance of these systems depends on a number of factors, including the characteristics of the phosphor screen, the choice of CCD and the method used to optically couple the phosphor to the CCD.

A CCD is an integrated circuit formed by depositing a series of electrodes, called 'gates' on a semiconductor substrate to form an array of metal-oxide-semiconductor (MOS) capacitors [22].

CCDs are particularly well suited to digital radiography because of their high spatial resolution capability, wide dynamic range and high degree of linearity with incident signal.

\subsubsection{Flat panel}

The active matrix flat panel technology is the most promising digital radiographic technique $[25,26]$.

The active matrix detector is based on large glass substrates on which imaging pixels are deposited.

This flat panel plate consists of a matrix of approximately 5 million photodiodes that form the readout for each image. The charge produced on the diode in response to light emitted from the phosphor surface is collected and digitized [1].

\section{Quality Control (QC)}

QCs are fundamental to guarantee that the radiological equipment performs consistently, with standard and constant physical and technical operational parameters.

The technological advances of the past ten years have revolutionised imaging techniques for diagnostics. As a consequence, QC procedures need to be updated to suit the new technologies and related protocols. This is particularly true for mammographic equipment, for which the physical parameters to be monitored to guarantee high-quality imaging are identified in specific documents.

The European Protocol for "Quality Control of the Physical and Technical Aspects of Mammography Screening" [9] gives guidance on physical, technical and dose measurements, and the periodicity of the corresponding tests to be performed as part of mammography screening programmes.

On the other hand, in the case of the Italian regulation, the relevant legislation (D.L.vo 187/00) was approved before the commercialisation of CR and digital mammography. Therefore, guidelines and procedures for $\mathrm{CR}$ and digital mammography are missing. 
This shortcoming is particularly relevant in the case of mammography because it is well known that both image quality and breast dose depend on the equipment used and the radiographic technique employed.

For a complete and accurate estimate of image quality and delivered dose, the following components and system parameters should be monitored [9]:

- X-ray generation and exposure control system

- Bucky and image receptor

- Film processing (for screen-film systems)

- Image processing (for digital systems)

- System properties (including dose)

- Monitors and printers (for digital systems)

- Viewing conditions

\begin{tabular}{|c|c|c|c|c|}
\hline & & Screen-film mammography & \multicolumn{2}{|c|}{ Digital mammography } \\
\hline \multirow{6}{*}{\multicolumn{2}{|c|}{ 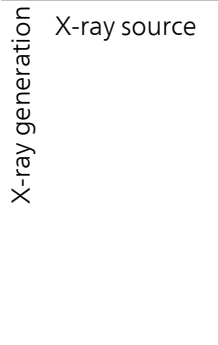 }} & \multicolumn{3}{|c|}{ Focal spot size } \\
\hline & & \multicolumn{3}{|c|}{ Source-to-image distance } \\
\hline & & \multicolumn{3}{|c|}{ Alignment of $\mathrm{X}$-ray field/image receptor } \\
\hline & & \multicolumn{3}{|c|}{ Film/bucky edge } \\
\hline & & \multicolumn{3}{|c|}{ Radiation leakage } \\
\hline & & \multicolumn{3}{|c|}{ Output } \\
\hline \multirow{3}{*}{\multicolumn{2}{|c|}{ Tube voltage }} & \multicolumn{3}{|c|}{ Reproducibility } \\
\hline & & \multicolumn{3}{|c|}{ Accuracy } \\
\hline & & \multicolumn{3}{|c|}{ HVL } \\
\hline & \multirow[t]{6}{*}{ AEC } & Central opt. dens. control settings & \multicolumn{2}{|c|}{$\begin{array}{l}\text { Exposure control steps: central } \\
\text { value }\end{array}$} \\
\hline & & Opt. dens. control step & \multicolumn{2}{|c|}{$\begin{array}{l}\text { Exposure control steps: } \\
\text { difference per step }\end{array}$} \\
\hline & & Target opt. dens. control settings & \multicolumn{2}{|l|}{------ } \\
\hline & & Short-term reproducibility & \multicolumn{2}{|c|}{ Short-term reproducibility } \\
\hline & & \multirow[t]{2}{*}{ Long-term reproducibility } & \multirow[t]{2}{*}{$\begin{array}{l}\text { Long-term } \\
\text { reproducibility }\end{array}$} & $\begin{array}{l}\text { Variation in } \\
\text { SNR }\end{array}$ \\
\hline & & & & $\begin{array}{l}\text { Variation in } \\
\text { dose }\end{array}$ \\
\hline
\end{tabular}




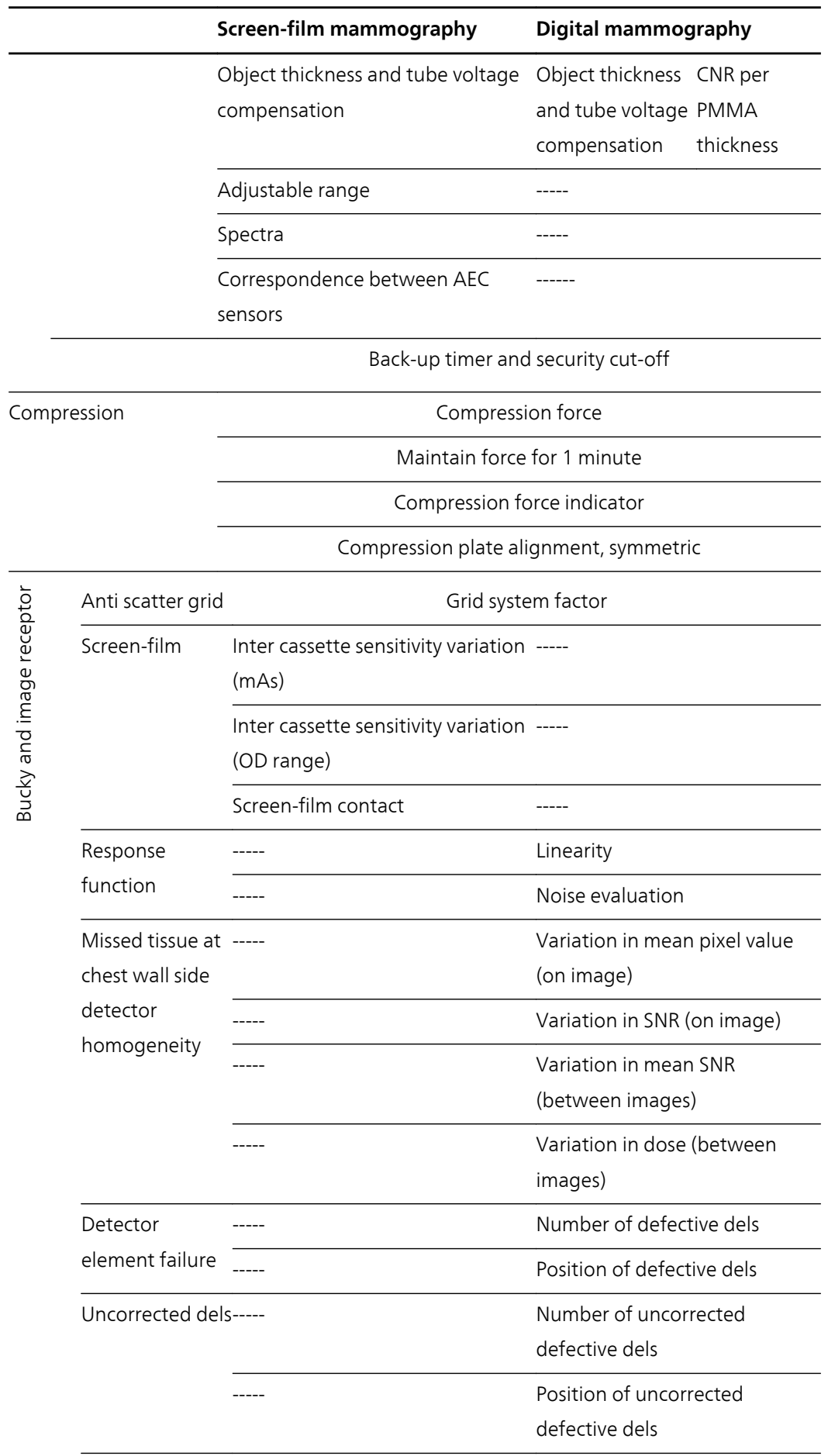




\begin{tabular}{|c|c|c|}
\hline & Screen-film mammography & Digital mammography \\
\hline \multirow{2}{*}{$\begin{array}{l}\text { Inter plate } \\
\text { sensitivity } \\
\text { variations }\end{array}$} & ----- & Variation in SNR \\
\hline & $\begin{array}{ll}--- \\
---\end{array}$ & Variation in dose \\
\hline Dosimetry & \multicolumn{2}{|c|}{ Glandular dose per PMMA thickness } \\
\hline \multirow[t]{7}{*}{ Image quality } & \multicolumn{2}{|c|}{ Threshold contrast visibility } \\
\hline & \multicolumn{2}{|c|}{ Exposure time } \\
\hline & Spatial resolution & MTF and NPS \\
\hline & ----- & Scanning time \\
\hline & ----- & Geometric distortion \\
\hline & ----- & Artifact evaluation \\
\hline & ---- & Ghost image factor \\
\hline
\end{tabular}

Table 1. Operational parameters relevant to analogue and digital mammographs [9].

Some of the above components are suitable only for analogue systems, others only for digital ones, and some are common to both systems although requiring dedicated QC procedures.

With reference to QCs for mammography, the EU legislation is subdivided in two parts: Section $2 \mathrm{a}$ for screen-film mammography, Section $2 \mathrm{~b}$ for digital mammography. In both cases, several measurements should be undertaken by medical physicists. The components that are common to both analogue and digital mammographic systems are listed in Table 1 with corresponding operational parameters specific for the two cases. As expected, the methodology to be used for QC in the two different cases are substantially different particularly with respect to image quality monitoring.

For example, in the case of traditional, analogue mammography, spatial resolution and threshold contrast visibility can be used to uniquely characterise the image quality. On the other hand, in digital mammography image quality is assessed by monitoring the Modulation Transfer Function (MTF), Noise Power Spectrum (NPS) and Nyquist frequency. MTF represents the efficiency or fan imaging system in reproducing subject contrast at various spatial frequencies [7, 20,27]. The Nyquist frequency, instead, indicates the maximum spatial resolution that can be visualized in an image. NPS provides information on noise at different spatial frequencies. In digital mammography, in fact, spatial resolution is obtained from MTF and Nyquist frequency.

The combination of MTF and NPS gives the Detective Quantum Efficiency (DQE), regarded as the best overall indicator of the image quality of digital radiographic systems. DQE is the efficiency with which a detector uses the incident photons to form an image [28]. Systems with higher DQE can produce higher quality images, at the same dose. Further, there are also other parameters that need to be monitored in digital techniques to defined the image quality. These are listed in Table 2. 


\begin{tabular}{ll}
\hline Metric & Performance attribute \\
\hline MTF & Resolution properties of the image/detector/system \\
\hline NPS & Noise properties of the image/detector/system \\
\hline DQE & SNR transfer properties of the detector \\
\hline eDQE & SNR transfer properties of the system \\
\hline Dark noise & Noise in the absence of signal \\
\hline Uniformity & Signal uniformity in the absence of an object \\
\hline Exposure Indicator & Accuracy of exposure indication by the system \\
\hline Linearity & Exposure response behavior of the system \\
\hline High-contrast resolution & Ability of the system to represent high-contrast patterns \\
\hline Low-contrast resolution & Ability of the system to represent low-contrast patterns \\
\hline Distortion & Geometrical accuracy of images \\
\hline Artifact & Non-uniform artifactual features in the images \\
\hline Ghosting & Appearance of shadows of prior images on subsequent images \\
\hline Throughput & Speed by which a system can sequentially capture images \\
\hline Normal exposure & Target exposure values for clinical use reflecting system speed \\
\hline
\end{tabular}

Table 2. List of parameters for digital image quality control [27].

The problem is to define a unified protocol that can be applied to any (analogue, CR, digital) type of mammographic system.

On the basis of procedures developed previously [29, 30], and to minimise problems arising from the use of different QC procedure to monitor different physical parameters for analogue and digital mammographs it is proposed here to monitor only parameters related to the beam at the output of the RX tube. The resulting QC procedure is then flexible and applicable universally to any type of mammograph.

The only additional pieces of equipment needed to execute the proposed $\mathrm{QC}$ is a phantom coupled to a solid-state exposure meter (PHAN-EX).

The phantom is a $4.5 \mathrm{~cm}$ thick block of PMMA, simulating a standard breast, including details simulating those of clinical interest (micro-calcification, tumoral mass, fibrous structures). This is coupled to an RX exposure meter composed of a photodiode and a digital counter, thus capable of measuring the exposure and the quality of the mammographic image [31]. The proposed protocol was tested on different (analogue and digital) mammographs, to assess its versatility and accuracy, independent of the physical characteristics of the mammographic system. Results on AEC tests obtained from the implementation of the proposed protocol implemented on analogue and digital mammographs, are presented and discussed in Section 3. 


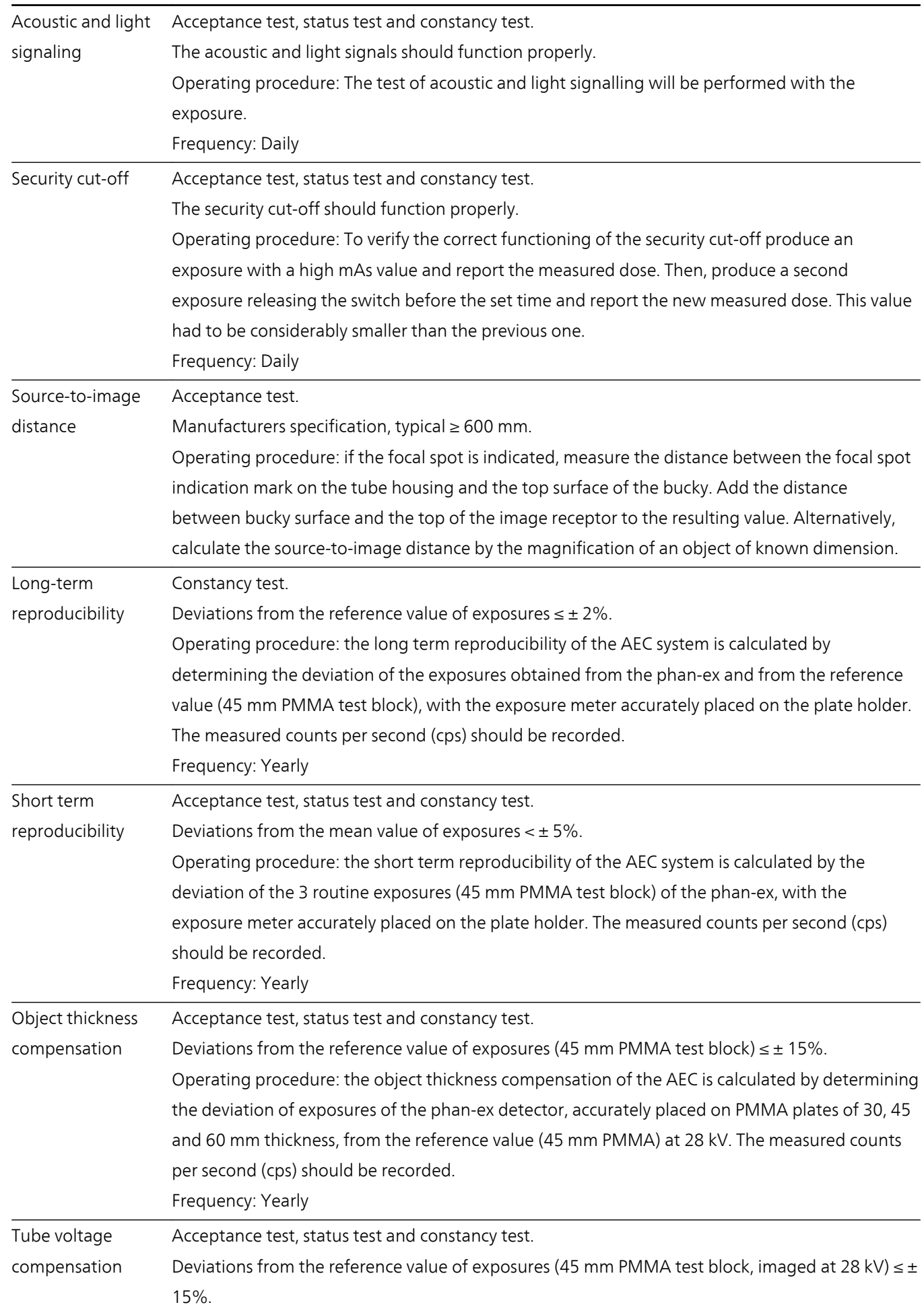


Operating procedure: the tube voltage compensation of the AEC is calculated by imaging the 45 mm PMMA test block, setting the tube voltage at $26 \mathrm{kV}, 28 \mathrm{kV}$ and $30 \mathrm{kV}$, with the exposure meter of the phan-ex accurately placed on the test block. The measured counts per second (cps) should be recorded.

Frequency: Yearly

Difference per step Acceptance test, status test and constancy test.

All the deviation in the measured exposures between successive steps: $0.1-0.2$ per step.

Operating procedure: The optical density control step can be determined by placing the phan-ex on a $45 \mathrm{~mm}$ PMMA plate and taking an exposure at all possible steps, setting the operating voltage at $28 \mathrm{kV}$. The measured counts per second (cps) should be recorded.

Frequency: Yearly

Uniformity Acceptance test, quality control.

Parallel to the axis tube, the exposure value should decrease by $30-35 \%$ at a height of $12 \mathrm{~cm}$ from the chest wall. Perpendicularly to the axis tube, a typical value of exposure decrease is $<7 \%$ from the centre of the $X$-ray field to $10 \mathrm{~cm}$, for each side.

Operating procedure: Beam uniformity can be determined by positioning the exposure meter on a $45 \mathrm{~mm}$ PMMA plate, first at the centre of the PMMA plate and, successively, at the top, right, bottom and left of the test block. Image the plate and report the measured counts per second (cps).

Frequency: Yearly

Spatial resolution Acceptance test, status test and constancy test

(at high frequency) Spatial resolution should be $\geq 12$ line pairs per $\mathrm{mm}(\mathrm{lp} / \mathrm{mm}$ )

Operating procedure: It can be estimated by imaging two resolution lead bar patterns, up to 20 line pairs per $\mathrm{mm}(\mathrm{lp} / \mathrm{mm})$ each, placed on a $45 \mathrm{~mm}$-thick PMMA plate. Image the patterns using a Mo/Mo target-filter combination at $28 \mathrm{kV}$.

Frequency: Yearly

Threshold contrast Acceptance test, status test and constancy test.

visibility Minimum detectable contrast for a $5-6 \mathrm{~mm}$ detail $<1.3 \%$

Operating procedure: It can be estimated by imaging a suitable phantom containing 5-6 $\mathrm{mm}$ circular details. The phantom is accurately placed on a $45 \mathrm{~mm}$ PMMA plate. Image the phantom using a Mo/Mo target-filter combination at $28 \mathrm{kV}$.

Frequency: Yearly

Alignment of $\mathrm{X}$-ray Acceptance test, status test and constancy test.

field/image $\quad X$-rays must cover the film by no more than $5 \mathrm{~mm}$ outside the film parallel to the axis tube, receptor laterally $X$-rays must totally cover the film.

Operating procedure: The alignment of the $\mathrm{X}$-ray field and image receptor at the chest wall side can be determined by using two loaded cassettes and two $X$-ray absorbers. Produce an exposure Frequency: Every three months

Tube Voltage Acceptance test, status test.

Accuracy Accuracy for the range of clinically used tube voltages $(25-31 \mathrm{kV}):< \pm 1 \mathrm{kV}$.

Operating procedure: The equipment should be tested over the range of clinically used settings (typically $25-31 \mathrm{kV}$ ) at intervals of $1 \mathrm{kV}$. To determine the tube voltage accuracy, the kV-meter 
should be accurately placed. The resulting measured $\mathrm{kV}$ should be recorded. After having assessed that the differences between measured and nominal tube voltage values are within 1 $\mathrm{kV}$, the exposures can be repeated at $1 \mathrm{kV}$ intervals, after positioning the exposure meter, by recording the resulting counts per second (cps).

Constancy test.

Accuracy for the range of clinically used tube voltages ( $25-31 \mathrm{kV})$ : measured mGy vs nominal $\mathrm{kV}$ curve should be within the error bar.

Operating procedure: Adequately position the exposure meter and report the counts per second (cps) measured at intervals of $1 \mathrm{kV}$.

Frequency: Yearly

\begin{tabular}{ll}
\hline Tube Voltage & Acceptance test, status test and constancy test. \\
Reproducibility & Reproducibility (at $28 \mathrm{kV}):< \pm 0.5 \mathrm{kV}$. \\
& Operating procedure: To determine tube voltage reproducibility, accurately position the $\mathrm{kV}$ - \\
& meter and make at least three exposures at a fixed tube voltage that is normally used clinically \\
& (e.g. $28 \mathrm{kV})$. When the deviation from the mean value is $< \pm 0.5 \mathrm{kV}$ and repeat the exposures, \\
& after positioning the exposure meter, and record the resulting counts per second (cps). \\
& Constancy test. \\
& Reproducibility (at $28 \mathrm{kV}):< \pm 2 \%$. \\
& Operating procedure: Adequately position the exposure meter. Make at least three exposure at a \\
& fixed tube voltage that is normally used clinically (e.g. $28 \mathrm{kV}$ ) and report the measured counts per \\
& second (cps). \\
& Frequency: Yearly
\end{tabular}

Exposure time Acceptance test, status test and constancy test.

Exposure time needed to image a $45 \mathrm{~mm}$ PMMA phantom: $<2 \mathrm{sec}$.

Operating procedure: After accurately positioning the PMMA phantom and the sensor, the time for a routine exposure is measured.

Frequency: Yearly

\begin{tabular}{ll}
\hline Reference dose & Acceptance test, status test and constancy test. \\
& Entrance dose: $\leq 10 \mathrm{mGy}$ ( $40 \mathrm{~mm}$ PMMA test block); $12 \mathrm{mGy}$ (45 mm PMMA test block); $\leq 20$ \\
& mGy ( $50 \mathrm{~mm}$ PMMA test block). \\
& Operating procedure: Accurately position the exposure meter on the PMMA test block of known \\
& thickness. Report the counts per second (cps) measured at the entrance. \\
& Frequency: Yearly \\
\hline Output rate & Acceptance test, status test and constancy test. \\
& Output rate must be $</ 7.5 \mathrm{mGy} / \mathrm{s}$ (at the focus-to-film distance). \\
& Operating procedure: The output rate should be measured using a Mo/Mo target-filter \\
& combination at $28 \mathrm{kV}$, in the absence of scatter material and attenuation, and reporting the \\
& counts per second (cps). After calculating the exposure value, calculate the output rate at a \\
& distance equal to the focus-to-film distance (FFD). \\
& Frequency: Yearly
\end{tabular}

Average glandular Acceptance test, quality control.

dose (AGD) AGD $(45 \mathrm{~mm}$ PMMA): $<2 \mathrm{mGy}$. 
Operating procedure: After determining the tube load (mAs) necessary to image the phan-ex, accurately position the exposure meter on the $45 \mathrm{~mm}$ PMMA test block and report the measured counts per second (cps), without backscattering. After calculating the exposure value, calculate the output rate at a distance equal to the focus-to-film distance (FFD) and convert this value into the average glandular dose.

Frequency: Yearly

Grid system factor At acceptance and when dose or exposure time increases suddenly.

Grid system factor must be $\leq 3$.

Operating procedure: The grid system factor can be estimated by accurately positioning the phan-ex and measuring counts per second (at $28 \mathrm{kV}$ ), without compression, and with and without the grid system.

\begin{tabular}{ll}
\hline Grid imaging & Acceptance test, status test and constancy test. \\
& No significant non uniformity \\
& Operating procedure: image the bucky at the lowest position of the AEC-selector, without \\
& PMMA. Verify the image uniformity. \\
& Frequency: Yearly \\
\hline Back-up timer & Acceptance test, quality control. \\
& The back-up timer should function properly. \\
& Operating procedure: Make an exposure of a 1 mm lead sheet and verify if the AEC system \\
& terminates the exposure. \\
& Frequency: Yearly \\
& Acceptance test, status test and constancy test. \\
& For $28 \mathrm{kV}$ Mo/Mo target-filter combination the HVL must be between 0.30 and 0.40 mm Al \\
& equivalent. \\
& Operating procedure: Position the exposure detector at the reference ROI (since the HVL is \\
position-dependent) on top of the bucky. Place the compression device halfway between focal & spot and detector. Select a Mo/Mo target/filter combination, $28 \mathrm{kV}$ tube voltage and an \\
adequate tube loading (mAs-setting), and expose the detector directly. The filters can be placed \\
on the compression device and must intercept the whole radiation field. Use the same tube load \\
(mAs) setting and expose the detector through each filter. \\
Frequency: Yearly
\end{tabular}

Focal spot size At acceptance and when resolution has changed, quality control.

For $28 \mathrm{kV}$ Mo/Mo target-filter combination, focal spots size are reported in the following table.

\begin{tabular}{lll}
\hline \multirow{2}{*}{ Focal spot size } & \multicolumn{2}{l}{ Reference values } \\
\cline { 2 - 3 } & Length $(\mathrm{cm})$ & Width $(\mathrm{cm})$ \\
\hline $1 \times 1$ & $0.1+0.15$ & $0.1+0.15$ \\
\hline $2 \times 2$ & $0.2+0.3$ & $0.2+0.3$ \\
\hline $3 \times 3$ & $0.45+0.65$ & $0.3+0.45$ \\
\hline $4 \times 4$ & $0.6+0.85$ & $0.4+0.6$ \\
\hline
\end{tabular}

Operating procedure: Produce a magnified image of the pinhole and measure, on the image, the length and the width, in $\mathrm{cm}$. Repeat for all available focal spots.

Frequency: Yearly

Compression force Acceptance test, status test and constancy test. 
Maximum automatically applied force: $130-200 \mathrm{~N}$.

Operating procedure: The compression force can be estimated using a compression force test device or a bathroom scale.

Frequency: Yearly

Compression plate Acceptance test, status test and constancy test.

alignment The difference between the measured distances at the left and right side of the compression paddle should be $\leq 5 \mathrm{~mm}$ for symmetrical load.

Operating procedure: The alignment of the compression device at maximum force can be visualized and measured when a piece of foam-rubber is compressed.

Frequency: Yearly

Table 3. Proposed protocol for mammography QC and technical specification of the parameters to be monitored.

\section{Results and discussion}

The chosen protocol can be used equally for acceptance, status and constancy tests. It was successfully implemented for both analogue and digital mammographs.

In particular, it was implemented for constancy tests of all parameters relevant to the exposure, utilising the same phantom-exposure meter pair.

In addition to the protocol, Table 3, the QC report worksheet is proposed in which the raw results (counts per second, cps) can be reported, Fig. 2-3. The raw data is then elaborated to estimate the entrance dose.

The proposed protocol and QC report were tested on different (analogue and digital) mammographs, to assess their versatility and accuracy, independent of the physical characteristics of the mammograph.

As an example, the AEC test results obtained for a digital mammographic system are reported in Figs. 4-6 to show that the same protocol can also be used on digital instruments.

The results obtained from the object thickness compensation are represented in Fig. 4. In particular, in Fig. 4 (a), the value of the dose (mGy) normalised to the tube load value (mAs) for the reference PMMA test block thickness $(45 \mathrm{~mm})$, is constant and within the error bar $( \pm$ $15 \%$ ). The dose as a function of the PMMA plate thickness is presented in Fig. 4 (b). This curve shows that, with increasing dose, the normalised dose is constant, indicating the correct operation of the AEC system.

The results obtained from the tube voltage compensation are presented in Fig. 5 (a). Differently from the previous test, where the tube voltage was kept constant $(28 \mathrm{kVp})$ varying only the tube load, in this type of test two parameters are varied: tube voltage and tube load. Therefore, in this test the parameter chosen to assess the tube voltage compensation is the logarithm (base 10) of the dose. Also in this case the results show that the logarithm of the dose is within the limit values $( \pm 15 \%$ calculated for a reference tube voltage of $28 \mathrm{kVp}$ and for a $45 \mathrm{~mm}$ PMMA test block). 


\section{TEST RESULTS}

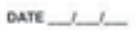

QCREPORT

Waccepravce Test

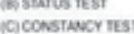

$a$
0
0

Monitored parameters

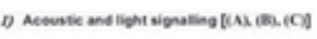

\begin{tabular}{|c|c|}
\hline 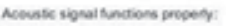 & peso 0 \\
\hline Lete signa Lectems presenty & mos 0 \\
\hline
\end{tabular}

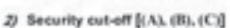

Espesure valies

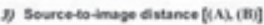

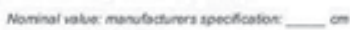

Focus indection bo ludy

Bucly bo camede for neceiver inage) __ on

Seurce bo image diatance $\quad$ on

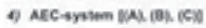

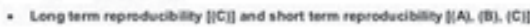

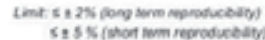

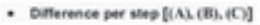

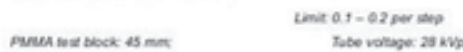

\begin{tabular}{|c|c|c|}
\hline sopp & Tute bas (im (an) & $\begin{array}{c}\text { Cowest per secind } \\
\text { (epes) }\end{array}$ \\
\hline-2 & & \\
\hline$\Rightarrow$ & & \\
\hline 0 & & \\
\hline$*$ & & \\
\hline$\cdot 2$ & & \\
\hline
\end{tabular}

9) Unibormity fas (bi) (C)

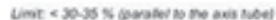

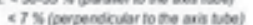

\begin{tabular}{|c|c|}
\hline Coposive meter poscon & 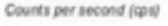 \\
\hline Contes & \\
\hline Top & \\
\hline Algent & \\
\hline Doteon & \\
\hline Let & \\
\hline
\end{tabular}

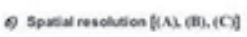

Troet ewer combination. Mb $\mathrm{M}_{0}$

Fute velage $28 \mathrm{kV}$

Resolven

$N_{0}$ 

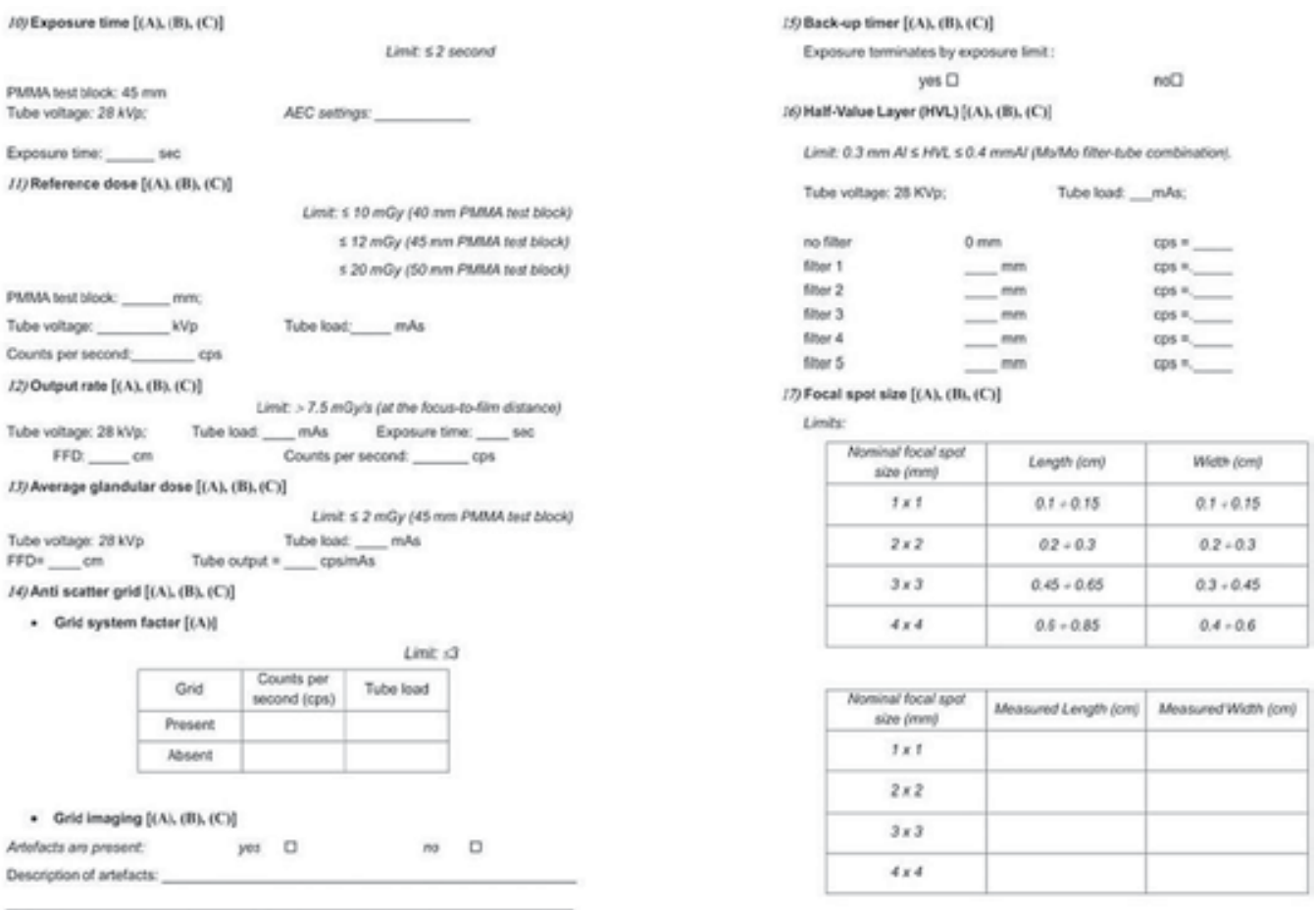

\begin{tabular}{|c|c|c|}
\hline 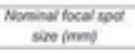 & Measoned lengen fions & 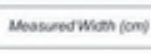 \\
\hline IXI & & \\
\hline $2 \times 2$ & & \\
\hline $3 \times 3$ & & \\
\hline $4 \times 4$ & & \\
\hline
\end{tabular}

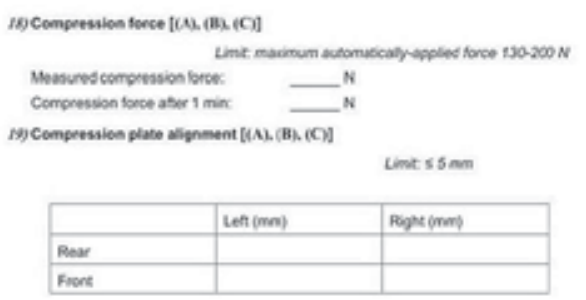

Figure 3. QC report worksheet for raw data recording (part 2).

The dose radiated by the AEC system as a function of the tube voltage is presented in Fig. 5 (b), as measured with the phan-ex. From the results of Fig 5 (b) it is noticed that as the tube voltage increases, the dose decreases, further confirming that the AEC system is functioning correctly.

Results from the test on the "difference per step" are reported in Fig. 6. Also in this case, the logarithm of the dose was calculated at each step. The obtained values are within the limit values $(0.2-0.4$ as the step difference was 2$)$, Fig. 6 (a). The corresponding values of the dose per step are reported in Fig. 6 (b).

For the short-term reproducibility test, exposure values were measured, from which the average dose value was determined with respect to the tube load supplied by the AEC system $(\mathrm{mGy} / \mathrm{mAs})$, Fig. 7 , to show the proposed unified protocol is equally applicable to analogue and digital mammographic system. 
Object Thickness Compensation

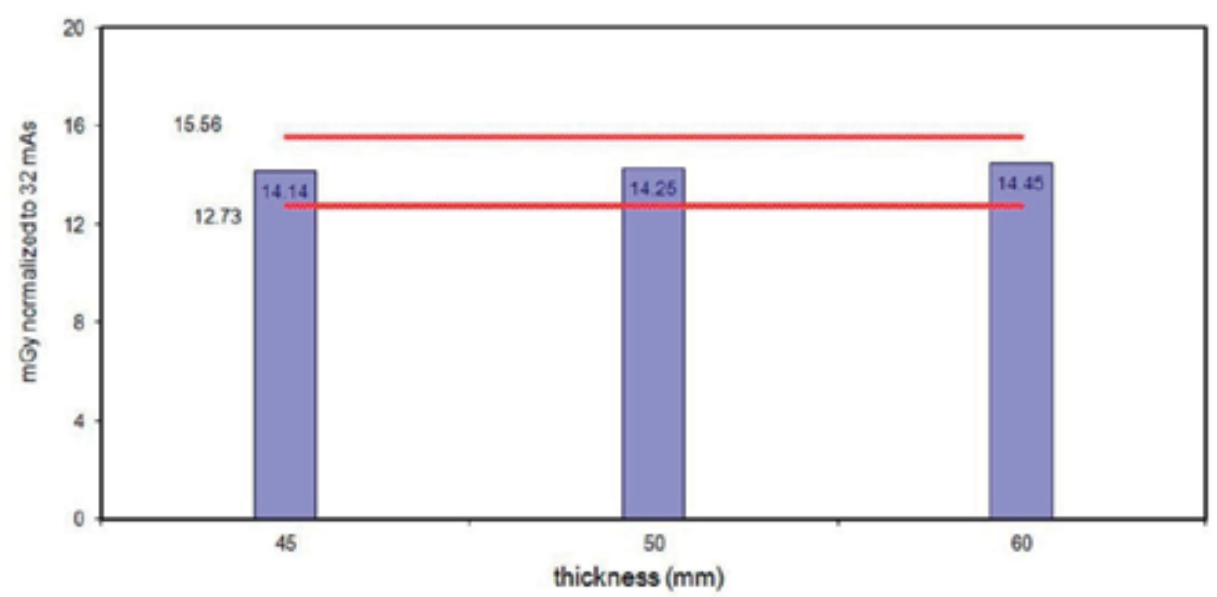

(a)

Object Thickness Compensation (Dose vs thickness)

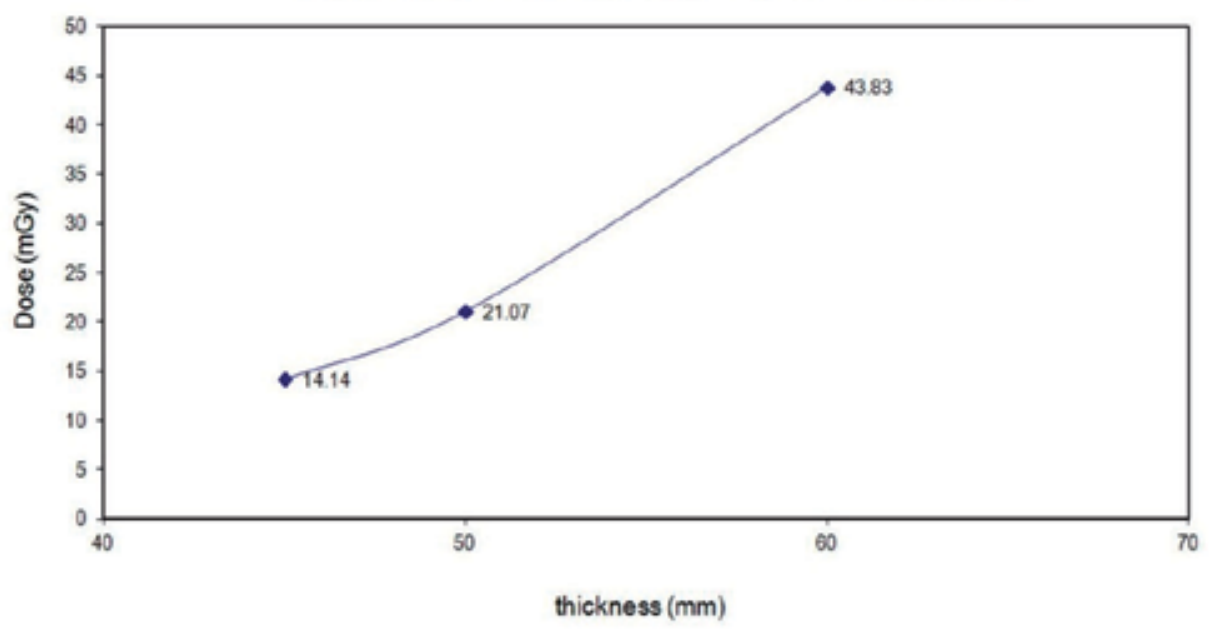

(b)

Figure 4. Results of object thickness compensation test: (a) the dose (mGy) normalised to the tube load value (mAs); (b) the dose ( $m G y)$ as a function of PMMA plate thickness.

The use of the phan-ex, coupled with the proposed protocol, is useful also to verify parameters related to the exposure such as tube voltage precision and accuracy, and exposure time.

Most importantly the proposed protocol permits the evaluation of the functional parameters of the instruments by utilising a single phantom, thus significantly reducing the number of additional dedicated equipment and simplifying the task of the Medical Physics Expert.

The results obtained from raw data analysis obtained following the proposed protocol were found to be consistent with those obtained from standard procedures [32-35], thus highlighting the usefulness and versatility of the proposed unified protocol to test all relevant param- 
eters in analogue and (direct or indirect) digital instruments. The simplification is even more relevant in the latter type of mammographs for which the QC procedures currently used present considerable difficulties in the interpretation of the measurement protocols.

The applicability of the proposed phantom can be further extended to the measurement of parameters other than those relevant to the exposure even for the next generation of mammographs which are still under development. One such instrument is the SYRMEP, equipped with a Si-based microstrip detector and a synchrotron X-Ray source characterised by superior performance with respect to typical X-Ray tubes [36].

\section{Tube Voltage Compensation}

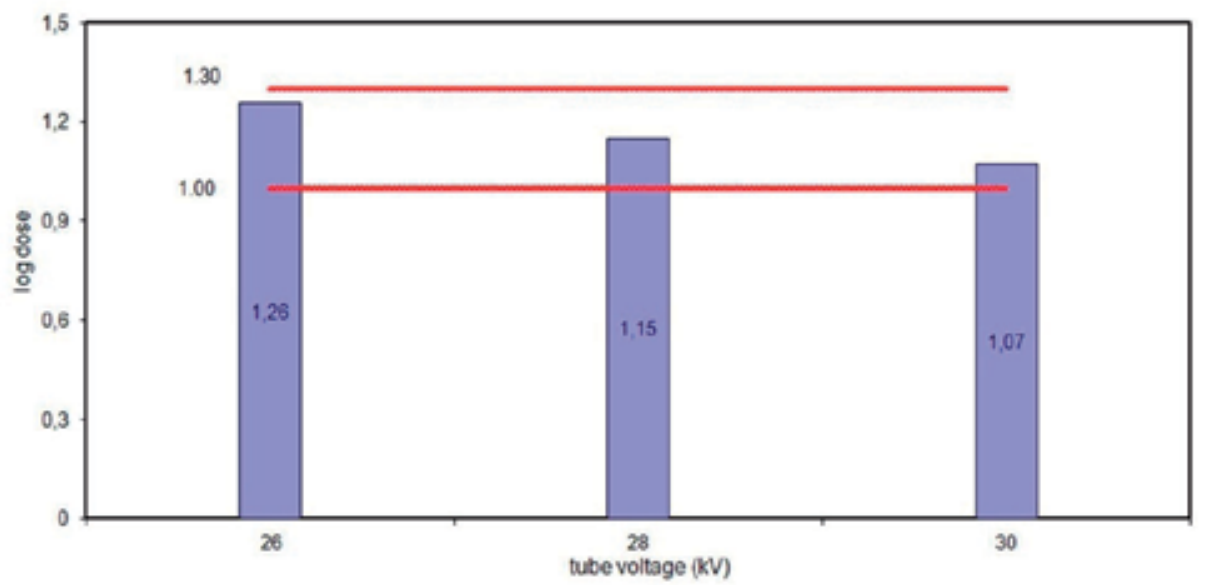

(a)

Tube Voltage Compensation (Dose vs Tube Voltage)

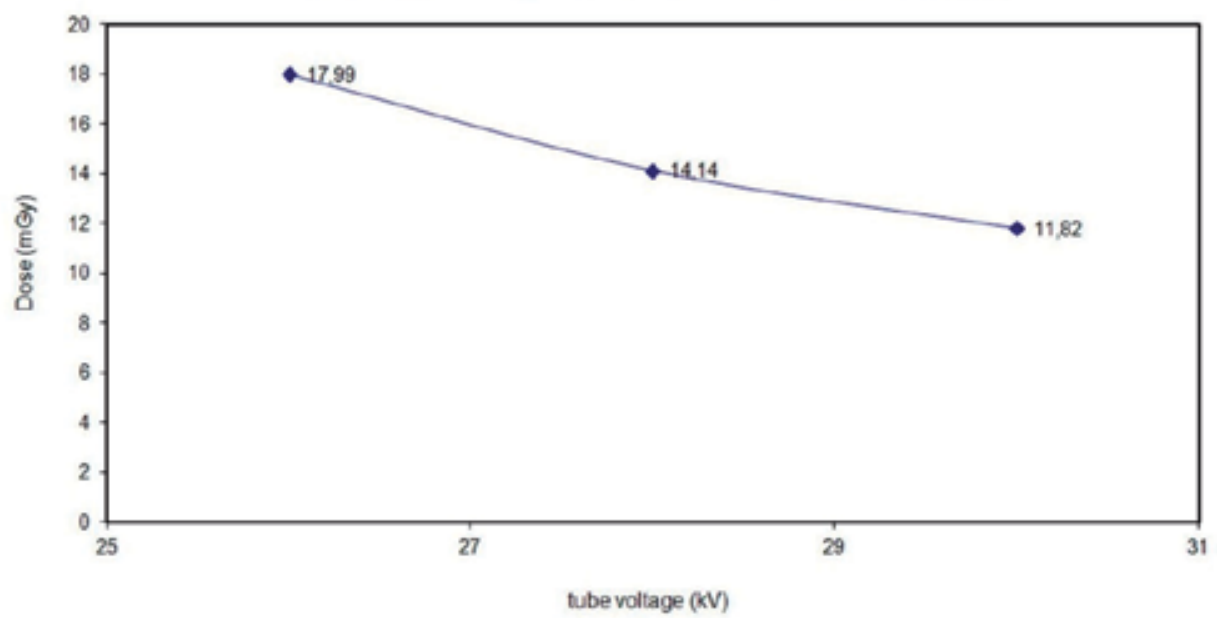

(b)

Figure 5. Results of tube voltage compensation test: (a) logarithm of the dose, red lines representing the limit values $( \pm 15 \%)$ with respect to $28 \mathrm{kVp}$ reference tube voltage; (b) the dose (mGy) as a function of tube voltage $(\mathrm{kVp})$. 


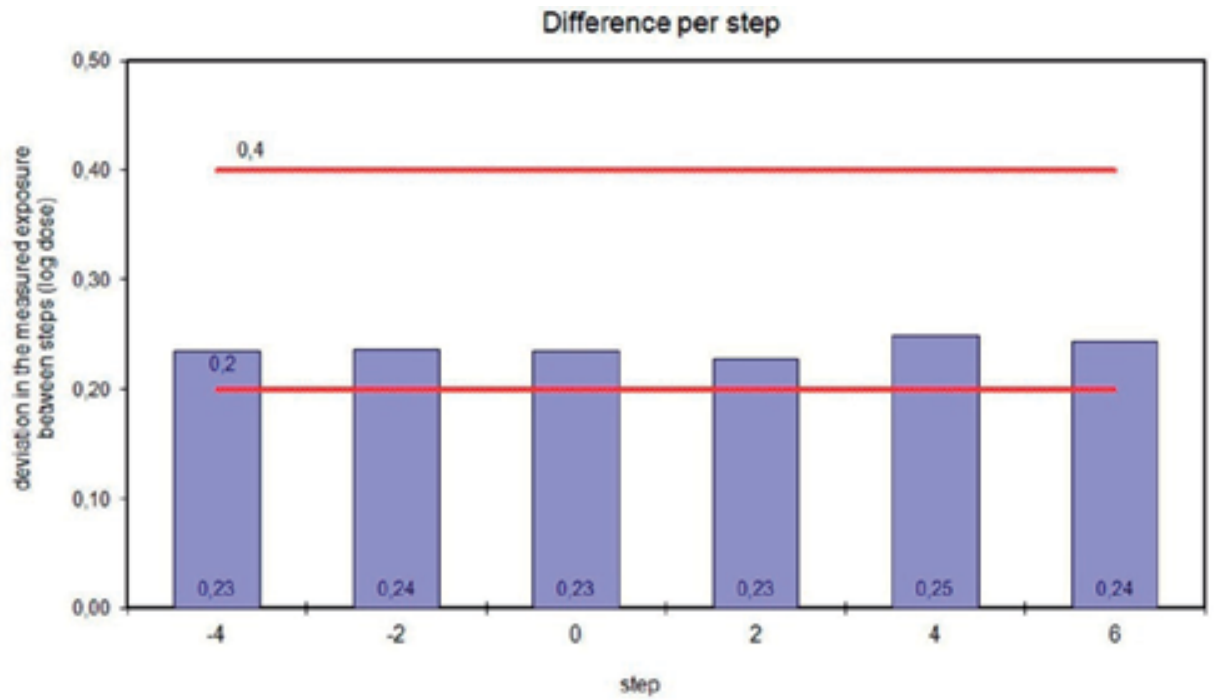

(a)

Difference per step (Dose vs steps)

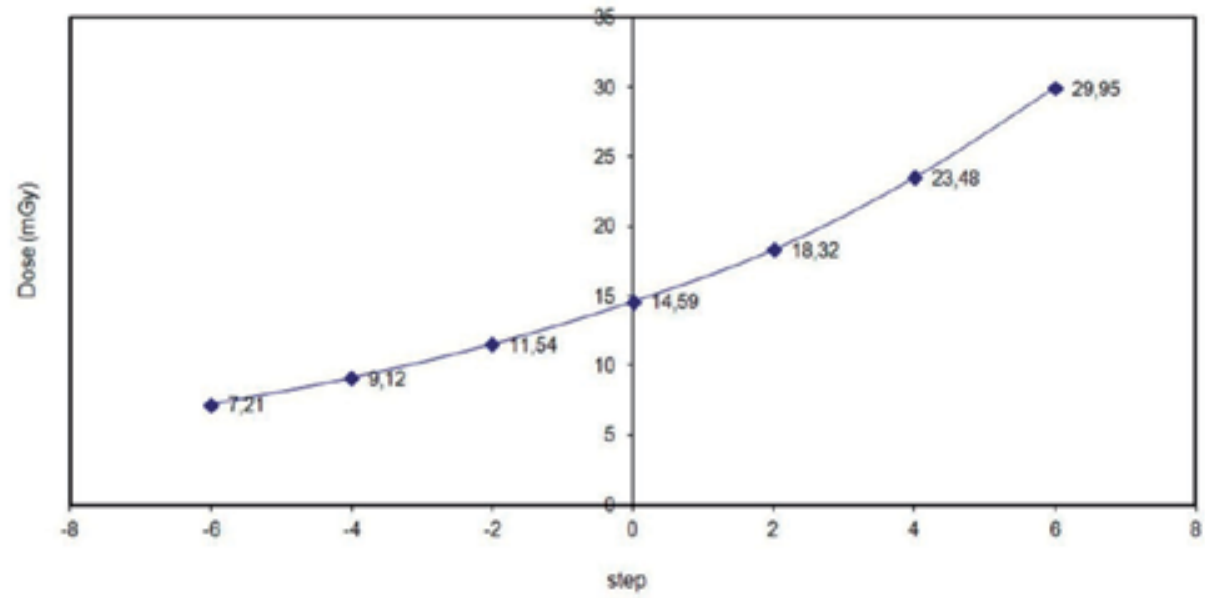

(b)

Figure 6. Results of difference per step test: (a) logarithm of the dose, red lines representing the limit values $(0.2-0.4$ per step); (b) the dose (mGy) per step. 


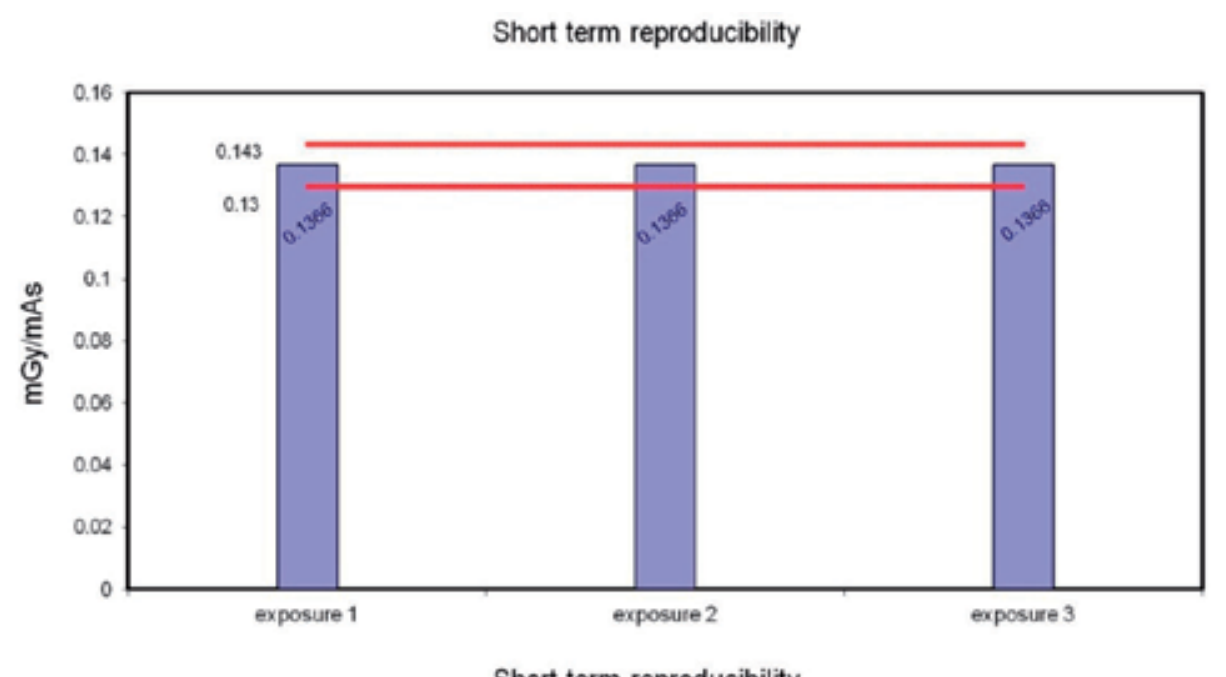

(a)

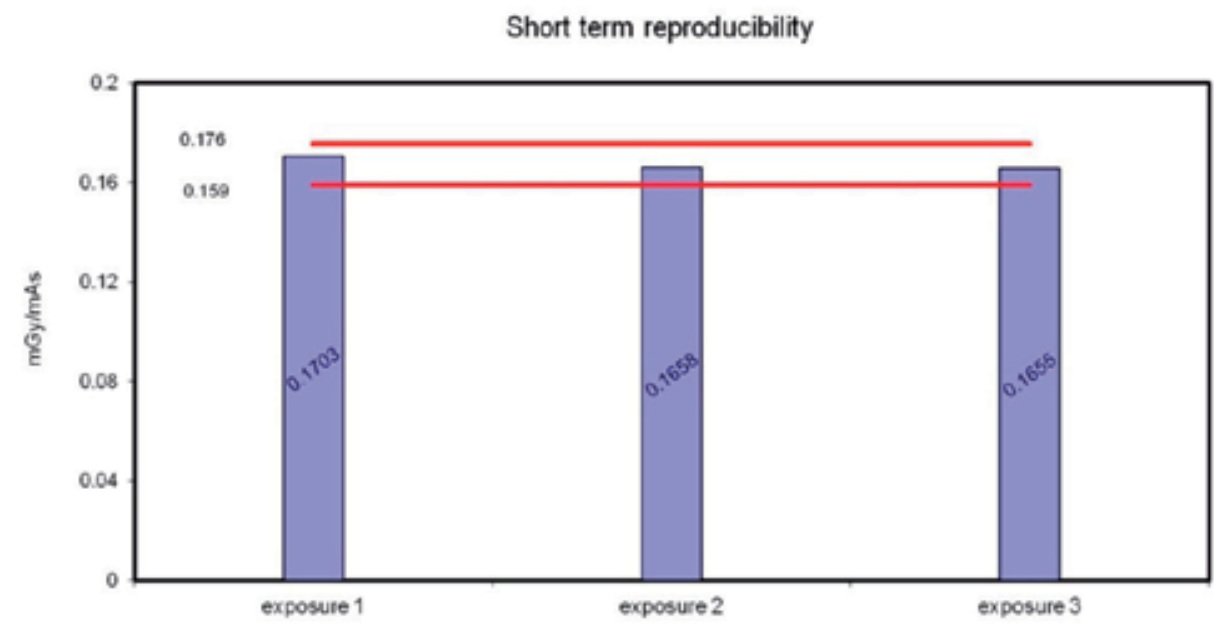

Figure 7. Results of short term reproducibility test, dose to tube load ratio (mGy/mAs) for three different exposures : (a) digital, (b) analogue mammograph;

\section{Author details}

Barbara Testagrossa ${ }^{1}$, Giuseppe Acri ${ }^{1}$, Federica Causa ${ }^{1}$, Raffaele Novario ${ }^{2}$, Maria Giulia Tripepi ${ }^{1}$ and Giuseppe Vermiglio ${ }^{1^{*}}$

*Address all correspondence to: vermigli@unime.it

1 Environmental, Healh, Social and Industrial Department - University of Messina, Italy

2 Department of Biotechnologies and Life Sciences- University of Insubria, Italy 


\section{References}

[1] Shah, A. J., Wang, J., Yamada, T., \& Fajardo, L. L. (2003). Digital Mammography: A Review of Technical Development and Clinical Applications. Clinical Breast Cancer, $4(1), 63-70$.

[2] Feig, S. A. (1988). Decreased breast cancer mortality through mammographic screening: results of clinical trials. Radiology, 167, 659-665.

[3] Skaane, P., \& Skjennald, A. (2004). Screen-film mammography versus full-filled digital mammography with soft-copy reading: screening program. The Oslo II study. Radiology, 232(1), 197-204.

[4] Huda, W., Sajewicz, A. M., \& Ogden, K. M. (2003). Experimental investigation of the dose and image quality characteristics of a digital imaging system. Medical Physics, 30(3), 442-448.

[5] Law, J. (2006). The development of mammography. Physics in Medicine and Biology, R155-R167.

[6] Noel, A., \& Thibauld, F. (2004). Digital detectors for mammography: the technical challenges. European Radiology, 14, 1990-1998.

[7] James, J. J. (2004). The current status of digital mammography. Clinical Radiology, 59, 1-10.

[8] Maidment, A. D. A. (2003). Digital mammography. Seminars in Roentgenology, 38(3), 216-230.

[9] Perry, N., Broeders, M., de Wolf, C., Törnberg, S., Holland, R., \& von Karsa, L., Ed. (2006). European guidelines for quality assurance in breast cancer screening and diagnosis ( $4^{\text {th }}$ Edition), Luxembourg, Office for Official Publications of the European Communities.

[10] Pisano, E. D., Cole, E. B., Hemminger, B. M., Yaffe, M. J., Aylward, S. R., Maidment, A. D. A., Johnston, E., Williams, M. B., Niklason, L. T., Conant, E. F., Fayardo, L. L., Kopans, D. B., Brown, M. E., \& Pizer, S. M. (2000). Image processing algorithms for digital mammography: a pictorial essay. Radio Graphics, 20, 1479-1491.

[11] Muller, S. (1999). Full-field digital mammography designed as a complete system. European Journal of Radiology, 31, 25-34.

[12] Feig, S. A., \& Yaffe, M. J. (1996). Current status of digital mammography. Seminars in Ultrasound, CT, and MRI, 17(5), 424-443.

[13] Cherie, M. Kuzmiak. (2012). Digital Mammography, Imaging of the Breast- Technical Aspects and Clinical Implication, Laszlo Tabar (Ed.), 978-9-53510-284-7, InTech, Available from, http://www.intechopen.com/books/imaging-of-the-breast-technical-aspectsand-clinical-implication/digital-mammography-chapteraccessed 5 June 2012. 
[14] Feig, S. A., \& Yaffe, M. J. (1998). Digital mammography. Radio Graphics, 18, 893-901.

[15] Screen-Film Radiography II. (2012). http://myweb.dal.ca/halem/phyc2250/09__Screen-Film_Radiography_II.pdf, accessed 25 June 2012.

[16] Pisano, E. D., \& Yaffe, M. J. (2005). State of the art: Digital Mammography. Radiology, 234(2), 353-362.

[17] Berns, E. A., Hendrick, R. E., Solari, M., Barke, L., Reddy, D., Wolfman, J., Segal, L., De Leon, P., Benjamin, S., \& Willis, L. (2006). Digital and screen-film mammography: comparison of image acquisition and interpretation times. American Journal of Roentgenology, 187, 38-41.

[18] Yaffe, in., Haus, A., \& Yaffe, M. J., Eds. (1992). Syllabus of Categorial Course on Technical Aspects of Mammography, Radiological Society of North America, Oak Book, IL.

[19] Säbel, M., \& Aichinger, H. (1996). Recent development in breast imaging. Physics in Medicine and Biology, 41, 315-368.

[20] Smith, A. P. (2003). Fundamentals of Digital Mammography: Physics, Technology and Practical Consideration. Radiology Management, 25(5), 18-24, 26-31.

[21] Rampado, O., Isoardi, P., \& Ropolo, R. (2006). Quantitative assessment of computed radiography quality control parameters. Physics in Medicine and Biology, 51, 1577-1593.

[22] Panagiotakis, G. (2012). Mammographic detectors. PhD Thesis, University of Patras, http://www.hep.upatras.gr/class/download/bio_sim_eik/mammographic_detectors.pdf, accessed 5 June 2012.

[23] Kruger, D. G., Abreu, C. C., Hendee, E. G., Kocharian, A., Peppler, W. W., Mistretta, C. A., \& Mac Donald, C. A. (1996). Imaging Characteristics of x-ray capillary optics in digital mammography. Medical Physics, 23(2), 187-196.

[24] Pisano, E. D., Yaffe, M. J., \& Kuzmiak, C. M. (2004). Digital mammography, Lippencott, Williams \& Wilkins, Philadelphia.

[25] Yaffe, M. J., \& Rowlands, J. A. (1998). X-ray detectors for digital radiography. Physics in Medicine and Biology, 42, 1-39.

[26] Kasap, S. O., \& Rowlands, J. A. (2000). X-ray photoconductors and stabilized a-Se for direct conversion digital flat-panel X-ray image detectors. Journal of Materials Science: Materials in Electronics, 11, 179-198.

[27] Samei, E., \& Ravin, C. E. (2008, March 12-13, 2008). Washington DC, USA. Assuring image quality for classification of digital chest radiographs: conference proceedings, NIOSH Scientific Workshop.

[28] Ranger, N. T., Samei, E., Dobbins, J. T., I. I. I., \& Ravin, C. E. (2007). Assessment of Detective Quantum Efficiency: intercomparison of a recently introduced international standard with prior methods. Radiology, 243(3), 785-795. 
[29] Vermiglio, G., Tripepi, M. G., Mannino, G., Sansotta, C., \& Testagrossa, B. (2005, 14-17 June 2005). Prove periodiche di funzionalità in mammografia mediante misure di esposizione. Verona, Italy. In: Polimetrica S.a.s. (ed.) Proceedings of $4^{\circ}$ Congresso $\mathrm{Na}$ zionale AIFM, 672-675.

[30] Testagrossa, B., Sansotta, C., Acri, G., Tripepi, M. G., \& Vermiglio, G. (2007, October 1-3 2007). Vasto Marina (CH), Paper presented at XXXIV Convegno AIRP “Sicurezza e qualità in radioprotezione". Fantoccio multiuso per controlli di qualità in mammografia tradizionale e digitale: conference proceedings.

[31] Controllo di qualità in mammografia PHAN-EX, Operating handbook.

[32] Italian Regulation 26 Maggio 2000, n. 187. Attuazione della direttiva 97/43/Euratom in materia di protezione sanitaria delle persone contro i pericoli delle radiazioni ionizzanti connesse ad esposizioni mediche. Gazzetta Ufficiale n. 157 del 7 luglio 2000. Suppl. Ordinario n. 105. http://www.camera.it/parlam/leggi/deleghe/00187dl.htm (accessed 25 June 2012).

[33] Linee Guida ANPEQ-ISPESL relative al controllo sugli impianti radiologici e accessori. (2004). http://www.anpeq.it/download/app_radiologiche.pdf, accessed 27 June 2012.

[34] Corrado, F., Gennaro, G., Golinelli, P., \& Rossetti, V. (2004). Protocollo Italiano per il controllo di qualità degli aspetti fisici e tecnici in mammografia. AIFM Report, http:// www.fisicamedica.it/aifm/report/2004_n1_ReportAIFM.pdf, accessed 27 June 2012.

[35] Norma CEI 62-27 ed.2 (CEI EN 60601-2-7). (1999). Apparecchi elettromedicali. Parte 2: Norme particolari per la sicurezza di generatori ad alta tensione dei generatori radiologici per diagnostica.

[36] Castelli, E. (2012). Un sistema di rivelazione per mammografia digitale con luce di sincrotrone. PhD Thesis, University of Trieste, http://www.infn.it/thesis/PDF/256-Bergamaschi-laurea.pdf, accessed 25 June 2012. 


\section{Section 5}

\section{Quality Control in Energy}



Chapter 15

\title{
The Quality Management of The R\&D in High Energy Physics Detector
}

\author{
Xuemin Zhu and Sen Qian \\ Additional information is available at the end of the chapter \\ http://dx.doi.org/10.5772/51434
}

\section{Introduction}

Particle physics, also recognized as high energy physics, is a basic subject focusing on the research of the elementary elements of materials and their mutual actions. One distinguished characteristic of particle physics study is that the experimental equipments involved are always huge and special ones. Therefore, big science projects, including the R\&D of large detectors, are usually required in high energy physics experiments. Those projects are complicated systematic engineering, involving many front and technology fields. It is impossible for a single institute to finish those large projects by its own. Cooperation among different institutes or organizations is necessary for big science projects in particle physics, especially international ones.

\section{1. The Institute of High Energy Physics [1]}

The Institute of High Energy Physics (IHEP) is the biggest and comprehensive fundamental research center in Chinese Academy of Science. The major research fields of IHEP are particle physics, accelerator physics and technologies, radiation technologies and application, Particle physics experiments and Accelerator physics and technology are two of the leading research areas. The main research facilities at IHEP include Beijing Electron Positron Collider (BEPC) and Beijing Spectrometer (BES), DayaBay Neutrino Experiment, Chinese Spallation Neutron Source, etc. IHEP has extensive cooperation with all high energy physics laboratories and participates in many important particle physics experiments in the world.

\subsection{The Beijing Electron Positron Collider [2] and the Beijing Spectrometer [3]}

The Beijing Electron Positron Collider (BEPC) consists of the injector, the storage ring, the transportation line, the Beijing Spectrometer (BES), the Beijing Synchrotron Radiation Facili- 
ty (BSRF) and the computer center. Beijing Spectrometer (BES) is a general purpose magnetic spectrometer in the South IP of the storage ring. The general layout of the BEPC is shown in Fig.1.

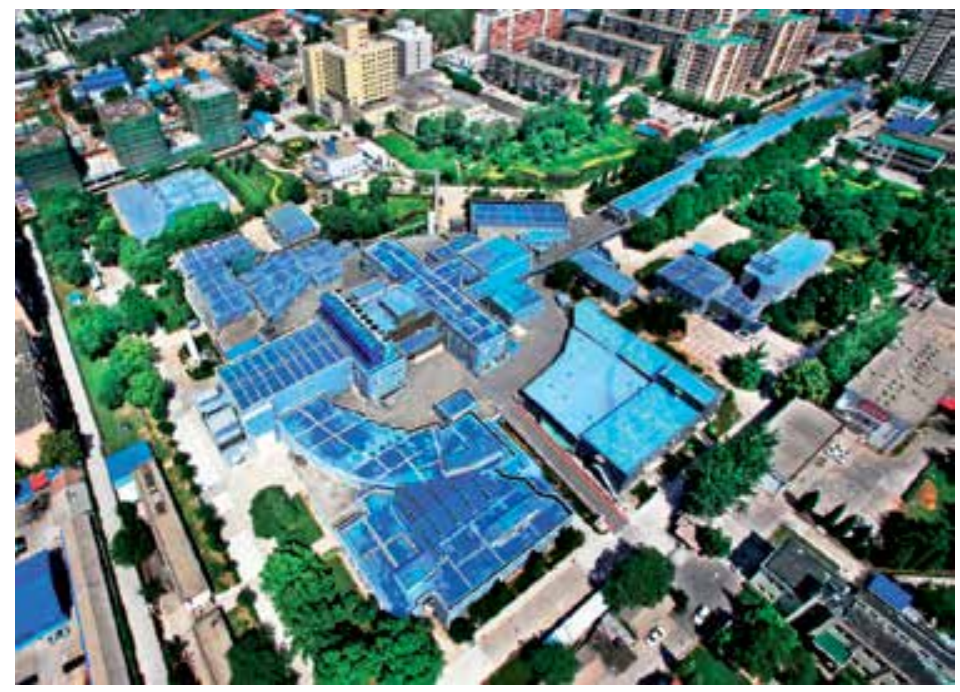

Figure 1. The airscape of the BEPC.

BEPC started construction in 1984 and the first electron-proton collider was produced in Oct. 1988. BEPCII was installed in 2003 and finished five years later in 2009. IHEP establishes comprehensive and long-term cooperation with high energy laboratories and universities all over the world, especially in USA, Japan and Europe. With the international cooperation, IHEP have gained huge success in 30 years. For example, IHEP took part in the research of CMS and ATLAS detectors of Large Hadron Collider (LHC), which is the world's largest, highest-energy particle accelerator and the collider at the beginning of 21 centuries, built by CERN [4]. BESIII is also organized by IHEP and participated by 51 institutions and universities around the world, 34 from Asian, 12 from Europe and 5 from USA.

\subsection{The Daya Bay Neutrino Experiment [5]}

The Daya Bay Neutrino Experiment is a neutrino-oscillation experiment designed to measure the mixing angle q13 using anti-neutrinos produced by the reactors of the Daya Bay $\mathrm{Nu}^{-}$ clear Power Plant (NPP) and the Ling Ao NPP.

The Daya Bay Neutrino Experiment is a major international joint research program, mainly organized by China working closely with researchers from other countries. In terms of both money and people, it is among the largest scientific collaborations between US and China. More than 200 scientists from China, include Hong Kong and Taiwan, the US, Russia, the Czech Republic are involved in the Daya Bay experiment. During the cooperation, China is in change of the laboratory construction, R\&D of Anti-neutrino detector (AD), Gd-loaded 
Liquid Scintillator, Muon Veto Detector, Readout Electronic and Data acquisition system (DAQ) etc. While America is in responsible of the construction of water Cherenkov detector and so on.

Scientists from the Chinese Academy of Sciences (CAS) and the U.S.-based Brookhaven National Laboratory and the Lawrence Berkeley National Laboratory will participate in the underground experiment. An international funding commission comes into existence in the funding agency to discuss fee issues and instruct the experiment process and fee management through experimental supervision organization. The project management of the Daya Bay Neutrino Experiment adopts the advanced and mature modern management idea used for managing large international joint project and big science experimental research project. An international cooperation group is built and management rules are made. Besides, a cooperation group commission is founded, during which executive board and spokesperson is elected for overall supervision of the whole project. The Daya Bay Neutrino Experiment is initiated in 2007 and finished in 2012.

\subsection{Chinese Spallation Neutron Source [6]}

Chinese Spallation Neutron Source (CSNS) is designed to build a device with the power of proton beam reaching up to $100 \mathrm{~kW}$ effective and the flux of pulsed neutrons coming out top in the world, along with other three spallation neutron sources built in America, Japan and British. CSNS is also a large cooperative project, supported by Chinese Academy of Sciences and Guangdong government. The normal operation for uses is foreseen in 2018. IHEP is the main construction institution in the project with the Institute of Physics Chinese Academy of Sciences as the co-operation unit. The construction team bring together three generations of outstanding scientific and technical researchers in China. An international CSNS neutron technology advisory committee is set up for reviewing the key experimental work. The experts of the advisory committee are from well-known laboratories in America, Japan, Germany, Australia and other countries.

\section{Introduction of Quality Management of Scientific Projects in IHEP}

During the process of big science project and research, IHEP has significant advantages in accelerator physics and technology, human resources, international cooperation and academic exchange. IHEP owns mature model and advanced experience in the quality management of scientific projects.

\subsection{Project Management System}

Before 2011, the project manager is responsible for the big science project management in IHEP. International cooperation group is formed and fees are under the sponsors' supervision and review. There is a perfect project management system, though without quality management system meeting international standards. 


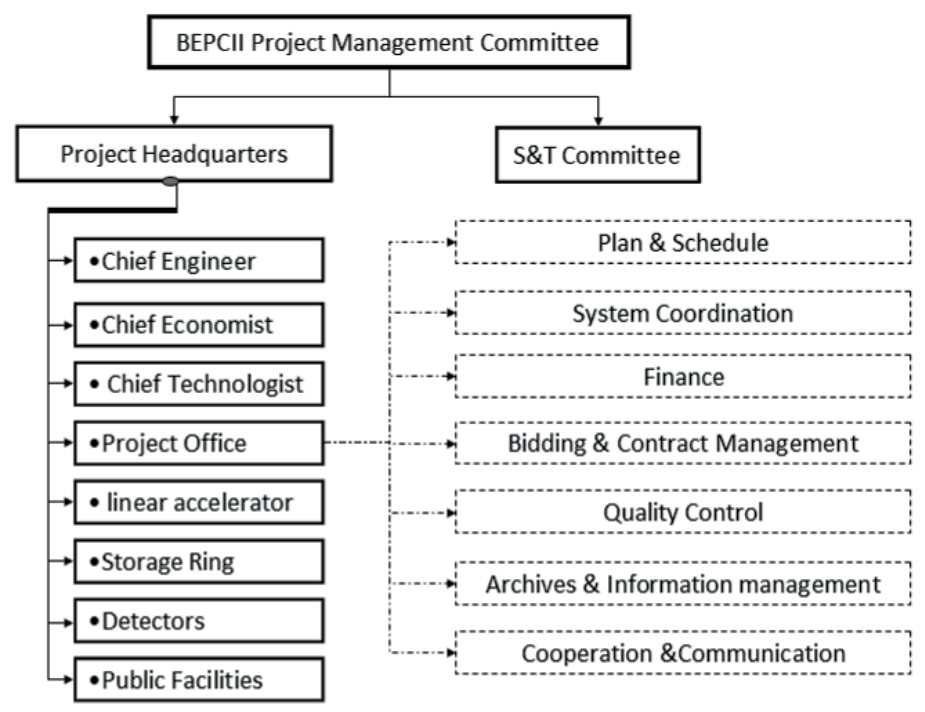

Figure 2. The Organization Chart of the BEPCII Project Management.

In the project management system shown in Fig.2, special-purpose management mechanism such as fund, purchasing, quality, safety and archive is established, with clear responsibilities and authorities. Besides, the internal communication mechanism and interface management mechanism are also set up. CPM Plan is adopted for fund and schedule management. To ensure the quality of the project, during the design and development process, experts are always invited for evaluation. And an international council committee is asked for review in terms of major international cooperation projects.

In fact, the requirements of the project management system have already displayed in the ISO 9001 quality management system. Though without a systematic quality manual and standards and lack of resource, purchasing and archive management. In the project management system, quality management is more focused on the management of various test guidelines and processing of key parts (including outsourced progress)

\subsection{Quality Management Systems}

The BEPCII project headquarters has placed great important on the quality management and published "BEPCII project management file" in 2002. In the file, responsibilities and rights of personnel, fund management, file number, document signing and alteration, early stages management, bidding and purchasing are described in detail.

At the beginning of 2005, during the construction of BEPCII project, the headquarters built a quality management system according to GB/T19001-2000(idt ISO9001:2000). Although the system doesn't get a national certification, it is completely in accordance with standards of quality management system requirements and it has played a very good effect. In 2009, 
BEPCII completed the construction task successfully by time, with high quality and budget under control.

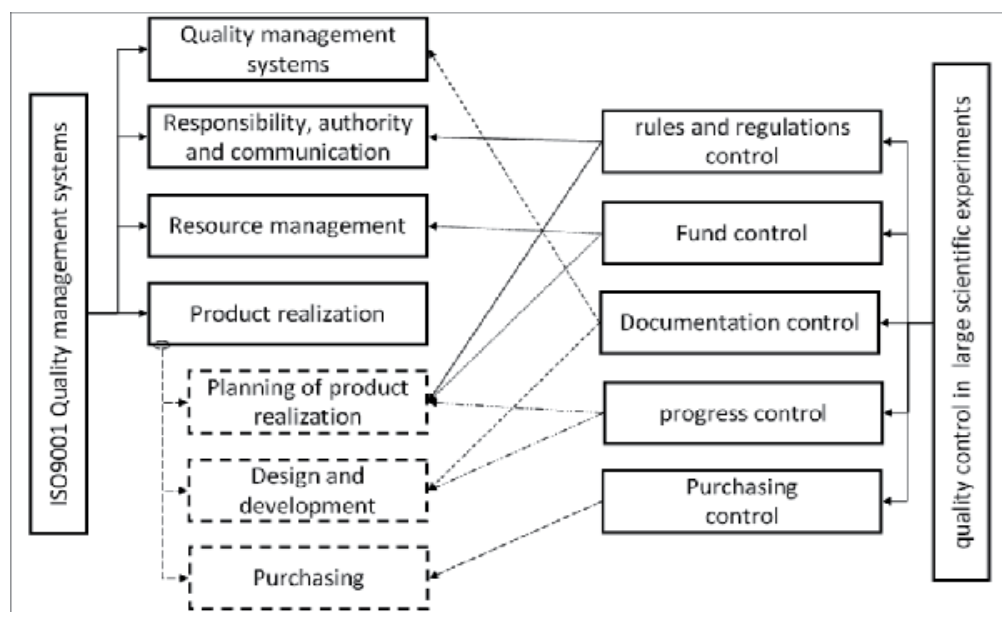

Figure 3. The Relationship between the quality control system in large scientific experiments and ISO9001 Quality management systems.

As described in Fig.3, The quality control in large scientific experiments corresponds with ISO9001 Quality management systems, which is classified according to the production. While, the quality control in large scientific experiments is classified according to the type of different work. The ISO9001 Quality management system is widely adopted by corporations all over the word and it's more normative.

In 2011, IHEP passed a national quality management certification system: GB/ T19001-2008(idt ISO90012008). After two years' development of quality management system from its very beginning to being passed, it has confirmed that IHEP has the ability to produce scientific production meeting requirements.

The set up of quality management system makes the project management procedure standard, and promotes the overall management level in IHEP. The clients' needs are fully met and the quality management of IHEP joined the line of international standard management. The role played by quality management in the scientific research, especially in the big science project, is invaluable and imponderable.

\section{Quality management in R\&D of BESIII detector}

The project of BESIII detector began its research and development, according to the scientific project management system and quality management system, like other big science projects. 


\subsection{Mechanism Management}

BESIII detector R\&D is part of BEPCII project. So the quality management of the detector research is responsible by the project director. As a whole, BESIII carries out the management system of BEPCII project headquarters strictly and makes some special mechanism to form a mechanism with a clear hierarchy. Quality technician are employed in the project.

BESIII detector R\&D project has outlined the responsibilities and rights of each person in charge with an appropriate staffing in the organization. The communication methods of the total and sub system and record control requirements are defined.

The director in charge of sub system is responsible for the implementation of the BESIII research plan, management, arrangement of related resources and coordination with scientific and technical issues. Each division leading person is specifically responsible for the respective task implementation plan. Members in the project cooperate with each other closely at reaching difficult goals. The whole project has the characteristic of unified task, defined responsibilities, reasonable arrangement and integrated resources.

The high energy physics experiment is a complex project, and the communication in different study cells seems more important. The Task Control Form is widely used in study works, and the forms are preserved and archived as records of the system.

\begin{tabular}{|c|c|c|c|c|}
\hline Subject & & & & \\
\hline \multicolumn{5}{|l|}{ Send to } \\
\hline From & & & Date & \\
\hline Serial No. & & & Pages & \\
\hline \multicolumn{5}{|l|}{ Attached } \\
\hline \multicolumn{5}{|l|}{ C.C. } \\
\hline \multicolumn{5}{|l|}{ Content } \\
\hline \multicolumn{5}{|l|}{ Jointly Sign } \\
\hline validation & Prepared by & Checked by & Examined by & Approved by \\
\hline \multicolumn{5}{|l|}{ Signature } \\
\hline Date & & & & \\
\hline
\end{tabular}

Figure 4. The task control form used by different teams.

Researchers in the project communicate with each other in time and have a regular meeting each or twice a week, to make sure the project is under schedule control and discuss some technical problems. Meeting minutes are kept as a reference. Sub-system will report the progress of the project and accept an inspection and evaluation regularly. 


\subsection{Fund Management}

Fund management is important for the whole management of scientific project. Appropriate fund use a basis for carrying out any high energy physics experiment smoothly. As for the $R \& D$ of BESIII detector, the experiment design and development planning will affect the rationality of the budget and fund use directly. They are also the important contents in the requirements of the quality management system

Funds come from Chinese Academy Sciences (CAS) allocation and self-provided funds in the BESIII project. At the end of the year, expenses are counted and reported to CAS and the project will receive examination and evaluation.

\subsection{Control of documents and records}

Control of documents and records is critical whether for scientific project management or for quality management. For high energy physic experiments, large and complicated equipments are usually involved. During the project design and scheme phase, rules for documents and records reserved need to be made clearly and principles for numbering and signing the documents and records need to be described specifically.

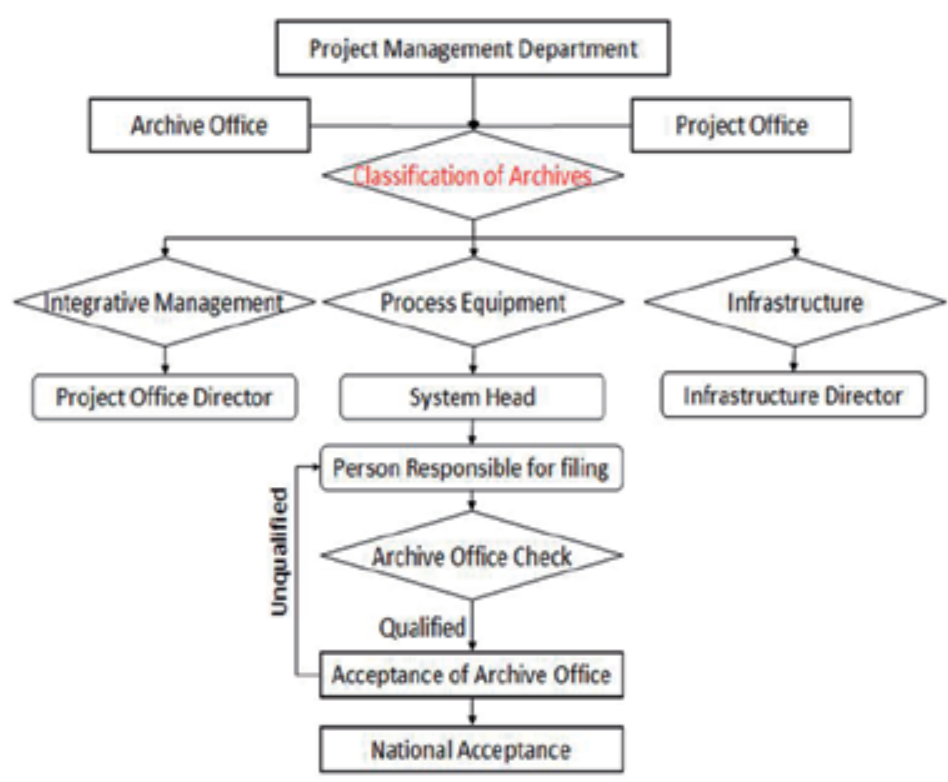

Figure 5. The workflow of archive management.

BEPCII project builds up a special mechanism of file control. Because BESIII is part of the whole project, the rules of document management are in accordance with the requirements of BEPCII. Documents and records need to be signed according to the regulations, in accord- 
ance with the whole project and effective as well. Documents and records need to be preserved and archived on a regular basis.

There are several characteristics in archive management work, especially for the high energy experiments. Firstly, this work must be arranged by the project management department at the beginning of the project. Secondly, the document and records which need to be preserved must be clearly described and the responsibility should be defined at the first time. Thirdly, the archive office, the project office and each member working for the project should cooperate to get the work done quickly and perfectly.

All the quality documents of the whole process of each single detector, from design, research, test, and acceptance are preserved according to the regulations. Technical specifications, interface of different tasks, diagrams, test reports are archived as written documents. Regular meeting minutes are kept also as archives. Those Documents and records can be used to track and follow the quality of the product in the whole process.

The running cycle of big science project, just like its construction cycle, is as long as to last more than ten years. Therefore, control of documents and records is very essential for the running and maintenance of the big science project, as an important prop and support.

\subsection{Schedule Management}

BESIII project has followed Critical Path Method (CPM) to control the schedule of the whole project. The plan in the CPM is in detail and convenient for check. It is easy for revision according to the actual process and make sure it is updated in time within the system.

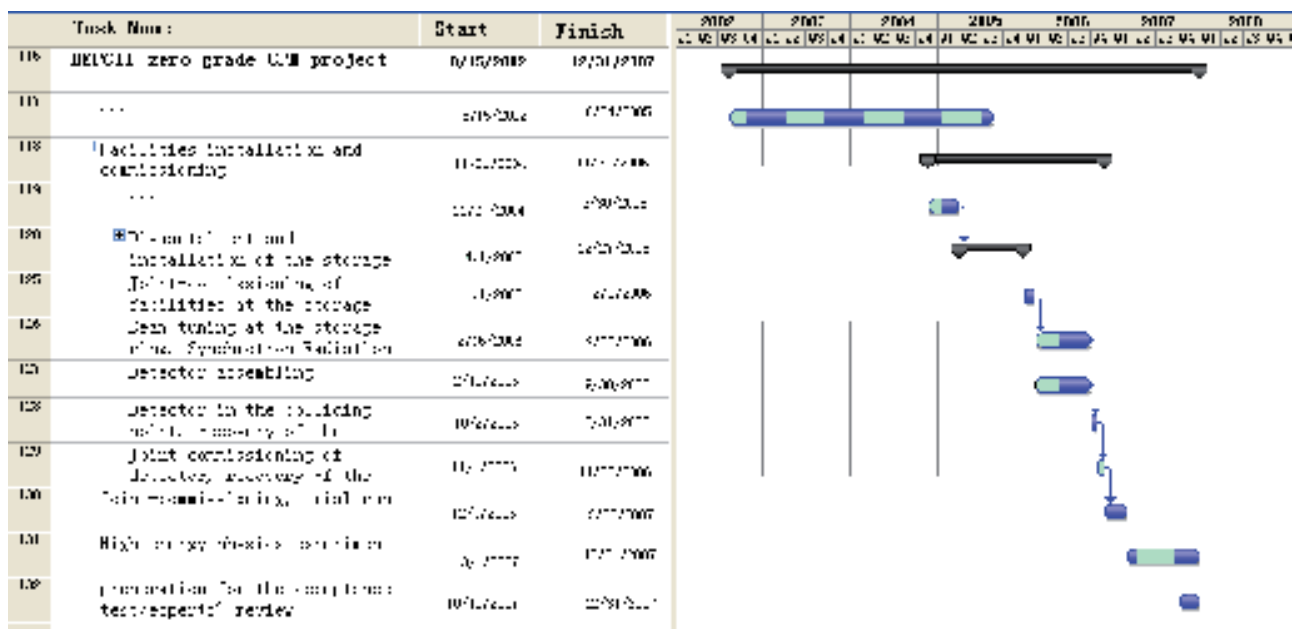

Figure 6. BEPCII zero-grade CPM project (partly,2002).

In order to give a better control of the schedule, CPM is classified. Any sub-system could make its own play and updates in time following the step of the total plan. Therefore inter communication plays an important role in the schedule management. In a word, CPM is a 
further refinement of the time arrangement of the project design report and makes the management of the project construction effective.

The CPM project is highly in accord with the practical progressand BEPCII zero-grade CPM project is modified frequently. The BEPCII project was finished in 2008 and was finally checked in 2009.

\subsection{Purchasing Management}

The R\&D of large detectors is involved with bulk purchase. In the BESIII project, purchasing management rules are made according to the relevant laws and regulations on acquisition. Purchasing and approval process are defined clearly. Bidding is strictly adopted in the project to save research money. An appropriate regulation in the purchasing process is a guarantee for carrying out the project under the budget..

Abroad purchase has a long life cycle, heads of procurement need to do significant preparatory work in advance, and the heads should be quite familiar with the procurement procedures in order to complete the purchase in time. The purchasing department published the flowchart to facilitate the work.

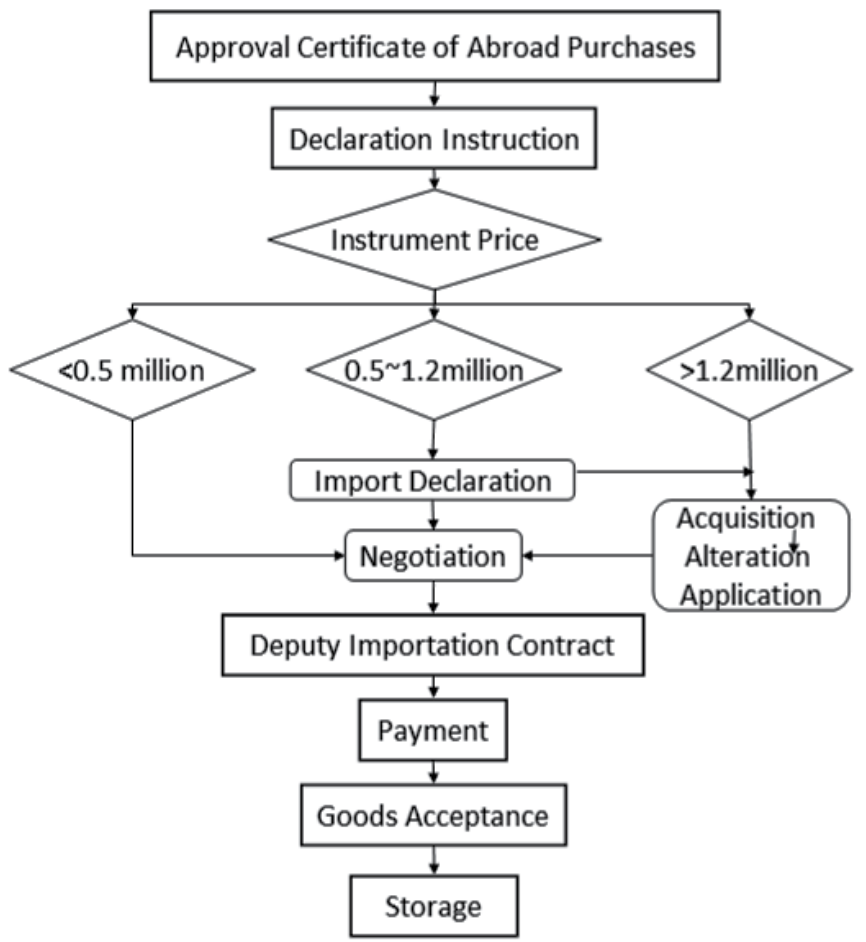

Figure 7. Abroad purchase flowchart. 


\section{BESIII-MUC Quality Management in R\&D of BESIII-MUC Detector}

\subsection{Introduction of BESIII Detector and MUC Detector}

The Beijing Spectrometer (BESIII) is designed to measure the properties of the particles produced in the collisions of electrons and positrons at BEPCII. The physics goal of the BESIII experiment is to conduct high statistics and highly precise studies on a number of physics topics in this energy region, including light hadron spectroscopy, charmonium spectra, charm meson decay properties, QCD, tau physics, rare decays, search for glueballs and other non-pure quark states [3].

The BESIII detector will consist of a $1 \mathrm{~T}$ superconducting solenoid magnet, a high precision main drift chamber (MDC), Time-Of-Flight counters (TOF), a CsI crystal Electromagnetic Calorimeter (EMC) and a muon identifier chamber (MUC) that is integrated in the iron magnetic field return yoke [7]. The muon identifier is the outer most subsystem of the BESIII detector [8], which is constructed by resistive plate chambers (RPCs, shown in Fig.8.a). 962 RPC are used in the whole MUC detector, which consists of 136 RPC superlayer modules (SM, shown in Fig.8.b). And the Fig.8.c shows the status of the MUC detector when it was finished it's barrel part assemblage. The Fig.8.d shown the designed construct of the BESIII MUC detector with the endcap and barrel parts.
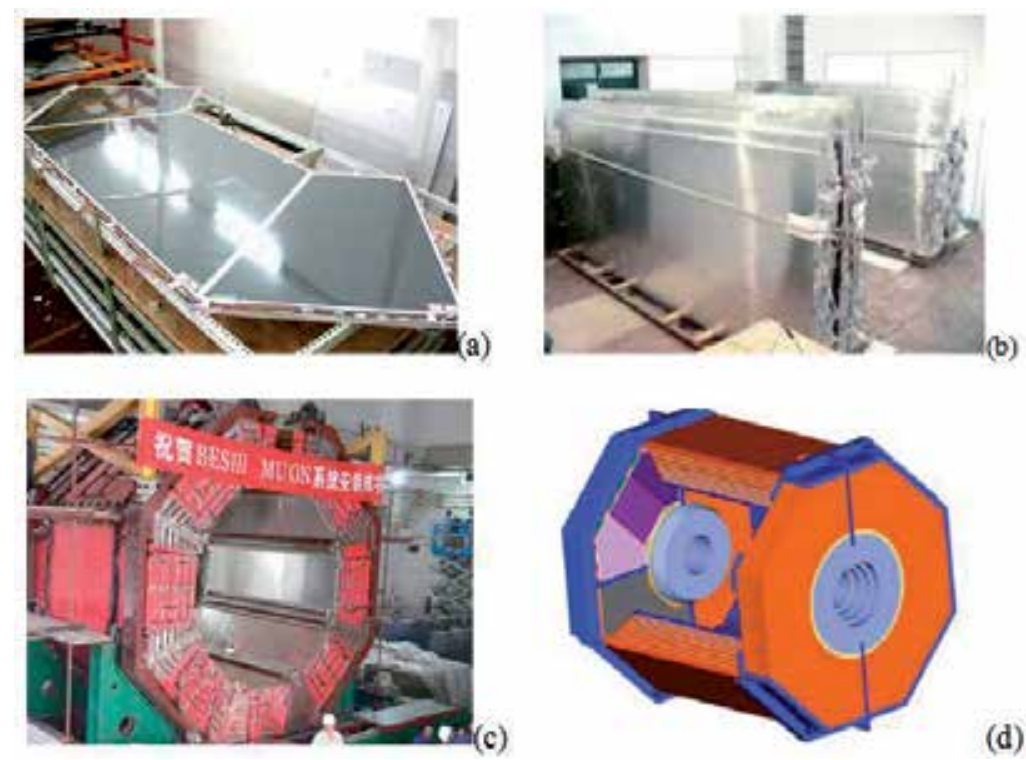

(d)

Figure 8. a). The RPC moduls, (b). The Suprlayer Modul, (c). The overview the barrel part of the MUC detector after it's assemblage, (d) The construct of the BESIII MUC detector. 


\subsection{Quality Management in the R\&D of BESIII-MUC Detector}

The whole process of R\&D of MUC detector include the design of the basic unit RPC, properties investigation, bulk production, SM design; design of MUC detector, installation debugging, running and maintenance.

Throughout the research process, the project director managed the project scientifically and effectively, with each research aspect considered carefully, comprehensively and deeply, and made some achievements. From the pre-research in 2003 to the formal data collection in 2009 , more than 30 papers have been published by the research group of MUC, covering the whole research process.

7 papers have been published in NIMA, as follows:

1. A new surface treatment for the prototype RPCs[9],

2. Cosmic ray test results on resistive plate chamber for the BESIII experiments [10]

3. The Design and Mass Production on RPC for the BESIII Experiment [11]

4. A monitor for the composition of the gas mixture of BESIII muon chambers [12]

5. First results of the RPC commissioning at BESIII [13]

6. The BESIII Muon Identification System [14]

7. An underground cosmic-ray detector made of RPC [15]

8 papers have been published in Chinese Physics $C$, as follows:

1. Cosmic Ray Test Station for BES® RPC [16]

2. Research and Development of Large Area Resistive Plate Chamber [17]

3. A Study of RPC Gas Composition using Daya Bay RPCs [18]

4. Quality control and database on RPC for the BES๑ experiment [19]

5. Test of BES๑ RPC in the avalanche mode [20]

6. Performance Study of RPC Prototypes for the BES๑ Muon Detector [21]

7. Study of the RPC-Gd as thremal neutron detector [22]

\begin{tabular}{cccccc}
\hline & Design & $\begin{array}{c}\text { Performance } \\
\text { Test }\end{array}$ & $\begin{array}{c}\text { Mass } \\
\text { Production }\end{array}$ & $\begin{array}{c}\text { Research } \\
\text { Work }\end{array}$ & Application \\
\hline RPC & $1 \mathrm{~b}$ & 2 acef & $3 \mathrm{~d}$ & 4 & $7 \mathrm{~g}$ \\
\hline SM & & & 6 & & \\
\hline MUC & & & 6 & 5
\end{tabular}

Table 1. The analysis of the manuscripts published by MUC group. 
As shown in Table 1, it is not difficult to come into conclusion that the whole R\&D of MUC detector applied scientific project quality management, which promotes the research work. In the phase of initial RPC research, the key point is on the study of the detector's performance test. It is the phase for building a standard quality management. After the acceptance of $\mathrm{RPC}$ and project review, mass production and SM reassembling come into being. In this phase, scientific quality control and management play a key role.

A perfect quality tracking system is established in each session, from the production and test of RPC, assembling and test of modules, to the installation and debugging of MUC detector, to ensure the supervision of the performance of detector is plausible.

Especially for the mass production of RPC and SM, before research and test, a database is built for storage related data and affording date support for quality control and final running \& maintenance.

\subsection{Summary}

All the requirements such as verification, validation, monitoring, measurement, inspection and test activities specific to the detector are described in the design report of the detector in detail. The report plays the same role as in making a particular quality control plan.

\begin{tabular}{|c|c|c|c|c|c|c|}
\hline$\square \mathbf{\square}$ & Design & Prototyp & $\begin{array}{c}\text { Mass } \\
\text { e productio } \\
n\end{array}$ & $\begin{array}{c}\text { Assemblag } \\
\text { e }\end{array}$ & Debug & Running \\
\hline $\begin{array}{c}\text { critical } \\
\text { characteristic }\end{array}$ & - & $\square$ & & & & \\
\hline $\begin{array}{c}\text { major } \\
\text { characteristic }\end{array}$ & - & $\mathbf{\square}$ & $\square$ & & & \\
\hline critical process & & घ & घ & & & \\
\hline article inspection & - & घ & घ & & & घ \\
\hline $\begin{array}{c}\text { quality } \\
\text { improvement }\end{array}$ & - & $\mathbf{\square}$ & & $\mathbf{\square}$ & & \\
\hline effectiveness & & & घ & घ & & \\
\hline traceability & & & - & - & - & - \\
\hline preventive action & & $\square$ & $\square$ & & घ & $\mathbf{\square}$ \\
\hline corrective action & & & & & घ & \\
\hline quality plan & & & $\square$ & - & & \\
\hline
\end{tabular}

Table 2. Quality management/control factor distribution of MUC detector.

The design report of the detector divide the R\&D process into several phases, including concept design, project design, sample trail-manufacture, product research and production, test, 
installation and debugging. In each phase, review and identification is defined. For important phases, such as aging test, assembly test and system test, detailed guidelines and instructor are written. As shown in Table 2, during the outsourcing process, key parts are defined, and acceptance rules are also clearly described. Control point is set up and design files are carried out strictly to ensure the product quality. More detailed could be found in table 2 for summary.

\section{Significance of Scientific Quality Management in Research}

\subsection{Promote Scientific Projects}

We could come into conclusion that scientific quality management can promote scientific projects to proceed successfully, in the following ways:

The schedule of the project could be arranged and controlled well, especially the adoption of CPM, which could provide a time map for the whole project. Throughout the four years' successful implementation and of BEPCII project, CPM plays an important role in the project acceptance in due. CPM was adjusted in time according to the project status, thus effective management and restriction was formed for all the related sub systems in the project.

The project has been implemented within the budget and cost was controlled. Purchasing procedures and approval process were strictly described, which played a role for the fair use of the fund.

Documents and records were kept in detail, as reference in the project to find the source of old problems and avoid new problems. Especially for those big scientific projects which will last more than ten years, files about interface management and quality management and various records are significant for the running and maintenance in the following work. They also act as important reference for the future project construction in high energy physics.

\subsection{Promote Scientific Research}

Scientific quality management could promote scientific research effectively. At the same time, as the development of scientific research, cooperation among researchers will be increased. It is good for the communication and exchange in the area of quality management and promotes the refining of the quality management system thus.

Experiences in big scientific project are good for the growth of young researchers. With participation in the R\&D of big science equipment under quality management system, researchers will learn how to organize and manage scientific programs or projects in future.

In an ongoing scientific project managed launched by IHEP, researchers are from participants in BESIII or DayaBay. Although it is non-international, at the beginning the project is managed as required in strict quality management, just like that in big science project. As the development of the project, communication and cooperation among other institutions 
both at home and abroad have increased. To coordinate the partnership among different organizations and unites, cooperation group is formed. As shown below, a formality management system and strictness organization is built, which lays a solid foundation for the sustainable development of cooperation group and joint research work in future, whose Organization Chart shown in fig.9 for example.

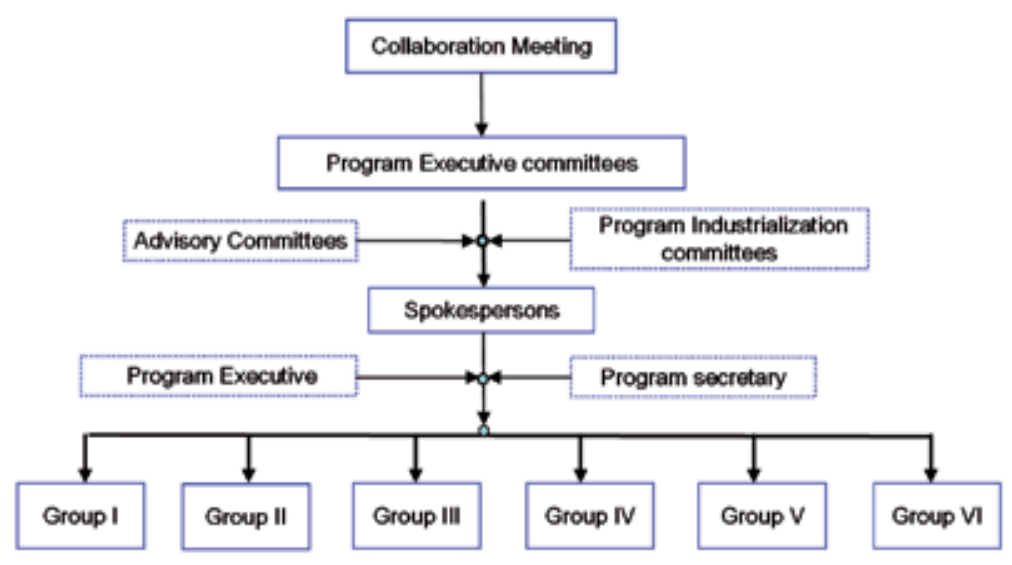

Figure 9. The organization of the BEPCII

\section{Conclusion}

Quality management plays a significant role both in project management and in the scientific research. With a scientific and comprehensive quality management system, big science project will be duly executed. The level of scientific projects will be greatly improved by the application and popularization of national and international quality standards.

\section{Acknowledgements}

In the process of writing this manuscript, we have received much understanding and support from many departments in our institute. We are particularly grateful to those staffs in the IHEP offices, archives, the purchasing department and the project teams for their strongly supported. Special thanks go to Prof. Zhao Jingwei for this support and encouragement to finish this work. 


\section{Author details}

Xuemin Zhu $^{1^{*}}$ and Sen Qian ${ }^{1^{*}}$

*Address all correspondence to: zhuxm@ihep.ac.cn

1 The Institute of High Energy Physics, Chinese Academy of Sciences, China

\section{References}

[1] The Institute of High Energy Physics. http://english.ihep.cas.cn/au/bi/.

[2] The BEPCII Project. http://english.ihep.cas.cn/rs/fs/bepc/index.html.

[3] The Beijing Spectrometer. http://bes3.ihep.ac.cn/orga/institute.htm.

[4] European Organization for Nuclear Research. http://public.web.cern.ch/public/.

[5] The Bay Reactor Neutrino Experiment. http://dayabay.ihep.ac.cn/twiki/bin/view/ Public/WebHome.

[6] China Spallation Neutron Source (CSNS). http://csns.ihep.ac.cn/english/index.htm.

[7] Tianchi, Z. (2010). Design and construction of the BESIII detector Nuclear. Nuclear Instruments and Methods in Physics Research A, 614, 345-399.

[8] Boxiang, Y. (2009). The construction of the BESIII experiment. Nuclear Instruments and Methods in Physics Research A, 598, 7-11.

[9] Jiawen, Z. (2005). A new surface treatment for the prototype RPCs. Nuclear Instruments and Methods in Physics Research A, 540(2005), 102-112.

[10] Jifeng, H. Cosmic ray test results on resistive plate chamber for the BESIII experiments. Cosmic ray test results on resistive plate chamber for the BESIII experiments.

[11] Jiawen, Z. (2007). The Design and Mass Production on RPC for the BESIII Experiment. Nuclear Instruments and Methods in Physics Research A, 580, 1250-1256.

[12] Sen, Q. (2008). A monitor for the composition of the gas mixture of BESIII muon chambers. Nuclear Instruments and Methods in Physics Research A, 595, 520-525.

[13] Yuguang, X. (2008). First results of the RPC commissioning at BESIII. Nuclear Instruments and Methods in Physics Research A, 595, 520-525.

[14] Sen, Q. (2010). The BESIII Muon Identification System. Nuclear Instruments and Methods in Physics Research A, 614, 196-205.

[15] Qingmin, Z. (2007). An underground cosmic-ray detector made of RPC. Nuclear Instruments and Methods in Physics Research A, 583, 278-284. 
[16] Qian, L. (2006). Cosmic Ray Test Station for BES॰ RPC. China Physics C (High Energy And Unclear Physics), 30(4).

[17] Jiawen, Z. (2003). Research and Development of Large Area Resistive Plate Chamber. China Physics C (High Energy And Unclear Physics), 27(7).

[18] Malie, H. (2010). Study of RPC gas composition using Daya Bay RPCs. China Physics C (High Energy And Unclear Physics), 34(8).

[19] Jifeng, H. (2008). Quality control and database on RPC for the BES॰ experiment. China Physics C (High Energy And Unclear Physics), 32(3).

[20] Jifeng, H. (2008). Test of BES॰ RPC in the avalanche mode. China Physics C (High Energy And Unclear Physics), 32(5).

[21] Yuguang, X. (2008). Performance Study of RPC Prototypes for the BES๑ Muon Detector. China Physics C (High Energy And Unclear Physics), 31(1).

[22] Sen, Q. (2009). Study of the RPC-Gd as thremal neutron detector. China Physics C (High Energy And Unclear Physics), 33(8). 
Quality Control in Cosmetics 



\title{
Cosmetics' Quality Control
}

\author{
Bruna Galdorfini Chiari, \\ Maria Gabriela José de Almeida, \\ Marcos Antonio Corrêa and Vera Lucia Borges Isaac
}

Additional information is available at the end of the chapter

http://dx.doi.org/10.5772/51846

\section{Introduction}

The quality of a cosmetic product, in the same way as to other kind of products, is initially defined by the manufacturer that chooses the features that a product should present. On the other hand, the quality control of a product aims to verify if all of these defined features are in accordance with the standard definitions and if it will be maintained during the shelf life of the product (Shewhart, 1980).

The quality control of cosmetics is important to ensure the efficacy and safety of products and its raw-materials. Due to the rapid growth that cosmetic industries have exhibit all over the world, efficient, low cost and rapid methods to assay cosmetics' quality control are a priority. Some current techniques used by the cosmetic industry can be applied to the evaluation of cosmetics' quality control in an efficient manner, such as: rheology, sensory analysis and small angle X-ray scattering (SAXS).

Sensory analysis is a powerful tool, since there is no equipment able to measure the human feelings. It applies experimental design and statistical analysis to obtain information about a product in relation to what people feel when use or consume a product, in other words, it is used to indicate consumer acceptance of a particular product. It can be understood as the discipline that interprets, assess and measures characteristics of a product, after stimulating people in relation to their vital senses, as vision, touch, smell and taste (Stone et al., 1992). It is widely used in food industry and recently, it has also been applied in the cosmetic industry (Almeida et al., 2008; Aust et al., 1987; Backe et al., 1999; Lee et al., 2005; Parente et al., 2005; Wortel et al., 2000). 
The sensory analysis can be applied in the research and development of a new cosmetic (Isaac et al., 2012a), in controlling the manufacturing process to evaluate raw-materials quality and, even, to make possible the substitution of a raw-material of a product that is traditional in the market without changes in the product's features (Meilgaard et al., 1991; Muñoz et al., 1993).

The application of sensory analysis could be related to the product control, referring to the storage, packaging and maintenance of sensory quality in relation to time and temperature (Muñoz et al., 1993), since these factors can change a sensory attribute that the product present originally (Zague, 2008) and people who participates of the sensorial panel could realize the changes in the sensorial attributes. Another function of this important tool is to performance comparative tests between competing products.

Another tool that could be applied to evaluate cosmetics' quality control is the rheology, which studies the flow and deformation of fluids. It has been used in research laboratories and industries as a tool for characterizing ingredients and products, and to predict the performance of products and consumer acceptance.

Rheology has been widely used because, by means of this tool, the researcher can determine physicochemical properties of a product. Constructing a rheogram, it is possible to check the flow curve, evaluate if there is a yield stress and a hysteresis area, which appears to be related to the release of drugs and actives. It is also possible to construct a creep and recovery curve obtaining information about viscoelasticity of each system.

Specifically, in relation to the quality control of cosmetics, specifically, rheology can be applied to help in determining the stability of products by means of the apparent viscosity measured periodically in a determined period exposing the samples to stress conditions (high and low temperatures, solar irradiation), and to monitor the flow characteristics during the shelf life or in the stability assay of a product.

The SAXS technique have being used for the analysis of cosmetics, in order to evaluate the presence of liquid crystalline structures, called liquid-crystals, which are known to increase the stability of formulations becoming, therefore, desirable in cosmetics (Makai et al., 2003).

Combining these three tools, it is possible to test the quality of cosmetics with a rich range of data, and obtain a deep characterization of the system. The results contribute to determining product use, or even, they provide indication of what need to be done to develop a product with predetermined characteristics.

\section{Sensory analysis}

Sensory analysis is defined by Piana et al. (2004) as the examination of a product through the evaluation of the attributes perceptible by the five sense organs (organoleptic attributes), such as color, odor, taste, touch, texture and noise, allowing the establishment of the organoleptic profile of diverse products, including cosmetics. 
The sensory analysis was first applied to the food industry, but the high advance in other areas, such as the cosmetic and pharmaceutical industries, and the important data obtained with the sensory analysis, demanded this useful technique to describe what the consumers fell.

An important advantage of the use of sensory analysis in the quality control of a cosmetic product is that it yields a complex analysis in relation to all sensorial attributes that a product could present, it means that, the volunteer who participates of the sensorial panel is able to give information about the fragrance, the sensation, the appearance, the consistence, and other features that this person experience when use such product. The description of these characteristics by means of equipment would be an arduous work and would provide not sufficient or not valuable data when compared to the data provided by the human senses. Beyond that, the acquisition of this equipment could be of high cost when compared to the sensory analyses' costs (Ross, 2009).

The association of data obtained from sensory analysis and instrumental analysis (especially physicochemical analysis) provides great information and a more complete profile of the product (Ross, 2009).

Nowadays, there are companies specialized in perform sensory analysis of cosmetic products, and thus, they could be contracted to perform this study for cosmetic industries that don't have a sector trained to do it.

The sensorial performance of cosmetics is essential to the acceptance of consumers (Almeida et al., 2008; Fouéré et al., 2005; Lee et al., 2005; Proksch, 2005), thus, especial attention should be given to this subject.

The sensorial features of a formulation are mainly related to the raw-materials and package (Dooley et al., 2009). The raw-materials influence directly in what the consumer feels when applies the cosmetic. The emollients, for example, are raw-materials of marked influence in the tactile sense (Parente et al., 2008; Gorcea and Laura, 2010). Other raw-materials are available at the market and are commercialized to be used in formulations as sensorial modifiers. The main representatives of this kind of product are the silicones and Polymethyl Methacrylate (Ozkan et al., 2012).

The package influences in the first impression of the consumer about a product, since the first sense used to choose a cosmetic in the market is the vision. After, the smell is used too. The tact is not involved in the first purchase attitude, but it will define if a consumer will become a loyal consumer.

In this context, it is possible to verify that the sensorial features of a cosmetic are of great importance in the success of it in the market.

Thus, the sensorial analysis could help a company to define the attributes that a product should or not present beyond the characteristics and intensity of these attributes.

Another point is that these desired sensorial characteristics should be maintained during the cosmetic shelf life. To obtain that, the raw-materials used should be of good quality, the 
manufacture practices should be appropriate, the preservatives used need to be efficient and the formulation should be stable.

In conclusion, the sensorial analysis is an indispensable technique to help the formulator to evaluate the quality of its new product, in relation to its sensorial characteristics and to its stability, testing if the product will keep the nice sensorial feelings that transmit to the consumer during the time of use. This tool is helpful to the research and development area of a company which aims to obtain good quality products of high acceptance by the consumers. The suitable application of sensory evaluation could avoid the outlay of a company with the launching of a product in the market that was rejected by the volunteers of the preliminary study.

Currently, the sensorial analysis have gained more scientific rigor due to the need to offer to the consumers products that meet their expectations and due to the high competition between the major industries of this sector.

To perform the sensorial analysis with rigor and organization, the laboratory destined to it must have the following areas:

A room destined to the analyst who leads the team (Figure 1a)

A conference room (Figure 1b)

A room for the samples preparation (Figure 1c)

An area to the analyses with the volunteers (Figure 1d)

The laboratory should be located in an easy access place.

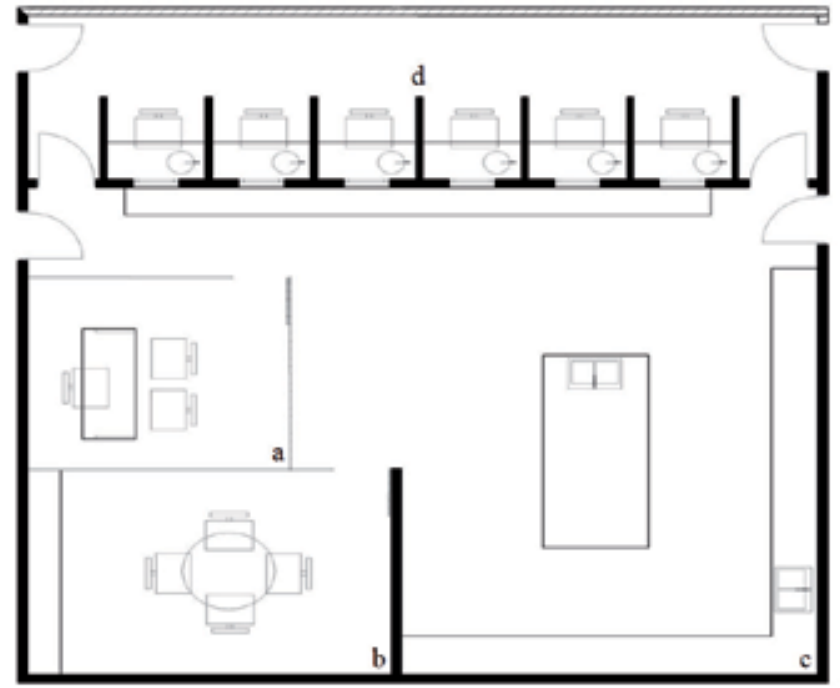

Figure 1. An example of layout of a sensorial analysis laboratory (Isaac et al., 2012a). 
The area where will be performed the analyses should be divided in individual cabins (Figure 2) with a window, where the analyst must offer the samples to the volunteer, sink and faucet, to the volunteer use when necessary (Isaac et al., 2012).

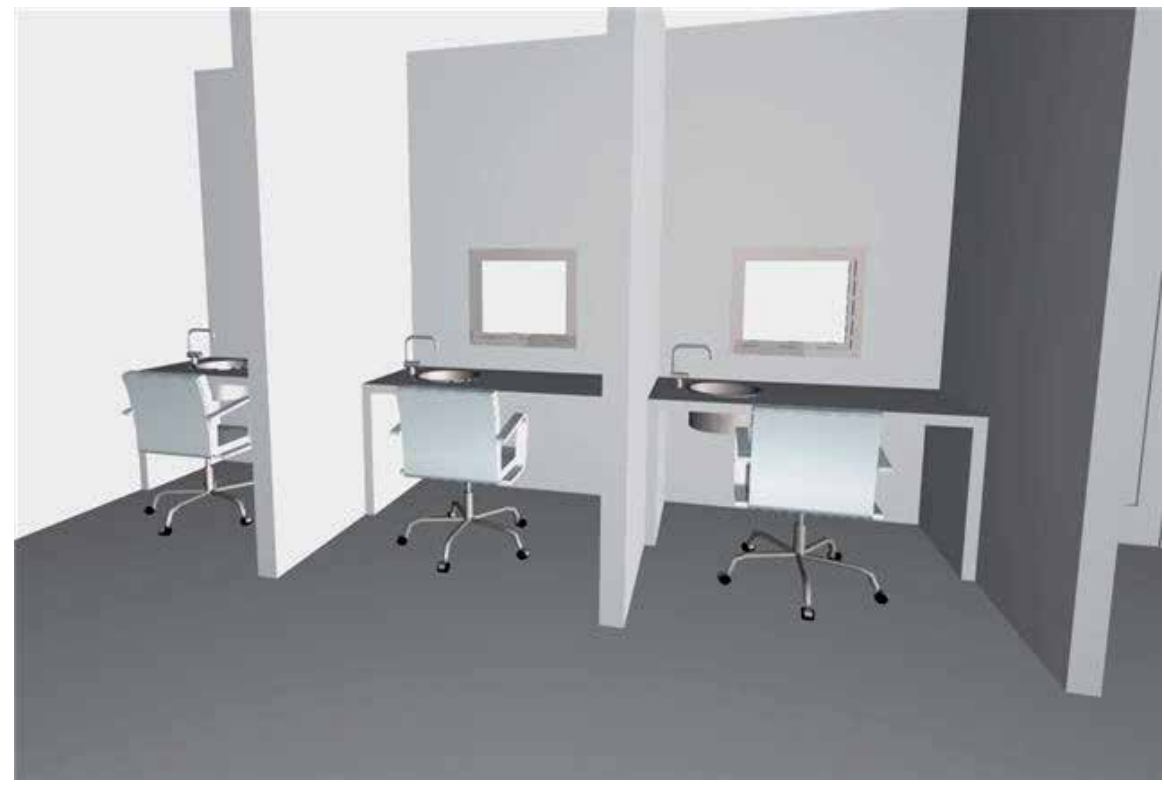

Figure 2. An example of layout of sensorial analysis cabine (Isaac et al., 2012a).

The cabins must be ventilated and odor free, to avoid interferences in the analyses. The temperature and humidity should be controled around $22{ }^{\circ} \mathrm{C}$ and at $45 \%$ of humidity (Isaac et al., 2012).

It is recommended that the walls and furniture of the rooms are colored with neutral and light colors to not disturb the attention of the volunteers and to not interfere in the attributes analyzed by the vision, such as color and appearance of the product.

The volunteers should not smoke, should be healthy, with ease of memorization and communication.

In the study, the volunteers judges could be an experienced judge or not, depending on the kind of evaluation and the answers that the professional team needs to obtain. In the case of utilization of sensorial analysis in the quality control of a cosmetic, usually the volunteers are regular users of the product in analysis, since they need to be familiarized with the characteristics of the product and have sensibility to perceive slight modification on it. When the aim of the sensorial analysis is to evaluate the acceptance of a product that should be launched in the market, it is recommended that the volunteers are potencial users of this new product, orienting the formulator to make changes in the formulation and guiding the company to evaluate if the costs of the product launch are recommended or not. 
There are four different methods to perform the sensorial analysis that are most used, they are: affective, discriminative, descriptive (Aust et al., 1987) and methods to evaluate the effective of the product.

Independent of the method of sensory analysis suitable for each evaluation, the professional team should use printed questionnaires to obtain the answers from each volunteer. The use of printed questionnaires avoids the contact between the professional and the volunteers preventing that the professional is biased in his responses, beyond that, it facilitates the data collection.

In the elaboration of these questionnaires the professional team should use suitable lexicons for each class of product, for example, the lexicons used to the evaluation of lip products are different from that used for corporal lotions (Dooley et al., 2009). Some researches had developed suitable lexicons for different classes of cosmetic products (Civille and Dus, 1991; Wortel and Wiechers, 2000; Dooley et al., 2009). The manner as the volunteer is questioned is fundamental to obtain the information required from them. An inadequate formulary could invalidate a sensory evaluation. It is interesting also, that a description of all descriptors attributed to the formulation being provided to the volunteer, for example: "Thickness: Viscosity of the cream when picking up from the container", "Ease of spreading: Ease of rubbing the sample over the skin", "Absorption: Ease of absorption of the product through the skin", "Residue: Amount of product left on the skin after application" (Parente et al., 2010).

The affective methods represent the consumer opinion and evaluate how much consumers like or dislike a product. It is a quantitative method that is performed in order to know the consumers preferences (Aust et al., 1987). This technique could be applied in the development of new products and when it is necessary to replace a constituent of a formulation without loss of the product quality. It could be performed in two different ways: offering two different samples to the volunteer asking him about what sample he prefers between them or using a hedonic scale for the volunteer attributes grades of intensity of its acceptation in relation to the sample.

The hedonic scale either can be presented to the panel of evaluators in different manners, as shown in Figure 3.

The affective methods provide quantitative data and allow more than one attribute in each sample being evaluated at the same time.

The discriminative test is better represented by the Triangular test. It allows differentiating one between three different samples and is very useful in shelf life studies and in the quality control of cosmetics. The ideal is to perform this evaluation with twelve to forty volunteers, who will receive the three samples and should indicate the different one between them (Zenebon et al., 2008).

The descriptive tests provide a broad sensory description about the product that is being evaluated (Almeida et al., 2008), helping to predict the consumer acceptance and what consumers think about such product (Almeida et al., 2006; Aust et al., 1987). 


\title{
Option 1 Do you like the color of the product?
}

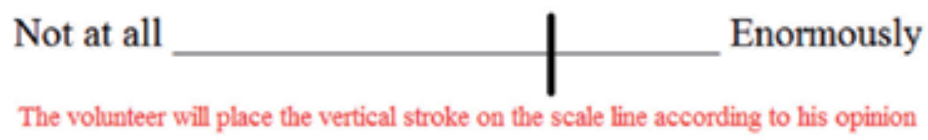

\section{Option 2 How much do you like the color of the product?}

\author{
() 1: most pleasant \\ () 2: very pleasant \\ () 3 : moderately pleasant \\ () 4: slightly pleasant \\ () 5 : neither pleasant nor unpleasant \\ () 6: slightly unpleasant \\ () 7: moderately unpleasant \\ () 8: very unpleasant \\ () 9: most unpleasant \\ The volunteer will mark with a cross the scale according to his opnion
}

Figure 3. Examples of presentation of hedonic scale (Olshan et al., 2000; Barkat et al., 2003).

The tests to evaluate the effective of the products should be performed in true conditions of use and the volunteer may use only the product that is being assessed. These tests could be conduct by the evaluation of dermatologists, by the evaluation of volunteers, and even, by the measurement of one parameter by un equipment, such as the equipment that measures hydration, sebum and transepidermal water loss, to define if a product is really effective. Based on these clinical evaluations, a company could create an efficacy claim to the product (Wortel and Wiechers, 2000).

The sensory analysis could be also applied when a cosmetic industry needs to replace a rawmaterial of a commercialized product without changes in the performance of it. This replacement could be originated by many factors, such as the reduction of costs, problems with the firm who provides this raw-material problems with same raw material which causes irritation, comedogenicity or other problems that affect the consumer. In this field, the sensorial analysis helps the formulator, who proposes different raw materials as substitute, to evaluate if the consumer will notice the adaptation in the cosmetic product.

The statistical analysis is indispensable in the sensory studies. The sensory analysis data should be evaluated transforming them in scores which allows the application of statistical analysis to calculate the mean and standard deviation of the results, and the determination if the difference between the scores obtained is statistically significant. Graphics, tables and preference maps could be elaborated with the results obtained to facilitate the analysis of the data by the professional team.

The sensory analysis is especially indispensable in the industries of fragrances and perfumes, and because of that, high-resolution instrumental methods for evaluation of flavor 
and aroma have been developed and between them are the breath analysis via mass spectrometry (Dijksterhuis and Piggott, 2001; Ross, 2009). Instrumental measurements are thought to be objective, representing an independent fact or truth, however, the human smell sense is irreplaceable, being considered by Ross (2009) not necessarily valid because instrumental methods cannot account for the complexity of human perception.

Nevertheless, rheological studies have been applied to objectify the sensations when cosmetic emulsions are applied to the skin (Brummer and Godersky, 1999).

\section{Rheology}

Rheology is a tool widely applied in the food, petrochemical and pharmaceutical industries, but to the cosmetic industry it is incipient yet. Until now, the majority of cosmetic industries use viscometers to guarantee that the viscosity of different batches of a product is maintained.

This chapter was elaborated in order to show that many other rheological characteristics could be used to evaluate and to predict the stability of cosmetic products and could be applied to compare competing products in the market and to assay if a change in the composition will cause alterations that could be perceived by the consumer.

First, it is necessary to define the three parameters of most importance in rheology: shear stress, shear rate and viscosity. Shear stress can be defined as a force applied in an area. Shear rate is the ratio of the velocity of material to its distance from a stationary object (Naé, 1993). The shear rate can be calculated by the ratio between the velocity and the layer or film thickness. In a lipstick application, for example, with a velocity estimated in $5 \mathrm{~cm} / \mathrm{s}$ and a layer thickness of $0.1 \mathrm{~mm}$, the ratio (shear rate) is $5.10^{2} \mathrm{~s}^{-1}$. Finally, the viscosity can be defined as the resistance to flow. Thus, a viscous product presents smaller flow than others.

Concluding, rheology is the study of deformation and flow of materials under external forces. Some equations and the units of these parameters are (Naé, 1993):

$$
\sigma=\mathrm{F} / \mathrm{A}
$$

Where:

$\sigma=$ shear stress $\left(\mathrm{Pa}=\mathrm{kg} \cdot \mathrm{m}^{-1} \cdot \mathrm{s}^{-2}\right)$

$\mathrm{F}=$ force $\left(\mathrm{N}\right.$ or $\left.\mathrm{kg} \cdot \mathrm{m} \cdot \mathrm{s}^{-2}\right)$

$A=\operatorname{area}\left(\mathrm{m}^{2}\right)$

The viscosity can be defined as the ratio between shear stress and shear rate:

$$
\eta=\sigma / \dot{\gamma}
$$


Where:

= viscosity

$=$ shear stress $(\mathrm{Pa})$

$\dot{\gamma}=$ shear rate $\left(\mathrm{s}^{-1}\right)$

Since the unit of shear stress is $\mathrm{Pa}$ and the unit of deformation is $\mathrm{s}^{-1}$, the unit of viscosity is Pa.s. These parameters are involved in scientific measurements of rotational assays.

Using controlled shear rate and measuring shear stress is possible to carry out rotational assays, and determine flow curves and describe the models: Newtonian or non-Newtonian and, among the last one, plastic, pseudoplastic, dilatant, tixotropic and reopetic fluids. Newtonian fluids are materials that present constant viscosity, independent of time and temperature. These materials present flow curves with proportionality between shear stress and shear rate. The Figure 4 represents the flow curve of a Newtonian material.

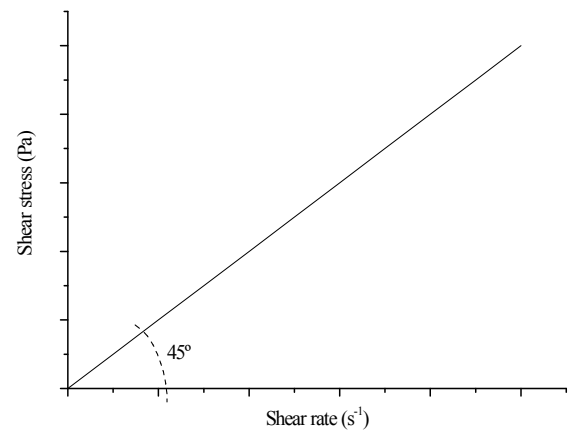

Figure 4. Flow curve of a Newtonian material.

In the case of non-Newtonians materials, this proportionality between shear stress and shear rate does not happen.

If in the beginning of the flow curve there is an increasing in the shear stress but the shear rate is equal to zero, and after to it is verified a Newtonian flow, this material is called plastic. This initial shear stress with shear rate equal to zero is called yield value and it represents the shear stress necessary for the material flow. The Figure 5 represents a plastic material. The yield value is related to the energy required to deform the material sufficiently so that they can flow. The value of the yield stress can be determined by measuring the deformation of the material as a function of the applied stress (Abdel-Rahem et al., 2005).

For non-Newtonian materials time-dependents, if the viscosity decreases with the shear rate, the material is called pseudoplastic and if the viscosity increases, the material is called dilatant. On the other hand, if the material is time-independent, it will be called tixotropic if the viscosity decreases with the shear rate or reopetic if the viscosity increases with the shear 
rate (Naé, 1993). When the ascending and the descending curves of the flow curve do not overlap it shows thixotropy which is a desirable feature for cosmetics and semisolid drug carriers for topical application (Lippacher et al., 2004). The Figures 6, 7, 8 and 9 represent the flow curves of non-Newtonian materials (Naé, 1993).

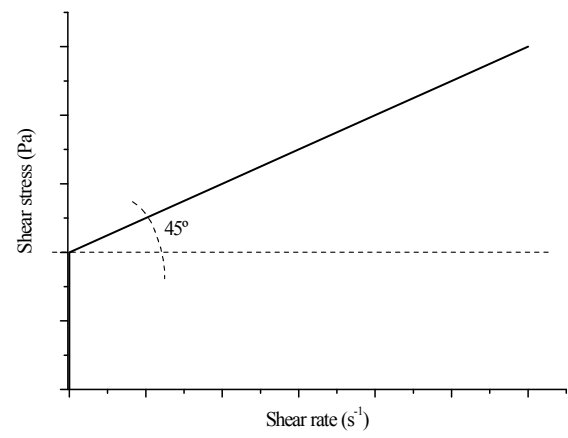

Figure 5. Flow curve of a plastic material.

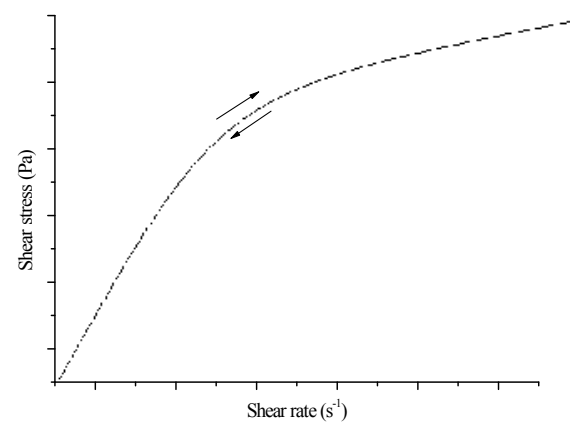

Figure 6. Flow curve of a pseudoplastic material.

For screening purposes and in the initial phases of the formulation development, the rheological tests proved to be very useful for the study of stability.

In a stability assay to determine the shelf life of a recently developed product, the formulation should be exposed to stress conditions, such as storage at $-5^{\circ} \mathrm{C}, 45^{\circ} \mathrm{C}$, and cycles of -5 ${ }^{\circ} \mathrm{C}$ during 24 hours followed by exposure to $45^{\circ} \mathrm{C}$ during more 24 hours. This procedure is done in order to induce the appearance of instability signals in the formulations, where can be cited the darkening of the formulation, the precipitation of a constituent, the phase separation in the case of emulsions, and other signals. These stressing conditions are kept for a period around 2 or 3 months. 


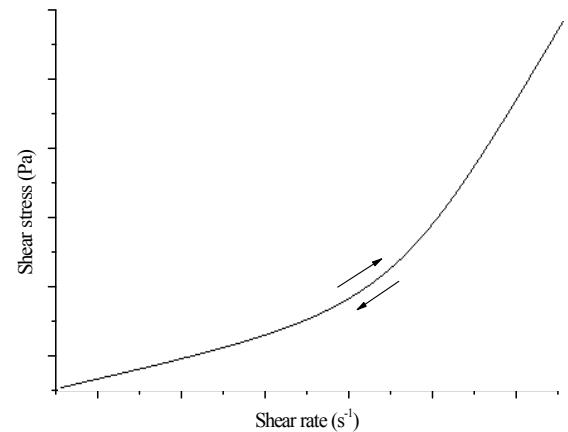

Figure 7. Flow curve of a dilatant material.

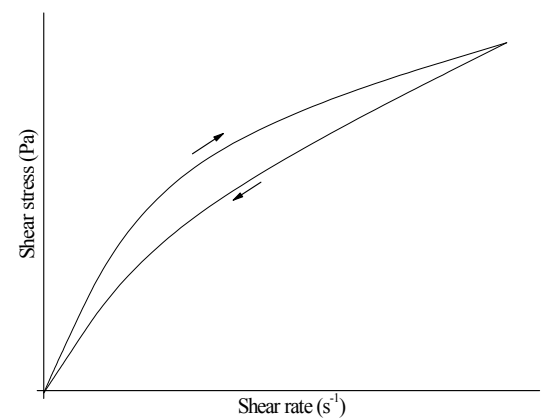

Figure 8. Flow curve of a thixotropic material.

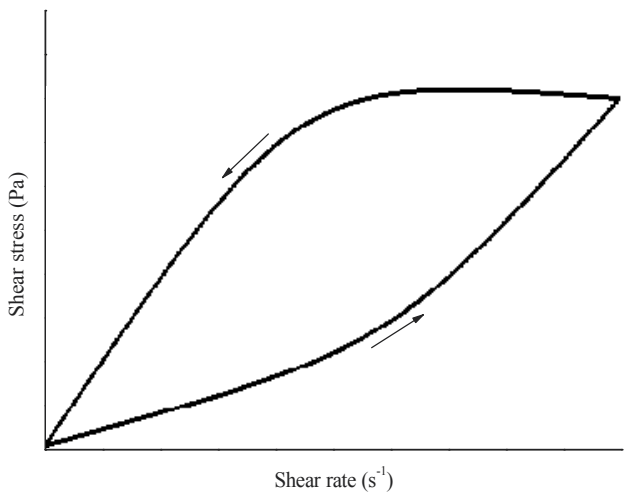

Figure 9. Flow curve of a reopetic material. 
It is usually measured the viscosity of the stressed formulations periodically during the stability assay. It could be done by means of a viscometer or by using a rheometer.

With a viscometer, it is possible to carry out rotational assays or measurements by steadystate flow. On the other hand, the rheometer allows the development of oscillatory assays or dynamic measurements (Biradar, 2009).

When using an oscillatory rheometer it is necessary to carry out a flow curve assay and determine the apparent viscosity of the formulation in a defined shear rate. It is recommended to use the higher shear rate in the ascendant curve of the flow curve, since in this point the sample is in a suitable condition, it means that the formulation is not starting to flow and is not excessively sheared (Figure 10).

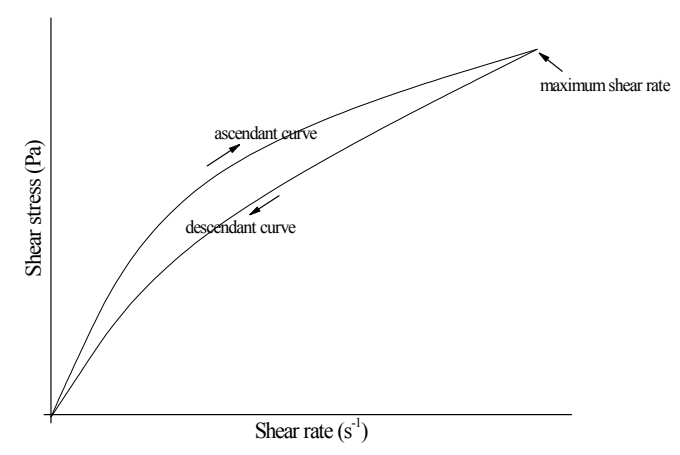

Figure 10. Example of a flow curve indicating the condition to the measurement of apparent viscosity.

In some papers, the flow curves have been plotted as viscosity as a function of shear stress instead of the traditional approach of plotting it versus shear rate because has been previously found that such curves are more discriminating and give better results for evaluation (Roberts, 2001; Samavati, 2011).

After obtaining, periodically, the minimum apparent viscosity of the samples exposed to stress conditions during a period, they should be compared with the initial value, and also compared the viscosity values of the control with the samples exposed to stress conditions, which allows the verification of the increase, decrease or maintenance of this attribute of the formulations.

Further exploiting the same assay, it is possible to calculate the hysteresis area of the formulation in each flow curve performed during the stability assay. The hysteresis loop areas can be obtained through a three-step experiment: upward curve, plateau, downward (Benchabane and Bekkour, 2008) and represents a way to measure, indirectly, the spreadability of the formulation, so it is possible to define if the formulation losses or gains easiness on spreadability during the shelf life. How much bigger is the hysteresis area, higher is the spreadability. 


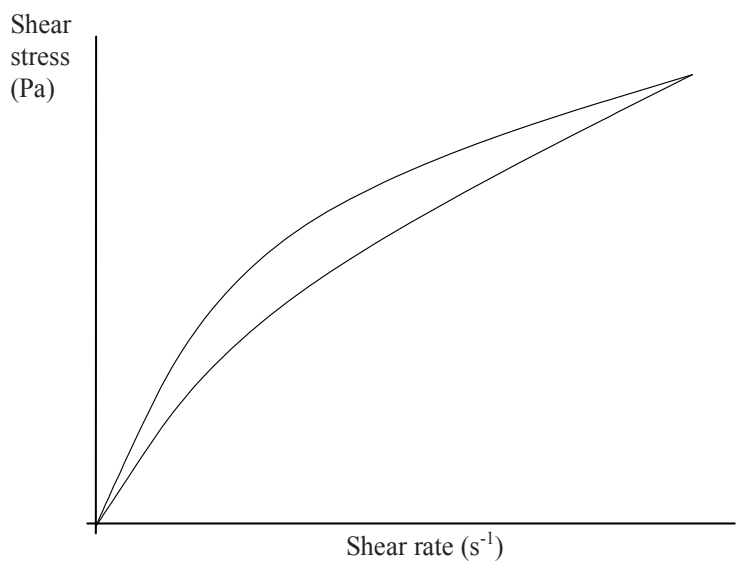

Figure 11. Flow curve with hysteresis area.

Using the flow curve is possible to compare two samples in relation to its hysteresis area and viscosity. A simple way to verify what formulation have a higher viscosity is by simple observation of the rheogram, since the curve that forms a bigger inclination in relation to the $x$ axis of the graphic is the one with higher viscosity. On Figure 12 is showed an example of it, where sample 2 is more viscous than sample 1 . It happens because the tangent of the angle formed is correspondent to the viscosity of the formulation in each shear rate.

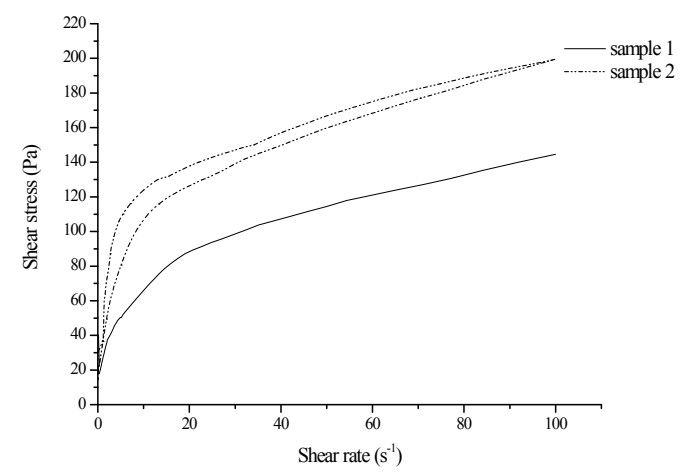

Figure 12. Comparison between flow curves of different samples (a thixotropic and a plastic fluid).

Beyond the different sensorial features caused by the differences in viscosity is known that the viscosity of emulsioned systems is one of the factors that retards or avoids the phase separation processes. The coalescence of dispersed phase can be due to the emulsifier agent and can be related to an instability because of low viscosity of dispersed phase (Corrêa \& Isaac, 2012). This low viscosity can occur because of high shear stress (Samavati et al., 2011). 
In general, for emulsioned systems, the continuos phase is shear thinning, which means that its viscosity decreases with the increasing on shear rate and viscoelastic, which means that it has viscous and elastic components (Tadros, 2004).

An example of the verification of differences in viscosity and thixotropy between two samples is shown on Figure 13.

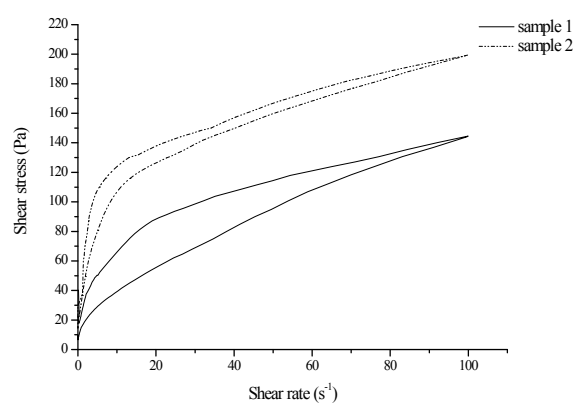

Figure 13. Comparison between flow curves of different samples.

Sample 1 is less viscous but more thixotropic than formulation 2. This simple verification gives to the analyst wide information, depending on what he needs.

Lescanne et al. (2004) studied organogels and aging properties of them. Organogels can be obtained by precipitation processes. These authors verified that, when aggregates are formed by the cooling rate, can be observed a elastic behavior, however, these aggregates can be aligned in the direction of the flow without lost the structure and when the flow is stopped, the aggregates are quickly rearranged and it inducing an thixotropic behavior. When the hot solution is introduced between the flat and the conical plates of the rheometer cell it is cooled to $5^{\circ} \mathrm{C}$ with a cooling rate of $20^{\circ} \mathrm{C} / \mathrm{min}$, during the first hour of the gel life, it was measured the elastic properties of a gel as a function of time just after the cooling. Five minutes after its formation, the gel was submitted to a periodic stress $(0.5 \mathrm{~Pa})$ at a constant frequency $(f=1 \mathrm{~Hz})$. The authors showed that the shear moduli are constants and the aging phenomenon did not modify the elastic properties at least in a period of $1 \mathrm{~h}$. However, when more than a week of aging is waited the samples lost most of its elastic properties.

The flow curve is a rotational assay, but using a rheometer it is possible to perform oscillatory assays too. Among the oscillatory assays are stress sweep and the frequency sweep assays.

The elastic (storage) modulus $G^{\prime}$ and the viscous (loss) modulus $G^{\prime \prime}$ are determined as a function of frequency or stress. The elastic modulus is a measure of energy stored and recovered per cycle of deformation and represents the solid-like component of a viscoelastic material. If a sample is elastic or highly structured then the elastic modulus will be high. The 
viscous modulus is a measure of the energy lost per cycle and represents the liquid-like component. If a sample is viscous the viscous modulus will be high.

In the stress sweep analyses, the structure of the sample is progressively destroyed by applying oscillations with an increasing stress amplitude at a fixed frequency (Callens et al., 2003). The linear viscoelasticity region occurs over that region of strain where the complex modulus is independent of the strain (Hemar, 2000). The linear viscoelastic region is determined by the maximum stress which can be applied without affecting $G^{\prime}$ and $G^{\prime \prime}$. Furthermore, the relative magnitude of the moduli is a qualitative indication for the structure in the sample. Two different situations can occur: $G^{\prime}>G^{\prime \prime}$ for a network consisting of secondary bonds and $G^{\prime} \leq G^{\prime \prime}$ for a physically entangled polymer solution (Callens et al., 2003).

Frequency sweep tests are performed in the linear viscoelastic region of each sample, keeping the structure of the system intact during the measurement. By performing such small stress amplitude oscillations at a whole range of frequencies, the type of network structure can be revealed. The main difference between a network of secondary bonds and one of physical entanglements is located in the low frequency range: in an entangled network the polymers can disentangle if the available time is long enough (low frequency). In a network with secondary bonds the bonds are fixed irrespective of the time scale. This results for an entangled solution in a limiting slope of 2 for $G^{\prime}$ and 1 for $G^{\prime \prime}$ at low frequency in a log-log plot of moduli versus frequency, while at intermediate frequency a plateau develops. For a network of secondary bonds an almost constant value of $G^{\prime}$ and $G^{\prime \prime}$ is observed over the whole frequency range, with the value of $G^{\prime}$ exceeding that of $G^{\prime \prime}($ Callens et al., 2003; Madsen et al., 1998).

The stress sweep is important to evaluate the linear viscoelastic region of a sample that is a range of shear stress in which the formulation does not suffer profound alterations on it structure, being not disrupted. When a shear stress of the linear viscoelastic region is applied in an oscillatory assay, only the intermolecular and interparticle forces are being evaluated (Martin, 1993). To determine the linear viscoelastic region, the oscillating stress sweeps are carried out for the most extreme values. These measurements are used to determine where the reological properties are independent of the applied stress and the identify the critical rheological properties (Tuarez, 2011).

Knowing the values of shear stress that do not cause the disrupt in the formulation by means of the stress sweep, the analyst could perform a frequency sweep of the formulation. The frequency sweep is carried out in a constant shear stress found in the linear viscoelastic region. With this assay it is possible to evaluate the elastic or storage modulus $\left(G^{\prime}\right)$ and the viscous or loss modulus $\left(G^{\prime \prime}\right)$. The cosmetic excipients most used, emulsions and gels, are often viscoelastic samples. The viscoelastic samples when evaluated by means of the frequency sweep present $G^{\prime}$ and $G^{\prime \prime}$ values. When the $G^{\prime}$ value is higher than $G^{\prime \prime}$ it is an indicative that the formulation is more elastic than viscous. It is a characteristic of gels.

Emulsions which exhibits $G^{\prime}$ values higher than $G^{\prime \prime}$ (Figure 14) are described as more stable than formulations with $G^{\prime \prime}$ values higher than $G^{\prime}$ (Figure 15), since they tends to recovery its initial structure faster and more efficiently than the others, and are less susceptible to the 
gravitational forces which retards or avoids the coalescence process and the phase separation of emulsions (Alam and Aramaki, 2009). So, the $G^{\prime}$ values higher than $G^{\prime \prime}$ in emulsions is a desirable feature, being an indicative of stability of the cosmetic system.

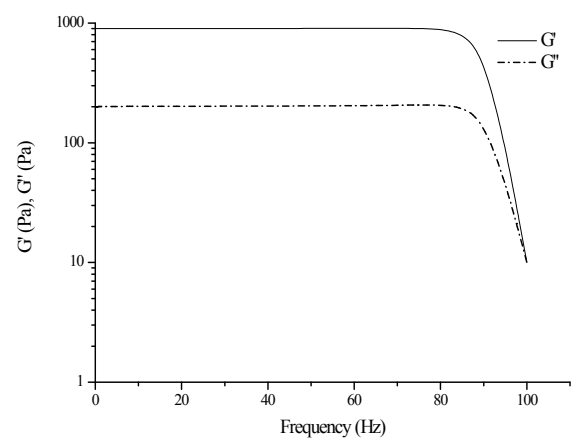

Figure 14. A frequency sweep example $\left(G^{\prime}>G^{\prime \prime}\right)$.

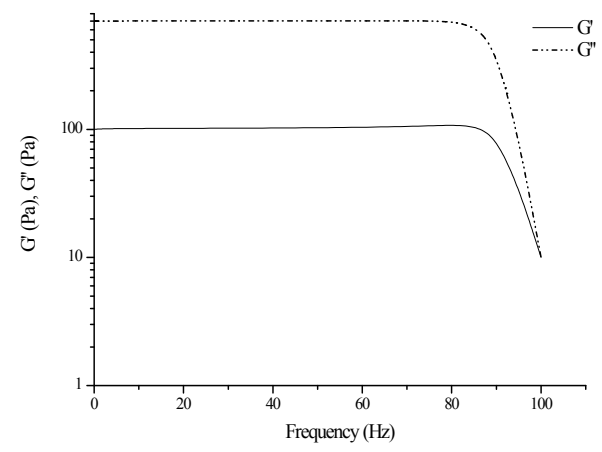

Figure 15. A frequency sweep example $\left(G^{\prime \prime}>G^{\prime}\right)$.

Another assay that could be conducted using an oscillatory rheometer is the creep and recovery assay. It is done by submitting the samples to a constant shear stress during a period, and after, removing this shear stress and monitoring the formulation in relation to the deformation (measured by the compliance - J) during the same period. The compliance parameter is the resulting strain divided by the applied stress (Koop, 2009; Toro-Vazquez et al., 2010). If the compliance parameter is the relationship between strain and the applied stress, the strain is dimensionless and stress is measured in $\mathrm{Pa}$, then, the compliance can be measured in $1 / \mathrm{Pa}$.

In the example showed on the Figure 16 the samples were submitted to a shear stress during 300 seconds, and after removing this shear stress it was monitored during more 300 seconds. 
Analyzing the result obtained in the first 300 seconds is verified that sample 1 exhibited lower compliance values than sample 2, which represents a higher difficult on being deformed than sample 1. The difficult on being deformed is always linked to higher viscosity values.

In the second part of the assay, where the shear stress imposed to the sample is removed, represented in the graphic by the time 301 to 600 seconds, is verified the viscoelastic properties of the samples. Formulations that are able to recovery its initial structure or part of it exhibit a gradually decrease in the compliance values. On Figure 17 there is an example of a formulation that is not a viscoelastic sample, it means that it do not exhibits storage modulus, and is not able to recovery its structure when the shear stress is ceased.

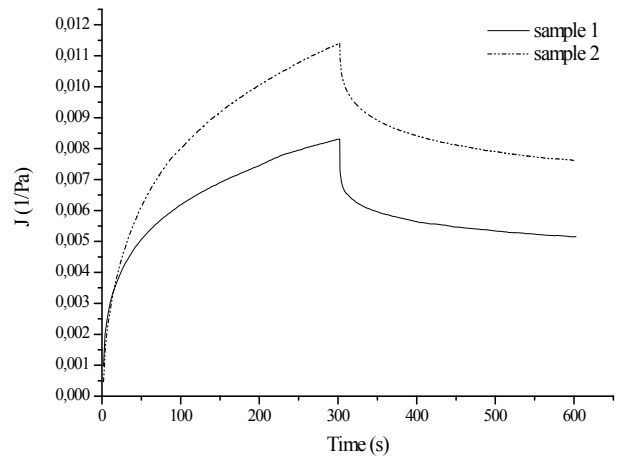

Figure 16. A creep and recovery example of viscoelastic samples.

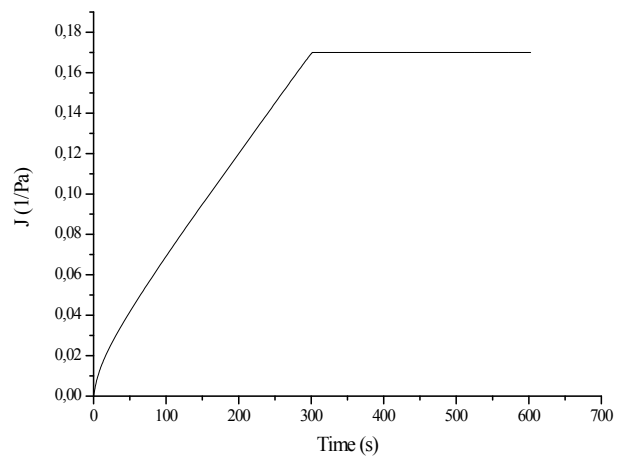

Figure 17. A creep and recovery example of a non-viscoelastic sample.

In addition, the rheology can be used to evaluate the stability over the time by dynamic and oscillatory rheological measurements (Pénzes et al., 2004; Vasiljevic et al., 2006) and the release of active principles. According to Martinez et al. (2007), the transdermal absorption of 
topically administered drugs depends on the rate of release and the permeability of them into the skin and also of the viscosity of the formulation (Martinez et al., 2007).

Thus, it is possible to say that different categories of products should present peculiar rheological properties inherent to its application (Gregolin et al., 2010).

In this way, the rheology can influence the diffusion coefficient, altering the release and permeation of cosmetics active substances (Welin-Berger et al., 2001; A-sadutjarit et al., 2005; Vasiljevic et al., 2006). Some authors have related the influence of rheological characteristics on the release profiles and consequently in the permeation of active substances in the skin; thus, the addition of thickening agents or attainment of a weak-gel because of physical entanglement of polymer chains must be considered in the choice of cosmetics bases (Spiclin, et al., 2003). Thus, rheology can help in the assay of release and permeation in the skin. Some studies have been published about it.

So, in a short way, the rheology is a valuable tool that helps in the quality control of cosmetics, being used in the stability tests, in the comparison between competing samples, in the comparison between an original product and a product with an alteration in a constituent, and in the development of new products, aiming to develop cosmetic with rheological characteristics which indicate stability.

\section{Small Angle X-ray Scattering (SAXS)}

The use of this technique in determining the quality control of a cosmetic is closely related to the stability of the product, which could be improved with the presence of liquid crystals.

Liquid crystals are described as a state of matter between solids and liquids, it means that, they are fluid like liquids but are organized like solids, being called mesophases (Marsh, 1973; Kelker and Hatz, 1980; Müller-Goymann, 2004). These organization contributes to the highly stability of systems.

The formation of liquid crystals in emulsions could be induced by some components present in this system, such as surfactants (Müller-Goymann, 2004). So, what happens is that it is possible to find a peculiar system that is not a simple emulsion and not a genuine liquid crystal, but an emulsioned system that contains liquid crystals, commonly lamellar structures, that are formed around of the inner phase of the emulsion (Oka et al., 2008), making difficult the coalescence, flocculation and the separation of the oily and water phases, what makes the system formed more stable than a simple emulsion (Figures 18 and 19). Flocculation is defined as the formation of aggregates of droplets of an emulsion under the influence of interparticle colloidal forces which are net attractive (Dickinson, 1992) and the formation of lamellar structures avoid or prevent the occurrence of this phenomenon. The formation of lamellar structures is essential to obtain emulsified oil/water systems finely dispersed, with balanced hydrophilic-lipophilic properties, resulting in minimal interfacial tension between aqueous and oily phases, thus contributing to the stability of the system (Engels et al., 1995). Previous studies have also shown that it is possible to make correlation between SAXS and 
rheological analysis, since were verified that the thicker the interlamellar water layers, the higher the viscosity of the cream (Eccleston et al., 2000). Thus, liquid crystals could be responsible by the emulsion stabilization and by the increasing in the viscosity (Klein, 2002), being the presence of this structures desirable in cosmetic emulsions which could be an indicative of quality of them.

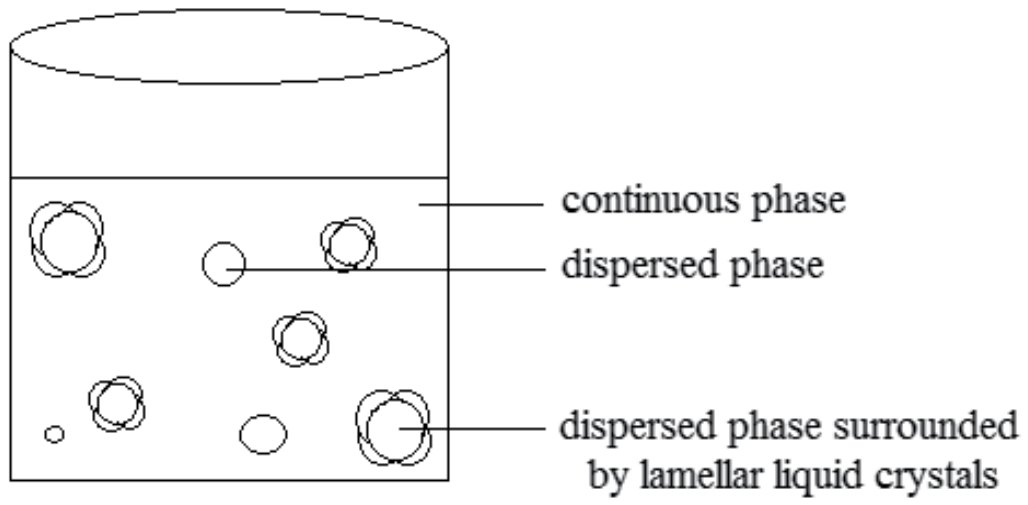

Figure 18. Scheme of a cosmetic emulsion containing liquid crystals.
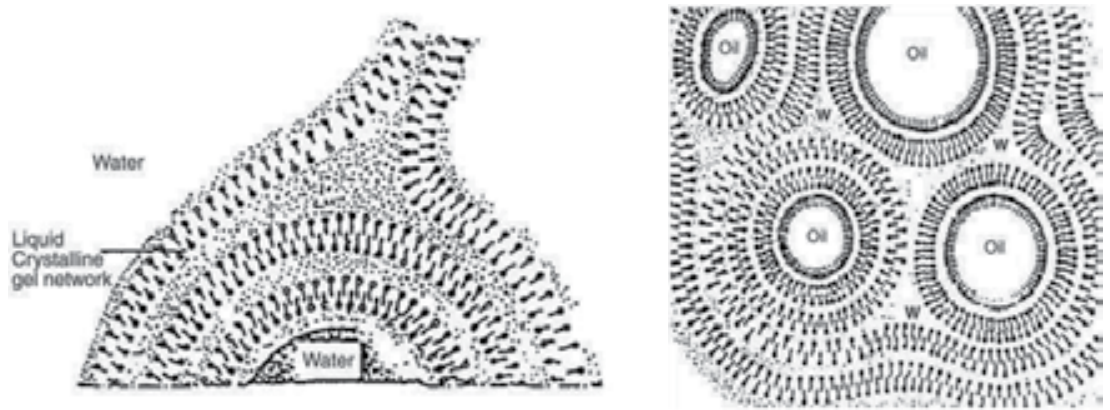

Figure 19. Schemes of the microscopic visualization of lamellar gel networks surrounding emulsion droplets proposed by Klein (2002).

This kind of structure is more commonly found in cosmetics due to the high diversity of components used in it in order to obtain a moisturizer, emollient, humectant, good sensory and, above all, stable cosmetic. In other pharmaceutical forms, usually are used a less diversified composition, which gives a system easier to understand, described as emulsion or liquid crystal, or even, a gel, a suspension, etc. The quantity of these lamellar structures, found in cosmetic emulsions, probably is dependent of three main factors: the raw-materials, the amount of it used and the process of preparation, where should be cited, the temperature and the speed of agitation. 
In cosmetics, other kinds of systems could be used, such as genuine liquid crystals aiming to explore its characteristics of controlled delivery systems.

There are different kinds of liquid crystals and different classifications, but this chapter has not the function of describe them, since it have been done by many authors (Bechtold, 2005; Formariz et al., 2005; Atkins and Jones, 2006), the aim was to demonstrate the importance of these structures in the maintenance of the cosmetics' quality. Nevertheless, according to the literature data (Klein, 2002) and to our experience in this subject, it is possible to say that the lamellar arrangement is the most commonly found in cosmetic emulsions.

An initial analysis of the presence of liquid crystals in a cosmetic emulsion could be done using a polarized light microscope, but it should be confirmed and better analyzed by means of Small Angle X-Ray Scattering. When a microscope slide containing a sample of the system is studied and it presents structures that reflect the incident light, it is an evidence of the presence of liquid crystals (Figure 20). So, they should be submitted to SAXS analysis to confirm this expectation (Savic et al., 2011).

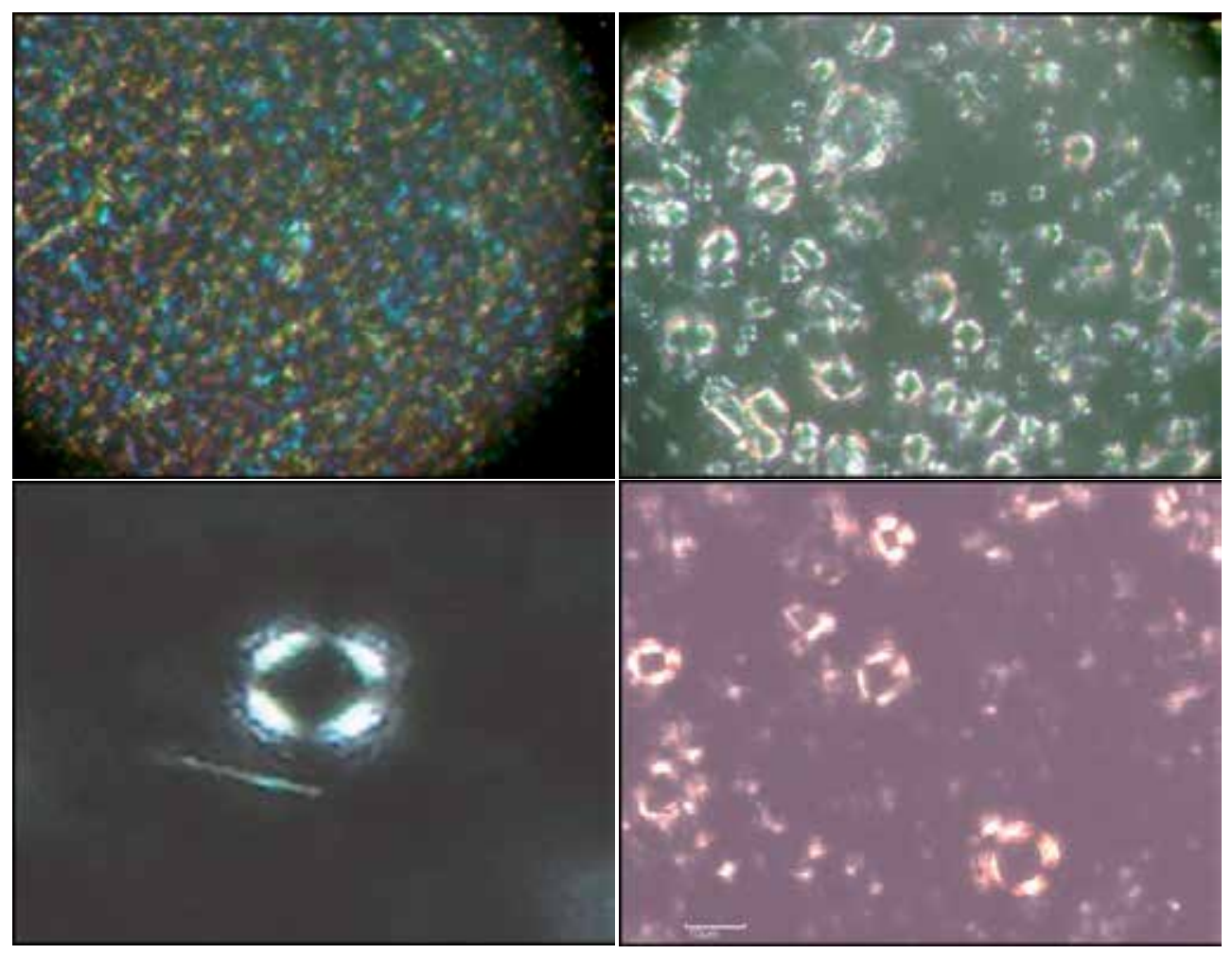

Figure 20. Photomicrographs of liquid-crystal present in emulsions evaluated by polarized light microscope.

The SAXS method requires a synchrotron light source that is formed by means of a particle accelerator, and using a monochromatic beam, that is used to irradiate the sample. After 
that, the scattering of the rays in small angle should be analyzed (Glatter and Kratky, 1982; Urban, 2004; Koch, 2010). Liquid crystals can be analyzed by SAXS since they are able to disperse the X-rays focused on it. In the SAXS line is used an X-rays detector and an multichannel analyzer to capture the intense of the SAXS measures $(I(q))$ in function of the modulus of the scattering vector (q) (Glatter and Kratky, 1982; Molina et al., 2006; Koch, 2010).

Analyzing the data obtained (Figure 19), the $d$ value obtained represents the distance between the particles able to scatter the X-rays. It is calculated by the equation: $d=2 \pi / q$ max, where $q$ max, is the maximum intensity of scattering (Craievich, 2002). The relation between the $d$ values obtained indicates the type of arrangement found in the system (Glatter and Kratky, 1982; Craievich, 2002; Alexandridis et al., 1998).

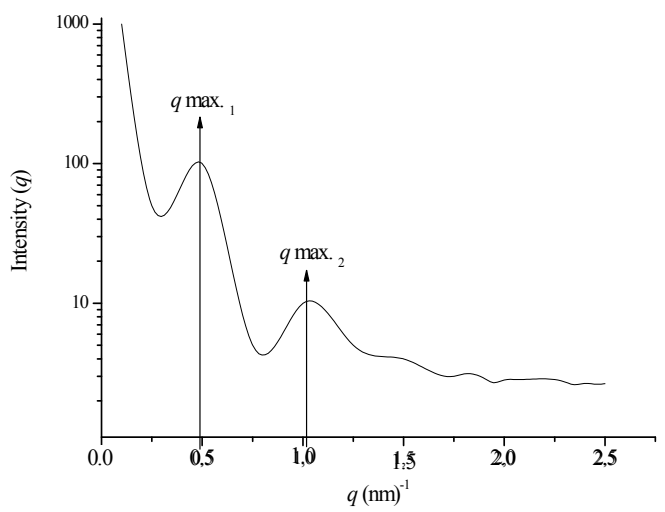

Figure 21. Hypothetical SAXS curve.

In the case of the hypothetical curve showed in Figure 19, $d_{1} / d_{2}$ would result in 2 , which describe lamellar structures (Alexandridis et al., 1998).

Beyond the advantages already mentioned, in a research conducted by Moaddel and Friberg (1995), the authors showed that the presence of lamellar liquid crystals in an emulsion avoids the water evaporation rate in this system, thus contributing in another way to the stability and maintenance of the cosmetic quality.

According to the advantages obtained with the presence of liquid crystals, these mesophases can be of great importance to the Cosmetic Industry in the development of very stable cosmetics and, the SAXS technique, an efficient tool to confirm the presence of these desirable structures that helps in the maintenance of cosmetics' quality control.

Camerel et al. (2003) pointed the importance in correlate the microstructure of a colloidal suspension with its rheological behavior to define its better use in industry and in life, beyond that, according to these authors there are few reports correlating these analyses. 
Our research group has invested in researches to assess the stability of cosmetics (Isaac et al., 2008); evaluating of the influence of the addition of thickening agents in creams using rheological measurements (Isaac et al., 2012a); evaluating the thickeners' influence on the rheological properties of a cosmetic (Isaac et al., 2012b,c); proposing alternative methods to assay the efficacy and safety of them (Chiari et al., 2012a; Chiari et al., 2012b) and using of the sensory analysis in the cosmetics development (Isaac et al., 2012a) which, in different points of view of what was demonstrated in this chapter, also influence in the product quality.

\section{Conclusion}

This chapter aimed to show the facility that some simple or advanced techniques already used, sometimes to other finalities, could offer to the quality control of cosmetic products. The sensory analysis, rheology and SAXS technique have earned attention due to the important contribution that they can offer to the cosmetic area.

\section{Author details}

Bruna Galdorfini Chiari, Maria Gabriela José de Almeida, Marcos Antonio Corrêa and Vera Lucia Borges Isaac*

*Address all correspondence to: veraisaac@fcfar.unesp.br

Faculdade de Ciências Farmacêuticas, UNESP - Univ Estadual Paulista, Departamento de Fármacos e Medicamentos, Laboratório de Cosmetologia - LaCos, Araraquara, Laboratório de Cosmetologia, São Paulo, Brazil

\section{References}

[1] Abdel-Rahem, R., Gradzielski, M., \& Hoffmann, H. (2005). A novel viscoelastic system from a cationic surfactant and a hydrophobic counterion. Journal of Colloid and Interface Science, 288, 570-582.

[2] Alam, M.M., \& Aramaki, K. (2009). Glycerol effects on the formation and rheology of hexagonal phase and related gel emulsion, J. Colloid Interface Sci., 336, 820-826.

[3] Alexandridis, P., Olsson, U., \& Lindman, B. (1998). A record nine different phases (four cubic, two hexagonal, and one lamellar lyotropic liquid crystalline and two micellar solutions) in a ternary isothermal system of an amphiphilic block copolymer and selective solvents (water and oil). Langmuir, 14, 2627-2638.

[4] Almeida, I.F., Gaio, A.R., \& Bahia, M.F. (2006). Estimation of hedonic responses from descriptive skin sensory data by chi squared minimization. J. Sens. Stud., 21(1), 2-19. 
[5] Almeida, I.F., Gaio, A.R., \& Bahia, M.F. (2008). Hedonic and descriptive skinfeel analysis of two oleogels: comparison with other topical formulations. J. Sens. Stud., 23(1), 92-113.

[6] A-Sadutjarit, R., Sirivat, A., \& Vayumhasuwan, P. (2005). Viscoelastic properties of carbopol 940 gels and their relationships to piroxicam diffusion coefficients in gel bases. Pharmaceutical Research, 22(12), 2134-2140.

[7] Atkins, P., \& Jones, L. (2006). Princípios de química: questionando a vida moderna e o meio ambiente. Porto Alegre: Bookmam. 3ed., p. 293-295 and 300-302.

[8] Aust, L.B., Oddo, P., Wild, J.E., Mills, O.H., \& Deupree, J.S. (1987). The descriptive analysis of skin care products by a trained panel of judges. J. Soc. Cosmet. Chem., 38, 443-448.

[9] Backe, I., Meges, S., Lauze, C., Macleod, P., \& Dupuy, P. (1999). Sensory analysis of four medical spa spring waters containing various mineral concentrations. Int. J. Dermatol., 38(10), 784-786.

[10] Barkat, S., Thomas-Danguin, T., Bensafi, M., Rouby, C., \& Sicard, G. (2003). Odor and color of cosmetic products: correlations between subject judgement and autonomous nervous system response. International Journal of Cosmetic Science, 25, 273-283.

[11] Bechtold, I. H. (2005). Liquid crystals: A complex system of simple application. Rev. Bras. Ensino Física, 27(3), 333-342.

[12] Benchabane, A., \& Bekkour, K. (2008). Rheological properties of carboxymethyl cellulose (CMC) solutions. Colloid Polymer Science, 286, 1173-1180.

[13] Biradar, S.V., Dhumal, R.S., \& Paradkar, A. (2009). Rheological investigation of selfemulsification process. Journal of Pharmacy and Pharmaceutical Science, 12(1), 17-31.

[14] Brummer, R., \& Godersky, S. (1999). Rheological studies to objectify sensations occurring when cosmetic emulsions are applied to the skin. Colloids and Surfaces A: Physicochemical and Engineering Aspects, 152, 89-94.

[15] Callens, C., Ceulemans, J., Ludwig, A., Foreman, P., \& Remon, J.P. (2003). Rheological study on mucoadhesivity of some nasal powder formulations. European Journal of Pharmaceutics and Biopharmaceutics, 55, 323-328.

[16] Camerel, F., Gabriel, J.C.P., Batail, P., Panine, P., \& Davidson, P. (2003). Combined SAXS - Rheological studies of liquid-cristalline colloidal dispersions of mineral particles. Langmuir, 19, 10028-10035.

[17] Chiari, B.G., Magnani, C., Salgado, H.R.N., Côrrea, M.A., \& Isaac, V.L.B. (2012a). Estudo da segurança de cosméticos: presente e futuro. Revista Brasileira de Ciências Farmacêuticas Básica e Aplicada, 33(2).

[18] Chiari, B.G., Martini, P.C., Moraes, J.D.D., Andréo, R., Corrêa, M.A., Cicarelli, R.M.B., \& Isaac, V.L.B. (2012b). Use of HepG2 cells to assay the safety of cosmetic active substances. International Journal of Research in Cosmetic Science, 2(2), 8-14. 
[19] Civille, C.V., \& Dus, C.A. (1991). Evaluating tactile properties of skincare products: A descriptive analysis technique. Cosmet. Toilet., 106, 83-88.

[20] Corrêa, M.A., \& Isaac, V.L.B. (2012). Emulsões. In: CORRÊA, M.A. Cosmetologia: ciência e técnica. São Paulo: Medfarma, p. 337-381.

[21] Craievich, A. F. (2002). Synchrotron SAXS studies of nanostructured materials and colloidal solutions. Materials Research Rev., 5(1), 1-11.

[22] Dickinson, E. (1992). Structure and composition of adsorbed protein layers and the relationship to emulsion stability. J. Chem. Soc. Faraday Trans., 88(20), 2973-2983.

[23] Dijksterhuis, G. B., \& Piggott, J. R. (2001). Dynamic methods of sensory analysis. Trends in Food Science and Technology, 11, 284-290.

[24] Dooley, L.M., Adhikari, K., \& Chambers IV, E. (2009). A general lexicon for sensory analysis of texture and appearance of lip products. J. Sens. Stud., 24(4), 581-600.

[25] Eccleston, G.M., Behan-Martin, M.K., Jones, G.R., \& Towns-Andrews, E. (2000). Synchrotron X-ray investigations into the lamellar gel phase formed in pharmaceutical creams prepared with cetrimide and fatty alcohols. International Journal of Pharmaceutics, 203, 127-139.

[26] Engels, T., Förster, T., \& Von Rybinski, W. (1995). The influence of coemulsifier type on the stability of oil-in-water emulsions. Colloids Surfaces A: Physicochemical and Engineering Aspects, 99, 141-149.

[27] Formariz, T.P., Urban, M.C.C., Da Silva Júnior, A.A., Gremião, M.P.D., \& De Oliveira, A.G. (2005). Microemulsões e fases líquidas cristalinas como sistemas de liberação de fármacos. Revista Brasileira de Ciências Farmacêuticas, 41(3), 301-313.

[28] Fouéré, S., Adjadj, L., \& Pawin, H. (2005). How patients experience psoriasis: results from a European survey. J. Eur. Acad. Dermatol. Venereol., 19(3), 2-6.

[29] Glatter, O., \& Kratky, O. (1982). Small-Angle X-ray Scattering, Academic Press, New York.

[30] Gorcea, M., \& Laura, D. (2012). Evaluating the physiochemical properties of emollient esters for cosmetic use. Cosmetics and Toiletries, 125(12), 26-33.

[31] Gregolin, M.T., Chiari, B.G., Ribeiro, H.M., \& Isaac, V.L.B. (2010). Rheological Characterization of hydrophilic gels. Journal of Dispersion Science and Technology, 31, 820-825.

[32] Hemar, Y., \& Horne, D.S. (2000). Dynamic rheological properties of highly concentrated protein-stabilized emulsions. Langmuir, 16(7), 3050-3057.

[33] Isaac, V.L.B., Cefali, L.C., Chiari, B.G., Almeida, M.G.J., Ribeiro, H. M., \& Corrêa, M.A. (2012c) Effect of various thickening agents on the rheological properties of $\mathrm{O} / \mathrm{W}$ emulsions containing non-ionic emulsifier. Journal of Dispersion Science and Tech- 
nology. Available at: 10.1080/01932691.2012.695952

[34] Isaac, V.L.B., Cefali, L.C., Chiari, B.G., Oliveira, C.C.L.G., Salgado, H.R.N., \& Corrêa, M.A. (2008). Protocolo para ensaios físico-químicos de estabilidade de fitocosméticos. Rev. Ciênc. Farm. Básica Apl., 29(1), 81-96.

[35] Isaac, V., Chiari, B.G., Magnani, C., \& Corrêa, M.A. (2012a). Análise sensorial como ferramenta no desenvolvimento de cosméticos. Revista de Ciências Farmacêuticas Básica e Aplicada, 33 (in press).

[36] Isaac, V.L.B., Moraes, J.D.D., Chiari, B.G., Guglielmi, D.A.S., Cefali, L.C., Rissi, N.C., \& Corrêa, M.A. (2012b). Determination of the real influence of the addition of four thickening agents in creams using rheological measurements. Available at: http:// www.tandfonline.com/doi/full/10.1080/01932691.2012.683759

[37] Kelker, H., \& Hatz, R. (1980). Handbook of Liquid Crystals, Verlag Chemie, Weinheim, Germany.

[38] Klein, K. (2002) Liquid crystals and emulsions: a wonderful marriage. Chapter 26, p. 265-269. In: Skin Barrier: Chemistry of Delivery Systems. Available at: http:// www.alluredbooks.com/sample_pages/skin_barr_chem_skin_deli_syst_ch26.pdf.

Acessed on july, 2012.

[39] Koch, M.H.J. (2010). SAXS Instrumentation for Synchrotron Radiation then and now. XIV International Conference on Small-Angle Scattering (SAS09). Journal of Physics: Conference Series 247.

[40] Koop, H.S., Praes, C.E.O., Reicher, F., Petkowicz, C.L.O., \& Silveira, J.L.M. (2009). Rheological behavior of gel of xanthan with galactomannan: effect of hydroalcoholicascorbic acid. Materials Science and Engineering C, 29, 559-63.

[41] Lee, I-S., Yang, H-M., Kim, J-W., Maeng, Y-J., Lee, C-W., Kang, Y-S., Rang, M-J., \& Kim H-Y. (2005). Terminology development and panel training for sensory evaluation of skin care products including aqua cream. J. Sens. Stud., 20(5), 421-433.

[42] Lescanne, M., Grondin, P., D’Aléo, A., Fages, F., Pozzo, J.L., Moundain Monval, O., Reinheimer, P., \& Colin, A. (2004). Thixotropic organogels based on a simple N-hydroxyalkyl amide: rheological and aging properties. Langmuir, 20(8), 3032-3041.

[43] Lippacher, A., Müller, R.H., \& Mäder, K. (2004). Liquid and semisolid SLNe dispersions for topical application: rheological characterization. European Journal of Pharmaceutics and Biopharmaceutics, 58, 561-567.

[44] Madsen, F., Eberth, K., \& Smart, J.D. (1998). A rheological assessment of the nature of interactions between mucoadhesive polymers and a homogenized mucus gel. Biomaterials, 19, 1083-1092.

[45] Makai, M., Csányi, E., Németh, Z.S., Pálinkás, J., \& Erós, I. (2003). Structure and drug release of lamellar liquid crystals containing glycerol. Int. J. Pharm., 256, 95-107. 
[46] Marsh, H. (1973). Carbonization and liquid-crystal (mesophase) development: Part 1. The significance of the mesophase during carbonization of coking coals. Fuel, 52, 205-212.

[47] Martin, A. (1993). Physical Pharmacy, fourth ed., Lea \& Febiger, Philadelphia.

[48] Martinez, M.A.R., Gallardo, J.L.V., Benavides, M.M., López-Duran, J.D.G., \& Lara, V.G. (2007). Rheological behavior of gels and meloxicam release. International Journal of Pharmaceutics, 333, 17-23.

[49] Meilgaard, M., Civille, G.V., \& Carr, B.T. (1991). Consumer test and in-house panel acceptance tests. In: Meilgaard, M., Civille, G.V., Carr, B.T. Sensory evaluation techniques. Florida: CRC Press, p. 142-7, 281.

[50] Moaddel, T., \& Friberg SE. (1995). Phase equilibria and evaporation rates in a four component emulsion. J. Disp. Sci. Technol., 16, 69-97.

[51] Molina, C., Dahmouche, K., Hammer, P., Bermudez, V.Z., Carlos, L.D., Ferrari, M., Montagna, M., Gonçalves, R.R., De Oliveira, L.F.C., Edwards, H.G.M., Messaddeq, Y., \& Ribeiro, S.J.L. (2006). Structure and Properties of $\mathrm{Ti}^{{ }^{4+}-U r e a s i l}$ Organic-Inorganic Hybrids, J. Braz. Chem. Soc., 17(3), 443-452.

[52] Müller-Goymann, C.C. (2004). Physicochemical characterization of colloidal drug delivery systems such as reverse micelles, vesicles, liquid crystals and nanoparticles for topical administration. European Journal of Pharmaceutics and Biopharmaceutics 58, 343-356.

[53] Muñoz, A.M., Civille, G.V., \& Carr, B.T. (1993). Sensory evaluation in quality control. New York: Van Nostrand Reinhold, p. 240.

[54] Naé, H.N. (1993). Introduction to rheology. In: Laba, D. Rheological propertie of cosmetics and toiletries. New York: Marcel Dekker, $426 \mathrm{p}$.

[55] Oka, T., Miyahara, R., Teshigawara, T., \& Watanabe, K. (2008). Development of novel cosmetic base using sterol surfactante. I. Preparation of novel emulsified particles with sterol surfactant. Journal of Oleo Science, 57(10), 567-575.

[56] Olshan, A.A., Kohut, B.E., Vincent, J.W., Borden, L.C., Delgado, N., Qaqish, J., Sharma, N.C., \& Mcguire, J.A. (2000). Clinical effectiveness of essential oil-containing dentifrices in controlling oral malodor. American Journal of Dentistry, 13, 18C-22C.

[57] Ozkan, S., Gillece, T.W., Senak, L., \& Moore, D.J. (2012). Characterization of yield stress and slip behaviour of skin/hair care gels using steady flow and LAOS measurements and their correlation with sensorial attributes. International Journal of Cosmetic Science, 34, 193-201.

[58] Parente, M.E., Ares, G., \& Manzoni, A.V. (2010). Application of two consumer profiling techniques to cosmetic emulsions. Journal of Sensory Studies, 25, 685-705.

[59] Parente, M.E., Gámbaro, A., \& Ares, G. (2008). Sensory characterization of emollients. Journal of Sensory Studies 2, 149-161. 
[60] Parente, M.F., Gambaro, A., \& Solana, G. (2005). Study of sensory properties of emollients used in cosmetics and their correlation with physicochemical properties. J Cosmet Sci., 56(3), 175-182.

[61] Pénzes, T., Csóka, I., \& Eros, I. (2004). Rheological analysis of the structural properties effecting the percutaneous absorption and stability in pharmaceutical organogels. Rheological Acta, 43, 457-63.

[62] Piana, M.L., Oddo, L.P., Bentabol, A., Bruneau, E., Bogdanov, S., \& Guyot Declerck, C. (2004). Sensory analysis applied to honey: state of the art. Apidologie, 35, S26-S37.

[63] Proksch, E., \& Lachapelle, J.M. (2005). The management of dry skin with topical emollients: recent perspectives. J. Dtsch. Dermatol. Ges., 10(5), 768-774.

[64] Roberts, G.P., Bames, H.A., \& Carew, P. (2001). Modelling the flow behavior of very shear-thinning liquids. Chem. Eng. Sci., 56, 5617-5623.

[65] Ross, C.F. (2009). Sensory science at the humane-machine interface. Trends in Food Science \& Technology, 20, 63-72.

[66] Samavati, V., Emam-Djomeh, Z., Mohammadifar, M.A., Omid, M., \& Mehdinia, A.L.I. (2011). Stability and rheology of dispersions containing polysaccharide, oleic acid and whey protein isolate. Journal of Texture Studies, p. 1-14.

[67] Savic, S., Lukic, M., Jaksic, I., Reichl, S., Tamburic, S., \& Müller-Goymann, C. (2011). An alkyl polyglucoside-mixed emulsifier as stabilizer of emulsion systems: The influence of colloidal structure on emulsion skin hydration potential. Journal of Colloid and Interface Science, 358, 182-191.

[68] Shewhart, W.A. (1980). Economic control of quality of manufactured product, American Society for Quality Control.

[69] Spiclin, P, Homar, M, Valant, A.Z., \& Gasperlin, M. (2003). Sodium ascorbyl phosphate in topical microemulsions. International Journal of Pharmaceutics, 256, 65-73.

[70] Stone, H.S., \& Sidel, J.L. (1992). Sensory evaluation practices. San Diego, CA: Academic Press.

[71] Tadros, T. (2004). Application of rheology for assessment and prediction of the longterm physical stability of emulsions. Advances in Colloid and Interface Science, 108 $109,227-258$.

[72] Toro-Vazquez, J.F., Morales-Rueda, J., Ajay Mallia, V., \& Weiss, R.G. (2010). Relationship between molecular structure and thermo-mechanical properties of candelilla wax and amides derived from (R)-12-hydroxystearic acid as gelators of safflower oil. Food Biophysics, 5, 193-202.

[73] Tuarez, E.P., Sadtler, V., Marchal, P, Choplin, L., \& Salager, J.L. (2011). Making use of formulation-composition map to prepare highly concentrated emulsions with particular rheological properties. Ind. Eng. Chem. Res., 50, 2380-87. 
[74] Urban, M. C. C. (2004). Desenvolvimento de sistemas de liberação micro e nanoestruturados para administração cutânea do acetato de dexametasona. 2004. Dissertação (Mestrado em Ciências Farmacêuticas) - Faculdade de Ciências Farmacêuticas, Universidade Estadual Paulista Júlio de Mesquita Filho, Araraquara.

[75] Vasiljevic, D., Parojcic, J., Primorac, M., \& Vuleta, G. (2006). An investigation into the characteristics and drug release properties of multiple W/O/W emulsion systems containing low concentration of lipophilic polymeric emulsifier. International journal of Pharmaceutics, 309, 171-177.

[76] Welin-Berger, K., Neelissen, J.A.M., \& Bergenstahl, B. (2001). The effect of rheological behavior of a topical anaesthetic formulation on the release and permeation rates of the active compound. European Journal of pharmaceutical Sciences, 13, 309-18.

[77] Wortel, V.A.L., \& Wiechers, J.W. (2000). Skin sensory performance of individual personal care ingredients and marketed personal care products. Food Qual. Pref., 11(1-2), 121-127.

[78] Zague, V., Nishikawa, D.O., Silva, D.A., Baby, A.R., Behrens, J.H., Kaneko, T.M., \& Velasco, M.V.R. (2008). Influence of storage temperature on cooling intensity of topical emulsions containing encapsulated menthol. J. Sens. Stud., 23(1), 26-34.

[79] Zenebon, O., Pascuet, N.S., \& Tiglea, P. (2008). Métodos físico-químicos para análise de alimentos. Instituto Adolfo Lutz (São Paulo). On line version. Available at: http:// www.crq4.org.br/sms/files/file/analisedealimentosial_2008.pdf 
Sops: What Are They Good For? 



\section{Standard Operating Procedures (What Are They Good For ?)}

Isin Akyar

Additional information is available at the end of the chapter

http://dx.doi.org/10.5772/50439

\section{Introduction}

Standardization is defined as an activity that gives rise to solutions for repetitive application to problems in various disciplines. Generally, the activity constitudes the process of establishing (determining, formulating, and issuing) and implementing standards. Thus, standards are the perfect result of a standardization activity and inside the context of quality systems consist of quality documents or documents related to the quality system. High levels of quality are important to accomplish Company business objectives. Quality, a source of competitive benefit, should stay a symbol of Company products and services. High quality is not an additional value; it is an important elemantary necessity. Each employee in all organizational units is responsible for guaranteeing that their work processes are effective and continually getting better. Top management should provide the training and an appropriate motivating environment to support teamwork both inside and across organizational units for employees to advance processes. Ultimately, everyone in an institution is responsible for the quality of its products and services. An institution in the role of a sponsor of clinical trials can best achieve its business objectives by establishing and managing robust quality systems with their integral quality documents including standard operating procedures (SOPs) (Manghani, K. 2011). The Quality Management system must evolve by trial and error, with enlarging experience, by group discussions and with changing understanding. In the beginning, attention will be focused on basic operational SOPs, afterwards moving to record keeping (as more and more SOPs are issued) and filling gaps as practice admits missing links in the chain of Quality Assurance. Essentially problems will turn up. One way to react to them is to talk with people in other laboratories who have faced similar problems. It 
should not be forgotten that Quality Management is a tool rather than a goal. The goal is quality performance of the laboratory. The philosopher Kant saw autonomy as self-government originning from morality, with morality proceeding from knowledge and self-discipline. Conger \& Kanungo noted that an appropriate level of authority, discretion, formalization, and rule structure is a requirement for worker empowerment, which we see as consistent with the concept of self-government. Merriam-Webster defined autonomy as 'the quality or state of being self-governing; especially: the right of self-government; self-directing freedom and especially moral independence'. Necessitated SOP use will be absolutely related to the sense of self-determination experienced by workers. Worker participation in SOP advancenment and clarification controls the affiliation between required SOP use and the sense of self-determination experienced by workers.

Standard Operating Procedures (SOP) is a process document that describes in detail the way that an operator should perform a given operation. SOPs involve the purpose of the operation, the equipment and materials required, how to perform the set-up and operations required for the process, how to perform the maintenance and shutdown operations carried out by the worker, a description of safety issues, trouble-shooting, a list of spare parts and where to find them, illustrations, and checklists. The SOP is one of many process documents which is needed for consistent operation of a given process, with other documents involving process flow charts, material specifications, and so forth.

The purpose of SOPs today is to guarantee that all workers are performing tasks in the same way, which is a needed for condition to get expected output from the process. When all workers perform their tasks constantly, it becomes possible to run controlled experiments to test the impact of changing various process parameters. When a process change is shown to improve process performance, SOPs are updated and workers are trained to the new procedures. All over the process, it is adorable to involve workers in SOP development and to praise worker ideas for the SOP improvement. For constant organizational advance, organized processes need to be constantly improved, hence necessitating ideas from those workers using those procedures. Ideas are not creative simply because they deviate from organized knowledge; ideas are creative when they are novel and suitable to the task at hand. Workers may have many ideas; nevertheless, what they choose to do with their ideas will depend on various organizational and individual-difference factors. The most important factor, however, for the advancement of creative behaviours is worker intrinsic motivation- a sine qua non of worker creative contribution. By the help of confirmatory factor analysis, the Spreitzer construct validated the four dimensions of intrinsic motivation (i.e. psychological authorization): (a) Competence (example item includes 'I am confident about my ability to do my job'); (b) Meaning (example item includes 'The work I do is very important to $\mathrm{me}^{\prime}$; (c) Impact (example item includes 'I have a great deal of control over what happens in my department'; (d) Self-determination (example item includes 'I can decide on my own how to go about doing my work'). Furthermore, Spreitzer argued and empirically established that an antecedent condition to innovation (i.e. creativity) and effectiveness is intrinsic motivation (De Trevil et al. 2005). 


\section{Overview}

The quality documents constitudes of Company policies, quality management plan, SOPs, working instructions, conventions, guidelines, forms, templates, logs, tags and labels. They are organized by consensus and approved by a nominated body and they provide for common and repeated use, rules, guidelines or characteristics for activities or their results with a view to promote transparency, consistency, reproducibility, interchangeability and to facilitate communication. The hierarchy and types of quality documents relevant to quality systems will depend upon Company business objectives and business model. SOPs are Level 2 quality documents and, along with other related quality documents, guarantee the efficacy and effectiveness of quality systems (Manghani, K. 2011). Standard operating procedures (SOPs) are a vital component in any quality management system (Hattamer-Apostel, R. 2001). Every good quality system is based on its Standard Operating Procedures (SOPs) (Saxena). The advancement and use of SOPs are a necessary part of a successful quality system as it supplies individuals with the information to carry out a job adequately, and aids precision in the quality and integrity of a product or end-result (United States Environmental Protection Agency, 2007). They assign all processes involved in an organization (Frank, D. 2010). A quality system is defined as the organizational structure, responsibilities, processes, procedures and resources for implementing quality management (Manghani, K. 2011).

Standard Operating Procedures are sets of instructions having the force of a directive, covering those features of operations which lend themselves to a definite or standardized procedure without loss of effectiveness (Saxena).

The purpose of a SOP is to reach out the operations correctly and always in the same manner. A SOP should be available at the place where the work is done". SOPs assist the progress of constant application of processes and procedures so even when there are changes in personnel, organizations avoid inconsistencies and safety risks (Frank, D. 2010). Standard operating procedures or SOPs are written step-by-step procedures that quality control $(\mathrm{QC})$, quality assurance (QA), and production units use in order to assure the accuracy and precision of the quantitative experimental results and materials that they generate and provide in support of other units. SOP's are needed to guarantee the continuity of processes to obtain quality performance and quality products/preparations (Natural Resources Management and Environment Dept.). SOP's are alive documents that detail written instructions describing specific steps to follow in all activities under defined conditions (Jain, SK. 2008). They are used to accomplish standardization when performing specific functions and is used to set out the way practice and procedures necessitated to be performed. SOPs are written instructions and records of procedures agreed and adopted as standard practice (Cardiff University, 2009). SOP's are necessary to guarantee the progression of processes to accomplish quality performance and quality products/preparations (Jain, SK. 2008). A Standard Operating Procedure (SOP) document is a routine or repetitive activity followed by an organization. SOPs describe both technical and administrative operational elements of an organization that would be managed under a Quality Assurance Project Plan and under an organization's Quality Management Plan (Almeida S.L.), (United States Environmental Protection Agency 2001). 
SOPs are determined to be specific to the organization whose activities are defined and assist that organization to maintain their quality control and quality assurance processes (United States Environmental Protection Agency 2001).

All organizations, businesses, etc. should have SOPs (Jain, SK. 2008). SOPs support employees with the information necessitated to perform their jobs regularly and help guarantee consistency in the quality of performance (Frank, D. 2010). SOPs are used by the governmental agencies, private industry, and academic laboratories by scientists and engineers from all of the science, technology, engineering, and mathematical disciplines. SOPs can also be intensely valuable in academic laboratories and can be employed anytime there is process that likely more than one person will use in a research group (Natural Resources Management and Environment Dept.). SOPs are mainly associated with specific documentation necessities. It should not be forgotten that "If you don't document, it didn't happen! (Jain, SK. 2008). The International Conference on Harmonization Good Clinical Practice (ICH GCP) guideline ascertaines SOPs as "detailed, written instructions to achieve uniformity of the performance of a specific function". SOPs must be well written in order to supply an efficacious control of good clinical practice (GCP) and prevent errors from occurring, thereby lessening waste and rework. Poorly written SOPs are a source of misinformation. To be user friendly, they should be absolute, unambiguous and must be written in plain language. SOPs are controlled documents and are best written by persons involved in the activity, process or function that is required to be specified or covered in the SOP. SOPs must be reviewed prior to their approval for release, for adequacy, completeness and compliance with Company standards and all applicable legal, ethical and regulatory requirements. They must be checked out and updated as necessitated over their life cycle and any changes made to the SOPs must be re-approved. They must bear a revision status on them and their distribution must continually be documented and controlled. When obsolete SOPs are needed to be hold for any purpose, they should be suitably identified to prevent unintended use. Only relevant SOPs in their current version must be available at points of use and must remain legible. SOPs are mandatory for the implementation of GCP and other GxPs, namely, cGMP (Good Manufacturing Practice) and GLP (Good Laboratory Practice), within the scope of quality systems; therefore, it is well said that without SOPs there are no GxPs: no SOPs, no quality systems, and no GxPs (Manghani, K. 2011). SOPs are necessary for a clinical research organization whether it concerns a pharmaceutical company, a sponsor, a contract research organization, an investigator site, an Ethics Committee or any other party involved in clinical research to achieve maximum safety and efficiency of the performed clinical research Operatings. It is therefore a must that all people and sites involved in clinical studies (both at the sponsor and at the investigative sites) have suitable SOPs in place so as to conduct clinical research and to ensure compliance with the current regulations.

The presence of these quality documents is important when regulatory inspections (FDA, EMEA) take place since the most frequent reported deficiencies during inspections are the lack of written SOPs and/or the failure to adhere to them. The risk of GMP non-compliance is high at organizations with a poor suitability of specific SOPs and also if at all they are 
achiavable the staff or the people for whom they were written are not either following them. It therefore becomes very essential for the personnel to be trained on these SOPs so that they are absolutely aware of why and how SOPs can play important role in fulfilling the specific organizatory requirements from WHO, FDA, EMEA or other national health authorities. Health authorities world wide like the FDA or EMEA expect pharmaceutical, cosmetic and food producers to describe their manufacturing processes in written SOPs (GMP7.com). An organization's SOP manual is an important training document and provides workers with increased confidence, motivation and a sense of achievement (Frank, D. 2010). A SOP is a compulsory instruction. If deviations from this instruction are allowed, the conditions for these should be documented including who can give permission for this and what exactly the complete procedure will be. The original should rest at a secure place while working copies should be authenticated with stamps and/or signatures of authorized persons. The advancement and use of SOPS are a basic part of a successful quality system. It supplies information to perform a job regularly, and constantly in order to access pre-determined specification and quality end-result.

SOP clarifies the followings; what is the objective of SOP (Purpose), what are applicability and use of SOP (Scope)?, who will perform tasks (Responsibility), who will ensure implementation of procedure (Accountability), how tasks will be performed (Procedure).

\begin{tabular}{|c|c|}
\hline Responsibility & Responsible \\
\hline $\begin{array}{l}\text { Identifying the need for development or revision of a standard } \\
\text { operating procedure (SOP) and to convey that need to their } \\
\text { immediate supervisor and/or the QA Manager (QAM). }\end{array}$ & Staff \\
\hline $\begin{array}{l}\text { An individual SOP to include sufficient detail that the process or } \\
\text { procedure can be followed by another person when needed. }\end{array}$ & Author \\
\hline $\begin{array}{l}\text { Requesting peers to review the SOP to determine whether it } \\
\text { contains sufficient detail. }\end{array}$ & Author \\
\hline Reviewing and approving the SOP prior to its use. & $\begin{array}{l}\text { Immediate supervisor and the QA } \\
\text { Manager }\end{array}$ \\
\hline $\begin{array}{l}\text { Ensuring that the procedure or process follows the details noted } \\
\text { in the individual SOP and to detail in writing when the SOP or a } \\
\text { component of that SOP has not been followed. }\end{array}$ & Staff and the QAM \\
\hline $\begin{array}{l}\text { Ensuring that all routine operations and activities in their area } \\
\text { are documented by SOPs. }\end{array}$ & Manager \\
\hline $\begin{array}{l}\text { Overseeing the appropriate preparation, numbering, retention, } \\
\text { indexing, revision, and use of SOPs. }\end{array}$ & QAM \\
\hline
\end{tabular}

Table 1. Responsibility distribution in SOP. 
Procedures are not an end in themselves - they do not ensure good performance or results. More important are well-designed systems and processes, qualified employees, and a motivating company culture. Procedures provide process people - environment but do not create processes, qualified people, or a good working environment (Jain, SK. 2008). The responsibility distribution in a SOP is shown in Table 1.

\section{Purpose}

The purpose of SOP is to assign the procedures for the preparation, approval, distribution, amendment and storage of Standard Operating Procedures (Cardiff University, 2009). The purpose or objective of the procedure should express and expand well written title (Jain SK., 2008). SOPs serve as frame for organizational action - support direction and structure. They tell what, how, when, why, and who. (Iowa State University, 2010). In order to be active, SOPs need to define not only what needs to be, but who is qualified to carry it out, and under what conditions the procedure can be performed reliably (Levine D.I., 2010). They should aid constant conformance support data quality. They should be determined to be specific to the organization and assist that organization to obtain their quality control and quality assurance processes and ensure compliance (Almeida S.L.) SOPs specify the commonly recurring work processes that are to be conducted or followed inside an arrangement. They approve the way activities are to be performed to alleviate constant conformance to technical and quality system necessities and to provide data quality. They may define, for example, basic programmatic actions and technical actions such as analytical processes, and procedures for maintaining, calibrating, and using equipment. If not written appropriately, SOPs are of limited value. Additionally, the best written SOPs will fail if they are not followed. Therefore, the use of SOPs needs to be checked out and re-enforced by management, alternatively the direct supervisor. Current copies of the SOPs also need to be readily accessible for reference in the work areas of those individuals absolutely carrying out the activity, either in hard copy or electronic format, otherwise SOPs serve little purpose (United States Environmental Protection Agency, 2007).

\section{Benefits}

The improvement and use of SOPs promotes quality through consistent implementation of a process or procedure within the organization reduced work effort, along with advanced data comparability, credibility, and legal defensibility (Almeida S.L.) The details in an SOP standardize the process and support step-by-step how-to instructions that enable anyone within your operation to perform the task in a consistent manner (Iowa State University, 2010). They abbreviate difference and advance quality through constant impact of a process or procedure inside the organization, although there are temporary or permanent personnel changes. 
SOPs can signify agreement with organizational and governmental needs and can be used as a part of a personnel training program, since they should supply detailed work instructions. It minimizes opportunities for miscommunication and can address safety concerns.

When historical data are being estimated for current use, SOPs can also be very important for reconstructing project activities when no other references are accessible. Besides, SOPs are commonly used as checklists by inspectors when auditing procedures. Eventually, the benefits of a valid SOP are decreased work effort, along with developed comparability, credibility, and legal defensibility.

The advancement and use of SOPs is a basic part of a successful quality system. It supplies individuals with the information to perform a job regularly and aids constancy in the quality and integrity of a product or end-result through constant implementation of a process or procedure inside the arrangement.

SOPs can also be used as a part of a personnel training program, hence they should support detailed work instructions. When historical data are being assessed for current use, SOPs can be beneficial for reconstructing project activities. Additionally, SOPs are commonly used as checklists by inspectors when auditing procedures. Finally, the benefits of a valid SOP are minimized work effort, together with improved data comparability, credibility, and legal defensibility. SOPs are necessary even when published methods are being administered because cited published methods may not include appropriate information for conducting the procedure in-house.

For example, if the SOP is written for a standard analytical method, the SOP should designate the procedures to be followed in greater detail than appear in the published method, detailing how, if at all, the SOP differs from the standard method and any options, changes or alterations that the organization follows (United States Environmental Protection Agency, 2007). The significance regularly set up and managed quality control and quality assurance systems with their integral well-written SOPs and other quality documents for the achievement of Company business objectives cannot be ignored. They serve as a passport to success by assisting the Company to accomplish high-quality processes, procedures, systems, and people, with eventual high-quality products and services and enhancement of the following: Customer satisfaction, and therefore, customer loyalty and repeat business and referral; timely registration of drugs by eliminating waste and the requirement for rework; operational results such as revenue, profitability, market share and export opportunities; alignment of processes with achievement of better results; understanding and motivation of employees toward the Company quality policy and business objectives, as well as participation in continuous quality improvement initiatives; and confidence of interested parties in the effectiveness and efficiency of the Company as demonstrated by the financial and social gains from Company performance and reputation (Manghani, K. 2011). Benefits of SOPs are shown in Table 2. 


\section{Benefit}

To provide people with all the safety, health, environmental and functional information necessitated to perform a job properly.

To guarantee that production
operations are performed constantly to obtain quality control of processes and products.

To guarantee that processes continue uninterrupted and are completed on a prescribed schedule.

To guarantee that no failures occur in manufacturing and other processes that would harm anyone in the surrounding community.

To guarantee that acknowledged procedures are followed in compliance with company and government regulations.

To serve as a training document for
teaching users about the process for which the SOP was written.

To serve as a checklist for co-workers who observe job performance to reinforce proper performance.

To serve as a checklist for auditors.

\section{Explanation}

Placing value only on production while disregarding safety, health and environment is costly finally. It is better to train employees in all aspects of doing a job than to face accidents, fines and litigation later

Consumers, from individuals to companies, want products of consistent quality and specifications. SOPs specify job steps that help standardize products and consequently quality.

By following SOPs, you help to guarantee against process shutdowns caused by equipment failure or other facility damage

Following health and environmental steps in SOPs guarantees against spills and emissions that threaten plant neighbors and create community outrage

Well-written SOPs help to gurantee that government regulations are satisfied. They also show a company's good-faith intention to operate perfectly. Failure to write and use good SOPs only signals government regulators that your company is not serious about compliance.

Thorough SOPs can be used as the basis for supplying standardized training for employees who are new to a particular job and for those who need re-training.

The process of actively caring about fellow workers involves one worker coaching another in all aspects of proper job performance. When the proper procedures are outlined in a good SOP, any coworker can coach another to help improve work skills.

Auditing job performance is a process similar to observation mentioned in the previous item only it usually involves record keeping. SOPs should serve as a strong basis when detailed audit checklists are developed.

To serve as an historical record of the As people move from job to job inside and between companies, how, why and when of steps in an existing process so there is a factual basis for revising those steps when a unwritten knowledge and skills disappear from the workplace. Regularly maintained written SOPs can chronicle the best knowledge that can serve new workers when older ones move on. process or equipment are changed.

To serve as an explanation of steps in a Although accidents are unfortunate, view them as opportunities to process so they can be reviewed in learn how to improve conditions. A good SOP gives you a basis accident investigations. from which to being investigating accidents

(Jain, SK. 2008)

Table 2. Benefits Of SOPs. 


\section{Writing style}

SOPs should be written in a step-by-step, easy-to-read format by subject-matter experts who know the processes and the structure of the organization (Frank, D. 2010). They should be written by individuals aware of the activity and the organization's internal structure. These individuals are basically subject-matter experts who actually perform the work or use the process. A team accession can also be followed, particularly for multi-tasked processes where the experiences of a number of individuals are critical (United States Environmental Protection Agency, 2007).

Well-written SOPs should first shortly define the purpose of the work or process, involving any regulatory information or standards that are suitable to the SOP process, and the scope to show what is covered. Any specialized or different terms either in a separate definition section or in the suitable discussion section should be explained.

The information presented should be clear and easy to understand. The active voice and present verb tense should be used. SOP shall be simple and short. Information should be transported clearly and absolutely to remove any doubt as to what is needed. Flow charts should be used to illustrate the process being defined (Jain SK., 2008), (United States Environmental Protection Agency, 2007). (United States Environmental Protection Agency, 2001), (Almeida S.L.). It may be helpful to include additional experts to help gather information and to review, test and approve draft SOPs (Frank D., 2010).

The most commonly used method of task analysis is Hierarchical Task Analysis (HTA). Operating instructions should be close to the user and kept up to date. The following issues should be considered in evaluating operating procedure documentation:

1. There should be no easier, more dangerous opportunities than following the procedure.

2. There should be an appropriate QA system in place to guarantee that the procedures can be kept up to date and that any errors are rapidly detected and corrected.

3. The procedures should not be needlessly prescriptive. The best way of guaranteeing that procedures do not become overly prescriptive is through involving the operator during the design stage.

4. Procedures should contain information on the necessities for the wearing of personal protective equipment during the task.

5. Any risks to the operator should be documented at the start of the procedure, based on a risk assessment of the task.

6. An appropriate method of coding each procedure should be used.

7. Each time a procedure is produced it should be dated.

8. There should be no uncertainty between which procedures apply to which situations.

9. Procedures do not always have to be paper based. 
10. At the start of the procedure an overview of the task should be provided.

11. Prerequisites should presented clearly at the start of the procedure to guarantee that the operator can check that it is safe to proceed.

12. The most important information on the page should be defined and this should be designed to be the most prominent information.

13. Separate headings should be used to discriminate apparently between sub tasks.

14. Any warnings, cautions or notes should be placed immediately prior to the instruction step to which they refer.

15. Language should be kept as simple as possible, i.e. use nomenclature familiar to the operator.

16. The nomenclature should be consistent with that on controls or panels.

17. Symbols, colours, and shapes used for graphics should conform to industry standards (Health and Safety Executive).

\section{Preparation of SOP}

When actualizing a SOP one can choose number of different ways to organize and format them. There are some factors which determine what type of SOP to use or create: How many decisions will user need to make during process? How many steps and sub steps are there in procedure? Routine procedures that are short and necessitate few decisions can be written using simple steps format. Long procedures consisting of more than 10 steps, with few decisions should be written along with graphical format or hierarchical steps. Procedures that necessitate many decisions should be written along with flow chart. Requirement for document identification and control, accountability and traceability responsibility must be involved with every SOP; this can be obtained by supporting constant format.

The need for an SOP or the revision of an existing one should be identified by informing the appropriate supervisor. Written instructions on standardized procedures supply guidance to guarantee that activities are conducted in a constant way, hence leading to reliable product and service quality. SOPs should be prepared in full compliance with guidelines and organizations and must mirror current organizational practices (Hattamer-Apostel, R. 2001). Ideally, SOP's should be written by teams that involve some or all of the following people: Those who will perform the job, those who will perform maintenance on equipment involved in an SOP, engineers or others who design equipment and processes, technical initiator, safety personnel, environmental personnel, equipment manufacturers (Jain, SK. 2008). 


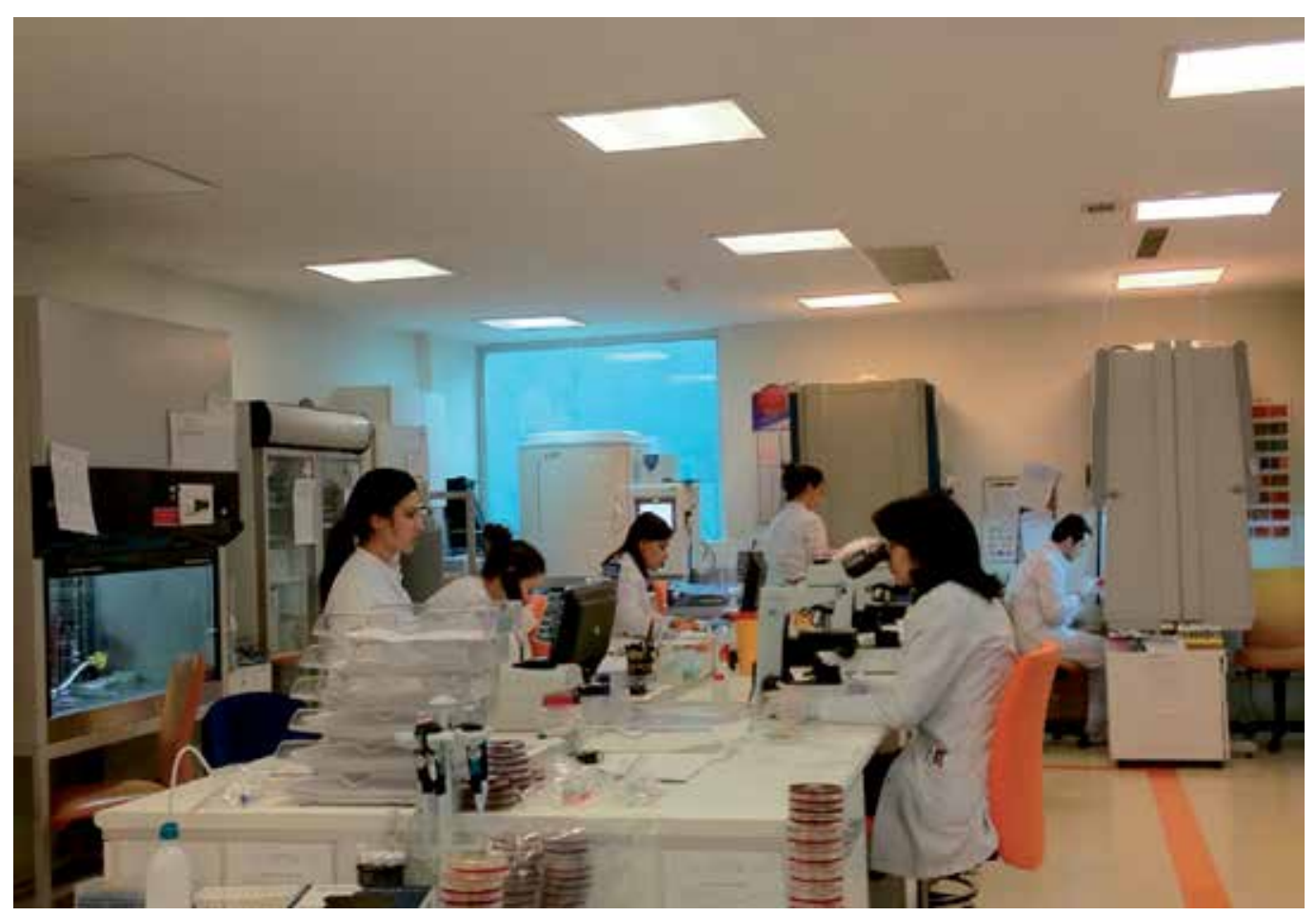

Figure 1. A SOP should be written by a team in that field.

\section{Implementing SOP}

The most substantial step for administering the SOP in working area, train or retrain the user. Every one should follow the procedure accurately with each and every step in detail, it is very significant to train the user otherwise individual may interpret meaning in different ways.

The trainer should share the reason WHY, SOP must performed correctly while training the user. People can follow better when they understand significance of procedure. Trainer should explain and demonstrate how each step in the SOP will be performed and should assure them this will increase Quality of product by providing safety and accuracy which will ultimately increase the confidence of the user.

The people in the writing team can write or edit parts of an SOP independently and then one person can combine the individual contributions. After combination the SOP should be circulated the draft SOP for review among the initiator before editing a final draft for review by supervisors and subsequent supervised testing by employees. Ideally a writing team should meet at least once in the beginning of a project to establish writing objectives, targets and responsibilities, but then can work semi-independently with one person serving as co- 
ordinator. SOPs should be checked out by several people qualified to assess the SOP in terms of its completeness and clarity of subject matter.

SOPs should at least mention:

a. who can or should make which type of SOP;

$b$. to whom proposals for a SOP should be submitted, and who estimates the draft;

c. the procedure of approval;

d. who decides on the date of implementation, and who should be informed;

e. how revisions can be made or how a SOP can be withdrawn.

It should be organized and recorded who is responsible for the proper distribution of the documents, the filing and administration (e.g. of the original and further copies). Finally, it should be indicated how frequently a valid SOP should be periodically evaluated (usually 2 years) and by whom. Only officially issued copies may be used, only then the use of the proper instruction is guaranteed (United States Environmental Protection Agency, 2007).

\section{SOP Review and approval}

SOPs should be reviewed (that is, validated) by one or more individuals with appropriate training and experience with the process (Almeida S.L.).

It is especially helpful if draft SOPs are completely tested by individuals other than the original writer before the SOPs are finalized. The completed SOPs then must be checked out and approved by peer reviewers, the QA Manager, and appropriate management prior to the use of the SOP. A set format in styling, information necessitated, and a numbering system is required, as well as biannual or annual review to ensure that the procedure is up-to-date. An archival system is needed to ensure that an historical record can be maintained and only current SOPs are available for staff use (United States Environmental Protection Agency, 2007).

The finalized SOPs should be approved as described in the organization's Quality Management Plan or its own SOP for preparation of SOPs. Generally the immediate supervisor, such as a section or branch chief, and the organization's quality assurance officer review and approve each SOP. Signature approval indicates that an SOP has been both reviewed and approved by management. When practical, use of electronic signatures, as well as electronic maintenance and submission, is an acceptable substitution for paper.

SOP general form defines an integrated system of management activities involving planning, implementation, documentation, assessment, and improvement to ensure that a process, or item, is of the type and quality needed for the project (United States Environmental Protection Agency, 2007), (Jain, SK. 2008). 


\section{Revising SOPS}

If the SOP does not definitely describe the procedure, then the SOP must be revised. Any change in the procedure must be included into the SOP. Nevertheless, prior to any change to the SOP, management must be advised of, and approve, the change.

If there are some errors in the finalized SOPs, such as typographical errors, printing errors, e.g., wrong page numbers or misaligned sentences) or any errors that do not affect the scope of the procedure, they may be correctly immediately and reprinted. These types of errors do not require full SOP revision, thus a revision number will not be generated and management approval is not needed. If the error occurs on the signature page then the signature page will be resigned. These types of corrections will be traceable since the historical file will reflect all corrections including typographical errors. Specifically, the historical SOP file will contain both the SOP with the correct page(s) as well as the page(s) containing the error. The page with the error will not be removed from the historical file. Additions can be made to an SOP via a clarification or an addendum. Explanations and addenda must be attached to the appropriate SOP until such time that the SOP can be revised. Usually, the revision will be organized during the biannual review process. When the SOP is revised, the revision number is updated. Revisions, explanations, and addenda are prepared by appropriate personnel, but must be approved by management. An SOP can be eliminated when it is no longer applicable. Management must approve the elimination of an SOP. Two or more SOPs can be consolidated; in this case one SOP supersedes the other, but management approval is required for consolidation of procedures. The signed revised SOP must be sent to the historical file for archiving (United States Environmental Protection Agency, 2007).

\section{Frequency of revisions \& Reviews}

SOPs necessitate to remain current to be useful. The review process should not be overly cumbersome to encourage timely review. Therefore, whenever procedures are changed, SOPs should be updated and re-approved. If desired, only the pertinent section of an SOP can be modified and indicate the change date/revision number for that section in the Table of Contents and the document control notation.

SOPs should also be reviewed systematically on a periodic basis, e.g. every 1-2 years, to ensure that the policies and procedures remain current and suitable, or to decide whether the SOPs are even needed. The review date should be added to each SOP that has been reviewed. If an SOP defines a process that is no longer followed, it should be removed from the current file and archived (Almeida S.L.) The frequency of review should be indicated by management in the organization's Quality (Jain, SK., 2008). 


\section{Checklists}

SOPs should describe how the checklist is to be prepared or on what it is to be based (Almeida S.L.)

Many activities use checklists to guarantee that steps are followed in order. Checklists are also used to document completed actions. Any checklists or forms involved as part of an activity should be referenced at the points in the procedure where they are to be used and then attached to the SOP (United States Environmental Protection Agency, 2007).

In some cases, detailed checklists are prepared specifically for a given activity. In those cases, the SOP should describe, at least generally, how the checklist is to be prepared, or on what it is to be based. Copies of specific checklists should be then maintained in the file with the activity results and/or with the SOP.

Many activities use checklists to guarantee that steps are followed in order. Checklists are also used to document completed actions. Any checklists or forms involved as part of an activity should be referenced at the points in the procedure where they are to be used and then attached to the SOP. In some cases, detailed checklists are prepared specifically for a given activity. In those cases, the SOP should describe, at least generally, how the checklist is to be prepared, or on what it is to be based. Copies of specific checklists should be then maintained in the file with the activity results and/or with the SOP. Remember that the checklist is not the SOP, but a part of the SOP (Jain SK., 2008).

\section{Document Control}

Each organization should develop a numbering system to systematically identify and label their SOPs, and the document control should be described in its Quality Management Plan. Usually, each page of an SOP should have control documentation notation. A short title and identification (ID) number can serve as a reference designation. The revision number and date are very useful in identifying the SOP in use when reviewing historical data and is critical when the requirement for observable records is included and when the activity is being reviewed (United States Environmental Protection Agency, 2007).

\section{SOP Document Tracking and Archival}

The organization should sustain a master list of all SOPs. This file or database should show the SOP number, version number, date of issuance, title, author, status, organizational division, branch, section, and any historical information regarding past versions. The QA Manager (or designee) is usually the individual responsible for sustaining a file listing all current quality-related SOPs used inside the organization. If an electronic database is used, automatic "Review SOP" notices can be sent. Note that this list may be used also when audits are being considered or when questions are raised as to practices being followed within the organization. 
The Quality Management Plan should indicate the individual (s) responsible for assuring that only the current version is used. That plan should also designate where, and how, outdated versions are to be maintained or archived in a manner to prevent their continued use, as well as to be available for historical data review.

Electronic storage and retrieval mechanisms are generally easier to access than a hard-copy document format. For the user, electronic access can be limited to a read-only format, thereby protecting against unauthorized changes made to the document (United States Environmental Protection Agency, 2007).

\section{SOP General Format}

This term describes an integrated system of management activities involving planning, implementation, documentation, assessment, and improvement to ensure that a process, or item, is of the type and quality needed for the project (Levine, D.I. 2010).

How should a SOP be organized? A SOP should be organized to ensure ease and efficiency in use and to be specific to the organization which develops it. There is no one 'correct' format; and internal formatting will vary with each organization and with the type of SOP being written.

How much detail needs to be included in a SOP? A SOP should be written with sufficient detail so that someone with a basic understanding of the field, can successfully reproduce the activity or procedure when unsupervised (United States Environmental Protection Agency, 2007).

The QA systems in place will be covered in general by 'standard operating procedures' (SOP) and will be made up of the following essential components:

SOPs should be organized to guarantee ease and efficiency in use and to be specific to the organization which develops it. There is no one "correct" format; and internal formatting will vary with each organization and with the type of SOP being written. Where possible break the information into a series of logical steps to avoid a long list. The level of detail provided in the SOP may differ based on, e.g., whether the process is critical, the frequency of that procedure being followed, the number of people who will use the SOP, and where training is not routinely available. A generalized format is discussed next (Levine, D.I. 2010).

Organization shall have SOP on Preparation, approval, revision and control of standard Operating Procedure for more excellent control and management of SOPs. Before finalizing and distributing SOPs, organizations should get the documentation reviewed and validated by people with training and experience on the processes. If the SOP does not definitely define the procedure, then the SOP must be revised. Any change in the procedure must be included into the SOP. After all, prior to any change to the SOP, management must be advised of, and approve, the change. Finalized SOPs, containing typographical errors, printing errors, e.g., wrong page numbers or misaligned sentences) or any errors that do not act on the scope of the procedure may be corrected immediately and reprinted.

An organization's SOPs should be written in a format that is tailored to the organization type and its unique requirements. In general, administrative/programmatic SOPs will consist of five elements: Title page, Table of Contents, Purpose, Procedures, Quality Assurance/ Quality Control, and Reference (Frank, D. 2010). General SOP format is shown in Table 3. 


\section{Element Explanation}

Title page The SOP should be arranged to guarantee ease and efficiency in use and to be specific to the organization (Almeida S.L.) Each SOP produced will be issued with a unique SOP number for reference purposes. This will be located in the table on the front page and in the footer of the document. This number will state where the SOP originated, the year it was produced, the SOP number and also state the version number. The SOP reference and effective date should be included in the footer on each page of the SOP. The first page or cover page of each SOP should contain the following information: a title that clearly identifies the activity or procedure, an SOP identification (ID) number, date of issue and/or revision, the name of the applicable agency, division, and/or branch to which this SOP applies, and the signatures and signature dates of those individuals who prepared and approved the SOP. Electronic signatures are satisfactory for SOPs obtained on a computerized database. (Jain SK., 2008), (United States Environmental Protection Agency, 2001), (Almeida S.L.), (Frank D., 2010). The Author shall be the individual primarily responsible for writing the SOP.

Chapter pages: Chapter pages can help divide content by area or task type. Chapter pages serve as mini title pages introducing each section and indicate dates for the most recent revisions.

Title - a clear, brief title describing the aim of the SOP and the conditions under which it can be accurately used (Levine D.I., 2010). The title should use directive language to declare what is being done to what (United States Environmental Protection Agency, 2007). Each SOP should be given a unique name which captures the significance of the practice described (Levine D.I. ,2010).

Table of Table of contents is not required if SOP is three pages or less.

Contents A table of contents may be necessitated for quick reference, particularly if the SOP is long, for locating information and to designate changes or revisions made only to certain sections of an SOP. Denotes changes or revisions made only to certain sections of a SOP (Almeida S.L.), (United States Environmental Protection Agency, 2007).

Definitions There should be a part defining any words, phrases, or acronyms having special meaning or application (United States Environmental Protection Agency 2001).

Purpose Each chapter should first briefly describe the purpose of the work or process, including any regulatory information or standards that are appropriate to the process (Levine, D.I. 2010). It is recommended to include criteria for the control of the described system during operation.

Procedures In general there are four major types of procedure: Procedures that supply general operating guidance; an aid to meeting operating aims; mandatory and prescribe behaviour; and used as a training tool.

The key to any program striving for quality is the set of Standard Operating Procedures (SOPs) that describe how work is to be done. The procedure section will identify how the aims will be achieved. This will clearly indicate a step by step description of how the procedure to be followed. Steps should include products and equipment required, possible obstacles, personnel qualifications and safety considerations. For lengthy process descriptions, a flow chart might be necessary to define processes that often involve interferences or variances. 
Procedures If calculations are involved in analyzing the data, then an example of the calculation should be

(continued) provided. Figures and tables showing laboratory apparatus, representative data, etc. can be included here (Levine, D.I. 2010).

Once the necessity for a particular SOP is organized, it should be drafted immediately. SOPs are drafted by laboratory or supervisory staff qualified to perform the procedure. Next the SOP is reviewed by other staff, where possible, and then approved by the QA Manager (QAM) and management, such as immediate supervisor. Circulation to staff members for review/comment is advisable prior to acquiring management approval. The SOPs should be written to define study methods or procedures in sufficient detail so as to guarantee the quality and integrity of the data or procedure to be followed. When writing SOPs, the detail used may include both procedural requirements (exact instructions) and guidance information (general information) on the procedure. Procedural requirements must be followed accurately, while guidance information is used to help perform the procedure; it is not a mandatory requirement and, therefore, it does not have to be followed exactly. Procedural requirements can be distinguished from guidance elements, based on the context they are used. Office standard format for margins, font, and font size should be followed. Official SOPs will have a colored header and footer on each page, dated signatures on the front title page, and be printed on ivory colored paper with a watermark. An outline format should be used and include alpha and/or numeric characters are to be included to indicate levels of information (United States Environmental Protection Agency, 2007).

A SOP should be written as soon as the need for a standard written procedure for an activity is required (Cardiff University, 2009). How much someone knows about an entire process or job affects the way he or she does that job. Incorporate safety, health and environment into the traditional how-to-operate or how-to-do steps. Based on best practice/standards, the procedure should be written in specific detail to ensure that the procedure can be repeated in a reproducible fashion to include the order of steps that should be followed, the times allowed for each step (as needed) and the temperatures at which the steps are performed.

It should be kept in mind that many people do not read all the steps before starting on step one. ASOP should be written as long as necessary for a specific job. People tend to ignore long SOPs because they cannot remember more than 6 to 12 steps. If the SOP goes beyond 10 steps, the following solutions should be considered; The long SOPs should be broken into several logical subjob SOPs, an accompanying shortened SOP should be written that lists only the steps but not detailed explanations of those steps, and the long-form SOP should be made as a training document or manual to supplement the shorter sub-job SOPs mentioned earlier (Jain, SK. 2008).

All SOPs before implementation or after revision will be approved by the management committee before implementation. Previous versions of all documentation will be stored electronically, with only the current versions available in the biorepository file.

All SOPs will be checked out on an annual basis by the management committee. Protective equipment that should be worn by staff when performing the procedure described. A list of the equipment needed to perform the procedure. All materials and supplies should be recorded. The date the procedure was first introduced as well as the date of the most recent version. The date format should be based on the ddmmyyyy system where $d$ represents day, m represents month and $y$ represents year. 
Procedures Personnel Qualifications/Responsibilities (identifying any special qualifications users should have (continued) such as certification or training experience and/or any individual or positions having responsibility for the activity being described)(United States Environmental Protection Agency 2001)

Any related SOPs (of operations used in the present SOP); possible safety instructions should be added.

Generally there are four types of procedure: (Health and Safety Executive).

General operating guidance procedures

Procedures that an aid providing procedures to meeting operating aims

Mandatory and behaviour prescribing procedures.

Training tool procedures

Scope and Applicability-under what specific conditions can this protocol be used reliably; are there any known interferents or other limitations on the protocol's effective use?

Introduction-Appropriate background information on the system, methods, and instruments are used. The background section should plan the procedure and the specific aim of the SOP.

Materials and Supplies-There should be a list of any reagents involving names of suppliers used in this procedure. If the suppliers are obscure sources, a list of addresses and contact information should be supplied as well. Cautions - If there are some specific health and safety precautions they should be considered. For example, should gloves be worn? If so, what kind? How should spills, if they occur, be cleaned up? Are there any special procedures that should be followed in order to safely dispose of waste? (Levine, D.I. 2010). Some SOPs should be written for people who perform under different interpersonal circumstances, people who work alone, two or more people who work together as a team, for people who will control other people doing a job, for people who not familiar with rules generally understood by your employees (Jain, SK. 2008).

Well-written SOPs should first briefly describe the purpose of the work or process, including any regulatory information or standards that are appropriate to the SOP process, and the scope to indicate what is covered. Diagrams and flow charts should be used to help to break up long sections of text and to briefly summarize a series of steps for the reader(Almeida S.L.)

The age, education, knowledge, skill, experience and training, and work culture of the individuals should be considered who will be performing the SOP steps.

Criteria, checklists, or other standards should be applied during the procedure such as citing the document as guidance for reviewing SOPs Records Management (specifically,e.g., as forms to be used and locations of files.

Once writing of an SOP have been completed, there should be several workers test it and give you feedback.

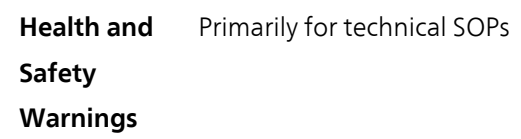


Quality The preparation of appropriate QC procedures (self-checks, such as calibrations, recounting, Assurance/ reidentification) and QC material (such as blanks - rinsate, trip, field, or method; replicates; splits; Quality spikes; and performance evaluation samples) that are needed to display successful performance of Control the method should be defined.

All SOPs should be checked out annually to certify all SOPs are in line with current processes, guidelines and regulations. They should be checked out in regards with the review date assigned and recorded on the front page of each SOP. The designated individuals will approve all SOP amendments. All significant amendments will be required 2 months in advance of the annual review. Any control steps and provisions for review or oversight should be defined prior to acceptance of the product or deliverable. This can involve test plans such as verification and validation plans for software or running a "spell-check" program on the finished document (United States Environmental Protection Agency 2001).

Finally, next all appropriate QA and quality control (QC) activities for that procedure should be defined, and list any cited or significant references (United States Environmental Protection Agency, 2007). Before finalizing and distributing SOPs, organizations must get the documentation reviewed and validated by people with training and experience on the processes. Additionally, it is a good idea to have the SOPs tested by staff who will be asked to comply with them. By following these steps, the author can identify missing information or needed revisions. Once SOPs are approved, they should be made readily available to facility management, building occupants and cleaning employees. The SOP will require final approval and authorization. The signature on an SOP will authoise the associated forms which should show an identical issue date to the SOP. When an SOP is issued and become effective, adequate time is required for training purposes. Finally, SOPs must remain current, so they should be updated and re-approved at least annually or whenever procedures change. Though the SOP development process takes time and effort, it can provide significant improvement to a cleaning organization's operational results and workers' understanding and job performance (Frank, D. 2010).

All SOPs are reviewed by the applicable supervisor at least every two years in order to maintain their relevancy. Names of those individuals who have reviewed and approved the SOP for use in the laboratory. Signatures and dates should be supplied whenever possible as well. For those SOPs which do not necessitate a revision, documentation attesting to that fact must be submitted to the QAM who in turn initials and dates the table located at the bottom of the title page of the original SOP (United States Environmental Protection Agency, 2007). All SOPs require version control to ensure that individuals are using the correct verision of SOP. It is good to practice to assign a document a version number, in the format $\mathrm{N}$. $\mathrm{n}$ where $\mathrm{N}$ represents a finalized document and $\mathrm{n}$ represents draft versions. Each new, approved and finalized document a major verison number.... should be assigned. When taking a document for revision or as draft, assign a new minor version. During the review cycle assign each new revision of the draft the next minor version, upon approval/finalization of the document assign the next major version (United States Environmental Protection Agency 2001), (Natural Resources Management and Environment Dept.).

References References relating to the development of the SOP are required to be listed. These may include other SOPs, regulatory guidelines and published papers etc. Documents listed in the SOP must be recorded in the appendices and listed accordingly (United States Environmental Protection Agency 2001). 
Contact list It should involve contact details for relevant individuals such as author of document.

Appendices This section should list appendices of other SOPs referenced in the document, or related to the procedure.

Distribution Once approved the original paper SOP folder, it will also contain supporting documentation relating to each approved SOP for referencing purposes. An electronic copy of the SOP will be held. Approved SOPs will be distributed in hard copy to PDs and will be published. The paper version of the abandoned SOP will be filled into the archived SOP folder. All SOPs will be checked out and approved annually before it is superseded, unless a specific reason for a 6 month review can be justified. All SOPs must be kept for the duration of the project.

When the Sop fulfils all the necessary requirements it is printed. The author hands over the manuscript (or the floppy disk with text) to the SOP administrator who is responsible for the printing. The number of copies is decided by him/her and the author. Copying SOPs is forbidden. Extra copies can be obtained from the SOP administrator. The author (or his successor) signs all copies in the presence of the administrator before distribution. As the new copies are distributed the old ones (if there was one) are taken in. For each SOP a list of holders is made. The holder signs for receipt of a copy. The list is kept with the spare copies. Users are responsible for proper keeping of the SOPs. If necessary, copies can be protected by a cover or foil, and/or be kept in a loose-leaf binding.

Appropriate SOPs will be placed in green binders to be found in a designated spot in each work area, e.g., laboratory, equipment rooms, the library, etc., and shall be available to staff and managers. These binders will not be located in the supervisor's office. Removal of an individual SOP requires completion of the sign-out located on the insider of the binder. The binder must not be removed from its designated spot by anyone other than the QAM or laboratory director. It is the responsibility of the QAM to update each binder as individual SOPs are revised. The staff is required to read any revised SOP within 7 working days of issuance if the SOP is applicable to their work. Reading of the updated SOP requires signature on the SOP review sheet (United States Environmental Protection Agency, 2007).

Archiving Proper archiving is essential for good administration of SOPs. All operating instructions should be kept up-to-date and be accesible to personnel. Good Laboratory Practice requires that all documentation pertaining to a test or investigation should be kept for a certain period. SOPs belong to this documentation. An historical file is created for each SOP that is approved by management and will be maintained in the company's archives by the QAM. The historical file will consist of the original signed SOP and all subsequent modifications thereof. Official SOPs will have both colored header and footer lines, and be printed on watermarked ivory colored paper. All copies of the original will be black and white, initialed, numbered, and placed in the appropriate binder located in each office. If a procedure is incorporated into another SOP (superseded), a copy of the superseded version is placed in the historical file of both SOPs (United States Environmental Protection Agency, 2007).

Table 3. General SOP Format. 


\section{Types of SOP}

Several categories and types of SOPs can be distinguished. The name "SOP" may not always be appropriate, e.g., the description of situations or other matters may better designated protocols, instructions or simply registration forms. Also worksheets belonging to an analytical procedure have to be standardized (to avoid jotting down readings and calculations on odd pieces of paper) (Almeida S.L.)

Some of the most important SOP types:

- Fundamental SOPs. These give instructions how to make SOPs of the other categories.

- Methodic SOPs. These describe a complete testing system or method of investigation.

- SOPs for safety precautions

- Standard procedures for operating instruments, apparatus and other equipment.

- SOPs for analytical methods.

- SOPs for the preparation of reagents.

- SOPs for receiving and registration of samples.

- SOPs for Quality Assurance.

- SOPs for archiving and how to deal with complaints.

Generally the SOPs may be written for any repetitive technical activity, as well as for any administrative procedure (Almeida S.L.).

SOPs may be written for any repetitive technical activity, as well as for any authoritative or functional programmatic procedure, that is being followed inside an organization. General guidance for preparing both technical and administrative SOPs follows and examples of each are located in the Appendix (United States Environmental Protection Agency, 2007).

\section{Guidelines for Technical SOP Text}

Technical SOP and Administrative SOP are typical structures of SOPs. Technical and administrative SOPs need to involve the specific steps aimed at initiating, coordinating, and recording and/or reporting the results of the activity, and should be tailored only to that activity.

A technical SOP is a standard operating procedure which involves environmental data generation, manipulation, or accumulation, e.g., an analytical process. Technical SOPs can be written for a wide variety of activities.

Examples are SOPs instructing the user how to perform a specific analytical method to be followed in the laboratory or field (such as field testing using an immunoassay kit), or how to collect a sample in order to preserve the sample integrity and representativeness (such as collection of samples for future analysis of volatile organic compounds or trace metals), or how to conduct a bioassessment of a freshwater site. Technical SOPs are also needed to cover ac- 
tivities such as data processing and evaluation (including verification and validation), modeling, risk assessment, and auditing of equipment operation. Citing published methods in SOPs is not always acceptable, because cited published methods may not contain pertinent information for conducting the procedure-in-house. Technical SOPs need to include the specific steps aimed at initiating, coordinating, and recording and/or reporting the results of the activity, and should be tailored only to that activity. Technical SOPs should fit within the framework presented here, but this format can be modified, reduced, or expanded as required.

\section{Guidelines for Administrative or Fundamental Programmatic SOP}

An administrative SOP is a standard operating procedure which does not include environmental data manipulation activities, e.g., how to conduct an inspection. As with the technical SOPs, these SOPs can be written for a wide variety of activities, e.g., reviewing documentation such as contracts, QA Project Plans and Quality Management Plans; inspecting (auditing) the work of others; determining organizational training needs; developing information on records maintenance; validating data packages; or describing office correspondence procedures.

Administrative SOPs need to include a number of specific steps aimed at initiating the activity, coordinating the activity, and recording and/or reporting the results of the activity, tailored to that activity. For example, audit or assessment SOPs should specify the authority for the assessment, how auditees are to be selected, what will be done with the results, and who is responsible for corrective action. Administrative SOPs should fit within the framework presented here, but this format can be modified, reduced, or expanded (United States Environmental Protection Agency, 2007).

\section{Conclusion}

Eventually, SOPs serve as a fundamental means of communication for all levels of the organization. Not only do they include employees departmentally, but they also allow management and employees to gain a cross-functional view of the organization. This attitude encourages employees to think about how process change may affect other functional areas. A good system forces employee to think through processes and examine how procedure might influence product, personnel, production, and equipment. It should not be forgotten that the "Best written SOPs will fail if they are not followed" (Hattamer-Apostel, R. 2001), (Jain, SK. 2008).

What happens to workers' intrinsic task motivation and creativity when they are required to follow SOPs in completing their tasks? Job design and work motivation theory literatures have suggested a negative relationship; the OM literature has suggested a positive relation. We suggest that the discussion has been hindered by differences in conceptualizing required SOP use, by not explicitly incorporating the multidimensional nature of intrinsic motivation into the analysis, by an ambiguous definition of autonomy, and by ignoring important contextual moderators. When these three elements are included in the discussion, we showed that the relationship between required SOP use and intrinsic motivation could theoretically 
be positive. Finally, our model high- lights the importance of worker participation. Production pressures, high capacity utilization, and lack of management - especially supervisor support are likely to reduce opportunities for worker participation, and hence lower intrinsic motivation and creativity (De Trevil et al. 2005).

\section{Example SOP}

TITLE: Preparation of the Perfect Cup of Coffee by the Drip Method

Date of Preparation: 11/29/05; Date of Revision: N/A; Revision No.: N/A

\section{Submitted by: Ay Dot Student; Approved by: Professor Ex}

Purpose: Provide an example of a standard operating protocol or SOP that can be appreciated by undergraduate research students from all academic disciplines.

Scope and Applicability: The following protocol can be used wherever quality coffee beans, good drinking water, and a drip coffee maker are available.

Introduction: Coffee is the beverage of choice of many college students. Properly prepared the beverage provides an invigorating and revitalizing effect. One of the most frequently used methods of preparation is the drip method. In this method, water, heated to near boiling temperatures, is slowly added to finely ground coffee beans held in a filter unit. The coffee beverage is collected below the filter unit in a glass carafe. Today this procedure is frequently accomplished using a semi-automated process in an electronic coffee maker. The procedure below outlines a reliable method for preparing drip coffee using any commercially available drip coffee maker, high quality ground coffee beans, and filtered water.

References: For information on coffee beans, the standard methods of preparation of coffee, and recipes see:

Materials and Supplies: Freshly ground Starbucks ${ }^{\circledR}$ coffee (any flavor you prefer; medium grind works best with most commercial coffee makers), commercial 4-c drip coffee maker including filter (gold mesh preferred but high quality paper filter may be used), good quality drinking water (Polar Springs ${ }^{\circledR}$, Brita ${ }^{\circledR}$-filtered, or similar quality source recommended), coffee cup, and additives (as desired: sugar or sugar alterative, cream or milk).

Cautions: Hot coffee can scald and burn. Water is an electrical conductor. If spills occur during the brewing process, wait until the brewing process is complete, turn of the electricity, and disconnect the unit from the electricity before attempting to clean up any spills. Accidental spills may be cleaned up with a kitchen sponge and dish washing detergent such as ....®. Used coffee grounds can be disposed of in the regular trash. Be sure to carefully read the directions that accompanied your coffee maker unit before attempting to use it. In particular, it is important to find out if your unit has (1) a pause feature that will allow you to remove the carafe while the coffee is brewing; and (2) an auto-off feature that turns off the heater unit located beneath the carafe at a set time after the coffee has been brewed.

Personnel Qualifications: No special knowledge or training is required to make coffee. However, due to the potential risk of burns, it is recommended that anyone performing this procedure who is less than ten years old be actively supervised by an adult. 
Protocol

1. Make sure that the coffee maker is off. Locate water reservoir unit on coffee maker and carefully add 4-cups of clean drinking water to the reservoir. Note that the outside or inside of most quality coffee makers' water reservoir units are marked for the user's convenience.

2. Locate the coffee filter assembly on the coffee unit. If you are preparing the standard 4-c carafe of coffee, carefully measure one coffee measure of ground coffee into your units coffee filter assembly. Note that one standard coffee measure is equivalent to $1 / 8-c$ of coffee. Close the coffee filter assembly.

3. Plug in the coffee maker and turn the unit on. Wait until the carafe located beneath the coffee filter unit is filled with coffee. Note that some units may have a "pause" feature that will allow you to temporarily remove the carafe and pour a cup of coffee while the unit is working. If you are unfamiliar with your unit, be sure to wait until the unit is done filtering before attempting to remove the carafe.

4. If coffee spills beneath the base of the carafe unit, be sure to turn off the unit and disconnect the electricity before attempting to clean up the spill.

5. Pour yourself a cup of coffee. Most coffee units will keep the carafe warm for a set period of time before turning off automatically. Some however, do not turn off automatically. Be sure to read your coffee maker's instructions beforehand. If in doubt, be sure to turn off the electricity to your unit after the brewing process is complete. (Levine D.I et al, 2010)

\section{Author details}

Isin Akyar*

Address all correspondence to: isinakyar@gmail.com

Acibadem University Faculty of Medicine Department of Microbiology, Turkey

\section{References}

[1] Almeida-Lynne, Sherri. Guidance for Preparing Standard Operating Procedures ppt. Available at: http://www.google.com.tr/\#hl=tr\&safe=active\&output=search\&sclient =psy-ab\&q=Guidance+for+Preparing+Standard+Operating+Proced.

[2] Cardiff University (2009). Standard operating procedure for the use or storage of human tissue for the purposes of research or education. Available at: http:// www.cf.ac.uk/govrn/cocom/resources/standard\%20Operating\%20procedures.pdf.

[3] De Trevil, Suzanne., Antonakis, John., \& Edelson, Norman. (2005). Can Standard Operating Procedures be Motivating? Reconciling Process Variability Issues and Behavioural Outcomes. Total Quality Management, 16(2), 231-41. 
[4] Frank, Dave. (2010). How to write SOPs that help increase consistency and improve performance quality in Standard Operating Procedures: A Writing Guide. Available at: http://www.cmmonline.com/management-training/article/standard-operatingprocedures-a-writing-guide.

[5] GMP7.com (GMP online consultancy). What is a standard operating procedure? Available at: http://www.gmp7.com/whatisastandardoperatingprocedure_cm 380.html.

[6] Hattamer-Apostel, Rita. (2001). Standard operating procedures-a novel perspective. The Quality Assurance Journal, 5(4), 207-219, DOI:10.1002/qaj.155, available at:, http:// onlinelibrary.wiley.com/doi/10.1002/qaj.155/abstract?systemMessage=Wiley+Online +Library+will+be+disrupted+3+Mar+from+.

[7] Health and Safety Executive. Operating procedures. Available at: http:// www.hse.gov.uk/comah/sragtech/techmeasoperatio.htm.

[8] Iowa State University (2010). Hotel, restaurant and management extension. Available at: http://www.extension.iastate.edu/HRIM/HACCP/restaurants.htm.

[9] Jain, Sanjay Kumar. (2008). Standard operating procedures (SOP) - Back Bone of Pharmaceutical Industries. Pharma info.net Available at: http://www.pharmainfo.net/reviews/standard-operating-procedures-sop-back-bone-pharmaceutical-industries.

[10] Levine, David I., \& Toffel, Michael W. (2010). Quality management and job quality: How the ISO 9001 standard for quality management systems affects employees and employers. Journal Management Science, 56(6), 978-96.

[11] Manghani, Kishu. (2011). Quality assurance: Importance of systems and standard operating procedures. Perspect Clin Res., 2(1), 34-37, Doi: 10.4103/2229-3485.76288.

[12] Natural Resources Management and Environment Dept. Guidelines for quality management in soil and plant laboratories. FAO corporate document repository. Available at: http://www.fao.org/docrep/W7295E/w7295e04.htm.

[13] Saxena, Akanksha. SOP Writing for Clinical Trials: Staff Training Aspects. International Biopharmaceutical Association Publication. Available at: http://www.ibpassociation.org/IBPA_articles/sop_writing.htm.

[14] United States Environmental Protection Agency (2001). Guidance for Preparing Standard Operating Procedures (SOPs)." 10 Aug. 2010. United States Environmental Protection Agency. EPA QA/G-6. Available at: http://www.cluin.org/download/toolkit/thirdednew/guidanceprepsops.pdf.

[15] United States Environmental Protection Agency (2007). Guidance for Preparing Standard Operating Procedures (SOPs) EPA QA/G-6. Available at: http:// www.epa.gov/quality/qs-docs/g6-final.pdf. 

Section 8

Quality Control in Clinical Laboratory Medicine 

Chapter 18

\title{
Postmortem DNA: QC Considerations for Sequence and Dosage Analysis of Genes Implicated in Long QT Syndrome
}

\author{
Stella Lai, Renate Marquis-Nicholson, \\ Chuan-Ching Lan, Jennifer M. Love, Elaine Doherty, \\ Jonathan R. Skinner and Donald R. Love
}

Additional information is available at the end of the chapter

http://dx.doi.org/10.5772/51685

\section{Introduction}

Long QT syndrome is a rare disorder of cardiac ion channels, characterised by a prolonged QT interval and T-wave abnormalities on electrocardiogram (ECG) and the occurrence of the ventricular tachycardia torsade de pointes. Sodium, potassium or calcium channels present in heart muscle may be affected, altering the regulation of electrical current in the cells [1-3]. Individuals with this condition will be predisposed to cardiac events such as arrhythmias and polymorphic ventricular tachycardia, which may lead, if untreated, to sudden cardiac death $[2,3]$. Thirteen genes are associated with the condition, and hundreds of mutations have been identified [3-5]. Currently, more than $95 \%$ of the pathogenic mutations listed in disease databases (Gene Connection For the Heart, http://www.fsm.it/cardmoc/; online $\mathrm{Hu}-$ man Gene Mutation Database, www.hgmd.cf.ac.uk/) are sequence variants (including point mutations and small insertions or deletions), but the importance of whole or multi-exon deletions and duplications has more recently been recognised [6] and it is now recommended to use both sequence and dosage techniques in order to provide comprehensive analysis [3].

In New Zealand, the majority of specimens referred for Long QT syndrome diagnostic testing are retrieved after death. Postmortem specimens are often difficult to handle as they are usually either tissue samples or severely haemolysed blood. The extracted DNA is frequently of low quality, due to the presence of unwanted material such as short fragments produced by degradation and chemical modifications from oxidation and hydrolysis processes [7]. As a result, only short sequences can be reliably amplified [7]. Moreover, capillary-based 
sequencing, the gold standard first-line diagnostic test for Long QT syndrome, is very sensitive to the presence of contaminants, such as proteins, RNA and residual salt. The presence of such contaminants leads to poor quality electropherograms for analysis, which tend to be compromised by the presence of dye blobs, C-shoulders and a variable degree of baseline noise (Figure 1). Although the QC requirements for array comparative genomic hybridisation $(\mathrm{aCGH})$, which can be used to detect whole exon deletion and duplication mutations, are less stringent than those for sequencing, contamination or degradation of sample DNA can lead to suboptimal efficiency of labelling and hybridisation. Such difficulties arising in the practical procedure mean that the analysis of postmortem DNA can be time-consuming and challenging, and obtaining high quality data within a reasonable timeframe can be extremely difficult.
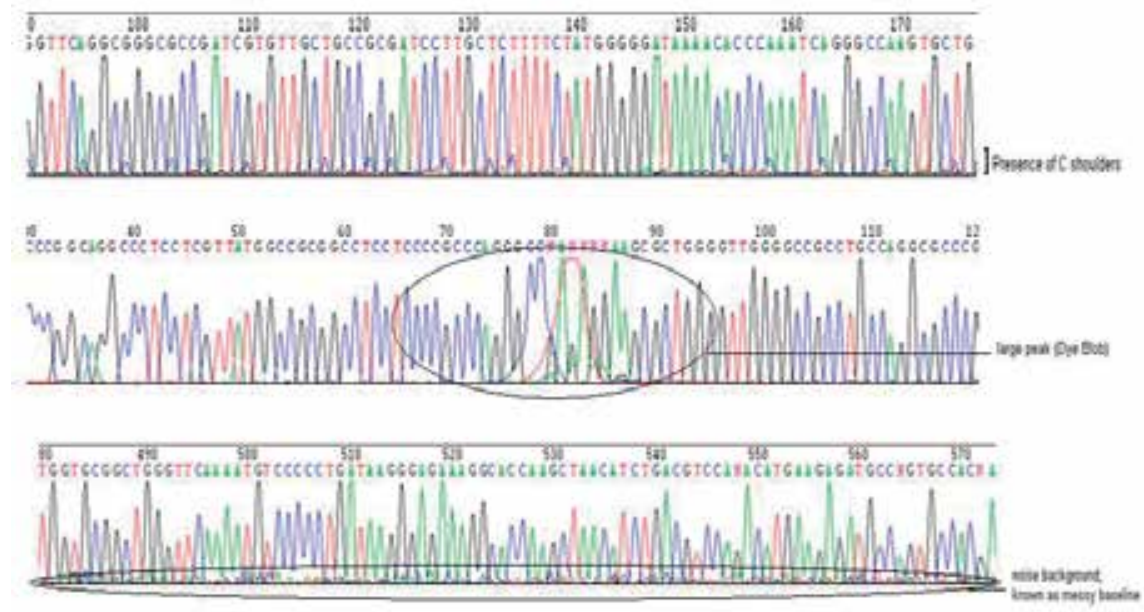

Figure 1. Electropherograms showing poor quality sequence data.

Long QT syndrome affects 1 in 2000 individuals [8] and contributes to 15-25\% of sudden unexplained death in 1-40 year olds $[9,10]$ and $10 \%$ of sudden unexplained deaths in infancy (SIDs) [11]. As a consequence, it is an important differential diagnosis to be considered in all cases of autopsy negative young sudden death. Molecular genetic testing is essential to make a postmortem diagnosis, given that screening for the electrocardiogram characteristics is no longer possible after death. Historically the turnaround time for diagnosis has been up to six months, due to the large number of genes to be analysed and the difficulties inherent in dealing with postmortem specimens (as detailed above). However, the demand by coroners for diagnostic laboratories to undertake more rapid analysis has been steadily increasing. A protocol tailored specifically to the treatment of postmortem specimens is necessary to meet this demand. Within our laboratory, we have successfully developed a robust process for sequence and dosage analysis of postmortem samples and have achieved an excellent turnaround time of 6-12 weeks. Here, we address the critical QC parameters that should be considered in order to obtain high quality data for rapid, accurate analysis. The discussion 
presented below concerns both sequence analysis and dosage analysis. The former uses conventional sequencing technology, while the latter involves the simultaneous high resolution screening of deletion and duplication mutations in multiple cardiac genes as opposed to the more conventional multiplex ligation dependent probe amplification (MLPA) technique, which many diagnostic laboratories still use [6].

\section{Materials and methods}

\subsection{Sequencing}

\subsubsection{Primer design}

We have used two approaches to design primers flanking each of the coding exons of the genes KCNQ1 (LQT1), KCNH2 (LQT2), SCN5A (LQT3), KCNE1 (LQT5), KCNE2 (LQT6), and KCNJ2 (LQT7), including at least 50 base pairs of the flanking intronic regions. The first used the primer design program called PrimerQuest (Integrated DNA Technologies Inc; http:// www.idtdna.com/Scitools/Applications/Primerquest/Advanced.aspx). This program allows the region in a sequence file to be specified, against which primers are designed to flank the targeted region. The designed primers for each exon are then checked in silico for annealing characteristics using the Macintosh-based program, Amplify. Finally, all primers were checked for single nucleotide polymorphisms (SNPs) using the software tool available from the National Genetic Reference Laboratory, Manchester (http://ngrl.man.ac.uk/SNPCheck/ SNPCheck.html). This bioinformatics program uses the current National Center for Biotechnology Information (NCBI) build of the human genome and the current release of the Single Nucleotide Polymorphism database (dbSNP) to identify the position in the sequence where the primers bind and to detect any known SNPs at these sites

Following the above approach, we developed an alternate design protocol [12,13]. Each mRNA sequence of interest was identified through the public UCSC genome browser page http://genome.ucsc.edu. This website provides a direct link to ExonPrimer for the design of primers specific to the mRNA sequence. ExonPrimer uses exon position information provided by the UCSC genome browser in combination with the primer design tool Primer3 to create primer pairs according to set parameters, while avoiding pairing to homologous regions within the genome. Exon and amplicon size are provided and multiple alternative primer sets are given. Following primer design, all primers were checked for single nucleotide polymorphisms (SNPs), as described above. Following a negative SNP check the primer sequences were evaluated using the UCSC genome browser to confirm the identification of single amplicons. Each primer was then tailed with an M13 sequence and manufactured by Integrated DNA Technologies Inc or Life Technologies. In this way, primers are designed to allow amplification of all exons of interest and the corresponding splice sites using a single set of PCR conditions such that PCR (and subsequent sequencing) can be performed under identical conditions within a 96-well PCR plate. 


\subsubsection{DNA extraction}

Genomic DNA (gDNA) was extracted from peripheral blood leucocytes (EDTA blood samples) using the Gentra Puregene DNA Extraction kit (Qiagen), according to the manufacturer's instructions.

A standard phenol/chloroform protease protocol was used to extract gDNA from postmortem tissue specimens. A small section $(2 \mathrm{~mm} \times 2 \mathrm{~mm} \times 4 \mathrm{~mm})$ is usually cut from frozen tissue and diced as finely as possible using a scalpel blade. The tissue is placed into a $1.5 \mathrm{ml}$ microcentrifuge tube with $450 \mu \mathrm{l}$ of $1 x$ TES Buffer (1M NaCl, $0.5 \mathrm{M}$ Tris- $\mathrm{HCl}, 10 \mathrm{mM}$ EDTA), $60 \mu \mathrm{l}$ of $20 \mu \mathrm{g} / \mu \mathrm{l}$ Proteinase K (Roche) and $10 \mu \mathrm{l}$ of $10 \%$ SDS, and incubated overnight with vigorous shaking. Following digestion, an equal volume of phenol is added and the sample is vortexed vigorously. Once homogenous, the sample is centrifuged to separate the layers and the top aqueous layer is removed and transferred to a fresh $1.5 \mathrm{ml}$ centrifuge tube. An equal volume of chloroform is then added to the aqueous layer. This is vortexed, centrifuged, and the aqueous (top) layer again transferred to a fresh tube. A $2 x$ volume of $100 \%$ ethanol is added to the aqueous layer to precipitate the DNA, followed by centrifugation at $13,000 \mathrm{rpm}$ and the supernatant is removed. The pellet is then washed with $70 \%$ ethanol and re-centrifuged for 2 minutes at 13,000rpm. The supernatant is again removed, and the pellet air dried prior to re-suspension in TE buffer (10mM Tris-HCl, 1mM EDTA, $\mathrm{pH}$ 7.0-8.0).

The quality and quantity of extracted gDNA is measured using a NanoDrop ND-1000 Spectrophotometer.

\subsection{3. $P C R$}

PCR amplification is performed in a final $25 \mu \mathrm{l}$ reaction volume with the following reagents: Faststart buffer (Roche Applied Science), $2 \mathrm{mM} \mathrm{MgCl}_{2}$ (Roche Applied Science), M13-tailed forward and reverse primers at $0.8 \mu \mathrm{M}$ each (synthesised by Integrated DNA Technologies Inc), $0.4 \mathrm{mM}$ dNTPs (GE Healthcare Ltd), 1 unit Faststart Taq DNA polymerase (Roche Applied Science) and 5 $\mu$ l GC-rich solution (Roche Applied Science). 50ng of gDNA is included in each reaction. PCR amplification is carried out with the following conditions: denaturation at $95^{\circ} \mathrm{C}$ for 5 minutes, followed by 35 cycles of $94^{\circ} \mathrm{C}$ for 45 seconds, $60^{\circ} \mathrm{C}$ for 30 seconds and $72^{\circ} \mathrm{C}$ for 30 seconds, with a final extension of $72^{\circ} \mathrm{C}$ for 10 minutes.

\subsubsection{Sequencing (Figure 2)}

$5 \mu \mathrm{L}$ of each PCR is cleaned with ExoSAP-IT (Affymetrix, USB) prior to bidirectional DNA sequencing using M13 forward and reverse primers and Big-Dye Terminator v3.0 (Applied Biosystems Ltd). $20 \mu \mathrm{l}$ of each sequenced product is manually purified using the CleanSEQ Sequencing Purification System (Agencourt Bioscience). Four different drying times prior to elution (20 minutes, 24 hours, four days and seven days) were assessed to establish an optimal drying time for generating high quality sequencing data. $15 \mu \mathrm{L}$ of purified product was then subjected to capillary electrophoresis using the Applied Biosystems model 3130xl Genetic Analyzer. 
The analysis of sequence traces is performed using Variant Reporter v1.0 (Applied Biosystems). Variant Reporter uses advanced algorithms and quality metrics to automate the detection of variants and to streamline the analysis process.

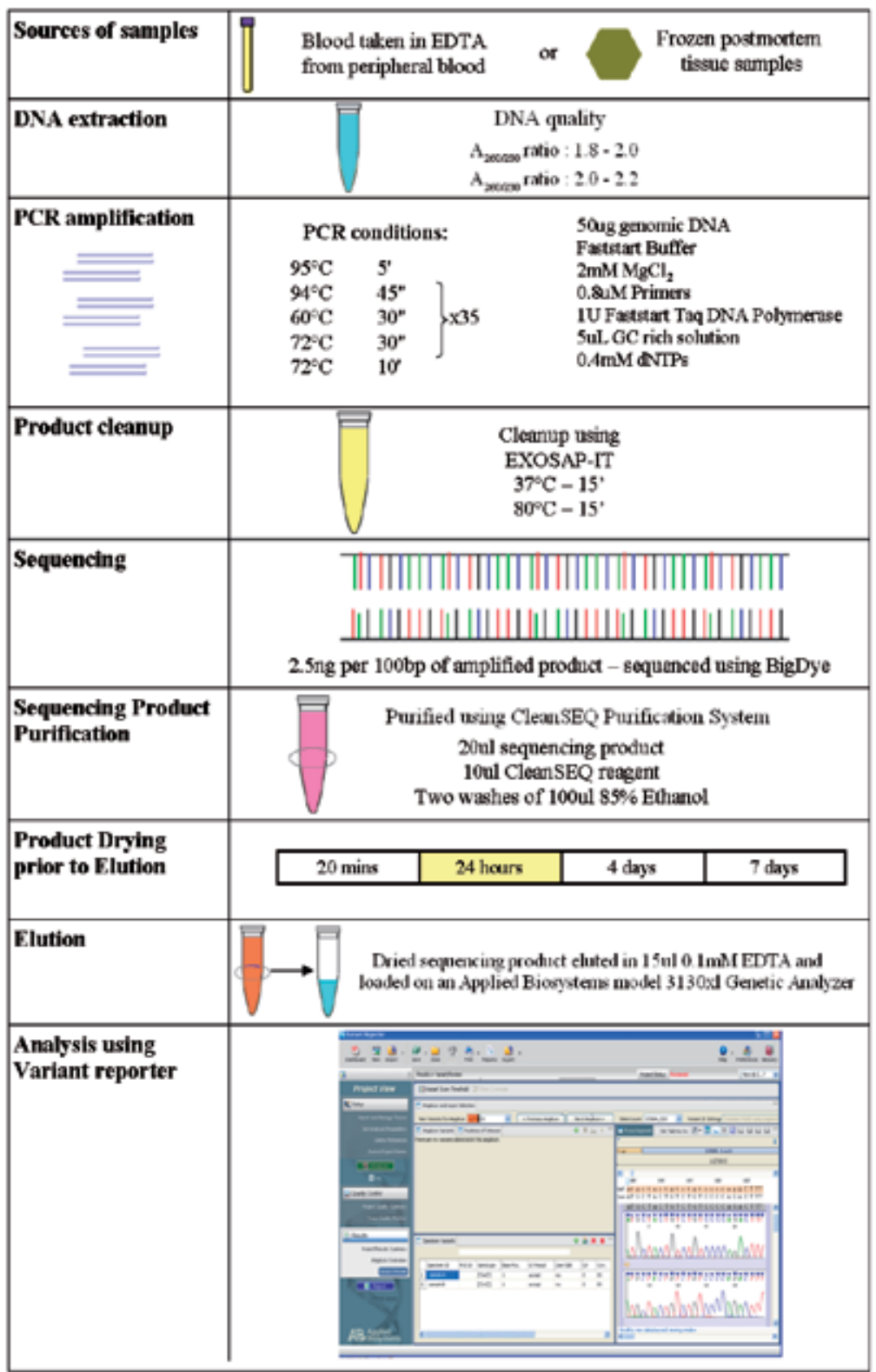

Figure 2. Flowchart of the sequencing method used in our laboratory. 


\subsection{Array comparative genomic hybridization (aCGH)}

A Roche NimbleGen 12x135K Custom CGH Array was used for dosage analysis. This bespoke CGH array has been designed to screen for dosage changes within the genes responsible for LQT1-12 (KCNQ1, KCNH2, SCN5A, ANK2, KCNE1, KCNE2, KCNJ2, CACNA1C, CAV3, SCN4B, AKAP9, and SNTA1), the LQT-associated genes GPD1L, KCNE3, SCN1B, $S C N 3 B, C A C N B 2$, and the CPVT1 (RYR2) and CPVT2 (CASQ2) genes.

Two hundred and fifty nanograms of gDNA are processed according to the manufacturer's instructions (NimbleGen Array User's Guide: CGH and CNV Arrays v8.0; http:// www.nimblegen.com). In brief, extracted gDNA from samples and Promega controls are denatured in the presence of a Cy3- (test) or Cy5- (control) labelled random primers and incubated with the Klenow fragment of DNA polymerase, together with dNTPs (5mM of each $\mathrm{dNTP}$ ), at $37^{\circ} \mathrm{C}$ for 2 hours. The reaction is terminated by the addition of $21.5 \mu \mathrm{L}$ of $0.5 \mathrm{M}$ EDTA, prior to isopropanol precipitation and ethanol washing. Following quantification, the test and sex-matched control samples are combined in equimolar amounts and applied to one of the twelve arrays on a microarray slide. Hybridisation is carried out in a Roche NimbleGen Hybridisation Chamber for a period of 48 hours. Slides are washed and scanned using a NimbleGen MS 200 Microarray Scanner. Array image files (.tif) produced by the MS 200 Data Collection Software are imported into DEVA v1.2.1 (Roche NimbleGen Inc) for analysis. Each genomic region exhibiting a copy number change within one of the LQT genes of interest were examined using the UCSC genome browser (http://genome.ucsc.edu/) to determine the location and significance of the change.

\section{Results}

In order to overcome the historical difficulties faced when performing Long QT syndrome testing using postmortem specimens and meet the demand by coroners for rapid results, we addressed the following parameters:

\subsection{DNA purity}

Since poor quality gDNA leads to suboptimal PCR amplification affecting downstream applications, the purity of gDNA is an important criterion for success in generating high quality sequencing data $[14,15]$. A NanoDrop ND-1000 spectrophotometer was used to measure the quality and quantity of the extracted gDNA. The ratio of absorbance at $260 \mathrm{~nm}$ and $280 \mathrm{~nm}$ (A260/280 ratio) is used to assess the purity of gDNA, which should be in the range of 1.8 to 2.0 to be accepted as pure gDNA. The ratio of absorbance at $260 \mathrm{~nm}$ and $230 \mathrm{~nm}$ (A260/230 ratio) is used as a secondary measure of nucleic acid purity, and for pure gDNA should be in the range of 2.0-2.2 [16]. Postmortem gDNA isolated using a standard phenol/ chloroform protease protocol may contain residual phenol, chloroform or ethanol. These contaminants inhibit the activity of DNA polymerase in downstream applications (Figures 3-5) [14], so the purity of the gDNA must be strictly monitored. If a suboptimal A260/280 or A260/230 ratio indicates that the gDNA is of low quality, a secondary cleanup process 
should be considered. In our laboratory, we perform this cleanup using another phenol extraction and ethanol precipitation to further purify the gDNA sample [17].

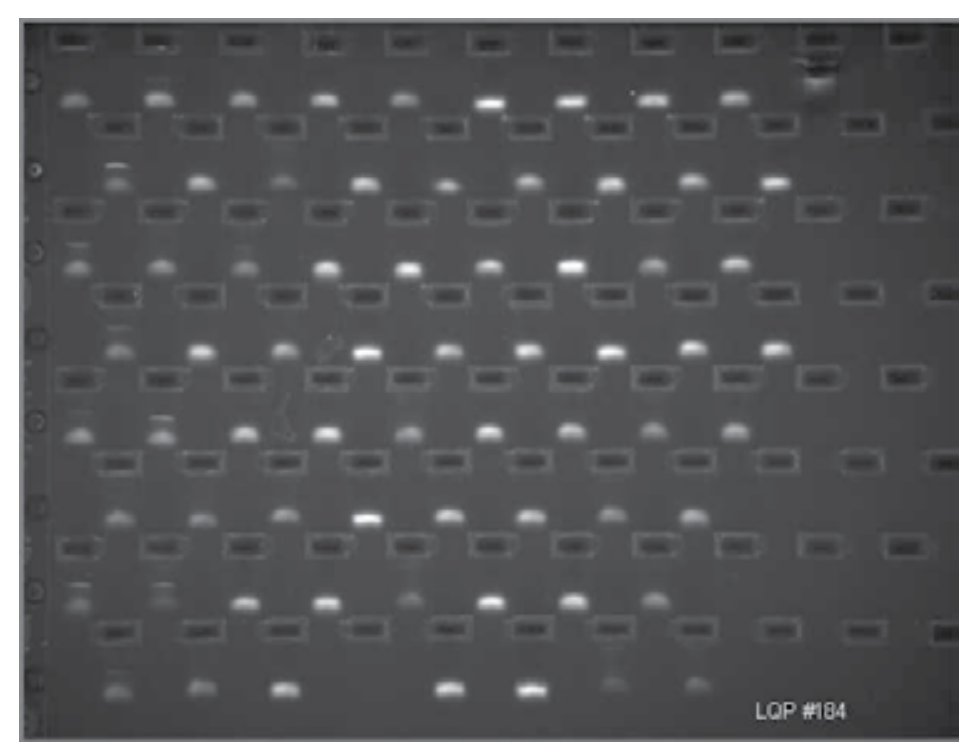

Figure 3. Agarose gel image of PCR amplification from an impure DNA with an acceptable $A_{260 / 280}$ ratio (1.88) but an acceptable $A_{260 / 230}$ ratio (2.5). NOTE: very few exons amplified effectively and strong primer dimers are visible.

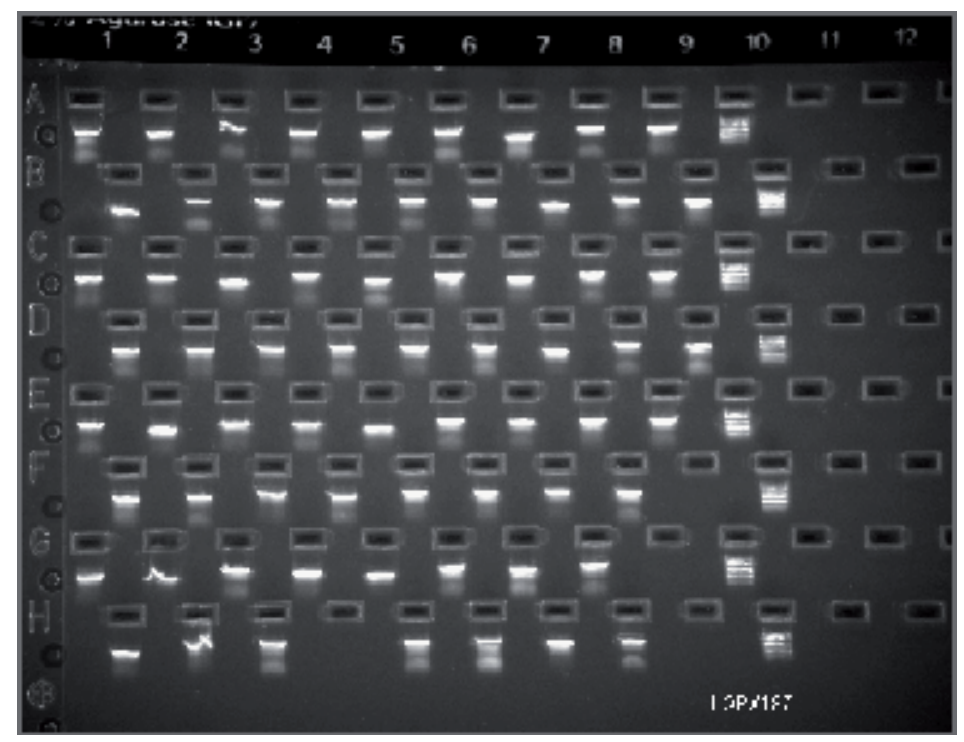

Figure 4. Agarose gel image of PCR products amplified from the same DNA sample after purification. NOTE: sUccessful amplification and significantly reduced primer dimers. 


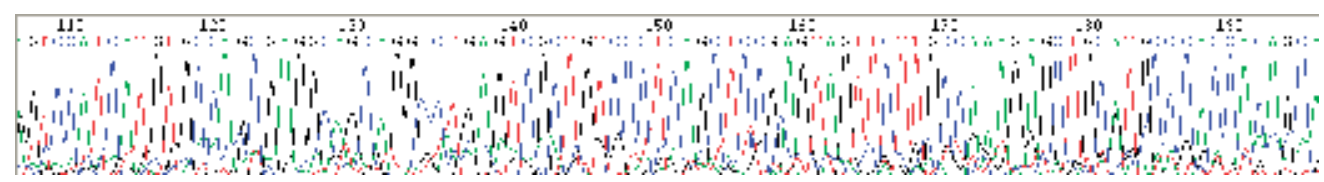

Figure 5. Electropherogram generated from an impure DNA sample with an acceptable $A_{260 / 280}$ ratio (1.95) but an unacceptable $A_{260 / 230}$ ratio (0.95). NOTE: significant baseline noise and data unable to be analysed.

\subsection{Amount of DNA template used in a sequencing reaction}

The extent of dilution of the cleaned PCR product prior to sequencing determines the amount of DNA template used in the sequencing reaction, which can affect the data quality for analysis [15]. The presence of excessive DNA template in a sequencing reaction will lead to rapidly progressive signal loss on the electropherogram (Figure 6), while using insufficient DNA template in a sequencing reaction will result in weak signal strength and a loss of peak shape (Figure 7), causing difficulties in basecalling and accurate analysis [15]. In order to obtain high quality data with good signal strength, the amplified product should be diluted in $\mathrm{ddH}_{2} \mathrm{O}$ to as close as possible to $2.5 \mathrm{ng}$ per $100 \mathrm{bp}$.

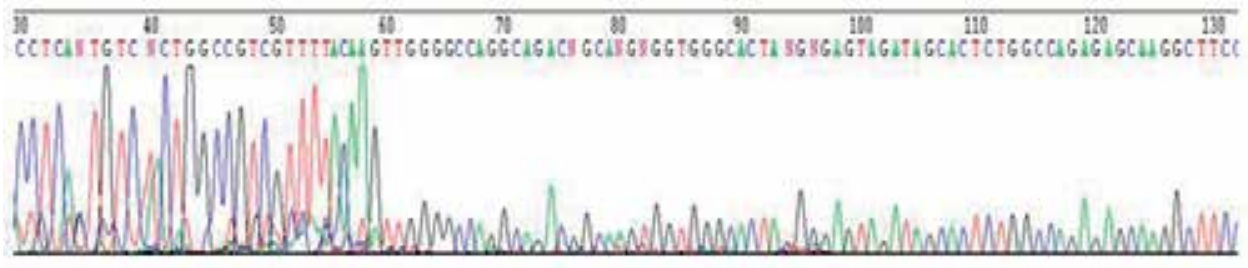

Figure 6. Electropherogram begins with strong high peaks, which fade rapidly.

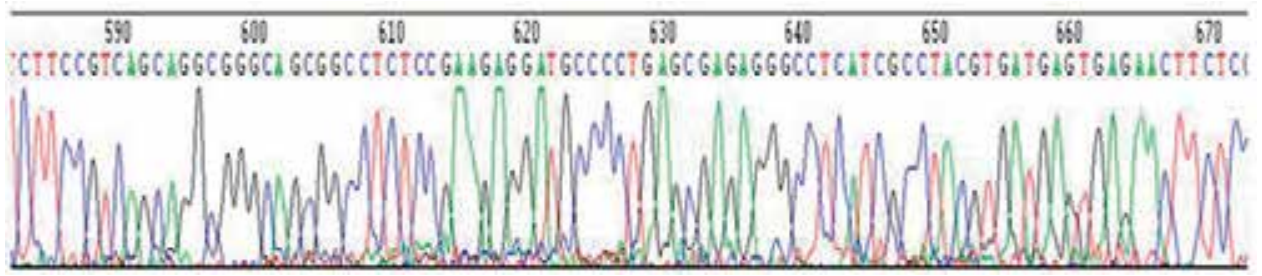

Figure 7. Electropherogram with increased background noise and loss of sharp peak shape. 


\subsection{CleanSEQ treatment of sequencing reactions}

The sequenced product is purified using an Agencourt CleanSEQ system, which uses SPRI (Solid Phase Reversible Immobilization) magnetic bead-based technology. According to the manufacturer's recommendation, the sequencing product should be cleaned using undiluted CleanSEQ reagent, but in our experience, postmortem DNA samples should be cleaned using CleanSEQ reagent diluted 1:2 in $\mathrm{ddH}_{2} \mathrm{O}$ (figures 8,9).

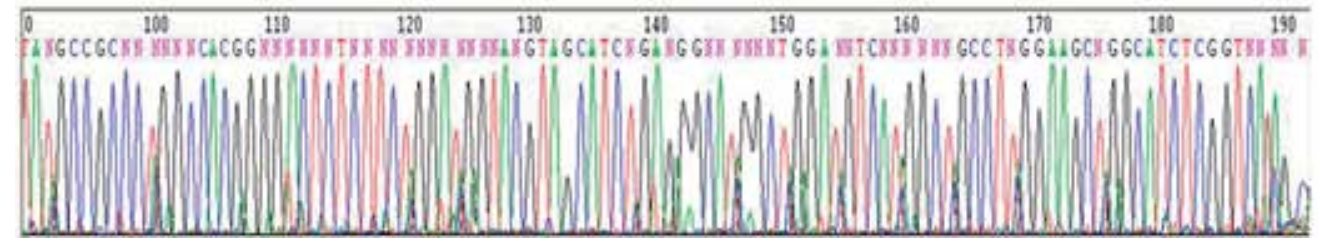

Figure 8. Electropherogram obtained from sequencing product cleaned with $10 \mu \mathrm{l}$ of undiluted CleanSEQ reagent: serious baseline noise and incorrect basecalling.

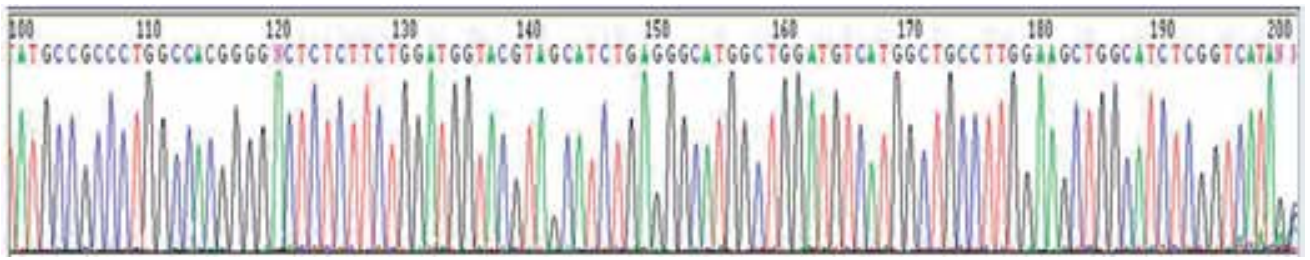

Figure 9. Electropherogram obtained from sequencing product cleaned using diluted CleanSEQ reagent: good quality trace.

\subsection{Drying of DNA-bound beads prior to elution}

The length of drying time following ethanol washing and prior to elution of the sequencing product from the CleanSEQ beads is one of the critical parameters for obtaining high quality sequence data. We assessed 4 different drying periods: 20 minutes, 24 hours, four days and seven days.

A drying time of 20 minutes, as recommended by the manufacturer, is frequently associated with the presence of large 'dye blobs', most probably as a result of residual ethanol in the eluted product (Figure 10); this problem resolves if drying time is extended to 24 hours (Figure 11). However, variable baseline noise on the electropherogram will be evident if the drying time exceeds four days (Figure 12); further increased background noise and loss of resolution occur when the drying time exceeds seven days (Figure 13). 


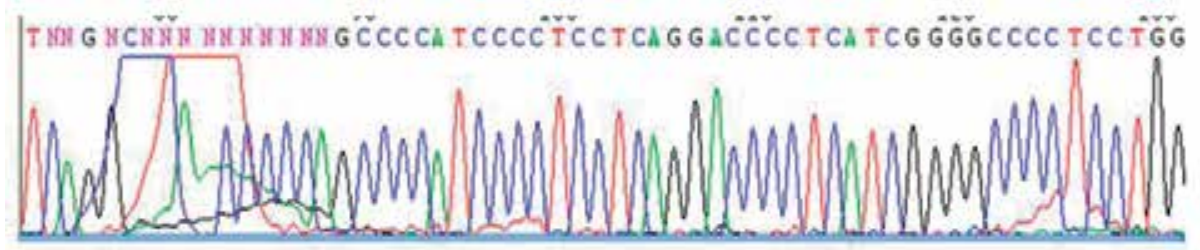

Figure 10. Electropherogram of purified products dried for 20 minutes prior to elution; presence of large dye blob.

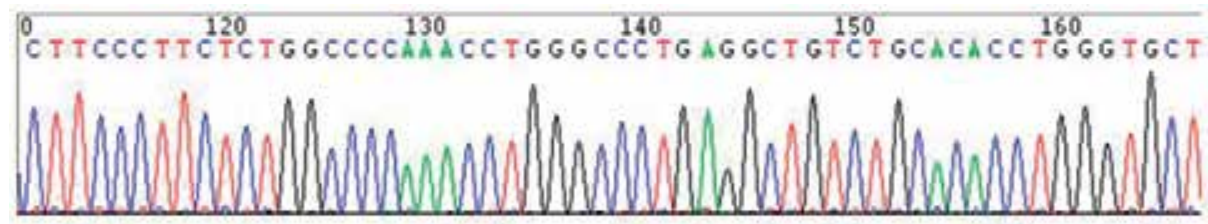

Figure 11. Electropherogram of purified products dried for 24 hours prior to elution; free of dye blobs, minimal baseline noise.

Figure 12. Electropherogram of purified products dried for 4 days prior to elution; presence of shoulders and messy baseline.

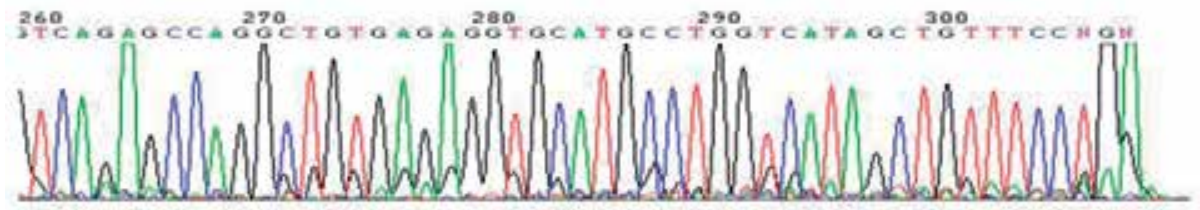

Figure 13. Electropherogram of purified products dried for 7 days prior to elution; extremely messy baseline and early loss of resolution. 


\section{Discussion}

\subsection{DNA quality}

\subsubsection{Sequence analysis}

The gDNA used in a PCR should be as pure as possible in order to optimise the quality of the template that will be used in downstream applications. The purity of DNA is assessed in our laboratory using a NanoDrop ND-1000 spectrophotometer. It is important to consider both A260/280 and A260/230 as poor DNA quality will affect downstream applications $[16,18]$. An unacceptable A260/280 or A260/230 ratio indicates the presence of contaminants in the DNA; an abnormal A260/230 indicates the presence of residual phenol or other chemical from the extraction process, while an abnormal A260/280 most frequently indicates the presence of protein $[16,18]$. If any of the ratios appear to be abnormal, DNA purification should be considered before processing any further.

\subsubsection{Dosage analysis (aCGH)}

The quality requirements for gDNA used in an aCGH assay are not as stringent as those for a sequencing assay. An awareness of the presence of significant degradation is important; however, partial compensation for this can be made by increasing the volume of gDNA used in the amplification and labelling step. In our laboratory, we have found that doubling the volume of gDNA when degradation is visible on the 'check gel' (present in lanes 5-8 of Figure 14) is an effective counter-measure. The adequacy of amplification is measured using a NanoDrop ND-1000 spectrophotometer in order to allow the hybridisation of equimolar amounts of test and control DNA to the array slide. A post-amplification concentration of greater than $2500 \mathrm{ng} / \mu \mathrm{l}$ is ideal for further processing; a sample with a post-amplification concentration of less than $1500 \mathrm{ng} / \mu \mathrm{l}$ is unlikely to produce good quality data for analysis. Therefore, if there is sufficient volume of gDNA available, a repeat amplification with an increased volume of template gDNA should be undertaken.

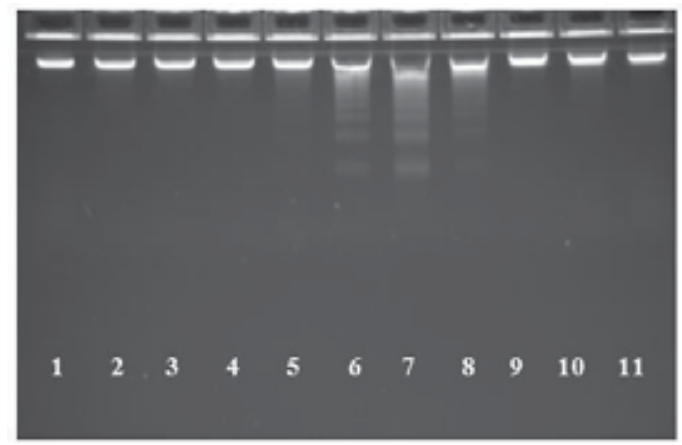

Figure 14. Agarose gel (2\%) check of gDNA quality prior to aCGH. 


\subsection{Amount of DNA template used in a sequencing reaction}

The DNA template should be diluted to $2.5 \mathrm{ng}$ per $100 \mathrm{bp}$ prior to sequencing. Incorrect quantification and dilution will alter the amount of input DNA template in a sequencing reaction, which can lead to problematic data for analysis. In our laboratory, we use Variant Reporter ${ }^{\mathrm{TM}}$ (Applied Biosystems Ltd) for automated analysis of sequence data. The signal strength of the sequence data is automatically assessed. Data with good signal strength is above the value of 200 in the Variant Reporter ${ }^{\mathrm{TM}}$ software. Sequence data with weak signal strength, a value below 100, indicates insufficient DNA template has been used in the sequencing reaction. Noisy background and a loss of sharp peak shape are also commonly evident. A repeat treatment with EXOSAP-IT and less extensive dilution of product is necessary to avoid inaccurate analysis using sequence data with unacceptable quality. In contrast, when excess DNA template is used in a sequencing reaction, a rapidly progressive signal loss is seen on the electropherogram. In this instance, trace quality can be improved by diluting the eluted product with water and reloading the sample on the capillary sequencing platform (we use an Applied Biosystems model 3130xl Genetic Analyzer).

\subsection{CleanSEQ treatment of sequencing reactions}

Agencourt CleanSEQ is routinely used to purify the sequenced products. According to the manufacturer's recommendation, $20 \mu \mathrm{l}$ of sequencing product should be cleaned with $10 \mu \mathrm{l}$ of CleanSEQ reagent. However, we found that this leads to an excess of CleanSEQ beads in relation to the amount of sequence product, therefore unwanted short fragments (e.g unincorporated dNTPs and excess primers) were able to bind to beads during purification, and the quality of sequence data generated was consequently reduced. We found that diluting the CleanSEQ beads 1:2 with $\mathrm{ddH}_{2} \mathrm{O}$ leads to much higher quality data.

\subsection{Drying of DNA-bound beads prior to elution}

This drying step is a critical QC consideration that significantly affects sequence quality. When the purified product is dried for 24 hours prior to elution, the subsequent electropherograms are free of dye blobs, C-shoulders and baseline noise, and consistently pass all QC metrics in Variant Reporter.

A drying time of 20 minutes, as suggested by manufacturer, was found to be problematic, causing the presence of dye blobs. Dye blobs are a common artefact due to either the presence of excess unincorporated dye or residual ethanol following product purification [15]. Previously, operators have focused on purifying sequenced products using an accu- 
rate final concentration of ethanol in an attempt to achieve consistency of evaporation/ drying between sequencing runs. Ethanol is highly volatile and it is therefore difficult to achieve consistency - even the short period of time in which the vessel containing the ethanol is open to allow access can result in a decrease in concentration. Here, we showed that the issue of dye blobs can be resolved by simply extending the drying time to 24 hours, allowing adequate time for all ethanol to evaporate completely, despite any minor differences in the concentration of ethanol used.

On the other hand, we found that the quality of sequence data is adversely affected if the drying time is extended to more than 4 days. This suggests that the stability of purified sequencing products is another factor that affects data quality. High quality sequence data was obtained with a drying time of 24 hours; however, the sequences were suboptimal when elution and electrophoresis were carried out at day 4 or day 7 of drying. This indicates that purified sequencing product is most stable for the first 24 hours and that consideration of the sample stability should be taken into account when aiming for high quality data.

\subsection{Analysis parameters}

\subsubsection{Sequencing}

All sequence traces are analysed in our laboratory using Variant Reporter Software v1.0 (Applied Biosystems). The current CMGS best practice guidelines for Sanger sequence analysis in diagnostic laboratories [19] recommend a PHRED score of at least 20 for bidirectional data (corresponding to $99 \%$ confidence that the base is called correctly), and a PHRED score of at least 30 for unidirectional data $(99.9 \%$ confidence that each base is called correctly). The procedure we describe above produces bidirectional sequence data that meet our laboratory's even more stringent analysis criteria: a Variant Reporter trace score of 35 , corresponding to a false base call rate of $0.031 \%$.

\subsubsection{Dosage analysis}

In order to provide simultaneous dosage analysis of all the Long QT syndrome genes, we developed a custom designed Roche NimbleGen 12x135K CGH array. As part of the validation of this array we analysed twenty patients with known copy number abnormalities [20]. Analysis of the data from these individuals with known copy number changes allowed precise threshold criteria to be developed. It was determined that a $\log _{2}$ ratio $\leq-0.4$ over 6 contiguous probes is indicative of a deletion, and a $\log _{2}$ ratio of $\geq 0.4$ over 15 contiguous probes indicative of a duplication. 


\subsection{Other parameters}

It is important to monitor other QC parameters when it comes to sequence analysis of Long QT specimens: the freshness of the Polymer (POP-7) and the usage of the capillaries in the Applied Biosystems model 3130xl Genetic Analyzer.

Polymer (POP-7) is used to separate DNA fragments on genetic analyzers and the polymer remains stable for up to 7 days [15]. The capillary in the genetic analyzer should be replaced after 1000 injections. Within our laboratory, we perform a regular weekly maintenance of the analyzer, and the capillary is replaced when 600 injections are reached. From past experience, both the freshness of polymer and the usage of the capillary play a vital role in the data quality. Electropherograms with reduced resolution and peak shape are produced when either of these two parameters is suboptimal. In order to achieve high quality data, both freshness of polymer and usage of the capillary should be closely monitored.

\section{Conclusions}

We have successfully established a robust method for processing postmortem specimens for Long QT diagnostic testing in a timely manner. The electropherograms in Figure 15 are indicative of the high quality data routinely produced, despite the limitations inherent in the types of specimens that are referred. The blood sample used to extract gDNA for sequencing in this example was heavily haemolysed on arrival at the laboratory.

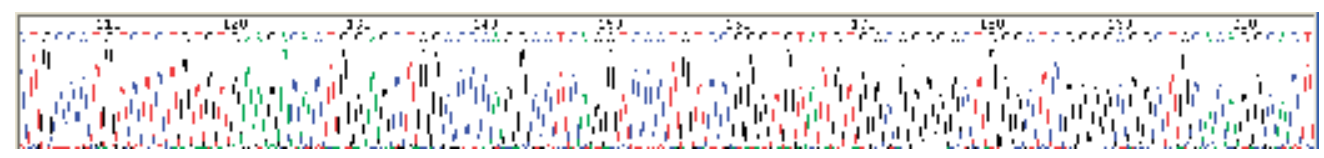

Figure 15. High quality sequence data generated from a haemolysed blood specimen; flat baseline along with distinct and evenly spaced peaks allow accurate basecalling.

The QC parameters described above should be monitored closely in order to consistently achieve optimal results (Figure 16). Although the basic procedure is essentially the same as that routinely used for sequence and aCGH analysis, the poor quality of postmortem specimens as a source of template DNA mean that particular attention needs to be paid to each step, in particular the critical initial assessment of DNA quality, the addition of the appropriate volume of template DNA to the sequencing reaction, and the drying time of the beads used in the purification procedure prior to elution. 


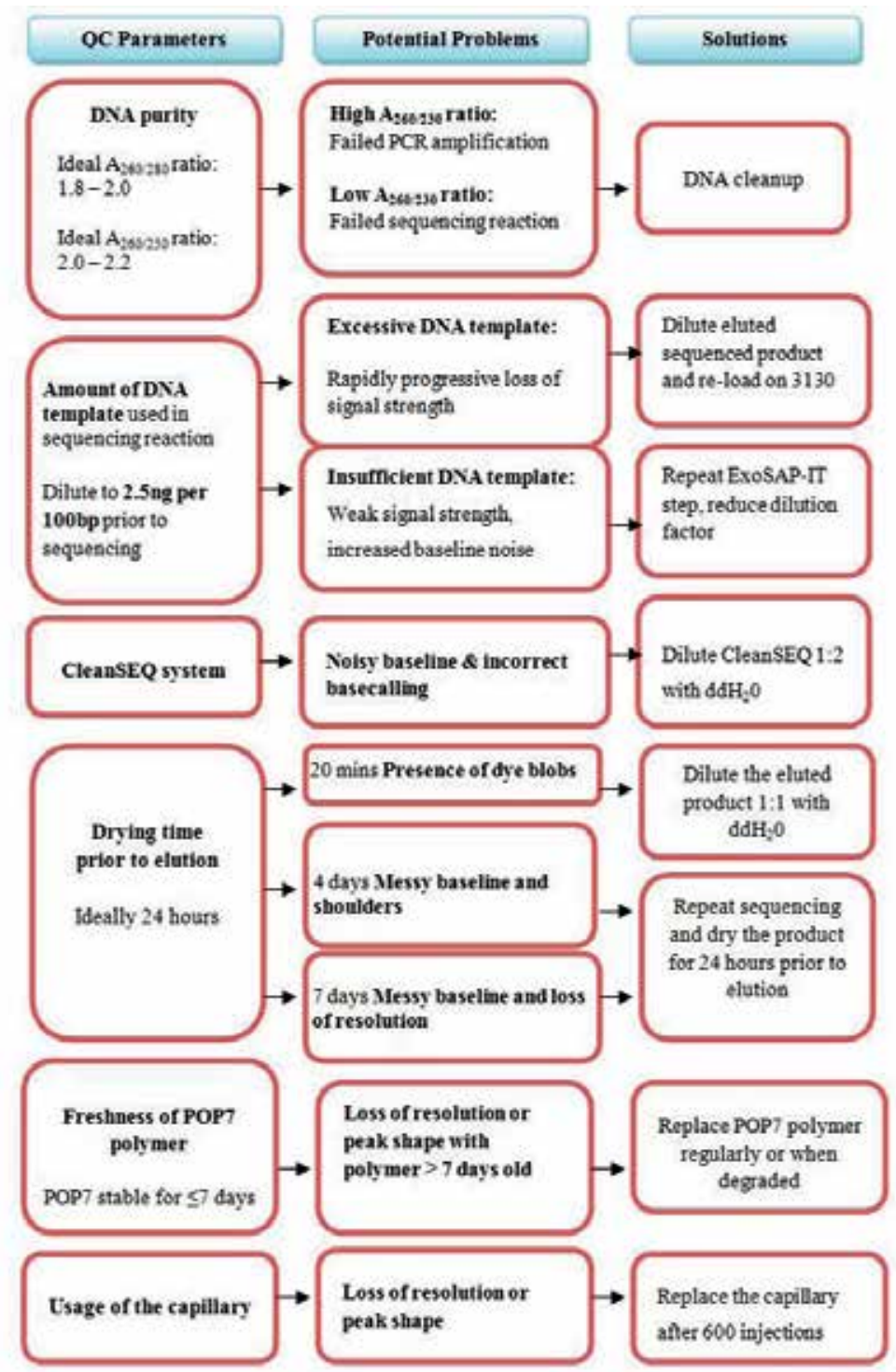

Figure 16. Flowchart of potential QC problems and their solutions.

\section{Acknowledgements}

We acknowledge the assistance of Dr Andrew Dodd in the initial design of primers against the coding regions of the LQT genes, and Mr Daniel Lai for his assistance in optimizing aspects of our amplicon clean-up. 


\section{Author details}

Stella Lai ${ }^{1}$, Renate Marquis-Nicholson ${ }^{1,2^{*}}$, Chuan-Ching Lan ${ }^{1}$, Jennifer M. Love ${ }^{1}$, Elaine Doherty ${ }^{1}$, Jonathan R. Skinner ${ }^{3}$ and Donald R. Love ${ }^{1,2^{*}}$

*Address all correspondence to: donaldl@adhb.govt.nz

1 Diagnostic Genetics, Auckland City Hospital, New Zealand

2 School of Biological Sciences, The University of Auckland, New Zealand

3 Inherited Disease Group New Zealand, Paediatric Cardiac Services, Starship Childrens' Hospital, New Zealand

\section{References}

[1] Hunter, J. D., Sharma, P., \& Rathi, S. (2008). Long QT syndrome. Contin Educ Anaesth Crit Care Pain., 8, 67-70.

[2] Modell, S. M., \& Lehmann, M. H. (2006). The long QT syndrome family of cardiac ion channelopathies: a HuGE review. Genet Med., 8(3), 143-55.

[3] Alders, M., \& Mannens, M. M. A. M. (2003). Romano-Ward Syndrome. Feb 20 [Updated 2012 May 31]., In: Pagon RA, Bird TD, Dolan CR, et al., editors. GeneReviews ${ }^{\mathrm{TM}}$ [Internet]. Seattle (WA): University of Washington, Seattle;, 1993Available from:, http:// www.ncbi.nlm.nih.gov/books/NBK1129/.

[4] Yang, Y., Yang, Y., Liang, B., Liu, J., Li, J., Grunnet, M., Olesen, S. P., Rasmussen, H. B., Ellinor, P. T., Gao, L., Lin, X., Li, L., Wang, L., Junjie, J. X., Liu, Y. Y., Liu, Y. Y., Zhang, S. S., Lian, D. D., Peng, L. Y., Jespersen, T., \& Chen, Y. H. (2010). Identification of a Kir3.4 mutation in congenital long QT syndrome. Am. J. Hum. Genet., 86, 872-880.

[5] Dan, M., \& Roden, M. D. (2008). Long-QT Syndrome. N Engl J Med., 358, 169-176.

[6] Eddy, C. A., MacCormick, J. M., Chung, S. K., Crawford, J. R., Love, D. R., Rees, M. I., et al. (2008). Identification of large gene deletions and duplications in KCNQ1 and KCNH2 in patients with long QT syndrome. Heart Rhythm, 5(9), 1275-1281.

[7] Bonin, S., Petrera, F., Niccolini, B., \& Stanta, G. (2003). PCR analysis in archival post mortem tissues. Mol Pathol., 56, 184-186.

[8] Schwartz, P. J., Stramba-Badiale, M., Crotti, L., Pedrazzini, M., Besana, A., Bosi, G., et al. (2009). Prevalence of the congenital long-QT syndrome. Circulation, 120(18), 1761-1767. 
[9] Skinner, J. R., Crawford, J., Smith, W., Aitken, A., Heaven, D., \& Evans, CA. (2011). Prospective, population-based long QT molecular autopsy study of postmortem negative sudden death in 1 to 40 year olds. Heart Rhythm, 8(3), 412-419.

[10] Gladding, P. A., Evans, C. A., Crawford, J., Chung, S. K., Vaughan, A., Webster, D., et al. (2010). Posthumous diagnosis of long QT syndrome from neonatal screening cards. Heart Rhythm, 7(4), 481-486.

[11] Skinner, J. R. (2010). Sudden Unexplained Death in Infancy and Long QT syndrome. Current Pediatric Reviews, 6, 48-55.

[12] Doherty, E., Marquis-Nicholson, R., Love, J. M., Brookes, C., Prosser, D., \& Love, D. R. (2011). Primer Design to Sequence Analysis- a Pipeline for a Molecular Genetic Diagnostic Laboratory, Applications and Experiences of Quality Control, Ognyan Ivanov (Ed.). 978-9-53307-236-4, InTech, Available from, http://www.intechopen.com/ books/applications-and-experiences-of-quality-control/primer-design-to-sequenceanalysis-a-pipeline-for-a-molecular-genetic-diagnostic-laboratory.

[13] Lai, D., \& Love, D. R. (2012). Automation of a primer design and evaluation pipeline for subsequent sequencing of the coding regions of all human Refseq genes. Bioinformation, 8(8), 363-366.

[14] Verhagen, O. J., Wijkhuijs, A. J., van der Sluijs-Gelling, A. J., Szczepanski, T., van der Linden-Schrever, B. E., Pongers-Willemse van, M. J., Wering, E. R., van Dongen, J. J., \& van der Schoot, C. E. (1999). Suitable DNA isolation method for the detection of minimal residual disease by PCR techniques. Leukemia, 13(8), 1298-1299.

[15] Applied Biosystems Chemistry Guide, second edition. (2009). DNA Sequencing by Capillary Electrophoresis. Available from:, http:// www3.appliedbiosystems.com/cms/groups/mcb_support/documents/generaldocuments/cms_041003.pdf.

[16] T009 - Technical Support Bulletin and 260/230Ratios. (2008). Available from:, http:// www.nanodrop.com/Library/T009-NanoDrop\%201000-\&-NanoDrop\%208000-Nucleic-Acid-Purity-Ratios.pdf.

[17] Powell, R., \& Gannon, F. (2002). Purification of DNA by phenol extraction and ethanol precipitation. Oxford Practical Approach Series, Oxford University Press., Available from:, http://fds.oup.com/www.oup.co.uk/pdf/pas/9v1-7 -3.pdf.

[18] T042 - Technical Bulletin. (2011). Assessment of Nucleic Acid Purity. Available from:, http://www.nanodrop.com/Library/T042-NanoDrop-Spectrophotometers-NucleicAcid-Purity-Ratios.pdf.

[19] Ellard, S., Charlton, R., Yau, M., Gokhale, D., Taylor, G., Wallace, A., \& Ramsden, S. C. (2009). Practice guidelines for Sanger Sequencing Analysis and Interpretation. Available from:, http://www.cmgs.org/BPGs/pdfs\%20current\%20bpgs/Sequencingv2.pdf. 
[20] Marquis-Nicholson, R., Doherty, E., Thrush, A., Love, J. M., Lan-C, C., George, A. M., \& Love, D. R. (2012). Array-based identification of copy number changes: simultaneous gene-focused and low resolution whole genome analysis. Sultan Qaboos University Medical Journal, submitted. 
Chapter 19

\title{
Quality Assurance in Antimicrobial Susceptibility Testing
}

\author{
Onur Karatuna \\ Additional information is available at the end of the chapter \\ http://dx.doi.org/10.5772/51998
}

\section{Introduction}

Most of the clinically important bacteria causing infections in humans are capable of exhibiting resistance to antimicrobial agents commonly used for the treatment. Therefore, upon isolation of the organism in the clinical microbiology laboratory, characterization frequently also employs tests to detect its antimicrobial susceptibility. Thus, the report produced by clinical microbiology laboratory for the physician, also includes organism's susceptibility profile to different antimicrobials along with its identification [1]. Antimicrobial susceptibility testing (AST) is performed on bacteria that are isolated from clinical specimens to determine if the bacterial etiology of concern can be killed or inhibited by antimicrobial drugs that are potential choices for therapy, at the concentrations of the drugs that are attainable at the site of infection using the dosing regimen indicated in the drug product's labeling. The results of AST are generally reported with interpretive categories. The category "susceptible" indicates that the bacteria are inhibited by the usually achievable concentrations of antimicrobial agent when the dosage recommended to treat the site of infection is used. The "intermediate" category defines the bacteria for which the response rates to usually attainable blood and tissue levels of antimicrobial agent are lower compared to susceptible isolates. The intermediate category plays the role of a buffer zone between the susceptible and resistant categories, but also indicates a number of other possibilities; the antimicrobials which are concentrated at the site of infection may be regarded as options for treatment (e.g., nitrofurantoin for the urinary tract infections). The "resistant" category, however, defines the bacteria which are not inhibited by the usually achievable concentrations of the agent with normal dosage regimens and that the clinical efficacy of the agent against the isolate may not be sufficient [2]. Clinicians consider these interpretations to determine which antimicrobial agent might be effective in treating the particular patient. The primary role of routine microbiology laboratories is to provide accu- 
rate and timely antimicrobial susceptibility test results for guiding the treatment of infectious diseases. In order to achieve that, the microbiologist should inform the clinician about whether an infectious agent is present in the patient's specimen and which antimicrobial agent should provide the optimum therapy. Although the importance of antimicrobial susceptibility testing is well established, the procedure itself is very sensitive to changes in the environment and test conditions. Therefore, it is crucial that each variable in the procedure should be standardized and carefully controlled. With more reliable susceptibility results, infectious disease specialists and public health leaders can be able to recognize emerging resistance and novel resistance patterns. Additionally, the results of AST can be applied to define the agent of choice for empirical therapy, establish institutional or nationwide policies for prescribing of antibiotics, conduct epidemiological studies or resistance surveillance, and to evaluate the efficacy of newly developed agents. Owing to numerous variables that may affect the results, rigorous quality control is of utmost importance for susceptibility testing. Properly performed quality control would aid in providing accurate, reproducible and timely results. In this chapter the components of a quality assurance program for antimicrobial susceptibility testing will be highlighted.

\section{Overview of the antimicrobial susceptibility testing methodologies}

Fleming was first to report the inhibitory effect of penicillin on agar by observing a zone of growth inhibition of staphylococcal colonies grown next to a Penicillium contaminant on an agar plate. Fleming also made two significant contributions to the field of AST in the 1920s. In 1924, he introduced the use of the ditch plate technique for evaluating antimicrobial qualities of antiseptic solutions [3]. Fleming's second contribution to modern AST was the development of broth dilution technique using turbidity as an end-point determination [4]. Filter paper disks incorporating penicillin were utilized by Vincent \& Vincent for assaying this newly discovered compound in 1940s [5]. Agar dilution AST method was also described in the 1940s [6]. At an early stage, it was realized that there were many variables affecting AST methods [7]. In 1961, World Health Organization (WHO) published a report on standardization of AST methodology [8]. The broad application of AST was introduced to clinical laboratories by the efforts of Bauer, Kirby and co-workers, with the method known as KirbyBauer disk diffusion method which is still the most widely used AST technique in the world [9]. Bergeron \& Ouellette highlighted the shortcomings of the phenotypic approach to AST and concluded that different bacterial species have different susceptibilities to the same antibiotic, and that there is no international aggreement on breakpoints for interpretation of antimicrobial susceptibility tests [10]. The need for developing standardized AST methods became a necessity soon after antibiotics became commercially available. During World War II, following penicillin, other antibiotics were discovered and used. Altough these new antibiotics were regarded as "wonder drugs" at the time of their introduction, emergence of resistant strains followed. With the emergence of bacterial resistance to antimicrobials and the changing properties of different bacteria to different classes of antimicrobials, the need for the performance of AST on pathogens became a practical necessity. 
Nationwide attempts were made to standardize AST methodologies; Clinical and Laboratory Standards Institute (CLSI, formerly NCCLS) (USA) [11], Werkgroep Richtlijnen Gevoeligheidsbepalingen (Netherlands) [12], Comité de l'Antibiogramme de la Société Française de Microbiologie (France) [13], the Swedish Reference Group for Antibiotics (Sweden) [14], Deutsches Institut für Normung (Germany) [15], the British Society for Antimicrobial Chemotherapy (UK) [16], they all published guidelines to improve the methodology and interpretation of AST. Recently, the European Committee on Antimicrobial Susceptibility Testing (EUCAST), a non-profit organization under the auspices of European Society of Clinical Microbiology and Infectious Diseases (ESCMID), developed and published AST guidelines. Breakpoint and QC tables for disk diffusion and minimum inhibitory concentration (MIC) testing can freely be accessed on organization's website [17].

In clinical laboratories, widely adopted AST methods are disk diffusion and broth dilution methods. In disk diffusion method, disks impregnated with antimicrobial agents are used. The disks are placed onto agar plates which are preinoculated with the suspension of the microorganism being tested. The basic principle of the disk diffusion method is the diffusion of the antimicrobial agent into the medium which occurs when the disks come into contact with the moist surface of the plate. The concentration of the agent reduces logarithmically as the distance from the disk is increased. After the incubation period the plates are observed for the circular inhibiton zone created around the disk which is due to the inhibitory effect of the antimicrobial agent on the microorganism. Within the zone the concentration of the agent is sufficient to inhibit growth, whereas at the point where the concentration of the agent is no longer enough to inhibit growth, the organism is able to grow and forms a lawn of bacteria around the disk. To interprete the test results, the radius of the inhibition zone is measured and compared against the predefined values provided by the guidelines [18]. The most widely used guidelines are the CLSI and EUCAST guidelines [2, 17]. CLSI divides the results into three categories for most of the organism-agent combinations; susceptible, intermediate and resistant, whereas EUCAST uses only two categories, susceptible and resistant.

In the dilution methods, however, the susceptibility of the microorganisms to antimicrobial agents is determined whether in tubes (macrobroth dilution method) or in microtube wells molded into a plastic plate (microbroth dilution method). Both broth dilution methods use the same principle; first serial two-fold dilutions of the antimicrobial agent to be tested are made in the tubes/wells containing broth, and then same amount of bacterial suspension is distributed on each tube/well. At the end of the incubation period, the tubes/wells are examined for turbidity which is the indicator of bacterial growth in broth. The tubes/wells remain clear where the concentration of the agent is high enough to inhibit the bacterial growth, whereas at lower concentrations of the agent, the bacteria may grow which causes the tube/ well become turbid. The lowest concentration of antimicrobial agent that prevents the in vitro growth of bacteria is defined as the minimal inhibitory concentration (MIC) [18]. As in the disk diffusion method, the MIC values are compared against the predefined values provided by the guidelines and their intrepretive category is determined and reported. 


\section{Quality assurance program for antimicrobial susceptibility testing}

Clinical microbiology laboratories are an integral part of the total healthcare delivery system. Quality assurance (QA) is the overall process by which a laboratory can verify that a laboratory does its job well. While QA and quality control (QC) share the similar purposes, their meanings and functions are different [19]. QA can be defined as the overall program by which the quality of the test results can be guaranteed [20]. It evaluates and ensures that procedures provide relevant and timely data in the delivery of healthcare services. QA is primarily concerned with broader measures and monitors the performance of laboratory in total and covers all three phases of testing; pre-analytical, analytical and post-analytical. QC, in the other hand, is responsible for monitoring of the analytical phase of testing only and ensures that the daily tests are working properly [21]. QC and QA, only together provide measures for controlling how correct the tests are being performed because QC by itself often does not detect problems in time to prevent harmful results. For example, if $>5 \%$ of $E n$ terobacter, Serratia, or Citrobacter isolates are susceptible to ampicillin, it likely indicates a problem with insufficient inoculum [22]. Although daily or weekly QC test results are in acceptable limits, such an error can be overlooked until enough data have been accumulated and evaluated which can sometimes take weeks.

Standard processes are required to establish quality measures to be monitored. Standardization of AST has been achieved by CLSI, and in part by EUCAST. The processes defined in CLSI guidelines help clinical laboratories to perform QC tests, measure their results and provide corrective action recommendations covering a broad spectrum of error types. Each laboratory should establish its own quality requirements for testing processes. Only with established quality goals, laboratories can determine whether acceptable quality is being achieved, identify processes that are not performing satisfactorily and are in need of improvement, or to plan new processes to reach a specified level of quality [21]. And to ensure that all the established quality goals are achieved, a comprehensive QA program should be functional in a clinical laboratory.

The major components of a comprehensive QA program for AST, with the relative amount of effort required to be spent on each component given in parantheses, can be listed as follows [23]:

- Clinically relevant testing strategies (15\%)

- Testing of reference QC strains (15\%)

- Technical competency (15\%)

- Organism antibiogram verification (15\%)

- Supervisor review of results (15\%)

- Procedure manual (10\%)

- Cumulative antibiogram (5\%) 
- Proficiency surveys (5\%)

- Other $(5 \%)$

The goals of the QC program as set by the CLSI [24,25] includes to monitor the following:

- the precision (repeatability) and accuracy of AST procedures

- the performance of reagents used in the tests

- the performance of persons who carry out the tests and read the results

The continuous monitorization of the performance is best achieved, but not limited to, by the testing of QC strains.

\subsection{Developing relevant antimicrobial susceptibility testing strategies}

Only organisms likely to be the cause of an infection should be tested for antimicrobial susceptibility which necessitates the differentiation should be done between the normal flora that resides at the site of the infection and the actual organism causing the infection. Some important factors are to be considered to decide which bacterium or bacteria from a clinical specimen must be included in the AST; such as the body site from which the organism was isolated, the presence of other bacteria and the quality of the specimen from which the organism was grown, the host's status, the ability of the bacterial species to cause infection at the body site from which the specimen was obtained, etc. [1, 26].

\subsection{Selecting antimicrobials to test and to report}

Each laboratory is unique in its capability, resources, level of experience or institutional needs. Therefore, the decision of which antimicrobials to test depends on each laboratory's specifications and cannot be generalized. The decision involves the opinions of infectious diseases specialist and the pharmacist and should also be in concordance with the hospital formulary. Generally, a laboratory defines 10 to 15 antimicrobial agents for routine testing against various organisms or organism groups, which is called antimicrobial panel or battery. In CLSI's M100 documents Table 1A (Suggested Groupings of Antimicrobial Agents With FDA Clinical Indications That Should Be Considered for Routine Testing and Reporting on Nonfastidious Organisms by Clinical Microbiology Laboratories in the United States) is a valuable source of information to refer to when such tables are to be created at the local level [2]. Because the identity of the bacterial isolate is often not known at the time the AST is performed, some drugs, which are inappropriate to report for that particular isolate, may be tested. These results, however, should be supressed in the final report.

The goal of the clinical microbiology laboratory is to create a report which will direct the clinician to use the least toxic, most cost-effective and most clinically effective agent that is available. This is accomplished by using the selective-reporting protocol provided by the CLSI. CLSI categorizes antimicrobial agents generally into four groups, Group A, B, C and U. Group A includes the primary agents whose results to be reported first. The results of 
Group B drugs should be selectively reported because these are generally broader spectrum agents. However, if the isolate is resistant to the primary agents, the patient cannot tolerate drugs in Group A, the infection has not responded to the therapy with the primary agents, a secondary agent would be a better clinical choice for the particular infection or that the patient has organisms isolated from another site also, and a secondary agent might be more appropriate for treating both organisms, then the results of Group B drugs can be reported [26]. Group C includes alternative or supplemental agents for special cases; such as resistant strains, for patients allergic to primary drugs, for treatment of unusual isolates or for epidemiological purposes. And finally, Group $U$, includes the agents that are used only or primarily in the treatment of urinary tract infections (e.g., nitrofurantoin, norfloxacin).

Selective-reporting, also called cascade-reporting, improves the clinical relevance of the reports produced and minimizes the selection of multiresistant strains by avoiding the use of broad spectrum agents when narrow spectrum option is susceptible.

\subsection{Standardization of the antimicrobial susceptibility testing methodology}

The procedural steps of each method must be followed strictly in order to obtain reproducible results. Standardization of AST methodology helps to optimize bacterial growth conditions so that the inhibition of growth can be attributed to the antimicrobial agent and the effects of nutrient limitations, temperature differences or other environmental conditions can be eliminated. And it also optimizes conditions for maintaining antimicrobial integrity and activity so that the failure to inhibit bacterial growth can be attributed to the organism's resistance mechanisms [1].

The standardized components of AST include:

Bacterial inoculum size: Preparation of the inoculum is one of the most critical steps in any susceptibility test method. Inoculum suspensions are prepared using either a log-phase or direct-colony suspension. When direct-colony suspension method is used, 4 to 5, fresh (16to 24-hour old) colonies, rather than a single colony, should be selected to minimize the possibility of testing a susceptible colony only and missing the resistant mutants dispersed in other colonies. McFarland turbidity standards are used to standardize the number of bacteria in the inoculum. McFarland standards can be prepared by adding specific volumes of $1 \%$ sulfuric acid and $1.175 \%$ barium chloride to obtain a barium sulfate solution with a specific optical density. The most commonly used is the McFarland 0.5 standard, which provides turbidity comparable with that of a bacterial suspension containing approximately $1.510^{8}$ $\mathrm{CFU} / \mathrm{mL}$ (CFU: colony-forming unit). Once standardized, the inoculum suspensions should be used within 15 minutes of preparation. False-susceptible results may occur if too few bacteria are tested, and false-resistant results may be the outcome of testing too many bacteria [26].

Growth medium: The most frequently used growth media are Mueller-Hinton broth and Mueller-Hinton agar. The standardized variables regarding these media should include; its formulation, $\mathrm{pH}$, cation concentration and thymidine content, thickness of agar (disk diffusion test), and supplements such as blood and serum. 
Incubation conditions (atmosphere, temperature, duration): Different organisms require different incubation conditions. Moreover, some antimicrobial agents require different incubation length or temperature than the other disks used for the same organism (e.g., oxacillin with Staphylococcus spp.). The user should refer to CLSI M100 tables which give detailed testing conditions for each organism or organism group [2].

Antimicrobials concentrations to be tested: The contents of antimicrobial disks in disk diffusion test and concentrations of antibiotic solutions to be tested in dilution tests are also included in CLSI documents [2].

\subsection{Quality control testing with reference quality control strains}

Routine QC testing with a range of QC strains is the backbone of the internal QC testing. QC strains are well characterized organisms with defined susceptibility or resistance mechanisms to the antimicrobial agent(s) tested. Testing of QC strains helps to concurrently monitor the performance of the test and ensures that the test is being performed properly. The results obtained with the QC strains should be in predefined, acceptable ranges; for disk diffusion test, between the predefined inhibition zone diameters, and for MIC tests in predefined MIC ranges. If deviations from the acceptable limits are observed, it indicates unacceptable performance and the source(s) of the error should be investigated. CLSI recommends to use various QC strains for different aspects of AST. The list of QC strains can be found in the M100 tables which are updated on a yearly basis. Because of the introduction of new drugs, the changes effecting the existing drugs, or the emergence of new resistance mechanisms which should be investigated by the laboratory, the users are always referred to the latest update available. The QC strains recommended by CLSI are divided in two as being regular "QC strains" and „supplemental QC strains“. Each laboratory performing AST with CLSI's reference methods should include QC strains in regular QC tests, however, the supplemental strains are only required if they are used to assess a new test, for training new personnel, investigation of special susceptibility or resistance characteristics, etc., and are not required to be included in the routine QC of AST [2].

CLSI's European counterpart, EUCAST, also publishes guidelines for the use of QC strains for AST, however, compared with the comprehensive battery of QC strains suggested by the CLSI, EUCAST is limited to six QC strains at the moment [27]. The guidelines of EUCAST are continously evolving and on areas where EUCAST's experience is not able to cover yet, EUCAST does not refrain from making referrals to relevant CLSI documents. However, one big difference between the QC strains recommended by CLSI and EUCAST is that, EUCAST's recommendation for Haemophilus influenzae NCTC 8468 in contrast to CLSI's H. influenzae ATCC ${ }^{\circ}$ 49247. The strain EUCAST chose as a QC strain is susceptible to $\beta$-lactam antibiotics whose inhibition zones are easier to read than the ATCC $\AA$ strain which is a $\beta$ lactamase negative, ampicillin resistant (BLNAR) strain. The suggested QC strains by CLSI with their specifications are listed in Table 1 [2]. 


\section{QC Strain}

Escherichia coli ATCC ${ }^{\circledR} 25922$

Escherichia coli ATCC ${ }^{\circledast} 35218$

\section{Test(s), for which strain is primarily used}

Disk diffusion and MIC of Enterobacteriaceae, Pseudomonas aeruginosa, Acinetobacter spp., Burkholderia cepacia,

Stenotrophomonas maltophilia

MIC of other non-Enterobacteriaceae

Screening and confirmatory tests for ESBLs (negative)

Disk diffusion and MIC of Neisseria meningitidis (for ciprofloxacin, nalidixic acid, minocycline, and sulfisoxazole)

Disk diffusion and MIC for $\beta$-lactam/ $\beta$-lactamase inhibitor combination drugs of Enterobacteriaceae, Pseudomonas aeruginosa, Acinetobacter spp., Burkholderia cepacia, Stenotrophomonas maltophilia, Staphylococcus spp.

MIC for $\beta$-lactam/ $\beta$-lactamase inhibitor combination drugs of other non-Enterobacteriaceae

Testing of amoxicillin-clavulanic acid for Haemophilus spp.

\begin{tabular}{|c|c|}
\hline Klebsiella pneumoniae ATCC ${ }^{\oplus} 700603$ & Screening and confirmatory tests for ESBLs (positive) \\
\hline \multirow[t]{2}{*}{ Klebsiella pneumoniae ATCC ${ }^{\circledast}$ BAA-1705 } & Confirmatory test for suspected carbapenemase production in \\
\hline & Enterobacteriaceae (MHT positive) \\
\hline \multirow[t]{2}{*}{ Klebsiella pneumoniae ATCC ${ }^{\circledast}$ BAA-1706 } & Confirmatory test for suspected carbapenemase production in \\
\hline & Enterobacteriaceae (MHT negative) \\
\hline \multirow[t]{3}{*}{ Pseudomonas aeruginosa ATCC ${ }^{\circledR} 27853$} & Disk diffusion and MIC of Pseudomonas aeruginosa, Acinetobacter \\
\hline & spp., Burkholderia cepacia, Stenotrophomonas maltophilia \\
\hline & MIC of other non-Enterobacteriaceae \\
\hline \multirow[t]{8}{*}{ Staphylococcus aureus ATCC ${ }^{\circledR} 25923$} & Disk diffusion of Staphylococcus spp. and Enterococcus spp. \\
\hline & $\begin{array}{l}\text { Screening test for } \beta \text {-lactamase production of Staphylococcus aureus } \\
\text { group and coagulase negative Staphylococci (negative) }\end{array}$ \\
\hline & Screening test for mecA-mediated oxacillin resistance using \\
\hline & cefoxitin in Staphylococcus aureus group and coagulase negative \\
\hline & Staphylococci (mecA negative; disk diffusion susceptible) \\
\hline & Screening test for inducible clindamycin resistance in \\
\hline & Staphylococcus aureus group and coagulase negative Staphylococci \\
\hline & with disk diffusion ( $\mathrm{D}$-zone test) (negative) \\
\hline
\end{tabular}
Screening test for high-level mupirocin resistance in Staphylococcus aureus group (mupA negative; disk diffusion susceptible)

Staphylococcus aureus ATCC ${ }^{\circledast} 29213$ 


\section{QC Strain}

\section{Test(s), for which strain is primarily used}

Screening test for $\beta$-lactamase production in Staphylococcus aureus group and coagulase negative Staphylococci (positive)

Screening test for oxacillin resistance in Staphylococcus aureus group (susceptible)

Screening test for mecA-mediated oxacillin resistance using cefoxitin in Staphylococcus aureus group (mecA negative; MIC susceptible)

Screening test for inducible clindamycin resistance in Staphylococcus aureus group, coagulase negative Staphylococci and Streptococcus spp. $\beta$-hemolytic group with broth microdilution (no growth)

Screening test for high-level mupirocin resistance in Staphylococcus aureus group (mupA negative; MIC susceptible)

Staphylococcus aureus ATCC ${ }^{\circledR} 43300$ Screening test for oxacillin resistance in Staphylococcus aureus group (resistant)

Screening test for mecA-mediated oxacillin resistance using cefoxitin in Staphylococcus aureus group (disk diffusion and MIC) and coagulase negative Staphylococci (disk diffusion) (mecA positive)

Staphylococcus aureus ATCC ${ }^{\circledR}$ BAA-976 Screening test for inducible clindamycin resistance in Staphylococcus aureus group, coagulase negative Staphylococci and Streptococcus spp. $\beta$-hemolytic group with broth microdilution (no growth)

Staphylococcus aureus ATCC ${ }^{\circledR}$ BAA-977 Screening test for inducible clindamycin resistance in Staphylococcus aureus group, coagulase negative Staphylococci and Streptococcus spp. $\beta$-hemolytic group with broth microdilution (growth)

Staphylococcus aureus ATCC ${ }^{\circledR}$ BAA-1708 Screening test for high-level mupirocin resistance in Staphylococcus aureus group (mupA positive; disk diffusion and MIC resistant)

\begin{tabular}{|c|c|}
\hline \multirow[t]{2}{*}{ Enterococcus faecalis ATCC ${ }^{\circledR} 29212$} & MIC of Enterococcus spp. \\
\hline & $\begin{array}{l}\text { Screening test for vancomycin MIC } \geq 8 \mu \mathrm{g} / \mathrm{mL} \text { in Staphylococcus } \\
\text { aureus group (susceptible) }\end{array}$ \\
\hline & Screening test for high-level aminoglycoside resistance in \\
\hline & $\begin{array}{l}\text { Enterococcus spp. (disk diffusion, broth microdilution, agar dilution: } \\
\text { susceptible) }\end{array}$ \\
\hline
\end{tabular}




\begin{tabular}{|c|c|}
\hline QC Strain & Test(s), for which strain is primarily used \\
\hline & $\begin{array}{l}\text { Screening test for vancomycin resistance in Enterococcus spp. (agar } \\
\text { dilution: susceptible) checking that medium is acceptable for testing } \\
\text { sulfonamides, trimethoprim, and trimethoprim/sulfamethoxazole }\end{array}$ \\
\hline \multirow[t]{3}{*}{ Enterococcus faecalis ATCC ${ }^{\oplus} 51299$} & $\begin{array}{l}\text { Screening test for vancomycin MIC } \geq 8 \mu \mathrm{g} / \mathrm{mL} \text { for Staphylococcus } \\
\text { aureus group (resistant) }\end{array}$ \\
\hline & $\begin{array}{l}\text { Screening test for high-level aminoglycoside resistance in } \\
\text { Enterococcus spp. (broth microdilution, agar dilution: resistant) }\end{array}$ \\
\hline & $\begin{array}{l}\text { Screening test for vancomycin resistance in Enterococcus spp. (agar } \\
\text { dilution: resistant) }\end{array}$ \\
\hline Haemophilus influenzae ATCC ${ }^{\circledR} 49247$ & $\begin{array}{l}\text { Disk diffusion and MIC of Haemophilus spp. (BLNAR; } \beta \text {-lactamase } \\
\text { negative, ampicillin resistant) }\end{array}$ \\
\hline Haemophilus influenzae ATCC ${ }^{\oplus} 49766$ & $\begin{array}{l}\text { Disk diffusion and MIC of Haemophilus spp. with selected } \\
\text { cephalosporins ( } \beta \text {-lactamase positive) }\end{array}$ \\
\hline Haemophilus influenzae ATCC ${ }^{\circledR} 10211$ & $\begin{array}{l}\text { Checking growth capabilities of medium used for disk diffusion and } \\
\text { MIC tests for Haemophilus spp. }\end{array}$ \\
\hline Neisseria gonorrhoeae ATCC ${ }^{\circledR} 49226$ & $\begin{array}{l}\text { Disk diffusion and MIC of Neisseria gonorrhoeae (CMRNG; } \\
\text { chromosomally mediated (penicillin) resistant N. gonorrhoeae) }\end{array}$ \\
\hline \multirow[t]{2}{*}{ Streptococcus pneumoniae ATCC ${ }^{\circledR} 49619$} & $\begin{array}{l}\text { Disk diffusion and MIC of Streptococcus pneumoniae (penicillin } \\
\text { intermediate), Streptococcus spp. } \beta \text {-hemolytic group Streptococcus } \\
\text { spp. viridans group and Neisseria meningitidis }\end{array}$ \\
\hline & $\begin{array}{l}\text { Screening test for inducible clindamycin resistance in Streptococcus } \\
\text { spp. } \beta \text {-hemolytic group with disk diffusion (D-zone test) and broth } \\
\text { microdilution (negative) }\end{array}$ \\
\hline Bacteroides fragilis ATCC ${ }^{\circledast} 25285$ & MIC of anaerobes \\
\hline Bacteroides thetaiotaomicron ATCC ${ }^{\circledR} 29741$ & MIC of anaerobes \\
\hline Clostridium difficile ATCC ${ }^{\circledast} 700057$ & MIC of anaerobes \\
\hline Eubacterium lentum ATCC ${ }^{\circledast} 43055$ & MIC of anaerobes \\
\hline
\end{tabular}

Table 1. Quality Control Strains Suggested for Antimicrobial Susceptibility Testing by CLSI

\subsection{Selection, obtaining and maintenance of reference QC strains}

When selecting QC strains for routine internal QC testing; the strains that most closely resemble the patient's isolate should be tested [23]. This will provide that the drugs planned to be tested for the patient can be concomitantly tested with the QC strain. Additionally, same materials and testing conditions used for the clinical isolates can be evaluated. Before obtaining the QC strains, laboratories should decide which strains do fit best to the laborato- 
ry's procedures. For example, if a laboratory does not perform Modified Hodge Test (MHT) to confirm suspected carbapenemase production in Enterobacteriaceae, the Klebsiella pneumoniae ATCC® BAA-1705 (MHT-positive) and Klebsiella pneumoniae ATCC® BAA-1706 (MHTnegative) strains are not necessary for that particular laboratory. QC organisms susceptible to the tested antimicrobials are generally used but resistant QC strains are also necessary when testing for special resistance mechanisms.

The QC strains can be obtained from various suppliers and in many formats. What important is, no matter in what format the strain has been received, the initial reconstitution should be performed according to supplier's recommendations. For long term storage, stock cultures can be stored in a suitable stabilizer (e.g., trypticase soy broth with 10 to $15 \%$ glycerol, $50 \%$ fetal calf serum in broth, defibrinated sheep blood or skim milk) at $-20^{\circ} \mathrm{C}$ or below (preferably at $-60^{\circ} \mathrm{C}$ or below). To obtain working control cultures, subcultures from the permanent stock culture are made onto agar plates. Isolated colonies (4 to 5) are selected and subcultured to an agar slant (trypticase soy agar slants for non-fastidious organisms and chocolate agar slants for fastidious organisms) and incubated overnight. These working cultures on agar slants are stored at $2-8^{\circ} \mathrm{C}$, for no more than three successive weeks. New working control cultures should be prepared at least monthly from permanent stock cultures. Prior to QC testing, growth from an agar slant is subcultured to agar plates and incubated overnight. To use for QC testing, 4 to 5 isolated colonies from the plate are selected. A new working culture should be prepared each day the QC test is being performed $[2,23]$.

Working control cultures can be used to monitor precision (repeatability) and accuracy of the AST as long as no significant change in the mean zone diameter or MIC value, not attributable to faulty methodology, is observed. Laboratories usually do not have problems with the maintenance of susceptible QC strains owing to the stability of these strains, however, QC strains with particular resistance mechanisms are harder to maintain since they may be less genetically stable. Repeated subcultures can cause the loss of resistance mechanisms and unsatisfactory performances can be experienced. Documented problems have arisen with the QC strains which carry their specific resistance mechanism on a plasmid (e.g., E. coli ATCC® 35218 and K. pneumoniae ATCC® 700603) [2]. Suboptimal storage conditions and repeated cultures may cause the spontaneous loss of the plasmid encoding the $\beta$-lactamase and off-the-limit results may be encountered.

\subsection{Frequency of QC testing}

Appropriate QC organisms should be tested daily for all antimicrobial agents routinely included in the antimicrobial battery until a laboratory achieves "satisfactory performance". CLSI makes the definition of "satisfactory performance" as obtaining unacceptable results in no more than 1 out of 20 or 3 out of 30 results obtained in consecutive test days for each antimicrobial agent/organism combination. Once this satisfactory performance is obtained, a laboratory can convert from daily QC testing to weekly QC testing. As long as all QC test results are within the acceptable limits, the laboratory can continue weekly testing, however on occasions when a modification in the test is made, consecutive QC testing is required (Table 2., adapted from reference 2). 


\begin{tabular}{|c|c|}
\hline $\operatorname{Day}(s)^{*}$ & Modification in the Test \\
\hline \multirow[t]{4}{*}{1} & $\begin{array}{l}\text { Start to use new shipment or lot number of disks/MIC } \\
\text { panels or prepared agar plates }\end{array}$ \\
\hline & Start to use disks from a new manufacturer \\
\hline & Expand or reduce the dilution range in MIC testing \\
\hline & Repair of instrument that affects the AST results \\
\hline \multirow[t]{3}{*}{5} & $\begin{array}{l}\text { Start to use prepared agar plates (disk diffusion), broth or } \\
\text { agar (MIC) from a new manufacturer }\end{array}$ \\
\hline & $\begin{array}{l}\text { Convert inoculum preparation/standardization method } \\
\text { from visual adjustment of turbidity to use a photometric } \\
\text { device which has its own QC protocol }\end{array}$ \\
\hline & Update of the software which affects the AST results \\
\hline \multirow[t]{4}{*}{20 or 30} & $\begin{array}{l}\text { Use new method for MIC test (e.g., convert from visual } \\
\text { reading to instrument reading of panel, convert from } \\
\text { overnight to rapid MIC test) }\end{array}$ \\
\hline & Use new manufacturer of MIC test \\
\hline & $\begin{array}{l}\text { Change method of measuring zones in disk diffusion test } \\
\text { (e.g., start using an automated zone reader) }\end{array}$ \\
\hline & $\begin{array}{l}\text { Convert inoculum preparation/standardization method to } \\
\text { a method that is dependent on user technique }\end{array}$ \\
\hline
\end{tabular}

* Number of days of consecutive QC testing required

Table 2. Required Quality Control Frequency after Modifications in the Test

For both, disk diffusion and MIC testing, addition of any new antimicrobial agent to the existing panel requires 20 or 30 consecutive days of satisfactory testing before it can be tested on a weekly schedule.

\subsection{Corrective action}

Corrective action is defined as the "action to eliminate the cause of a detected nonconformity or other undesirable situation" [28] and in regard to AST, is needed whenever any of the weekly QC results are not within the acceptable limits. The factors causing for the deviation in the results are various but can be divided in two as being results due to identifiable errors and results with no error identified [24, 25]. Identifiable errors, also named obvious errors, are easy to detect and also easy to correct. Most usual reasons causing for identifiable errors include; use of the wrong disk, use of the wrong QC strain, contamination of the strain or media, use of the wrong incubation temperature or conditions. If the reason causing the outof-range results is one of the identifiable errors, the test must be carried out again the day the error is observed. If results of the repeat test are in acceptable limits, no further correc- 
tive action is necessary. On the other hand, if the reason causing for the error cannot be identified, the test must be carried out again the day the error is observed, preferably with a new working culture or subculture, but should also be monitored for a total of five consecutive test days. During five consecutive days, if all results are within the acceptable limits no additional corrective action is required. However, if any of the results are outside the acceptable limits, additional corrective action is required. At this point, a systematic error, rather than a random should be suspected and the components of AST should be thoroughly investigated. The reasons include; wrong measurement, clerical errors, problems in the adjustment of turbidity, past expiration date materials, failure in providing proper growth conditions (temperature, atmosphere), improper storage of disks, contamination of QC strain, loss of characteristics, inoculum prepared from an old plate ( $>24$ hours), etc.. In order to start to routine QC testing, satisfactory performance for another 20 or 30 consecutive days is required once the reason causing the error is detected and corrected.

When an out-of-range QC results necessitates a corrective action, the factors listed in Table 3 should be considered for troubleshooting (Table 3., adapted from references 24 and 25).

\begin{tabular}{|c|c|}
\hline QC Strain & Use of the wrong QC strain \\
\hline & Improper storage \\
\hline & Inadequate maintenance (e.g., use of the same working culture for \\
\hline & $>1$ month) \\
\hline & Contamination \\
\hline & Nonviability \\
\hline & Changes in the organisms (e.g., mutation, loss of plasmid) \\
\hline \multirow[t]{6}{*}{ Testing supplies } & Improper storage or shipping conditions \\
\hline & Contamination \\
\hline & Use of a defective agar plate (too thick or too thin) \\
\hline & Inadequate volume of broth in tubes or wells \\
\hline & Use of damaged plates, panels, cards, tubes (e.g., cracked, leaking) \\
\hline & Use of expired materials \\
\hline \multirow[t]{9}{*}{ Testing process } & Use of the wrong incubation temperature or conditions \\
\hline & Inoculum suspensions were incorrectly prepared or adjusted \\
\hline & $\begin{array}{l}\text { Inoculum prepared from a plate incubated for the incorrect length } \\
\text { of time }\end{array}$ \\
\hline & Inoculum prepared from differential or selective media containing \\
\hline & anti-infective agents or other growth-inhibiting compounds \\
\hline & Use of wrong disk/reagents, ancillary supplies \\
\hline & Improper disk placement (e.g., inadequate contact with the agar) \\
\hline & Incorrect reading or interpretation of test results \\
\hline & Transcription error \\
\hline Equipment & Not functioning properly or out of calibration (e.g., pipettes) \\
\hline
\end{tabular}

Table 3. Factors Frequently Causing Out-of-range Results 


\subsection{Documentation of the quality control test results}

Results from all QC tests should be documented on a QC log sheet [23]. On this log sheet information regarding the following are required: the date, the technician who performed the test, antimicrobial agents used (potency, lot, expiration date, etc.), media used (lot, expiration date, etc.). Once the log sheet has been filled by the technician who performed and read the test, a second technician, or the supervisor, should check the results. Also, corrective actions taken, if any, and their outcomes should be noted.

A useful and simple way of monitoring QC results is to use the Shewhart diagram, in which the daily readings are plotted on a chart with upper and lower control limits marked [29]. It provides the visual assessment of the results but can also provide in depth information if a more formal mathematical approach is followed [20]. An example of presenting daily QC results on a Shewhart diagram is given in Figure 1. The famous rules of Westgard and Klee [30] can be easily adopted to the QC of disk diffusion test in which the control diameters are treated as mean \pm 2 SD [20].

One QC result lies outside the limits (Westgard rule $1_{2 \mathrm{~s}}$ ): It is a warning, whether it's a random error or the beginning of an emerging problem. Routine test results for that day may be reported if there is no other evidence of problems in the current tests. It does not require corrective action by itself, unless the result is far out of range or there are other indications of a problem.

Two consecutive QC results are outside the limits in the same side of the mean of the range (Westgard rule $2 \mathrm{2s}$ ): Indicates an error in the test methodology (a systematic error), corrective action is required.

Ten consecutive QC results falling on one side of the mean (Westgard rule $10_{\ddot{x}}$ ): Results may be accepted but this likely indicates a systematic problem which should be acted on.

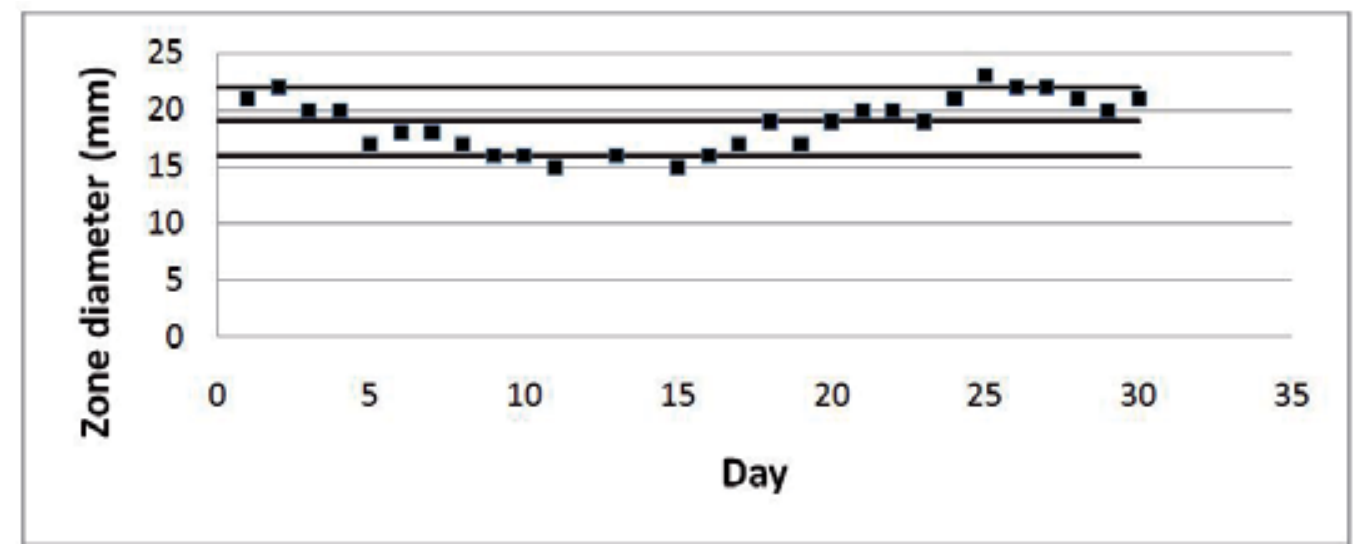

Figure 1. Example for daily disk diffusion QC results for Escherichia coli ATCC ${ }^{\circledR} 25922$ vs. ampicillin plotted on a Shewhart diagram (acceptable zone limits: $16-22 \mathrm{~mm}$ ). 


\subsection{Organism - Antimicrobial susceptibility test result verification}

One of the most widely used supplemental QC measure is the use of susceptibility test results to verify results generated on patient results. Species with "typical“ antibiograms are useful in verification of the identification as well as the susceptibility results. CLSI suggests some results to be confirmed before they are reported, these mostly include rare resistance phenotypes. The rare resistance phenotypes are divided in three categories; Category I; not reported or only rarely reported to date, Category II; uncommon in most institutions, and Category III; may be common, but is generally considered of epidemiological concern. Since category I includes the least encountered and most significant results, it is highly important to detect these results before being reported unnoticed and to follow the necessary steps for the verification. Unusual resistance phenotypes which require confirmation are given in Table 3 (adapted from reference 2).

\begin{tabular}{|c|c|}
\hline Category & Observed susceptibility result \\
\hline \multirow[t]{5}{*}{$\mathbf{I}$} & $\begin{array}{l}\text { NS to carbapenems, extended-spectrum cephalosporins or } \\
\text { fluoroquinolones in } \mathrm{H} \text {. influenzae }\end{array}$ \\
\hline & $\begin{array}{l}\text { NS to extended-spectrum cephalosporins, meropenem or } \\
\text { minocycline, R to ampicillin or penicillin in } N \text {. meningitidis }\end{array}$ \\
\hline & NS to linezolid or vancomycin in S. pneumoniae \\
\hline & $\begin{array}{l}\text { NS to ampicillin, penicillin, extended-spectrum } \\
\text { cephalosporins, daptomycin, ertapenem, meropenem, } \\
\text { linezolid or vancomycin in } \beta \text {-hemolytic group } \\
\text { Streptococcus }\end{array}$ \\
\hline & $\begin{array}{l}\text { NS to daptomycin, ertapenem, meropenem, linezolid, or } \\
\text { vancomycin, R to quinupristin-dalfopristin in viridans } \\
\text { group Streptococcus }\end{array}$ \\
\hline \multirow[t]{7}{*}{ II } & I or R to carbapenems in Enterobacteriaceae \\
\hline & $\begin{array}{l}\text { I or R to 3rd generation cephalosporins or } \\
\text { fluoroquinolones in Salmonella and Shigella spp. }\end{array}$ \\
\hline & R to colistin/polymyxin in A. baumannii \\
\hline & I or R to colistin/polymyxin in $P$. aeruginosa \\
\hline & I or R to trimethoprim-sulfamethoxazole in S. maltophilia \\
\hline & $\begin{array}{l}\mathrm{R} \text { to amoxicillin-clavulanic acid, } \mathrm{R} \text { to ampicillin without } \\
\text { accompanying } \beta \text {-lactamase production in } \mathrm{H} \text {. influenzae }\end{array}$ \\
\hline & $\begin{array}{l}\text { NS to extended spectrum cephalosporins in } N \text {. } \\
\text { gonorrhoeae }\end{array}$ \\
\hline
\end{tabular}




\begin{tabular}{|c|c|}
\hline Category & Observed susceptibility result \\
\hline & $\begin{array}{l}\text { I to ampicillin, penicillin, I or R to rifampin, NS to } \\
\text { azithromycin in N. meningitidis }\end{array}$ \\
\hline & R to linezolid, NS to daptomycin for Enterococcus spp. \\
\hline & $\begin{array}{l}\text { NS to daptomycin, } \mathrm{R} \text { to linezolid, I or R to quinupristin- } \\
\text { dalfopristin, vancomycin } \mathrm{MIC}=4 \mu \mathrm{g} / \mathrm{mL} \text { or vancomycin } \\
\mathrm{MIC} \geq 8 \mu \mathrm{g} / \mathrm{mL} \text { for } S \text {. aureus }\end{array}$ \\
\hline & $\begin{array}{l}\text { NS to daptomycin, I or R to quinupristin-dalfopristin or } \\
\text { vancomycin, R to daptomycin in coagulase-negative } \\
\text { Staphylococcus spp. }\end{array}$ \\
\hline & $\begin{array}{l}\text { I or R to fluoroquinolone, imipenem, meropenem, } \\
\text { quinupristin-dalfopristin, rifampin in S. pneumoniae }\end{array}$ \\
\hline & $\begin{array}{l}\text { I or } \mathrm{R} \text { to quinupristin-dalfopristin in } \beta \text {-hemolytic group } \\
\text { Streptococcus }\end{array}$ \\
\hline \multirow[t]{9}{*}{ III } & $\begin{array}{l}\text { R to amikacin, gentamicin, and tobramycin in } \\
\text { Enterobacteriaceae }\end{array}$ \\
\hline & $\begin{array}{l}\text { I or R to extended spectrum cephalosporins in E. coli, } \\
\text { Klebsiella spp. or P. mirabilis }\end{array}$ \\
\hline & I or R to carbapenem in A. baumannii \\
\hline & $\begin{array}{l}\mathrm{R} \text { to amikacin, gentamicin, and tobramycin, or } \\
\text { carbapenem in } P \text {. aeruginosa }\end{array}$ \\
\hline & I or R to fluoroquinolone in N. gonorrhoeae \\
\hline & $\begin{array}{l}\text { I or R to chloramphenicol or fluoroquinolone in } N \text {. } \\
\text { meningitidis }\end{array}$ \\
\hline & $\begin{array}{l}\mathrm{R} \text { to vancomycin or high-level aminoglycoside in } \\
\text { Enterococcus spp. }\end{array}$ \\
\hline & R to oxacillin in S. aureus \\
\hline & $\begin{array}{l}\mathrm{R} \text { to amoxicillin, penicillin or extended spectrum } \\
\text { cephalosporins in S. pneumoniae using nonmeningitis } \\
\text { breakpoints }\end{array}$ \\
\hline
\end{tabular}

NS; nonsusceptible, l; intermediate, R; resistant

Table 4. Unusual Resistance Phenotypes Which Require Confirmation

The general approach to be followed is, for all three categories, to confirm the identification of the organism and the AST. If the results are confirmed, the infection control should be informed about the case. 


\subsection{Real-time review of results}

Accuracy of the susceptibility test results should be continously monitored. This is mostly accomplished by daily reviewing of the data that is being produced. Profiles which are likely, somewhat likely, somewhat unlikely and nearly impossible should be identified, whether manually or with the help of a software programmed to recognize different patterns of susceptibility data [1]. Prompt recognition of unusual resistance or inconsistent susceptibility helps the laboratory to timely confirm the susceptibility results. In order to confirm the results, first step is to exclude the transcriptional and reading errors and make sure of the purity of the inoculum which has been tested. If no errors are found in the previous steps, the identification of the organism should be confirmed and the susceptibility test be repeated, preferably with another method. In cases where no errors are detected and the unusual resistance is confirmed, the clinician may be warned and measures can be taken to limit the spread of this unusual resistance.

\subsection{Education}

Education is an important component of the QA process. Having knowledge about the methods also provides the understanding of their limitations and pitfalls. A well-educated technician may timely recognize atypical results and is aware of the approach to follow for the resolution and avoidance of errors [20]. A very efficient way of training in-service personnel is the end-point interpretation control [24, 25]. Laboratory workers, who perform AST, are provided with a set of selected disk diffusion plates and are asked to read the results. The recorded results are then compared by an experienced reader, e.g., the laboratory director, and the individual performances of each technician is evaluated and if necessary, corrected. It significantly helps to minimize variation in the interpretation of zone sizes among laboratory workers.

\subsection{External quality assessment}

In external quality assessment (EQA) programs, a central laboratory distributes test strains with known susceptibility profiles to all participant laboratories. Each participating laboratory tests and reports the results to the central laboratory. Once all the results are returned from participants, the central laboratory evaluates the results and prepares a feedback report. The benefit of participating in such program is that each individual laboratory can assess ist own performance compared with other laboratories, at national and international levels, it functions as an educational tool, and also provides the evidence of performance required by the accrediting bodies. On the other hand, the number of strains distributed in a year is relatively small, which brings the disadvantage of the rare errors going unnoticed [20]. Also, in contrast to internal QC, which is capable of acting on problems encountered on daily basis, it takes quite a while for the EQA feedback reports to be sent to the participating laboratories, thus corrective action is delayed.

\subsection{Internal quality assessment}

Internal quality assessment (IQA) is a complementary activity to EQA in which routine tests are repeated on the same day as the original, but this time, with the identity of the specimen 
blinded. After the reports are produced, the results are compared and discrepancies noted. This activity helps to monitor the precision and accuracy of the test procedure and may highlight problem areas not detected by other QC methods. It monitors not only the performance of the test and reagents, but also the performance of the persons carrying out the tests [20]. The EQA and the IQA are complementary activities, while IQA focuses on monitoring a single laboratory on a daily basis, EQA compares the performance of different laboratories and is important for maintaining long-term accuracy of the AST methods employed [21].

\subsection{Proficiency testing programs}

They are a type of EQA in which simulated patient specimens are sent to participating laboratories. Again, the reports are produced by each laboratory, and returned to the central laboratory for evaluation. In the United States, government mandates that clinical laboratories be accredited and licensed. The government and licensing agencies are using proficiency testing as an objective method for the accreditation of laboratories [21]. In 1988, the U.S. Congress passed the Clinical Laboratory Improvement Amendment (CLIA '88) which mandated proficiency testing (PT) as a major part of the laboratory accreditation process [31]. The initial CLIA ' 88 proposal called for two PT specimens per year but final legislative rule, published in 2003, expanded this to study five samples three times per year. The definition of failure is defined as two of five incorrect results on two of the three consecutive PT surveys [32].

\section{Quality control of automated antimicrobial susceptibility test systems}

According to the work load and the resources a laboratory has, a laboratory can choose to use one of many types of commercial automated antimicrobial susceptibility test systems. Most of these systems use the principle of turbidimetric detection of bacterial growth in a broth medium by use of a photometer which periodically examines the test wells [26]. The most widely used systems in the world are VITEK 2 System (bioMérieux Vitek, Hazelwood, MO), BD Phoenix System (BD Diagnostic Systems, Sparks, MD), MicroScan WalkAway SI (Siemens Healthcare Diagnostics, Sacramento, CA) and TREK Sensititre (ARIS 2X, Trek Diagnostic Systems, Cleveland, $\mathrm{OH}$ ). Each device has its own QC procedure and commercial susceptibility testing devices are not addressed in CLSI standards. CLSI only describes methods regarding generic reference procedures, however these reference methods are used by the US Food and Drug Administration before clearence is given to a commercial system for marketing in the US to evaluate its performance.

\section{Conclusion}

Although great improvement has been done in AST methodology and automated susceptibility systems have been introduced which provide same-day results, it should be considered that there are still many variables not covered by the standard methods. First of all, the 
laboratory test conditions are far different from in vivo conditions where the organism and the antimicrobial agent do actually interact. Factors, such as bacterial inoculum size, $\mathrm{pH}$, cation concentration and oxygen tension differ greatly depending on the site of infection [1]. In spite of all these limitations, the clinical microbiology laboratory should follow the most upto-date guidelines to serve the patients in the best possible way. With a well constructed QA program in operation, a laboratory should aim to ensure that the right test is carried out on the right specimen, and that the right result and right interpretation is delivered to the right person at the right time.

\section{Author details}

Onur Karatuna

Acibadem University, Istanbul, Turkey

\section{References}

[1] Laboratory Methods and Strategies for Antimicrobial Susceptibility Testing. (2007). In: Forbes B. A., Sahm D. F., Weissfeld A. C. (ed.) Bailey \& Scott's Diagnostic Microbiology. 12th ed. St. Louis, MO: Mosby Elsevier; 187-214.

[2] CLSI. (2012). Performance Standards for Antimicrobial Susceptibility Testing; Twenty-Second Informational Supplement. CLSI document M100-S22. Wayne, PA: Clinical and Laboratory Standards Institute.

[3] Fleming, A. (1924). A comparison of the activities of antiseptics on bacteria and on leucocytes. Proceedings of the Royal Society of London, Series B 96, 171-80.

[4] Fleming, A. (1929). On the antibacterial action of cultures of a penicillium, with special reference to their use in the isolatation of B. influenzae. British Journal of Experimental Pathology 110, 226-36.

[5] Vincent, J. G., Vincent, H. V. (1944). Filter paper disc modification of the Oxford cup penicillin determination. Proceedings of the Society for Experimental Biology and Medicine 55, 162-4.

[6] Schmith, K., Reymann, F. E. (1940). Experimentelle og kliniske undersogelser over gonococcers folsomhed overfor sulfapyridin. Nordisk Medicin 8, 2493-9.

[7] Waterworth, P. M. (1951). A comparative study of methods of testing sensitivity to antibiotics and of the factors influencing the results. Journal of Medical Laboratory Technology 9, 65-85. 
[8] World Health Organization. (1961). Standardization of Methods for Conducting Microbic Sensitivity Tests. Second Report of the Expert Committee on Antibiotics. WHO Technical Report Series, No. 210. WHO, Geneva.

[9] Bauer, A. W., Kirby, W. M. M., Sherris, J. C., Turck, M. (1966). Antibiotic susceptibility testing by a standardized single disk method. American Journal of Clinical Pathology 45, 493-6.

[10] Bergeron, M. G., Ouellette, M. (1998). Preventing antibiotic resistance through rapid genotypic identification of bacteria and of their antibiotic resistance genes in the clinical microbiology laboratory. Journal of Clinical Microbiology 36, 2169-72.

[11] National Committee for Clinical Laboratory Standards. (1975). Performance Standards for Antimicrobial Disk Susceptibility Tests; Approved Standard M2-A7 ASM-2. NCCLS, Villanova, PA.

[12] Werkgroep Richtlijnen Gevoeligheidsbepalingen Report. (1990). Standaardisatie van Gevoeligheidsbepalingen. WRG, Bilthoven.

[13] Comité de l'Antibiogramme de la Société Française de Microbiologie. (1996). Clinical Microbiology and Infection 2, Suppl 1, S1-49.

[14] The Swedish Reference Group for Antibiotics. (1997). A revised system for antibiotic sensitivity testing. Scandinavian Journal of Infectious Diseases Suppl. 105.

[15] Deutsches Institut für Normung. (2000). Methoden zur Empfindlichkeitsprüfung von bakteriellen Krankheitserregern (außer Mykobakterien) gegen Chemotherapeutika. DIN 58940.

[16] Andrews, J. M. For the BSAC Working Party on Susceptibility Testing. (2000). BSAC standardized disc susceptibility testing method. Journal of Antimicrobial Chemotherapy 48, Suppl. 1, 43-57.

[17] European Comittee on Antimicrobial Susceptibility Testing - EUCAST. (2012). http:// www.eucast.org (accessed 30 July 2012).

[18] Antimicrobial Susceptibility Testing. (2006). In: Winn W., Allen S., Janda W., Koneman E., Procop G., Schreckenberger P., Woods G. (ed.) Koneman's Color Atlas and Textbook of Diagnostic Microbiology. 6th ed. Baltimore, MD: Lippincott Williams \& Wilkins; 945-1021.

[19] August M. J., Hindler J. A., Huber T. W., Sewell D. L. (1990). Cumitech 3A. Quality Control and Quality Assurance Practices in Clinical Microbiology. Coordinating ed. Weissfeld S. A. Washington, D.C.: American Society for Microbiology.

[20] King A., Brown D. F. J. (2001). Quality assurance of antimicrobial susceptibility testing by disc diffusion. Journal of Antimicrobial Chemotherapy ,48, Suppl. S1, 71-6.

[21] Westgard J. O., Klee G. G. (2006). Quality Management. In: Burtis C. A., Ashwood E. R., Bruns D. E. (ed.) Tietz Textbook of Clinical Chemistry and Molecular Diagnostics. 4th ed. St. Louis, MO: Elsevier Saunders; 485-529. 
[22] Washington J. A. (1988). Current problems in antimicrobial susceptibility testing. Diagnostic Microbiology and Infectious Diseases 9, 135-8.

[23] Rankin I. D. (2005). Quality Assurance/Quality Control (QA/QC) In: Coyle M. B. (ed.) Manual of Antimicrobial Susceptibility Testing. 1st ed, Washington, D.C.: American Society for Microbiology; 63-89.

[24] CLSI. (2012). Performance Standards for Antimicrobial Disk Susceptibility Tests; Approved Standard-Eleventh Edition. CLSI document M02-A11. Wayne, PA: Clinical and Laboratory Standards Institute.

[25] CLSI. (2012). Methods for Dilution Antimicrobial Susceptibility Tests for Bacteria That Grow Aerobically; Approved Standard-Ninth Edition. CLSI document M07-A9. Wayne, PA: Clinical and Laboratory Standards Institute.

[26] Marsik F. J. (2011). Antimicrobial Susceptibility Testing. In: Mahon C. R., Lehman D. C., Manuselis G. (ed.) Textbook of Diagnostic Microbiology. 4th ed. Maryland Heights, MO: Saunders Elsevier; 276-314.

[27] European Comittee on Antimicrobial Susceptibility Testing. (2012). EUCAST recommended strains for internal quality control. Version 2.1, valid from 2012-06-29. http:// www.eucast.org/fileadmin/src/media/PDFs/EUCAST_files/Disk_test_documents/ EUCAST_QC_tables_2.1_120629_errata.pdf (accessed 30 July 2012).

[28] ISO. (2000). Quality Management Systems-Fundamentals and Vocabulary. ISO 9000. Geneva: International Organization for Standardization.

[29] Shewhart W. A. (1931). Economic control of quality of the manufactured product. New York: Van Nostrand.

[30] Westgard J. O., Barry P. L., Hunt M. R., Groth T. A. (1981). A multirule Shewhart chart for quality control in clinical chemistry. Clinical Chemistry 27, 493-501.

[31] US Department of Health and Human Services. (1992). Clinical Laboratory Improvement Amendments of 1988; Final Rules and Notice. 42 CFR Part 493. The Federal Register 57, 7188-288.

[32] US Centers for Medicare \& Medicaid Services (CMS). (2003). Medicare, Medicaid, and CLIA Programs: Laboratory Requirements Relating to Quality Systems and Certain Personnel Qualifications. Final Rule. The Federal Register 16, 3640-714. 

Chapter 20

\title{
The Investigation of Gene Regulation and Variation in Human Cancers and Other Diseases
}

\author{
Shihori Tanabe and Sun Ha Jee \\ Additional information is available at the end of the chapter \\ http://dx.doi.org/10.5772/51271
}

\section{Introduction}

Dynamic regulation of genes is an important part of the cell life cycle in health and disease. The regulation includes the variety and alteration of genome and gene expression, and the concept such as quality of genome will be useful to predict and assess the developmental stages of the cells, disease status and drug sensitivity. Recent technologies and worldwide sequencing projects have revealed 26,383 annotated genes in the 2.91-Gigabase human genome [1,2]. The main molecular functions of the annotated genes, as categorized by Gene Ontology (GO), are enzyme, signal transduction, nucleic acid binding, cell adhesion, chaperone, cytoskeletal structural protein, extracellular matrix, immunoglobulin, ion channel, motor, structural protein of muscle, protooncogene, select calcium binding protein, intracellular transporter, and transporter [1,3]. Despite a wealth of knowledge, the function of $42 \%$ of the annotated genes remains unknown [1]. When the human genome sequence was published in 2001 [1], there were a predicted 39,114 genes, of which 59\% were of unknown function. According to the International Human Genome Sequencing Consortium, the number of identified genes is approximately 32,000 , of which $51 \%$ show a match within InterPro, a database that integrates diverse information about protein families, domains, and functional sites [2-5]. In 2001, InterPro combined sequence and pattern information from four databases (PRINTS, PROSITE, Pfam, Prosite Profile); however, it now includes information from an additional eight databases (SMART, ProDom, PIRSF, SUPERFAMILY, PANTHER, CATH-Gene3D, TIGRFAM, and HAMAP) [2,4-16]. In [2], the InterPro entries are collapsed into 12 broad categories: cellular processes, metabolism, DNA replication/modification, transcription/translation, intracellular signaling, cell-cell communication, protein folding and degradation, transport, multifunctional proteins, cytoskeletal/structural, defense and immunity, and miscellaneous function. The 
rate of single nucleotide polymorphism (SNP) variation has been reported as 1 in 1250 base pairs [1] and more than 1.4 million SNPs have been identified [2] (Table 1).

\begin{tabular}{clc}
\hline Size of the genome & $2.91 \mathrm{Gbp}$ & {$[1]$} \\
\hline Number of annotated genes & 26,383 & {$[1]$} \\
\hline $\begin{array}{c}\text { Main molecular functions of } \\
\text { annotated genes }\end{array}$ & $\begin{array}{l}\text { enzyme, signal transduction, nucleic acid binding, cell adhesion, chaperone, cytoskeletal structural protein, } \\
\text { extracellular matrix, immunoglobulin, ion channel, motor, structural protein of muscle, protooncogene, } \\
\text { select calcium binding protein, intracellular transporter, transporter }\end{array}$ & {$[1]$} \\
\hline $\begin{array}{c}\text { Percentage of annotated genes } \\
\text { with unknown function }\end{array}$ & $42 \%$ & [1] \\
\hline $\begin{array}{c}\text { Number of hypothetical and } \\
\text { annotated genes }\end{array}$ & 39,114 & {$[1]$} \\
\hline $\begin{array}{c}\text { Percentage of hypothetical and } \\
\text { annotated genes with unknown function }\end{array}$ & $59 \%$ & {$[1]$} \\
\hline Number of identified genes & approx. 32,000 & {$[2]$} \\
\hline Percentage of matches with & $51 \%$ & {$[2]$} \\
\hline InterPro & $1 / 1250 \mathrm{bp}$ & {$[1]$} \\
\hline Rate of SNP variation & more than 1.4 million & [2] \\
\hline SNPs identified & & \\
\hline
\end{tabular}

Table 1. Genomic and gene characteristics revealed by the Human Genome Project.

Among the databases combined in InterPro (Table 2), PRINTS, PROSITE, and Pfam contain protein families in which the homology between each protein is predicted by the degree of sequence similarity [8]. The others-SMART, ProDom, PIRSF, SUPERFAMILY, PANTHER, CATH-Gene3D, TIGRFAM, and HAMAP [4-16]-have unique characteristics and URLs, and have been developed sharing information among each other and incorporating information from GO. In detail, PRINTS is a collection of diagnostic protein family "fingerprints", which are groups of conserved motifs, evident in multiple sequence alignments [6]; PROSITE is a protein domain database for functional characterization and annotation that consists of documentation entries describing protein domains, families, and functional sites as well as associated patterns and profiles to identify them [7]; Pfam contains collections of protein families, each represented by multiple sequence alignments and hidden Markov models, available via servers in the UK, the USA, and Sweden [8]; SMART (Simple Modular Architecture Research Tool) is an online resource for the identification and annotation of protein domains and the analysis of protein domain architectures [9]; ProDom is a comprehensive set of protein domain families generated automatically from the UniProt database [10]; PIRSF is a classification system that reflects evolutionary relationships among fulllength proteins and domains [11]; SUPERFAMILY is a database of structural and functional annotation for all proteins and genomes [12]; PANTHER is a classification system that clas- 
sifies genes by their functions using published experimental evidence and evolutionary relationships to predict function even in the absence of direct experimental evidence [13]; CATH-Gene3D is a comprehensive database of protein domain assignments for sequences from the major sequence databases [14]; TIGRFAM is a collection of protein family definitions built to aid high-throughput annotation of specific protein functions [15]; and HAMAP is composed of two databases: the proteome database and the family database, and of an automatic annotation pipeline mainly focused on microbial proteomes [16]. Hidden Markov models are usually used for the database algorithm.

\begin{tabular}{|c|c|c|c|}
\hline $\begin{array}{l}\text { Database } \\
\text { Name }\end{array}$ & Context & URL & Reference \\
\hline PRINTS & $\begin{array}{l}\text { a collection of diagnostic protein family } \\
\text { "fingerprints" which are groups of conserved motifs, evident in multiple sequence } \\
\text { alignments }\end{array}$ & $\begin{array}{l}\text { http://www.bioinf.manchester.ac.uk/ } \\
\text { dbbrowser/PRINTS/index.php }\end{array}$ & {$[6]$} \\
\hline PROSITE & $\begin{array}{l}\text { a protein domain database for functional characterization and annotation which } \\
\text { consists of documentation entries describing protein domains, families and functional } \\
\text { sites as well as associated patterns and profiles to identify them }\end{array}$ & | http://prosite.expasy.org/ & [7] \\
\hline Pfam & $\begin{array}{l}\text { a database of collection of protein families, each represented by multiple sequence } \\
\text { alignments and hidden Markov models, available via servers in the } \\
\text { UK, the USA and Sweden }\end{array}$ & $\begin{array}{l}\text { http://pfam.sanger.ac.uk/http:// } \\
\text { pfam.janelia.org/http://pfam.sbc.su.se/ }\end{array}$ & [8] \\
\hline SMART & $\begin{array}{l}\text { an online resource for the identification and annotation of protein domains and the } \\
\text { analysis of protein domain architectures, of which abbreviation } \\
\text { is Simple Modular Architecture Research Tool }\end{array}$ & http://smart.embl.de/ & [9] \\
\hline SUPERFAMILY & $\begin{array}{l}\text { a database of structural and functional annotation } \\
\text { for all proteins and genomes }\end{array}$ & http://supfam.org/SUPERFAMILY/ & [12] \\
\hline PANTHER & $\begin{array}{l}\text { the classification system which classifies genes by } \\
\text { their functions using published scientific experimental evidence and evolutionary } \\
\text { relationships to predict function even in the absence of direct experimental evidence }\end{array}$ & http://www.pantherdb.org/ & [13] \\
\hline CATH-Gene3D & $\begin{array}{l}\text { a comprehensive database of protein domain assignments for sequences from the } \\
\text { major sequence databases }\end{array}$ & http://gene3d.biochem.ucl.ac.uk/ & [14] \\
\hline
\end{tabular}




\begin{tabular}{llll}
\hline $\begin{array}{l}\text { Database } \\
\text { Name }\end{array}$ & Context & URL & Reference \\
\hline TIGRFAM & $\begin{array}{l}\text { a collection of protein family definitions built to aid } \\
\text { in high-throughput annotation of specific protein functions }\end{array}$ & $\begin{array}{l}\text { http://www.jcvi.org/cgi-bin/tigrfams/ } \\
\text { index.cgi }\end{array}$ & {$[15]$} \\
\hline HAMAP & $\begin{array}{l}\text { a system which composed of two databases, the proteome database and the family } \\
\text { database, and of an automatic annotation pipeline }\end{array}$ & http://hamap.expasy.org/ & {$[16]$} \\
\hline
\end{tabular}

Table 2. Database information.

\section{Gene regulation}

\subsection{Gene markers for cancer and cancer stem cells}

Several molecular markers of cancer have been identified [17]. Metastatic cancer cells can transfer into bodily fluids through the cellular epithelia, which enables the detection of cancer markers in bodily fluids such as blood plasma, urine, or saliva [17]. The different types of cancer markers include genomic DNA point mutations, microsatellite alterations, promoter hypermethylation, viral sequences, aberrant chromosomal copy number, chromosomal translocations, deletions, or loss of heterozygosity, telomere extension, alterations in RNA or protein expression, and mitochondrial DNA mutations [17].

Molecular markers of cancer include TP53 (encoding p53), which has been shown to be mutated in head and neck, lung, colon, pancreatic, and bladder cancer [17,18]; colon, lung, esophagus, breast, liver, brain, reticuloendothelial tissue, and hematopoietic tissue cancers [19]; and bladder cancer [20]. Mutation of the epidermal growth factor receptor (EGFR) gene is an important predictive/prognostic factor for EGFR-tyrosine kinase inhibitor therapy in non-small cell lung cancer [21]. RAS oncogene mutations have been identified in colorectal tumors [22]. Microsatellites, which are tandem iterations of simple di-, tri-, or tetranucleotide repeats, have been reported to be unstable in some inherited diseases and in some types of cancer [23], including head and neck, lung, breast, and bladder cancer $[17,23]$.

The expression levels of the cell cycle-related proteins p21 (CDKN1A), p53 (TP53), cyclin D1 (CCND1), and aurora kinase A (AURKA) may be used as prognostic markers to predict recurrence in stage II and stage III colon cancer [24]. In addition, markers of the epithelialmesenchymal transition (EMT)-such as reduced expression of keratins, a switch from ECadherin to N-Cadherin, and enhanced migration in D492M cells-might be a useful marker in breast cancer [25]. Furthermore, expression of the stem cell markers cytokeratins 15 and 19 was altered in squamous cell carcinoma: cytokeratin 15 levels were decreased and the localization of cytokeratin 19 was altered [26]. KLK3, which encodes prostate-specific antigen, a member of the kallikrein family of serine proteases, is a biomarker for prostate cancer detection and disease monitoring [27,28]. Mitochondrial DNA mutations have been associated with bladder, head and neck, lung, colorectal, and pancreatic cancer [29-32] (Table 3). 
Highly parallel identification of cancer-related genes using small hairpin RNA screening has revealed that the expression of known and putative oncogenes, such as EGFR, KRAS, MYC, $B C R-A B L, M Y B, C R K L$, and $C D K 4$ that are essential for cancer proliferation, is altered in cancer cells [33]. Other genes such as PTPN1, NF1, SMARCB1, and SMARCE1 have been identified as essential for the imatinib response of leukemia cells, and TOPOIIA expression is involved in resistance to etoposide, an anti-topoisomerase II agent, in small cell lung cancer [33-36].

\begin{tabular}{|c|c|c|}
\hline Marker & Cancer Type & Reference \\
\hline \multirow{3}{*}{ TP53 mutation } & head and neck cancer & {$[18]$} \\
\hline & bladder cancer & {$[20]$} \\
\hline & $\begin{array}{l}\text { lung cancer (small cell lung cancer and non-small cell lung cancer); breast, colon, esophagus, liver, } \\
\text { bladder, ovary, and brain cancers; sarcomas, lymphomas, and leukemias }\end{array}$ & {$[19]$} \\
\hline EGFR mutation & non-small cell lung cancer & {$[21]$} \\
\hline RAS mutation & colorectal tumors & {$[22]$} \\
\hline \multirow[t]{2}{*}{ DNA microsatellite alterations } & bladder cancer & {$[23]$} \\
\hline & colon cancer & {$[24]$} \\
\hline alteration in cytokeratin mRNA & squamous cell carcinoma & {$[26]$} \\
\hline alteration in kallikrein mRNA & prostate cancer & {$[27]$} \\
\hline \multirow{3}{*}{ mitochondrial DNA mutations } & bladder cancer, head and neck cancer, lung cancer & [29] \\
\hline & colorectal tumors & [30], [32] \\
\hline & pancreatic cancer & [31] \\
\hline
\end{tabular}

Table 3. Genomic markers of cancer.

\subsection{Genes related to cell proliferation}

Cyclins, which regulate the cell cycle, play important roles in cell proliferation and the uncontrolled cell proliferation that is the most important factor in tumorigenesis [37]. Tumor cells accumulate mutations that result in constitutive mitogenic signaling and defective responses to anti-mitogenic signals that contribute to unscheduled proliferation [38]. In cancer, unscheduled proliferation, genomic instability, and chromosomal instability are the three major factors in cell cycle dysregulation [38]. Regulation of the cell cycle is mainly conducted by complexes of cyclins and cyclin-dependent kinases [38]. Cyclin D1 in cell migration and proliferation is temporo-spatially separated by its biphasic expression induced by thrombin, a G protein-coupled receptor agonist, which is mediated by nuclear factor of activated $\mathrm{T}$ cells $\mathrm{c1}$ (NFATC1) and signal transducer and activator of transcription 3 (STAT3) [39]. Cyclin D1 regulates kinase activity and the $\mathrm{G}_{1}-\mathrm{S}$ phase tran- 
sition in the cell cycle; deregulated cyclin D1 expression is well documented in breast, colon, and prostate cancers $[39,40]$. The expression of cyclin D1 is regulated by several factors including cytokines such as interleukin 3 and interleukin 6 via STAT3 and STAT5, or extracellular matrix factors such as collagen, fibronectin, and vitronectin, which activate focal adhesion kinase upon integrin clustering, and hepatocyte nuclear factor 6 [41]. Cyclin D1 is a crucial regulator of Wnt- and Notch-regulated development [41,42]. The binding of Wnt to its receptor, Frizzled, causes release of $\beta$-catenin to translocate from the cytoplasm to the nucleus, where it forms a complex with the ternary complex factor and/or the lymphoid enhancer-binding factor [41,43]. Cyclin D1 is induced by overexpression of $\beta$-catenin, which is a major component of adherens junctions that link the actin cytoskeleton to members of the cadherin family of transmembrane cell-cell adhesion receptors. It plays an important role in linking the cytoplasmic side of cadherin-mediated cell-cell contacts to the actin cytoskeleton [43]. Betacatenin is upregulated in colorectal cancer, which is considered to trigger cyclin D1 gene expression followed by uncontrolled progression of the cell cycle [43]. In addition, $\beta$-catenin plays another role in signaling that involves transactivation, in complex with transcription factors of the lymphoid enhancing factor family in the nucleus [43]. The pathway involving $\beta$-catenin/LEF1 and elevation of cyclin D1 might be crucial for tumorigenesis [43]. Inhibiting EglN2, a member of the EglN (also called PHD or HPH) family of prolyl hydroxylases that regulates the heterodimeric transcription factor hypoxia-inducible factor (HIF), causes a decrease in the expression of its interaction partner cyclin D1 in cancer cells and impairs the cells' ability to proliferate in vivo [44].

Progression of the eukaryotic cell cycle is driven by cyclin-dependent protein kinases (CDKs), which are binding partner of cyclins. The CDK oscillator acts as the primary organizer of the cell cycle [45]. Phosphorylation of cyclin-Cdk complexes is one of the primary mechanisms of cell cycle regulation [46]. Cyclins are degraded by ubiquitinmediated proteolysis [46]. The ubiquitylation and degradation of cyclin 1 and cyclin 2 are mediated by the SCF complex, a multi-subunit ubiquitin ligase that contains Skp1, a member of the cullin family (Cdc53) and an F-box protein, as well as a RING-finger-containing protein [46]. CDKs including CDK1, CDK2, CDK4, CDK6, and CDK11 have various functions that have been investigated using loss-of-function, target validation, and gain-of-function mouse models [38]. CDK1 is a mitotic CDK, also known as cell division control protein 2 (CDC2). It is one of the master regulators of mitosis as it controls the centrosome cycle as well as mitotic onset; deficiency in CDK1 results in embryonic lethality in the first cell divisions $[38,47]$. CDK2, CDK4, and CDK6 are interphase CDKs that are not essential for the mammalian cell cycle; they are, however, required for the proliferation of specific cell types [38]. Deficiency in CDK2, CDK4, and CDK6 caused mid-gestation embryonic lethality because of hematopoietic defects $[38,47]$.

\subsection{Genes related to cell differentiation}

Inhibitor of differentiation 1 (Id1) is associated with the induction of cell proliferation and invasion [48], as well as the invasive features of cancer and the EMT [48]. The HOX genes 
encode homeodomain-containing transcription factors involved in the regulation of cellular proliferation and differentiation during embryogenesis [49]. The expression of HOXA1, which plays an important role in proliferation, apoptosis, adhesion, invasion, the EMT, and anchorage-independent growth, was significantly increased in oral squamous cell carcinoma compared with in healthy oral mucosa [49], and it might be a useful prognostic marker for patients with this disease [49].

Wnt/ $\beta$-catenin signaling controls skeletal development and differentiation [50]. The initiating step of skeletal development is mesenchymal condensation, during which mesenchymal progenitor cells are at least bipotentiate [50]. Osteochondral progenitor cells differentiate into osteoblasts instead of chondrocytes when $\mathrm{Wnt} / \beta$-catenin signaling is activated [50]. In vitro models using human pluripotent stem cell-derived neural progenitor cells have been used to examine whether G11778A-mutated mitochondrial DNA, which is associated with Leber's hereditary optic neuropathy, might be involved in the differentiation of neural progenitor cells into neurons, oligodendrocytes, and astrocytes [51]. The differentiation of neural progenitor cells can be visualized by staining for the neuronal marker class III betatubulin [51]. Alternative splicing of exons play an important role in cellular differentiation and pathogenesis [52]. Alternative splicing in colorectal cancer and renal cell cancer samples has been analyzed by the Bioinformatics Exon Array Tool (BEAT, http://beat.ba.itb.cnr.it/) using an Affymetrix GeneChip Exon Array [52]. When the dataset was analyzed using GO terms, the cell differentiation (GO:0030154)-related gene delta-like 1 (Drosophila) (DLL1) was found to be involved in colorectal cancer [52].

\subsection{Genes related to apoptosis}

Cell proliferation and death are regulated by various molecules. Recently, microRNAs have been revealed to play important roles during death receptor-mediated apoptosis (programmed cell death) [53]. Transfection with miR-133b caused a proapoptotic effect on tumor necrosis factor alpha (TNF $\alpha$ )-stimulated HeLa cells [53]: the expression of apoptosis regulatory proteins such as transgelin 2 (TAGLN2), myosin, heavy chain 9, non-muscle (MYH9), cytoskeleton-associated protein 4 (CKAP4), polypyrimidine tract binding protein 1 (PTBP1), glutathione-S-transferase pi 1 (GSTP1), and copine III (CPNE3) were down-regulated compared with in control cells [53]. The BCL protein family plays a major role in regulation of the apoptotic cascade [54]. BCL2-associated protein (BAX) promotes apoptosis and delays disease progression, and has been associated with longer disease-free survival in patients with a number of gastrointestinal cancers, such as esophageal, stomach, small intestine, and colon cancer; moreover, high BCL6 expression is correlated with worse prognosis in patients with other gastrointestinal tumors, such as esophageal adenocarcinoma [54]. There are two major cell death pathways that transduce the effects of various death inducers: the extrinsic death pathway that is mediated through cell death receptors of the TNF receptor family, such as the Fas receptor; and the intrinsic death pathway that proceeds through mitochondria [55]. The expression of apoptosis signal-regulating kinase (ASK1), which plays an important role as a mitogen-activated protein kinase kinase kinase in apoptosis signaling, is in- 
creased in gastric cancer [56]. Furthermore, the levels of cyclin D1 and phosphorylated JNK were higher in gastric cancer than in non-tumor epithelium [56]. ASK1 may play a role in the development of gastric cancer [56].

\subsection{Detection of cell proliferation or apoptosis}

Several methods have been suggested for the diagnosis of cancer [57]. Protein markers for cancer include prostate-specific antigen for prostate cancer, CA125 for ovarian cancer, carcinoembryonic antigen for colon cancer, human chorionic gonadotropin for trophoblastic cancer, and a-fetoprotein for hepatocellular carcinoma and germ cell tumors [57]. Assays to detect telomerase activity in clinical samples include the TRAP (telomere repeat amplification protocol) assay, which involves protein extraction and subsequent primer-directed PCR amplification of telomere extensions [57].

Assays for the detection of kinases that regulate cell growth, proliferation, differentiation, and metabolism have been developed [58]. The assay technology includes fluorescence polarization to detect protein phosphorylation, scintillation proximity to detect protein dephosphorylation by phosphatases, fluorescence resonance energy transfer to detect protein cleavage or modification, immunosorbent assays to detect phosphorylation state, luciferasebased ATP detection to detect the kinase-dependent depletion of ATP, luminescent oxygen channeling to detect phosphorylation, time-resolved fluorescence resonance energy transfer to detect phosphopeptide formation, and enzyme fragment complementation to detect molecular interactions with kinases [58,59]. Cell proliferation can also be determined by the tetrazolium hydroxide (XTT) cell proliferation assay, in which absorbance is measured by an ELISA reader under 490-nm-wavelength light (Biological Industries) [60].

Cell proliferation assays and apoptosis assays have been used to examine the effects of inhibitors on cancer cells [61]. The cell proliferation of Neuro-2A cells, neuroblastoma cells, can be determined using the CellTiter 96 Aqueous Non-Radioactive Cell Proliferation Assay reagent (Promega) [61]. A colony formation assay using Neuro-2A cells was used to determine the effect of an inhibitor of GSK-3 $\beta$ [61]. In this experiment, colonies were allowed to form for 10 days, after which the cells were fixed with $70 \%$ ethanol and stained with $1 \%$ methylene blue. Apoptosis was then measured by flow cytometry using an Annexin V-allophycocyanin (APC) /propidium iodide (PI) detection kit (BD PharMingen) [61]. Apoptosis was also determined using 4'6-diamidino-2-phenylindole (DAPI) staining, observing apoptotic nuclear morphology, and immunoblotting with antibodies to $\beta$-catenin, X-linked inhibitor of apoptosis, and BCL2 [61]. Cell cycle analysis using PI to quantify the proportions of cells in the $G_{1} / G_{0}$ or $G_{2}-M$ phases was used to examine cell cycle status [61].

Viable cells can be determined using MTT (3-(4,5-dimethylthiazol-2-yl)-2,5-diphenyltetrazolium bromide) colorimetric assays [62]. Absorbance at $570 \mathrm{~nm}$ is used to detect the incorporation of MTT. Apoptosis can also be determined by caspase activation using an anti-poly ADP-ribose polymerase (PARP) antibody [62]. Viable cells can also be determined using a 3-(4,5-dimethyl-thiazol-2yl)-5-(3-carboxymethoxyphenyl)-2-(4-sulfophenyl)-2H-tetrazolium (MTS) kit (Promega) [63]. The terminal transferase dUTP nick end labeling (TUNEL) assay is commonly used to detect apoptosis [63]. Harvested cells are resuspended in DNA labeling solution 
consisting of TdT reaction buffer, TdT enzyme, and BrdUTP, then stained with PI to detect a fluorescein isothiocyanate-labeled anti-BrdU antibody [63]. Cell viability and proliferation assays were used to validate internal tandem duplication mutations in FLT3 as a therapeutic target for human acute myeloid leukemia [64]. Cell viability and proliferation can be determined using a Vi-cell XR automated cell viability analyzer (Beckman Coulter) [64].

\section{Genomic variation in disease}

\subsection{Genome-wide association studies in cancer}

Despite extensive research efforts for several decades, the genetic basis of common human diseases such as cancers remains largely unknown [65]. Genome-wide association studies (GWAS) have emerged as an important tool for the discovery of genomic regions that harbor genetic variants conferring risk for various cancers [66,67]. Family-based linkage studies and studies comprising tens of thousands of gene-based SNPs can also assay genetic variation across the genome [68], but the National Institutes of Health guidelines for GWAS require a sufficient density of genetic markers to capture a large proportion of the common variants in the study population, measured in enough individuals to provide sufficient power to detect variants of modest effect [67]. The recent success of GWAS can be attributed to the convergence of new technologies that can genotype hundreds of thousands of SNPs in hundreds or thousands of samples [66,69].

GWAS have been conducted in the five of the most common cancer types: breast, prostate, colorectal, lung, and melanoma (Table 4) and have identified more than 20 novel disease loci, confirming that susceptibility to these diseases is polygenic [70]. For many years, human genetics has been used to map rare mutations with large effect sizes in families or genetically homogeneous populations, such as $B R C A 1 / B R C A 2$ mutations in Ashkenazi women with breast cancer and ovarian cancer [71]. A number of SNPs have now been associated with breast cancer; for example, a SNP in intron 2 of the FGFR2 gene, which encodes a receptor tyrosine kinase that is amplified and overexpressed in 5-10\% of breast tumors [72,73], and SNPs on chromosomes $16 \mathrm{q}$ and $5 \mathrm{q}$. The locus on $16 \mathrm{q}$ contains a gene TNRC9 and a hypothetical gene LOC643714. The function of TNRC9 is unknown but the presence of an HMG box motif suggests that it might act as a transcription factor. The $5 \mathrm{q}$ locus includes MAP3K1, which encodes a protein involved in signal transduction (but not previously known to be involved in cancer) and two other genes: MGC33648 and MIER3. In addition, several of the breast cancer loci appear to be associated with specific subtypes of the disease. In particular, the FGFR2 association is strongly associated with estrogen receptor-positive breast cancer, while the TNRC9 SNP is associated with both estrogen receptor-positive and -negative breast cancer [74,75]. It is surprising that none of the strongest associations map to regions harboring estrogen/progesterone genes in women of European background, particularly because a GWAS in Asian women reported a convincing association with markers near the estrogen receptor alpha (ESR1) gene [76]. In prostate cancer, the first and most important region to emerge was $8 \mathrm{q} 24$. This region was first associated with prostate cancer through 
linkage studies by the deCode group, was followed up by association analyses [77], and has been confirmed in subsequent GWAS [78-81]. Another signal, on chromosome 10q13, points to a variant in the promoter of the MSMB gene, which encodes the PSP94 protein; this is now under intense investigation as a biomarker for prostate cancer [80,81].

In general, the susceptibility alleles discovered thus far are common-that is, with a frequency in one or more population of $>10 \%$, and each allele confers a small contribution to the overall risk of the disease. For nearly all regions conclusively identified by GWAS, the effect sizes per allele are estimated at $<1$.3. It was not anticipated that GWAS in certain cancers would yield many novel regions when other cancers strongly associated with particular environmental exposures have yielded so few regions. For example, prostate cancer, breast cancer, and colon cancer have been associated with 29, 13, and 10 regions of the genome, respectively, while there are only three associated regions for lung cancer in smokers, and three for bladder cancer despite analysis of sufficiently large data sets [67]. Several GWAS for lung cancer have identified the same locus on $15 q 25$, suggesting that this is an important susceptibility locus for this disease [82-87]. This locus contains the nicotinic acetylcholine receptor subunit genes CHRNA3 and CHRNA5, suggesting that susceptibility may be mediated through smoking behavior $[86,87]$.

GWAS represent an important advance in discovering genetic variants influencing disease but have important limitations. There is a high potential for false-positive results, they do not yield information on gene function, they are insensitive to rare and structural variants, they require large sample sizes, and incur possible biases because of case and control selection and genotyping errors [88]. Clinicians and scientists must understand the unique aspects of these studies and be able to assess and interpret GWAS results for themselves and their patients. However, at present these studies mainly represent a valuable discovery tool for examining genomic function and clarifying pathophysiological mechanisms. However, through GWAS, the identification of variants, genes, and pathways involved in multiple cancers offers a potential route to new therapies, improved diagnosis, and better disease prevention [65].

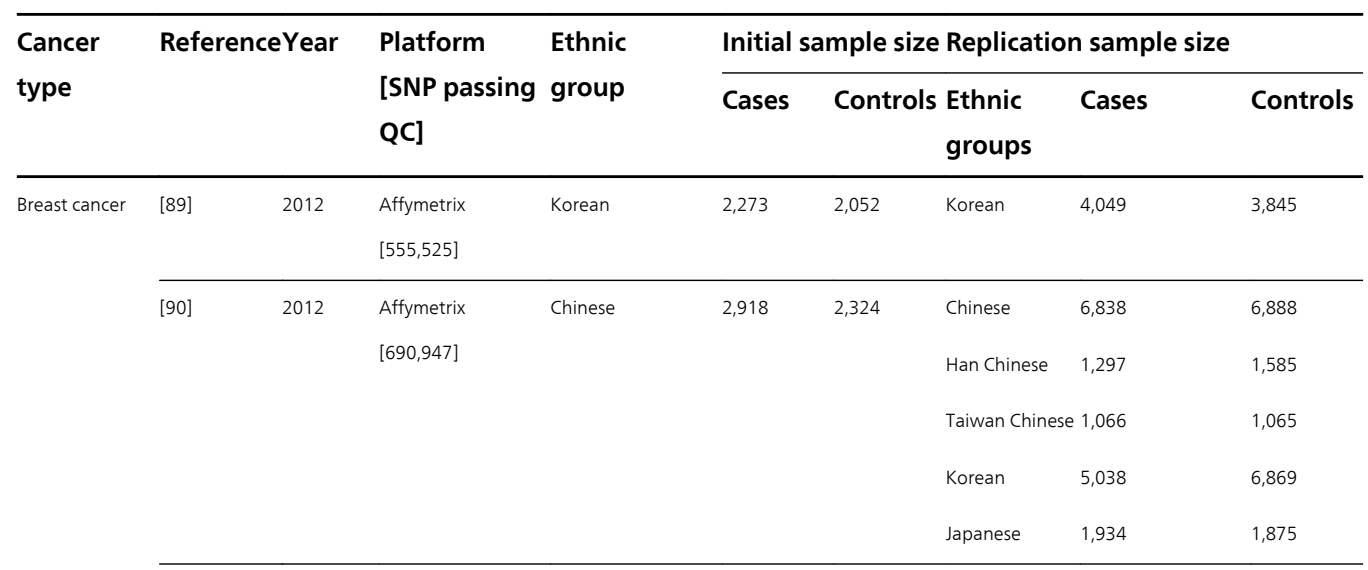




\begin{tabular}{|c|c|c|c|c|c|c|c|c|c|}
\hline \multirow{2}{*}{$\begin{array}{l}\text { Cancer } \\
\text { type }\end{array}$} & \multicolumn{2}{|c|}{ ReferenceYear } & \multirow{2}{*}{$\begin{array}{l}\text { Platform } \\
\text { [SNP passing } \\
\text { QC] }\end{array}$} & \multirow{2}{*}{$\begin{array}{l}\text { Ethnic } \\
\text { group }\end{array}$} & \multicolumn{5}{|c|}{ Initial sample size Replication sample size } \\
\hline & & & & & Cases & \multicolumn{2}{|c|}{$\begin{array}{r}\text { Controls Ethnic } \\
\text { groups }\end{array}$} & Cases & Controls \\
\hline & {$[91]$} & 2012 & $\begin{array}{l}\text { Affymetrix } \\
{[613,031]}\end{array}$ & Chinese & 1,950 & - & Chinese & 4,160 & - \\
\hline & {$[92]$} & 2012 & $\begin{array}{l}\text { Illumina } \\
{[470,796]}\end{array}$ & Japanese & 240 & - & Japanese & 222 & - \\
\hline & {$[93]$} & 2011 & $\begin{array}{l}\text { Affymetrix } \\
{[684,457]}\end{array}$ & East Asian & 2,062 & 2,066 & East Asians & 15,091 & 14,877 \\
\hline & [94] & 2011 & $\begin{array}{l}\text { Affymetrix } \\
{[782,838]}\end{array}$ & European & 302 & 321 & European & 1,153 & 1,215 \\
\hline & [95] & 2011 & $\begin{array}{l}\text { Illumina } \\
{[\sim 296,114]}\end{array}$ & British & 1,694 & 2,365 & $\begin{array}{l}\text { British } \\
\text { European }\end{array}$ & $\begin{array}{l}7,317 \\
1,145\end{array}$ & $\begin{array}{l}8,124 \\
1,142\end{array}$ \\
\hline & {$[96]$} & 2010 & $\begin{array}{l}\text { Illumina } \\
{[285,984]}\end{array}$ & Swedish \& Finnish & 617 & 4,583 & European & 1,001 & 7,604 \\
\hline & {$[97]$} & 2010 & $\begin{array}{l}\text { Affymetrix } \\
{[592,163]}\end{array}$ & European & 899 & 804 & European & 1,264 & 1,222 \\
\hline & {$[98]$} & 2010 & $\begin{array}{l}\text { Illumina } \\
{[285,984]}\end{array}$ & European & 2,702 & 5,726 & European & 7,386 & 7,576 \\
\hline & [99] & 2010 & $\begin{array}{l}\text { Illumina } \\
{[582,886]}\end{array}$ & UK & 3,659 & 4,897 & European & 12,576 & 12,223 \\
\hline & {$[100]$} & 2010 & $\begin{array}{l}\text { Illumina } \\
{[528,252]}\end{array}$ & British & 1,145 & - & British & 4,335 & - \\
\hline & {$[101]$} & 2009 & $\begin{array}{l}\text { Illumina } \\
{[528,173]}\end{array}$ & & 1,145 & 1,142 & & 8,625 & 9,657 \\
\hline & {$[102]$} & 2009 & $\begin{array}{l}\text { Affymetrix } \\
\text { [up to } 607,728 \text { ] }\end{array}$ & Chinese & 1,505 & 1,522 & Chinese & 1,554 & 1,576 \\
\hline & {$[103]$} & 2008 & $\begin{array}{l}\text { Affymetrix } \\
{[200,220]}\end{array}$ & & 30 & 30 & - & - & - \\
\hline & {$[104]$} & 2008 & $\begin{array}{l}\text { Affymetrix } \\
{[492,900]}\end{array}$ & Ashkenazi Jewish & 249 & 299 & $\begin{array}{l}\text { Ashkenazi } \\
\text { Jewish }\end{array}$ & 1,193 & 1,166 \\
\hline & {$[105]$} & 2007 & $\begin{array}{l}\text { Affymetrix } \\
{[70,897]}\end{array}$ & Framing-ham & 1,345 & - & - & - & - \\
\hline & {$[106]$} & 2007 & $\begin{array}{l}\text { Perlegen } \\
{[205,586]}\end{array}$ & & 390 & 634 & & 26,646 & 24,889 \\
\hline & {$[107]$} & 2007 & $\begin{array}{l}\text { Illumina } \\
{[528,173]}\end{array}$ & & 1,145 & 1,142 & & 1,176 & 2,072 \\
\hline
\end{tabular}




\begin{tabular}{|c|c|c|c|c|c|c|c|c|c|}
\hline \multirow{2}{*}{$\begin{array}{l}\text { Cancer } \\
\text { type }\end{array}$} & \multicolumn{2}{|c|}{ ReferenceYear } & \multirow{2}{*}{$\begin{array}{l}\text { Platform } \\
\text { [SNP passing } \\
\text { QC] }\end{array}$} & \multirow{2}{*}{$\begin{array}{l}\text { Ethnic } \\
\text { group }\end{array}$} & \multicolumn{5}{|c|}{ Initial sample size Replication sample size } \\
\hline & & & & & Cases & Controls & $\begin{array}{l}\text { Ethnic } \\
\text { groups }\end{array}$ & Cases & Controls \\
\hline \multirow[t]{11}{*}{ Prostate cancer } & [108] & 2012 & $\begin{array}{l}\text { Illumina } \\
{[509,916]}\end{array}$ & European & 1,176 & 1,101 & European & 1,964 & 3,172 \\
\hline & [109] & 2012 & $\begin{array}{l}\text { Affymetrix } \\
\text { \& Illumina } \\
{[1,117,531]} \\
\text { (imputed) }\end{array}$ & & 4,723 & 4,792 & & - & - \\
\hline & [110] & 2011 & $\begin{array}{l}\text { NR } \\
\text { [2.6 million] } \\
\text { (imputed) }\end{array}$ & European & 6,621 & 6,939 & $\begin{array}{l}\text { European } \\
\text { Japanese } \\
\text { Chinese } \\
\text { African } \\
\text { American }\end{array}$ & $\begin{array}{l}22,957 \\
285 \\
135 \\
112\end{array}$ & $\begin{array}{l}23,234 \\
298 \\
135 \\
298\end{array}$ \\
\hline & [111] & 2011 & $\begin{array}{l}\text { Illumina } \\
{[571,243]}\end{array}$ & European & 2,782 & 4,458 & European & $\begin{array}{l}7,140 \\
8,217\end{array}$ & $\begin{array}{l}5,455 \\
6,732\end{array}$ \\
\hline & {$[112]$} & 2011 & $\begin{array}{l}\text { Illumina } \\
{[1,047,198]}\end{array}$ & African American & 3,425 & 3,290 & $\begin{array}{l}\text { African } \\
\text { American } \\
\text { Senegalese } \\
\text { Ghanaian } \\
\text { Barbadian }\end{array}$ & $\begin{array}{l}1,275 \\
86 \\
271 \\
246\end{array}$ & $\begin{array}{l}1,695 \\
414 \\
968 \\
253\end{array}$ \\
\hline & [113] & 2011 & $\begin{array}{l}\text { Affymetrix } \\
{[387,384]}\end{array}$ & European & 202 & 100 & European & 1,122 & 1,167 \\
\hline & [114] & 2010 & $\begin{array}{l}\text { Affymetrix } \\
{[419,613]}\end{array}$ & Caucasian & 222 & 415 & Caucasian & 500 & 155 \\
\hline & [115] & 2010 & $\begin{array}{l}\text { Illumina } \\
{[510,687]}\end{array}$ & Japanese & 1,583 & 3,386 & Japanese & 3,001 & 5,415 \\
\hline & [116] & 2009 & $\begin{array}{l}\text { Illumina } \\
\text { [541,129] }\end{array}$ & European & 1,854 & 1,894 & $\begin{array}{l}\text { European, } \\
\text { Chinese, } \\
\text { Japanese, } \\
\text { African } \\
\text { American, } \\
\text { Latino, and } \\
\text { Hawaiian }\end{array}$ & 19,879 & 18,761 \\
\hline & [117] & 2009 & $\begin{array}{l}\text { Illumina } \\
{[310,520]}\end{array}$ & Icelandic & 1,968 & 35,382 & European & 11,806 & 12,387 \\
\hline & [118] & 2008 & $\begin{array}{l}\text { Illumina } \\
{[541,129]}\end{array}$ & European & 1,854 & 1,894 & & 3,268 & 3,366 \\
\hline
\end{tabular}




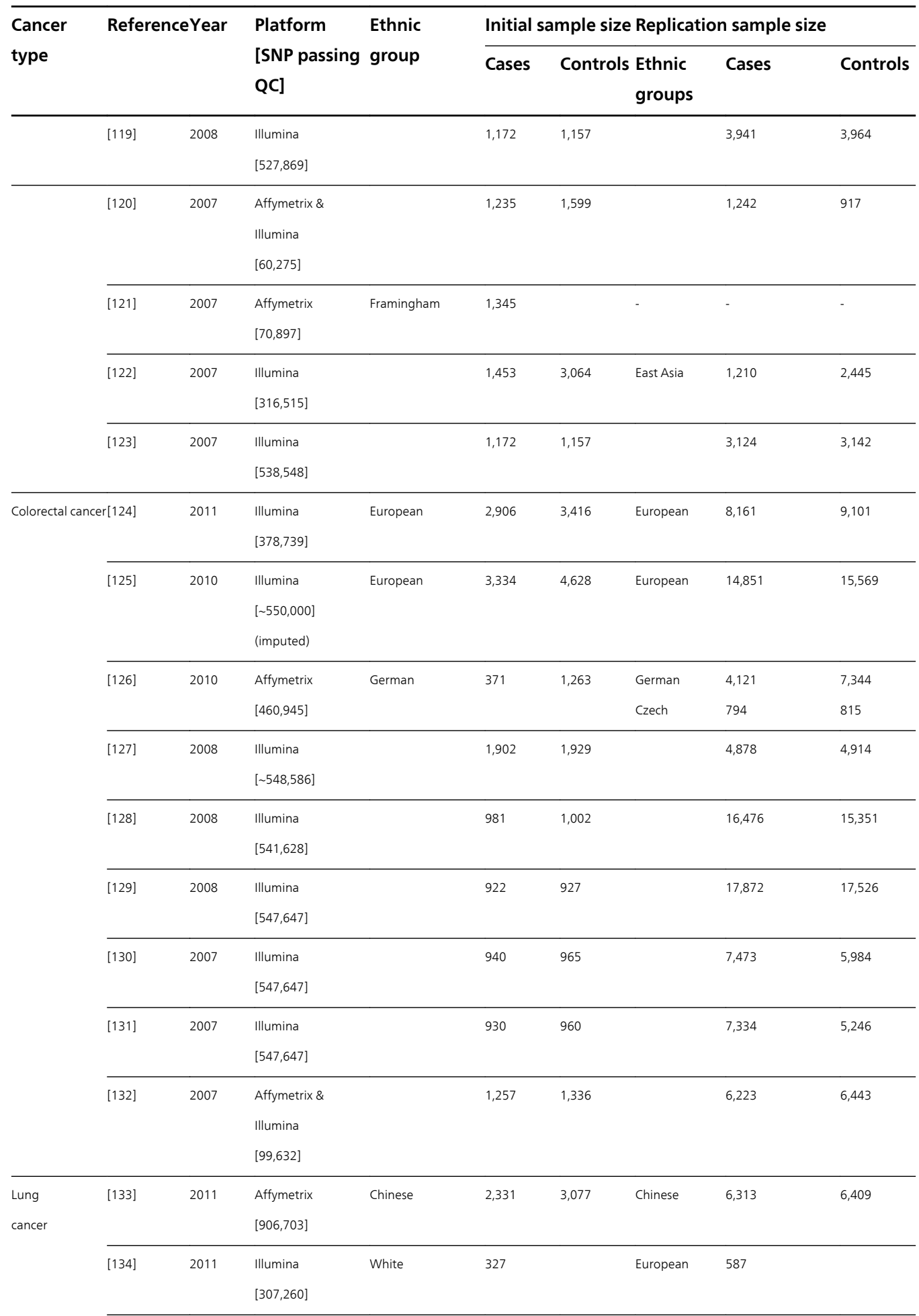




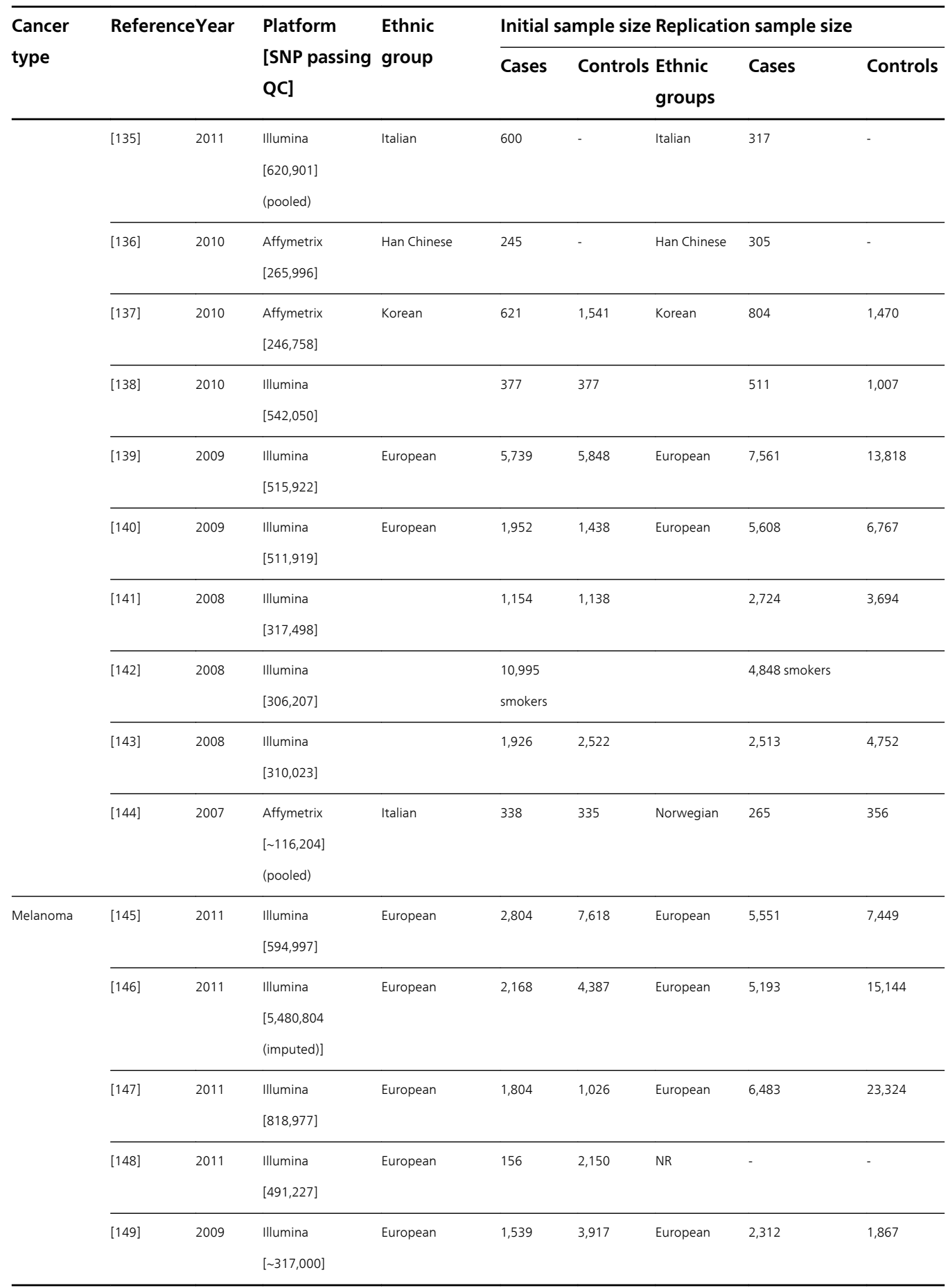

Table 4. Summary of GWAS for the five of the most common types of cancer. 


\subsection{Genetic risk score in cancer and diabetes}

Type 2 diabetes mellitus and cancers are major health problems worldwide $[150,151]$. The recent increase in the prevalence of these diseases is largely attributable to environmental factors. However, convincing evidence shows that genetic factors may play an important role in these diseases [152,153]. Recent GWAS have led to the identification of a series of SNPs that are robustly associated with either the risk of diabetes or cancers [151,154-159]. For type 2 diabetes mellitus, common SNPs have been identified in the PPARG, KCNJ11, and TCF7L2 genes, and have been widely replicated in populations of various ethnicities [160-162]. Other potential new loci include HHEX, CDKAL1, CDKN2A/B, IGF2BP2, SLC30A8, and WFS1 [65,155-159,163,164]. A number of SNPs have been identified as associated with breast cancer risk, including FGFR2, CASP8, ERBB4, TAB2, BARX2, TMEM45B, ESR1, FGFR2, TNRC9, MAP3K1, MGC33648, MIER3, and RAD51L1 [74,75,151] (Table 5).

Combining multiple loci with modest effects into a global genetic risk score (GRS) might improve the identification of those at risk for common complex diseases such as type 2 diabetes and cancers [165-167]. Several studies have developed methods to predict the risk of certain diseases, such as coronary heart disease, type 2 diabetes, and breast cancer, aggregating information from multiple SNPs into a single GRS [151,168,169]. For example, in the Atherosclerosis Risk in Communities study, the aggregation of multiple SNPs into a single GRS was responsible for improving the prediction of coronary heart disease incidence [168]. In a study that used a GRS to determine the risk of type 2 diabetes in US men and women, individuals in the highest quintile of GRS had a significantly increased risk of type 2 diabetes compared with those in the lowest quintile; however, the addition of a GRS to the conventional model consisting of lifestyle risk factors only increased the area under the curve by only $1 \%$ (AUC=0.78). In this instance, the GRS was determined to be useful only when combined with the body mass index or a family history of diabetes [169]. For breast cancer, a GRS was created using 14 SNPs previously associated with breast cancer, and was substantially more predictive of estrogen receptor-positive breast cancer than of estrogen receptornegative breast cancer, particularly for absolute risk [151]. Further studies are needed to confirm whether a GRS improves disease risk prediction.

The GRS is calculated on the basis of reproducible tagging of SNP-associated loci reaching genome-wide levels of significance. The GRS can be created by two methods: a simple count method (count GRS) and a weighted method (weighted GRS) [169,170]. Both methods anticipate each SNP to be independently associated with risk. An additive genetic model is used for each SNP, applying a linear weighting of 0,1 , or 2 to genotypes containing 0,1 , or 2 risk alleles, respectively. This model is known to perform well even when the true genetic model is unknown or wrongly specified [171]. The count model assumes that each SNP in the panel contributes equally to the disease risk and is calculated by summing the values for each of the SNPs. The weighted GRS is calculated by multiplying each B-coefficient, the estimates resulting from an analysis carried out on variables that have been standardized, by the number of corresponding risk alleles $(0,1$, or 2$)$. 
450 Latest Research into Quality Control

\begin{tabular}{|c|c|c|c|c|c|c|}
\hline \multirow{3}{*}{$\begin{array}{l}\text { Disease } \\
\text { Type } 2 \\
\text { diabetes }\end{array}$} & $\begin{array}{l}\text { Reference } \\
{[165]}\end{array}$ & $\begin{array}{l}\text { Year } \\
2008\end{array}$ & \multicolumn{2}{|c|}{ Ethnic group Participants } & $\begin{array}{l}\text { No. of SNPs } \\
18\end{array}$ & $\begin{array}{l}\text { Genes found from GWAS } \\
\text { NOTCH2 (rs10923931), }\end{array}$ \\
\hline & {$[165]$} & 2008 & Framingham & 2,377 diabetic patients & 18 & $\begin{array}{l}\text { NOTCH2 (rs10923931), } \\
\text { BCL11A (rs10490072), } \\
\text { THADA (rs7578597), } \\
\text { IGF2BP2 (rs1470579), } \\
\text { PPARg (rs1801282), } \\
\text { ADAMTS9 (rs4607103), } \\
\text { CDKAL (rs7754840), } \\
\text { VEGFA (rs9472138), } \\
\text { JAZF1 (rs86475), } \\
\text { SLC30A8 (rs13266634), } \\
\text { CDKNA/2B (rs10811661), } \\
\text { HHEX (rs1111875), } \\
\text { CDC123, CAMK1D (rs12779790), } \\
\text { TCF7L2 (rs7903146), } \\
\text { KCNJ11 (rs5219), INS (rs689), } \\
\text { DCD (rs1153188), } \\
\text { TSPAN8, LGR5 (rs7961581) }\end{array}$ \\
\hline & [169] & 2009 & European & $\begin{array}{l}\text { 2,809 diabetic } \\
\text { patients \& } \\
3,501 \text { health } \\
\text { controls }\end{array}$ & 10 & $\begin{array}{l}\text { WFS1 (rs10010131), } \\
\text { HHEX (rs1111875), } \\
\text { CDKAL1 (rs7756992), } \\
\text { IGF2BP2 (rs4402960), } \\
\text { SLC30A8 (rs13266634), } \\
\text { CDKN2A/B (rs10811661), } \\
\text { TCF7L2 (rs12255372), } \\
\text { PPARG (rs1801282), } \\
\text { KCNJ11 (rs5219) }\end{array}$ \\
\hline Breast cancer & [151] & 2010 & UK & $\begin{array}{l}10,306 \\
\text { breast cancer } \\
\text { patients \& } \\
10,393 \text { controls }\end{array}$ & 14 & $\begin{array}{l}\text { FGFR2 (rs2981582), } \\
\text { TNRC9 (rs3803662), } \\
2 \text { 2q35 (rs13387042), } \\
\text { MAP3K1 (rs889312), } \\
8 q 24 \text { (rs13281615), } \\
\text { 2p (rs4666451), } \\
5 p a s(\text { rs981782), } \\
\text { CASP8 (rs104548), } \\
\text { LSP1 (rs3817198), } \\
5 p \text { (rs30099), } \\
\text { TGFB1 (rs198/2073), } \\
\text { ATM (rs1800054), } \\
\text { TNRC9 (rs8051542), } \\
\text { TNRC9 (rs12443621) }\end{array}$ \\
\hline
\end{tabular}

Table 5. Studies using a genetic risk score for cancers and diabetes, comprising SNPs identified in GWAS. 


\subsection{Cancer Cell Line Encyclopedia}

The Cancer Cell Line Encyclopedia (CCLE) has made predictive modeling of anticancer drug sensitivity a realistic proposition, by determining genomic markers of drug sensitivity in cancer cells [172,173]. The CCLE contains information from 947 human cancer cell lines including data on gene expression, chromosomal copy number, and massively parallel sequencing data. It has been used to identify genetic, lineage-specific, and gene expressionbased predictors of drug sensitivity [172]. This has revealed, for example, that the plasma cell lineage is correlated with sensitivity to IGF1 receptor inhibitors, aryl hydrocarbon receptor $(A H R)$ expression is associated with MEK inhibitor efficacy in NRAS-mutant lines, and SLFN11 expression is associated with sensitivity to topoisomerase inhibitors [172]. Genomic markers of drug sensitivity in cancer cells have also been systematically identified using the Genomics of Drug Sensitivity in Cancer database (http://www.cancerRxgene.org) [173]. These databases will enable to overview genome quality.

\section{Conclusion}

There are dramatic changes in the genomes of cancer cells, which vary according to cancer subtype. Integrative and wide investigations of cancer cell genomes have revealed mutations and alterations in gene expression that are associated with the disease. Databases that include abundant data related to gene and protein conformation, gene expression, and genomic mutations enable the construction of dynamic cellular simulations and disease models. New sequencing tools such as next-generation sequencing will reveal new horizons in the prediction of disease and drug sensitivity, which play an important role in personalized medicine. Appropriate translation of the abundance of information to clinical practice is one of most important future challenges for medicine. The quality of genome would be one of the important factors for detecting the development of the disease.

\section{Acknowledgements}

The authors are grateful to all those who helped with preparation of the manuscript. In particular, we thank Jaeseong Jo for his great assistance.

\section{Author details}

Shihori Tanabe ${ }^{1^{*}}$ and Sun Ha Jee ${ }^{2}$

*Address all correspondence to: stanabe@nihs.go.jp

1 National Institute of Health Sciences, Tokyo, Japan

2 Institute for Health Promotion, Yonsei University, Seoul, Korea 


\section{References}

[1] Venter, J. C., Adams, M. D., Myers, E. W., Li, P. W., Mural, R. J., Sutton, G. G., et al. (2001). The sequence of the human genome. Science, 291(5507), 1304-1351.

[2] Lander, E. S., Linton, L. M., Birren, B., Nusbaum, C., Zody, M. C., Baldwin, J., et al. (2001). Initial sequencing and analysis of the human genome. Paper presented at International Human Genome Sequencing Consortium. Nature, 409(6822), 860-921.

[3] Ashburner, M., Ball, C. A., Blake, J. A., Botstein, D., Butler, H., Cherry, J. M., et al. (2000). Gene ontology: tool for the unification of biology. The Gene Ontology Consortium. Nat. Genet., 25(1), 25-29.

[4] Hunter, S., Apweiler, R., Attwood, T. K., Bairoch, A., Bateman, A., Binns, D., et al. (2009). InterPro: the integrative protein signature database. Nucleic Acids Res., 37, D211-D215.

[5] Hunter, S., Jones, P., Mitchell, A., Apweiler, R., Attwood, T. K., Bateman, A., et al. (2012). InterPro in 2011: new developments in the family and domain prediction database. Nucleic Acids Res., 40, D306-D312.

[6] Attwood, T. K., Coletta, A., Muirhead, G., Pavlopoulou, A., Philippou, P. B., Popov, I., et al. (2012). The PRINTS database: a fine-grained protein sequence annotation and analysis resource--its status in 2012. Database, Oxford, bas019.

[7] Sigrist, C. J. A., Cerutti, L., de Castro, E., Langendijk-Genevaux, P. S., Bulliard, V., Bairoch, A., et al. (2010). PROSITE, a protein domain database for functional characterization and annotation. Nucleic Acids Res., 38, D161D166.

[8] Punta, M., Coggill, P. C., Eberhardt, R. Y., Mistry, J., Tate, J., Boursnell, C., et al. (2012). The Pfam protein families database. Nucleic Acids Res., 40, D290-D301.

[9] Letunic, I., Doerks, T., \& Bork, P. (2012). SMART7: recent updates to the protein domain annotation resource. Nucleic Acids Res., 40, D302-D305.

[10] Bru, C., Courcelle, E., Carrère, S., Beausse, Y., Dalmar, S., \& Kahn, D. (2005). The ProDom database of protein domain families: more emphasis on 3D. Nucleic Acids Res., 33, D212-D215.

[11] Nikolskaya, A. N., Arighi, C. N., Huang, H., Barker, W. C., \& Wu, C. H. (2007). PIRSF family classification system for protein functional and evolutionary analysis. Evol. Bioinform, Online, 2, 197-209. 
[12] de Lima, Morais. D. A., Fang, H., Rackham, O. J. L., Wilson, D., Pethica, R., Chothia, C., et al. (2011). SUPERFAMILY 1.75 including a domain-centric gene ontology method. Nucleic Acids Res., 39, D427-D434.

[13] Mi, H., Dong, Q., Muruganujan, A., Gaudet, P., Lewis, S., \& Thomas, P. D. (2010). PANTHER version 7: improved phylogenetic trees, orthologs and collaboration with the Gene Ontology Consortium. Nucleic Acids Res., 38, D204-D210.

[14] Lees, J., Yeats, C., Perkins, J., Sillitoe, I., Rentzsch, R., Dessailly, B. H., et al. (2012). Gene3D: a domain-based resource for comparative genomics, functional annotation and protein network analysis. Nucleic Acids Res., 40, D465-D471.

[15] Selengut, J. D., Haft, D. H., Davidsen, T., Ganapathy, A., Gwinn-Giglio, M., Nelson, W. C., et al. (2007). TIGRFAMs and Genome Properties: tools for the assignment of molecular function and biological process in prokaryotic genomes. Nucleic Acids Res., 35, D260-D264.

[16] Lima, T., Auchincloss, A. H., Coudert, E., Keller, G., Michoud, K., Rivoire, C., et al. (2009). HAMAP: a database of completely sequenced microbial proteome sets and manually curated microbial protein families in UniProtKB/ Swiss-Prot. Nucleic Acids Res., 37, D471-D478.

[17] Sidransky, D. (2002). Emerging molecular markers of cancer. Nat. Rev. Cancer, 2(3), 210-219.

[18] Brennan, J. A., Mao, L., Hruban, R. H., Boyle, J. O., Eby, Y. J., Koch, W. M., et al. (1995). Molecular assessment of histopathological staging in squamous-cell carcinoma of the head and neck. N. Engl. J. Med., 332(7), 429-435.

[19] Hollstein, M., Sidransky, D., Vogelstein, M., \& Harris, C. C. (1991). p53 mutations in human cancers. Science, 253(5015), 49-53.

[20] Sidransky, D., Von Eschenbach, A., Tsai, Y. C., Jones, P., Summerhayes, I., Marshall, F., et al. (1991). Identification of p53 gene mutations in bladder cancers and urine samples. Science, 252(5006), 706-709.

[21] Rossi, A., \& Galetta, D. (2012). Biomarkers for the targeted therapies of nonsmall cell lung cancer. Current Biomarker Findings, 2, 7-17.

[22] Sidransky, D., Tokino, T., Hamilton, S. R., Kinzler, K. W., Levin, B., Frost, P., et al. (1992). Identification of ras oncogene mutations in the stool of patients with curable colorectal tumors. Science, 256(5053), 102-105.

[23] Gonzalez-Zulueta, M., Ruppert, J. M., Tokino, K., Tsai, Y. C., Spruck, I. I. I. C. H., Miyano, N., et al. (1993). Microsatellite instability in bladder cancer. Cancer Res., 53(23), 5620-5623. 
[24] Belt, E. J. T., Brosens, R. P. M., Delis-van Diemen, P. M., Bril, H., Tijssen, M., van Essen, D. F., et al. (2012). Cell cycle proteins predict recurrence in stage II and III colon cancer. Ann. Surg. Oncol., DOI: 10.1245/ s10434-012-2216-7, Published online: 04 Feb 2012.

[25] Sigurdsson, V., Hilmarsdottir, B., Sigmundsdottir, H., Fridriksdottir, A. J. R., Ringnér, M., Villadsen, R., et al. (2011). Endothelial induced EMT in breast epithelial cells with stem cell properties. PLoS One, 6(9), e23833.

[26] Abbas, O., Richards, J. E., Yaar, R., \& Mahalingam, M. (2011). Stem cell markers (cytokeratin 15, cytokeratin 19 and p63) in in situ and invasive cutaneous epithelial lesions. Mod. Pathol., 24(1), 90-97.

[27] Kote-Jarai, Z., Amin, Al., Olama, A., Leongamornlert, D., Tymrakiewicz, M., Saunders, E., et al. (2011). Identification of a novel prostate cancer susceptibility variant in the KLK3 gene transcript. Human Genet., 129(6), 687-694.

[28] Schröder, F., Hugosson, J., Roobol, M. J., Tammela, T. L. J., Ciatto, S., Nelen, V., et al. (2012). ERSPC Investigators. Prostate-cancer mortality at 11 years of follow-up. N. Engl. J. Med., 366(11), 981-990.

[29] Fliss, M. S., Usadel, H., Caballero, O. L., Wu, L., Buta, M. R., Eleff, S. M., et al. (2000). Facile detection of mitochondrial DNA mutations in tumors and bodily fluid. Science, 287(5460), 2017-2019.

[30] Polyak, K., Li, Y., Zhu, H., Lengauer, C., Willson, J. K. V., Markowitz, S. D., et al. (1998). Somatic mutations of the mitochondrial genome in human colorectal tumours. Nat. Genet., 20(3), 291-293.

[31] Lam, E. T., Bracci, P. M., Holly, E. A., Chu, C., Poon, A., Wan, E., et al. (2012). Mitochondrial DNA sequence variation and risk of pancreatic cancer. Cancer Res., 72(3), 686-695.

[32] Skonieczna, K., Malyarchuk, B. A., \& Grzybowski, T. (2012). The landscape of mitochondrial DNA variation in human colorectal cancer on the background of phylogenetic knowledge. Biochim. Biophys. Acta, 1825(2), 153-159.

[33] Luo, B., Cheung, H. W., Subramanian, A., Sharifnia, T., Okamoto, M., Yang, X., et al. (2008). Highly parallel identification of essential genes in cancer cells. Proc. Natl. Acad. Sci., USA, 105(51), 20380-20385.

[34] Nitiss, J. L., Liu, Y., \& Hsiung, Y. (1993). A temperature sensitive topoisomerase II allele confers temperature dependent drug resistance on amsacrine and etoposide: a genetic system for determining the targets of topoisomerase II inhibitors. Cancer Res., 53(1), 89-93.

[35] Osheroff, N. (1989). Effect of antineoplastic agents on the DNA cleavage/ religation reaction of eukaryotic topoisomerase II: inhibition of DNA religation by etoposide. Biochemistry, 28(15), 6157-6160. 
[36] Hande, K. R. (1998). Etoposide: four decades of development of a topoisomerase II inhibitor. European J. Cancer, 34, 1514-1521.

[37] Sherr, C. J. (1996). Cancer cell cycles. Science, 274(5293), 1672-1677.

[38] Malumbres, M., \& Barbacid, M. (2009). Cell cycle, CDKs and cancer: a changing paradigm. Nat. Rev. Cancer, 9(3), 153-166.

[39] Kundumani-Sridharan, V., Quyen, D. V., Subramani, J., Singh, N. K., Chin, Y. E., \& Rao, G. N. (2012). Novel interactions between NFATc1 (nuclear factor of activated T cells c1) and STAT-3 (signal transducer and activator of transcription-3) mediate G protein-coupled receptor agonist, thrombin-induced biphasic expression of cyclin D1, with first phase influencing cell migration and second phase directing cell proliferation. J. Biol. Chem., 287(27), 22463-22482.

[40] Landis, M. W., Pawlyk, B. S., Li, T., Sicinski, P., \& Hinds, P. W. (2006). Cyclin D1-dependent kinase activity in murine development and mammary tumorigenesis. Cancer Cell, 9(1), 13-22.

[41] Klein, E. A., \& Assoian, R. K. (2008). Transcriptional regulation of the cyclin D1 gene at a glance. J. Cell Sci., 121, Pt 23, 3853-3857.

[42] Hsu, W., Shakya, R., \& Costantini, F. (2001). Impaired mammary gland and lymphoid development caused by inducible expression of Axin in transgenic mice. J. Cell Biol., 155(6), 1055-1064.

[43] Shtutman, M., Zhurinsky, J., Simcha, I., Albanese, C., D’Amico, M., Pestell, R., et al. (1999). The cyclin D1 gene is a target of the beta-catenin/LEF-1 pathway. Proc. Natl. Acad. Sci., USA, 96(10), 5522-5527.

[44] Zhang, Q., Gu, J., Li, L., Liu, J., Luo, B., Cheung, H., et al. (2009). Control of cyclin D1 and breast tumorigenesis by the EglN2 prolyl hydroxylase. Cancer Cell, 16(5), 413-424.

[45] Coudreuse, D., \& Nurse, P. (2010). Driving the cell cycle with a minimal CDK control network. Nature, 468(7327), 1074-1079.

[46] Bloom, J., \& Cross, F. R. (2007). Multiple levels of cyclin specificity in cellcycle control. Nat. Rev. Mol. Cell Biol., 8(2), 149-160.

[47] Santamaría, D., Barrière, C., Cerqueira, A., Hunt, S., Tardy, C., Newton, K., et al. (2007). Cdk1 is sufficient to drive the mammalian cell cycle. Nature, 448(7155), 811-815.

[48] Tobin, N. P., Sims, A. H., Lundgren, K. L., Lehn, S., \& Landberg, G. (2011). Cyclin D1, Id1 and EMT in breast cancer. BMC Cancer, 11, 417.

[49] Bitu, C., Destro, M., Carrera, M., Silva, S., Graner, E., Kowalski, L. P., Soares, F., et al. (2012). HOXA1 is overexpressed in oral squamous cell 
carcinomas and its expression is correlated with poor prognosis. BMC Cancer, $12,146$.

[50] Yang, Y. (2012). Wnt signaling in development and disease. Cell E Bioscience, 2,14 .

[51] Iyer, S., Xiao, E., Alsayegh, K., Eroshenko, N., Riggs, M. J., Bennett, J. P., et al. (2012). Mitochondrial gene replacement in human pluripotent stem cellderived neural progenitors. Gene Ther., 19(5), 469-475.

[52] Consiglio, A., Carella, M., De Caro, G., Delle, Foglie. G., Giovannelli, C., Grillo, G., et al. (2012). BEAT: Bioinformatics Exon Array Tool to store, analyze and visualize Affymetrix GeneChip Human Exon Array data from disease experiments. BMC Bioinformatics, 13(Suppl 4), S21.

[53] Patron, J. P., Fendler, A., Bild, M., Jung, U., Müller, H., Arntzen, M., et al. (2012). MiR-133b targets antiapoptotic genes and enhances death receptorinduced apoptosis. PLoS One, 7(4), e35345.

[54] Alevizos, L., Gomatos, I. P., Smparounis, S., Konstadoulakis, M. M., \& Zografos, G. (2012). Review of the molecular prolife and modern prognostic markers for gastric lymphoma: how do they affect clinical practice? Can. J. Surg., 55(2), 117-124.

[55] Aoudjit, F., \& Vuori, K. (2012). Integrin signaling in cancer cell survival and chemoresistance. Chemother. Res. Pract., 2012, 283181.

[56] Hayakawa, Y., Hirata, Y., Nakagawa, H., Sakamoto, K., Hikiba, Y., Kinoshita, H., et al. (2011). Apoptosis signal-regulating kinase 1 and cyclin D1 compose a positive feedback loop contributing to tumor growth in gastric cancer. Proc. Natl. Acad. Sci., USA, 108(2), 780-785.

[57] Cairns, P., \& Sidransky, D. (1999). Molecular methods for the diagnosis of cancer. Biochim. Biophys. Acta, 1423(2), C11-C18.

[58] Glickman, J. F., Mc Gee, J., \& Napper, A. (2004). Assay development for protein kinase enzyme. Assay Guidance Manual [Internet]. Sittampalam GS, Weidner J, Auld D, et al., editors, Bethesda (MD): Eli Lilly \& Company and the National Center for Advancing Translational Sciences, 2012.

[59] Eglen, R. M., \& Singh, R. (2003). Beta galactosidase enzyme fragment complementation as a novel technology for high throughput screening. Comb. Chem. High Throughput Screen., 6(4), 381-387.

[60] Baran, Y., Zencir, S., Cakir, Z., Ozturk, E., \& Topcu, Z. (2011). Imatinibinduced apoptosis: a possible link to topoisomerase enzyme inhibition. J. Clin. Pharm. Ther., 36(6), 673-679.

[61] Dickey, A., Schleicher, S., Leahy, K., Hu, R., \& Hallahan, D. (2011). Thotala DK GSK-3 $\beta$ inhibition promotes cell death, apoptosis, and in vivo tumor 
growth delay in neuroblastoma Neuro-2A cell line. J. Neurooncol., 104(1), 145-153.

[62] Wang, L., Hurley, D. G., Watkins, W., Araki, H., Tamada, Y., Muthukaruppan, A., et al. (2012). Cell cycle gene networks are associated with melanoma prognosis. PLoS One, 7(4), e34247.

[63] Kim, J., Chae, M., Kim, W. K., Kim, Y., Kang, H. S., Kim, H. S., et al. (2011). Salinomycin sensitizes cancer cells to the effects of doxorubicin and etoposide treatment by increasing DNA damage and reducing p21protein. $\mathrm{Br}$. J. Pharmacol., 162(3), 773-784.

[64] Smith, C. C., Wang, Q., Chin, C., Salerno, S., Damon, L. E., Levis, M. J., et al. (2012). Validation of ITD mutations in FLT3 as a therapeutic target in human acute myeloid leukaemia. Nature, 485(7397), 260-263.

[65] Wellcome Trust Case Control Consortium. (2007). Genome-wide association study of 14,000 cases of seven common diseases and 3,000 shared controls. Nature, 447(7145), 661-668.

[66] National Institutes of Health. (2007). Policy for sharing of data obtained in NIH supported or conducted genome-wide association studies (GWAS). Federal Regist., 72(166), 49290-49297, http://www.grants.nih.gov/grants/guide/ notice-files/NOT-OD-07-088.html, accessed 3 July 2012.

[67] Chung, C. C., Magalhaes, W. C., Gonzalez-Bosquet, J., \& Chanock, S. J. (2010). Genome-wide association studies in cancer--current and future directions. Carcinogenesis, 31(1), 111-120.

[68] Hampe, J., Franke, A., Rosenstiel, P., Till, A., Teuber, M., Huse, K., et al. (2007). A genome-wide association scan of nonsynonymous SNPs identifies a susceptibility variant for Crohn disease in ATG16L1. Nat. Genet., 39(2), 207-211.

[69] Manolio, T. A. (2010). Genomewide association studies and assessment of the risk of disease. N. Engl. J. Med., 363(2), 166-176.

[70] Easton, D. F., \& Eeles, R. A. (2008). Genome-wide association studies in cancer. Hum. Mol. Genet., 17(R2), R109-R115.

[71] Miki, Y., Swensen, J., Shattuck-Eidens, D., Futreal, P. A., Harshman, K., Tavtigian, S., et al. (1994). A strong candidate for the breast and ovarian cancer susceptibility gene BRCA1. Science, 266(5182), 66-71.

[72] Adnane, J., Gaudray, P., Dionne, C. A., Crumley, G., Jaye, M., Schlessinger, J., et al. (1991). BEK and FLG, two receptors to members of the FGF family, are amplified in subsets of human breast cancers. Oncogene, 6(4), 659-663. 
[73] Greenman, C., Stephens, P., Smith, R., Dalgliesh, G. L., Hunter, C., Bignell, G., et al. (2007). Patterns of somatic mutation in human cancer genomes. Nature, 446(7132), 153-158.

[74] Garcia-Closas, M., Hall, P., Nevanlinna, H., Pooley, K., Morrison, J., Richesson, D., et al. (2008). Heterogeneity of breast cancer associations with five susceptibility loci by clinical and pathological characteristics. PloS Genet., 4(4), e1000054.

[75] Antoniou, A. C., Spurdle, A. B., Sinilnikova, O. M., Healey, S., Pooley, K. A., Schmutzler, R. K., et al. (2008). CIMBA. Common breast cancer-predisposition alleles are associated with breast cancer risk in BRCA1 and BRCA2 mutation carriers. Am. J. Hum. Genet., 82(4), 937-948.

[76] Zheng, W., Long, J., Gao, Y. T., Li, C., Zheng, Y., Xiang, Y. B., et al. (2009). Genome-wide association study identifies a new breast cancer susceptibility locus at 6q25.1. Nat. Genet., 41(3), 324-328.

[77] Amundadottir, L. T., Sulem, P., Gudmundsson, J., Helgason, A., Baker, A., Agnarsson, B. A., et al. (2006). A common variant associated with prostate cancer in European and African populations. Nat. Genet., 38(6), 652-658.

[78] Yeager, M., Orr, N., Hayes, R. B., Jacobs, K. B., Kraft, P., Wacholder, S., et al. (2007). Genome-wide association study of prostate cancer identifies a second risk locus at 8q24. Nat. Genet., 39(5), 645-649.

[79] Gudmundsson, J., Sulem, P., Manolescu, A., Amundadottir, L. T., Gudbjartsson, D., Helgason, A., et al. (2007). Genome-wide association study identifies a second prostate cancer susceptibility variant at 8q24. Nat. Genet., 39(5), 631-637.

[80] Eeles, R. A., Kote-Jarai, Z., Giles, G. G., Olama, A. A., Guy, M., Jugurnauth, S. K., et al. (2008). Multiple newly identified loci associated with prostate cancer susceptibility. Nat. Genet., 40(30), 316-321.

[81] Thomas, G., Jacobs, K. B., Yeager, M., Kraft, P., Wacholder, S., Orr, N., et al. (2008). Multiple loci identified in a genome-wide association study of prostate cancer. Nat. Genet., 40(3), 310-315.

[82] Chanock, S. J., \& Hunter, D. J. (2008). Genomics: when the smoke clears. Nature, 452(7187), 537-538.

[83] Hung, R. J., Mc Kay, J. D., Gaborieau, V., Boffetta, P., Hashibe, M., Zaridze, D., et al. (2008). A susceptibility locus for lung cancer maps to nicotinic acetylcholine receptor subunit genes on 15q25. Nature, 452(7187), 633-637.

[84] Mc Kay, J. D., Hung, R. J., Gaborieau, V., Boffetta, P., Chabrier, A., Byrnes, G., et al. (2008). Lung cancer susceptibility locus at 5p15. Nat. Genet., 40(12), 1404-1406. 
[85] Wang, Y., Broderick, P., Webb, E., Wu, X., Vijayakrishnan, J., Matakidou, A., et al. (2008). Common 5p15and 6p21.33 variants influence lung cancer risk. Nat. Genet., 40(12), 1407-1409.

[86] Bierut, L. J., Madden, P. A., Breslau, N., Johnson, E. O., Hatsukami, D., Pomerleau, O. F., et al. (2007). Novel genes identified in a high-density genome wide association study for nicotine dependence. Hum. Mol. Genet., 16(1), 24-35.

[87] Caporaso, N., Gu, F., Chatterjee, N., Sheng-Chih, J., Yu, K., Yeager, M., et al. (2009). Genome-wide and candidate gene association study of cigarette smoking behaviors. PloS One, 4(2), e4653.

[88] Pearson, T. A., \& Manolio, T. A. (2008). How to interpret a genome-wide association study. JAMA, 299(11), 1335-1344.

[89] Kim, H. C., Lee, J. Y., Sung, H., Choi, J. Y., Park, S. K., Lee, K. M., et al. (2012). A genome-wide association study identifies a breast cancer risk variant in ERBB4 at 2q34: results from the Seoul Breast Cancer Study. Breast Cancer Res., 14, R56.

[90] Long, J., Cai, Q., Sung, H., Shi, J., Zhang, B., Choi, J. Y., et al. (2012). Genome-wide association study in east Asians identifies novel susceptibility loci for breast cancer. PLoS Genet., 8(2), e1002532.

[91] Shu, X. O., Long, J., Lu, W., Li, C., Chen, W. Y., Delahanty, R., et al. (2012). Novel genetic markers of breast cancer survival identified by a genome-wide association study. Cancer Res., 72(5), 1182-1189.

[92] Kiyotani, K., Mushiroda, T., Tsunoda, T., Morizono, T., Hosono, N., Kubo, M., et al. (2012). A genome-wide association study identifies locus at 10q22 associated with clinical outcomes of adjuvant tamoxifen therapy for breast cancer patients in Japanese. Hum. mol. genet., 21(7), 1665-1672.

[93] Cai, Q., Long, J., Lu, W., Qu, S., Wen, W., Kang, D., et al. (2011). Genomewide association study identifies breast cancer risk variant at 10q21.2: results from the Asia Breast Cancer Consortium. Hum. Mol. Genet., 20(24), 4991-4999.

[94] Sehrawat, B., Sridharan, M., Ghosh, S., Robson, P., Cass, C. E., Mackey, J. R., et al. (2011). Potential novel candidate polymorphisms identified in genome-wide association study for breast cancer susceptibility. Hum. Genet., $130(4), 529-537$.

[95] Fletcher, O., Johnson, N., Orr, N., Hosking, F. J., Gibson, L. J., Walker, K., et al. (2011). Novel breast cancer susceptibility locus at 9q31.2: results of a genome-wide association study. J. Natl. Cancer Inst., 103(5), 425-435. 
[96] Li, J., Humphreys, K., Darabi, H., Rosin, G., Hannelius, U., Heikkinen, T., et al. (2010). A genome-wide association scan on estrogen receptor-negative breast cancer. Breast Cancer Res., 12(6), R93.

[97] Gaudet, M. M. , Kirchhoff, T., Green, T., Vijai, J., Korn, J. M., Guiducci, C., et al. (2010). Common genetic variants and modification of penetrance of BRCA2-associated breast cancer. PloS Genet., 6(10), e1001183.

[98] Li, J., Humphreys, K., Heikkinen, T., Aittomäki, K., Blomqvist, C., Pharoah, P. D., et al. (2011). A combined analysis of genome-wide association studies in breast cancer. Breast Cancer Res Treat., 126(3), 717-727.

[99] Turnbull, C., Ahmed, S., Morrison, J., Pernet, D., Renwick, A., Maranian, M., et al. (2010). Genome-wide association study identifies five new breast cancer susceptibility loci. Nat. Genet., 42(6), 504-507.

[100] Azzato, E. M., Pharoah, P. D., Harrington, P., Easton, D. F., Greenberg, D., Caporaso, N. E., et al. (2010). A genome-wide association study of prognosis in breast cancer. Cancer Epidemiol. Biomarkers Prev., 19(4), 1140-1143.

[101] Thomas, G., Jacobs, K. B., Kraft, P., Yeager, M., Wacholder, S., Cox, D. G., et al. (2009). A multistage genome-wide association study in breast cancer identifies two new risk alleles at 1p11and 14q24.1 (RAD51L1). Nat. Genet., 41(5), 579-584.

[102] Zheng, W., Long, J., Gao, Y. T., Li, C., Zheng, Y., Xiang, Y. B., et al. (2009). Genome-wide association study identifies a new breast cancer susceptibility locus at 6q25.1. Nat. Genet., 41(3), 324-328.

[103] Kibriya, M. G., Jasmine, F., Argos, M., Andrulis, I. L., John, E. M., ChangClaude, J., et al. (2009). A pilot genome-wide association study of early-onset breast cancer. Breast Cancer Res. Treat., 114(3), 463-477.

[104] Gold, B., Kirchhoff, T., Stefanov, S., Lautenberger, J., Viale, A., Garber, J., et al. (2008). Genome-wide association study provides evidence for a breast cancer risk locus at 6q22.33. Proc. Natl. Acad. Sci., USA, 105(11), 4340-4345.

[105] Murabito, J. M., Rosenberg, C. L., Finger, D., Kreger, B. E., Levy, D., Splansky, G. L., et al. (2007). A genome-wide association study of breast and prostate cancer in the NHLBI's Framingham Heart Study. BMC Med. Genet., 8(Suppl 1), S6.

[106] Easton, D. F., Pooley, K. A., Dunning, A. M., Pharoah, P. D., Thompson, D., Ballinger, D. G., et al. (2007). Genome-wide association study identifies novel breast cancer susceptibility loci. Nature, 447(7148), 1087-1093.

[107] Hunter, D. J., Kraft, P., Jacobs, K. B., Cox, D. G., Yeager, M., Hankinson, S. E., et al. (2007). A genome-wide association study identifies alleles in FGFR2 
associated with risk of sporadic postmenopausal breast cancer. Nat. Genet., 39(7), 870-874.

[108] Tao, S., Feng, J., Webster, T., Jin, G., Hsu, F. C., Chen, S. H., et al. (2012). Genome-wide two-locus epistasis scans in prostate cancer using two European populations. Hum. Genet., 131, 1225-1234.

[109] Tao, S., Wang, Z., Feng, J., Hsu, F. C., Jin, G., Kim, S. T., et al. (2012). A genome-wide search for loci interacting with known prostate cancer riskassociated genetic variants. Carcinogenesis, 33(3), 598-603.

[110] Kote-Jarai, Z., Olama, A. A., Giles, G. G., Severi, G., Schleutker, J., Weischer, M., et al. (2011). UK Genetic Prostate Cancer Study Collaborators/British Association of Urological Surgeons' Section of Oncology; UK ProtecT Study Collaborators, The Australian Prostate Cancer BioResource; PRACTICAL Consortium. Seven prostate cancer susceptibility loci identified by a multi-stage genome-wide association study. Nat. Genet., 43(8), 785-791.

[111] Schumacher, F. R., Berndt, S. I., Siddiq, A., Jacobs, K. B., Wang, Z., Lindstrom, S., et al. (2011). Genome-wide association study identifies new prostate cancer susceptibility loci. Hum. Mol. Genet., 20(19), 3867-3875.

[112] Haiman, C. A., Chen, G. K., Blot, W. J., Strom, S. S., Berndt, S. I., Kittles, R. A., et al. (2011). Genome-wide association study of prostate cancer in men of African ancestry identifies a susceptibility locus at 17q21. Nat. Genet., 43(6), 570-573.

[113] Fitz, Gerald L. M., Kwon, E. M., Conomos, M. P., Kolb, S., Holt, S. K., Levine, D., et al. (2011). Genome-wide association study identifies a genetic variant associated with risk for more aggressive prostate cancer. Cancer Epidemiol. Biomarkers Prev., 20(6), 1196-1203.

[114] Penney, K. L., Pyne, S., Schumacher, F. R., Sinnott, J. A., Mucci, L. A., Kraft, P. L., et al. (2010). Genome-wide association study of prostate cancer mortality. Cancer Epidemiol. Biomarkers Prev., 19(11), 2869-2876.

[115] Takata, R., Akamatsu, S., Kubo, M., Takahashi, A., Hosono, N., Kawaguchi, T., et al. (2010). Genome-wide association study identifies five new susceptibility loci for prostate cancer in the Japanese population. Nat. Genet., 42(9), 751-754.

[116] Eeles, R. A., Kote-Jarai, Z., Al Olama, A. A., Giles, G. G., Guy, M., Severi, G., et al. (2009). UK Genetic Prostate Cancer Study Collaborators/British Association of Urological Surgeons' Section of Oncology; UK ProtecT Study Collaborators; PRACTICAL Consortium, Easton DF Identification of seven new prostate cancer susceptibility loci through a genome-wide association study. Nat. Genet., 41(10), 1116-1121. 
[117] Gudmundsson, J., Sulem, P., Gudbjartsson, D. F., Blondal, T., Gylfason, A., Agnarsson, B. A., et al. (2009). Genome-wide association and replication studies identify four variants associated with prostate cancer susceptibility. Nat. Genet., 41(10), 1122-1126.

[118] Eeles, R. A., Kote-Jarai, Z., Giles, G. G., Olama, Guy. M., Jugurnauth, S. K., et al. (2008). UK Genetic Prostate Cancer Study Collaborators; British Association of Urological Surgeons' Section of Oncology; UK ProtecT Study Collaborators. Multiple newly identified loci associated with prostate cancer susceptibility. Nat. Genet., 40(3), 316-321.

[119] Thomas, G., Jacobs, K. B., Yeager, M., Kraft, P., Wacholder, S., Orr, N., et al. (2008). Multiple loci identified in a genome-wide association study of prostate cancer. Nat. Genet., 40(3), 310-315.

[120] Duggan, D., Zheng, S. L., Knowlton, M., Benitez, D., Dimitrov, L., Wiklund, F., et al. (2007). Two genome-wide association studies of aggressive prostate cancer implicate putative prostate tumor suppressor gene DAB2IP. J. Natl. Cancer Inst., 99(24), 1836-1844.

[121] Murabito, J. M., Rosenberg, C. L., Finger, D., Kreger, B. E., Levy, D., Splansky, G. L., et al. (2007). A genome-wide association study of breast and prostate cancer in the NHLBI's Framingham Heart Study. BMC Med. Genet., 8(Suppl 1), S6.

[122] Gudmundsson, J., Sulem, P., Manolescu, A., Amundadottir, L. T., Gudbjartsson, D., Helgason, A., et al. (2007). Genome-wide association study identifies a second prostate cancer susceptibility variant at 8q24. Nat. Genet., 39(5), 631-637.

[123] Yeager, M., Orr, N., Hayes, R. B., Jacobs, K. B., Kraft, P., Wacholder, S., et al. (2007). Genome-wide association study of prostate cancer identifies a second risk locus at 8q24. Nat. Genet., 39(5), 645-649.

[124] Peters, U., Hutter, C. M., Hsu, L., Schumacher, F. R., Conti, D. V., Carlson, C. S., et al. (2012). Meta-analysis of new genome-wide association studies of colorectal cancer risk. Hum. Genet., 131(2), 217-234.

[125] Houlston, R. S., Cheadle, J., Dobbins, S. E., Tenesa, A., Jones, A. M., Howarth, K., et al. (2010). Meta-analysis of three genome-wide association studies identifies susceptibility loci for colorectal cancer at 1q41, 3q26.2, 12q13.13 and 20q13.33. Nat. Genet., 42(11), 973-977.

[126] Lascorz, J., Försti, A., Chen, B., Buch, S., Steinke, V., Rahner, N., et al. (2010). Genome-wide association study for colorectal cancer identifies risk polymorphisms in German familial cases and implicates MAPK signalling pathways in disease susceptibility. Carcinogenesis, 31(9), 1612-1619. 
[127] Houlston, R. S., Webb, E., Broderick, P., Pittman, A. M., Di Bernardo, M. C., Lubbe, S., et al. (2008). Meta-analysis of genome-wide association data identifies four new susceptibility loci for colorectal cancer. Paper presented at International Colorectal Cancer Genetic Association Consortium. Nat. Genet., 40(12), 1426-1435.

[128] Tenesa, A., Farrington, S. M., Prendergast, J. G., Porteous, M. E., Walker, M., Haq, N., et al. (2008). Genome-wide association scan identifies a colorectal cancer susceptibility locus on $11 \mathrm{q} 23$ and replicates risk loci at 8q24 and 18q21. Nat. Genet., 40(5), 631-637.

[129] Tomlinson, I. P., Webb, E., Carvajal-Carmona, L., Broderick, P., Howarth, K., Pittman, A. M., et al. (2008). A genome-wide association study identifies colorectal cancer susceptibility loci on chromosomes 10p14and 8q23.3. Nat. Genet., 40(5), 623, 630.

[130] Broderick, P., Carvajal-Carmona, L., Pittman, A. M., Webb, E., Howarth, K., Rowan, A., et al. (2007). CORGI Consortium. A genome-wide association study shows that common alleles of SMAD7 influence colorectal cancer risk. Nat. Genet., 39(11), 1315-1317.

[131] Tomlinson, I., Webb, E., Carvajal-Carmona, L., Broderick, P., Kemp, Z., Spain, S., et al. (2007). A genome-wide association scan of tag SNPs identifies a susceptibility variant for colorectal cancer at 8q24.2. Nat. Genet., 39(8), 984-988.

[132] Zanke, B. W., Greenwood, C. M., Rangrej, J., Kustra, R., Tenesa, A., Farrington, S. M., et al. (2007). Genome-wide association scan identifies a colorectal cancer susceptibility locus on chromosome 8q24. Nat. Genet., 39(8), 989-994.

[133] Hu, Z., Wu, C., Shi, Y., Guo, H., Zhao, X., Yin, Z., et al. (2011). A genomewide association study identifies two new lung cancer susceptibility loci at $13 q 12.12$ and 22q12.2 in Han Chinese. Nat. Genet., 43(8), 792-796.

[134] Wu, X., Ye, Y., Rosell, R., Amos, C. I., Stewart, D. J., Hildebrandt, et., \& al, . (2011). Genome-wide association study of survival in non-small cell lung cancer patients receiving platinum-based chemotherapy. J. Natl. Cancer Inst., 103(10), 817-825.

[135] Frullanti, E., Galvan, A., Falvella, F. S., Manenti, G., Colombo, F., Vannelli, A., et al. (2011). Multiple genetic loci modulate lung adenocarcinoma clinical staging. Clin. Cancer Res., 17(8), 2410-2416.

[136] Wu, C., Xu, B., Yuan, P., Miao, X., Liu, Y., Guan, Y., et al. (2010). Genomewide interrogation identifies YAP1 variants associated with survival of smallcell lung cancer patients. Cancer Res., 70(23), 9721-9729. 
[137] Yoon, K. A., Park, J. H., Han, J., Park, S., Lee, G. K., Han, J. Y., et al. (2010). A genome-wide association study reveals susceptibility variants for non-small cell lung cancer in the Korean population. Hum. Mol. Genet., 19(24), 4948-4954.

[138] Li, Y., Sheu, C. C., Ye, Y., de Andrade, M., Wang, L., Chang, S. C., et al. (2010). Genetic variants and risk of lung cancer in never smokers: a genomewide association study. Lancet Oncol., 11(4), 321-330.

[139] Landi, M. T., Chatterjee, N., Yu, K., Goldin, L. R., Goldstein, A. M., Rotunno, M., et al. (2009). A genome-wide association study of lung cancer identifies a region of chromosome 5 p15associated with risk for adenocarcinoma. Am. J. Hum. Genet., 85(5), 679-691.

[140] Broderick, P., Wang, Y., Vijayakrishnan, J., Matakidou, A., Spitz, M. R., Eisen, T., et al. (2009). Deciphering the impact of common genetic variation on lung cancer risk: a genome-wide association study. Cancer Res., 69(19), 6633-6641.

[141] Amos, C. I., Wu, X., Broderick, P., Gorlov, I. P., Gu, J., Eisen, T., et al. (2008). Genome-wide association scan of tag SNPs identifies a susceptibility locus for lung cancer at 15q25.1. Nat. Genet., 40(5), 616-622.

[142] Thorgeirsson, T. E., Geller, F., Sulem, P., Rafnar, T., Wiste, A., Magnusson, K. P., et al. (2008). A variant associated with nicotine dependence, lung cancer and peripheral arterial disease. Nature, 452(7187), 638-642.

[143] Hung, R. J., Mc Kay, J. D., Gaborieau, V., Boffetta, P., Hashibe, M., Zaridze, D., et al. (2008). A susceptibility locus for lung cancer maps to nicotinic acetylcholine receptor subunit genes on 15q25. Nature, 452(7187), 633-637.

[144] Spinola, M., Leoni, V. P., Galvan, A., Korsching, E., Conti, B., Pastorino, U., et al. (2007). Genome-wide single nucleotide polymorphism analysis of lung cancer risk detects the KLF6 gene. Cancer Lett., 251(2), 311-316.

[145] Barrett, J. H., Iles, M. M., Harland, M., Taylor, J. C., Aitken, J. F., Andresen, P. A., et al. (2011). GenoMEL Consortium. Genome-wide association study identifies three new melanoma susceptibility loci. Nat. Genet., 43(11), 1108-1113.

[146] Mac Gregor, S., Montgomery, G. W., Liu, J. Z., Zhao, Z. Z., Henders, A. K., Stark, M., et al. (2011). Genome-wide association study identifies a new melanoma susceptibility locus at 1q21.3. Nat. Genet., 43(11), 1114-1118.

[147] Amos, C. I., Wang, L. E., Lee, J. E., Gershenwald, J. E., Chen, W. V., Fang, S., et al. (2011). GenoMEL Investigators. Genome-wide association study identifies novel loci predisposing to cutaneous melanoma. Hum. Mol. Genet., 20(24), 5012-5023. 
[148] Teerlink, C., Farnham, J., Allen-Brady, K., Camp, N. J., Thomas, A., Leachman, S., et al. (2012). A unique genome-wide association analysis in extended Utah high-risk pedigrees identifies a novel melanoma risk variant on chromosome arm 10q. Hum. Genet., 131(1), 77-85.

[149] Bishop, D. T., Demenais, F., Iles, M. M., Harland, M., Taylor, J. C., Corda, E., et al. (2009). Genome-wide association study identifies three loci associated with melanoma risk. Nat. Genet., 41(8), 920-925.

[150] King, H., Aubert, R. E., \& Herman, W. H. (1998). Global burden of diabetes, 1995-2025: prevalence, numerical estimates, and projections. Diabetes Care, 21(9), 1414-1431.

[151] Reeves, G. K., Travis, R. C., Green, J., Bull, D., Tipper, S., Baker, K., et al. (2010). Incidence of breast cancer and its subtypes in relation to individual and multiple low-penetrance genetic susceptibility loci. JAMA, 304(4), 426-434.

[152] Kaprio, J., Tuomilehto, J., Koskenvuo, M., Romanov, K., Reunanen, A., Eriksson, J., et al. (1992). Concordance for type 1 (insulin-dependent) and type 2 (non-insulin-dependent) diabetes mellitus in a population-based cohort of twins in Finland. Diabetologia, 35(11), 1060-1067.

[153] Risch, N. (1990). Linkage strategies for genetically complex traits. I. Multilocus models. Am. J. Hum. Genet., 46(2), 222-228.

[154] Grant, S. F., Thorleifsson, G., Reynisdottir, I., Manolescu, A., Sainz, J., Helgason, A., et al. (2006). Variant of transcription factor 7-like 2 (TCF7L2) gene confers risk of type 2 diabetes. Nat. Genet., 38(3), 320-323.

[155] Sladek, R., Rocheleau, G., Rung, J., Dina, C., Shen, L., Serre, D., et al. (2007). A genome-wide association study identifies novel risk loci for type 2 diabetes. Nature, 445(7130), 881-885.

[156] Saxena, R., Voight, B. F., Lyssenko, V., Burtt, N. P., de Bakker, P. I., Chen, H., et al. (2007). Genome-wide association analysis identifies loci for type 2 diabetes and triglyceride levels. Science, 316(5829), 1331-1336.

[157] Scott, L. J., Mohlke, K. L., Bonnycastle, L. L., Willer, C. J., Li, Y., Duren, W. L., et al. (2007). A genome-wide association study of type 2 diabetes in Finns detects multiple susceptibility variants. Science, 316(5829), 1341-1345.

[158] Zeggini, E., Weedon, M. N., Lindgren, C. M., Frayling, T. M., Elliott, K. S., Lango, H., et al. (2007). Wellcome Trust Case Control Consortium (WTCCC). Replication of genome-wide association signals in UK samples reveals risk loci for type 2 diabetes. Science, 316(5829), 1336-1341.

[159] Zeggini, E., Scott, L. J., Saxena, R., Voight, B. F., Marchini, J. L., Hu, T., et al. (2008). Meta-analysis of genome-wide association data and large-scale 
replication identifies additional susceptibility loci for type 2 diabetes. Nat. Genet., 40(5), 638-645.

[160] van Dam, R. M., Hoebee, B., Seidell, J. C., Schaap de, Bruin. T. W., \& Feskens, E. J. (2005). Common variants in the ATP-sensitive $\mathrm{K}+$ channel genes KCNJ11 (Kir6.2) and ABCC8 (SUR1) in relation to glucose intolerance: population-based studies and meta-analyses. Diabet. Med., 22(5), 590-598.

[161] Ludovico, O., Pellegrini, F., Di Paola, R., Minenna, A., Mastroianno, S., Cardellini, M., et al. (2007). Heterogeneous effect of peroxisome proliferatoractivated receptor gamma2 Ala12 variant on type 2 diabetes risk. Obesity (Silver Spring), 15(5), 1076-1081.

[162] Cauchi, S., El Achhab, Y., Choquet, H., Dina, C., Krempler, F., Weitgasser, R., et al. (2007). TCF7L2 is reproducibly associated with type 2 diabetes in various ethnic groups: a global meta-analysis. J. Mol. Med., 85(7), 777-782.

[163] Steinthorsdottir, V., Thorleifsson, G., Reynisdottir, I., Benediktsson, R., Jonsdottir, T., Walters, G. B., et al. (2007). A variant in CDKAL1 influences insulin response and risk of type 2 diabetes. Nat. Genet., 39(6), 770-775.

[164] Sandhu, M. S., Weedon, M. N., Fawcett, K. A., Wasson, J., Debenham, S. L., Daly, A., et al. (2007). Common variants in WFS1 confer risk of type 2 diabetes. Nat. Genet., 39(8), 951-953.

[165] Meigs, J. B., Shrader, P., Sullivan, L. M., Mc Ateer, J. B., Fox, C. S., Dupuis, J., et al. (2008). Genotype score in addition to common risk factors for prediction of type 2 diabetes. N. Engl. J. Med., 359(21), 2208-2219.

[166] Weedon, M. N., Mc Carthy, M. I., Hitman, G., Walker, M., Groves, C. J., Zeggini, E., et al. (2006). Combining information from common type 2 diabetes risk polymorphisms improves disease prediction. PloS. Med., 3(10), e374.

[167] Wray, N. R., Goddard, M. E., \& Visscher, P. M. (2007). Prediction of individual genetic risk to disease from genome-wide association studies. Genome Res., 17(10), 1520-1528.

[168] Morrison, A. C., Bare, L. A., Chambless, L. E., Ellis, S. G., Malloy, M., Kane, J. P., et al. (2007). Prediction of coronary heart disease risk using a genetic risk score: the Atherosclerosis Risk in Communities Study. Am. J. Epidemiol., 166(1), 28-35.

[169] Cornelis, M. C., Qi, L., Zhang, C., Kraft, P., Manson, J., Cai, T., et al. (2009). Joint effects of common genetic variants on the risk for type 2 diabetes in U.S. men and women of European ancestry. Ann. Intern. Med., 150(8), 541-550. 
[170] Ripatti, S., Tikkanen, E., Orho-Melander, M., Havulinna, Silander. K., Sharma, A., et al. (2010). A multilocus genetic risk score for coronary heart disease: case-control and prospective cohort analyses. Lancet, 376(9750), 1393-1400.

[171] Balding, D. J. (2006). A tutorial on statistical methods for population association studies. Nat. Rev. Genet., 7(10), 781-791.

[172] Barretina, J., Caponigro, G., Stransky, N., Venkatesan, K., Margolin, Kim. S., et al. (2012). The Cancer Cell Line Encyclopedia enables predictive modelling of anticancer drug sensitivity. Nature, 483(7391), 603-607.

[173] Garnett, N. J., Edelman, E. J., Heidorn, S. J., Greenman, C. D., Dastur, A., Lau, K. W., et al. (2012). Systematic identification of genomic markers of drug sensitivity in cancer cells. Nature, 483(7391), 570-575. 



\title{
Quality Control Considerations for Fluorescence In Situ Hybridisation of Paraffin-Embedded Pathology Specimens in a Diagnostic Laboratory Environment
}

\author{
Lisa Duffy, Liangtao Zhang, Donald R. Love and \\ Alice M. George
}

Additional information is available at the end of the chapter

http://dx.doi.org/10.5772/51266

\section{Introduction}

Paraffin FISH testing is the application of the fluorescence in situ hybridisation (FISH) methodology to formalin fixed paraffin embedded sections (FFPE), and has proven a powerful tool for both histopathologists and cytogeneticists. Pathologists use the method to confirm or exclude a histological diagnosis, to differentiate between tumour subtypes, or as a confirmatory tool where the tissue morphology is poor or the immunohistochemistry (IHC) staining is uninformative [1]. Similarly, cytogeneticists find it useful when the tissue sample is insufficient or unsatisfactory for conventional culture methods, or when such methods fail to yield a result. The method can also be used to confirm abnormalities found in other tissue samples. Paraffin testing has a further advantage over conventional cytogenetic and molecular testing methods, as it can localize the anomaly within specific cells or tissue areas, and this provides the ability to study anomalies at a single cell level [2,3], unlike DNA techniques that pool DNA from hundreds of different cells $[1,3]$.

Compared to FISH testing on conventional suspension samples (Figure 1), paraffin FISH can be labour intensive and highly variable due to differing fixation times between samples and referring histology labs, and the interpretation may be limited due to truncation of signal and overlapping cells $[1,4]$. 


\section{Paraffin pretreatment steps}
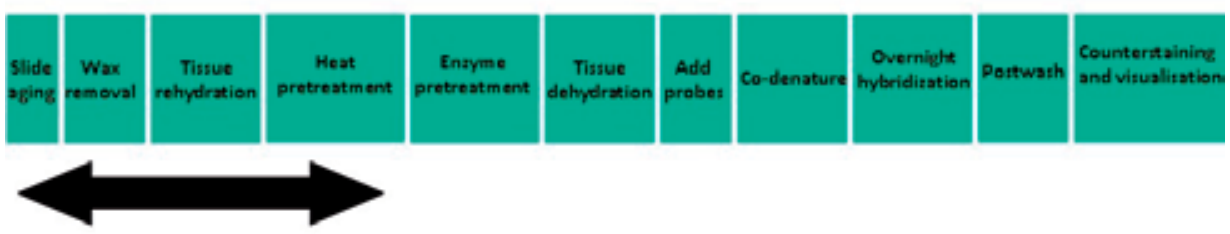

Additional steps for paraffin pretreatment

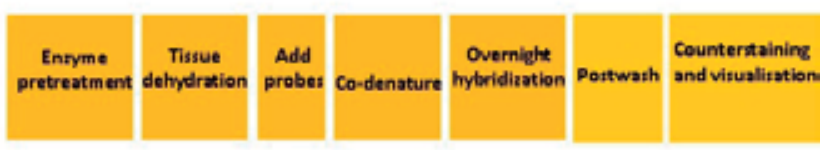

\section{Suspension pretreatment steps}

Figure 1. A comparison of the paraffin pre-treatment process with the conventional FISH pre-treatment process on suspension semples.

For these reasons, it must be considered separately from the conventional suspension FISH method, and while it can be used as either a stand-alone technique, or an adjunct to conventional cytogenetics techniques [5], it must be noted that due to the use of interphase nuclei, a prior knowledge of the anomaly of interest is required.

PROCESS

1. Sample received at laboratory

2. H+E slide made and assessed by laboratory staff

3. H+E slide assessed by pathologist

4. Target area on $\mathrm{H}+\mathrm{E}$ marked by pathologist

5. FISH slide set up and processed

6. Postwash and analysis of slide

\section{PROBLEM}

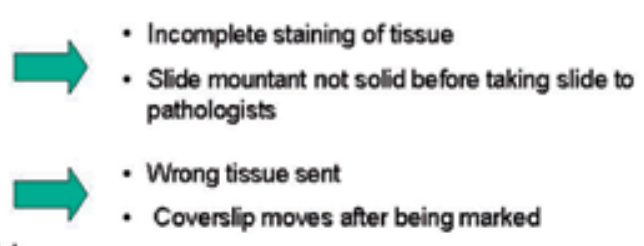

- Tissue sections fall off during pretreatment,

- Different tissue cuts from block on each slide.

- Different orientation of tissue on slide to $\mathrm{H}+\mathrm{E}$ (affects transfer of target area).
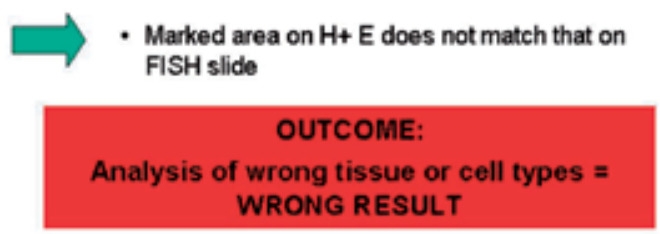

Figure 2. Errors that occur during the paraffin pre-treatment process.

The basic premise of the method involves establishing the area of interest for testing on the $\mathrm{H}+\mathrm{E}$ stained pathology slide, and transferring this area to an unstained paraffin slide, which is then 
pretreated, probed and co-denatured using the traditional FISH methodology [6,7]. However, one of the most crucial factors for paraffin analysis is the assessment of the correct target area before beginning the procedure - without this, an erroneous result may occur (Figure 2), which may be costly to patients if it results in the appropriate treatment being with held [1].

For this reason, robust internal and external quality control procedures are required for diagnostic paraffin FISH testing and the exclusion of non-target tissue before analysis decreases the likelihood of an incorrect result due to an analysis error [1]. This protocol therefore aims to provide a guide to some of the considerations and troubleshooting that are necessary when using the method for diagnostic medical testing. It is adapted from the method used by the Diagnostic Genetics Department, LabPlus at Auckland City Hospital, New Zealand. There are a number of variations to the basic FISH method that can be used depending on the nature and number of samples being processed, and new technology has also been developed to automate the process (Xmatrix, Abbott Molecular). In this protocol however, we have suggested extra steps that are designed to help improve the quality of the testing procedure for diagnostic use. Probes used for diagnostic testing are commercially available and may be downloaded and gathered from the websites of companies such as Abbott Molecular, Cytocell, Zytovision or Kreatech Diagnostics.

\section{Method}

One slide (2-5 micron thickness usually) is needed per probe or probe set, and if a haematoxylin and eosin $(\mathrm{H}+\mathrm{E})$ slide is not provided by pathologists, an extra slide must also go through the deparaffinisation steps before staining with the Shandon Rapid-Chrome ${ }^{\mathrm{TM}}$ Frozen Section Staining kit (alternatively the individual stain kit components can be made from powder).

A. Slide aging

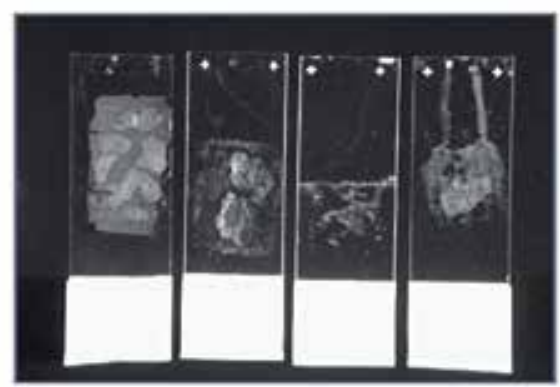

Not aged
B. Slide pre-treatment

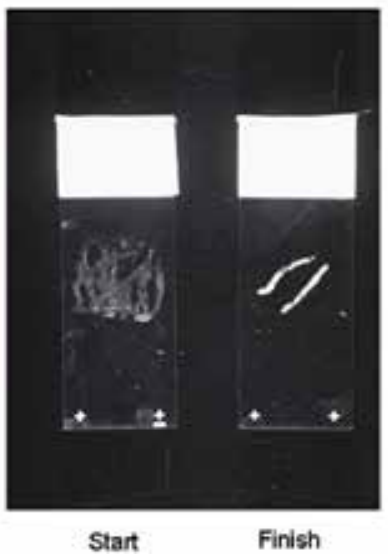

Figure 3. Slide pretreatment steps for paraffin FISH. (A) Appearance of unstaind paraffin slides after aging in a $60^{\circ} \mathrm{C}$ oven - note melted or "bubbled" appearance. (B) Unstained paraffin slides and after the pre-treatment steps. 
1. Deparaffinisation (approx. 60 minutes); see Figure 3

a. Leave slide/s on the hotplate/in the oven at approximately $65^{\circ} \mathrm{C}$ for $30-60$ minutes for aging (Figure 3).

b. Perform deparaffinization by placing slide/s in xylene for at least 10 minutes in the fume hood, with intermittent shaking.

c. Rehydrate slide/s by placing them for 2 minutes in each of $100 \%, 80 \%$, and $70 \%$ ethanol solutions, followed by deionised water at room temperature.

2. Haemotoxylin and Eosin (H+E) slides; see Figure 4

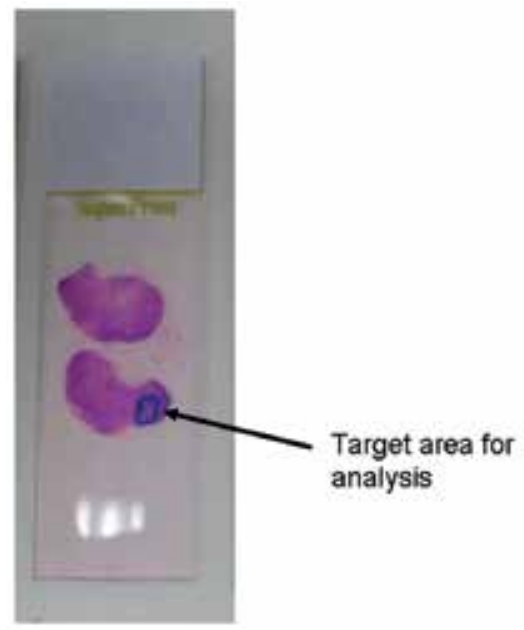

Figure 4. A haemotoxylin and eosin $(\mathrm{H}+\mathrm{E})$ stained slide with the target area for analysis marked by a pathologist.

a. Take rehydrated slide/s and stain using the Shandon Rapid-Chrome ${ }^{\mathrm{TM}}$ Frozen Section Staining kit and mount the slide using Shandon Mount.

b. Leave slides on the hotplate for at least $30 \mathrm{~min}$ to dry the mountant.

c. Check slides for stain quality under a light microscope.

d. Take slide/s to pathologist for marking (Figure 5).

3. Heat Pre-treatment (approx. 30 minutes)

a. Add $35 \mu \mathrm{l}$ of heat pre-treatment solution (Invitrogen Tissue Pre-treatment Kit) to the slide/s, cover with a 22x22mm (or bigger sized cover slip) glass cover slip and seal with rubber cement. Alternatively slides can be heat-pre-treated in coplin Jar at $95^{\circ} \mathrm{C}$ or pressure cooker.

b. Heat slide/s on the thermal cycler for $15-60$ minutes at $95^{\circ} \mathrm{C}$ (The time is dependent on the type of tissues and length of formalin fixation). 
c. On completion, immerse slide/s with cover slip in deionised water to cool down and gently remove the cover slip.

d. Wash briefly in a coplin jar of deionised water at room temperature and drain off excessive water.

4. Enzyme Digestion (approx. 40 minutes).

a. Add an appropriate amount $(\sim 15 \mu \mathrm{l})$ of enzyme reagent (Invitrogen Tissue Pre-treatment Kit) to the slide/s, depending on the size of hybridisation area, and cover with a square of parafilm.

b. Incubate slide/s for $15-45$ minutes in a humidified chamber at $37^{\circ} \mathrm{C}$ (This time is dependent on the type of tumours and length of formalin fixation).

c. Remove cover slip/s and wash briefly in a coplin jar of deionised water at room temperature.

d. Dehydrate slide/s for 2 minutes each in each of $70 \%, 80 \%$ and $100 \%$ ethanol solutions and air dry at room temperature. Please note that a different ethanol series is used for the dehydration steps to avoid reagent contamination issues.

e. Check the tissue morphology of the pre-treated slide looks the same as that of the H+E.

f. The pre-treated paraffin slide/s should then be carefully matched against the marked H\&E slide/s, and the area for testing transferred to the pre-treated slide/s using a marker pen initially, followed by the diamond-tipped engraver. This means that the area can still be visualised after the post-wash steps.
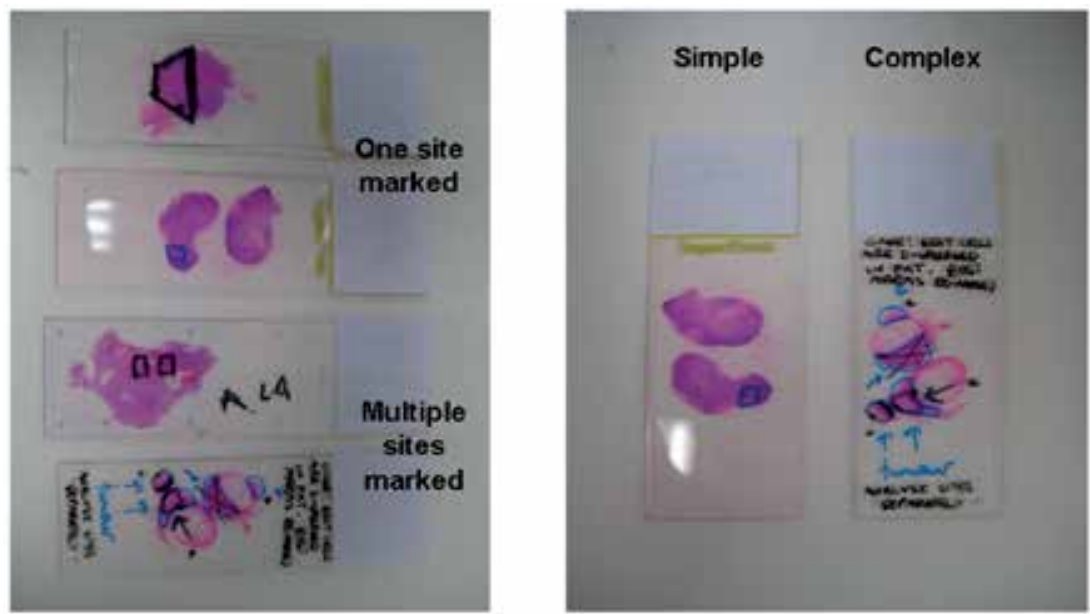

Figure 5. Haemotoxylin and eosin $(\mathrm{H}+\mathrm{E})$ stained slides marked with the target area for analysis. This reduces the volume of probe necessary and ensures that non-target tissue is excluded as much as possible before the FISH analysis procedure. 

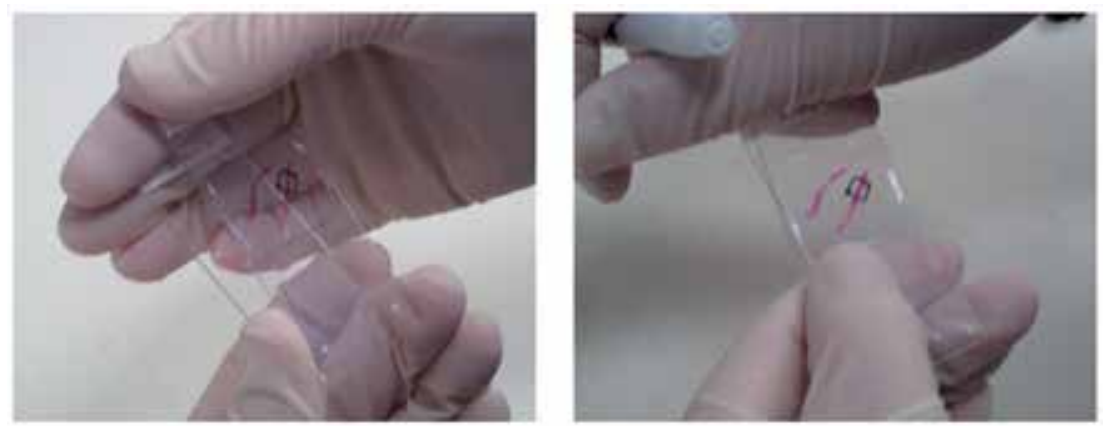

Figure 6. Transfer of target area for analysis from the $\mathrm{H}+\mathrm{E}$ slide to the pre-treated FISH slide prior to the probing steps.
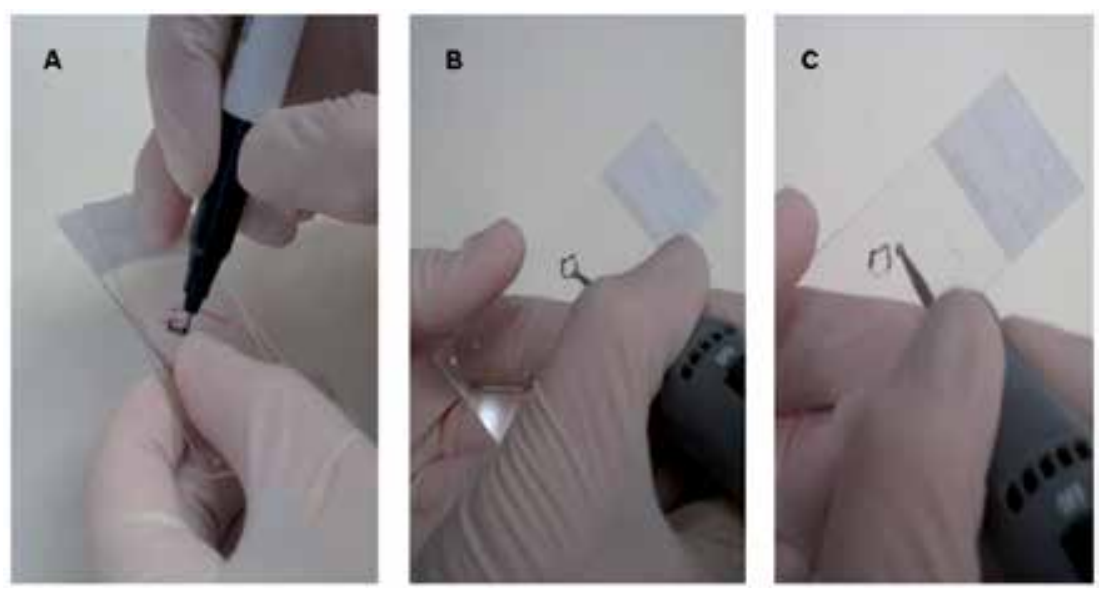

Figure 7. Engraving of target area on to the pre-treated paraffin FISH slide. (A) Draw target area onto bottom of slide with fix-resistant pen. (B \& C) Engrave marked area onto bottom of slide using diamond-tipped engraver to keep area visible after post-wash steps.

5. Probe preparation (approx. 10 minutes)

Use Ready-To-Use probes or refer to the probe preparation protocol outlined by the manufacturer.

6. Co-denaturation and hybridization (approx. 25 minutes)

a. Apply an appropriate amount $(2-10 \mu \mathrm{l})$ of probe mix to the hybridization site marked on each slide, depending on the size of cover slip being used, and seal with rubber cement. Leave the slide/s in the incubator or in a drawer at room temperature for a few minutes to allow the rubber cement to dry before placing them in the thermal cycler.

b. Denature slide/s together with probe mix for $10-20 \mathrm{~min}$ at $85^{\circ} \mathrm{C}$ or $5-10 \mathrm{~min}$ at $95^{\circ} \mathrm{C}$.

c. After co-denaturation, slide/s may be placed in a humidified box in the incubator at $37^{\circ} \mathrm{C}$ for at least $12-16$ hours, usually no more than 72 hours. 
7. Post Hybridization Wash (5 Minutes)

a. Briefly soak slide/s in 2xSSC and gently remove rubber cement.

b. Wash slide/s in $0.4 \times S S C / 0.03 \%$ Tween 20 (or NP40) at $72^{\circ} \mathrm{C}$ for $2 \mathrm{~min}$.

c. Place slide/s in 2xSSC/0.01\% Tween 20 (or NP40) for $1 \mathrm{~min}$.

d. Briefly drain slide/s, apply DAPI counter stain and put cover slip on.

e. Visualize FISHed-slide/s under fluorescence microscope.

When using indirectly labelled commercial probes that require antibody detection, signal detection must be done according to the manufacturer's instructions.

8. Analysis and interpretation; see Figure 8.

a. With a pathologist's consultation, check the H+E slide on a transmitted light microscope to assess whether the sample contains a mixture of cell types, as this may affect the interpretation of the FISH signal pattern.

b. Check the paraffin FISH slide on a fluorescence microscope using the 10x objective to ensure the area marked on the slide approximately matches that on the $\mathrm{H}+\mathrm{E}$ slide.

c. Using two observers, analyse a minimum of at least 8 representative sites within the marked region (a minimum of 4 different areas per observer), scoring only cells that show both the target and control loci. Analysis of areas of areas where the cells are not overlapped is preferable, and a third analyst is required where there is discordance between two observers.

\section{Each analyst must:}

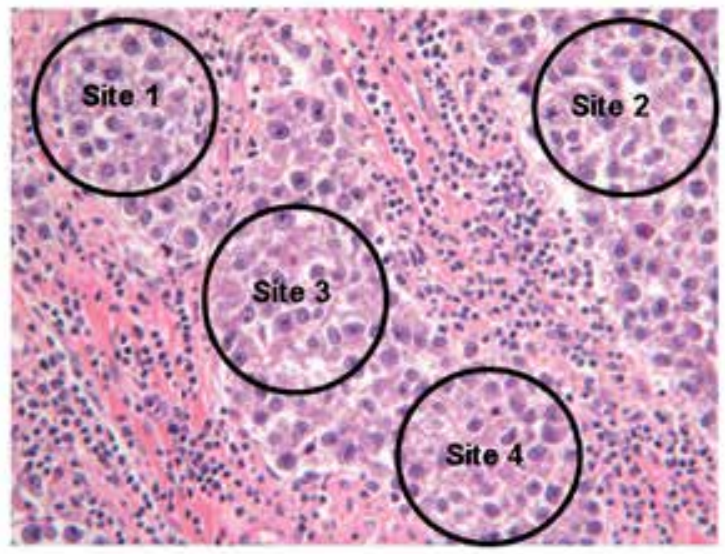

1. Check histology slide for mixed cell population

2. Check paraffin FISH slide to ensure that area matches

3. Score areas with minimal overlap

4. Observers score a minimum of 8 representative sites in marked area (different QAP programmes may use fewer number of sites.

Figure 8. Analysis principles for paraffin FISH slides. 


\section{Troubleshooting}

Problem: Unclear whether slides have been aged before arrival, as repeating this step may decrease the hybridization efficiency of the probe.

[Step 1]

Solution: Although some waxes do not change in appearance, pre-aged slides generally have a bubbled or melted appearance of the wax compared to the smooth appearance of non-aged slides in general (N.B: some wax types do not change in appearance so this is a rule of thumb only).

Problem: The use of xylene to remove the wax from around the sample is not ideal as xylol is highly toxic.

[Step 1]

Solution: An alternative to xylene is HemoDe from Scientific Safety Solvents.

Problem: Finding that the wrong tissue was sent by the referring laboratory.

[Step 2]

Solution: Ask for a copy of the pathology report to be sent with all samples, and get pathologists to ring the referring laboratory to request the appropriate sample for testing.

Problem: Incomplete staining of the $\mathrm{H}+\mathrm{E}$ slide causing correct target area to be missed by pathologist.

[Step 2]

Solution: Slides should be quality checked before taking them to a pathologist. Check the stain by eye to see if there are obvious colour differences across the slide - if one of the stains has been missed in an area it will appear either a dull purple (eosin missed) or a dull pink (haematoxylin missed or there is a problem with the $\mathrm{pH}$ of the bluing reagent) compared to the rest of slide. If there are any doubts, ask a histopathology technologist for assistance.

Problem: Cover slip moves after the slide has been marked because mountant is not completely hardened. This causes the target area to move.

[Step 2]

Solution: Leave the slides on the hotplate for a longer period of time, or change mountant to a faster drying version such as Entellan (Note: it is not possible to remove the Entellan with methanol after it has been cover slipped, hence why DPX is the preferred mountant).

The Rapid-Chrome ${ }^{\mathrm{TM}}$ Frozen Section Staining kit uses Shandon Mount; however alternatives such as Entellan are available.

Problem: Disappearance of tissue on slide during dehydration steps.

[Steps 3 and 4] 
Solution: The ethanol series (in step 1) is necessary to rehydrate the tissue for the enzyme solution to act on, and may cause the tissue to become translucent, however it will become white again once the slide is dehydrated.

Problem: Scratching or loss of tissue during washing steps. Small tissue samples (e.g. core biopsies) may become fragile during the pretreatment steps and fall off the slide.

[Steps 3 and 4]

Solution: As the tissue becomes soft during pre-treatment it may easily fall off or get scratched; coplin jars of deionised water can be used to dip slides into rather than the more aggressive use of squirter bottles or running tap water (do not leave the pre treated slide in water for a long time, especially for a core biopsy or a tiny sample). The size of the tissue gives a good indication as to the fragility of the tissue, so this should be taken into account before beginning the pre treatment steps. Increasing the ageing step may also help to fix the tissue to the slide better, although it may also decrease the hybridization efficiency of the probe to the sample. Alternatively, skipping the heat pretreatment step and doing a reduced enzyme treatment on the sample may combat this.

Problem: The tissue does not look the same as the H+E slide after dehydration steps.

[Step 4]

Solution: This can either be due to loss of tissue during pretreatment or different cuts through the tissue block. Untreated slides should be closely examined to find one that appears to match the pretreated slide and a new $\mathrm{H}+\mathrm{E}$ slide created using this slide. See also steps for reducing the loss of tissue during pretreatment.

Problem: Transfer of area is difficult due to a slight difference in the morphology of the tissue in different layers of the tissue section, or different orientation of tissue on pre-treated slide to that of the $\mathrm{H}+\mathrm{E}$ slide.

[Step 4]

Solution: If the morphology of tissue on the pre-treated slide looks different to that of the $\mathrm{H}$ + E slide, check it against the remaining untreated slides to see if it looks like tissue has been lost during the pre-treatment procedure. If tissue has been lost, simply start the procedure over again with a new slide. If the morphology of the tissue appears different between the untreated slides, ask a pathologist for help selecting an appropriate slide to pre-treat, and try to find two similar untreated slides. Pre-treat one and make the other into an H+E slide to allow for more accurate marking.

Problem: There is more than one target area marked on slide - is more probe required?

[Step 4 and 5]

Solution: Assess the size of the areas - if there are several small areas, the total volume of probe does not need to be increased, simply aliquot the volume of probe equally over the different areas and place a small cover slip over each. More than one aliquot of probe is only required if the areas are greater than can be covered by a $13 \mathrm{~mm}$ diameter cover slip. 
Problem: The hybridisation buffer for a probe runs out.

\section{[Step 5]}

Solution: As hybridisation buffers are all fairly similar, it is fine to use the buffer of similar probe as a substitution. Alternatively, hybridization mix can be made up:

Hybridization mix

(10\% dextran sulphate, $50 \%$ formamide in $2 x S S C, 0.1 \%$ SDS, $\mathrm{pH} 7.0$ )

1. Mix $12.5 \mathrm{ml}$ formamide, $2.5 \mathrm{ml} 20 \mathrm{xSSC}$ pH7.0 and $10 \mathrm{ml}$ MilliQ water. Adjust $\mathrm{pH}$ to 7.0 with $\mathrm{HCl}$ then transfer to a $50 \mathrm{ml}$ Falcon tube.

2. Add $2.5 \mathrm{mg}$ dextran sulphate and place on a roller mixer at room temperature for 1-2 hours.

3. Add $25 \mu \mathrm{l}$ Tween 20 and invert to mix.

4. Aliquot $500 \mu \mathrm{l}$ into sterile eppendorf tubes. Store at $-20^{\circ} \mathrm{C}$ and use a fresh aliquot each time.

Problem: A thermal cycler is not available for use.

[Step 6]

Solution: Denaturation of the slide(s) can be done separately using 70\% formamide/2xSSC, as it gives better quality denaturation although the downside is that it is highly toxic. The hybridisation steps can also be done adequately in a programmable system (e.g. Thermobyte).

Problem: The cover slip is hard to remove before the post wash steps.

[Step 7]

Solution: Place slide in 2xSSC solution and agitate gently after removing the rubber cement, and then remove cover slip. If the cover slip is still stuck to slide, slide the blade of a scalpel under one corner of the slide and lift gently before immersing the slide in a 2xSSC solution and agitating it gently. This may need to be repeated several times if the cover slip remains stuck.

Problem: Weak or patchy signal quality.

[Step 8]

Solution: This can be difficult to fix, as it primarily occurs as a result of poor handling and fixation of tissue prior to receiving the sample for FISH testing [8,9]. Different tissue samples may require the pretreatment times to be varied [10]. The heat pretreatment buffer prepares the tissue for the enzyme to act on and the enzyme degrades the cellular material away from the DNA, in order to allow the probe to anneal to the chromatin. Variation of either or both these times is effective, and the steps may be repeated on the probed slide to reduce the need for lengthy pretreatment times on a new slide. Bone samples such as trephines may show poor hybridization efficiency of the probe, and require hydrogen chloride treatment, unless the sample has already been decalcified prior to arrival. 
Poor signal quality may also be a result of incorrect post wash stringency. There is an alternative wash technique that uses $50 \%$ formamide/2xSSC to increase the stringency of the wash. However, this is not always ideal, as it significantly increases the length of the post wash, and also uses formamide which is extremely toxic [11].

Problem: High levels of cross hybridization due to non-specific binding of probes.

[Step 8]

Solution: This is due to incorrect stringency of the post wash [1]. For a quick fix, slides can be rewashed using the quick wash procedure reported here, or alternatively washing at a higher temperature or use of a different post wash procedure can be tried [11].

Problem: Cells only show one signal colour.

[Step 8]

Solution: Only cells showing both the control and target loci should be scored (e.g. 2R2G), so if both the control probe and the probe for the region of interest are on the same chromosome, it is most likely to be due to poor hybridisation of one of the probes. First check to see using single colour filters whether the signal colour is present but weak - if it is, repeat the pre-treatment and hybridisation steps again on the same slide (for a shorter time e.g. 15/15 buffer: enzyme treatment).

Problem: Using an indirectly labeled probe and can't get a good signal quality.

[Step 8]

Solution: In most cases, amplification with only a primary antibody is necessary, and further amplification can also increase the level of background on the slide(s). However if the signal is not bright enough, carefully remove the cover slip, rinse slide in 1xPBS (or SSC) and perform further amplification steps with secondary or tertiary antibodies as many times as necessary. After adding each antibody, slides should be covered with parafilm and incubated in a humidified chamber at $37^{\circ} \mathrm{C}$ for 5 minutes before being washed in $4 \times S S C / 0.05 \%$ Tween 20 for 2 minutes. Then mount with $8 \mu \mathrm{l}$ Vectashield antifade solution with DAPI.

Problem: Distinguishing between real signal and background or 'rubbish' on slide.

[Step 8]

Solution: Look at the signal intensity on single colour filters - rubbish generally appears to be brighter and shinier compared to real signals, and background will appear fuzzy and indistinct compared to real signal. High background may be due to the slides not being properly sealed with rubber cement during the pretreatment steps, as this allows the solution to evaporate and the tissue to dry out.

Problem: High background on the slides when analyzing.

[Step 8]

Solution: High background may be due to insufficient removal of material during the pretreatment steps. With high case numbers, solutions can become contaminated, therefore the 
solutions in the pretreatment steps need to be changed regularly, and it pays to have an additional coplin jar of $100 \%$ ethanol to dip the slides into after the xylol step in order to reduce contamination from the xylol solution. Alternatively, background may be due to the cover slip not being sealed properly during the pretreatment and co-denaturation steps, causing the tissue to dry out. By placing the slide in the incubator to allow the rubber cement to dry before these steps, this effect can be reduced. The use of a glass coverslip rather than a plastic coverslip also helps, as plastic acts as an insulator, and therefore will hold the temperature and increase the drying of the tissue.

The use of detergents in the post wash steps also helps to solubilize proteins, and if Tween20 is not effective, then NP-40 can also be used.

Problem: There is a mixed cell population in the marked target area (e.g. Tumour cells with non-target lymphocytes also present); see Figure 9.

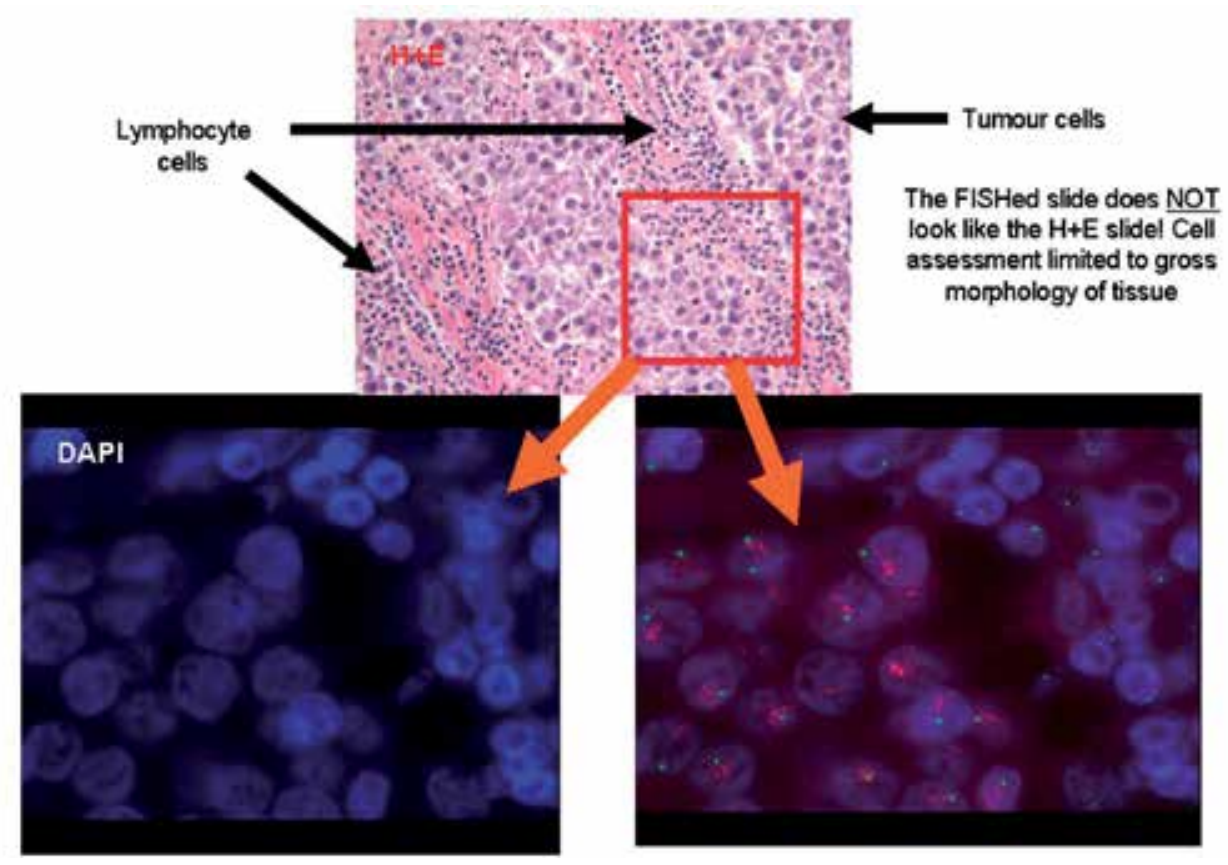

Figure 9. The analysis of slides with mixed tissue populations.

[Step 8]

Solution: Check the H+E slide first before analysing the FISH slide to see whether there is clustering of cell types, or differences in morphology between the different cell types. Then scan the marked target area on the FISH slide using the 10x objective to find areas which appear to be targeted cells and switch to a higher objective for confirmation and then analyse using appropriate filter. Consideration of accidental analysis of non-target cells must also be taken into account when interpreting such cases, therefore increasing the number of cells or sites analysed will increase the accuracy of the analysis. Alternatively, it may be pos- 
sible to get a pathologist to mark several smaller sites containing only target cells, as this reduces the risk of error before beginning the analysis.

Problem: Target area marked is very small, so it is difficult to test a variety of areas.

[Step 8]

Solution: While this makes analysis difficult, switching to the 10x objective and moving the stage to a different position will reduce the likelihood of reanalyzing the same cells. Numerical scoring is also preferable in such a case, as it provides a reliable basis for interpretation.

Problem: The cells are highly dispersed or highly clustered, making analysis difficult.

[Step 8]

Solution: Select good areas where the cells are not overlapping using the DAPI filter and use numerical scoring of individual cell signal patterns (this may mean increasing the number of sites examined if the cells are widely dispersed). If a gene rearrangement probe is being used, it may be sufficient just to report the presence or absence of a rearrangement without doing individual cell analysis.

Problem: Distinguishing between real loss and gain of signal compared to artefact.

[Step 8]

Solution: If the target abnormality is either a gain (trisomy/tetrasomy) or loss (deletion) of a signal, it pays to establish thresholds using normal control slides to estimate the level of artefactual gain or loss of signal, and to check the manufacturer's product information to see if splitting of the probe or non-target binding/polymorphisms are common with the probe. The variance in the signal patterns can also be checked - if the percentage of cells showing a $1 \mathrm{R} 2 \mathrm{G}$ signal pattern is roughly equivalent to those showing a $2 \mathrm{R} 1 \mathrm{G}$ signal, then it is reasonable to assume that it is due to artefactual truncation of signal.

Problem: There is discordance between analysts.

[Step 8]

Solution: Get a third analyst to score the sample. If two analysts have similar results, discard the third analysis, or if all three give different results, take an average of all three results to allow robust interpretation. If the three results differ hugely, it is preferable to confirm the result with a secondary probe where possible, or request a repeat sample from another block. Where the interpretation is still not clear, the case can be reported as inconclusive or failed.

Problem: A low level abnormality, multiple clones or mosaicism is suspected.

[Step 8]

Solution: Where the result is not straightforward use quantitative scoring and use appropriate thresholds for interpretation. Paraffin FISH is not the most suitable method of detection for these cases, although methods that involve taking thicker slices of the section have been developed [12]. 


\section{Conclusion}

The role of pathologists is crucial to the analysis of paraffin FISH sections from the beginning of the process. They can help to eliminate very basic laboratory errors, such as identifying whether incorrect tissue has been sent prior to processing the slides, and can also help to identify the appropriate target tissue within the paraffin section prior to analysing the sample, so that inappropriate tissues can be reduced or eliminated. When analysing products of conception, the fetal component can be very small compared to the maternal component, and without guidance of pathologists, an erroneous result may occur. Similarly, in breast cancer samples, it is important to eliminate areas of contained carcinoma (in situ components such as DCIS and LCIS) and lymphocytes, as these may result in false positive or negative results, which can be deleterious if treatments such as Herceptin are then withheld from the patient. Some samples such as lymphomas or graft versus host disease may require extensive guidance from pathologists as knowledge of the disease characteristics will allow for highly targeted analysis. In follicular lymphoma, the follicles need to be identified so that centrocytes and centroblasts are targeted for analysis, and normal lymphocytes and reactive cells are avoided when analysing the sample (Swerdlow et al. 2008). For this reason, it is best to include a variety of areas to get a representative result. It should be noted that external quality assurance programmes may differ in the number of sites required for analysis. Generally speaking, fewer sites are required, if initially the non-target tissue is eliminated.

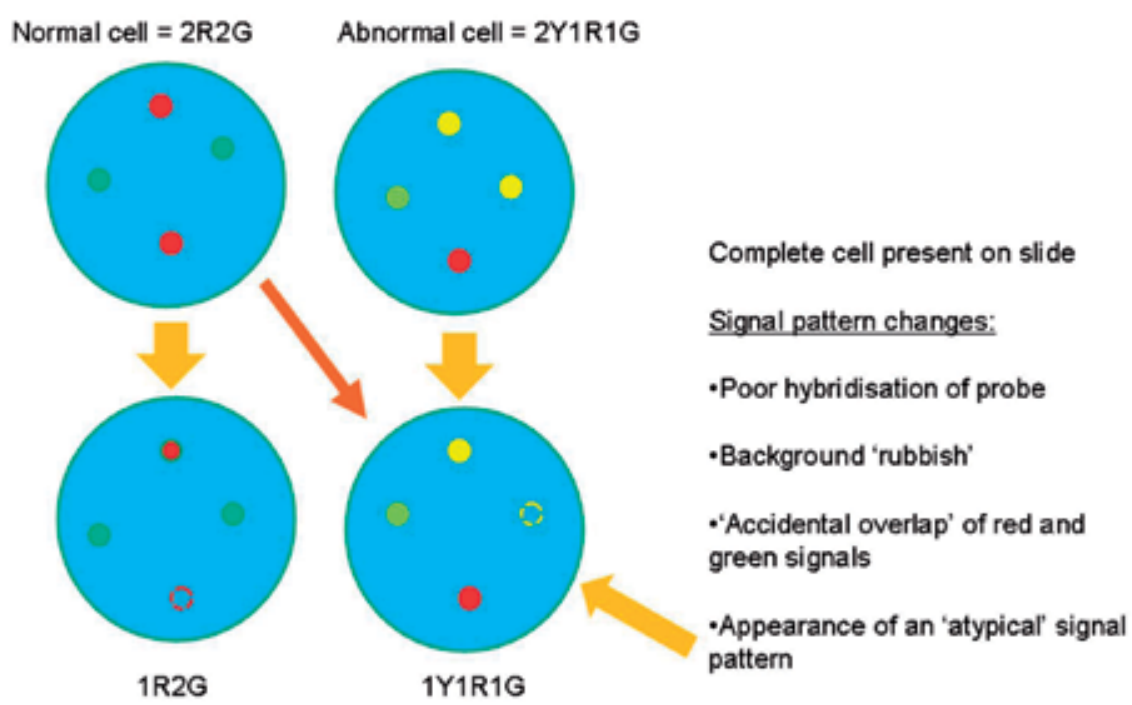

Figure 10. Artefactual signal changes on suspension FISH slides.

Despite such assistance however, care must also be taken during the analysis of paraffin samples, as in many cases it is impossible to completely remove the non-target tissue from the area of interest. It is therefore important to check the $\mathrm{H}+\mathrm{E}$ slide before beginning the 
analysis, as this will give an indication as to whether the sample is made up solely of target tissue, or whether it contains a mixture of target and non-target tissue that must be taken into account when making the final interpretation.

\section{Same issues as suspension samples BUT extra issues also present}

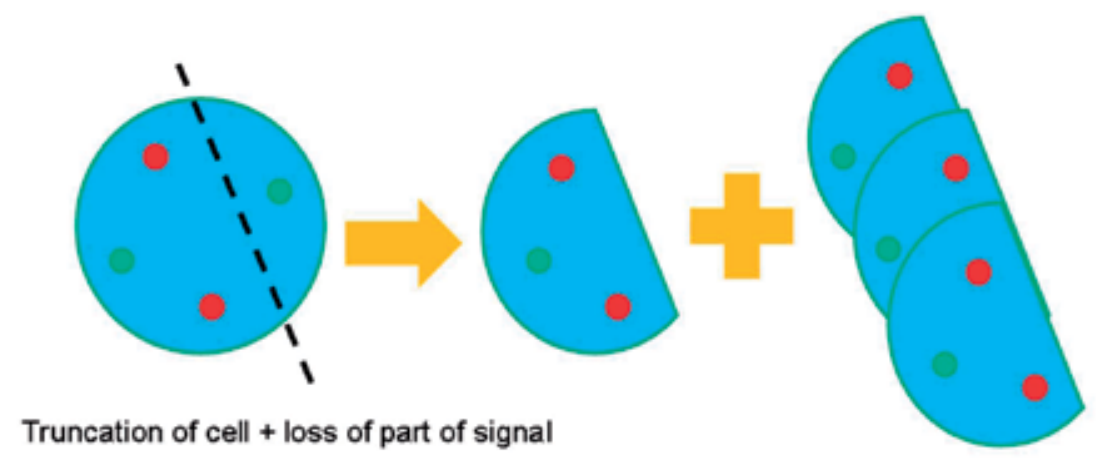

Overlapping of truncated cells

Figure 11. Artefactual considerations for paraffin FISH samples - truncation and overlapping of cells in specimen.

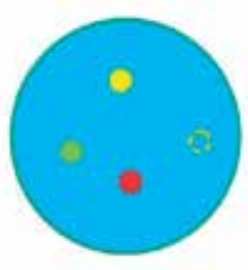

Poor hybridisation
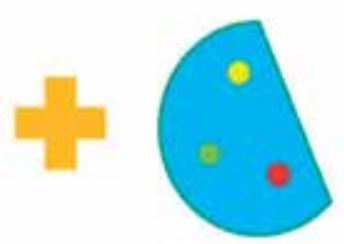

Truncation

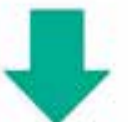

1Y2G1R

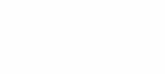

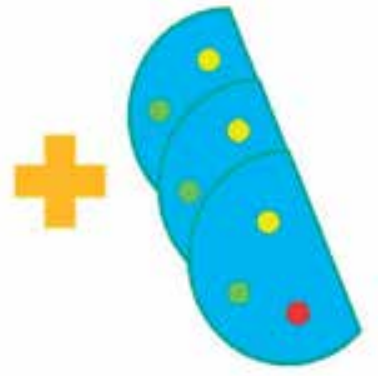

Cell overlap

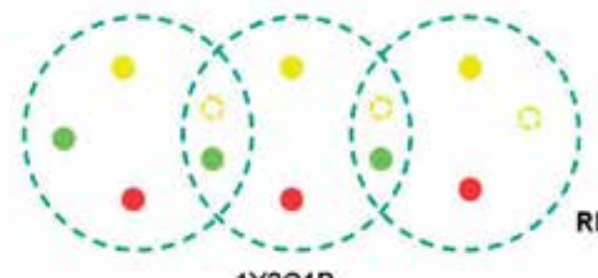

1Y2G1R
IY1G1R

RESULT $=$ ATYPICAL

SIGNALS

Figure 12. The need for thresholds for paraffin FISH analysis.

Due to both the potential for analysis of the incorrect target cells as outlined, and the artefactual variation that can arise when using the FISH technique [13], it is necessary to establish 
robust thresholds to guide the interpretation of results. Signal pattern changes can occur due to poor hybridization of probe, background 'rubbish-autofluorescence' or 'accidental overlap' of red and green signals (Figure 10).

These can lead to the appearance of false or atypical signal patterns; therefore thresholds need to be established to distinguish between false positives and negatives. Paraffin analysis requires higher thresholds than those for suspension cultures, as there is the additional complication of overlap and truncation of cells [1,12], causing artefactual gain or loss of signals (Figures 11 and 12).

Thresholds are of particular importance when dealing with cases that show atypical, non-target (e.g. unexpected loss or increase of copy number instead of a gene rearrangement) or low level abnormalities, or those where mosaicism or multiple clones appear to be present, as it is unclear in most cases as to how they may impact on patient treatment. While paraffin FISH is usually not the most appropriate way to deal with such cases, but when tissue is scarce or has already been processed, it can sometimes be the only option for testing. Numerical scoring of the tissue in such cases will give an indication of the major signal pattern(s) and the level of variation inherent in the tissue, particularly in tumours where there can be concurrent increase in the ploidy level, together with loss or gain of the target loci. This will allow a judgment to be made about whether the variation is likely to be artefactual or not, as false aneuploidies will show almost equivalent levels of loss between target and control loci.

Due to the potential complexities of paraffin analysis, the use of both cytogenetic and pathology external quality control programs such as the College of American Pathologists (CAP) and Australasian Society of Cytogeneticists (ASoC) is recommended, as it allows quality issues to be addressed from both the cytogenetic and pathology perspectives. This provides a balanced perspective on the degree of analytical stringency that is required prior to releasing result.

\section{Appendices}

\section{Materials}

Reagents

Biotin and Digoxygenin

Bovine serum albumin (BSA) Deionised water

Enzyme reagent (Invitrogen cat \#00-8401)

Ethanol (70\%, 80\% and 100\%)

Heat pre-treatment solution pH7.0 (Invitrogen cat \#00-8401)

Hybridisation buffer

Non ionic detergent: NP40 (Vysis 30-80482). Store in $-20^{\circ} \mathrm{C}$.

Phosphate Buffered Saline (PBS) 
$0.01 \%$ pepsin/ $\mathrm{HCl}$ solution

Purified $\mathrm{H}_{2} \mathrm{O}$

DNA probes

Shandon Rapid-Chrome ${ }^{\mathrm{TM}}$ Frozen Section Staining kit

2 XSC/0.1\% Tween 20

$0.4 \times S S C / 0.3 \%$ Tween20 solution

2xSSC/0.01\% Tween20 solution

Vectashield antifade mounting solution with $1.5 \mu \mathrm{g} / \mathrm{ml}$ DAPI (Vector laboratories Cat \# $\mathrm{H}-1200)$. Store in the dark at $4^{\circ} \mathrm{C}$.

Xylol

Equipment

Atlas cooler box

Blotting paper

Centrifuge -Heraeus Biofuge Pico

Coverslips (13mm diameter round, 22x22mm and $24 \times 50 \mathrm{~mm}$ )

Diamond pen or diamond-tipped engraver -Easy Marker Engraver (Taiwan)

Eppendorf tubes

Fix-resistant marker pen

Fluorescence microscope - Zeiss Axio Imager.M1 microscope, Zeiss Axioplan microscope, Olympus BX60 fluorescence microscope

Glass coplin jars

H\&E slide

Hotplate

Humidified box

Incubator - Contherm Scientific NZ

Parafilm

Pipettes (2 ul and 100ul)

Pipette tips

Poly-lysine slides (with tissue sections of 2-5 $\mu$ m thickness)

Rubber cement - Weldtite Vulcanising Rubber Solution 
Safety goggles

Scalpel

Scissors

Slide drying racks

Thermal cycler - MJC Research PTC- 100 and PTC-200 Peltier Thermal Cyclers

Transmitted light microscope (Zeiss)

Fine tweezers (2 pairs)

Water bath - Grant Instruments (Cambridge)

Recipes

Biotin- and Avidin-conjugated antibodies

Store antibodies as $20 \mu \mathrm{l}$ aliquots at $4^{\circ} \mathrm{C}$ in sterile eppendorf tubes. Do not freeze.

Texas Red Avidin DCS (Biotek/Vector Laboratories Cat \#A-2016).

Add $0.5 \mathrm{ml}$ of MilliQ water to $1 \mathrm{mg}$ lyophilised antibody for a final concentration of $2 \mathrm{mg} / \mathrm{ml}$.

Fluorescein Avidin DCS (Cell sorter grade), (Biotek/Vector Laboratories Cat \#A-2011).

$2 \mathrm{mg} / \mathrm{ml}$ stock solution aliquotted at $20 \mu \mathrm{l}$ and stored in the dark at $4^{\circ} \mathrm{C}$. Dilute $1: 400$ in $4 \times \mathrm{SSC} / 1 \%$ BSA immediately prior to use.

Biotinylated goat anti-avidin D (Biotek/Vector Laboratories Cat \#BA0300).

Add $1 \mathrm{ml}$ of MilliQ water to $0.5 \mathrm{mg}$ lyophilised antibody for a final concentration of $0.5 \mathrm{mg} / \mathrm{ml}$.

Bovine Serum albumin (BSA)

$1 \%$ BSA in $4 x$ SSC. Dissolve $0.25 \mathrm{~g}$ of BSA (Sigma A-7030) in $25 \mathrm{ml} 4 \times \mathrm{xSC} \mathrm{pH} 7.0$. Store at $4^{\circ} \mathrm{C}$ for up to 1 month.

FITC - conjugated anti-digoxygenin antibodies

Store antibodies as $50 \mu \mathrm{l}$ aliquots at $4^{\circ} \mathrm{C}$ in sterile eppendorf tubes. Do not freeze.

Anti-digoxigenin-fluorescein, FAB fragments (Boehringer Mannheim Cat \#1207741).

Add $1 \mathrm{ml}$ of MilliQ water to $200 \mu$ g lyophilised antibody for a final concentration of $0.2 \mathrm{mg} / \mathrm{ml}$.

Rabbit fluorescein anti-sheep IgG(H+L) (Biotek/Vector Laboratories Cat \#FI-6000).

Add $1 \mathrm{ml}$ of MilliQ water to $1.5 \mathrm{mg}$ lyophilised antibody for a final concentration of $1.5 \mathrm{mg} / \mathrm{ml}$.

Goat fluorescein anti-rabbit IgG(H+L) (Biotek/Vector Laboratories Cat \#FI-1000). 
Add $1 \mathrm{ml}$ of MilliQ water to $1.5 \mathrm{mg}$ lyophilised antibody for a final concentration of $1.5 \mathrm{mg} / \mathrm{ml}$.

Ethanol 100\% Molecular biology grade.

Ethanol $80 \%$ Mix ethanol absolute (molecular biology grade) and distilled water in a 4:1 ratio $(\mathrm{v} / \mathrm{v})$.

Ethanol 70\% Mix ethanol absolute (molecular biology grade) and distilled water in a 7:3 ratio $(\mathrm{v} / \mathrm{v})$.

Hydrochloric acid $(\mathrm{HCl})$

$0.2 \mathrm{M} \mathrm{HCl}$. Add $2.4 \mathrm{ml}$ of $5 \mathrm{~N} \mathrm{HCl}$ to $60 \mathrm{mls}$ of MilliQ water.

$0.01 \mathrm{~N} \mathrm{HCI}$. Add $1 \mathrm{~mL}$ of $5 \mathrm{~N} \mathrm{HCl}$ to $499 \mathrm{mLs}$ of distilled water. Store at room temperature for up to 1 year.

Phosphate buffered saline (PBS)

1xPBS. $\mathrm{Ca}^{++}$and $\mathrm{Mg}^{++}$free. Dissolve $8.0 \mathrm{~g}$ sodium chloride, $0.2 \mathrm{~g}$ potassium chloride, $2.89 \mathrm{~g}$ $\mathrm{Na}_{2} \mathrm{HPO}_{4} \cdot 12 \mathrm{H}_{2} \mathrm{O}$ and $0.2 \mathrm{~g} \mathrm{KH} \mathrm{PO}_{4}$ in order in $750 \mathrm{ml}$ of MilliQ water. Adjust the volume to 1 litre and autoclave. Store at room temperature.

Pre-treatment reagents for paraffin embedded tissue - Zymed (Invitrogen) Spotlight ${ }^{\mathrm{TM}} \mathrm{HER} 2 \mathrm{CISH}$ kit (84-0146)

Reagent A. 1 litre of heat pretreatment solution, pH 7.0 (Ready-To-Use).

Reagent B. $5 \mathrm{ml}$ of enzyme pretreatment reagent (Ready-To-Use).

Saline sodium citrate (SSC)

20xSSC (7.0). Dissolve 175.3g sodium chloride and $88.2 \mathrm{~g}$ trisodium citrate in $800 \mathrm{ml}$ MilliQ water. (or use SSC that comes with the Vysis kits; add 4 bottles to make 1L), pH to 7.0 and adjust the final volume to 1 litre. Autoclave and store at room temperature.

4xSSC (pH7.0). Add $200 \mathrm{ml}$ of $20 x S S C$ to $700 \mathrm{ml}$ MilliQ water. $\mathrm{pH}$ to 7.0 and adjust the final volume to 1 litre. Autoclave and store at room temperature.

4XSSC/0.05\% Tween20. Add 500 $\mu 1$ Tween20 to 1 litre of 4xSSC. Mix well.

2XSSC/0.1\% NP40. Add 1mL of NP40 to 1L of 2XSSC (pH7.0)

2xSSC (pH7.0). Add 100ml 20xSSC (pH 7.0) to $800 \mathrm{ml} \mathrm{MilliQ} \mathrm{water.} \mathrm{pH}$ to 7.0 and adjust the final volume to 1 litre. Autoclave and store at room temperature.

1xSSC (pH 7.0). Add 50ml of 20xSSC (pH 7.0) to 950ml of milliQ water. Adjust the $\mathrm{pH}$ to 7.0, autoclave and store at room temperature.

0.4XSSC/0.3\% NP40 (Quickwash buffer). Add 20ml of 20xSSC and 3ml of NP40 to $900 \mathrm{ml}$ MilliQ water. Adjust the $\mathrm{pH}$ to 7.0 and final volume to 1 litre. Store at room temperature. 


\section{Caution}

All reagents are potentially hazardous. Appropriate safety procedures must be followed when handling these materials. Avoid contact with skin and mucous membranes, and heating of slides should be performed in a fume hood, as formalin fixed specimens may produce toxic fumes when heated during processing. For more information consult the Hazardous Substances Data Bank (HSDB) - http://toxnet.nlm.nih.gov/cgi-bin/sis/htmlgen?HSDB.

Formamide: perform steps involving formamide in hood to avoid inhalation of fumes Xylene: perform steps involving xylene in hood to avoid inhalation of fumes

Commercial probes and hybridisation buffer solutions: Wear gloves at all times, and when co-denaturing probes use a fume hood, as formamide may be present in probe mixtures and give off toxic fumes.

\section{Author details}

Lisa Duffy ${ }^{1}$, Liangtao Zhang ${ }^{1}$, Donald R. Love ${ }^{1,2}$ and Alice M. George $\mathrm{e}^{*}$

*Address all correspondence to: AliceG@adhb.govt.nz

1 LabPlus, Auckland City Hospital, Auckland, New Zealand

2 School of Biological Sciences, The University of Auckland, New Zealand

\section{References}

[1] Pfeifer, J. D. (2006). Molecular Genetic Testing in Surgical Pathology, Lippincott Williams and Wilkens, 474.

[2] Vorasanova, S. G., Yurov, Y. B., \& Iourov, I. Y. (2010). Human interphase chromosomes: a review of available molecular cytogenetic technologies. Molecular Cytogenetics, 3(1), 1-15.

[3] Maierhofer, C., Gangnus, R., Diebold, J., \& Speicher, M. R. (2003). Multicolour deconvolution microscopy of thick biological specimens. Am. J. Path., 162(2), 373-379.

[4] Ventura, R. A., Martin-Subero, J. I., Jones, M., Mc Parland, J., Gesk, S., Mason, D. Y., \& Siebert, R. (2006). FISH Analysis for the Detection of Lymphoma-Associated Chromosomal Abnormalities in Routine Paraffin-Embedded Tissue. J. Med. Diagn., 8(2), 141-151.

[5] George, T. I., Wrede, J. E., Bangs, C. D., Cherry, A. M., Warnke, R. A., \& Arber, D. A. (2005). Low-Grade B-Cell Lymphomas With Plasmacytic Differentiation Lack PAX5 Gene Rearrangements. J. Med. Diag., 7(3), 346-351. 
[6] Naeim, F., Rao, P. N., Song, S., \& Grody, W. W. (2008). Principles of Molecular Techniques. In: Naeim F, Rao PN, Song S, Grody WW, editors. Hematopathology: Morphology, Immunophenotype, Cytogenetics and Molecular Approaches, Elsevier Inc., Chapter 4, 72-74.

[7] Solovei, I., Grasser, F., \& Lanctôt, C. (2007). FISH on Histological Sections. Cold Spring Harb. Protoc., doi:10.1101/pdb.prot4729.

[8] Varella-Garcia, M. (2006). Stratification of non-small cell lung cancer patients for therapy with epidermal growth factor receptor inhibitors: the EGFR fluorescence in situ hybridization assay. Diagnostic Pathology, 1, 19 .

[9] Kaeda, S. (2009). Molecular Pathology and Genetic Testing from the Perspective of a Commercial Laboratory. Connection, 7-11.

[10] Srinivasan, S., Sedmak, D., \& Jewell, S. (2002). Effect of fixatives and tissue processing on the content and integrity of nucleic acids. Am. J. Path., 161(6), 1961-1971.

[11] Barch, M. J., Knutsen, T., \& Spurbeck, J. (1997). Molecular Cytogenetics: Definitions, Clinical Aspects, and Protocols. In: The AGT Cytogenetics Laboratory Manual (Third Edition), Lippincott-Raven, Chapter 13, 557-595.

[12] Thompson, C., Le Boit, P. E., Nederlof, P. M., \& Gray, J. W. (1994). Thick-section fluorescence in situ hybridization on formalin-fixed, paraffin embedded archival tissue provides a histogenetic profile. Am. J. Path., 144(2), 237-243.

[13] Iourov, I. Y., Soloviev, I. V., Vorsanova, S. G., Monakhov, V. V., \& Yurov, Y. B. (2005). An approach for quantitative assessment of fluorescence in situ hybridization (FISH) signals for applied human molecular cytogenetics. J. Histo. \& Cyto., 53(3), 401-408.

[14] Swerdlow, S. H., Campo, E., Harris, N. L., Jaffe, E. S., Pileri, S. A., Stein, H., Thiele, J., \& Vardiman, J. W. (2008). WHO classification of tumours of Haematopoeitc and Lymphoid Tissues (4th Edition), International Agency for Research on Cancer (IARC), 220-226. 

Chapter 22

\title{
Quality Control of Biomarkers: From the Samples to Data Interpretation
}

\author{
F. G. Ravagnani, D. M. Saidemberg, A. L. C. Faria, \\ S. B. Sartor, D. N. Oliveira and R. R. Catharino \\ Additional information is available at the end of the chapter \\ http://dx.doi.org/10.5772/51555
}

\section{Introduction}

The recent advances in biotechnology and the improved understanding of disease's mechanisms and pathophsyology have strongly shifted the treatment paradigm of empiric knowledge to targeted therapy. Science has enhanced its ability to guide application of new and existing treatments with development, assay verification, biological validation and application of biomarkers; however, in order to be successful, it is needed a thorough understanding of the relationship between the choice of a biomarker and its influence on the treatment effects. [1]

Current biochemical and molecular biological knowledge states that genetic information flows from genomic DNA to mRNA transcripts, which are then translated to proteins; this class of molecules, which also include enzymes, directly influence the concentrations of their substrates and products, which are integrating parts in several tightly-controlled metabolic pathways. Finally, the existence and multiple interactions of these low-molecular weight metabolites within a cell, tissue, or organism, generates a phenotype. [2]

Metabolome, the link between phenotype and genotype, is the last comprehensive grouping for downstream products of the genome and contemplates the total complement of all the low-molecular weight molecules (metabolites) in a cell, tissue, or organism, required for growth, maintenance, or basal function in any given specific physiological state. [3] The potential size of the metabolome is arguable, as studies suggest more and more that an important role is played by residing microflora and its metabolic products. [2]

The monitoring of metabolite changes has been the primary indicator of disease, and has made it possible to diagnose it in individuals. For that reason, the measurement of metabo- 
lites has become an essential part of clinical practice. Employing a wide range of biological fluids, such as blood (including both plasma and serum), saliva, cerebrospinal fluid (CSF), synovial fluid, urine, semen, and tissue homogenates have ensured the widespread use of metabolites as a very powerful diagnostic tool. [4]

Despite significant advances in analytical technologies the past few years, the discovery of metabolomic biomarkers in biological fluids still remains a challenge. As discussed, metabolome plays an important role in biological systems, hence, are attractive candidates to understand disease phenotypes. [5-6] It represents a diverse group of low-molecular weight structures including lipids, amino acids, peptides, nucleic acids, organic acids, vitamins, thiols, carbohydrates and a few others. [7]

Biomarkers are defined as "characteristics that are objectively measured and evaluated as indicators of normal biological processes, pathogenic processes or pharmacological responses to therapeutic intervention". They can be categorized as biomarkers of exposure, biomarkers of effect and biomarkers of susceptibility. [8] Those characteristics are informative for clinical outcome and can be broadly understood as prognostic or predictive biomarkers. [9-10]

Along the variety of chemical classes and physical properties that constitute metabolites, as well as the dynamic range of metabolite concentrations across large orders of magnitude, it becomes clear why it is necessary to employ an extensive array of analytical techniques in metabolomic research, for it represents a comprehensive method for metabolite assessment. [11-12]

Enabling the parallel assessment of the levels of a broad number of endogenous and exogenous metabolites, it has been demonstrated to have great impact on investigation of physiological status, diseases diagnosis, biomarker discovery and identification of disrupted pathways due to disease or treatment. [13-14]

\section{Mass Spectrometry and Biomarkers}

\subsection{Mass spectrometry in metabolomics}

Nowadays, mass spectrometry is one of the most promising approaches for quantifying and qualifying known and unknown specific molecules within a very complex sample, and for elucidating the structure and chemical properties of different compounds. A mass spectrometer consists of three major components: (1) Ion Source: For producing gaseous ions from the substance being studied, some examples are electron impact (EI), chemical ionization (CI), electrospray ionization (ESI), atmospheric pressure chemical ionization (APCI), atmospheric pressure photon ionization (APPI), thermospray ionization (TSI), among others; (2) Analyzer: For resolving or separating ions according to their mass-to-charge ratios, some analyzer examples are: quadrupole, time of flight, ion traps, Fourier transform ion cyclotron resonance, orbitrap, among others; (3) Detector system: For detecting the ions and recording the relative abundance of each of the resolved ionic species, for example: electron multiplier, microchannel plate detector, Daly detector, Faraday cup, among others. The mass spectrom- 
etry technique relies on the capacity of converting neutral molecules into gaseous ions, with or without fragmentation, which are then characterized by their mass to charge ratios $(\mathrm{m} / \mathrm{z})$ and relative abundances. The introduction of a sample into the system, which can be a gas chromatography or liquid chromatography system is necessary to allow the study of different structures and ionic forms.

Historically, most studies with metabolites have been performed with a combination of high resolution capillary gas chromatography, combined with electron impact ionization mass spectrometry (GC-MS). This configuration allowed, for decades, the separation and identification of key micromolecules from complex mixtures, including fatty acids, amino acids, and organic acids in biofluids, generating diagnostic information for several metabolic disorders in qualitative and quantitative pathways [15-16].

Despite its age, GC is still a very useful and informative technique that seems to be far away from retirement; however, there are some limitations in relation to the size and metabolite types that can be analyzed by this technique, and the extensive sample preparation for this purpose. This resulted in the use of nuclear magnetic resonance (NMR) as a tool for metabolite profiling; however, besides the richness of information about molecular structures obtained by this approach, NMR has low sensitivity, allowing just the most abundant compounds to be identified. In contraposition of GC-MS and NMR, the mass spectrometry with a high performance liquid chromatographic system (LC-MS), and the possibility of tandem mass spectrometry (LC-MS) as post-source fragmentation, especially after soft ionization techniques, offers the possibility of analyzing a wide range of polar and medium polarity compounds with good quantification, sensibility and reproducibility [16].

According to Birkemeier et al. (2005) [17], the metabolomic approaches are in dynamic development and a diversity of synonyms have been suggested, such as metabonomics, metabolite profiling (fingerprinting), among others. Several analytical platforms have been introduced, including spectroscopies using diverse electromagnetic wavelengths, like metabolite profiling with the use of infrared spectroscopy (IR), near infrared (NIR), or ultraviolet (UV), besides gas chromatography coupled to mass spectrometry (GC-MS), liquid chromatography with electrospray ionization mass spectrometry (LC-ESIMS), capillary electrophoresis with mass spectrometry (CE-MS) or liquid chromatography with nuclear magnetic resonance (LC-NMR), and these are only a few examples of the technologies involved with metabolomic studies. There is not a single approach to analyze the wide range of chemically different biomolecules, but it is important to choose the technology that fits better to your target molecules [17].

Hollywood et al. (2006) [18] have summarized the main metabolomic strategies:

1. Metabolomic target analysis, which is a more restrict approach. For example, the metabolites originated from a particular enzymatic system after any kind of biotic or abiotic disturbance.

2. Metabolite profiling, which is focused in a group of specific metabolites, for example, lipids associated to a determined metabolic pathway; or related with clinical and pharmaceutical analyses, to map drug metabolism in an organism. This strategy can be also applied with 
other approaches, e.g.: a. "Metabolite fingerprinting", this approach is used in order to classify samples based both in their biological relevance to the organism, and in their origin. The fingerprinting technology is fast, but not necessarily gives specific information about metabolites. $b$. "Metabolite footprinting", exometabolome or secretome, this is similar approach to the fingerprinting, however the target now is a non-invasive analysis, in order to identify the extracellular metabolites. This technique is generally employed to the study of culture cells, with the advantage of not needing to extract the metabolites, and not having to interrupt the metabolism in a given moment before the analysis. Otherwise, this technique can be used for analysing the secretion of any organism, including the secretome of human embryos before in vitro fertilization, with the purpose of finding viable embryos and general disease biomarkers.

3. Metabolomics itself, which is the comprehensible analysis of the whole metabolome (all the mensurable metabolites), under a specific analysis condition. This term is frequently mistaken with metabonomics, a technique that focus in a wider profile of metabolites involved with different metabolic pathways interacting under the effect of some external stimuli, including diseases, drugs, toxins, among other.

\subsection{MALDI AND MALDI-Imaging}

Matrix-assisted laser desorption/ionization (MALDI) is an ionization method with common applications to high mass biomolecules, being a key technique in mass spectrometry (MS), and more traditionally to the proteomics field. MALDI-MS is extremely sensitive, easy-toapply, and relatively tolerant to contaminants [19]. Its high-speed data acquisition and large-scale, off-line sample preparation has made it once again the focus for high-throughput proteomic analyses. These and other unique properties of MALDI offer new possibilities in applications such as rapid molecular profiling and imaging by MS [19].

More recently, there is a growing focus on the use of MALDI ionization system to the analysis of small molecules, however it is important to take into consideration that the coupling of LC-MALDI is a more delicate issue than the coupling of HPLC with other ionization sources such as ESI, because MALDI, based on desorption of molecules from a solid surface layer, is a priori not compatible with LC or CE [20]. A simple alternative to this limitation is the automatic deposition of fractions from a chromatographic separation on a MALDI-TOF target. More advanced techniques have been developed recently: electrospray deposition, electrically mediated deposition, rotating ball inlet, continuous vacuum deposition, and continuous off-line atmospheric-pressure deposition. The current interfacing improvements will surely expand the use of LC-MALDI in the metabolomic area [20,21].

Another good advantage of MALDI ionization is the possibility of obtaining tissue imaging. This is a new technology that allows the simultaneous investigation of the content and temporal/spatial distribution of molecules within a tissue section, enabling to find the exact localization of any biomarker of interest for the prediction of pathologies and for the discovery of future secondary complications originated from different metabolic disease [22]. 
One of the most common applications for this new approach besides the well described proteomics application is the identification of membrane lipids, which have been successfully analyzed by different authors for several biological tissues. MS imaging of cryosections of mature cotton embryos revealed a distinct, heterogeneous distribution of molecular species of triacylglycerols and phosphatidylcholines, the major storage and membrane lipid classes in cotton embryos. Other lipids were imaged, including phosphatidylethanolamines, phosphatidic acids, sterols, and gossypol, indicating the broad range of metabolites and applications for this chemical visualization approach [23].

There are several possibilities for MALDI imaging technology; however applications to the study of small molecule biomarkers are becoming an interesting novel possibility for this ionization method, mainly when considering the development of new matrices which generate low noise levels in the low $\mathrm{m} / \mathrm{z}$ range of the spectra. Bnabdellah et al. (2009) [24] have described the detection and identification of 13 primary metabolites (AMP, ADP, ATP, UDP-GlcNAc, among others), directly from rat brain sections by chemical mass spectrometry imaging. Matrix-assisted laser desorption/ionization tandem mass spectrometry (MALDI-MS/MS) was combined with 9-aminoacridine as a powerful matrix in this study.

Metabolite distribution via imaging mass spectrometry (IMS) is an increasingly utilized tool in the field of neurochemistry. As most previous IMS studies analyzed the relative abundances of larger metabolite species, it is important to expand its application to smaller molecules, such as neurotransmitters [25]. However, it has been pointed out two technical problems that must be resolved to achieve neurotransmitter imaging, the lower concentrations of bioactive molecules, compared with those of membrane lipids, require higher sensitivity and/or signal-to-noise $(\mathrm{S} / \mathrm{N})$ ratios in signal detection, and the rapid molecular turnover of the neurotransmitters; thus, tissue preparation procedures should be performed carefully to minimize postmortem changes [25].

Furthermore, matrix-assisted laser desorption/ionization (MALDI) imaging mass spectrometry has attracted great interest for monitoring drug delivery and metabolism. Since this emerging technique enables simultaneous imaging of many types of metabolite molecules, MALDI-IMS can visualize and distinguish the parent drug and its metabolites. As another important advantage, changes in endogenous metabolites in response to drug administration can be mapped and evaluated in tissue sections [26].

Another applications of MALDI and MALDI imaging to the study of small molecule biomarkers are the use of the method for detecting drug-related degradation products [27] analysis of drugs from intact biological samples and crude extracts, a method that can be applied to rapid drug screening and precise identification of toxic substances in poisoning cases and postmortem examinations [28], the application of MALDI imaging mass spectrometry to the study of elevated nigral levels of dynorphin neuropeptides in L-DOPA-induced dyskinesia in rat model of Parkinson's disease [29], it is also possible to point out the recent advances in the field of lipidomics and oxidative lipidomics based on the applications of mass spectrometry and imaging mass spectrometry as they relate to studies of phospholipids in traumatic brain injury [30] and the using of proteomic or lipidomic signatures for discovery and spatial mapping of molecular disturbances within the microenvironment of chronic wounds using MALDI imaging technology [31]. 


\subsection{Orbitrap}

The orbitrap mass analyzer is a powerful and relatively new technology, which operates in the absence of any magnetic or $r f$ fields. In this analyzer, ion stability is achieved only due to ions orbiting around an axial electrode. Orbiting ions also perform harmonic oscillations along the electrode with frequency proportional to $(\mathrm{m} / \mathrm{z})-1 / 2$. These oscillations are detected using image current detection and are transformed into mass spectra using fast FT, similarly to FT-ICR [32]. In an orbitrap, ions are injected tangentially into the electric field between the electrodes and trapped because their electrostatic attraction to the inner electrode is balanced by centrifugal forces. Thus, ions cycle around the central electrode in rings. In addition, the ions also move back and forth along the axis of the central electrode. Therefore, ions of a specific mass-to-charge ratio move in rings which oscillate along the central spindle. The frequency of these harmonic oscillations is independent of the ion velocity and is inversely proportional to the square root of the mass-to-charge ratio $(\mathrm{m} / \mathrm{z})$. The entire instrument operates in LC/MS mode (1 spectrum/s) with nominal mass resolving power of 60000 and uses automatic gain control to provide high-accuracy mass measurements, within 2 ppm using internal standards and within $5 \mathrm{ppm}$ with external calibration. The maximum resolving power exceeds 100000 (Full Width at Half-Maximum - FWHM). Rapid, automated datadependent capabilities enable real-time acquisition of up to three high-mass accuracy MS/MS spectra per second $[32,33]$.

Some recent applications of this mass analyzer in the search of biomarkers include the ontissue digestion of proteins followed by detection of the resulting peptides, taking advantage of the high resolution obtained. Trypsin was applied by a spraying device for MALDI imaging experiments in a LTQ-Orbitrap mass spectrometer. The mass accuracy under imaging conditions was better than 3 ppm RMS. This allowed for confident identification of tryptic peptides by comparison with liquid chromatography/electrospray ionization tandem mass spectrometry (LC/ESI-MS/MS) measurements of an adjacent mouse brain section [34].

Another possible application for this mass analyzer is the monitoring of metabolites in human urine, approximately 970 metabolite signals with repeatable peak areas could be putatively identified in human urine, by elemental composition assignment within a 3 ppm mass error. The ability of the methodology for the verification of non-molecular ions, which arise from adduct formation, and the possibility of distinguishing isomers could also be demonstrated. Careful examination of the raw data and the use of masses for predicted metabolites produced an extension of the metabolite list [35].

Orbitrap mass analyzer has been also successfully applied to the monitoring of environmental contamination. The use of pharmaceuticals in livestock production is a potential source of surface water, groundwater and soil contamination. A rapid, versatile and selective multimethod was developed and validated for screening pharmaceuticals and fungicides compounds, in surface and groundwater, in one single full-scan MS method, using benchtop UHPLC-Exactive Orbitrap MS at 50,000 (FWHM) resolution. It demonstrates that the ultrahigh resolution and reliable mass accuracy of Exactive Orbitrap MS permits the detection of pharmaceutical residues in a concentration range of 10-100 ng. $\mathrm{L}^{-1}$, applying a post-target screening approach, in the multi-method conditions [36]. 
Other recent applications of orbitrap mass analyzer in the search of biomarkers include: the analysis of serotonin and related compounds in urine and the identification of a potential biomarker for attention deficit hyperactivity/hyperkinetic disorder [37,38]; the quantitative profiling of phosphatidylethanol molecular species, which are a group of aberrant phospholipids formed in cell membranes in the presence of ethanol by the catalytic action of the enzyme phospholipase D on phosphatidylcholine in human blood, by liquid chromatography high resolution mass spectrometry performed on an LTQ-Orbitrap XL hybrid mass spectrometer equipped with an electrospray ionization source operated in negative ion mode [39]; frozen sections (12 $\mu \mathrm{m}$ thick) of an ex vivo tissue sample set comprising primary colorectal adenocarcinoma samples and colorectal adenocarcinoma liver metastasis samples were analyzed by negative ion desorption electrospray ionization (DESI), with spatial resolution of $100 \mu \mathrm{m}$ using a computer-controlled DESI imaging stage mounted on a high resolution orbitrap mass spectrometer. DESI-IMS data were found to predominantly feature complex lipids, including phosphatidyl-inositols, phophatidyl-ethanolamines, phosphatidyl-serines, phosphatidyl-ethanolamine plasmalogens, phosphatidic acids, phosphatidyl-glycerols, ceramides, sphingolipids, and sulfatides among others, were identified based on their exact mass and MS/MS fragmentation spectra [40]; among several other applications of this promising technology to the discovery of important biomarkers in different biological systems, taking advantage of the high resolution and speed for LC-MS of this new analytical system.

\subsection{Gas Chromatography}

Gas chromatography (GC) can be understood as the chromatographic technique in which a gas is the mobile phase and, since 1952, when the first paper in this field was published, GC has always been considered simple, fast and applicable to the separation of many volatile materials, especially petrochemicals, for which distillation was the preferred method of separation at that time. Now, GC is a very important technique, and global market for instruments is estimated around to US\$ 1 billion or over 30,000 instruments annually [41].

Chromatography is the separation process of a mixture into individual components; through the separation process, each component in the sample can be identified (qualitatively) and measured (quantitatively). There are several kinds of chromatographic techniques with theirs corresponding instruments, and gas chromatography is one of those techniques. GC is used for compounds that are thermally stable and volatile - or that can become volatilizable. Because of its simplicity, sensitivity and effectiveness in separating components, GC is one of the most important tools in chemistry. The principle of basic operation of this instrument involves the evaporation of the sample in a heated inlet port (injector), separation of the components in a mixture employing a prepared column specially and detection of each component by a specific detector. At the end of the process, the amplified detector signals are often recorded and evaluated by integrator software, calculating the analytical results. The sample is introduced into a stream of inert gas, the carrier gas, and transported through the column by its flow. The column can be a packed column or a capillary column, depending on the properties of the sample. As the gas flow passes through the column, the components of the sample move in velocities that are influenced by the degree of interaction of each component with the stationary phase in the column. Consequently, the different 
components are separated. Since the processes are temperature-dependent, the column is usually contained in a thermostat-controlled oven. Once that the components are eluted from the column, they can be quantified by a suitable detector and/or be collected for further analysis. There are some types of detectors and the choice of the ones depends on the type of components that will be detected and measured. The most common detectors are: flame ionization detectors (FIDs), thermal conductivity detectors (TCDs), electron capture detectors (ECDs), alkali flame ionization detectors - also called nitrogen/phosphorous detectors (NPDs), flame photometric detectors (FPDs) and photo ionization detectors (PIDs). Several of these are further described in separate leaflets [41,42].

GC is a widely used method for separating and analyzing organic compounds. There are a variety of applications for gas chromatography in every laboratory and in different processes within several industries. In chemical, petrochemical and pharmaceutical industries we can have measurements of any kind of organic compounds, such as process control as well as product control. Also for environmental measurements: aromatic pollutants in air and water, detection and measurement of pesticides, etc. Beside the wide application of GC, there are a few examples of applications on which this analysis technique plays an important role $[43,44,45,46]$.

The detection of reliable biomarkers is a major research activity within the field of proteomics and a growing trend on metabolomics. A biomarker can be a single molecule or set of molecules that can be used to differentiate between normal and diseased states and can be separated and detected by Gas Chromatography - Mass Spectrometry (GC/MS). This combined technique is used to identify the presence of different substances in a given sample.

Kuhara et al. (2011) [47] has used a GC/MS-based approach to investigate the metabolome in urine of patients whom had been previously diagnosed with citrin deficiency. In this noninvasive technique, urine metabolic profiling provided should assist in the rapid and more reliable differential chemical diagnosis of citrin deficiency from other hyperammonemic syndromes.

Another application of GC/MS in biomarker analysis is its application on the studies of volatile organic compounds (VOCs). These compounds are exhaled in breath and provide valuable information about the human health status. The composition of the breath is variable and depends on the disease's characteristics; for example, a sweetened smell indicates diabetes, while the odor of rotten eggs, which are caused by sulfur-containing compounds, suggests liver problems [48,49]. Rudnicka (2011) [50] employed solid phase micro-extraction technique and gas chromatography coupled to time of flight in mass spectrometry (GCTOF/MS) for the analysis of VOCs on exhaled air from patients with lung cancer and healthy persons. The total number of identified compounds in breathing samples equal 55 and the compound that enables as an indication of lung cancer was isopropyl alcohol.

These studies show how highly important and relevant are the studies on the use of chromatographic techniques for biomarker analysis and identification. It shows a wide range of applications in a field not yet fully developed, which still may be a very suitable area for new ideas and uses for the next couple decades. 


\section{Statistical and chemometrical analysis of biomarkers}

In metabolomics, as well as in other branches of science and technology, there is a steady trend towards the use of more variables (properties) to characterize observations (e.g., samples, experiments, time points). Often, these measurements can be arranged into a data table, where each row constitutes an observation and the columns represent the variables or factors we have measured (e.g., wavelength, mass number, chemical shift, etc). This development generates huge and complex data tables, which are hard to summarize and overview without appropriate tools. Recently, with development of "omics" technologies (metabolomics, proteomics, foodomics, genomics, etc), the adoption of chemometric methods has been playing a very important role in planning and analyzing the obtained results. That includes efficient and robust methods for modeling and analysis of complex chemical or biological data tables that produce interpretable and reliable models capable of handling incomplete, noisy, and collinear data structures. These methods include principal component analysis (PCA) and partial least squares (PLS). It is also completely important to emphasize that chemometrics also provides a straightforward way to collect relevant information through statistical experimental design (SED) [51,52,53].

Multivariate statistical analysis such as Principal Components Analysis (PCA) is probably the most widely used technique for analyzing metabolomics. PCA technique is robust and objective and it is an appropriate way to reduce data sets containing high numbers of variables. By reducing the number of original variables to a smaller number of independent variables, this approach highlights fundamental differences between groups of variables. PCA has been extensively used in metabonomics literature. Despite apparent satisfying published results, the known large sensitivity of PCA to noise can suggest that improvements are expected with more robust methods to identify biomarkers in noisy data. Moreover, the traditional use of PCA remains highly questionable: biomarkers are identified from the loadings of the two first principal components, while the two first components do not necessarily contain the most relevant variations between altered and normal spectra. Sometimes, the results of the initial unsupervised analysis are confirmed by a second supervised analysis. This one employs classification methods as Partial Least Squares (PLS), SIMCA and neural networks, allowing firstly to separate normal and altered spectra, and secondly to identify more robust biomarkers [54,55].

Other data analysis methods frequently employed for disease diagnosis and biomarker identification in metabolomics are Univariate Testing, Soft independent modeling of class analogy (SIMCA), Linear discriminant analysis (LDA), Partial least squares discriminant analysis (PLS-DA), Orthogonal projection to latent structures discriminant analysis, (OPLS-DA), Neural networks (NN), Self organizing maps (SOM) and Support vector machines (SVM). Regardless of the chosen method, both statistical and biological validations are critical. Multivariate methods are of special importance to metabolomics since one biomarker often will not be sufficiently specific for a given condition by itself. There is a wide range of methods and it is natural that this can seem confusing to the non-specialist. The literature has already shown in previous works that it is more important that the chosen method is used correctly than the methodology itself. The reason for this is that all methods are data-driven, and since the parameter definition is through pre-processing, the contained features are static. Many statisti- 
cal methods will highlight the same metabolites with similar classification ability. It is clear, however, that pre-processing and scaling of the data can lead to dramatically different results, both with regard to chosen biomarkers and classification ability of the model [53].

\section{Bibliography}

Aitio A, Apostoli P. Quality assurance in biomarker measurement. Toxicol Lett. 1995 May; 77(1-3):195-204.

Aitio A, Bernard A, Fowler BA, Nordberg G. Biological Monitoring and Biomerkers. Handbook on the Toxicology of Metals. Third Edition. 2007; Chapter 4: 65-78.

Christians U, Klepacki J, Shokati T, Klawitter J, Klawitter J. Mass spectrometry-based multiplexing for the analysis of biomarkers in drug development and clinical diagnostics - How much is too much ? Microchem. J. 2012, doi:10.1016/j.microc.2012.02.001

Hendriks MMWB, Eeuwijk FA, Jellema RH, Westerhuis JA, Reijmers TH, Hoefsloot HCJ, Smilde AK. Data-processing strategies for metabolomics studies. Trends in Analytical Chemistry. 2011; 30 (10): 1685-98.

Holland NT, Smith MT, Eskenazi B, Bastaki M. Biological sample collection and processing for molecular epidemiological studies. Mutat Res. 2003 Jun;543(3):217-34.

Lampe JW, Rock CL. Biomarkers and biological indicators of chance. Nutrition in the Prevention and Treatment of Disease. 2001; Chapter 10:139-153.

Lee JW, Hall M. Method validation of protein biomarkers in support of drug development or clinical diagnosis/prognosis. J Chromatogr B Analyt Technol Biomed Life Sci. 2009 May 1;877(13):1259-71.

Roux A, Lison D, Junot C, Heilier JF. Applications of liquid chromatography coupled to mass spectrometry-based metabolomics in clinical chemistry and toxicology: A review. Clin Biochem. Jan;44(1):119-35.

\section{Author details}

F. G. Ravagnani ${ }^{1,2}$, D. M. Saidemberg ${ }^{1}$, A. L. C. Faria ${ }^{1}$, S. B. Sartor ${ }^{1}$, D. N. Oliveira ${ }^{1}$ and

R. R. Catharino ${ }^{1^{*}}$

*Address all correspondence to: rrc@fcm.unicamp.br

1INNOVARE Biomarkers Laboratory, Department of Clinical Pathology, School of Medical Sciences, University of Campinas, Brazil

2 Laboratory of Bioenergetics, Department of Clinical Pathology, School of Medical Sciences, University of Campinas, Brazil 


\section{References}

[1] Lee, J., et al. (2011). Proteomics and biomarkers in clinical trials for drug development. 74(12), 2632-264.

[2] Roberts, L. D., Souza, A. L., Gerszten, R. E., \& Clish, C. B. (2012). Targeted Metabolomics. Current Protocols in Molecular Biology, Unit 30.2.

[3] Goodacre, R. (2003). Metabolic Profiling: Its Role in Biomarker Discovery and Gene Function Analysis. Kluwer Academic Publishers, London.

[4] Ryan, D., Robards, K., Prenzler, P. D., \& Kendall, M. (2011). Recent and potential developments in the analysis of urine: a review. Anal Chim Acta, 684, 8-20.

[5] Koulman, A., Lane, G. A., Harrison, S. J., \& Volmer, D. A. (2009). From differentiating metabolites to biomarkers. Anal Bioanal Chem, 394, 663-70.

[6] de la Luz-Hernández, K. R., Rojas-del Calvo, L., Rabasa-Legón, Y., Lage-Castellanos, A., Castillo-Vitlloch, A., Díaz, J., et al. (2008). Metabolic and proteomic study of NS0 myeloma cell line following the adaptation to protein-free medium. J Proteomics, 71, 133-47.

[7] Ryan, D., Robards, K., Prenzler, P. D., \& Kendall, M. (2011). Recent and potential developments in the analysis of urine: a review. Anal Chim Acta, 684, 8-20.

[8] Atkinson, A. J. C. W., De Gruttola, V., De Mets, D. L., Downing, G. J., Hoth, D. F., Oates, J. A., et al. (2001). Biomarkers and Surrogate Endpoints: Preferred Definitions and Conceptual Framework. Clin Pharmacol Ther, 69, 89-95.

[9] Simon, R., \& Altman, D. G. (1994). Statistical aspects of prognostic factor studies in oncology. Br J Cancer, 69, 979-85.

[10] Sargent, D. J., Conley, B. A., Allegra, C., \& Collette, L. (2005). Clinical Trial designs for predictive marker validation in cancer treatment trials. J Clin Oncol, 23, 2020-7.

[11] Nicholson, J. K., \& Lindon, J. C. (2008). Systems biology: metabonomics. Nature, 455, 1054-1056.

[12] Arakaki, A. K., Skolnick, J., \& McDonald, J. F. (2008). Marker metabolites can be therapeutic targets as well. Nature, 456, 443.

[13] de la Luz-Hernández, K. R., Rojas-del Calvo, L., Rabasa-Legón, Y., Lage-Castellanos, A., Castillo-Vitlloch, A., Díaz, J., et al. (2008). Metabolic and proteomic study of NS0 myeloma cell line following the adaptation to protein-freemedium. J Proteomics, 71, 133-47.

[14] Schauer, N., Steinhauser, D., Strelkov, S., Schomburg, D., Allison, G., Moritz, T., Lundgren, K., Roessner-Tunali, U., Forbes, M. G., Willmitzer, L., Fernie, Ar., \& Kopka, J. (2005). GC-MS libraries for the rapid identification of metabolites in complex biological samples. FEBS Letters, 579, 1332-1337. 
[15] Want, E. J., O'maille, G., Smith, Brandon. T. R., Uritboonthai, W., Qin, C., Trauger, S. A., \& Siuzdak, G. (2006). Solvent-Dependent Metabolite Distribution, Clustering, and Protein Extraction for Serum Profiling with Mass Spectrometry. Anal Chem, 78, 743-752.

[16] Birkemeyer, C., et al. (2005). Metabolome analysis: the potential of in vivo labeling with stable isotopes for metabolite profiling. Trends Biotechnol, 23, 28-33.

[17] Hollywood, K., Brison, D., \& Goodacre, R. (2006). Metabolomic: current technologies and future trends. Proteomics, 6, 4716-4723.

[18] Cramer, R. (2009). MALDI MS. Methods Mol Biol, 564, 85-103.

[19] Villas-Bôas, S. G., Mas, S., Akesson, M., Smedsgaard, J., \& Nielsen, J. (2005). Mass spectrometry in metabolome analysis. Mass spectrometry reviews, 24(5), 613-646.

[20] Wehr, T. (2003). Coupling liquid-phase separations and MALDI-MS. LCGC North America, 10, 974-982.

[21] Seeley, E. H., \& Caprioli, R. M. (2011). MALDI imaging mass spectrometry of human tissue: method challenges and clinical perspectives Trends. Biotechnol, 29(3), 136-146.

[22] Horn, P. J., Korte, A. R., Neogi, P. B., Love, E., Fuchs, J., Strupat, K., Borisjuk, L., Shulaev, V., Lee, Y. J., \& Chapman, K. D. (2012). Spatial mapping of lipids at cellular resolution in embryos of cotton. Plant Cell, 24(2), 622-36.

[23] Benabdellah, F., Touboul, D., Brunelle, A., \& Laprévote, O. (2009). In situ primary metabolites localization on a rat brain section by chemical mass spectrometry imaging. Anal Chem, 81(13), 5557-60.

[24] Sugiura, Y., Zaima, N., Setou, M., Ito, S., \& Yao, I. (2012). Visualization of acetylcholine distribution in central nervous system tissue sections by tandem imaging mass spectrometry. Anal Bioanal Chem, In press.

[25] Sugiura, Y., \& Setou, M. (2010). Imaging mass spectrometry for visualization of drug and endogenous metabolite distribution: toward in situ pharmacometabolomes. $J$ Neuroimmune Pharmacol, 5(1), 31-43.

[26] Huang, J. T., Hannah-Qiuhua, L., Szyszka, R., Veselov, V., Reed, G., Wang, X., Price, S., Alquier, L., \& Vas, G. (2012). Molecular imaging of drug-eluting coronary stents: method development, optimization and selected applications. J Mass Spectrom, 47(2), 155-62.

[27] Kuwayama, K., Tsujikawa, K., Miyaguchi, H., Kanamori, T., Iwata, Y. T., \& Inoue, H. (2012). Rapid, simple, and highly sensitive analysis of drugs in biological samples using thin-layer chromatography coupled with matrix-assisted laser desorption/ionization mass spectrometry. Anal Bioanal Chem., 402(3), 1257-67.

[28] Ljungdahl, A., Hanrieder, J., Fälth, M., Bergquist, J., \& Andersson, M. (2011). Imaging mass spectrometry reveals elevated nigral levels of dynorphin neuropeptides in LDOPA-induced dyskinesia in rat model of Parkinson's disease. PLoS One, 6(9):e25653. 
[29] Sparvero, L. J., Amoscato, A. A., Kochanek, P. M., Pitt, B. R., Kagan, V. E., \& Bayir, H. (2010). Mass-spectrometry based oxidative lipidomics and lipid imaging: applications in traumatic brain injury. J Neurochem., 115(6), 1322-36.

[30] Taverna, D., Nanney, L. B., Pollins, A. C., Sindona, G., \& Caprioli, R. (2011). Multiplexed molecular descriptors of pressure ulcers defined by imaging mass spectrometry. Wound Repair Regen., 19(6), 734-44.

[31] Makarov, A. (2000). Electrostatic Axially Harmonic Orbital Trapping: A High-Performance Technique of Mass Analysis. Anal. Chem., 72 (6), 1156-1162.

[32] Makarov, A., Denisov, E., Kholomeev, A., Balschun, W., Lange, O., Strupat, K., \& Horning, S. (2006). Performance Evaluation of a Hybrid Linear Ion Trap/Orbitrap Mass Spectrometer. Anal. Chem., 78 (7), 2113-2120.

[33] Schober, Y., Guenther, S., Spengler, B., \& Römpp, A. (2012). High-resolution matrixassisted laser desorption/ionization imaging of tryptic peptides from tissue. Rapid Commun Mass Spectrom., 26(9), 1141-6.

[34] Zhang, T., Creek, D. J., Barrett, M. P., Blackburn, G., \& Watson, D. G. (2012). Evaluation of coupling reversed phase, aqueous normal phase, and hydrophilic interaction liquid chromatography with Orbitrap mass spectrometry for metabolomic studies of human urine. Anal Chem., 84(4), 1994-2001.

[35] Chitescu, C. L., Oosterink, E., de Jong, J., \& Stolker, L. A. A. (2012). Accurate mass screening of pharmaceuticals and fungicides in water by U-HPLC-Exactive Orbitrap MS. Anal Bioanal Chem, In press.

[36] Moriarty, M., Lee, A., O'Connell, B., Kelleher, A., Keeley, H., \& Furey, A. (2011). Development of an LC-MS/MS method for the analysis of serotonin and related compounds in urine and the identification of a potential biomarker for attention deficit hyperactivity/hyperkinetic disorder. Anal Bioanal Chem., 401(8), 2481-93.

[37] Moriarty, M., Lehane, M., O’Connell, B., Keeley, H., \& Furey, A. (2012). Development of a nano-electrospray MSn method for the analysis of serotonin and related compounds in urine using a LTQ-orbitrap mass spectrometer. Talanta, 90, 1-11.

[38] Nalesso, A., Viel, G., Cecchetto, G., Mioni, D., Pessa, G., Favretto, D., \& Ferrara, S. D. (2011). Quantitative profiling of phosphatidylethanol molecular species in human blood by liquid chromatography high resolution mass spectrometry. J Chromatogr A., 1218(46), 8423-31.

[39] Gerbig, S., Golf, O., Balog, J., Denes, J., Baranyai, Z., Zarand, A., Raso, E., Timar, J., \& Takats, Z. (2012). Analysis of colorectal adenocarcinoma tissue by desorption electrospray ionization mass spectrometric imaging. Anal Bioanal Chem, In press.

[40] McNair, H. M., \& Miller, J. M. (2009). Basic gas chromatography. Hoboken, N.J., John Wiley \& Sons. 
[41] Barry, R. L. G. E. F. (2004). Modern Practice of Gas Chromatography. Hoboken, NJ, Wiley and Sons.

[42] Fenoll, J., Hellin, P., et al. (2007). Multiresidue method for analysis of pesticides in pepper and tomato by gas chromatography with nitrogen, Äìphosphorus detection. Food Chemistry, 105(2), 711-719.

[43] Shahdousti, P., Mohammadi, A., et al. (2007). Determination of valproic acid in human serum and pharmaceutical preparations by headspace liquid-phase microextraction gas chromatography-flame ionization detection without prior derivatization. Journal of Chromatography B-Analytical Technologies in the Biomedical and Life Sciences, 850(1-2), 128-133.

[44] Botalova, O., Schwarzbauer, J., et al. (2009). Identification and chemical characterization of specific organic constituents of petrochemical effluents. Water Res, 43(15), 3797-3812.

[45] Tran, K., Eide, D., et al. (2012). Finding of pesticides in fashionable fruit juices by LC, ̈̈iMS/MS and GC, ÄiMS/MS. Food Chemistry, 134(4), 2398-2405.

[46] Kuhara, T., Ohse, M., et al. (2011). A GC/MS-based metabolomic approach for diagnosing citrin deficiency. Analytical and Bioanalytical Chemistry, 400(7), 1881-1894.

[47] Deng, C., Zhang, J., et al. (2004). Determination of acetone in human breath by gas chromatography, Mass spectrometry and solid-phase microextraction with on-fiber derivatization. Journal of Chromatography B, 810(2), 269-275.

[48] Buszewski, B., Kesy, M., et al. (2007). Human exhaled air analytics: biomarkers of diseases. Biomed Chromatogr, 21(6), 553-566.

[49] Rudnicka, J., et al. (2011). Determination of volatile organic compounds as biomarkers of lung cancer by SPME-GC-TOF/MS and chemometrics. Journal of Chromatography B-Analytical Technologies in the Biomedical and Life Sciences, 879(30), 3360-3366.

[50] Lindon, J. C., Nicholson, J. K., et al. (2007). The handbook of metabonomics and metabolomics. Amsterdam; Oxford, Elsevier.

[51] Trygg, J., Holmes, E., et al. (2007). Chemometrics in metabonomics. Journal of Proteome Research, 6(2), 469-479.

[52] Madsen, R., Lundstedt, T., et al. (2010). Chemometrics in metabolomics--a review in human disease diagnosis. Anal Chim Acta, 659(1-2), 23-33.

[53] Holmes, E., \& Antti, H. (2002). Chemometric contributions to the evolution of metabonomics: mathematical solutions to characterising and interpreting complex biological NMR spectra. Analyst., 127(12), 1549-1557.

[54] Rousseau, R., Govaerts, B., et al. (2008). Comparison of some chemometric tools for metabonomics biomarker identification. Chemometrics and Intelligent Laboratory Systems, 91(1), 54-66. 



\section{Edited by Isin Akyar}

Quality control has an emerging importance in every field of life. Quality control is a process that is used to guarantee a certain level of quality in a product or service. It might include whatever actions a business deems necessary to provide for the control and verification of certain characteristics of a product or service. With the improvement of technology everyday we meet new and complicated devices and methods in different fields. Quality control should be performed in all of those new techniques. In this book "Latest Research Into Quality Control” our aim was to collect information about quality control in many different fields. The aim of this book is to share useful and practical knowledge about quality control in several fields with the people who want to improve their knowledge. 